\section{Pacific Northwest}

National Laboratory

Operated by Battelle for the

U.S. Department of Energy

\title{
Estimate of Hanford Waste Rheology and Settling Behavior
}

\author{
A. P. Poloskịi \\ J. M. Tingey \\ B. E. Wells \\ L. A. Mahoney \\ Pacific Northwest National Laboratory \\ M. N. Hall \\ G. L. Smith \\ S. L. Thomson \\ Bechtel National Inc.
M. E. Johnson
M. A. Knight
J. E. Meacham
M. J. Thien
CH2M HILL \\ J. J. Davis \\ Department of Energy - Office of River Protection \\ Y Onishi \\ Yasuo Onishi Consulting, LLC
}

October 2007

Prepared for the U.S. Department of Energy

under Contract DE-AC05-76RL01830 


\title{
DISCLAIMER
}

This report was prepared as an account of work sponsored by an agency of the United States Government. Neither the United States Government nor any agency thereof, nor Battelle Memorial Institute, nor any of their employees, makes any warranty, express or implied, or assumes any legal liability or responsibility for the accuracy, completeness, or usefulness of any information, apparatus, product, or process disclosed, or represents that its use would not infringe privately owned rights. Reference herein to any specific commercial product, process, or service by trade name, trademark, manufacturer, or otherwise does not necessarily constitute or imply its endorsement, recommendation, or favoring by the United States Government or any agency thereof, or Battelle Memorial Institute. The views and opinions of authors expressed herein do not necessarily state or reflect those of the United States Government or any agency thereof.

\author{
PACIFIC NORTHWEST NATIONAL LABORATORY \\ operated by \\ BATTELLE \\ for the \\ UNITED STATES DEPARTMENT OF ENERGY \\ under Contract DE-AC05-76RL01830 \\ Printed in the United States of America \\ Available to DOE and DOE contractors from the \\ Office of Scientific and Technical Information, \\ P.O. Box 62, Oak Ridge, TN 37831-0062; \\ ph: (865) 576-8401 \\ fax: (865) 576-5728 \\ email: reports@adonis.osti.gov
}

Available to the public from the National Technical Information Service, U.S. Department of Commerce, 5285 Port Royal Rd., Springfield, VA 22161

ph: (800) 553-6847

fax: (703) 605-6900

email: orders@ntis.fedworld.gov

online ordering: http://www.ntis.gov/ordering.htm

This document printed on recycled paper. 
PNNL-16857

WTP-RPT-154 Rev. 0

\section{Estimate of Hanford Waste Rheology and Settling Behavior}
A. P. Poloski
J. M. Tingey
B. E. Wells
L. A. Mahoney
Pacific Northwest National Laboratory

M. N. Hall

G. L. Smith

S. L. Thomson

Bechtel National Inc.

M. E. Johnson M. A. Knight

J. E. Meacham M. J. Thien

CH2M HILL

J. J. Davis

Department of Energy - Office of River Protection

Y Onishi

Yasuo Onishi Consulting, LLC

October 2007

Test specification: N/A

Test plan: N/A

Test exceptions: N/A

R\&T focus area: Pretreatment

Test Scoping Statement(s): SCN 007

Pacific Northwest National Laboratory

Richland, Washington, 99354 


\section{Completeness of Testing}

This report summarizes the results of analyses of data obtained from the referenced analyses of Hanford waste. The analyses and the report, which used data from, but did not involve laboratory testing, followed the quality assurance requirements outlined in Pacific Northwest Division's Waste Treatment Plant Support Project Quality Assurance Requirements and Description Manual and are now compiled under the quality assurance requirements of the PNNL River Protection Project - Waste Treatment Plant Support Program (RPP-WTP) Quality Assurance Plan, RPP-WTP-QA-001.

The descriptions provided in this report are an accurate account of both the conduct of the work and the data analyses performed. A summary of the analysis results is reported. Also reported are any unusual or anomalous occurrences that are different from expected results. The analysis results and this report have been reviewed and verified.

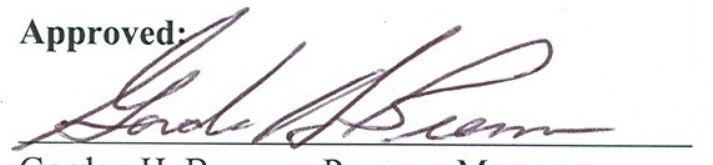

Gordon H. Beeman, Program Manager RPP-WTP Support Program

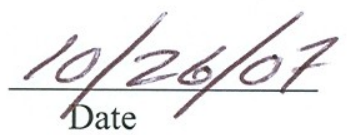




\section{Testing Summary}

The U.S. Department of Energy (DOE) Office of River Protection's Waste Treatment and Immobilization Plant (WTP) will process and treat radioactive waste that is stored in tanks at the Hanford Site. This report addresses the data analyses performed by the Rheology Working Group (RWG) and Risk Assessment Working Group. This group was composed of Pacific Northwest National Laboratory (PNNL), Bechtel National Inc. (BNI), CH2M HILL, DOE Office of River Protection (ORP), and Yasuo Onishi Consulting, LLC staff. The charter of the working group is the following:

1. To define the range of relevant waste properties that might be retrieved and handled at the Hanford Tank Farm.

2. To develop relationships that describe the solids settling and rheological behavior ranges for Hanford wastes.

The actual testing activities were performed and reported separately in referenced documentation. Because of this, many of the required topics below do not apply and are so noted.

\section{Test Objectives}

This section is not applicable. No testing was performed for this investigation.

\section{Test Exceptions}

This section is not applicable. No test specification as well as test exception applies to this investigation as there was no testing was performed.

\section{Results and Performance Against Success Criteria}

This section is not applicable. No success criteria were established as there was no testing performed for this investigation.

\section{Quality Requirements}

Since December 2001, Battelle - Pacific Northwest Division, under its use agreement with the Department of Energy (DE-AC05-76RL01831), has been providing support to BNI in accordance with the QA program approved under Subcontract No. 24590-101-TSA-W000-00004. This support has been provided under the WTP Support Project (WTPSP) QA Program and later the BNI Support Program (BNI-SP), for the technical support of the waste treatment plant being built in the 200 East area of the Hanford Site. In February 2007 the contract mechanism was switched to PNNL's Operating Contract DE-AC05-76RL01830, and the program was renamed the RPP-WTP Support Program.

The data represented in this report might refer to PNWD, PNNL, BNI-SP or WTPSP; both of these projects performed work to the same QA Program. As of February 2007, the Quality Assurance Program is described as follows: 
PNNL's Quality Assurance Program is based on requirements defined in U.S. Department of Energy (DOE) Order 414.1C, Quality Assurance, and 10 CFR 830, Energy/Nuclear Safety Management, Subpart A-Quality Assurance Requirements (a.k.a. the Quality Rule). PNNL has chosen to implement the requirements of DOE Order 414.1C and 10 CFR 830, Subpart A by integrating them into the Laboratory's management systems and daily operating processes. The procedures necessary to implement the requirements are documented through PNNL's Standards-Based Management System.

PNNL implements the RPP-WTP quality requirements by performing work in accordance with the River Protection Project - Waste Treatment Plant Support Program (RPP-WTP) Quality Assurance Plan (RPP-WTP-QA-001, QAP). Work will be performed to the quality requirements of NQA-1-1989 Part I, Basic and Supplementary Requirements, NQA-2a-1990, Part 2.7 and DOE/RW-0333P, Rev 13, Quality Assurance Requirements and Descriptions $(Q A R D)$. These quality requirements are implemented through the River Protection Project - Waste Treatment Plant Support Program (RPP-WTP) Quality Assurance Manual (RPP-WTP-QA-003, QAM).

This report is based on data from testing as referenced. The PNNL assumes that the data from these references has been fully reviewed and documented in accordance with the analysts' QA Programs. PNNL only analyzed data from the referenced documentation. At PNNL, the performed calculations, the documentation and reporting of results and conclusions were performed in accordance with the RPP-WTP Quality Assurance Manual (RPP-WTP-QA-003, QAM). Internal verification and validation activities were addressed by conducting an independent technical review of the final data report in accordance with PNNL procedure QA-RPP-WTP-604. This review verifies that the reported results are traceable, that inferences and conclusions are soundly based, and that the reported work satisfies the Test Specification Success Criteria. This review procedure is part of PNNL's RPP-WTP Quality Assurance Manual).

\section{Test Conditions}

The scope of the RWG effort is specified in the approved WTP issue response plan (24590-WTP-PLENG-06-0013) and defined in subcontractor change notice (SCN) 007 and Test Specification 24590-PTFTSP-RT-06-007, Rev 0.

Demonstrate the simulant properties used for testing bracket expected actual waste properties. For non-cohesive solids (Phase 1) this includes particle size, solids density, solids concentration, liquid density, and liquid viscosity. For cohesive solids (Phase 2) this includes bulk slurry density, particle size, particle density, slurry rheology (such as consistency and yield stress) and shear strength of settled, aged sediments, as well as settled layer (heel) thickness.

Waste received at the WTP will be subject to a feed specification supporting plant design and as agreed to in an Interface Control Document. This report compiles the existing Hanford Tank Farm rheological data addressed in italicized text above and establishes expected ranges for these properties for as-retrieved Hanford Tank Farm wastes. Various processes will be performed on these retrieved wastes which are expected to alter these property ranges from the as-retrieved conditions. Simulant development activities should focus on the expected properties of the waste streams under such processing conditions. 


\section{Simulant Use}

This section is not applicable. No testing was performed for this investigation.

\section{Results of Data Analysis}

The data discussed in this report can be applied to the feed systems of the WTP pretreatment facility. These data primarily consist of rheological and sedimentation data from Hanford tank farm core samples and core samples diluted with process water. An analysis of the affects of WTP process operations on these properties is not provided. Despite these limitations, three major process systems where these data apply are flow within process vessels, vertical piping, and horizontal piping. In these systems, several sludge configurations can be identified as potential operational scenarios. These scenarios and sludge configurations are shown in Figures S.1, S.2, and S.3 for process vessels (e.g. WTP EFRT issue M3), vertical piping, and horizontal piping (e.g. WTP EFRT issue M1).

Table S.1 provides a summary of the rheological parameters for each of the fluid layers shown in the associated process scenario figures. Dilution with water may result in rheological property maxima as zeta potential, particle size, and chemical composition of the solid and liquid phases change with dilution level. This table was compiled from the sections on sedimentation, shear strength, Bingham rheological parameters, and supernatant viscosity. In addition, the section discussing transient rheological modeling is summarized to provide an indication of the timescales for rheology regrowth at various process length scales. Process scales simulated include $0.1 \mathrm{~m}$ which is representative of process piping, $1 \mathrm{~m}$ represents small scale vessels, and $10 \mathrm{~m}$ represents large scale vessels. The timescales for each of the fluid layers to develop at each scale are shown schematically in Figure S.4. Timescales for the sediment heel to fully develop at different length scales are summarized in Table S.1.

Recovery from these slurry configurations will require the processing slurries through all of the parameters identified in Table S.1. Initially the sediment will be gelled to some extent and will possess a shear strength threshold that must be overcome. After the sediment is mobilized, the Bingham consistency and yield stress parameters will be elevated due to the large concentration of undissolved solids in the sediment bed. As mixing proceeds and supernatant liquid is blended into the sediment, the rheological properties will drop to the normal operation range when the slurry is fully suspended. This is shown graphically in Figure S.5. 
Normal operation with Newtonian fluid

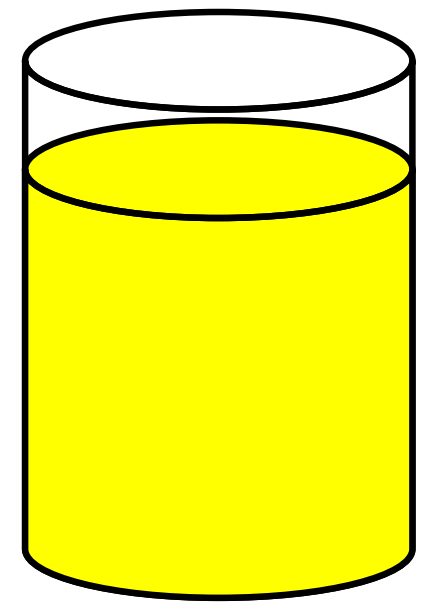

Process impacts:

- cloud height

- blend times

Off-normal operation with Newtonian fluid

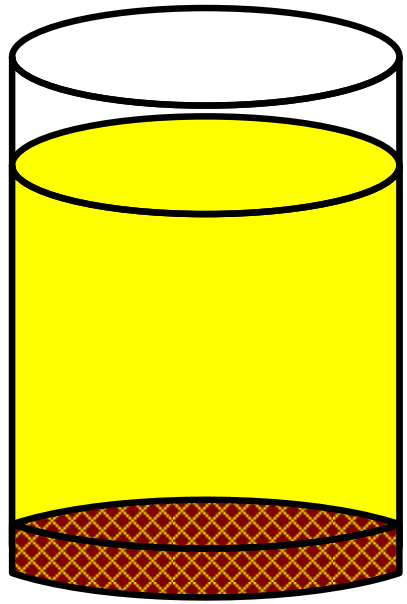

Process impacts:

- off-bottom resuspension of solids

- blend time of solids layer
Normal operation with non-Newtonian fluid

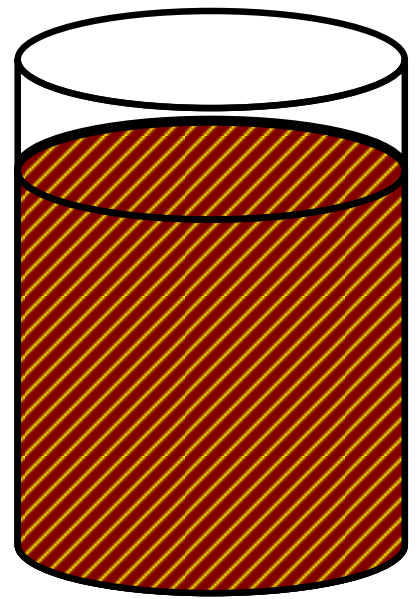

Process impacts:

- mixing performance

- gas retention \& release

Off-Normal operation with non-Newtonian fluid

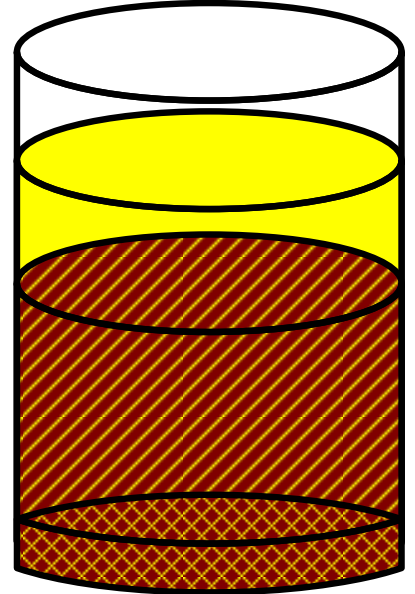

Process impacts:

- restart of mechanical agitators/PJMs

- off-bottom resuspension of solids

- blend time liquid/solids layer

- gas retention \& release

Solid - supernatant; diagonal - slurry; crosshatch - heel with coarse solids $(>74 \mu \mathrm{m} \&>2.7 \mathrm{~g} / \mathrm{cc})$

Figure S.1. Example Operational Scenarios for Process Vessels 
Normal operation with

Newtonian fluid

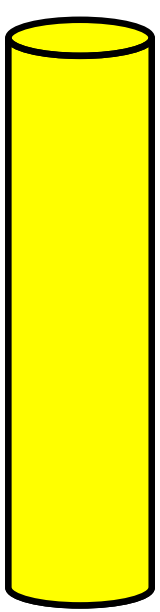

Process impacts:

- critical flow velocity
Normal operation with non-Newtonian fluid

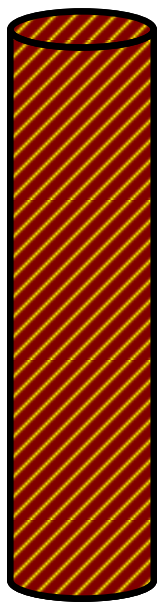

Process impacts:

- flow velocity \& pressure drop - flow regime
Off-normal operation with non-Newtonian fluid

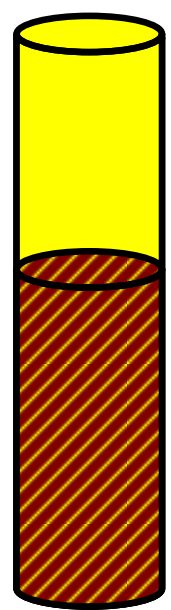

Process impacts:

- restart

- flushing effectiveness

Solid - supernatant; diagonal - slurry; crosshatch - heel with coarse solids $(>74 \mu \mathrm{m} \&>2.7 \mathrm{~g} / \mathrm{cc})$

Figure S.2. Example Operational Scenarios for Vertical Process Piping

Normal operation with Newtonian fluid

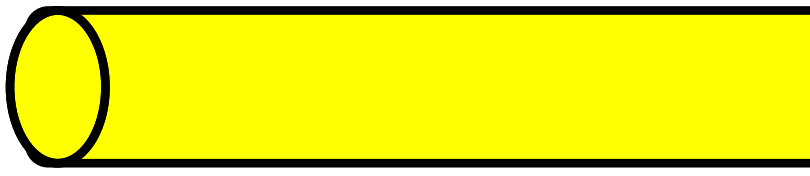

Process impacts:

- critical flow velocity

Off-normal operation with Newtonian fluid

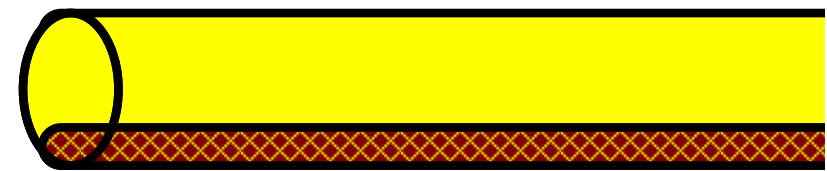

Process impacts:

- restart

- flushing effectiveness
Normal operation with non-Newtonian fluid

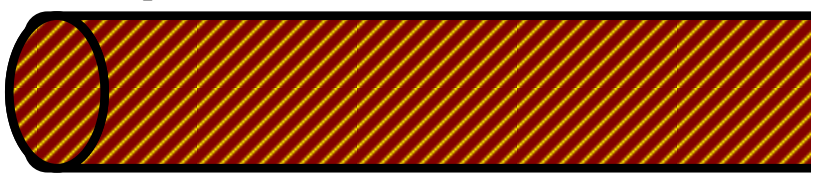

Process impacts:

- flow velocity and pressure drop

- flow regime

Off-Normal operation with non-Newtonian fluid

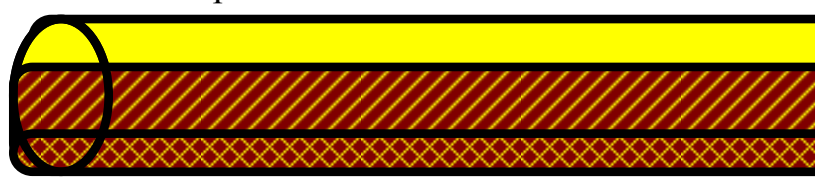

Process impacts:

- restart

- flushing effectiveness

Solid - supernatant; diagonal - slurry; crosshatch - heel with coarse solids $(>74 \mu \mathrm{m} \&>2.7 \mathrm{~g} / \mathrm{cc}$ )

Figure S.3. Example Operational Scenarios for Horizontal Process Piping 
Table S.1. Range of Rheological Parameters and Regrowth Times at Typical Process Scales

\begin{tabular}{|c|c|c|c|c|}
\hline Category & $\begin{array}{c}\text { Heel Shear } \\
\text { strength }\end{array}$ & $\begin{array}{l}\text { Slurry/Heel } \\
\text { Bingham } \\
\text { Yield Stress }\end{array}$ & $\begin{array}{l}\text { Slurry/Heel } \\
\text { Bingham } \\
\text { Consistency }\end{array}$ & $\begin{array}{c}\text { Supernatant } \\
\text { Viscosity }\end{array}$ \\
\hline $\operatorname{Min}^{(a)}$ & $40 \mathrm{~Pa}$ & $0 \mathrm{~Pa}$ & $1 \mathrm{cP}$ & $1 \mathrm{cP}$ \\
\hline Median $^{(a)}$ & $700 \mathrm{~Pa}$ & $1.5 \mathrm{~Pa}$ & $8 \mathrm{cP}$ & $8 \mathrm{cP}$ \\
\hline $\operatorname{Max}^{(\mathrm{a})}$ & $25,000 \mathrm{~Pa}$ & $40 \mathrm{~Pa}$ & $110 \mathrm{cP}$ & $30 \mathrm{cP}$ \\
\hline $\begin{array}{l}\text { Tank heel property after } 10 \text { hours of } \\
\text { sedimentation in process piping } \\
(0.1 \mathrm{~m} \text { sludge height })\end{array}$ & $25,000 \mathrm{~Pa}$ & $40 \mathrm{~Pa}$ & $110 \mathrm{cP}$ & $\mathrm{n} / \mathrm{a}$ \\
\hline $\begin{array}{l}\text { Tank heel property after } 100 \text { hours of } \\
\text { sedimentation in a medium-scale vessel } \\
(1 \mathrm{~m} \text { sludge height })\end{array}$ & $25,000 \mathrm{~Pa}$ & $40 \mathrm{~Pa}$ & $110 \mathrm{cP}$ & $\mathrm{n} / \mathrm{a}$ \\
\hline $\begin{array}{l}\text { Tank heel property after } 1000 \text { hours of } \\
\text { sedimentation in a large-scale vessel } \\
(10 \mathrm{~m} \text { sludge height })\end{array}$ & $25,000 \mathrm{~Pa}$ & $40 \mathrm{~Pa}$ & $110 \mathrm{cP}$ & $\mathrm{n} / \mathrm{a}$ \\
\hline
\end{tabular}




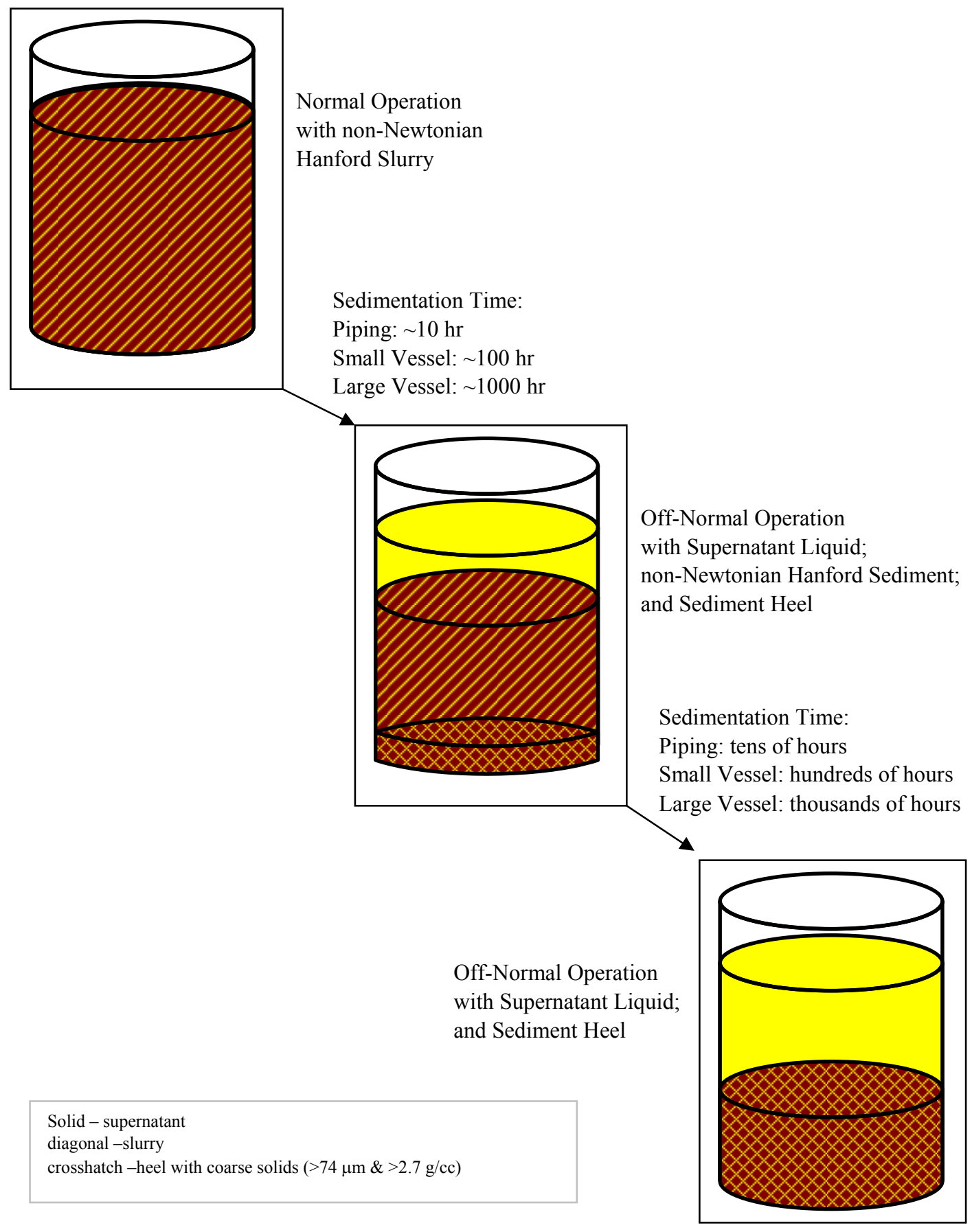

Figure S.4. Illustration of Development of Sludge Process Heel and Fully Settled Configuration at Various Process Scales 


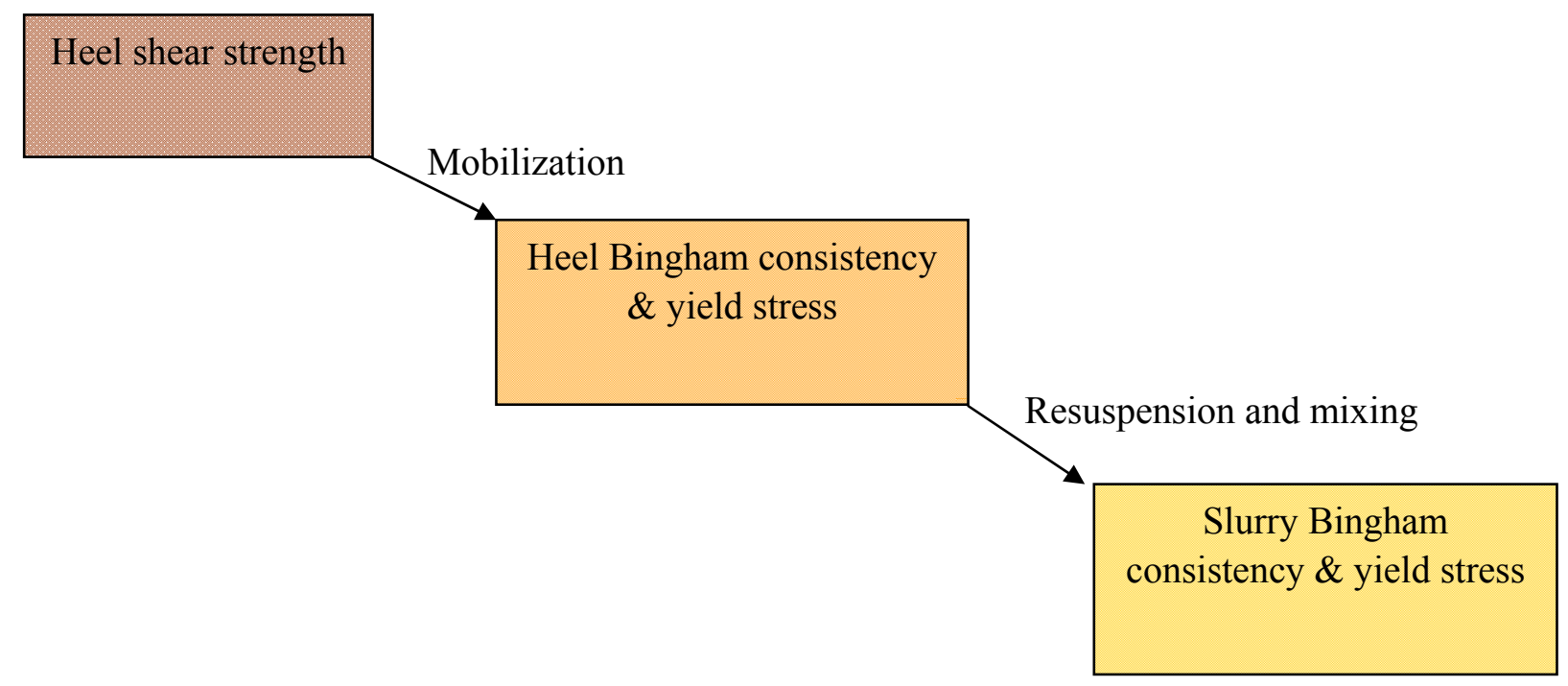

Figure S.5. Rheological Properties Encountered During Recovery from Process Upset Conditions

\section{Discrepancies and Follow-on Tests}

Rheology data and associated physical properties for Hanford tank wastes were compiled from all the readily available reports, but many gaps were observed when analyzing the data. These data include in situ as well as laboratory analysis of samples removed from the tanks. The gaps in the waste types analyzed are reported in each section of the report. Figure S.6 provides a summary of these gaps. The relative volume of wastes modeled for liquid viscosity, sedimentation, shear strength, rheological parameters (Bingham plastic model), and rheological parameters as a function of settling are plotted as a function of waste type. Additional testing of archive samples or samples being gathered on wastes that were collected as part of the analysis of the M-12 samples would fill in many of the gaps from current rheological and physical properties data. 


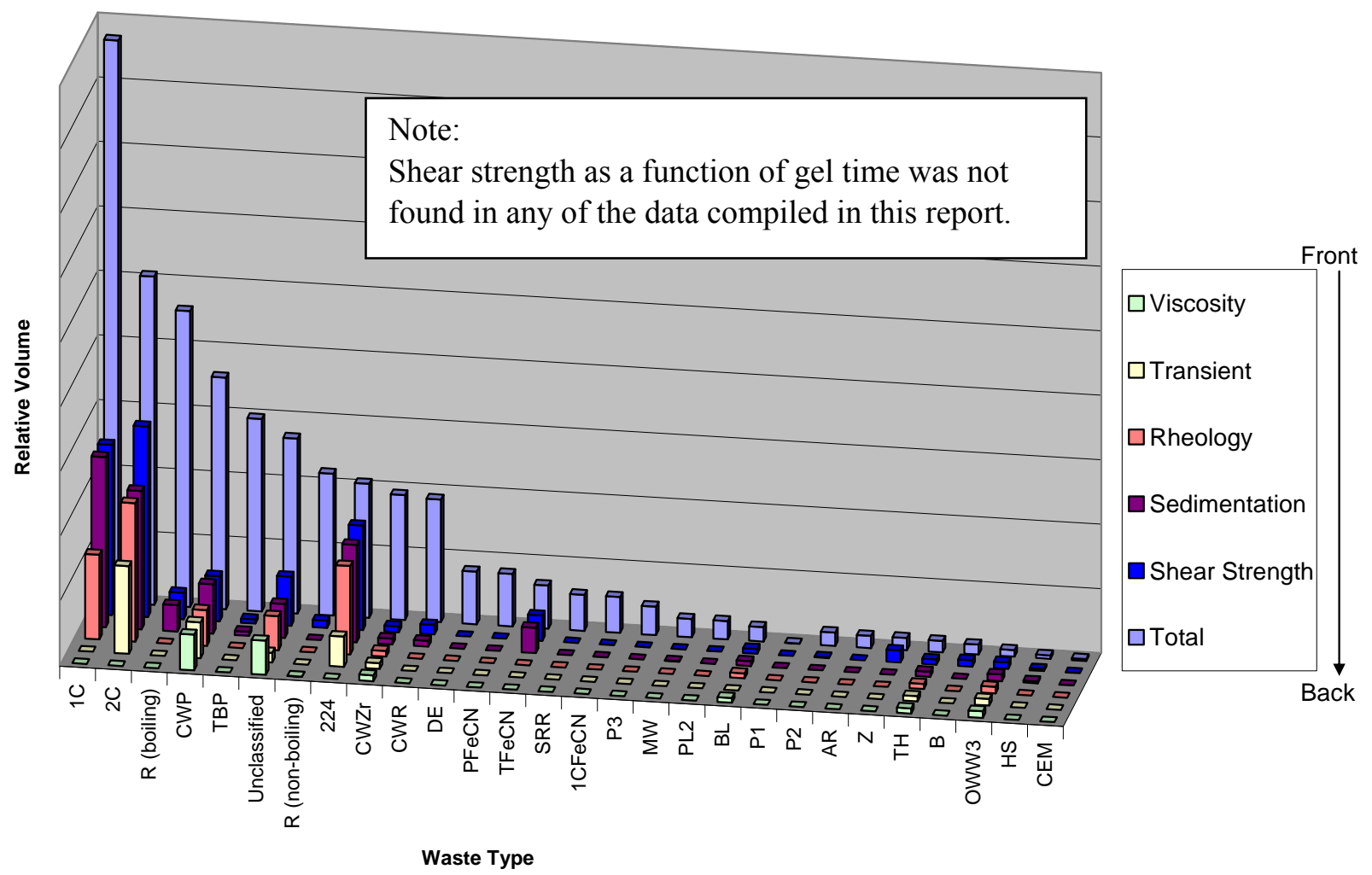

Figure S.6. Relative Volume of Waste Types Modeled Based on Waste Tank Data Available for Liquid Viscosity, Sedimentation, Shear Strength, Rheology, and Transient Modeling Compared with the Total Volume of Each Sludge Waste Type

\section{References}

Bechtel National, Inc. 2006. Issue Response Plan for Implementation of External Flowsheet Review Team (EFRT) Recommendations - M3, Inadequate Mixing System Design. 24590-WTP-PL-ENG-060013 Rev 000, Bechtel National, Inc., Richland, Washington.

Bechtel National, Inc. 2006. Scaled Testing to Determine the Adequacy of the WTP Pulse Jet Mixer Designs. 24590-PTF-TSP-RT-06-007 Rev 0, Bechtel National, Inc., Richland, Washington.

WTP/RPP-MOA-BNI-00007, Subcontractor Change Notice No. 007. 


\section{Acronyms and Abbreviations}

$\begin{array}{ll}\text { BBI } & \text { Best Basis Inventory } \\ \text { BNI } & \text { Bechtel National, Inc. } \\ \text { DOE } & \text { U.S. Department of Energy } \\ \text { ESP } & \text { Environmental Simulation Program } \\ \text { HLW } & \text { High Level Waste } \\ \text { PJM } & \text { Pulse Jet Mixer } \\ \text { PNNL } & \text { Pacific Northwest National Laboratory } \\ \text { QA } & \text { Quality Assurance } \\ \text { RPP } & \text { River Protection Project } \\ \text { TWINS } & \text { Tank Waste Information System } \\ \text { WTP } & \text { Waste Treatment Plant }\end{array}$




\section{Contents}

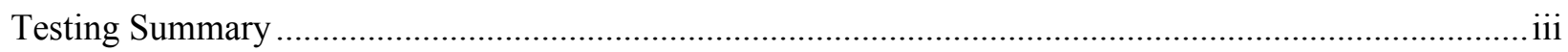

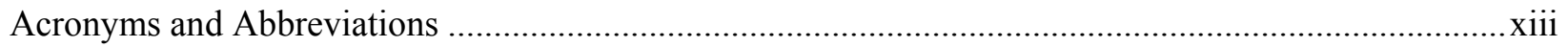

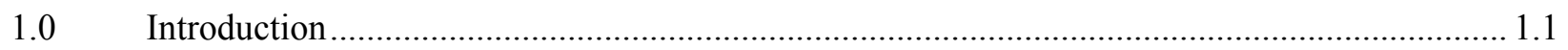

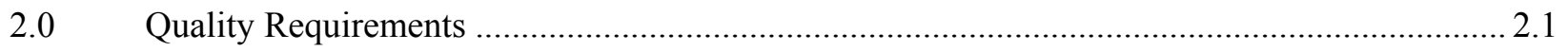

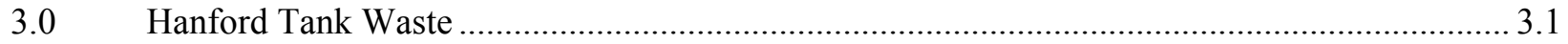

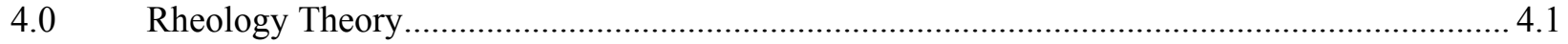

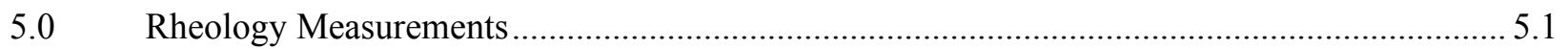

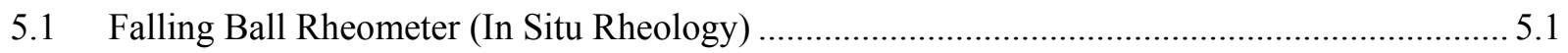

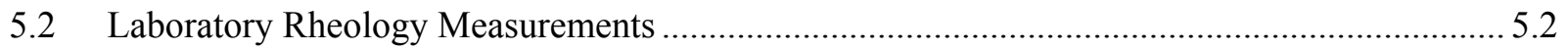

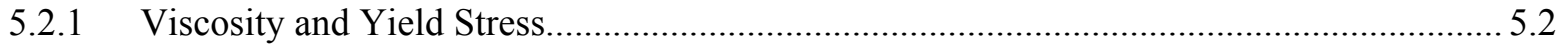

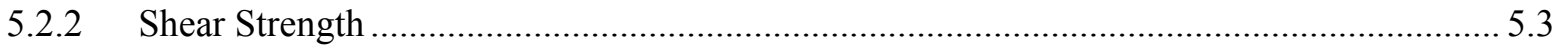

5.3 Shear Strength Calculation from Extrusion Data ................................................................. 5.4

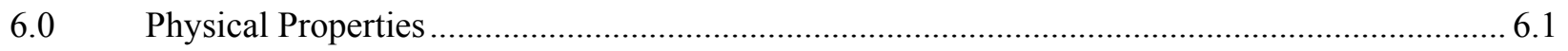

6.1 Density …

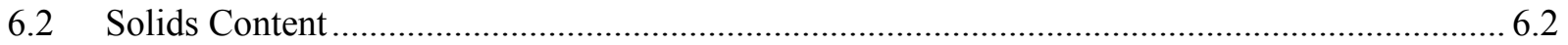

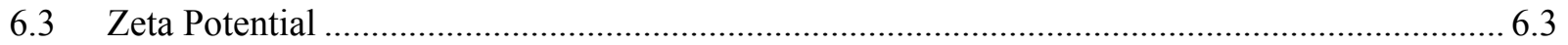

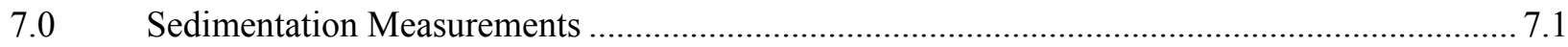

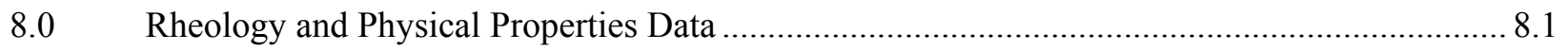

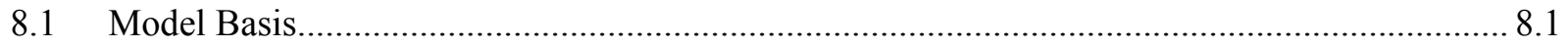

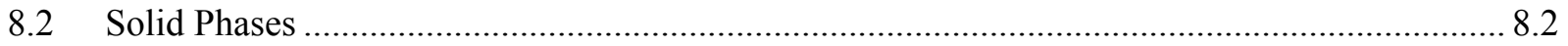

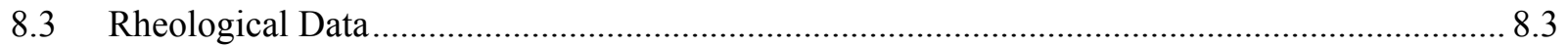

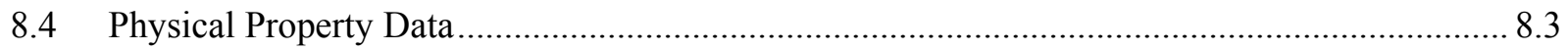

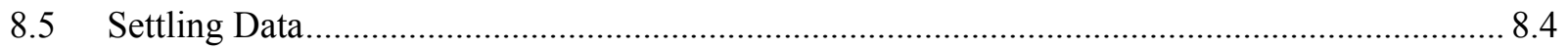

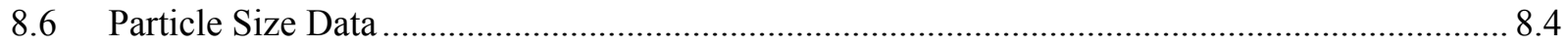

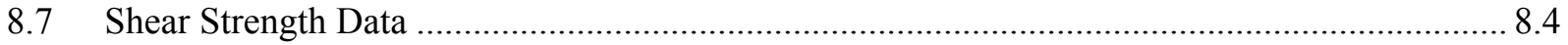

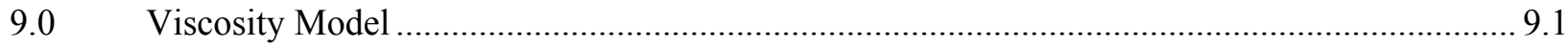

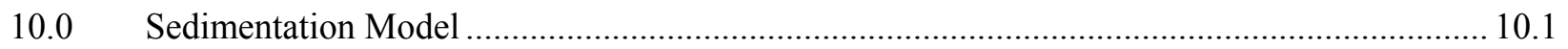

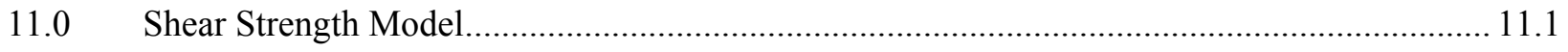

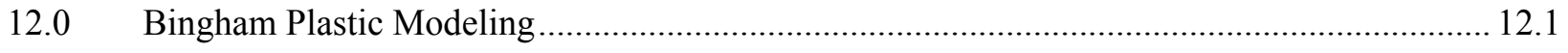

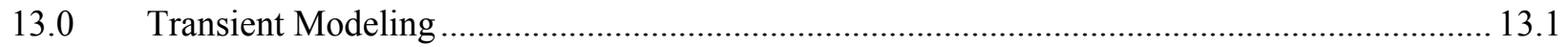

14.0 Rheology Summary and Processing Scenarios ................................................................. 14.1

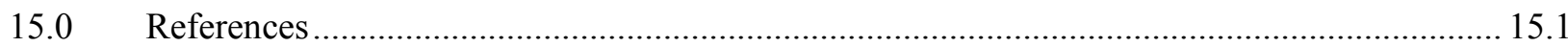

Appendix A - Tabulation of Available Rheology Data and Associated Physical and Chemical Data for Hanford Tank Wastes A.1 
Appendix B - Raw Data Used in Evaluating Correlations of the Bingham Plastic Model Parameters with

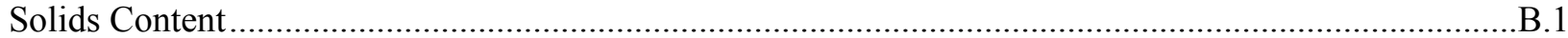

Appendix C - Correlations of the Bingham Plastic Model Parameters with Solids Content ..................... 1

Appendix D - Sedimentation Layer Properties as Sludge Settles Under Different Starting Heights....... D.1 


\section{Figures}

Figure S.1. Example Operational Scenarios for Process Vessels .........................................................vi

Figure S.2. Example Operational Scenarios for Vertical Process Piping ...............................................vii

Figure S.3. Example Operational Scenarios for Horizontal Process Piping ...........................................vii

Figure S.4. Illustration of Development of Sludge Process Heel and Fully Settled Configuration at

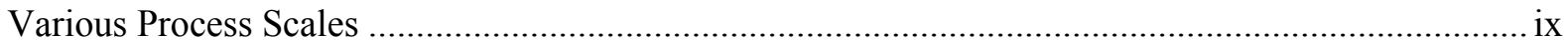

Figure S.5. Rheological Properties Encountered During Recovery from Process Upset Conditions ......... $\mathrm{X}$

Figure S.6. Relative Volume of Waste Types Modeled Based on Waste Tank Data Available for Liquid Viscosity, Sedimentation, Shear Strength, Rheology, and Transient Modeling Compared with the Total Volume of Each Sludge Waste Type.

Figure 3.1. Gaps in Rheology Data Available for Sludges in the Entire Hanford Tank Waste

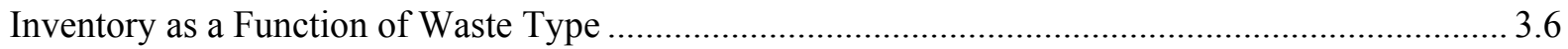

Figure 4.1. Diagram of Fluid Flow Between Stationary and Moving Plates ........................................ 4.1

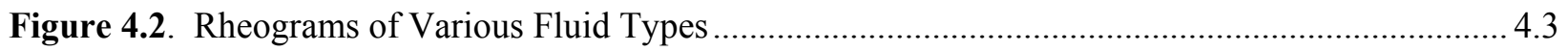

Figure 4.3. Example Flow Profiles for a Bingham Plastic Fluid (30 cP consistency, 30 Pa yield stress) in a 3-inch-ID Smooth Pipe at $90 \mathrm{gpm}$............................................................................. 4.5

Figure 4.4. Example Flow Profiles for a Newtonian Fluid (30 cP viscosity) in a 3-inch-ID Smooth Pipe at $90 \mathrm{gpm}$

Figure 5.1. Schematic of the Falling Ball Rheometer ..................................................................... 5.1

Figure 5.2. Rheogram of a Newtonian and Yield Pseudoplastic Fluid .............................................. 5.2

Figure 5.3. Typical Stress-Versus-Time Profile for a Shear Vane at Constant Shear Rate ................... 5.4

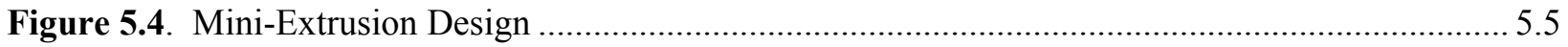

Figure 9.1. Viscosity of Water at Various Temperatures.................................................................. 9.1

Figure 9.2. Relative Waste Volumes Used in the Analysis of Liquid Viscosity as a Function of Sludge Waste Types........................................................................................................ 9.3

Figure 9.3. Viscosity of Hanford Supernatant at Various Densities and Temperatures......................... 9.4

Figure 9.4. Parity Plot of Measured and Predicted Hanford Supernatant Liquid .................................. 9.5

Figure 10.1. Gaps in Data Available for Sedimentation Modeling as a Function of Waste Type ......... 10.4

Figure 10.2. Predicted Sedimentation Curves for Various Waste Types at Different Scales ............... 10.5

Figure 11.1. Shear Strength as a Function of Gel Time for HLW Pretreated Sludge .......................... 11.1

Figure 11.2. Gel Time Constant Comparison of Various Particulate Suspensions and Temperatures $\left({ }^{\circ} \mathrm{C}\right) 11.4$

Figure 11.3. Shear Strength Summary for Various Hanford Waste Tanks and Types ........................ 11.5

Figure 11.4. Gaps in Data Available for Shear Strength Modeling as a Function of Waste Type..... 11.6 
Figure 12.1. Scatter Plot Showing the Range of Measured Bingham Parameters .....

Figure 12.2. Scatter Plot of Obtained Bingham Parameters at Various Solids Loadings

Figure 12.3. Maximum Measured Bingham Consistency for Various Hanford Tanks and Waste Types 12.3

Figure 12.4. Maximum Measured Bingham Yield Stress for Various Hanford Tanks and Waste Types 12.3

Figure 12.5. Gaps in Data Available for Rheological Correlations as a Function of Waste Type

Figure 12.6. Bingham Plastic Rheological Parameters as a Function of Solids Loading for Tank C-104 as an Example of an as-Received Hanford Core Sample.

Figure 12.7. Bingham Plastic Rheological Parameters as a Function of Solids Loading for Tank C-104 as an Example of a Hanford Core Sample Diluted with Water

Figure 12.8. Bingham Plastic Rheological Parameters as a Function of Solids Loading for a WaterDiluted Hanford Core Sample Showing Decreasing Rheology as Dilution Occurs....

Figure 12.9. Bingham Plastic Rheological Parameters as a Function of Solids Loading for a WaterDiluted Hanford Core Sample Showing a Rheological Peak as Dilution Occurs.... 12.11

Figure 13.1. Relative Volume of Waste Types Analyzed based on Waste Tank Data Available for Transient Modeling 13.5

Figure 13.2. Predicted Sludge Properties from Hanford Tank C-104 with Water Dilution at a Starting Slurry Height of $0.1 \mathrm{~m}$ with 33\% Volume Excess Supernatant from Fully Settled Configuration; Rheological Properties Taken at a Temperature Range of $20^{\circ}-35^{\circ} \mathrm{C}$. 13.6

Figure 13.3. $\quad$ Predicted Sludge Properties from Hanford Tank C-104 with Water Dilution at Starting Slurry Height of $1 \mathrm{M}$ with 33\% Volume Excess Supernatant from Fully Settled Configuration; Rheological Properties Taken at a Temperature Range of $20^{\circ}-35^{\circ} \mathrm{C}$ 13.7

Figure 13.4. $\quad$ Predicted Sludge Properties from Hanford Tank C-104 with Water Dilution at Starting Slurry Height of $10 \mathrm{M}$ with 33\% Volume Excess Supernatant from Fully Settled Configuration; Rheological Properties Taken at a Temperature Range of $20^{\circ}-35^{\circ} \mathrm{C}$ 13.8

Figure 13.5. Illustration of Development of Sludge Process Heel and Fully Settled Configuration at Various Process Scales

Figure 13.6. Rheological Properties Encountered During Recovery from Process Upset Conditions 13.10

Figure 14.1. Example Operational Scenarios for Process Vessels

Figure 14.2. Example Operational Scenarios for Vertical Process Piping

Figure 14.3. Example Operational Scenarios for Horizontal Process Piping

Figure 14.4. Relative Volume of Waste Types Modeled Based on Waste Tank Data Available for Liquid Viscosity, Sedimentation, Shear Strength, Rheology, and Transient Modeling Compared to the Total Volume of Each Sludge Waste Type. 14.4 


\section{Tables}

Table 3.1. List of Waste Type Definitions

Table 3.2. Primary and Secondary Waste Types for the Solids in Tanks with Rheological Data Available 3.2

Table 3.3. Primary and Secondary Waste Types for Liquids in Tanks with Rheological Data Available 3.3

Table 3.4. Comparison of Waste Type Groups ................................................................................... 3.5

Table 4.1. Typical Shear Rates in Food-Processing Applications …................................................... 4.2

Table 4.2. Viscosities of Several Common Newtonian Fluids................................................................. 4.3

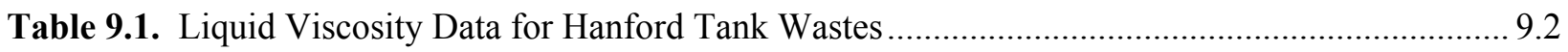

Table 10.1. Sedimentation Model Input and Fitting Parameters for Hanford Tank Waste................... 10.2

Table 10.2. Sedimentation Parameters for Each Waste Type .......................................................... 10.3

Table 11.1. Shear Strength Model Fit Parameters for Pretreated AZ-101 Sludge .............................. 11.2

Table 11.2. Shear Strength Rebuild Parameters for Various Materials.............................................. 11.3

Table 12.1. Bingham Model Parameters for Various Hanford Tanks............................................... 12.5

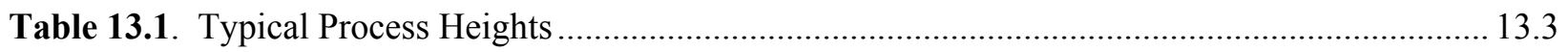

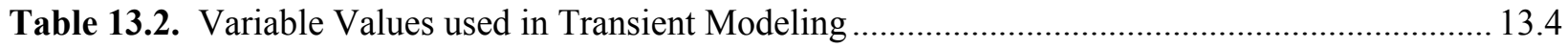

Table 14.1. Range of Rheological Parameters and Regrowth Times at Typical Process Scales .......... 14.1 


\subsection{Introduction}

The U.S. Department of Energy (DOE) Office of River Protection Waste Treatment and Immobilization Plant (WTP) is being designed and built to pretreat and then vitrify a large portion of the wastes in Hanford's 177 underground waste storage tanks. Once the material is transferred from the underground waste storage tanks to the WTP, the mixing systems at WTP must be capable of blending liquids, maintaining solids suspended, and resuspending settled solids.

In vessels where process streams are mixed, the liquids are blended to the degree required for processing and sampling. The pulse jet mixer (PJM) mixing test performance criteria require that that all points in the vessel must be reached during blending to ensure that there will be no zones where the material is not blended, and the slurry being blended into the vessel must achieve a sufficiently uniform vessel concentration.

The WTP PJM-mixed vessels do not have restrictive criteria on the degree of uniformity of solids concentration within the vessel liquid. However, the mixing must be sufficient to maintain the solids in suspension so that they do not accumulate on the bottom and can be transferred through the pump suction line. Solids suspended from the bottom of the vessel must be sufficiently lifted in a repeatable pattern so that they can be carried with the flowing fluid into the vessel suction line.

Mixing of tank waste slurries is also required for hydrogen control. During normal operations the vessels may be mixed intermittently, but must be mixed with a frequency to ensure that the hydrogen inventory is controlled. After a design basis event the important-to-safety air supply is limited and the PJMs will be operated intermittently. Between operating periods, solids will form a settled layer, which may have cohesive properties. The PJMs must be able to cause motion of the accumulated solids layer adequate to release hydrogen. Rheological properties of the suspending medium, solids suspensions, and settled solids are included in the parameters that define the ability of the PJM to blend, maintain solids suspensions, and resuspend settled solids.

Accurate rheological data of Hanford tank wastes at varying conditions including as a function of sedimentation are critical in validating the performance of the WTP-PJM mixing systems. This report provides a compilation of the available rheological data for Hanford tank wastes and empirical models describing this data. Rheological properties were modeled as a function of physical properties (volume percent settled solids, sedimentation rate, etc.) to provide a predictive tool for rheological behavior for different waste types under differing conditions.

This report presents the data sources considered and the development of the best-estimate data sets for rheological properties. The relation of the available data sets with regard to the insoluble solid inventory

at Hanford is discussed. Quantifiable uncertainties in the data are elucidated. Liquid viscosity, sedimentation, and rheological models are also presented. Conclusions and recommendations are presented based on the models and data available. 


\subsection{Quality Requirements}

Since December 2001, Battelle - Pacific Northwest Division, utilizing its use agreement with the Department of Energy (DE-AC05-76RL01831), has been providing support to Bechtel National, Inc. (BNI) in accordance with the Quality Assurance (QA) program approved under Subcontract No. 24590101-TSA-W000-00004. This support has been provided under the WTP Support Project (WTPSP) QA Program and later the BNI Support Program (BNI-SP) for the technical support of the waste treatment plant being built in the 200 East area of the Hanford Site. In February 2007, the contract mechanism was switched to Pacific Northwest National Laboratory (PNNL) Operating Contract, DE-AC05-76RL01830, and the program was renamed the RPP-WTP Support Program.

The data represented in this report might refer to PNWD, PNNL, BNI-SP or WTPSP; both of these projects performed work to the same QA Program. As of February 2007, the Quality Assurance Program is described as follows:

PNNL's Quality Assurance Program is based on requirements defined in U.S. Department of Energy (DOE) Order 414.1C, Quality Assurance, and 10 CFR 830, Energy/Nuclear Safety Management, Subpart A-Quality Assurance Requirements (a.k.a. the Quality Rule). PNNL has chosen to implement the requirements of DOE Order 414.1C and 10 CFR 830, Subpart A by integrating them into the Laboratory's management systems and daily operating processes. The procedures necessary to implement the requirements are documented through PNNL's Standards-Based Management System.

PNNL implements the RPP-WTP quality requirements by performing work in accordance with the River Protection Project - Waste Treatment Plant Support Program (RPP-WTP) Quality Assurance Plan (RPP-WTP-QA-001, QAP). Work will be performed to the quality requirements of NQA-1-1989 Part I, Basic and Supplementary Requirements, NQA-2a-1990, Part 2.7 and DOE/RW-0333P, Rev 13, Quality Assurance Requirements and Descriptions (QARD). These quality requirements are implemented through the River Protection Project - Waste Treatment Plant Support Program (RPP-WTP) Quality Assurance Manual (RPP-WTP-QA-003, QAM).

This report is based on data from testing as referenced. The PNNL assumes that the data from these references has been fully reviewed and documented in accordance with the analysts' QA Programs. PNNL only analyzed data from the referenced documentation. At PNNL, the performed calculations, the documentation and reporting of results and conclusions were performed in accordance with the RPP-WTP Quality Assurance Manual (RPP-WTP-QA-003, QAM). Internal verification and validation activities were addressed by conducting an independent technical review of the final data report in accordance with PNNL procedure QA-RPP-WTP-604. This review verifies that the reported results are traceable, that inferences and conclusions are soundly based, and that the reported work satisfies the Test Specification Success Criteria. This review procedure is part of PNNL's RPP-WTP Quality Assurance Manual). 


\subsection{Hanford Tank Waste}

Radioactive waste from the reprocessing of spent nuclear fuel on the Hanford Site was transferred to underground storage tanks. Four different chemical processes were used for reprocessing this spent nuclear fuel, and waste from each of these processes exists in these 177 underground storage tanks. The four processes used were the bismuth phosphate $\left(\mathrm{BiPO}_{4}\right)$ process, the tributyl phosphate (TBP) process, the reduction-oxidation (REDOX) process, and the plutonium-uranium extraction (PUREX) process. Wastes with different chemical composition and properties were generated in multiple steps of these processes, and modifications to the processes have resulted in multiple waste types. Some of this waste was treated in the underground storage tanks, resulting in additional waste types. Each waste type was made alkaline for storage in the steel tanks. Table 3.1 lists the waste type, acronym, and a brief description of each waste type. The definitions were adapted from Meacham (2003).

Table 3.1. List of Waste Type Definitions

\begin{tabular}{|c|c|}
\hline Waste Type & Definition \\
\hline $1 \mathrm{C}$ & $\mathrm{BiPO}_{4}$ first cycle decontamination waste (1944-1956) \\
\hline $1 \mathrm{CFeCN}$ & Ferrocyanide sludge from in-farm scavenging of 1C supernatants in TY-Farm (1955-1958) \\
\hline 224 & lanthanum fluoride process "224 Building" waste (1952-1956) \\
\hline $2 \mathrm{C}$ & $\mathrm{BiPO}_{4}$ second cycle decontamination waste (1944-1956) \\
\hline A1-SltCk & Saltcake from the first 242-A Evaporator campaign (1977-1980) \\
\hline A2-SltSlr & saltcake from the second 242-A Evaporator campaign (1981-1994). \\
\hline AR & Washed Plutonium-Uranium Extraction (PUREX) sludge (1967-1976) \\
\hline $\mathrm{B}$ & high-level acid waste from PUREX processed at B Plant for Sr recovery (1967-1972) \\
\hline $\mathrm{BL}$ & low-level waste from B Plant Sr and Cs recovery operations (1967-1976) \\
\hline CEM & Portland Cement \\
\hline CSR & Cesium recovery, supernatant from which Cs has been removed \\
\hline CWP & PUREX cladding waste (1956-1960 and 1961-1972) \\
\hline CWR & REDOX cladding waste, aluminum clad fuel (1952-1960 and 1961-1972) \\
\hline CWZr & zirconium cladding waste (PUREX and REDOX) \\
\hline$\overline{\mathrm{DE}}$ & diatomaceous earth \\
\hline HS & hot semi-works ${ }^{90} \mathrm{Sr}$ recovery waste $(1962-1967)$ \\
\hline MW & $\mathrm{BiPO}_{4}$ process metal waste (1944-1956) \\
\hline OWW3 & PUREX organic wash waste (1968-1972) \\
\hline P1 & PUREX HLW (1956-1962) \\
\hline $\mathrm{P} 2$ & PUREX HLW (1963-1967) \\
\hline P3 & PUREX HLW (1983-1988) \\
\hline $\mathrm{PFeCN}$ & Ferrocyanide sludge from in-plant scavenged supernatant (1954-1958) \\
\hline PL2 & PUREX low-level waste (1983-1988) \\
\hline R (boiling) & boiling REDOX HLW (1952-1966) \\
\hline $\mathrm{R}$ (non-boiling) & non-boiling REDOX HLW (1952-1966) \\
\hline R-SltCk & Saltcake from self-concentration in S- and SX-Farms (1952-1966) \\
\hline S1-SltCk & Saltcake from the first 242-S Evaporator campaign using 241-S-102 feed tank (1973-1976) \\
\hline S2-SltSlr & Saltcake from the second 242-S Evaporator campaign using 241-S-102 feed tank (1976-1980) \\
\hline SRR & HLW transfers (late B Plant operations) \\
\hline T2-SltCk & Saltcake from the second 242-T Evaporator campaign using 241-TX-118 feed tank (1965-1976) \\
\hline TBP & tributyl phosphate waste (from solvent based uranium recovery operations) \\
\hline TFeCN & ferrocyanide sludge produced by in-tank or in-farm scavenging \\
\hline $\mathrm{TH}$ & PUREX waste from processing of thoria targets \\
\hline $\mathrm{Z}$ & Z Plant waste \\
\hline
\end{tabular}


Rheological data is available on 40 tanks that contain several different waste types. The primary and secondary waste types for the solids in these 40 tanks, as described in the Tank Waste Information System (TWINS) database, are listed in Table 3.2. The waste types for the liquids in these tanks are listed in Table 3.3. Rheological measurements have been made on samples from the tanks that are listed in these tables, but the data have not been published or the published documents are not currently accessible. Additional effort will be needed to obtain and analyze the rheological data from these tanks.

Table 3.2. Primary and Secondary Waste Types for the Solids in Tanks with Rheological Data Available

\begin{tabular}{|c|c|c|}
\hline Tank & Primary Waste Type & "Secondary Waste Type \\
\hline A-101 & A1-SltCk & $\mathrm{P} 2$ \\
\hline AN-102 & A2-SltSlr & \\
\hline AN-103 & A2-SltSlr & -- \\
\hline AN-104 & A2-SltSlr & -- \\
\hline AN-105 & A2-SltSlr & - \\
\hline AN-107 & A2-SltSlr & -- \\
\hline AP-104 & \multicolumn{2}{|c|}{ No Insoluble Solids } \\
\hline AW-101 & A2-SltSlr & - \\
\hline AW-103 & CWZr & - \\
\hline AY-101 & $\mathrm{NA}^{(\mathrm{a})}$ & $-\overline{-}$ \\
\hline AY-102 & $\mathrm{NA}^{(\mathrm{a})}$ & $\mathrm{BL}$ \\
\hline AZ-101 & $\mathrm{P} 3$ & $\mathrm{NA}^{(\mathrm{a})}$ \\
\hline AZ-102 & P3 & PL2, SRR \\
\hline B-111 & $2 \mathrm{C}$ & $\mathrm{P} 2, \mathrm{~B}$ \\
\hline B-201 & 224 & - \\
\hline B-202 & 224 & 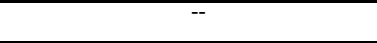 \\
\hline B-203 & 224 & $-\overline{-}$ \\
\hline BX-107 & $1 \mathrm{C}$ & - \\
\hline $\mathrm{C}-103$ & CWP & -- \\
\hline $\mathrm{C}-104$ & CWP & CWZr, OWW3, TH \\
\hline $\mathrm{C}-106$ & $\mathrm{NA}^{(\mathrm{a})}$ & \\
\hline $\mathrm{C}-107$ & $1 \mathrm{C}$ & CWP, SRR \\
\hline C-109 & $\mathrm{TFeCN}$ & $\mathrm{CWP}, 1 \mathrm{C}, \mathrm{HS}$ \\
\hline $\mathrm{C}-110$ & $1 \mathrm{C}$ & $=-$ \\
\hline $\mathrm{C}-112$ & $\mathrm{TFeCN}$ & 1C, CWP, HS \\
\hline$S-102$ & NA SltCk ${ }^{(b)}$ & R (non-boiling) \\
\hline S-104 & R (boiling) & CWR \\
\hline S-112 & S1-SltCk & R (non-boiling) \\
\hline SY-101 & S2-SltSlr & - \\
\hline SY-102 & $\mathrm{NA}^{(\mathrm{a})}$ & $\mathrm{Z}$ \\
\hline SY-103 & S2-SltSlr & 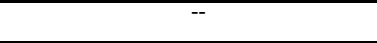 \\
\hline T-102 & CWP & MW \\
\hline T-104 & $1 \mathrm{C}$ & -- \\
\hline T-107 & $1 \mathrm{C}$ & CWP, TBP \\
\hline $\mathrm{T}-110$ & $2 \mathrm{C}$ & 224 \\
\hline $\mathrm{T}-111$ & 224 & $2 \mathrm{C}$ \\
\hline $\mathrm{T}-203$ & 224 & \\
\hline T-204 & 224 & 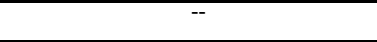 \\
\hline U-103 & S1-SltCk & S2-SltSlr, R (non-boiling) \\
\hline $\mathrm{U}-107$ & S2-SltSlr & CWR, T2-SltCk \\
\hline
\end{tabular}


Table 3.3. Primary and Secondary Waste Types for Liquids in Tanks with Rheological Data Available

\begin{tabular}{|c|c|c|}
\hline Tank & Primary Waste Type & Secondary Waste Type \\
\hline A-101 (interstitial only) & A1-SltCk & -- \\
\hline AN-102 & $\mathrm{NA}^{(\mathrm{a})}$ & - \\
\hline AN-103 & A2-SltSlr & -- \\
\hline AN-104 & A2-SltSlr & -- \\
\hline AN-105 & A2-SltSlr & - \\
\hline AN-107 & A2-SltSlr & -- \\
\hline AP-104 & $\mathrm{NA}^{(\mathrm{a})}$ & -- \\
\hline AW-101 & A2-SltSlr & - \\
\hline AW-103 & $\mathrm{NA}^{(\mathrm{a})}$ & A1-SltCk (interstitial) \\
\hline AY-101 & $\mathrm{NA}^{(\mathrm{a})}$ & -- \\
\hline AY-102 & $\mathrm{NA}^{(\mathrm{a})}$ & BL (interstitial) \\
\hline AZ-101 & P3 & -- \\
\hline AZ-102 & $\mathrm{P} 3$ & -- \\
\hline B-111 & CSR & -- \\
\hline B-201 & \multicolumn{2}{|c|}{ No Free Liquid } \\
\hline B-202 & \multicolumn{2}{|c|}{ No Free Liquid } \\
\hline B-203 & $\mathrm{NA}^{(\mathrm{a})}$ & -- \\
\hline BX-107 & \multicolumn{2}{|c|}{ No Free Liquid } \\
\hline C-103 & $\mathrm{NA}^{(\mathrm{a})}$ & -- \\
\hline C-104 & \multicolumn{2}{|c|}{ No Free Liquid } \\
\hline C-106 & $\mathrm{NA}^{(\mathrm{a})}$ & -- \\
\hline C-107 & \multicolumn{2}{|c|}{ No Free Liquid } \\
\hline C-109 & \multicolumn{2}{|c|}{ No Free Liquid } \\
\hline C-110 & $1 \mathrm{C}$ & -- \\
\hline C-112 & \multicolumn{2}{|c|}{ No Free Liquid } \\
\hline S-102 & \multicolumn{2}{|c|}{ No Free Liquid } \\
\hline S-104 (interstitial only) & R-SltCk & - \\
\hline S-112 & \multicolumn{2}{|c|}{ No Free Liquid } \\
\hline SY-101 & S2-SltSlr & -- \\
\hline SY-102 & $\mathrm{NA}^{(\mathrm{a})}$ & Z (interstitial only) \\
\hline SY-103 & S2-SltSlr & -- \\
\hline $\mathrm{T}-102$ & CSR & -- \\
\hline T-104 & \multicolumn{2}{|c|}{ No Free Liquid } \\
\hline T-107 & \multicolumn{2}{|c|}{ No Free Liquid } \\
\hline $\mathrm{T}-110$ & $2 \mathrm{C}$ & -- \\
\hline $\mathrm{T}-111$ & \multicolumn{2}{|c|}{ No Free Liquid } \\
\hline T-203 & \multicolumn{2}{|c|}{ No Free Liquid } \\
\hline T-204 & \multicolumn{2}{|c|}{ No Free Liquid } \\
\hline U-103 & S1-SltCk & -- \\
\hline U-107 (interstitial only) & S2-S1tSlr & T2-SltCk \\
\hline
\end{tabular}

Waste type definitions have evolved over time as additional information on the composition of wastes transferred to the Hanford tanks has been identified. The latest modifications were included in Revision 5 
of the Hanford Defined Waste (HDW) Model, which was published in February 2004 (Higley 2004). Most of these changes are included in the 2006 Best Basis Inventory (BBI), which is the database provided in TWINS and used in this report for determining the sludge volumes associated with each waste type. In the $2006 \mathrm{BBI}$, waste types $1 \mathrm{C} 1$ and $1 \mathrm{C} 2$ are combined as $1 \mathrm{C}$, and waste types $2 \mathrm{C} 1$ and $2 \mathrm{C} 2$ are combined as $2 \mathrm{C}$.

The 2002 BBI is used in the current Environmental Simulation Program (ESP) ${ }^{(a)}$ model; therefore, some of the wastes types in the 2006 BBI were combined to be consistent with the ESP model and previous reports. Waste types identified in the $2006 \mathrm{BBI}$ are compared with the waste types used in this report in Table 3.4. A few sludge, saltcake, and liquid layers in Hanford tanks have not been identified as a particular waste type and are listed as unclassified. The acronym NA is used for these unclassified wastes in the BBI presented in TWINS.

Twenty-nine of the 41 waste types described in Meacham (2003) are included in the tanks identified as having rheological data. Diatomaceous earth is included as a waste type in BBI but is not included in Meacham (2003); therefore, the total number of waste types is 42. Also included in TWINS are waste transfers and unclassified waste types. Only 26 of the 42 waste types are listed as sludge in TWINS, and these waste types are the focus of this study. Other waste types with rheological data are included in the data set to provide additional supporting data. Seven of the 26 sludge waste types are not represented in the rheology data set. These include $1 \mathrm{CFeCN}, \mathrm{AR}, \mathrm{DE}, \mathrm{P} 1, \mathrm{PFeCN}, \mathrm{CEM}$, and Z. Waste transfers are also not represented in the rheological data. The definitions of these waste types are included in Table 3.1 and listed in Table 3.4 .

REDOX high-level wastes (HLW) are classified as R1 and R2 in the 2006 BBI based on the date of waste generation, but these classifications do not indicate the thermal history of the REDOX waste, which is essential in determining whether gibbsite or boehmite is the predominant aluminum species in the waste. Therefore, REDOX HLW were reclassified as REDOX boiling and REDOX non-boiling waste types to provide waste type definitions that segregated the aluminum-containing sludges based on the predominant aluminum phase (gibbsite or boehmite). This reclassification was based on thermal history and aluminum leaching factors in these wastes, as described in Meacham (2003).

The 224 waste is currently in the Hanford baseline to be dried and transported to the Waste Isolation Pilot Plant (WIPP) as transuranic (TRU) waste. The inclusion of the 224 waste in this report raises the overall significance of the rheology characterization of the Hanford sludge. While this waste might not be a direct feed to WTP, it may be representative of sludge in other tanks that may be a feed to WTP. For example, Tanks T-110, T-111, and T-112 contain a blend of 224 and $2 \mathrm{C}$ waste that may not meet TRU waste specifications.

Rheology data available from tank waste core samples and falling ball rheometry are plotted as a function of waste type in Figure 3.1. The volume of waste in each tank for each waste type was determined using the TWINS database. Additional rheology data are being gathered on wastes that were collected as part of the analysis of the M-12 samples. The increase in the amount of rheology data that will be available based on these analyses is also shown in Figure 3.1.

(a) ESP was supplied and developed by OLI Systems, Inc., Morris Plains, New Jersey. 
Table 3.4. Comparison of Waste Type Groups

\begin{tabular}{|c|c|}
\hline $2006 \mathrm{BBI}$ & This Report \\
\hline \multicolumn{2}{|c|}{ Bismuth Phosphate Process Waste Types } \\
\hline MW1 & \multirow{2}{*}{ MW } \\
\hline MW2 & \\
\hline $1 \mathrm{C}$ & $1 \mathrm{C}$ \\
\hline $2 \mathrm{C}$ & $2 \mathrm{C}$ \\
\hline $224-1$ & \multirow{2}{*}{224} \\
\hline $224-2$ & \\
\hline \multicolumn{2}{|c|}{ Uranium Recovery and Scavenging Waste Types } \\
\hline $1 \mathrm{CFeCN}$ & $1 \mathrm{CFeCN}$ \\
\hline $\mathrm{PFeCN}$ & $\mathrm{PFeCN}$ \\
\hline TBP & TBP \\
\hline TFeCN & $\mathrm{TFeCN}$ \\
\hline \multicolumn{2}{|c|}{ REDOX Process Waste Types } \\
\hline $\mathrm{R} 1$ & \multirow{2}{*}{$\begin{array}{c}\mathrm{R} \text { (boiling) or } \\
\mathrm{R} \text { (non-boiling) }\end{array}$} \\
\hline R2 & \\
\hline CWR1 & \multirow{2}{*}{ CWR } \\
\hline CWR2 & \\
\hline \multicolumn{2}{|c|}{ PUREX Process Waste Types } \\
\hline P1 & P1 \\
\hline P2 & $\mathrm{P} 2$ \\
\hline P3AZ1 & \multirow{2}{*}{ P3 } \\
\hline P3AZ2 & \\
\hline CWP1 & \multirow{2}{*}{ CWP } \\
\hline CWP2 & \\
\hline CWZr1 & \multirow{2}{*}{ CWZr } \\
\hline CWZr2 & \\
\hline OWW3 & OWW3 \\
\hline PL2 & PL2 \\
\hline TH1 & \multirow{2}{*}{$\mathrm{TH}$} \\
\hline TH2 & \\
\hline \multicolumn{2}{|c|}{ Cesium and Strontium Recovery Waste Types } \\
\hline HS & $\mathrm{HS}$ \\
\hline AR & $\mathrm{AR}$ \\
\hline $\mathrm{B}$ & B \\
\hline $\mathrm{BL}$ & $\overline{\mathrm{BL}}$ \\
\hline CSR & CSR \\
\hline SRR & SRR \\
\hline \multicolumn{2}{|c|}{ Saltcake and Salt Slurries Waste Types } \\
\hline A1-SltCk & A1-SltCk \\
\hline A2-SltSlr & A2-SltSlr \\
\hline R-SltCk & R-SltCk \\
\hline S1-SltCk & S1-SltCk \\
\hline S2-S1tS1r & S2-SltS1r \\
\hline T2-SltCk & T2-SltCk \\
\hline \multicolumn{2}{|c|}{ Other Process Facility Wastes } \\
\hline $\mathrm{Z}$ & $\mathrm{Z}$ \\
\hline \multicolumn{2}{|c|}{ Miscellaneous Wastes } \\
\hline CEM & Portland Cement \\
\hline $\mathrm{DE}$ & $\mathrm{DE}$ \\
\hline
\end{tabular}




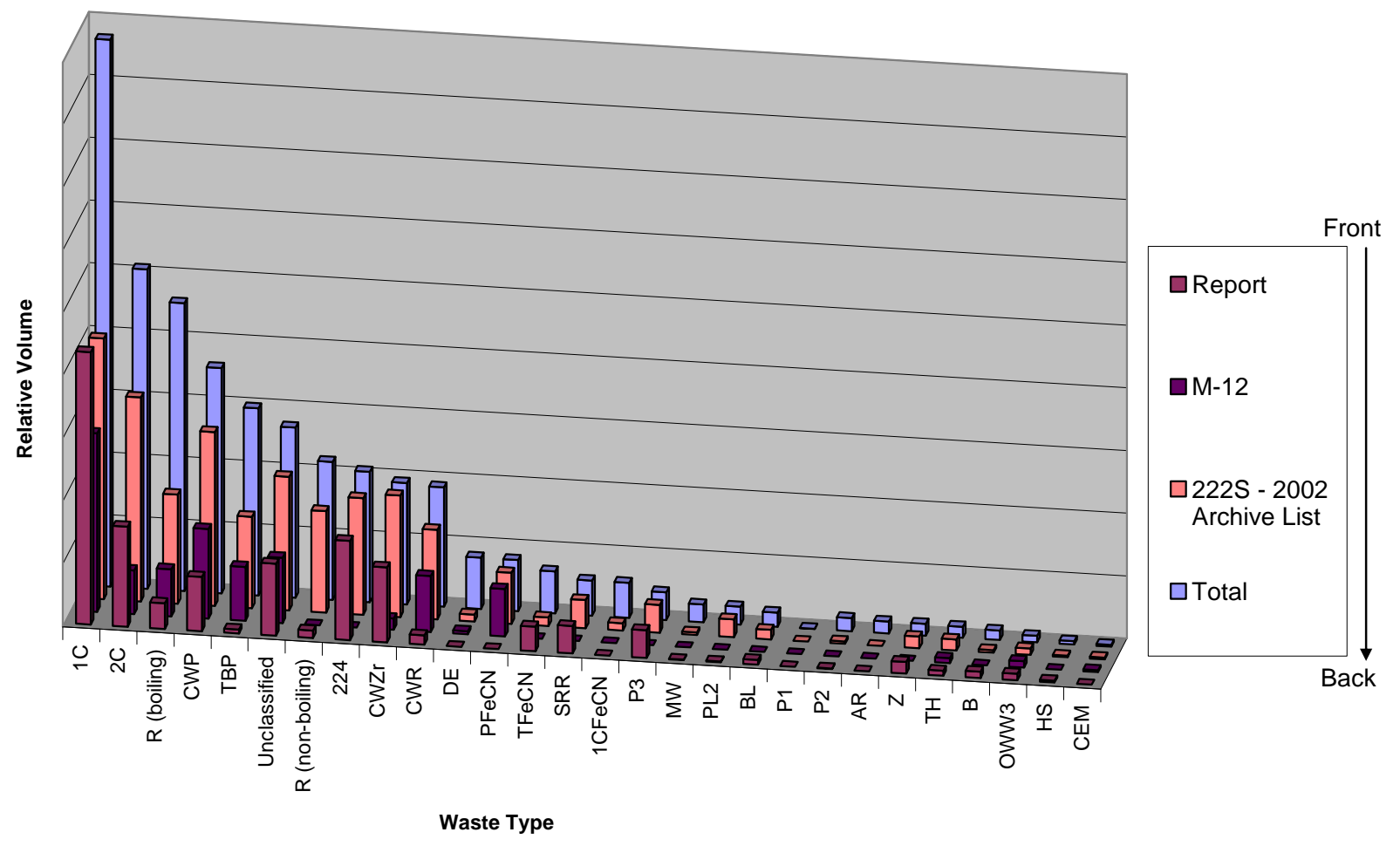

Figure 3.1. Gaps in Rheology Data Available for Sludges in the Entire Hanford Tank Waste Inventory as a Function of Waste Type 


\subsection{Rheology Theory}

Rheology is the study of the flow and deformation of materials. When a force (i.e., stress) is placed on an object, the object deforms or strains. Many relationships have been found relating stress to strain for various fluids. Flow behavior of a fluid can generally be explained by considering a fluid placed between two plates of thickness $\mathrm{x}$ (Figure 4.1). The lower plate is held stationary while a force, $\mathrm{F}$, is applied to the upper plate of area, A, that results in the plating moving at velocity, $\mathrm{v}$. If the plate moves a length, $\Delta L$, the strain, $\gamma$, on the fluid can be defined by Eq. (4.1).

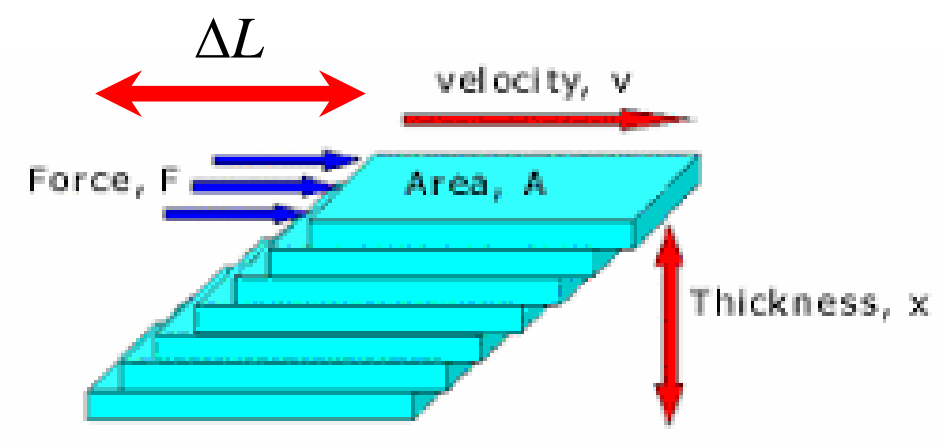

Figure 4.1. Diagram of Fluid Flow Between Stationary and Moving Plates

$$
\gamma=\frac{\Delta L}{\mathrm{x}}
$$

The rate of change of strain (also called shear rate), $\dot{\gamma}$, can be defined by Eq. (4.2). Because the shear rate is defined as the ratio of a velocity to a length, the units of the variable are the inverse of time, typically $\mathrm{s}^{-1}$.

$$
\dot{\gamma}=\frac{d \gamma}{d t}=\frac{d}{d t}\left(\frac{\Delta L}{\mathrm{x}}\right)=\frac{\mathrm{v}}{\mathrm{x}}
$$

Typical shear rates of food-processing applications can be seen in Table 4.1. Depending on the application, shear rates in the range of $10^{-6}$ to $10^{7} \mathrm{~s}^{-1}$ are possible. Human perception of a fluid is typically based on a shear rate of approximately $60 \mathrm{~s}^{-1}$.

The shear stress applied to the fluid can be found by Eq. (4.3). Because the shear stress is defined as the ratio of a force to an area, the units of the variable are pressures, typically expressed in $\mathrm{Pa}\left(\mathrm{N} / \mathrm{m}^{2}\right)$.

$$
\tau=\frac{\mathrm{F}}{\mathrm{A}}
$$


Table 4.1. Typical Shear Rates in Food-Processing Applications

\begin{tabular}{||l|c|l||}
\hline \multicolumn{1}{|c|}{ Situation } & $\begin{array}{c}\text { Shear Rate } \\
\text { Range (1/s) }\end{array}$ & \multicolumn{1}{c||}{ Typical Applications } \\
\hline $\begin{array}{l}\text { Sedimentation of particles in a } \\
\text { suspending liquid }\end{array}$ & $10^{-6}-10^{-3}$ & Medicines, paints, spices in salad dressing \\
\hline Leveling due to surface tension & $10^{-2}-10^{-1}$ & Frosting, Paints, printing inks \\
\hline Draining under gravity & $10^{-1}-10^{1}$ & Vats, small food containers \\
\hline Extrusion & $10^{0}-10^{3}$ & $\begin{array}{l}\text { Snack and pet foods, toothpaste, cereals, pasta, } \\
\text { polymers }\end{array}$ \\
\hline Calendering & $10^{1}-10^{2}$ & Dough sheeting \\
\hline Pouring from a bottle & $10^{1}-10^{2}$ & Foods, cosmetics, toiletries \\
\hline Chewing and swallowing & $10^{1}-10^{2}$ & Foods \\
\hline Dip coating & $10^{1}-10^{2}$ & Paints, confectionery \\
\hline Mixing and stirring & $10^{1}-10^{3}$ & Food processing \\
\hline Pipe flow & $10^{0}-10^{3}$ & Food processing, blood flow \\
\hline Rubbing & $10^{2}-10^{4}$ & Topical application of creams and lotions \\
\hline Brushing & $10^{3}-10^{4}$ & Brush painting, lipstick, nail polish \\
\hline Spraying & $10^{3}-10^{5}$ & Spray drying, spray painting, fuel atomization \\
\hline High-speed coating & $10^{4}-10^{6}$ & Paper \\
\hline Lubrication & $10^{3}-10^{7}$ & Bearings, gasoline engines \\
\hline \hline
\end{tabular}

The apparent viscosity of the fluid is defined as the ratio of the shear stress to shear rate (see Eq. 4.4). Often the shear stress and viscosity vary as a function of shear rate. Since the viscosity is defined as the ratio of shear stress to shear rate, the units of the variable are Pa.s. Typically, viscosity is reported in units of centipoise (cP; where $1 \mathrm{cP}=1 \mathrm{mPa} \bullet \mathrm{s})$.

$$
\eta(\dot{\gamma})=\frac{\tau(\dot{\gamma})}{\dot{\gamma}}
$$

For Newtonian fluids, the apparent viscosity is independent of shear rate (Eq. 4.5). Examples of the viscosity of common Newtonian materials can be seen in Table 4.2.

$$
\tau=\eta \dot{\gamma}
$$

where $\tau$ is the shear stress, $\eta$ is the Newtonian viscosity, and $\dot{\gamma}$ is the shear rate.

Fluids that do not behave as Newtonian fluids are referred to as non-Newtonian fluids. Rheograms or plots of shear stress versus shear rate are typically used to characterize non-Newtonian fluids. Examples of typical rheograms can be seen in Figure 4.2. 
Table 4.2. Viscosities of Several Common Newtonian Fluids

\begin{tabular}{||c|c||}
\hline Material & Viscosity at $\mathbf{2 0}^{\circ} \mathbf{C}(\mathbf{c P})$ \\
\hline Acetone & 0.32 \\
\hline Water & 1.0 \\
\hline Ethanol & 1.2 \\
\hline Mercury & 1.6 \\
\hline Ethylene Glycol & 20 \\
\hline Corn Oil & 71 \\
\hline Glycerin & 1,500 \\
\hline
\end{tabular}

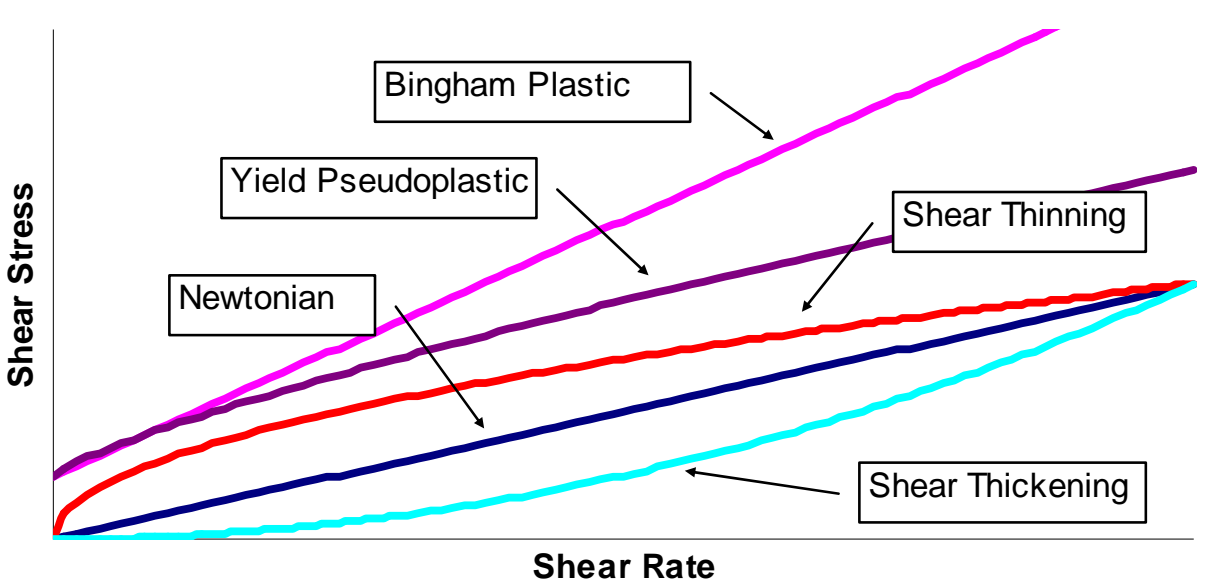

Figure 4.2. Rheograms of Various Fluid Types

Shear-thinning and shear-thickening fluids can be modeled by the Ostwald equation (Eq. 4.6). If $\mathrm{n}<1$, then the material is referred to as pseudoplastic (shear thinning). If $n>1$, then that material is referred to as dilatant (shear thickening). These fluids exhibit decreasing or increasing apparent viscosities as shear rate increases, depending on whether the fluid is shear thinning or shear thickening, respectively. Since shear-thickening flow behavior is rare, shear-thickening behavior is often an indication of possible secondary flow patterns or other measurement errors.

$$
\tau=m \dot{\gamma}^{n}
$$

where

$m \quad=$ the power law consistency coefficient

$n \quad=$ the power law exponent

$\dot{\gamma}=$ is the shear rate.

A rheogram for a Bingham plastic does not pass through the origin. When a rheogram possesses a non-zero y-intercept, the fluid is said to posses a yield stress. A yield stress is a shear-stress threshold that defines the boundary between solid-like behavior and fluid-like behavior. The fluid will not begin to flow 
until the yield stress threshold is exceeded. For Bingham plastic materials, once enough force has been applied to exceed the yield stress, the material approaches Newtonian behavior at high shear rates (Eq. 4.7).

$$
\tau=\tau_{B}+\eta_{P} \dot{\gamma}
$$

where $\tau_{B}$ is the Bingham yield stress, $\eta_{\mathrm{p}}$ is the plastic viscosity, and $\dot{\gamma}$ is the shear rate.

Fluids that exhibit a non-linear rheogram with a yield stress are typically modeled by the threeparameter Herschel-Bulkley equation (Eq. 4.8). Again, shear-thickening behavior is uncommon, and typically the Hershel-Bulkley power-law exponent is less than unity.

$$
\tau=\tau_{H}+k \dot{\gamma}^{b}
$$

where

$$
\begin{aligned}
\tau_{H} & =\text { yield stress } \\
\mathrm{k} & =\text { Herschel-Bulkley consistency coefficient } \\
\mathrm{b} & =\text { Hershel-Bulkley power law exponent } \\
\dot{\gamma} & =\text { shear rate. }
\end{aligned}
$$

An example of these rheological properties can be considered through a pipeline flow scenario through a 3-inch ID smooth pipe transporting 90 gallons per minute of fluid. This equates to an average pipeline velocity of $4.1 \mathrm{ft} / \mathrm{sec}$. The fluid is a Bingham plastic with a Bingham yield stress, $\tau_{B}$, of $30 \mathrm{~Pa}$, a Bingham consistency or plastic viscosity, $\eta_{\mathrm{p}}$, of $30 \mathrm{cP}$, and a slurry density of $1.2 \mathrm{~kg} / \mathrm{L}$. In this case, the fluid flow will be in the laminar regime with the velocity and apparent viscosity profiles shown in Figure 4.3. The flow profile reflects a "plug flow" regime where the center core of the fluid moves at constant velocity. This because the shear stress in this region does not exceed the yield stress of the fluid and acts as a solid material with an infinite apparent viscosity. At a radius of approximately 1.1 inches, the shear stress in the pipe exceeds the yield stress of the fluid and the fluid transitions from behaving as a solid to behaving as a "shear thinning" liquid. The apparent viscosity in the sheared region near the pipe wall (1.1-1.5 inch radius) drops from an infinite vale to approximately $100 \mathrm{cP}$ at the pipe wall. Pressure drop for flow under these conditions is calculated at $9 \mathrm{psig} / 100 \mathrm{ft}$ of straight horizontal pipe.

The case of a Newtonian fluid with the same pressure drop is then considered. At $90 \mathrm{gpm}$, a Newtonian viscosity of $300 \mathrm{cP}$ is required for a $9 \mathrm{psi} / 100 \mathrm{ft}$ pressure drop. The flow profiles for this system are shown in Figure 4.4. The flow profile shows a parabolic velocity profile that is characteristic of Newtonian, laminar pipe flows. The apparent viscosity in this case is constant at $300 \mathrm{cP}$ throughout the pipe radius. 


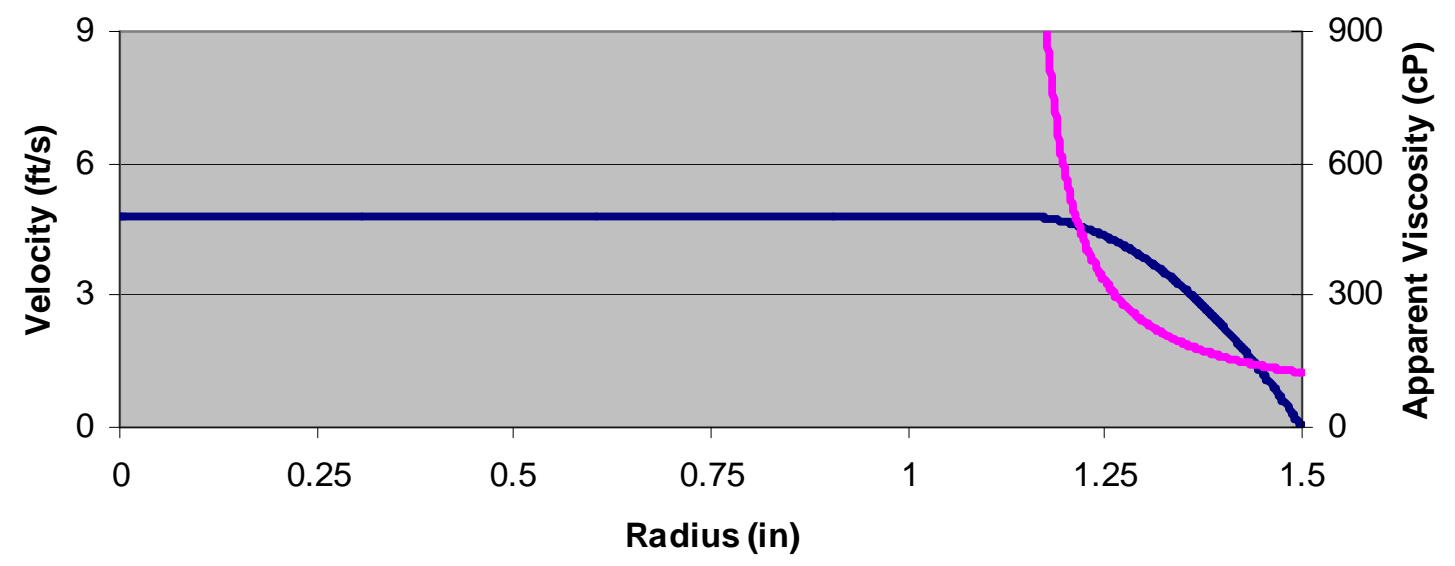

-Velocity —Apparent Viscosity

Figure 4.3. Example Flow Profiles for a Bingham Plastic Fluid ( $30 \mathrm{cP}$ consistency, 30 Pa yield stress) in a 3-inch-ID Smooth Pipe at 90 gpm

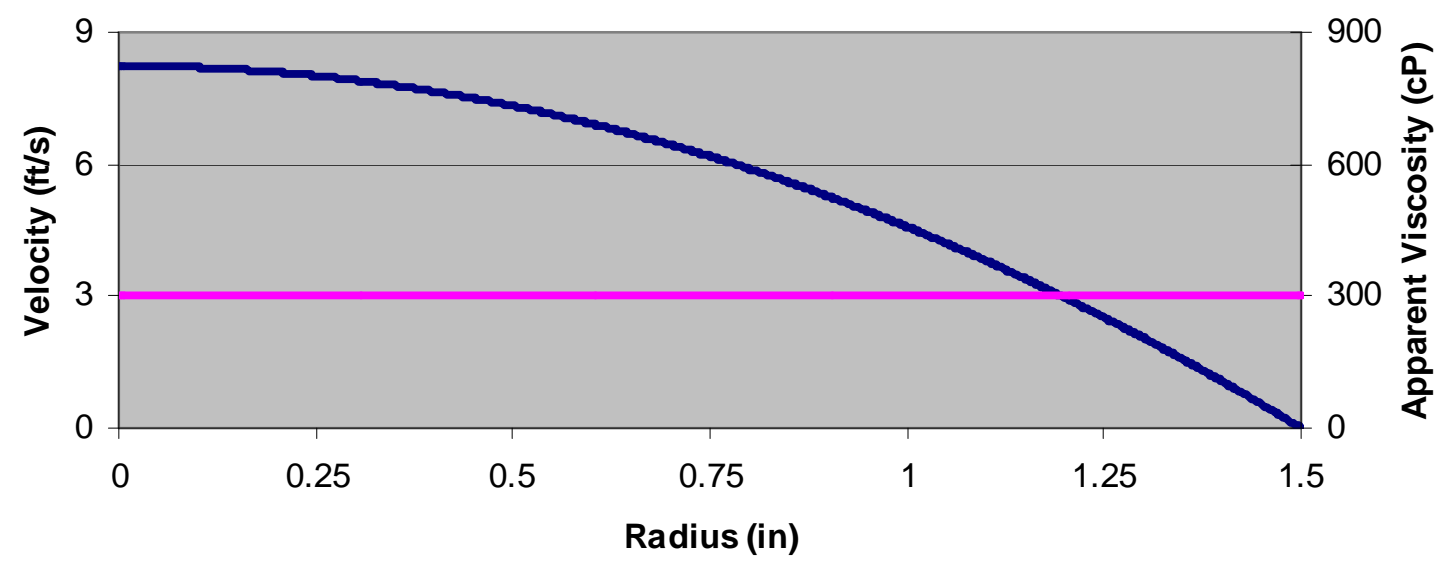

—Velocity —Apparent Viscosity

Figure 4.4. Example Flow Profiles for a Newtonian Fluid (30 cP viscosity) in a 3-inch-ID Smooth Pipe at $90 \mathrm{gpm}$ 


\subsection{Rheology Measurements}

Colloidal suspensions such as tank wastes exhibit a wide range of rheological behavior; therefore, rheological measurement of tank wastes requires a wide range of capabilities. Measurements of rheological properties of actual Hanford tank wastes have been performed in-situ using a falling ball rheometer and on core samples removed from the tanks using viscometers or rheometers. Rheological properties have also been calculated from extrusion data of tank waste core samples (slump tests).

\subsection{Falling Ball Rheometer (In Situ Rheology)}

The falling ball rheometer measures the drag force on a ball of known mass as it moves through the waste at various speeds from which rheology and density of the waste can be estimated. Physical models that form relationships are used to transform the drag force and velocity of the ball into fluid properties (density, viscosity, and yield strength). Different fluid types (Newtonian, Bingham plastic, or power law fluids) require different relationships. Details of the data reduction methodology are reported by Shephard et al. (1994).

The falling ball rheometer consists of a 71-N (16-lb), 9.12-cm diameter tungsten alloy ball tethered to a steel cable that is let out and retrieved from a spool at precise speeds using a computer-controlled drive system. A load cell measures the tension on the cable. A schematic of the system is shown in Figure 5.1.

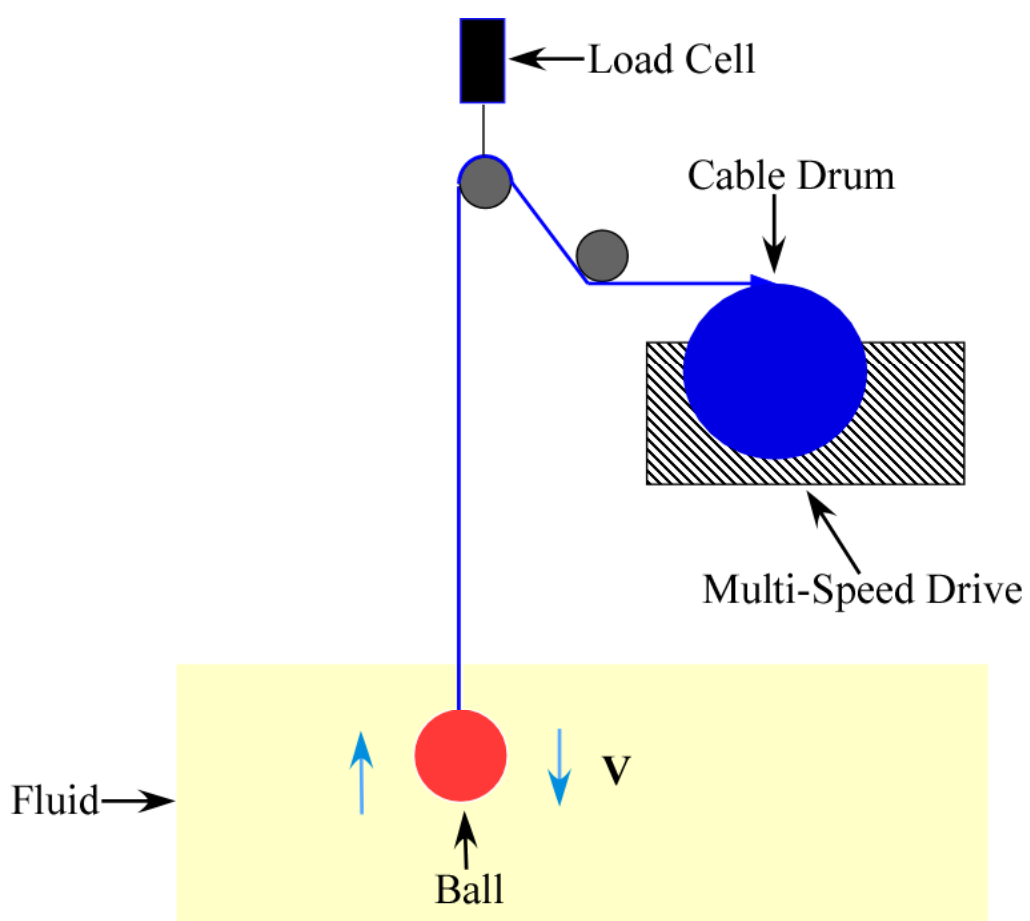

Figure 5.1. Schematic of the Falling Ball Rheometer 


\subsection{Laboratory Rheology Measurements}

The majority of the rheology data are obtained from measurements of samples removed from the Hanford tanks. These samples included push- and rotary-mode core samples, auger samples, and grab samples. Rheological properties of these samples were obtained using rheometers under varying conditions depending upon the viscosity of the sample. The measuring system of a rheometer consists of a fixed part and a rotating part. Rheological properties obtained by laboratory measurements include viscosity, yield stress, and shear strength. Viscosity and yield stress are determined from a plot of shear stress as a function of shear rate. Shear strength is obtained from measuring shear stress as a function of time at a low shear rate that is held constant.

\subsubsection{Viscosity and Yield Stress}

Viscosity and yield stress (also call yield strength) are measured by plotting shear stress as a function of shear rate (rheogram). An example of a typical rheogram is provided in Figure 5.2. Most of the rheograms for Hanford tank waste samples include a curve with increasing shear rate and a second curve with decreasing shear rate. Generally, some thixotropic behavior (i.e., time dependency and hysteresis) is observed in these two curves.

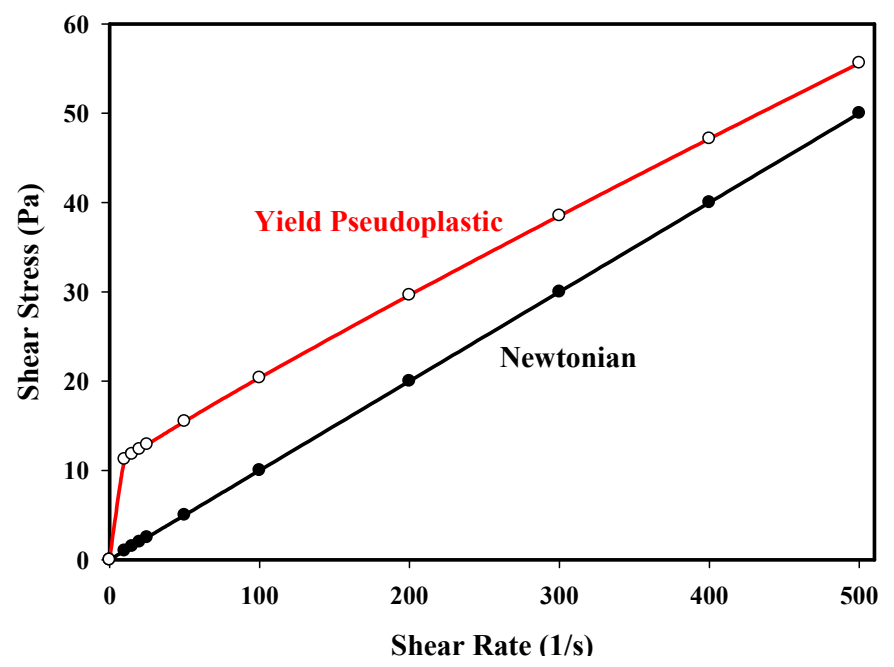

Figure 5.2. Rheogram of a Newtonian and Yield Pseudoplastic Fluid

Some rheometers measure shear stress as a function of shear rate (controlled-rate rheometers), while others measure shear rate as a function of shear stress (controlled-stress rheometers). Both types of systems were used to generate the data used in this report. Fixed and rotating parts of the rheometer varying according to the rheometer design and the viscosity of the sample. Both cone and plate and concentric cylinder geometries were used to measure tank waste samples. Rheograms were obtained at multiple temperatures and dilution levels (solids content) for many of the tank waste samples. Calibration of the systems was checked with Newtonian fluid standards of known viscosity. 
Empirical curve fits of the data in the rheograms were performed using well-accepted models. Four different models were used in these analyses. These models included Newtonian, Bingham Plastic, Power Law, and Herschel-Bulkley fits described in Section 4. Additional parameters are included in each of these successive curve fits to provide a better fit of the data. The mathematical equations for these curve fits are provided in Eq. (4.5) through (4.8).

Equation (4.5) is the Newtonian model. No yield stress is observed in Newtonian fluids, and viscosity is constant over the entire shear rate range. In this equation the slope of the line is the Newtonian viscosity.

Equation (4.7) is the Bingham plastic model where the fluid has a positive yield stress as indicated by a non-zero intercept with the ordinate (y-axis) followed by a linear increase in the shear stress as function of shear rate. The slope of this line is the Bingham viscosity. The difference between a Bingham plastic and a Newtonian fluid is the presence of a non-zero yield stress.

Equation (4.6) is the power law model (sometimes called the Ostwald equation). For Hanford tank wastes we limit the model fit to pseudoplastic or Newtonian materials (exponent less than or equal to one). When the exponent in the power law model is equal to one, the fluid is a Newtonian fluid and consistency coefficient is the Newtonian viscosity. A fluid with power law behavior does not have a yield stress.

Equation (4.8) is the Herschel-Bulkley model or yield power law curve fit. This model has the greatest number of parameters in the curve, but it is often more detailed than is needed to fit the data. In this model, a yield stress (non-zero intercept with the ordinate) is followed by pseudoplastic behavior (exponent is less than 1). If the yield stress is zero, this model becomes the power law model. If the exponent is one, this model becomes the Bingham plastic model.

\subsubsection{Shear Strength}

Shear strength was measured on core samples taken from the tanks using a shear vane of known dimension as the rotating part of the rheometer. Shear strength is a semi-quantitative measure of the force required to move the sample and is dependent on sample history. Shear strength can be measured directly by slowly rotating a vane immersed in the sample material and recording the resulting torque as a function of time. The measured torque is converted to a shear stress by Eq. 5.1 and 5.2.

$$
\tau=T / K
$$

where

$$
K=\frac{\pi D^{3}}{2}\left(\frac{H}{D}+\frac{1}{3}\right)
$$

where

$\tau=$ the calculated shear stress in Pascals

$\mathrm{T}=$ the measured torque in Newton-meters 
$\mathrm{K}=$ the shear vane constant in cubic meters

$\mathrm{D}=$ the shear vane diameter in meters

$\mathrm{H}=$ the shear vane height in meters.

A typical stress/time profile is shown in Figure 5.3. The profile shows an initial linear region $\left(\tau_{\mathrm{y}}\right)$ followed by a nonlinear region, a stress maximum $\left(\tau_{s}\right)$, and a stress decay region. The stress maximum is the transition between the visco-elastic and fully viscous flow. Shear strength is defined as the transition between these two flows and is measured at the stress maximum.

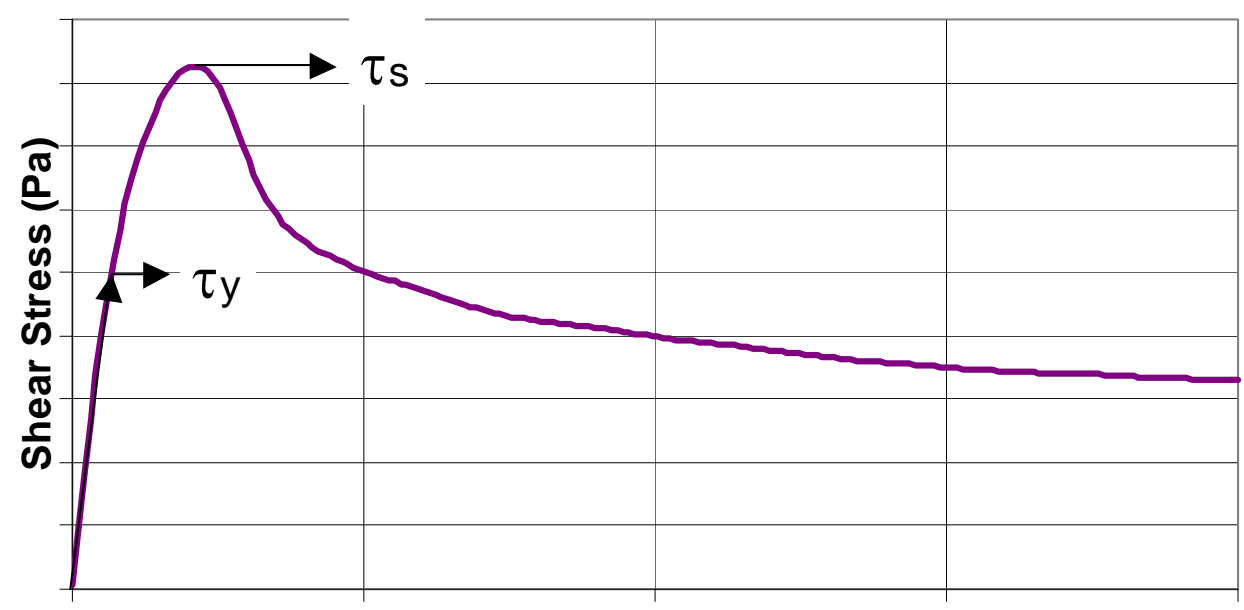

Time

Figure 5.3. Typical Stress-Versus-Time Profile for a Shear Vane at Constant Shear Rate

Shear strength was measured on core samples, tank composites, and dilutions that had measurable shear strengths. The diameter and height of the shear vane are typically 1.6 and $3.2 \mathrm{~cm}$, respectively, but other sizes have been used. Details of the vanes are available in the characterization reports. The rotation speed of the shear vane was constant (generally at $0.3 \mathrm{rpm}$ ).

To minimize history effects, the shear strength samples were often placed in the sample cup a minimum of 48 hours before the measurement. Sometimes the shear strength measurement was repeated one hour after the initial measurement to provide information about the effect of previous shear on the shear strength of these materials.

\subsection{Shear Strength Calculation from Extrusion Data}

Gauglitz and Aikin (1997) developed a methodology to estimate the shear strength of tank waste materials based on visual observations of horizontal extrusion behavior. A related core extrusion shear strength estimation technique was developed by Rassat et al. (2003). This technique is based strictly on extrusion length and was developed from the simulant extrusion results presented by Gauglitz and Aikin (1997). 
An extrusion system has also been developed to make these observations on smaller quantities of waste. In these measurements an infusion syringe pump was modified to mimic the extrusions performed on the core samples from the Hanford tanks (Figure 5.4).

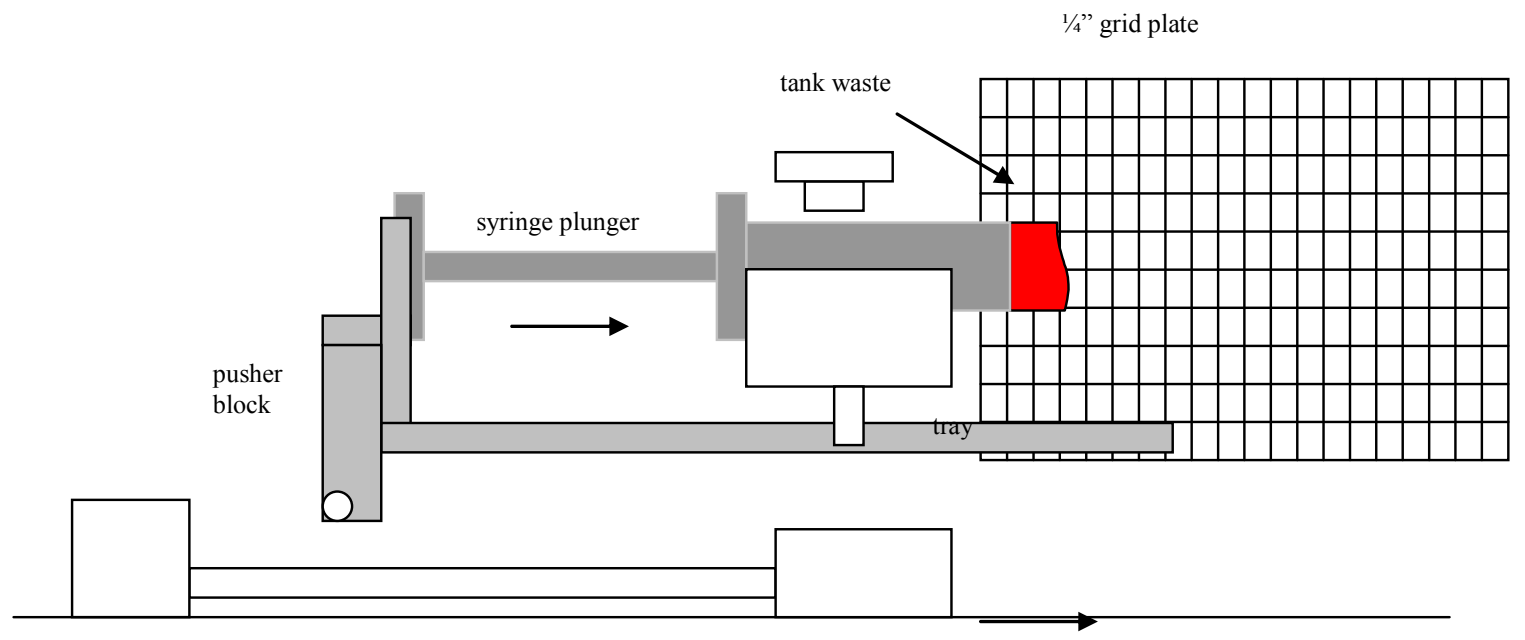

Figure 5.4. Mini-Extrusion Design

The pump was controlled by a microstepping motor drive. The motor drive pushed the pusher block against the syringe plunger, displacing the sample. The syringe pump was modified by the addition of a tray mounted directly to the pusher block. As the core was being extruded, the tray moved at the same speed the core was being pushed out.

For the extrusions, 10-mL Becton Dickinson syringes were used. The tips of the syringes were cut off so that the extrusion core would be the inner diameter of the syringe barrel. The cores were approximately $7 \mathrm{~cm}$ in length and $1.45 \mathrm{~cm}$ in diameter. The height from the bottom of the core to the tray was approximately $1.5 \mathrm{~cm}$. The rate at which the core was extruded was $0.5 \mathrm{in} . / \mathrm{min}$. The syringe was filled with the sample in $0.5-\mathrm{mL}$ increments. A microspatula was used to fill the syringe and remove voids in the sample. The syringes were filled a minimum of 72 hours prior to the extrusion. Parafilm was used to minimize drying of the sample. The samples were also placed in a closed plastic bag.

During the extrusions, video images were recorded and then analyzed to estimate the shear strength. To estimate the lengths of the extruded cores, a $6 \times 3$-inch grid plate was fabricated and attached to the syringe pump. The camera was placed directly in front of the syringe at a location to capture the entire extrusion. During the extrusions, the camera was not moved. 


\subsection{Physical Properties}

Often, physical properties were measured on the same samples used to make rheological measurements. These properties are crucial in correlating the rheological properties of the suspensions in the WTP mixing systems. The physical properties of particular importance in this study include density of the suspension, centrifuged supernatant, centrifuged and settled solids; total solids content in the suspension as well as in the centrifuged and settled solids (both volume and wt\% solids); dissolved and undissolved components of the total solids; and zeta potential. Very few data are available on zeta potential of Hanford tank wastes because of the high salt content in the supernatants, which make this measurement difficult on the standard zeta potential instruments available during characterization of these samples. Some of the other physical properties were not measured routinely on all samples; therefore, physical properties for many samples are incomplete.

\subsection{Density}

Density of the bulk sample, supernatant, centrifuged solids, and settled solids generally was calculated from volume and mass measurements. Details of these measurements are provided in the individual reports referenced for each sample. Generally, aliquots of the samples were placed in graduated centrifuge cones or in graduated cylinders and the sample was weighed. After allowing the samples to settle, the volume of the supernatant and solids was determined using the graduations on the centrifuge cones or graduated cylinders. When settled solids density was measured, the supernatant was decanted and placed in a graduated cylinder and weighed to determine the mass of the supernatant. The volume of the supernatant was also measured in the graduated cylinder. The settled solids were also weighed after decanting the supernatant. Settled solids and supernatant density were calculated by dividing the mass by the volume. Settled solids density was sometimes calculated without weighing the settled solids according to Eq. (6.1). In this calculation the density of the centrifuged supernatant was often used as the density of supernatant density used in Eq. (6.1).

$$
\rho_{\text {settled solids }}=\frac{m_{\text {sample }}-\left(\rho_{\text {sup }} \times V_{\text {sup }}\right)}{V_{\text {settled solids }}}
$$

where

$\rho_{\text {settled solids }}=$ the density of the settled solids

$\mathrm{m}_{\text {sample }} \quad=$ the mass of the sample used in the measurement

$\rho_{\text {sup }} \quad=$ the density of the settled supernatant

$\mathrm{V}_{\text {sup }} \quad=$ the volume of settled supernatant

$\mathrm{V}_{\text {settled solids }}=$ the volume of settled solids.

The supernatant was returned to the centrifuge cone, or a new aliquot of the sample was placed in a graduated centrifuge cone, and the sample was centrifuged to separate the liquid (supernatant) in the suspension from the solids. The sediment volume was measured on each sample at the completion of the centrifugation. The supernatant was decanted from the centrifuge cones and transferred to a graduated cylinder. The volume and mass of the decanted supernatant were measured to determine the supernatant 
density. The mass of the sediment remaining in the centrifuge cone was also measured. The densities of the supernatant, centrifuged solids, and bulk sample were often calculated from centrifugation data.

In situ liquid density data were obtained by the falling ball rheometer. A reference measurement of the ball in air is subtracted from the density measurements to adjust for the baseline forces associated with measurement. Both ball volume and buoyancy forces are used to calculate the density of waste, as shown in Eq. (6.2). This method is not applicable for materials with yield strengths because the strength of the material supports the ball resulting in an inaccurate buoyancy term (Stewart et al. 1996).

$$
\rho=\frac{B_{b}}{V_{b} \times g}
$$

where

$$
\begin{aligned}
& \rho=\text { the density of the liquid } \\
& \mathrm{B}_{\mathrm{b}}=\text { the buoyancy force on the ball } \\
& \mathrm{V}_{\mathrm{b}}=\text { the volume of the ball } \\
& \mathrm{g}=\text { the gravitational constant. }
\end{aligned}
$$

\subsection{Solids Content}

Weight percent total solids in the core samples was determined by either thermogravimetric analysis or simple gravimetric analysis methods. Both methods measure the change in the mass as the sample is heated. The mass loss is equated to the mass of water in the sample; therefore, the solids content is equal to the mass remaining in the sample after heating divided by the initial mass of the sample. Solids content is often reported as a percentage of the mass change instead of the fraction of the mass change. Solids content can also be reported in volume percent (volume of the dried solids divided by the volume of the initial sample reported as a percentage). Volume percent solids is often converted from the mass percent solids using the measured densities of the solids and supernatant.

In thermogravimetric analysis, approximately $25 \mathrm{mg}$ of each sample was placed in a platinum, aluminum, or stainless steel pan, and the temperature of the sample was increased from ambient to approximately $550^{\circ} \mathrm{C}$ at a constant rate (generally $5^{\circ} \mathrm{C} /$ minute). The mass of the sample (by thermogravimetric analysis) and the change in the temperature of the sample (by differential thermal analysis) in relation to a reference sample (an empty pan of the same metal) were monitored as a function of temperature. These analyses were performed in a flowing air or helium atmosphere. The calibration of the thermal analysis system was checked with a lead or indium standard and calibrated weights. The thermogravimetric approach is typically used to distinguish free water from waters of hydration and, possibly, decomposition of solids at the higher temperatures.

A simpler gravimetric weight percent solids measurement is made by drying samples of known mass in a drying oven at $105^{\circ} \mathrm{C}$. Greater than $2 \mathrm{~g}$ of material were weighed and then dried to obtain the total solids concentration. The mass of the sample before and after drying was measured.

Dissolved solids and undissolved solids content was calculated from the solids content measured in the supernatant (dissolved solids), total solids content (solids content of the bulk sample), and the fraction 
of supernatant in the sample measured after centrifugation or filtration. The equations used for calculating weight percent dissolved solids and undissolved solids are shown in Eq. (6.3) and (6.4), respectively. Weight percent undissolved solids is converted to volume percent using the densities of the undissolved solids and bulk sample.

$$
\begin{gathered}
\operatorname{DS}(w t \%)=\left(\frac{\text { Supernatant mass }}{\text { Sample mass }}\right) \times \text { Supernatant Solids Content } \\
U D S(w t \%)=T S(w t \%)-D S(w t \%)
\end{gathered}
$$

where

DS $=$ the percent dissolved solids

UDS $=$ the percent undissolved solids

TS $=$ the percent total solids.

\subsection{Zeta Potential}

Zeta potential is the electrostatic potential at the surface of shear and is calculated from the electrophoretic mobility (velocity per unit of electric field strength). The surface of shear is not the surface of the particle, but extends from the particle out into solution to include the solution that moves with the particle (hydrodynamic size). A positive mobility (zeta-potential) means the surface of the particle is positively charged, a negative mobility means the surface is negatively charged, and a mobility value of zero means that the velocity is zero, which implies that electrostatic repulsion is small.

A charged solid-liquid interface (electrical double layer) is created between the charged particle and the liquid. In this electrical double layer, the concentration of the counter ions (ions in solution with the opposite charge as the particle surface) is higher near the particle and decrease steadily to the concentration in the bulk liquid. The thickness of this double layer is dependent upon the temperature, the dielectric constant of the liquid, and the ionic strength of the bulk liquid. For 1:1 electrolytes such as $\mathrm{KCl}$ at a bulk concentration of $1 \mathrm{mM}$, the double-layer thickness is approximately $10 \mathrm{~nm}$. As the concentration of the electrolyte increases, the double layer thickness and the range of the repulsive forces between the molecules decreases.

Zeta potential is calculated from electrophoretic mobility using a theoretical model. Two classic models (Hückel and Smoluchowski equations) are used for these calculations. The Hückel equation is used for simple ions where the hydrodynamic radius is very small, and the Smoluchowski equation is used for very large particles. For most colloidal particles of interest dispersed in water, it is not easy to satisfy either model; therefore, mobility is related to zeta potential by a model-dependent function. Generally, these functions are more accurate for $1: 1$ electrolytes at $10^{-3}$ to $10^{-2} \mathrm{M}$ salt.

The zeta potential of tank waste was measured by the Brookhaven Instrument Corporation's ZetaPlus zeta potential analyzer. In this system, a laser beam passes through the dilute sample between two electrodes that create an electric field. The particles in solution scatter the laser light as they move in this electric field. 
The light that is scattered by the particles is Doppler-shifted because the scattering particles are moving in the electric field due to the charge on their surface. The Doppler shift is proportional to the velocity (both rate and direction) of the particles in this electric field, which is proportional to the electrophoretic mobility of the particles (Eq. 6.5).

$$
\mathbf{V}_{\mathbf{s}}=\mu_{\mathrm{e}} \mathbf{E}
$$

where

$\mathbf{V}_{\mathbf{s}}=$ the average velocity of the $\mathrm{TiO}_{2}$ particles

$\mu_{\mathrm{e}}=$ the electrophoretic mobility

$\mathbf{E}=$ the electric field.

Because the ionic strength in most tank waste samples is extremely high (salt contents $>1 \mathrm{M}$ ), zeta potential was difficult to measure in the standard instruments available when most samples were characterized. 


\subsection{Sedimentation Measurements}

Settling behavior of tank waste samples including dilutions of tank waste samples was determined by both gravity settling and centrifugation. Aliquots of the samples were allowed to settle in graduated cylinders or graduated centrifuge cones. The sediment volume and total volume of the sample aliquots were recorded as a function of time. In a few reports the height of the sediment bed and total sample were also recorded as a function of time. The samples were then centrifuged and the volume of the centrifuged supernatant and centrifuged solids were recorded. Details of the time and force used in centrifugation are reported in the individual characterization reports. The mass and volume of clear supernatant, centrifuged and settled solids, and total sample were generally determined for each aliquot at the completion of settling and centrifugation.

As the samples settled, an interface developed between the turbid suspension and clear supernatant. The sediment volume is the volume from the bottom of the suspension column to the interface between the clear supernatant and the cloudy suspension. Under the force of gravity, the solids in the suspension sank to the bottom of the cylinder, forming a sludge layer and a clear supernatant layer. The final sediment bed volume was measured after no significant change was observed in the height of this sludge layer over the prescribed time described in the individual characterization reports. The volume percent settled solids were then determined by dividing the final sediment bed volume by the total volume of the slurry.

The settling rate measured by this method is controlled by the settling rate of the smallest particles in the suspension. In a suspension with particles of uniform size, all of the particles will settle at the same rate, and a sharp boundary will exist between the clarified portion of the settling system and the fraction of the system where the particles are still settling. Hanford tank wastes are polydisperse systems, where each size fraction or material type (different particle densities) settles at its own characteristic velocity. The boundary between the clarified supernatant and the settling fraction of the system may be a diffuse region. The rate at which a particle settles in a suspending liquid depends on the size, shape, solvation, and density of the particle as well as the density and viscosity of the suspending medium. Stokes law provides a mathematical expression of the terminal settling velocity for spherical particles (Eq. 7.1). Asymmetry in the particle shape and solvation of the particle increase the friction factor of the settling particle, decreasing the settling rate of a particle of given mass compared to a spherical, unsolvated particle.

$$
v=\frac{2 r^{2}\left(\rho_{p}-\rho\right) g}{9 \eta}
$$

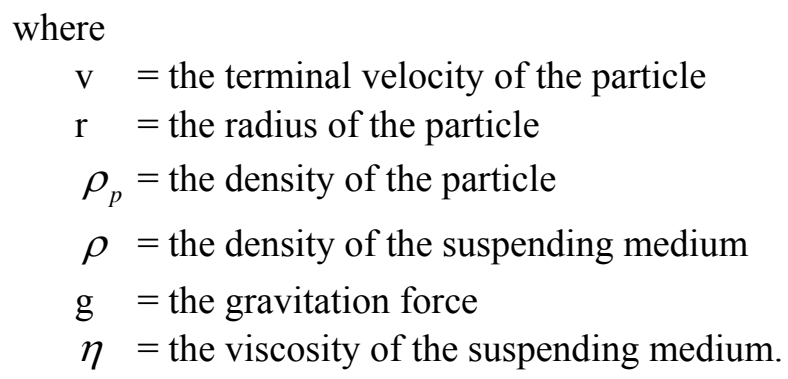


Interstitial liquid associated with the settled solids was further separated from the solids by centrifugation. The sediment volume was measured on each aliquot as function of time at a given force. Data for many samples were only recorded at a single duration. The volume percent centrifuged solids was then determined by dividing the sediment volume by the total volume of the slurry. The supernatant was decanted from the centrifuge cones, and the decanted supernatant and sediment were weighed. The weight percent supernatant and centrifuged solids were calculated by dividing their mass by the mass of the aliquot. 


\subsection{Rheology and Physical Properties Data}

Appendix A is a compilation of the available rheology data for Hanford tank wastes. These data include in situ as well as laboratory analysis of samples removed from the tanks. If rheology data were available for samples, the physical properties of these samples were included with the rheology data. Settling rate as a function of time was included when available. Composition of the solids phases as determined by ESP was also included for each rheology sample.

The tables in Appendix A are organized by tank with a column for each sample for which rheology was obtained. There is a brief description of each sample along with a reference for the source of the rheology data. The model or basis used for interpreting the solid composition data is based on the ionic strength of the supernatant. The choices for this basis included salty, salt free, washed, leached, liquid, and supernatant. No distinction is made between salt-free and washed or liquid and supernatant.

\subsection{Model Basis}

Salty is used to describe samples that were untreated; therefore, it is assumed that the supernatant and solids composition is not changed from what is observed in tank. Rheology measurements on many samples were performed at multiple temperatures, and measurements made near tank temperature will be closest to this approximation. As temperatures decrease, dissolved solids may precipitate, and the solid content may increase. At elevated temperatures, solids may dissolve in the suspending medium, and the concentration of undissolved solids may decrease.

Salt free and washed models describe samples that were washed or diluted with water or supernatant. Washing or water dilutions decrease the salts in solution and may result in decreased undissolved solids contents. Total solids content will also decrease upon washing or diluting the sample. Washing the waste may also selectively remove specific solids from the settled solids. Because of changes in the composition of the solids and supernatant, washing and diluting tank wastes may significantly affect the rheological properties of the samples.

Leached model describes those tank samples that were washed in dilute $\mathrm{NaOH} / \mathrm{NaNO}_{2}$, followed by washing in concentrated $\mathrm{NaOH}$ to remove $\mathrm{Cr}, \mathrm{Al}$, and $\mathrm{P}$ from the sample, and then washing with dilute $\mathrm{NaOH} / \mathrm{NaNO}_{2}$ to remove any leached $\mathrm{Cr}, \mathrm{Al}$, or $\mathrm{P}$ remaining in the sample. The removal of $\mathrm{Al}$ and $\mathrm{P}$ from the sample may significantly affect the rheology of the suspension.

Liquid and supernatant models were used to describe the supernatant obtained from centrifugation or settling of slurries. They also describe core and dip samples that consisted of liquid or supernatant. These samples provide additional information on the rheological properties of the suspending medium. 


\subsection{Solid Phases}

The average compositions of the solid and liquid phase in each tank were included in the tables in Appendix A. These compositions were derived from modeling carried out using the $\operatorname{ESP}(\mathrm{a})$ chemical thermodynamic model as described in WTP-RPT-153 (Wells et al 2007). The ESP predictions constitute the only phase composition information that 1) is available for all 177 tanks and 2) was prepared using a consistent method for all 177 tanks. It was therefore appropriate and advantageous to draw on this database in devising a sludge phase composition for transfer system design. However, this application of ESP had certain characteristics that should be noted:

- Compositions were calculated on a whole-tank basis, as if all the different layers of waste had been mixed and allowed to come to equilibrium.

- ESP is an equilibrium model and is not expected to predict the correct concentration of any compounds that have not yet come to equilibrium with an in-tank chemical environment different from those in which they formed (e.g., different temperature, $\mathrm{pH}$, etc.).

- In the 2002 study, certain compounds were excluded from precipitating to reflect kinetic limitations or sometimes to reduce computational time or avoid nonconvergence of the solution algorithm. A significant example is boehmite, which was excluded because, had it been included, it would have been thermodynamically preferred to gibbsite in all wastes. Because gibbsite is actually dominant due to kinetic constraints that prevent boehmite from forming at lower temperatures, the databank excluded boehmite from forming at a temperature less than $100^{\circ} \mathrm{C}$.

- Because of computational time constraints, Redox equilibrium was not calculated on a tankby-tank basis in the 2002 study; rather, expert judgment and generic-composition runs of ESP were used to fix the metal oxidation states in all tanks. Iron was fixed as $\mathrm{Fe}^{+3}$, manganese as $\mathrm{Mn}^{+2}$, chromium as $\mathrm{Cr}^{+3}$ or $\mathrm{Cr}^{+6}$, and so forth. Thus, the ESP predictions could not include compounds formed by metals in any other oxidation states.

- The study assigned compounds to the "trace analytes" (including thorium, cadmium, copper, tin, and many others) without employing the ESP model; thus, these metals are not present in the compounds in the ESP-predictions database.

- Thermodynamic data were not available for all the compounds that could potentially form in the tank waste, which led to the omission of some compounds.

The solids predicted by the ESP chemical thermodynamic model for the tank-average solid phase were taken as a best approximation, although [as noted in Wells et al. (2007)] there were cases in which the ESP model predicted solids that were not observed, and others in which observed solids were not predicted. Only one compound modification was made to the ESP output: all aluminum hydroxide predicted by ESP in tanks containing Redox boiling waste was substituted with boehmite on an equivalent-Al basis, as in Wells et al. (2007). Aluminum hydroxide/oxide predicted in other tanks was considered to be gibbsite.

(a) ESP was supplied and developed by OLI Systems, Inc., Morris Plains, New Jersey. 
The ESP model, as used, predicted the concentration of each solid in the waste on a normalized basis. In other words, the model predicted the relative masses of different solids, and the relative volumes and masses of total liquid and total solid, but not the absolute masses or volumes in a tank. The absolute mass of each solid-phase compound in a tank was calculated for the present study by combining the modified ESP results with BBI volumes, using Eq. (8.1):

$$
M_{S}=c_{S} V \phi_{E S P}
$$

where

$c_{S} \quad=$ mass of compound per volume of solid phase

$\mathrm{V}_{\mathrm{S}} \quad=$ solid phase volume in the tank

$\phi_{\mathrm{ESP}} \quad=$ ESP-predicted fraction of solid phase, average of all the waste in the tank

$\mathrm{V}=$ total waste volume in the tank as defined by the $2002 \mathrm{BBI}$.

The solid volume calculated by the above equation contains some uncertainty because of uncertainty in the parameters and because potential retained gas volume is not accounted for. The portion of the volume $V$ that is not solid phase is liquid volume, which includes both the interstitial liquid in the bulk solids layers and any supernatant that may be present. The volume of each compound in the solid phase was calculated by dividing the mass of each compound by the density. All the densities discussed were taken from the Handbook of Chemistry and Physics (CRC 1975), as in Wells et al. (2007), except where otherwise noted.

The ESP-predicted solid-phase compositions were on a "salty" basis; that is, the water-soluble sodium salt solids were included. These compositions were used to calculate a second composition that was on a "salt-free" basis, where only the insoluble metal compounds were retained in the solid phase.

Physical property measurements from tank characterization reports were used to provide the mass fraction of undissolved solids for a particular slurry sample. This was multiplied by the ESP solid phase speciation to produce the mass fraction of an ESP species in the slurry sample. Slurry densities from physical property measurements along with literature values for crystal density of the ESP phases were then used to convert the mass fraction to volume percent undissolved solids in the slurry sample.

\subsection{Rheological Data}

Rheological data included the temperature, instrument, source of the data, and all applicable model fits for the rheological data. Details about the rheological measurements can be obtained from the individual references. Correlation coefficients for each curve fit are also included with each model used for the available data. A description of the curve fits is provided in Section 4, Rheology Theory.

\subsection{Physical Property Data}

Physical property data may be obtained from a separate reference than was used to report the rheology data, but physical properties data do represent the properties of the sample used to perform the rheology. The physical properties reported included bulk, supernatant, centrifuged solids, settled solids, 
and particle (listed as solids) densities; volume and weight percent settled solids (also listed as settled supernatant which is just $100 \%$ minus the percent settled solids) and centrifuged solids; and solids content of the supernatant (dissolved solids), centrifuged sludge, and bulk sample (total solids); and the percent undissolved solids. Zeta potential is also listed in the table, but only minimal data were obtained on zeta potential of tank waste samples. A detailed description of these measurements is provided in Section 6 . If available, the temperature at which these physical properties were measured was also reported. Details of these data are provided in the reference associated with each sample.

\subsection{Settling Data}

Gravity settling data is reported as the volume of the sediment bed as a function of time (up to 72 hours) for each sample. The total volume of the aliquot in the settling column (initial volume) is also recorded for each sample. Settling data is also reported for multiple temperatures if it is available. A more detailed description of the method used for gravity settling is described in Section 7.

\subsection{Particle Size Data}

Particle size data used in this report are obtained from a report prepared earlier this year, Estimate of Hanford Waste Insoluble Solid Particle Size and Density Distribution (Wells et al. 2007). No modifications were made to the data reported in that document. The data reported in the tables are the particle diameter for cumulative volume percents ranging from 1 to $99 \%$.

\subsection{Shear Strength Data}

Shear strength data are reported as a function of gelation time. Only limited data were obtained at discrete gelation times. Most of the shear strength data were obtained for samples that had remained undisturbed for a minimum of three days. Inserting the shear vane did not constitute disturbing the sample for this report. A more detailed description of the methods used to obtain shear strength is provided in Section 5. The data reported include measurements from the ball rheometer and laboratory measurements using a vane geometry. Calculation of shear strength from core extrusions is not included in this report. 


\subsection{Viscosity Model}

Hanford liquid supernatant can be characterized rheologically as a Newtonian fluid. While the chemical composition of the aqueous waste has a significant effect on the viscosity of the supernatant liquid, the model described in this section attempts to model the Hanford supernatant viscosity as a function of only liquid density and temperature. Liquid density was selected as an independent variable in this model because it is relatively easy to measure and directly scales with dissolved species concentration.

Temperature is known to have a large effect on liquid viscosity. The Andrade correlation is a mathematical relationship often used when evaluating the relationship between increasing temperature and decreasing viscosity of liquids. It is not accurate for high-temperature fluids or highly polar mixtures. This equation is shown as Eq. (9.1):

$$
\eta=A e^{\frac{B}{T}}
$$

where

$$
\begin{array}{ll}
\eta & =\text { the Newtonian viscosity in } \mathrm{cP} \\
T & =\text { temperature in Kelvin }(\mathrm{K}) \\
A(\text { in } \mathrm{cP}) \text { and } B(\text { in } \mathrm{K}) & =\text { fitting parameters associated with molecular structure. }
\end{array}
$$

A plot of the viscosity of water as a function of temperature is shown in Figure 9.1. For water the Andrade equation parameters are $0.0019 \mathrm{cP}$ and $1800 \mathrm{~K}$ for $A$ and $B$, respectively.

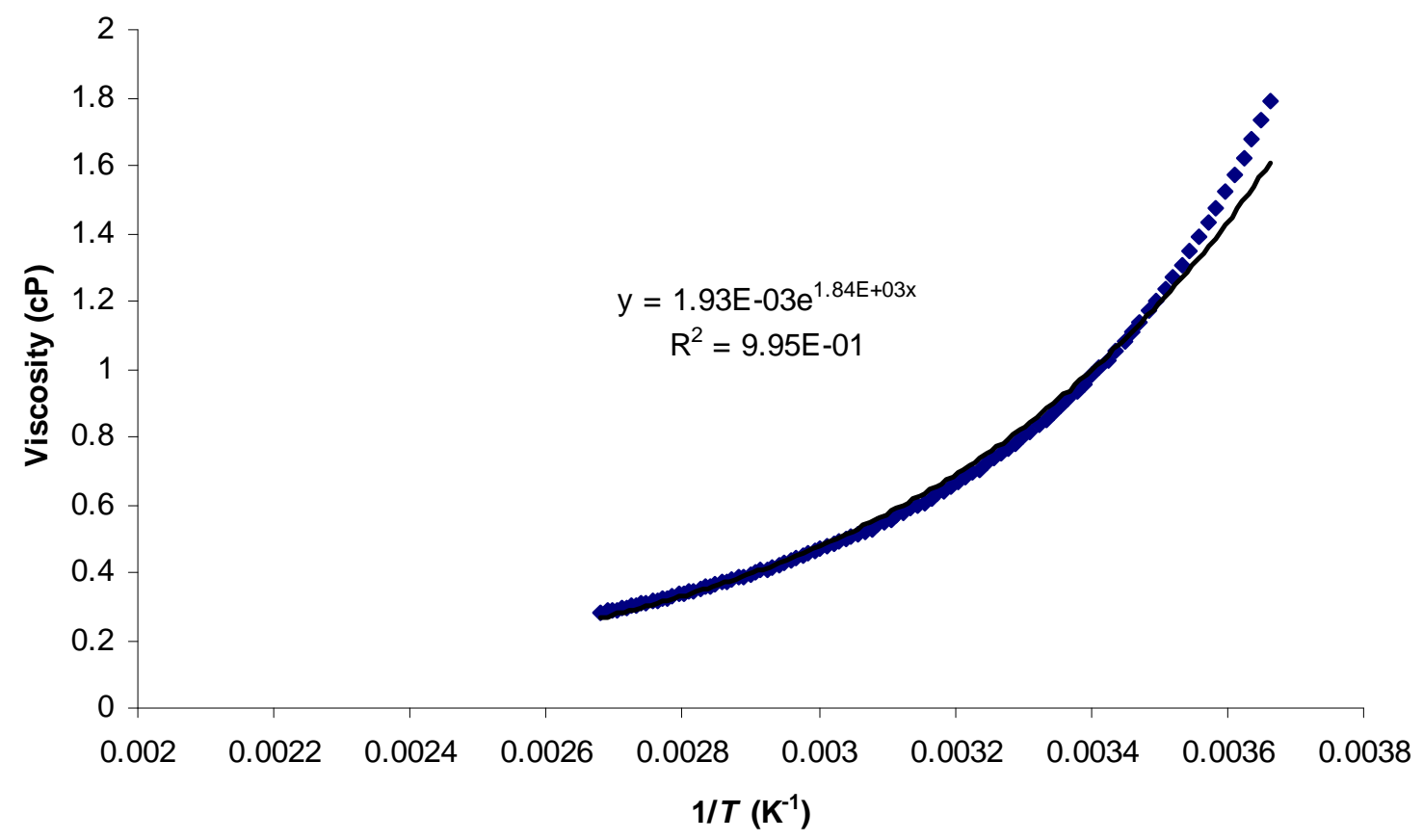

Figure 9.1. Viscosity of Water at Various Temperatures 
Liquid viscosity data from 8 different Hanford tanks was analyzed as a function of liquid density, temperature, and dilution with water (salt content). The tanks for which this data was available are listed in Table 9.1. Relative volumes of the waste types comprised by these tanks are shown in Figure 9.2. Several sludge waste types are not represented in this analysis due to a lack of appropriate data.

Table 9.1. Liquid Viscosity Data for Hanford Tank Wastes

\begin{tabular}{|c|c|c|c|c|}
\hline Tank & Density $(\mathrm{g} / \mathrm{mL})$ & Dilution & $\begin{array}{l}\text { Temperature } \\
\left({ }^{\circ} \mathrm{C}\right)\end{array}$ & Viscosity (cP) \\
\hline \multirow[t]{12}{*}{ 241-AN-103 } & 1.42 & \multirow{3}{*}{$\begin{array}{l}\text { Supernatant from } \\
80 \% \text { Dilution of Settled Solids }\end{array}$} & 29 & 11.6 \\
\hline & 1.42 & & 45 & 6.7 \\
\hline & 1.42 & & 65 & 4.6 \\
\hline & 1.46 & \multirow{3}{*}{$26 \%$ Dilution of Supernatant } & 29 & 7.8 \\
\hline & 1.46 & & 45 & 5.4 \\
\hline & 1.46 & & 65 & 3.7 \\
\hline & 1.52 & \multirow{3}{*}{$10 \%$ Dilution of Supernatant } & 29 & 8.8 \\
\hline & 1.52 & & 45 & 5.9 \\
\hline & 1.52 & & 65 & 4 \\
\hline & 1.58 & \multirow{3}{*}{ Undiluted Supernatant } & 29 & 27.4 \\
\hline & 1.58 & & 45 & 13 \\
\hline & 1.58 & & 65 & 7.6 \\
\hline \multirow{7}{*}{ 241-AN-104 } & 1.26 & Undiluted Permeate & 25 & 3.5 \\
\hline & 1.31 & \multirow{3}{*}{$\begin{array}{l}\text { Supernatant from } \\
80 \% \text { Dilution of Settled Solids }\end{array}$} & 25 & 17.5 \\
\hline & 1.31 & & 45 & 8.1 \\
\hline & 1.31 & & 65 & 5.4 \\
\hline & 1.42 & \multirow{3}{*}{ Undiluted Supernatant } & 25 & 16.9 \\
\hline & 1.42 & & 45 & 8.1 \\
\hline & 1.42 & & 65 & 4.6 \\
\hline \multirow[t]{6}{*}{ 241-AN-105 } & 1.35 & \multirow{3}{*}{$20 \%$ Dilution of Supernatant } & 25 & 9.4 \\
\hline & 1.35 & & 45 & 4.2 \\
\hline & 1.35 & & 65 & 2.4 \\
\hline & 1.42 & \multirow{3}{*}{ Undiluted Supernatant } & 25 & 12.3 \\
\hline & 1.42 & & 45 & 6.3 \\
\hline & 1.42 & & 65 & 3.5 \\
\hline \multirow{2}{*}{ 241-AP-104 } & 1.26 & \multirow{2}{*}{ Undiluted Supernatant } & 25 & 3.7 \\
\hline & 1.26 & & 40 & 2.4 \\
\hline \multirow[t]{9}{*}{ 241-AW-101 } & 1.38 & \multirow{3}{*}{$\begin{array}{c}\text { Supernatant from } \\
50 \% \text { Dilution of Tank Composite }\end{array}$} & 25 & 11.5 \\
\hline & 1.38 & & 45 & 5.4 \\
\hline & 1.38 & & 65 & 3.6 \\
\hline & 1.42 & \multirow{3}{*}{$\begin{array}{l}\text { Supernatant from } \\
80 \% \text { Dilution of Settled Solids }\end{array}$} & 25 & 6.0 \\
\hline & 1.42 & & 45 & 3.0 \\
\hline & 1.42 & & 65 & 1.8 \\
\hline & 1.52 & \multirow{3}{*}{ Undiluted Supernatant } & 25 & 22.0 \\
\hline & 1.52 & & 45 & 8.0 \\
\hline & 1.52 & & 65 & 5.4 \\
\hline \multirow{3}{*}{ 241-AY-102 } & 1.13 & \multirow{3}{*}{ Centrifuged Liquid Composite } & 27 & 1.8 \\
\hline & 1.13 & & 45 & 1.1 \\
\hline & 1.13 & & 65 & 0.3 \\
\hline \multirow[t]{3}{*}{$241-C-104$} & 1.24 & \multirow{3}{*}{ Undiluted Supernatant } & 25 & 1.3 \\
\hline & 1.24 & & 45 & 1.3 \\
\hline & 1.24 & & 65 & 1.4 \\
\hline \multirow{6}{*}{ 241-S-112 } & 1.16 & & 20 & 2.0 \\
\hline & 1.16 & $20 \%$ Dilution of 2 nd Dissolution Contact ${ }^{(\mathrm{a})}$ & 35 & 1.8 \\
\hline & 1.16 & & 50 & 1.4 \\
\hline & 1.36 & & 20 & 5.7 \\
\hline & 1.36 & $20 \%$ Dilution of 1 st Dissolution Contact ${ }^{(\mathrm{b})}$ & 35 & 4.0 \\
\hline & 1.36 & & 50 & 2.9 \\
\hline
\end{tabular}




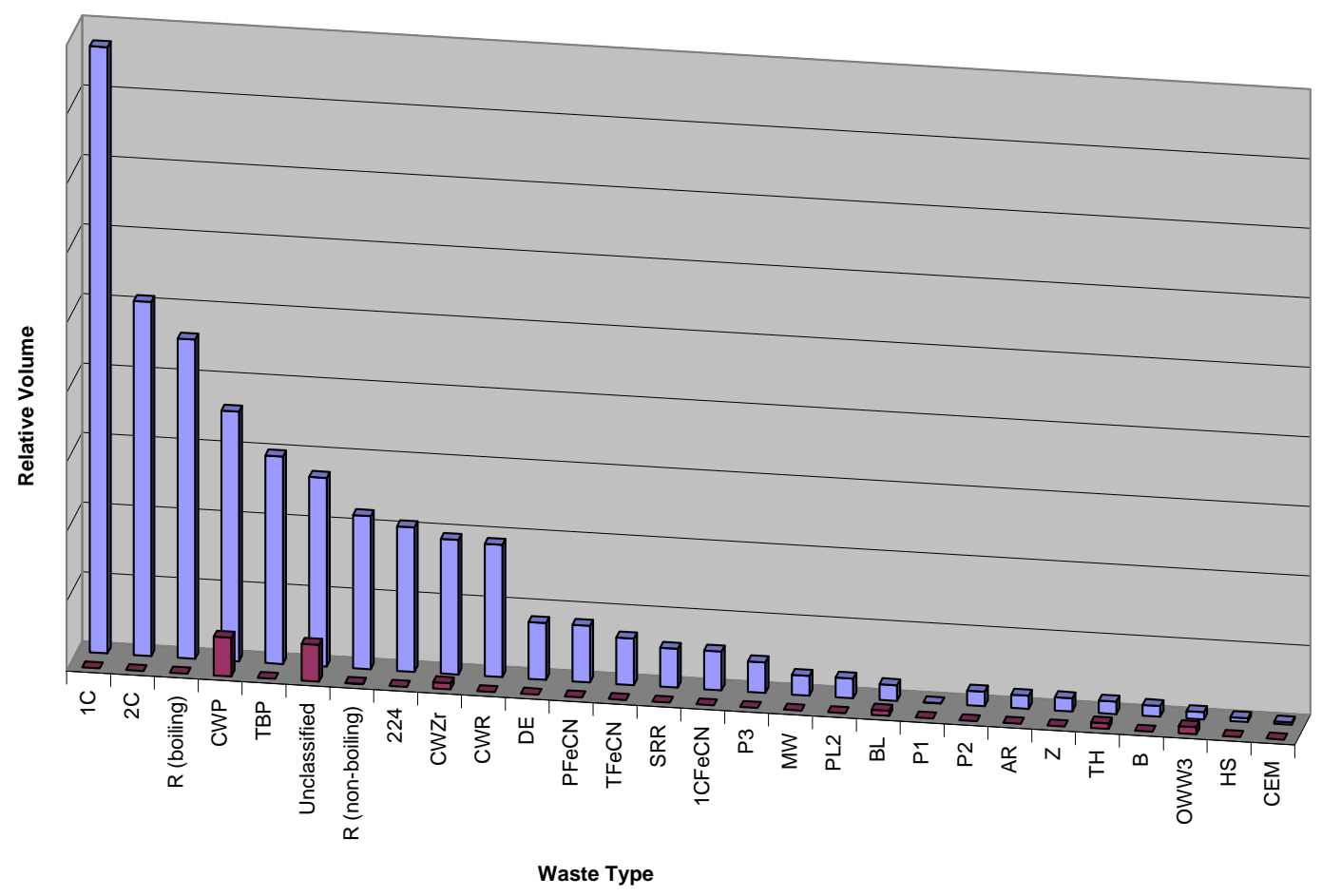

Figure 9.2. Relative Waste Volumes Used in the Analysis of Liquid Viscosity as a Function of Sludge Waste Types

Figure 9.3 is a plot of the Hanford liquid viscosity as a function of density. During the multidecade Hanford tank waste characterization effort, measurements were not performed at consistent temperatures. Therefore, data are grouped in $15^{\circ} \mathrm{C}$ temperature increments starting at $20^{\circ}$ to $35^{\circ} \mathrm{C}$ and ending at $80^{\circ}$ to $95^{\circ} \mathrm{C}$. In addition, the data are grouped according to whether the sample was from a tank waste composite [as-received (AR)] or diluted with water (Dil). Exponential fits to these data for each temperature group were also obtained and are shown in a $\log$ plot of viscosity as a function of liquid density minus one (second plot in Figure 9.3). These data indicate that liquid density should be maintained below 1.4 to $1.5 \mathrm{~g} / \mathrm{mL}$ to avoid exceeding the $15 \mathrm{cP}$ liquid viscosity bound found in Poloski et al. (2003a). The coefficients for these exponential fits were constrained to produce the viscosity of water according to the Andrade correlation when sample density is unity.

At constant density, the parameters of the exponential fits vary with temperature. Assuming that the model takes the form of the Andrade correlation at each liquid density, the viscosity of the supernatant in the Hanford tanks can be correlated to density according to:

$$
\eta=\left(m_{1} e^{b_{1}(\rho-1)}\right) e^{\left(m_{2}(\rho-1)+b_{2}\right) / T}
$$

where

$\eta=$ the Newtonian viscosity in $\mathrm{cP}$

$T=$ temperature in Kelvin

$\rho=$ the liquid density in $\mathrm{g} / \mathrm{mL}$

$m_{1}=0.00216 \mathrm{cP}$ 


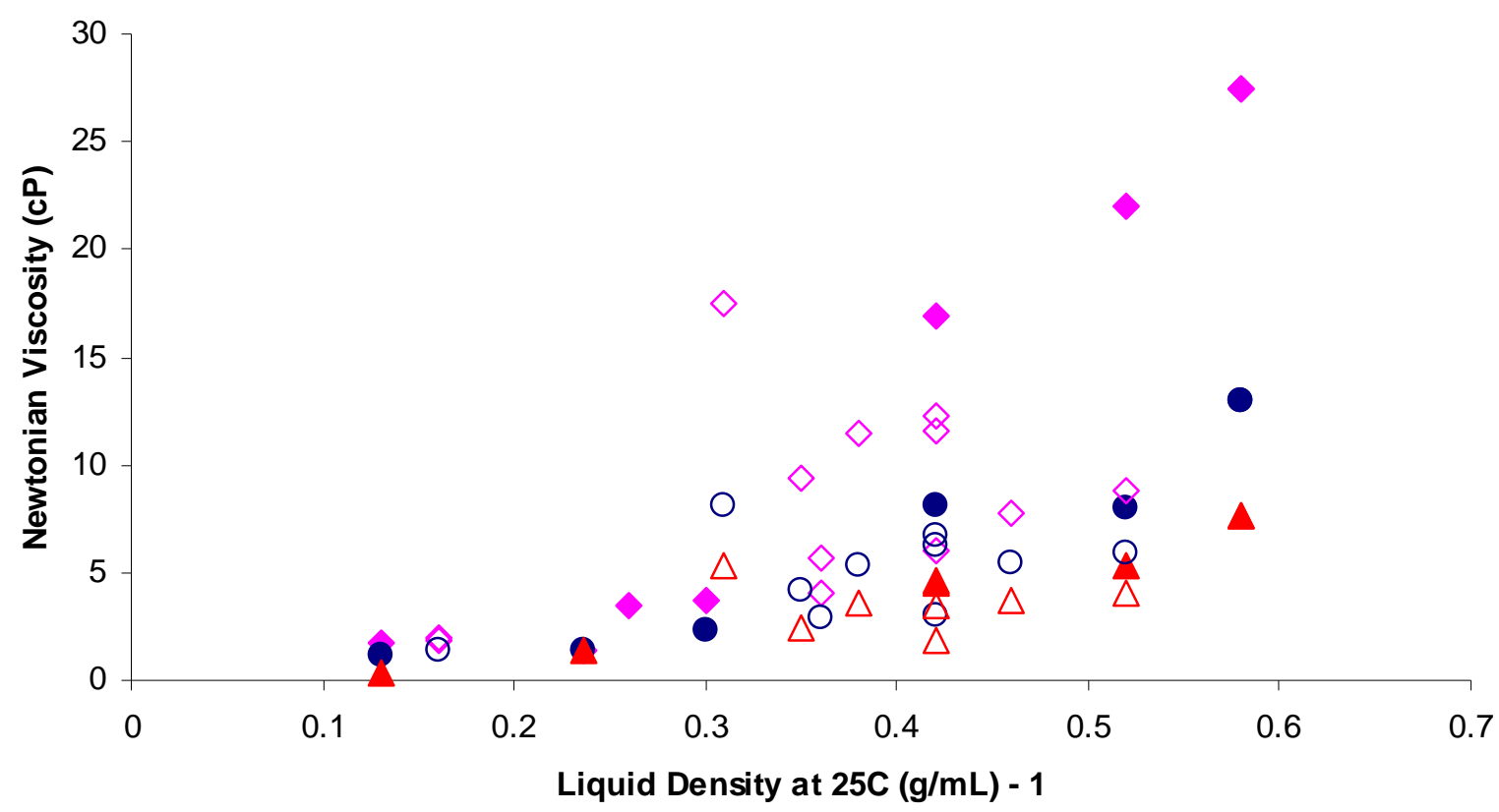

20-35C AR • 35-50C AR $\triangle 50-65 C$ AR $\diamond 20-35 C$ Dil $\circ 35-50 C$ Dil $\triangle 50-65 C$ Dil

(a) Data grouped according to dilution and temperature.

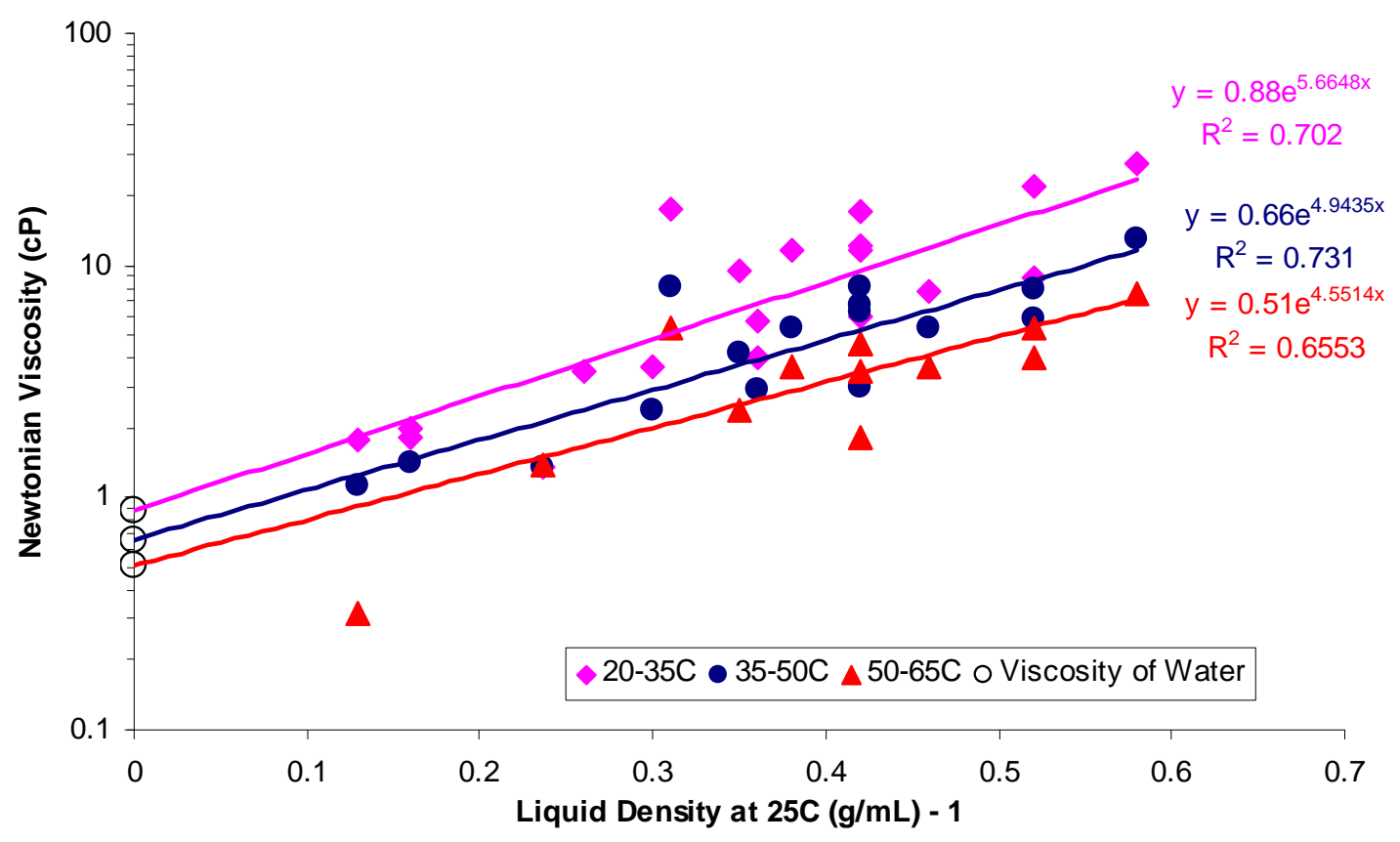

(b) Exponential fits at various temperatures

Figure 9.3. Viscosity of Hanford Supernatant at Various Densities and Temperatures 


$$
\begin{aligned}
& b_{1}=-6.69(\mathrm{~g} / \mathrm{mL})^{-1} \\
& m_{2}=3700 \mathrm{~K} \cdot(\mathrm{g} / \mathrm{mL})^{-1} \\
& b_{2}=1810 \mathrm{~K} .
\end{aligned}
$$

When the liquid density is unity, this equation reduces to the Andrade correlation for water.

Figure 9.4 is a parity plot of the measured and predicted viscosities. Based on this correlation, on average, measured viscosity is biased by $9 \%$.

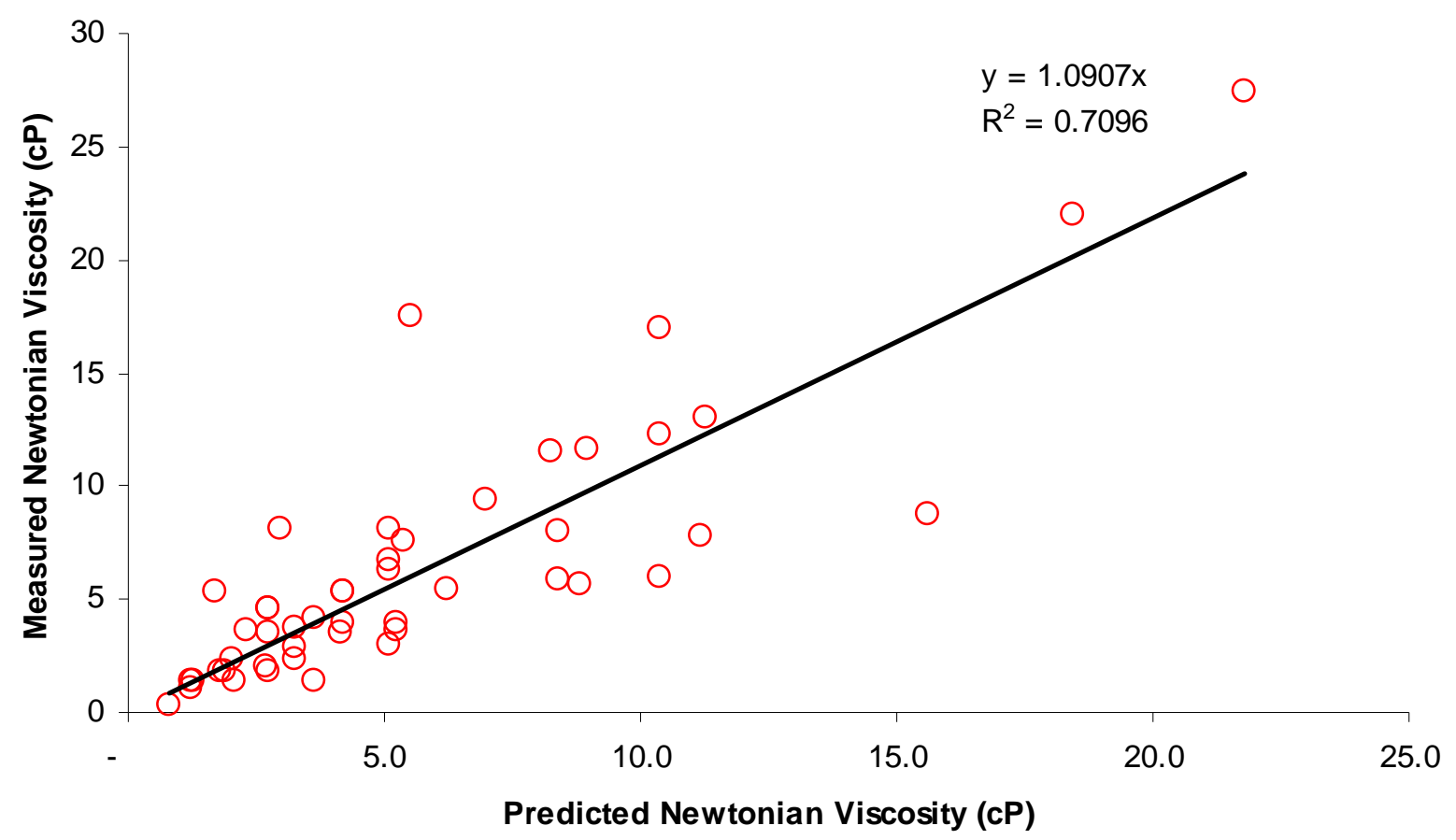

Figure 9.4. Parity Plot of Measured and Predicted Hanford Supernatant Liquid 


\subsection{Sedimentation Model}

Hanford tank waste was observed to settle in the "zone" or "hindered" settling regime. This regime occurs when interacting particles settle as a mass and is common in the wastewater industry. Hindered settling is characterized by the settled-solids layer height decreasing from the fully suspended volume to a final settled-solids volume. Several models have been developed to predict the performance of sludge in a settling system based on laboratory-scale sedimentation column data. One of the more recent models is described by Renko $(1996,1998)$ and is shown in Eq. (10.1).

$$
\frac{Z}{z_{0}}=\frac{C \phi_{0}}{\alpha}+\left(1-\frac{C \phi_{0}}{\alpha}\right) e^{\frac{-\alpha}{\phi_{0} z_{0}} t}
$$

where

$\mathrm{Z} \quad=$ the interface height $(\mathrm{m})$ at time $t$ in hours

$z_{0} \quad=$ the initial interface height $(\mathrm{m})$

$\phi_{0} \quad=$ the initial solids concentration of the suspended sample (volume fraction)

$\alpha$ and $C=$ fitting parameters in $\mathrm{m} / \mathrm{hr}^{-1}$.

When sedimentation begins, the interface height $(z)$ is equal to the initial interface height $\left(Z_{0}\right)$. As time goes to infinity, the interface height approaches $\frac{C \phi_{0} z_{0}}{\alpha}$. Because the average solids concentration varies with interface height, as shown in Eq. (10.2), the maximum solids concentration $\left(\phi_{\max }\right)$ achieved through sedimentation is $\frac{\alpha}{C}$.

$$
\phi=\frac{\phi_{0} z_{0}}{Z}
$$

Based on these relationships, Eq. (10.1) can be rewritten in terms of average solids concentration as shown in Eq. (10.3).

$$
\phi=\left[\frac{C}{\alpha}+\left(\frac{1}{\phi_{0}}-\frac{C}{\alpha}\right) e^{\frac{-\alpha}{\phi_{0} z_{0}} t}\right]^{-1}
$$

The data collected in this report contains 58 sedimentation curves over 17 waste tanks and eight waste types. Each sedimentation curve was fit to Eq. (10.1), and the fitting parameters $\alpha$ and $C$ are reported in Table 10.1. Values for the initial solids concentration $\left(\phi_{0}\right)$ were estimated from physical property data. Values for the initial sediment height $\left(z_{0}\right)$ were unknown for many of the data because they only reported volume (or vol\%) rather than height. However, most measurements were performed in small graduated cylinders or graduated centrifuge cones with a total volume of $20-50 \mathrm{~mL}$; therefore, an initial height of $10 \mathrm{~cm}$ was assumed in all calculations. This is similar to the data where sediment height as well as sediment volume was provided (Morrey and Tingey 1996). 
Table 10.1. Sedimentation Model Input and Fitting Parameters for Hanford Tank Waste

\begin{tabular}{|c|c|c|c|c|c|c|}
\hline Tank & Waste Type & $\begin{array}{c}\phi_{0} \\
\text { vol\% }\end{array}$ & $\begin{array}{c}\mathbf{z}_{0} \\
\mathbf{m m}\end{array}$ & $\begin{array}{c}C \\
\mathrm{~mm} / \mathrm{hr}\end{array}$ & $\begin{array}{c}\alpha \\
\mathbf{m m} / \mathbf{h r}\end{array}$ & $\begin{array}{r}\phi_{\max } \\
\mathbf{v o l \%} \\
\end{array}$ \\
\hline B-202 & 224 & $0.8 \%$ & 100 & 21.7 & 0.43 & $2.0 \%$ \\
\hline B-202 & 224 & $0.8 \%$ & 100 & 17.0 & 0.34 & $2.0 \%$ \\
\hline B-202 & 224 & $0.8 \%$ & 100 & 36.1 & 0.68 & $1.9 \%$ \\
\hline B-202 & 224 & $0.9 \%$ & 100 & 17.2 & 0.32 & $1.9 \%$ \\
\hline B-202 & 224 & $0.9 \%$ & 100 & 19.8 & 0.38 & $1.9 \%$ \\
\hline B-202 & 224 & $1.0 \%$ & 100 & 25.3 & 0.64 & $2.6 \%$ \\
\hline B-202 & 224 & $2.0 \%$ & 100 & 12.2 & 0.32 & $2.6 \%$ \\
\hline B-202 & 224 & $2.1 \%$ & 100 & 11.8 & 0.31 & $2.6 \%$ \\
\hline B-202 & 224 & $2.0 \%$ & 100 & 13.3 & 0.31 & $2.3 \%$ \\
\hline B-202 & 224 & $2.0 \%$ & 100 & 9.6 & 0.25 & $2.6 \%$ \\
\hline B-202 & 224 & $2.3 \%$ & 100 & 9.4 & 0.25 & $2.6 \%$ \\
\hline B-202 & 224 & $2.3 \%$ & 100 & 9.6 & 0.28 & $2.9 \%$ \\
\hline \multicolumn{4}{|c|}{ B-202 Average values $\rightarrow$} & 16.9 & 0.38 & $2.3 \%$ \\
\hline B-203 & 224 & $0.4 \%$ & 100 & 53.4 & 0.55 & $1.0 \%$ \\
\hline B-203 & 224 & $1.8 \%$ & 100 & 6.1 & 0.13 & $2.1 \%$ \\
\hline B-203 & 224 & $1.8 \%$ & 100 & 6.1 & 0.13 & $2.1 \%$ \\
\hline \multicolumn{4}{|c|}{ B-203 Average values $\rightarrow$} & 21.8 & 0.27 & $1.7 \%$ \\
\hline $\mathrm{T}-111$ & 224 & $0.1 \%$ & 100 & 9.4 & 0.01 & $0.1 \%$ \\
\hline $\mathrm{T}-111$ & 224 & $0.2 \%$ & 100 & 7.0 & 0.02 & $0.3 \%$ \\
\hline $\mathrm{T}-111$ & 224 & $1.9 \%$ & 100 & 1.9 & 0.05 & $2.6 \%$ \\
\hline \multicolumn{4}{|c|}{ T-111 Average values $\rightarrow$} & 6.10 & 0.027 & $1.0 \%$ \\
\hline $\mathrm{T}-203$ & 224 & $0.4 \%$ & 100 & 28.9 & 0.27 & $0.9 \%$ \\
\hline $\mathrm{T}-203$ & 224 & $1.3 \%$ & 100 & 4.7 & 0.07 & $1.4 \%$ \\
\hline \multicolumn{4}{|c|}{ T-203 Average values $\rightarrow$} & 16.8 & 0.17 & $1.2 \%$ \\
\hline $\mathrm{T}-204$ & 224 & $0.4 \%$ & 100 & 91.7 & 0.75 & $0.8 \%$ \\
\hline $\mathrm{T}-204$ & 224 & $1.5 \%$ & 100 & 4.4 & 0.07 & $1.7 \%$ \\
\hline \multicolumn{4}{|c|}{ T-204 Average values $\rightarrow$} & 48.1 & 0.41 & $1.3 \%$ \\
\hline BX-107 & $1 \mathrm{C}$ & $0.4 \%$ & 100 & 13.4 & 0.17 & $1.3 \%$ \\
\hline BX-107 & $1 \mathrm{C}$ & $0.5 \%$ & 100 & 26.0 & 0.29 & $1.1 \%$ \\
\hline BX-107 & $1 \mathrm{C}$ & $0.5 \%$ & 100 & 20.9 & 0.27 & $1.3 \%$ \\
\hline BX-107 & $1 \mathrm{C}$ & $2.2 \%$ & 100 & 12.4 & 0.50 & $4.1 \%$ \\
\hline BX-107 & $1 \mathrm{C}$ & $2.6 \%$ & 100 & 18.4 & 0.73 & $4.0 \%$ \\
\hline BX-107 & $1 \mathrm{C}$ & $2.7 \%$ & 100 & 13.9 & 0.56 & $4.0 \%$ \\
\hline \multicolumn{4}{|c|}{ BX-107 Average values $\rightarrow$} & 17.5 & 0.42 & $2.6 \%$ \\
\hline $\mathrm{C}-110$ & $1 \mathrm{C}$ & $0.3 \%$ & 100 & 9.7 & 0.08 & $0.8 \%$ \\
\hline $\mathrm{C}-110$ & $1 \mathrm{C}$ & $0.5 \%$ & 100 & 9.2 & 0.13 & $1.4 \%$ \\
\hline $\mathrm{C}-110$ & $1 \mathrm{C}$ & $1.5 \%$ & 100 & 7.8 & 0.17 & $2.2 \%$ \\
\hline $\mathrm{C}-110$ & $1 \mathrm{C}$ & $2.5 \%$ & 100 & 11.0 & 0.46 & $4.2 \%$ \\
\hline \multicolumn{4}{|c|}{$C-110$ Average values $\rightarrow$} & 9.4 & 0.21 & $2.2 \%$ \\
\hline T-107 & $1 \mathrm{C}$ & $3.4 \%$ & 100 & 10.7 & 1.02 & $9.6 \%$ \\
\hline $\mathrm{T}-107$ & $1 \mathrm{C}$ & $7.5 \%$ & 100 & 5.3 & 0.59 & $11.1 \%$ \\
\hline \multicolumn{4}{|c|}{ T-107 Average values $\rightarrow$} & 8.0 & 0.81 & $10.4 \%$ \\
\hline $\mathrm{T}-110$ & $2 \mathrm{C}$ & $0.3 \%$ & 100 & 20.1 & 0.25 & $1.2 \%$ \\
\hline $\mathrm{T}-110$ & $2 \mathrm{C}$ & $1.4 \%$ & 100 & 12.7 & 0.39 & $3.1 \%$ \\
\hline $\mathrm{T}-110$ & $2 \mathrm{C}$ & $3.5 \%$ & 100 & 1.1 & 0.05 & $4.5 \%$ \\
\hline \multicolumn{4}{|c|}{ T-110 Average values $\rightarrow$} & 11.3 & 0.23 & $2.9 \%$ \\
\hline
\end{tabular}


Table 10.1 (contd)

\begin{tabular}{|c|c|c|c|c|c|c|}
\hline Tank & Waste Type & $\begin{array}{c}\phi_{0} \\
\text { vol\% }\end{array}$ & $\begin{array}{c}\mathbf{z}_{0} \\
\mathbf{m m}\end{array}$ & $\begin{array}{c}C \\
\mathrm{~mm} / \mathrm{hr}\end{array}$ & $\begin{array}{c}\alpha \\
\mathbf{m m} / \mathbf{h r}\end{array}$ & $\begin{array}{c}\phi_{\max } \\
\mathbf{v o l} \%\end{array}$ \\
\hline AN-104 & A2SltSlr & $0.4 \%$ & 100 & 6.1 & 0.14 & $2.3 \%$ \\
\hline AN-104 & A2SltSlr & $0.9 \%$ & 100 & 7.4 & 0.68 & $9.2 \%$ \\
\hline AN-104 & A2SltSlr & $3.2 \%$ & 100 & 20.7 & 1.17 & $5.6 \%$ \\
\hline AN-104 & A2SltSlr & $4.5 \%$ & 100 & 19.8 & 1.05 & $5.3 \%$ \\
\hline \multicolumn{4}{|c|}{ AN-104 Average values $\rightarrow$} & 13.5 & 0.76 & $5.6 \%$ \\
\hline $\mathrm{AN}-105$ & A2SltSlr & $0.7 \%$ & 100 & 8.0 & 0.48 & $6.0 \%$ \\
\hline AN-105 & A2SltSlr & $4.2 \%$ & 100 & 1.8 & 0.54 & $30.1 \%$ \\
\hline AN-105 & A2SltSlr & $8.2 \%$ & 100 & 5.0 & 0.80 & $16.0 \%$ \\
\hline AN-105 & A2SltSlr & $10.5 \%$ & 100 & 3.9 & 0.99 & $25.1 \%$ \\
\hline \multicolumn{4}{|c|}{ AN-105 Average values $\rightarrow$} & 4.7 & 0.70 & $19.3 \%$ \\
\hline C-104 & CWP & $2.1 \%$ & 100 & 4.6 & 0.44 & $9.7 \%$ \\
\hline C-104 & CWP & $3.0 \%$ & 100 & 4.3 & 0.34 & $7.8 \%$ \\
\hline C-104 & CWP & $5.1 \%$ & 100 & 3.8 & 0.41 & $10.7 \%$ \\
\hline \multicolumn{4}{|c|}{$C-104$ Average values $\rightarrow$} & 4.2 & 0.40 & $9.4 \%$ \\
\hline $\mathrm{T}-102$ & CWP & $2.1 \%$ & 100 & 22.5 & 4.66 & $20.7 \%$ \\
\hline $\mathrm{T}-102$ & CWP & $4.7 \%$ & 100 & 37.6 & 10.33 & $27.5 \%$ \\
\hline \multicolumn{4}{|c|}{ T-102 Average values $\rightarrow$} & 30.1 & 7.5 & $24.1 \%$ \\
\hline S-104 & $\mathrm{R}$ (boiling) & $2.3 \%$ & 100 & 9.0 & 0.25 & $2.7 \%$ \\
\hline S-104 & R (boiling) & $2.8 \%$ & 100 & 8.6 & 0.37 & $4.3 \%$ \\
\hline S-104 & R (boiling) & $8.7 \%$ & 100 & 5.1 & 0.50 & $9.8 \%$ \\
\hline \multicolumn{4}{|c|}{$S-104$ Average values $\rightarrow$} & 7.6 & 0.37 & $5.6 \%$ \\
\hline C-109 & $\mathrm{TFeCN}$ & $2.2 \%$ & 100 & 16.1 & 0.82 & $5.1 \%$ \\
\hline C-109 & TFeCN & $3.5 \%$ & 100 & 14.3 & 0.58 & $4.1 \%$ \\
\hline \multicolumn{4}{|c|}{$C-109$ Average values $\rightarrow$} & 15.2 & 0.70 & $4.6 \%$ \\
\hline C-112 & TFeCN & $2.2 \%$ & 100 & 8.6 & 0.42 & $4.9 \%$ \\
\hline $\mathrm{C}-112$ & TFeCN & $5.9 \%$ & 100 & 1.9 & 0.17 & $8.8 \%$ \\
\hline \multicolumn{4}{|c|}{$C-112$ Average values $\rightarrow$} & 5.3 & 0.30 & $6.9 \%$ \\
\hline AY-102 & Unclassified & $7.8 \%$ & 100 & 6.0 & 0.80 & $13.3 \%$ \\
\hline
\end{tabular}

The data presented in Table 10.1 can be grouped according to waste type. A summary table of the fitting parameters for each waste type is shown in Table 10.2.

Table 10.2. Sedimentation Parameters for Each Waste Type

\begin{tabular}{|c|c|c|}
\hline Waste Type & $\operatorname{Avg~C~(mm/hr)~}$ & $\operatorname{Avg} \alpha(\mathrm{mm} / \mathrm{hr})$ \\
\hline 224 & $18.9 \pm 20.2$ & $0.3 \pm 0.2$ \\
\hline $1 \mathrm{C}$ & $13.2 \pm 5.9$ & $0.4 \pm 0.3$ \\
\hline $2 \mathrm{C}$ & $11.3 \pm 9.6$ & $0.2 \pm 0.2$ \\
\hline A2SltSlr & $9.1 \pm 7.2$ & $0.7 \pm 0.3$ \\
\hline CWP & $14.6 \pm 15.1$ & $3.2 \pm 4.4$ \\
\hline $\mathrm{R}$ (boiling) & $7.6 \pm 2.2$ & $0.4 \pm 0.1$ \\
\hline $\mathrm{TFeCN}$ & $10.2 \pm 6.4$ & $0.5 \pm 0.3$ \\
\hline Unclassified & $6.0 \pm$ not measured & $0.8 \pm$ not measured \\
\hline
\end{tabular}


Several of the waste types are not represented in this modeling activity because appropriate data was not available. The gaps in the waste types modeled are shown in Figure 10.1. The relative volume of each waste type in the17 waste tanks modeled (red bar in the front of the graph) is compared to the total volume of waste associated with those waste types (blue bar in the back of the graph). The volume of waste in each tank for each waste type was determined using the TWINS database.

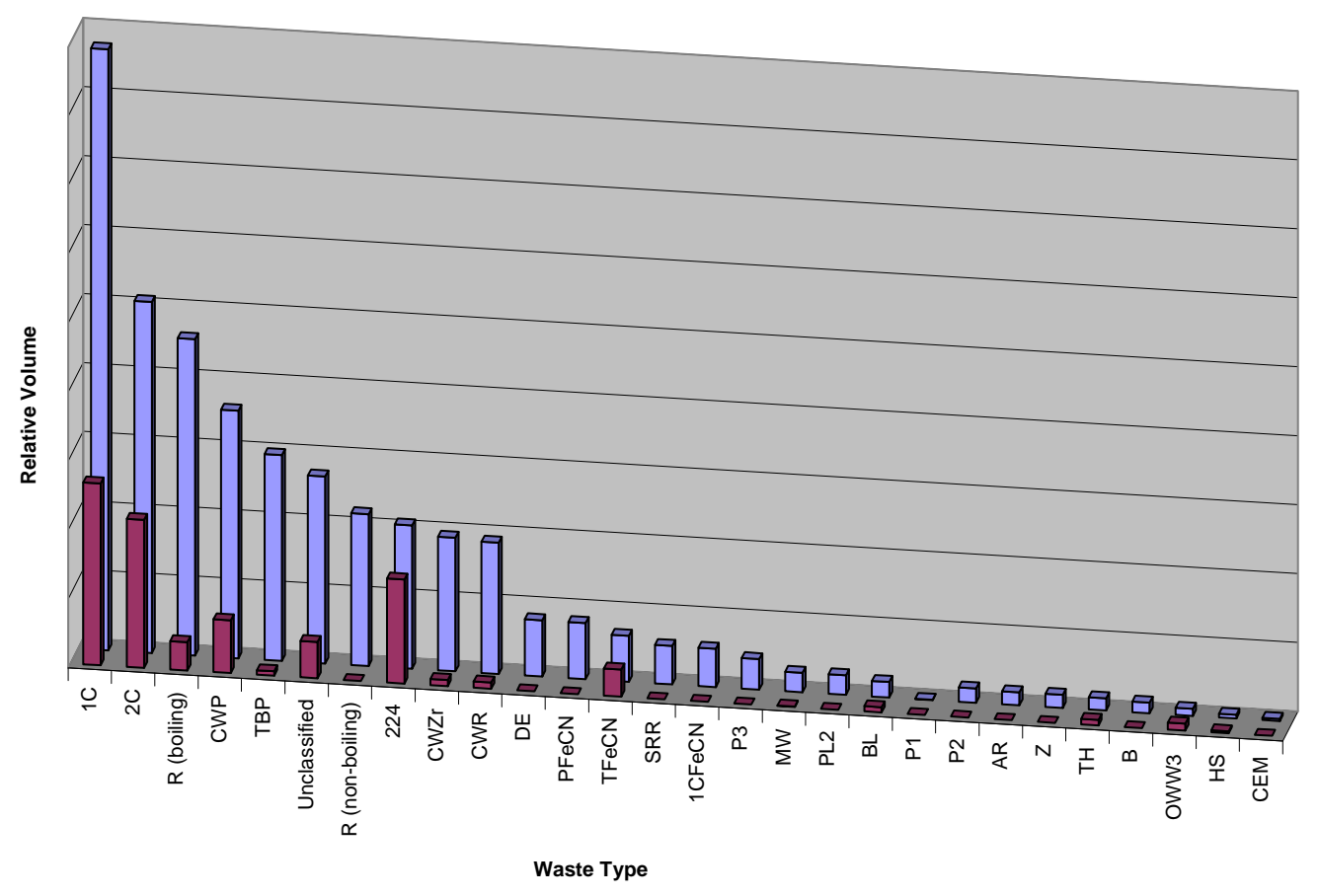

Figure 10.1. Gaps in Data Available for Sedimentation Modeling as a Function of Waste Type

From Eq. (10.1) and (10.3), initial interface height, $z_{0}$, and initial solids concentration, $\phi_{0}$, are input parameters to determine the interface level and solids concentration as a function of time. The average values from Table 10.1 were used to calculate interface height as a function of time at an initial solids concentration equivalent to a 3:1 dilution of the sediment volume at initial heights of $10 \mathrm{~cm}$ and $4 \mathrm{~m}$. The resulting plots shown in Figure 10.2 illustrate that small-scale laboratory experiments reach the fully settled configuration faster than plant-scale vessels. This can be explained by considering that the total distance the particles must travel to reach the full sediment configuration at plant scale is larger than the lab-scale experiments. 


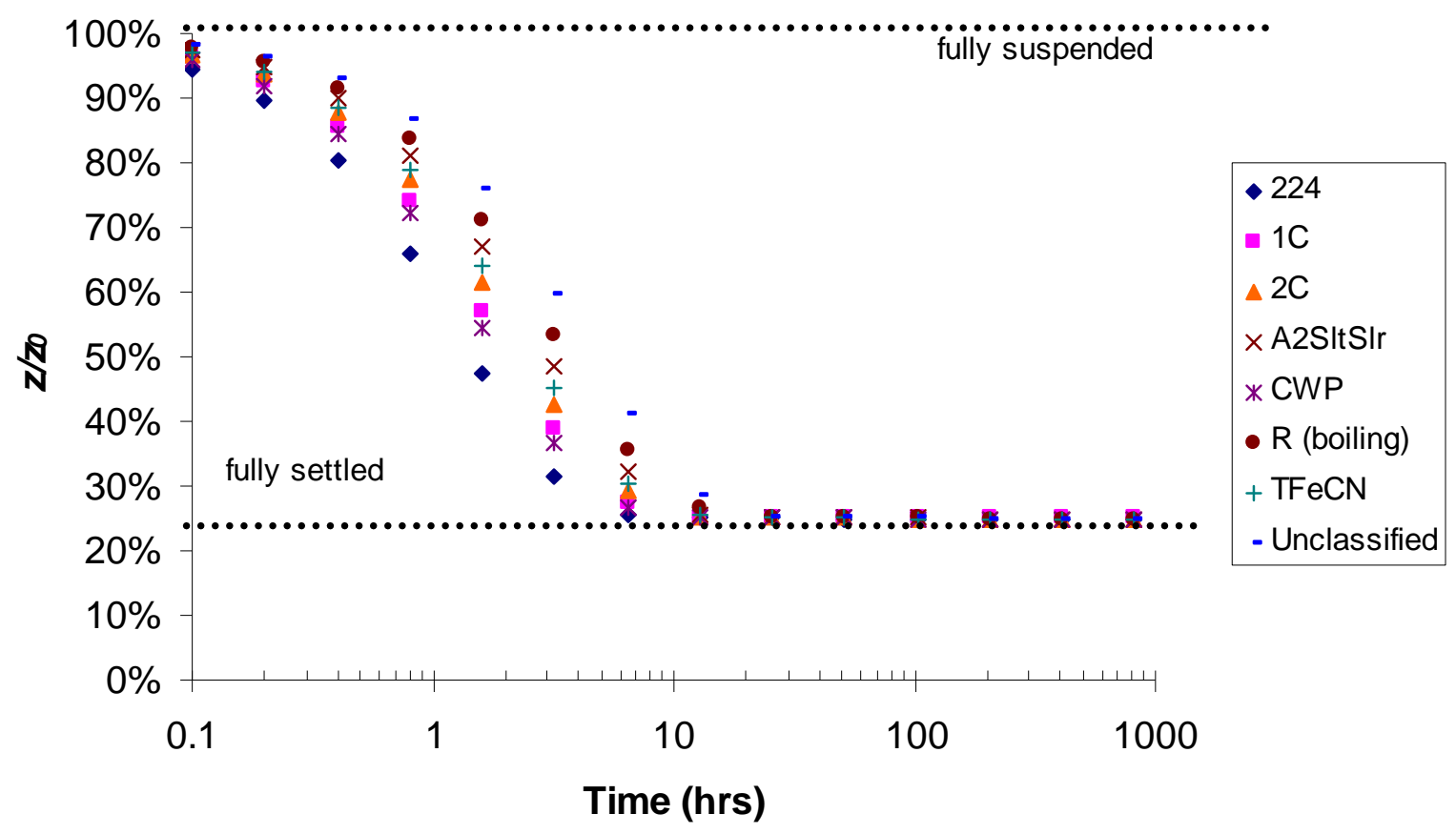

a) initial interface height is $z_{0}=10 \mathrm{~cm}$; initial solids concentration of the suspended sample is $\phi=\frac{\phi_{\max }}{4}$

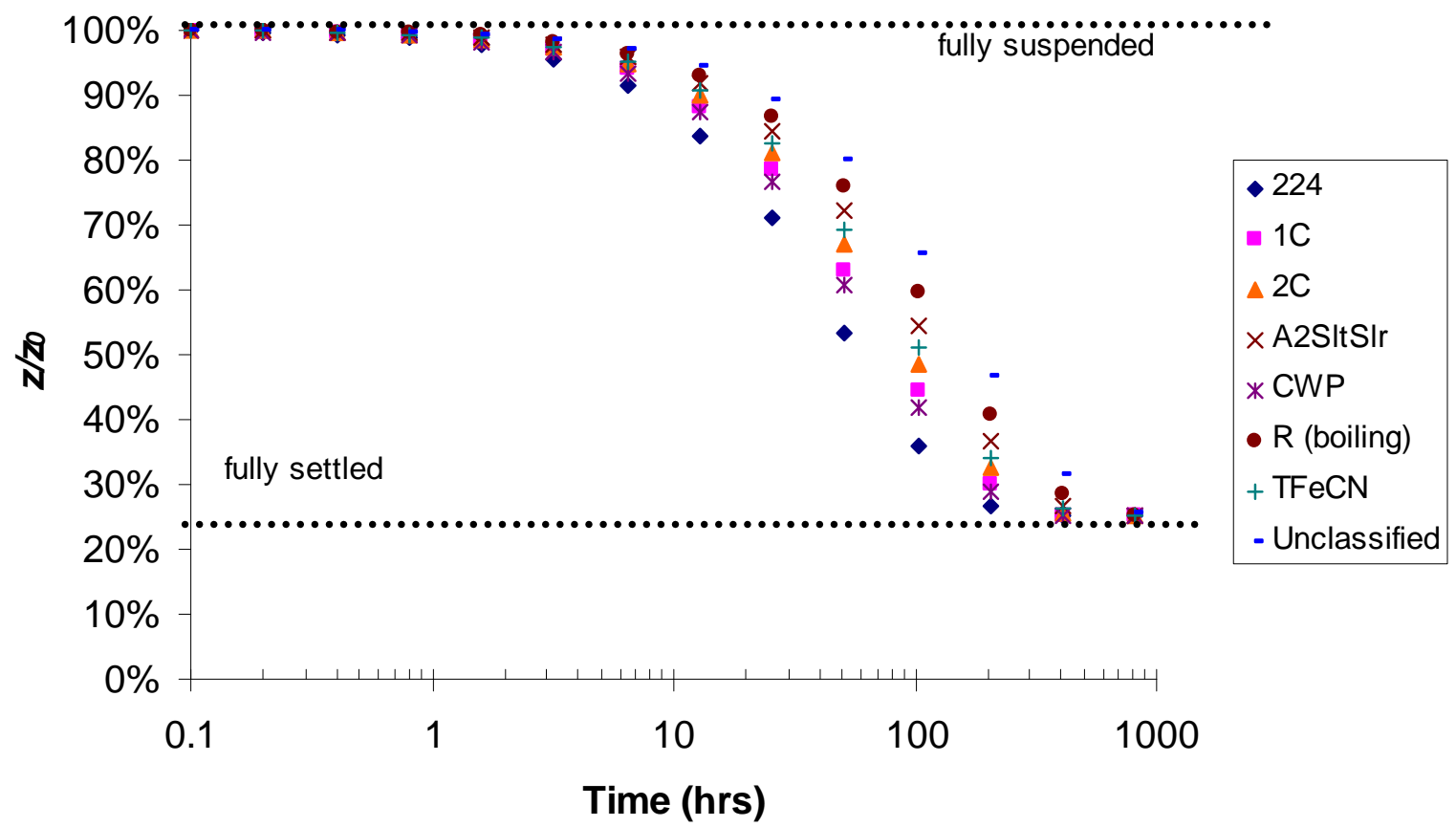

b) initial interface height is $z_{0}=4 \mathrm{~m}$; initial solids concentration of the suspended sample is $\phi=\frac{\phi_{\max }}{4}$

Figure 10.2. Predicted Sedimentation Curves for Various Waste Types at Different Scales 


\subsection{Shear Strength Model}

Shear strength is a transient property that grows during the time a sample remains quiescent. A model describing the growth of shear strength with respect to time is presented by Speers et al. (1987), who describe the shear strength rebuild behavior for several drilling mud slurries with a first-order rate model:

$$
\frac{G_{t}-G_{\infty}}{G_{0}-G_{\infty}}=e^{-k t}
$$

where

$$
\begin{array}{ll}
G_{t} & =\text { shear strength } \\
\mathrm{t} & =\text { gel time } \\
G_{0} & =\text { initial shear strength } \\
G_{\infty} & =\text { equilibrium shear strength } \\
\mathrm{k} & =\text { gel time constant. }
\end{array}
$$

Only limited data were available for shear strength as a function of gelation time, but this model appears to be a good fit to the AZ-101 pretreated sludge shear-strength data (Poloski et al. 2003b) shown in Figure 11.1. The model-fit parameters observed for the AZ-101 pretreated sludge are reported in Table 11.1. Using this model, the initial shear-strength parameter (14.2 Pa) should roughly agree with the measured rheological Bingham-yield-stress measurement (14.7 to 18.1 Pa) (Poloski et al. 2003b). This model indicates that the shear strength rebuilds immediately from the time that it remains unshared. The material is expected to reach $95 \%$ of its steady-state shear strength ( $31 \mathrm{~Pa}) 9$ hours from this time.

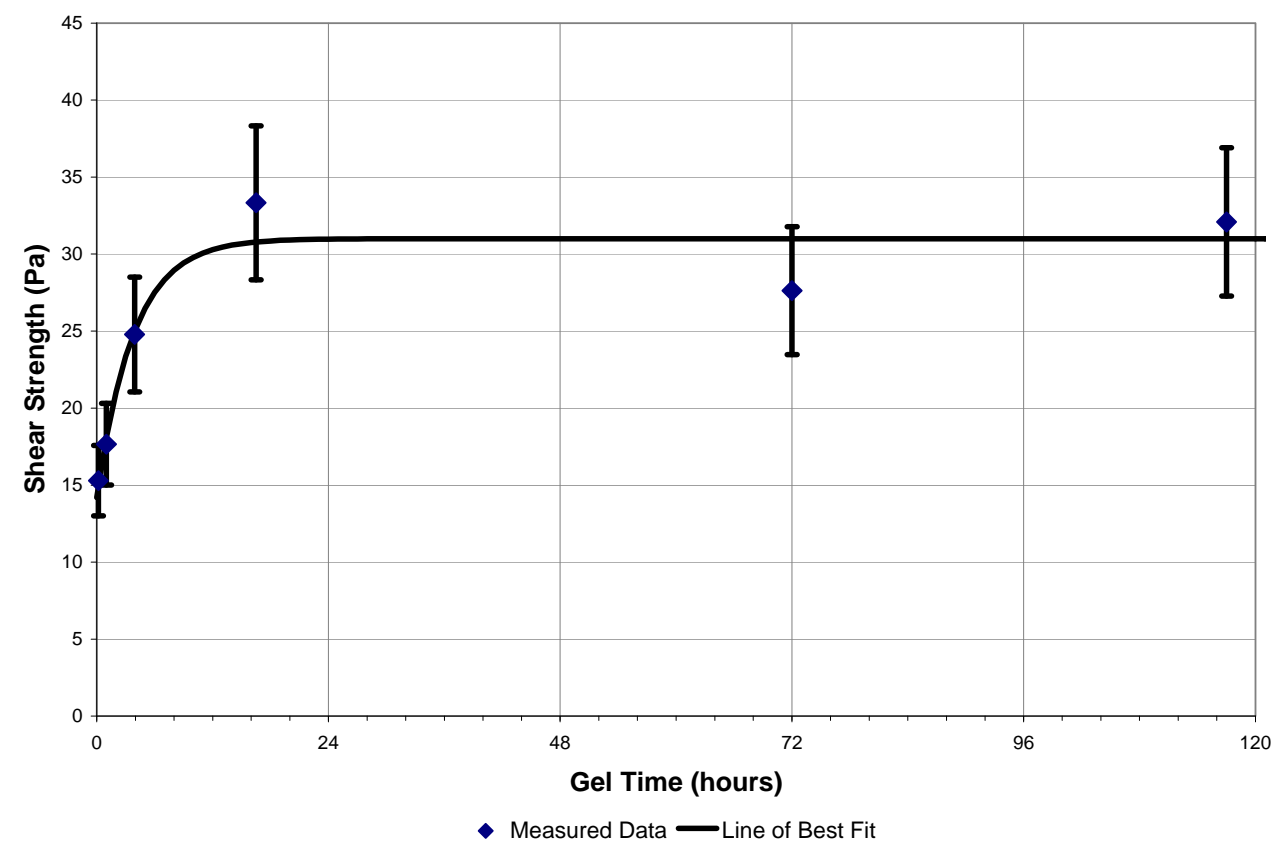

Figure 11.1. Shear Strength as a Function of Gel Time for HLW Pretreated Sludge 
Table 11.1. Shear Strength Model Fit Parameters for Pretreated AZ-101 Sludge

\begin{tabular}{|c|c|}
\hline Variable & Model Fit \\
\hline$G_{t}$ & See Figure 11.1 $\left(\mathrm{r}^{2}=0.929\right)$ \\
\hline $\mathrm{t}$ & 0 to 120 hours \\
\hline$G_{0}$ & $14.2 \mathrm{~Pa}$. \\
\hline$G_{\infty}$ & $31 \mathrm{~Pa}$ \\
\hline$k$ & $0.262 \mathrm{hr}^{-1}$ \\
\hline
\end{tabular}

These AZ-101 pretreated sludge shear strength data represent the only transient shear strength data found for Hanford waste suspensions. While other data exists to estimate the initial and steady-state shear strength values for several tank wastes, the only source for the gel time constant describing how quickly the shear strength rises to its steady state value is from AZ-101 pretreated sludge. A comparison of the AZ-101 pretreated sludge gel time constant against other suspensions either used in industry or for Hanford simulants is shown in Table 11.2. These data illustrate that the measured time constant of $0.262 \mathrm{hr}^{-1}$, while high, is comparable with other fine particulate slurry systems. A graphical view of the time it takes to reach $95 \%$ of the growth in shear strength for these systems is shown in Figure 11.2. The numbers closes to the $\mathrm{x}$-axis are the temperature in ${ }^{\circ} \mathrm{C}$ at which these shear strengths were measured. This figure portrays that the AZ-101 pretreated sludge material nearly reaches the equilibrium shear strength in approximately $10 \mathrm{hrs}$.

In the absence of actual shear strength as a function of time data, the transient shear strength model can be approximated through the following equation:

$$
\frac{G_{t}-\tau_{\infty}}{\tau_{O}^{B}-\tau_{\infty}} \cong e^{-k t}
$$

where

$G_{t}=$ shear strength

$\mathrm{t}=$ the gel time

$\tau_{O}^{B}=$ the Bingham yield stress

$\tau_{\infty}=$ the single point equilibrium shear strength measurement

$\mathrm{k}=$ the gel time constant.

In this model, the initial shear strength is replaced by the Bingham Plastic yield stress, $\tau_{O}^{B}$, while the equilibrium shear strength is approximated by a single point shear strength measurement, $\tau_{\infty}$, because most shear strength measurements were taken after the sample sat undisturbed for several days. Currently, a gel time constant of $0.262 \mathrm{hr}^{-1}$ is used based on the fit for the pretreated AZ-101 sludge. Until additional transient shear strength data are obtained, this gel time constant is recommended for Hanford tank waste. 
Table 11.2. Shear Strength Rebuild Parameters for Various Materials

\begin{tabular}{|c|c|c|c|c|c|}
\hline Material & $\begin{array}{c}\text { Temperature } \\
\left({ }^{\circ} \mathbf{C}\right)\end{array}$ & $\begin{array}{c}\text { Initial Shear } \\
\text { Strength, } \mathbf{G}_{0} \\
(\mathbf{P a})\end{array}$ & $\begin{array}{c}\text { Gel Time } \\
\text { Constant, } k \\
\left(\mathrm{hr}^{-1}\right)\end{array}$ & \begin{tabular}{|c|} 
Steady-State Gel \\
Strength, $G_{\infty}$ \\
$(\mathrm{Pa})$
\end{tabular} & $\begin{array}{c}\text { Correlation } \\
\text { Coefficient } \\
\left(\mathbf{r}^{2}\right) \\
\end{array}$ \\
\hline \multirow[t]{4}{*}{ Dispersed gel-chemical $^{(\mathrm{a})}$} & 20 & 2.4 & 0.00637 & 14.0 & 0.96 \\
\hline & 40 & 2.2 & 0.0253 & 4.7 & 0.986 \\
\hline & 60 & 1.8 & 0.028 & 4.2 & 0.985 \\
\hline & 80 & 1.8 & 0.0342 & 4.3 & 0.993 \\
\hline \multirow[t]{4}{*}{ Beaufort lignosulfonate $^{(a)}$} & 20 & 5.0 & 0.00609 & 16.5 & 0.908 \\
\hline & 40 & 1.6 & 0.0535 & 3.1 & 0.924 \\
\hline & 60 & 1.6 & 0.0165 & 4.8 & 0.933 \\
\hline & 80 & 1.4 & 0.0607 & 3.8 & 0.964 \\
\hline \multirow[t]{4}{*}{ E. Coast biovert ${ }^{(a)}$} & 20 & 2.7 & 0.00595 & 14.1 & 0.95 \\
\hline & 40 & 1.5 & 0.0467 & 7.8 & 0.991 \\
\hline & 60 & 1.7 & 0.0681 & 9.7 & 0.986 \\
\hline & 80 & 2.0 & 0.1 & 11.8 & 0.996 \\
\hline \multirow[t]{4}{*}{ Extended gel $^{(\mathrm{a})}$} & 20 & 5.8 & 0.0522 & 10.9 & 0.932 \\
\hline & 40 & 5.2 & 0.172 & 8.0 & 0.977 \\
\hline & 60 & 3.9 & 1.42 & 4.9 & 0.923 \\
\hline & 80 & 2.7 & 0.146 & 3.9 & 0.956 \\
\hline $\mathrm{Gel}^{(\mathrm{a})}$ no & 20 & 2.5 & 0.00237 & 16.6 & 0.962 \\
\hline$\# 2$ & 20 & 8.1 & 0.000378 & 16.6 & 0.956 \\
\hline$\# 3$ & 20 & 1.8 & 0.0154 & 15.0 & 0.986 \\
\hline$\# 4$ & 20 & 13.7 & 0.0215 & 24.5 & 0.981 \\
\hline$\# 5$ & 20 & 1.1 & 0.0166 & 13.6 & 0.99 \\
\hline$\# 6$ & 20 & 13.5 & 0.0137 & 34.0 & 0.958 \\
\hline$\# 7$ & 20 & 1.2 & 0.00264 & 14.3 & 0.958 \\
\hline$\# 8$ & 20 & 4.5 & 0.00767 & 16.0 & 0.93 \\
\hline$\# 9$ & 20 & 5.2 & 0.576 & 10.6 & 0.954 \\
\hline$\# 10$ & 20 & 3.1 & 0.0132 & 14.2 & 0.913 \\
\hline \multirow[t]{3}{*}{ Laponite PJM Simulant ${ }^{(\mathrm{b})}$} & 15 & 79.5 & 0.123 & 141.4 & 0.866 \\
\hline & 25 & 63.6 & 0.254 & 139.8 & 0.922 \\
\hline & 35 & 71.1 & 0.0761 & 149.7 & 0.995 \\
\hline \multirow[t]{3}{*}{$\begin{array}{l}\text { Kaolin-Bentonite PJM } \\
\text { Simulant }{ }^{(\mathrm{b})}\end{array}$} & 15 & 36.4 & 0.0193 & 70.0 & 0.916 \\
\hline & 25 & 41.4 & 0.0506 & 74.7 & 0.964 \\
\hline & 35 & 48.4 & 0.0168 & 97.2 & 0.905 \\
\hline $\begin{array}{l}\text { AZ-101 Pretreated (PT) } \\
\text { Sludge } \\
\end{array}$ & 30 & 14.2 & 0.262 & 31 & 0.929 \\
\hline $\begin{array}{l}\text { (a) Speers at al. (1987). } \\
\text { (b) Poloski et al. (2004). } \\
\text { (c) Poloski et al. (2003b). }\end{array}$ & & & & & \\
\hline
\end{tabular}

Values for the single point equilibrium shear strength, $\tau_{\infty}$, are provided in Figure 11.3. Falling ball rheometer and shear vane measurements were compiled, and the maximum measured value for each tank is shown in Figure 11.3. A breakdown of the primary sludge waste type is also provided. Saltcake waste 
types are stated in the absence of a sludge waste type. The gaps in the waste types represented in this shear strength data are shown in Figure 11.4. The relative volume of each waste type in the tanks where shear strength data was analyzed (red bar in the front of the graph) is compared to the total volume of waste associated with those waste types (blue bar in the back of the graph). Saltcake waste types are stated in the absence of a sludge waste type.

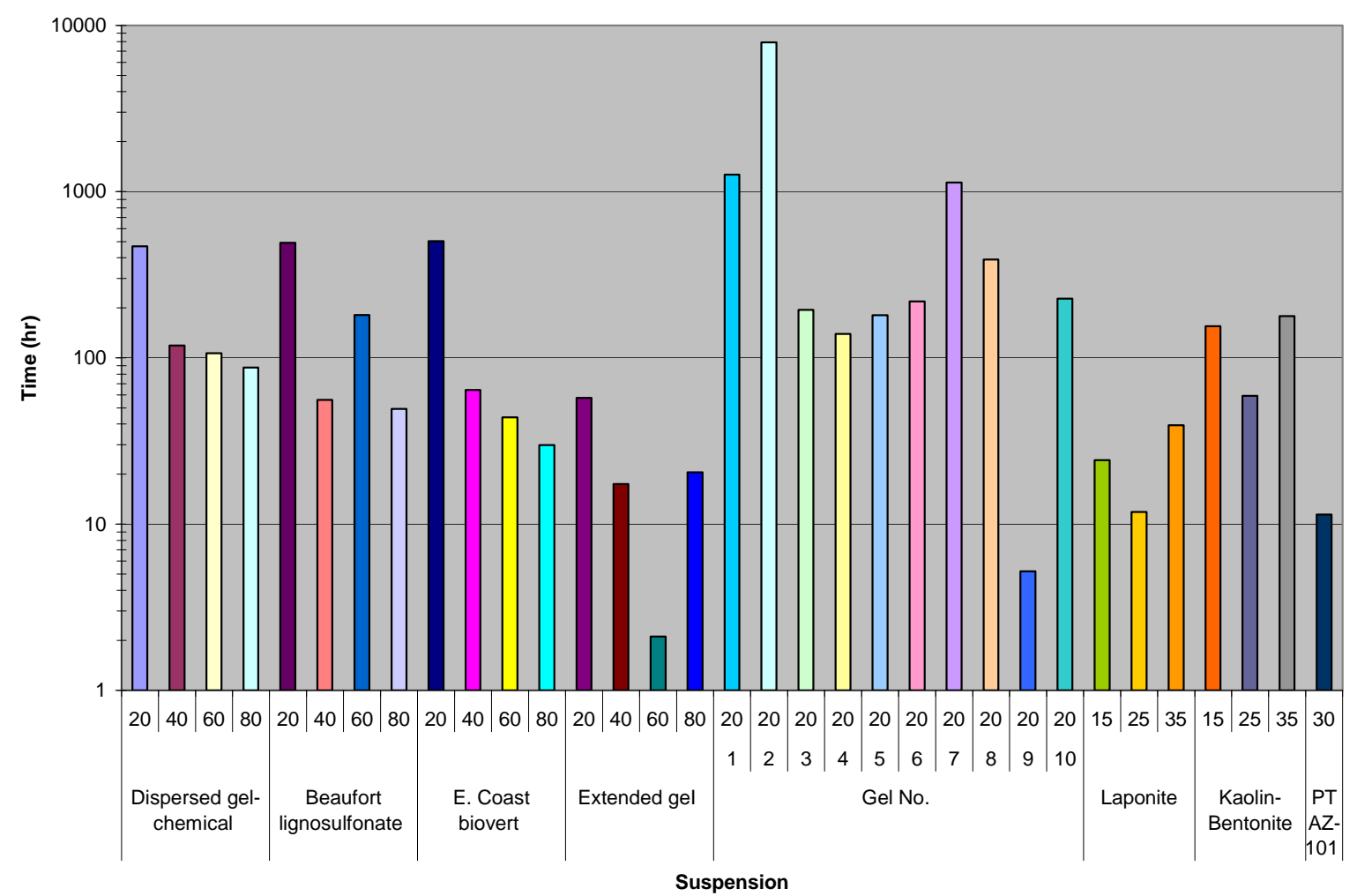

Figure 11.2. Gel Time Constant Comparison of Various Particulate Suspensions and Temperatures $\left({ }^{\circ} \mathrm{C}\right)$

An analysis of the slurry composition for the tanks identified with maximum shear strength for each waste type was performed. The analysis reveals that, with the exception of the saltcake wastes, shear strength increases with increasing concentration of the following species (in order of significance) zirconium compounds $>$ boehmite $>$ bismuth compounds $>$ gibbsite $>$ iron hydroxide. Silica and aluminosilicate content did not show a strong effect on shear strength. 


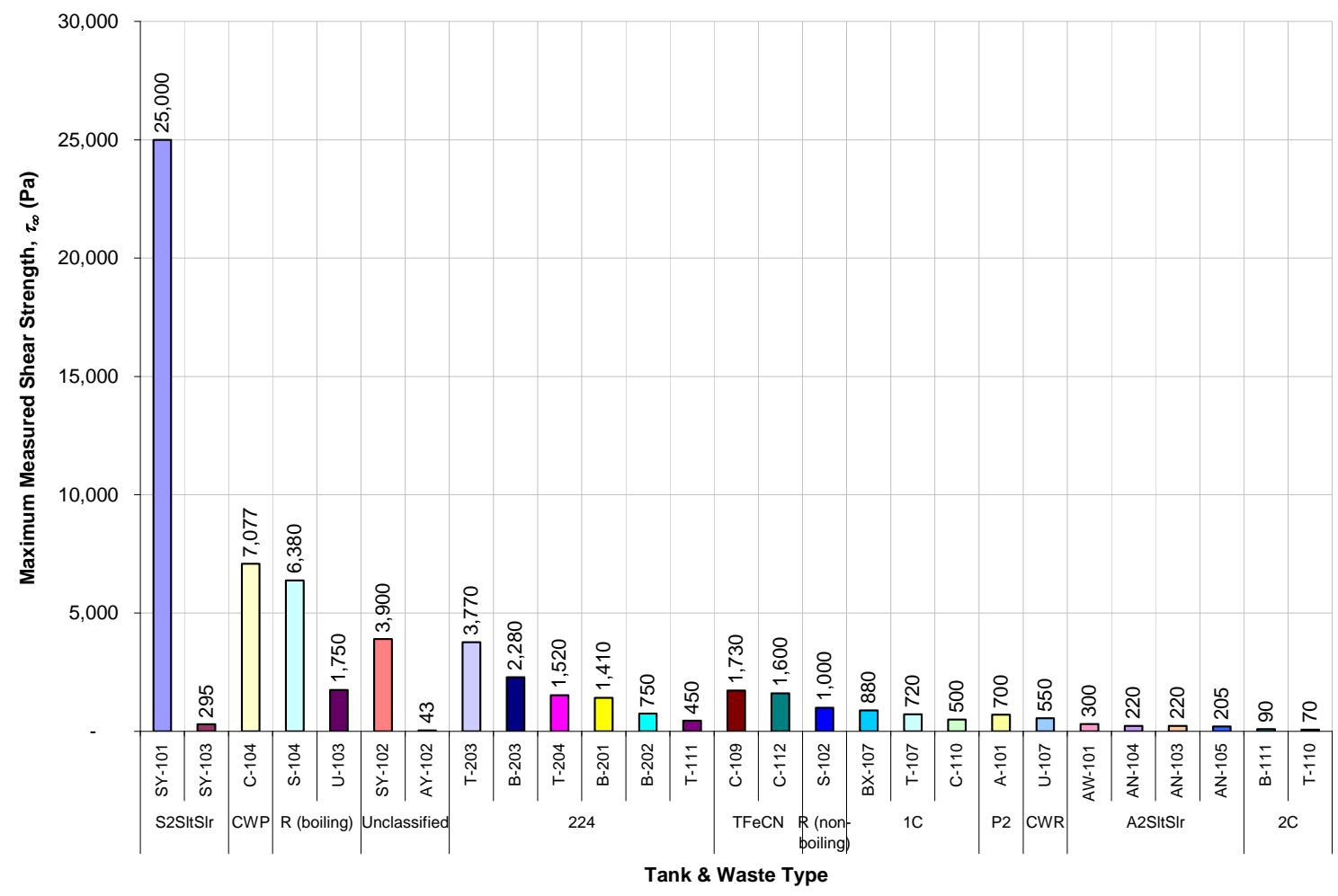

a) Maximum measured shear strength for each Hanford waste tank

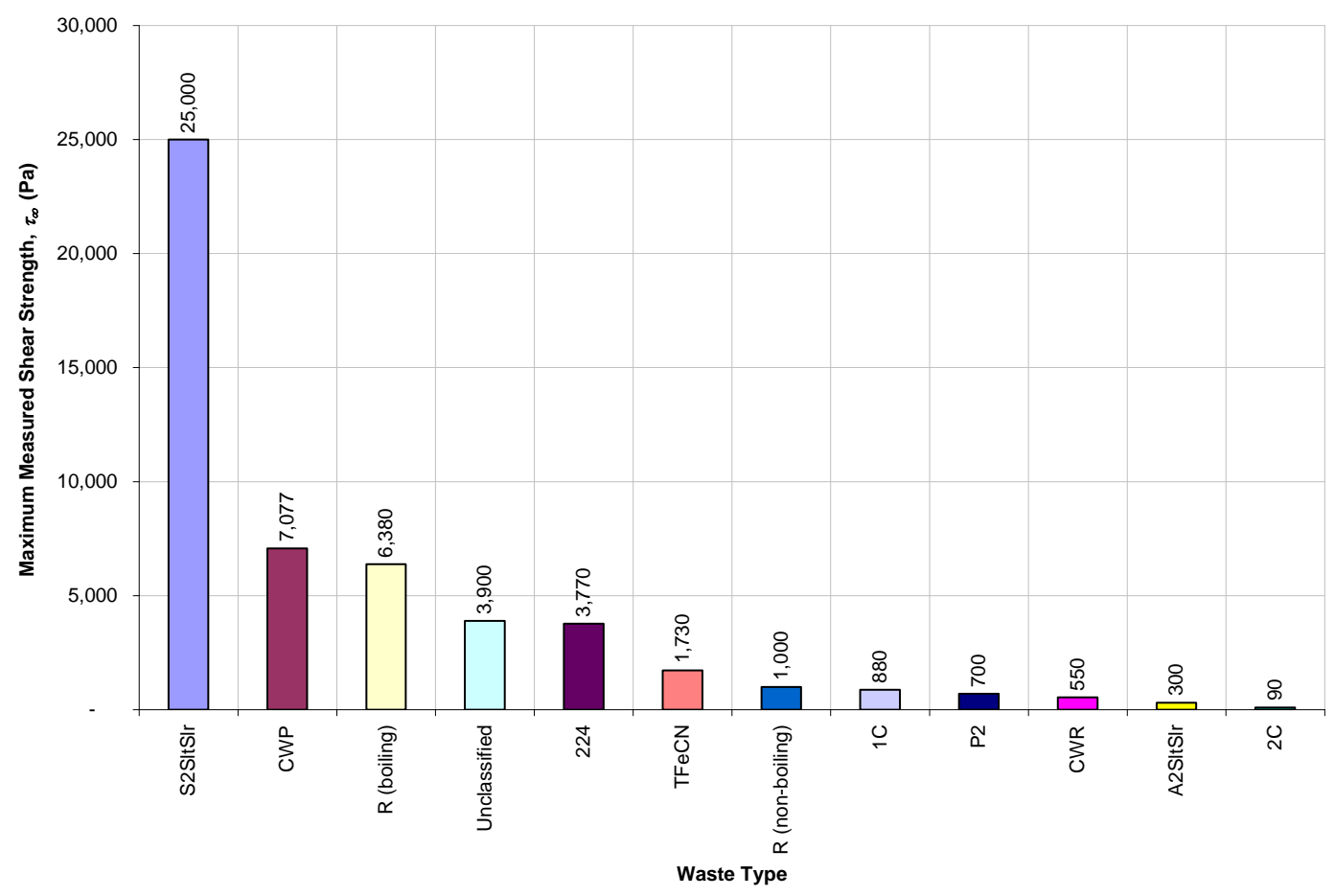

b) Maximum measured shear strength for each Hanford waste type

Figure 11.3. Shear Strength Summary for Various Hanford Waste Tanks and Types 


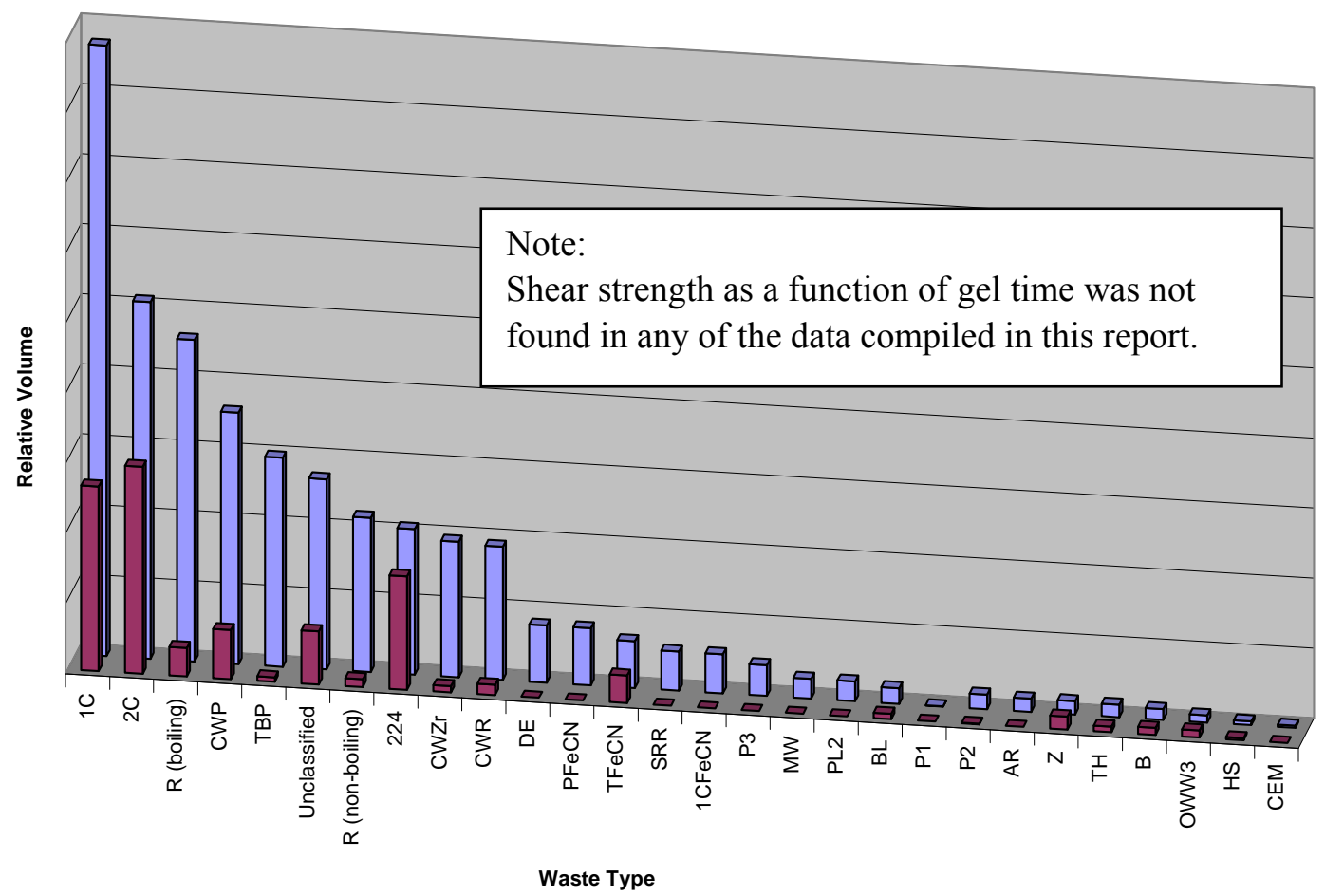

Figure 11.4. Gaps in Data Available for Shear Strength Modeling as a Function of Waste Type

This observation can be explained by a particle size argument. The zirconium compounds, boehmite, bismuth compounds, gibbsite, and iron hydroxide particles are generally submicron, colloidal particles with a large surface-to-mass ratio (Wells et al. 2007). The silica present in the tank waste is generally thought to come from Hanford sand with a much larger particle size (Wells et al. 2007). Zhou and coworkers (2001) present an equation that states at a given surface chemistry condition the shear strength of a non-Newtonian fluid varies according to:

$$
\tau_{\infty}=\frac{K}{d^{2}} \phi^{c}
$$

where

$K=$ a constant which relates bond strength to material properties and surface chemistry condition

$d=$ the particle diameter

$c \quad=$ a fitting parameter.

In this situation, increases in the relative amounts of $\mathrm{ZrO}_{2}$, boehmite, gibbsite, and iron hydroxide would reduce the overall particle diameter and increase shear strength. Adding silica would raise the particle diameter and reduce shear strength of the mixture. Bingham yield stress, $\tau_{O}^{B}$, can also be modeled with an equation of the same form as Eq. (11.3).

Saltcake waste had the largest measured shear strength, which is attributed to precipitated salt bridges between particles. It is unclear whether saltcake shear strength is reversible (will rebuild to these high levels). These bridges will likely require excessive time or a change in chemical composition to reform. 


\subsection{Bingham Plastic Modeling}

Hanford slurries can be characterized rheologically as non-Newtonian, Bingham plastic fluids. Bingham plastic model fits for 28 waste tanks from 12 sludge types covering a range of temperatures from $20^{\circ}$ to $95^{\circ} \mathrm{C}$ were obtained from the available literature. Figure 12.1 shows the range of Bingham parameters (Bingham consistency and yield stress) from approximately 300 flow curves. For reference, the HLW pretreated sludge boundaries discussed by Poloski and coworkers (2006a) are also placed on the figure. These data indicate that the Hanford tank feed has a greater tendency for exceeding the $30 \mathrm{cP}$ Bingham consistency parameter (15 measurements) than the Bingham yield stress ( 3 measurements). Only two measurements exceeded both the yield stress and consistency boundaries.

The Bingham plastic rheological parameters are a strong function of the undissolved solids loading. Figure 12.2 shows how the Bingham plastic parameters vary with undissolved solids concentration. Again, the HLW pretreated sludge rheological bounds discussed by Poloski et al. (2006a; WTP-RPT-112 Rev 0 ) are shown in the figure. In addition, the WTP contract solids processing specification of $16.7 \mathrm{wt} \%$ solids has been converted to an estimate of volume percent undissolved solids to provide a process throughput reference point. Data points in the lower right quadrant should have moderate rheology while meeting or exceeding contract throughput specifications. The lower left quadrant identifies slurries with moderate rheology that are within contract throughput specifications. The upper right quadrant identifies slurries with high rheology where further dilution is likely needed to lower slurry rheological properties and meet contract throughput specifications. The upper left quadrant identifies slurries with high rheology, where further dilution may lead to moderate rheology while meeting throughput specifications.

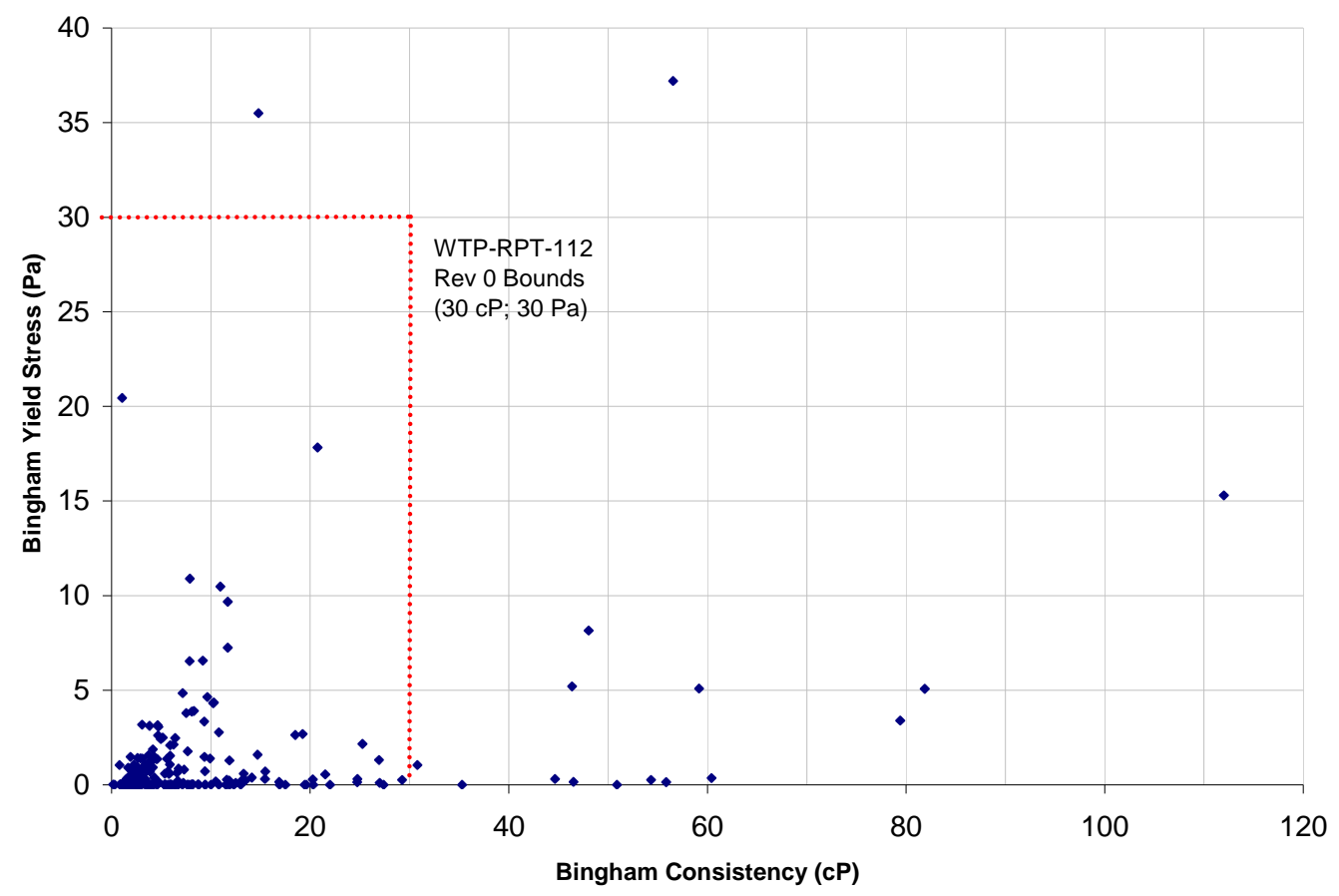

Figure 12.1. Scatter Plot Showing the Range of Measured Bingham Parameters 


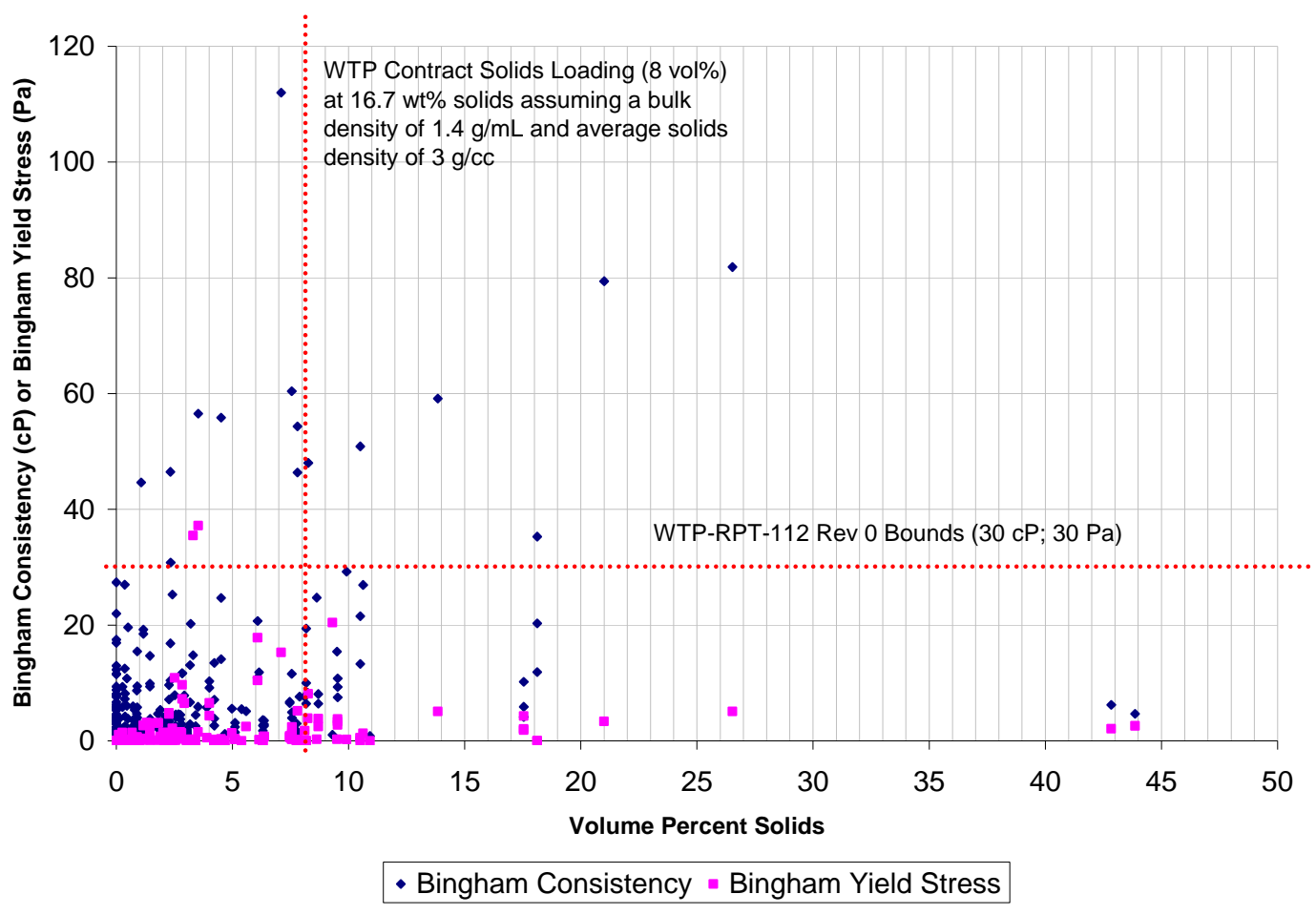

Figure 12.2. Scatter Plot of Obtained Bingham Parameters at Various Solids Loadings

While single points are shown on the scatter plot, each point actually lies on a series of dilution curves. Because of the exponential nature of rheological properties with respect to solids concentration, a small dilution can drastically lower the rheological properties of a fluid. Therefore, the high rheological point at $80 \mathrm{cP}$ consistency and $27 \mathrm{~Pa}$ yield stress should not be considered an anomaly. This data point demonstrates the extreme rheology for this class of material achievable from concentrating solids from a slurry.

Several of the data points in Figure 12.2 are for the same tank sample diluted with water to various solid contents. To gain an understanding of how the rheological parameters in the database vary by Hanford waste tank and waste type, Figures 12.3 and 12.4 were created based on the maximum measured Bingham parameters for each waste tank. Figure 12.3 illustrates that R-(boiling), TFeCN, saltcake, and 224 wastes tend to exhibit high Bingham consistency values; Figure 12.4 shows that TFeCN, R-(boiling), 224, and CWP wastes exhibit relatively high Bingham yield stress values.

Interestingly, TFeCN, R-(boiling), 224, and CWP wastes also appear to be among the wastes with the large measured shear strength values. Consequently, these waste types appear to have the largest degree of non-Newtonian behavior. Because these wastes tend to be relatively high in iron hydroxide, boehmite, aluminosilicate, zirconium compounds, and bismuth compounds, the trend observed between solids composition and shear strength appears to be applicable to flow curve behavior also.

While saltcake wastes have among the highest Bingham consistency values, they have moderate Bingham yield stress values. Again, this supports the hypothesis that the high shear strength for the saltcake wastes is a nonreversible artifact of salt bridging between particles. Once these salt bridges are broken the yield stress reduces to moderate levels. 


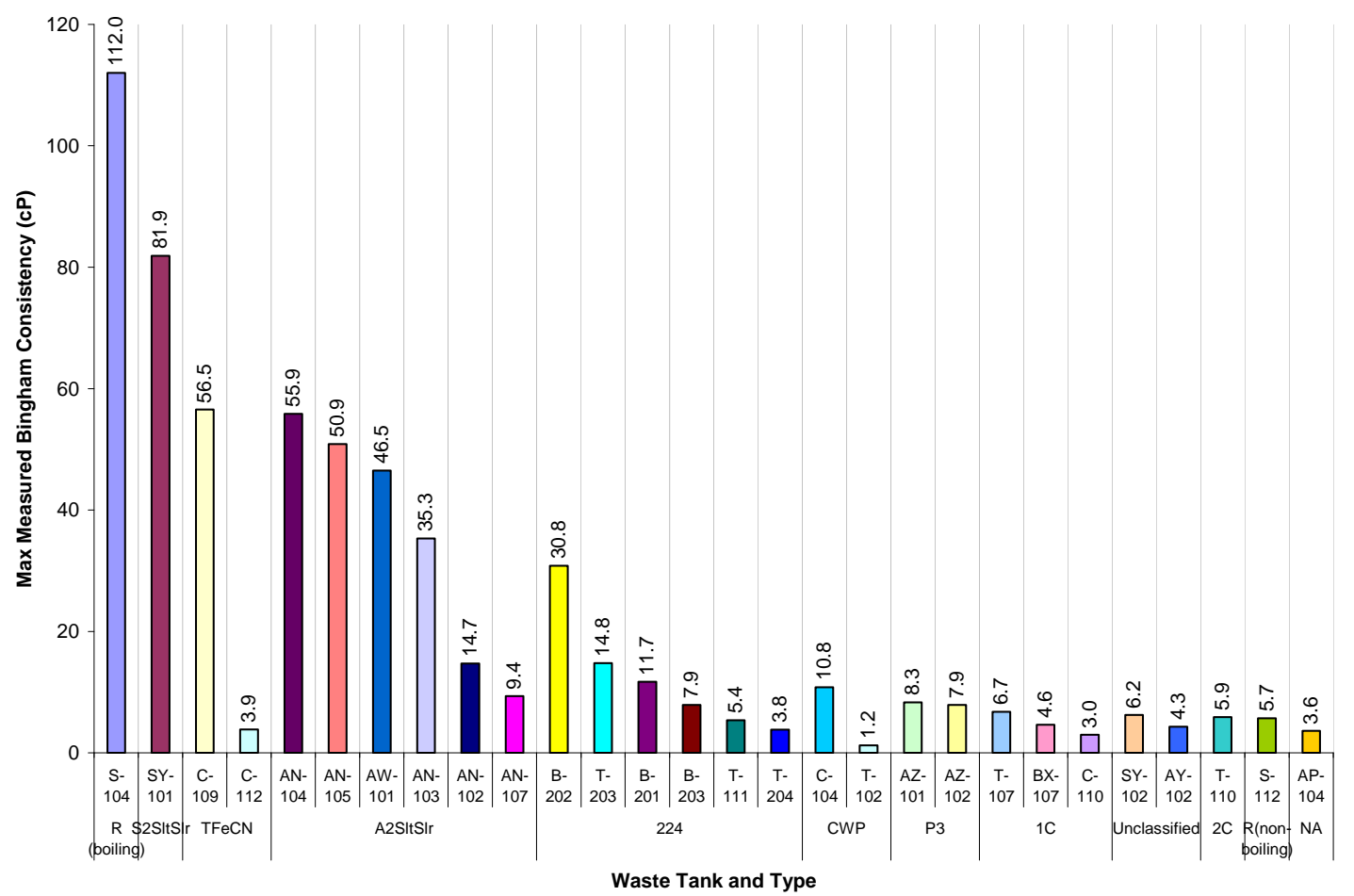

Figure 12.3. Maximum Measured Bingham Consistency for Various Hanford Tanks and Waste Types

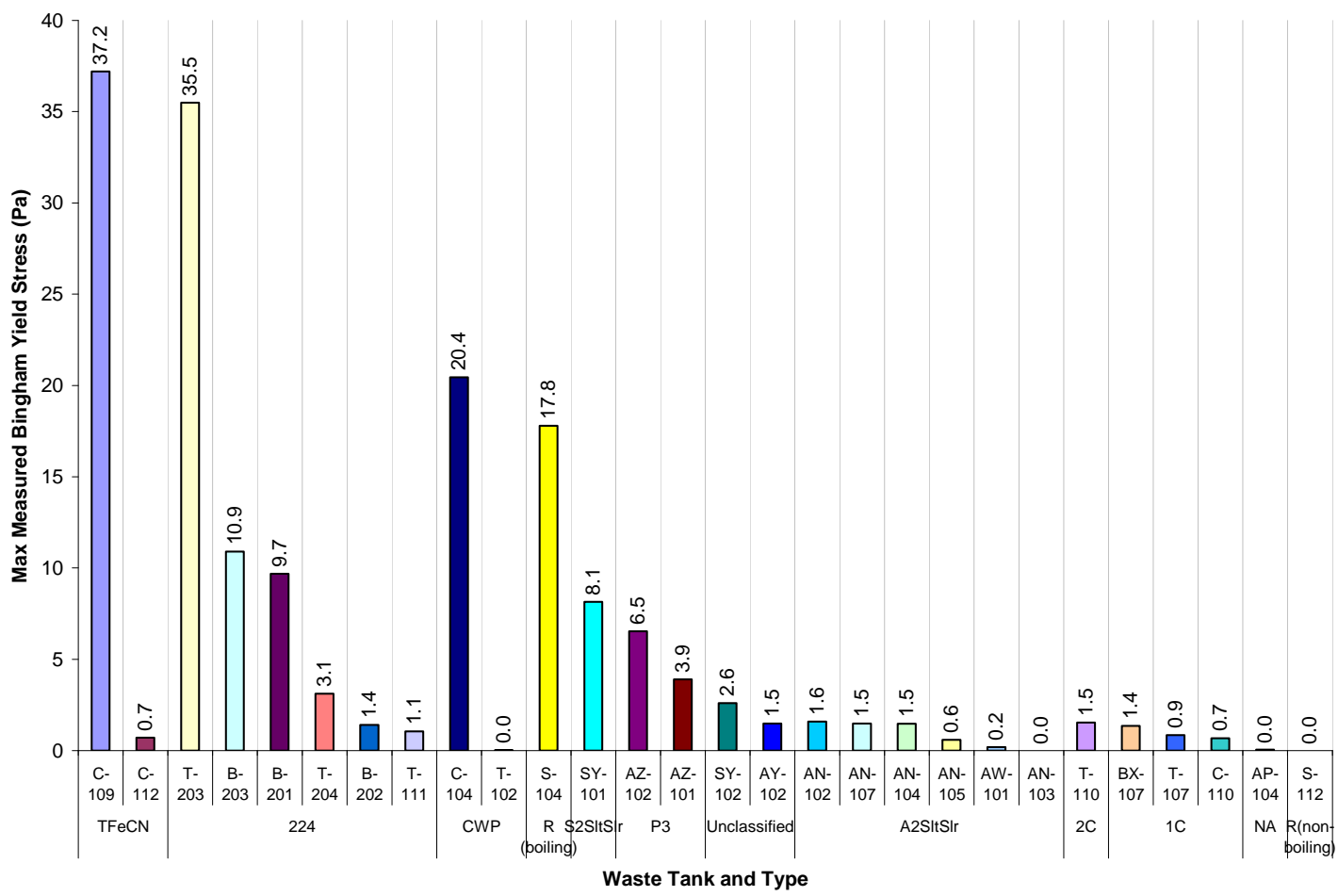

Figure 12.4. Maximum Measured Bingham Yield Stress for Various Hanford Tanks and Waste Types 
Ferraris and co-workers (2001) provide an equation that describes how the Bingham plastic consistency parameter varies with solids concentration:

$$
\eta_{P}=a e^{b \phi}
$$

where

$$
\begin{array}{ll}
\eta_{P} & =\text { the Bingham consistency in } \mathrm{cP} \\
\phi & =\text { the solids volume fraction } \\
a \text { and } b & =\text { fitting parameters. }
\end{array}
$$

Zhou and coworkers (2001) provide an equation that describes how the Bingham yield stress varies with solids concentration (Eq. 12.2). This equation is of similar form to the shear strength equation.

$$
\tau_{O}^{B}=c \phi^{d}
$$

$$
\begin{array}{ll}
\text { where } & \\
\tau_{O}^{B} & =\text { the Bingham yield stress } \\
\phi & =\text { the solids volume fraction } \\
c \text { and } d & =\text { fitting parameters. }
\end{array}
$$

These equations are typically valid only when the chemistry of the slurry is held constant while varying the solids loading. This can be achieved by diluting the slurry with decanted or separated interstitial liquid which is at equilibrium with the slurry solids. Unfortunately, this protocol was not observed and water was used as a diluent in all of the dilution studies cited in this report. Nonetheless, the data compiled in this report was modeled using Eq. (12.2) and (12.3) to find a correlation between rheological parameters and solids concentration.

Several correlations were found that had correlation coefficients $\left(r^{2}\right)$ above $0.7,17$ correlations were found for Bingham consistency and 10 correlations for Bingham yield stress. These data cover eight Hanford waste tanks, with five waste types represented including 224, 1C, 2C, A2SltSlr, and CWP. The parameters for these correlations are shown in Table 12.1. Gaps in waste types used in these rheological correlations are shown in Figure 12.5. Several of these correlations indicate that relatively high rheological parameters ( $>30 \mathrm{cp}$ or $30 \mathrm{~Pa}$ ) exist at low solids concentrations ( $>8$ vol\% undissolved solids). These fluids may pose throughput challenges to the WTP as additional dilution may be required to achieve moderate rheological parameters.

Plots presenting the raw data used to evaluate these correlations are shown in Appendix B, while plots of the data and correlations can be found in Appendix C. Example plots showing a good correlation with Eq. (12.2) and (12.3) are shown in Figures 12.6 and 12.7. Figure 12.6 shows the relationship between C-104 as-received tank core samples and solids concentration, while Figure 12.7 shows the same relationship for the water-diluted C-104 core samples. These data are a combination of tank core sample characterization reports and cross-flow filtration studies. A peak in the yield stress trend and minimum in the consistency trend are observed in this figure. 
Table 12.1. Bingham Model Parameters for Various Hanford Tanks

\begin{tabular}{|c|c|c|c|c|c|c|c|c|c|c|c|}
\hline $\begin{array}{l}\text { Waste } \\
\text { Type }\end{array}$ & $\begin{array}{l}\text { Waste } \\
\text { Tank }\end{array}$ & $\begin{array}{l}\text { Waste } \\
\text { Status }\end{array}$ & $\begin{array}{c}\text { Temperature } \\
\text { Category } \\
\left({ }^{\circ} \mathrm{C}\right)\end{array}$ & $\begin{array}{c}\text { Consistency } \\
\text { Parameter, } a \\
\text { (cP) }\end{array}$ & $\begin{array}{c}\text { Consistency } \\
\text { Parameter, } \\
\text { b (-) }\end{array}$ & 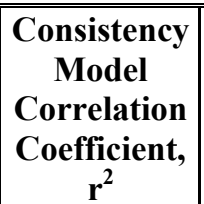 & $\begin{array}{c}\text { Predicted } \\
\text { Solids Loading } \\
\text { at } 30 \mathrm{cP} \\
\text { Consistency } \\
\text { (vol\%) }\end{array}$ & $\begin{array}{c}\text { Yield Stress } \\
\text { Parameter, } \\
c \\
(\mathbf{P a})\end{array}$ & $\mid \begin{array}{c}\text { Yield Stress } \\
\text { Parameter, } d \\
(-)\end{array}$ & $\begin{array}{c}\text { Yield Stress } \\
\text { Model } \\
\text { Correlation } \\
\text { Coefficient } \mathbf{r}^{2}\end{array}$ & \begin{tabular}{|c|} 
Predicted \\
Solids Loading \\
at 30 Pa Yield \\
Stress \\
(vol\%)
\end{tabular} \\
\hline 224 & B-202 & Washed & $20-35$ & 1.51 & 36 & 0.91 & 8.3 & $7.21 \mathrm{E}+04$ & 2.94 & 0.95 & 7.1 \\
\hline 224 & B-203 & Washed & $20-35$ & 1.30 & 72 & 1.00 & 4.4 & $1.31 \mathrm{E}+07$ & 3.80 & 1.00 & 3.3 \\
\hline 224 & $\mathrm{~T}-111$ & Washed & $20-35$ & 2.64 & 37 & 0.82 & 6.5 & $\mathrm{n} / \mathrm{r}$ & $\mathrm{n} / \mathrm{r}$ & $\mathrm{n} / \mathrm{r}$ & $\mathrm{n} / \mathrm{r}$ \\
\hline 224 & $\mathrm{~T}-203$ & Washed & $20-35$ & 1.08 & 79 & 1.00 & 4.2 & $2.50 \mathrm{E}+05$ & 2.60 & 1.00 & 3.1 \\
\hline 224 & B-202 & Washed & $80-95$ & 1.01 & 40 & 0.71 & 8.5 & $2.34 \mathrm{E}+04$ & 2.62 & 0.92 & 7.9 \\
\hline 224 & $\mathrm{~T}-111$ & Washed & $80-95$ & 1.16 & 43 & 0.90 & 7.6 & $\mathrm{n} / \mathrm{r}$ & $\mathrm{n} / \mathrm{r}$ & $\mathrm{n} / \mathrm{r}$ & $\mathrm{n} / \mathrm{r}$ \\
\hline $1 \mathrm{C}$ & BX-107 & Washed & $20-35$ & 1.64 & 37 & 0.98 & 7.9 & $\mathrm{n} / \mathrm{r}$ & $\mathrm{n} / \mathrm{r}$ & $\mathrm{n} / \mathrm{r}$ & $\mathrm{n} / \mathrm{r}$ \\
\hline $1 C$ & $B X-107$ & Washed & $80-95$ & 0.97 & 54 & 0.92 & 6.3 & $1.28 E+15$ & 9.53 & 1.00 & 3.7 \\
\hline $2 C$ & $T-110$ & Washed & 20-35 & 1.16 & 46 & 1.00 & 7.0 & $1.21 E+04$ & 2.68 & 1.00 & 10.7 \\
\hline A2SltSlr & AN-105 & Salt Cake & $20-35$ & 1.38 & 34 & 0.95 & 9.0 & $\mathrm{n} / \mathrm{r}$ & $\mathrm{n} / \mathrm{r}$ & $\mathrm{n} / \mathrm{r}$ & $\mathrm{n} / \mathrm{r}$ \\
\hline A2SltSlr & AN-105 & Salt Cake & $35-50$ & 3.37 & 17 & 0.93 & 13.0 & $\mathrm{n} / \mathrm{r}$ & $\mathrm{n} / \mathrm{r}$ & $\mathrm{n} / \mathrm{r}$ & $\mathrm{n} / \mathrm{r}$ \\
\hline A2SltSlr & AN-105 & Salt Cake & $50-65$ & 1.83 & 18 & 0.95 & 15.3 & $\mathrm{n} / \mathrm{r}$ & $\mathrm{n} / \mathrm{r}$ & $\mathrm{n} / \mathrm{r}$ & $\mathrm{n} / \mathrm{r}$ \\
\hline A2SltSlr & AN-104 & Washed & $20-35$ & 11.35 & 35 & 0.87 & 2.8 & $\mathrm{n} / \mathrm{r}$ & $\mathrm{n} / \mathrm{r}$ & $\mathrm{n} / \mathrm{r}$ & $\mathrm{n} / \mathrm{r}$ \\
\hline A2SltSlr & AN-104 & Washed & $35-50$ & 7.53 & 26 & 0.91 & 5.3 & $\mathrm{n} / \mathrm{r}$ & $\mathrm{n} / \mathrm{r}$ & $\mathrm{n} / \mathrm{r}$ & $\mathrm{n} / \mathrm{r}$ \\
\hline A2SltSlr & AN-104 & Washed & $50-65$ & 4.83 & 24 & 0.92 & 7.7 & $\mathrm{n} / \mathrm{r}$ & $\mathrm{n} / \mathrm{r}$ & $\mathrm{n} / \mathrm{r}$ & $\mathrm{n} / \mathrm{r}$ \\
\hline CWP & C-104 & Salt Cake & $20-35$ & 1.25 & 12 & 1.00 & 26.5 & $2.79 \mathrm{E}+02$ & 2.40 & 1.00 & 39.4 \\
\hline CWP & C-104 & Washed & $20-35$ & 0.78 & 28 & 1.00 & 13.2 & $4.13 \mathrm{E}+03$ & 3.11 & 1.00 & 20.5 \\
\hline CWP & C-104 & Washed & $35-50$ & 0.45 & 32 & 0.99 & 13.3 & $1.59 \mathrm{E}+04$ & 3.60 & 1.00 & 17.5 \\
\hline CWP & C-104 & Washed & $50-65$ & 0.36 & 32 & 0.99 & 13.9 & $4.90 \mathrm{E}+04$ & 4.03 & 1.00 & 15.9 \\
\hline S2SltSlr & SY-101 & Salt Cake & 20-35 & 43.05 & 3.7 & 0.91 & 0 & $n / r$ & $n / r$ & $n / r$ & $n / r$ \\
\hline S2SltSlr & \begin{tabular}{|l|} 
SY-101 \\
\end{tabular} & Salt Cake & $35-50$ & 16.47 & 6.4 & 0.95 & 9.4 & $7.14 \mathrm{E}+01$ & 1.98 & 0.99 & 64.6 \\
\hline S2SltSlr & \begin{tabular}{|l|} 
SY-101 \\
\end{tabular} & Salt Cake & $65-80$ & 8.32 & 14 & 0.76 & 9.0 & $1.19 \mathrm{E}+02$ & 1.58 & 0.65 & 41.9 \\
\hline S2SltSlr & $S Y-101$ & Salt Cake & $80-95$ & 16.18 & 13 & 1.00 & 4.7 & $n / r$ & $n / r$ & $n / r$ & $n / r$ \\
\hline Unclassified & AY-102 & Salt Cake & $50-65$ & 0.80 & 6.8 & 0.46 & 52.9 & $\mathrm{n} / \mathrm{r}$ & $\mathrm{n} / \mathrm{r}$ & $\mathrm{n} / \mathrm{r}$ & $\mathrm{n} / \mathrm{r}$ \\
\hline
\end{tabular}




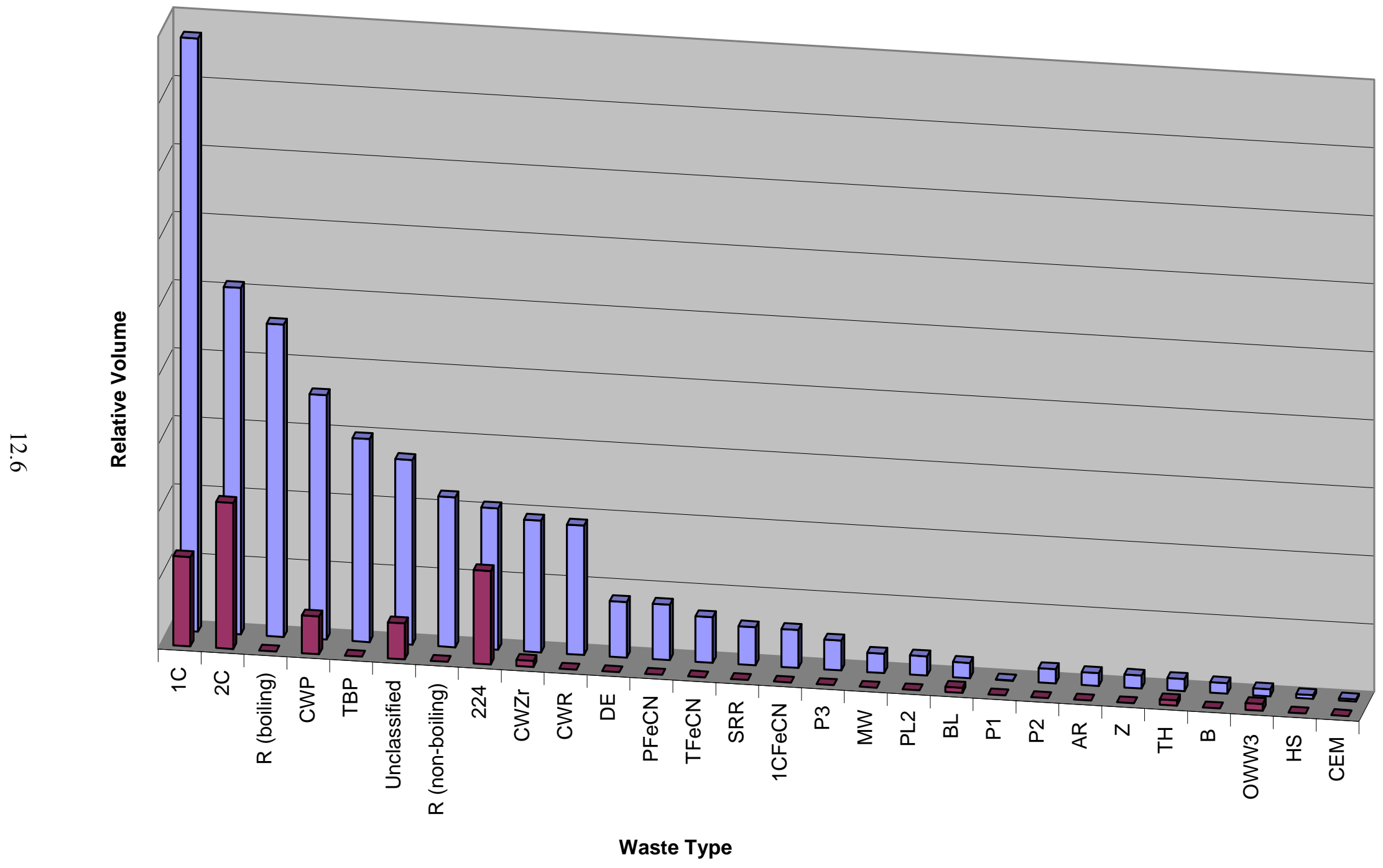

Figure 12.5. Gaps in Data Available for Rheological Correlations as a Function of Waste Type 

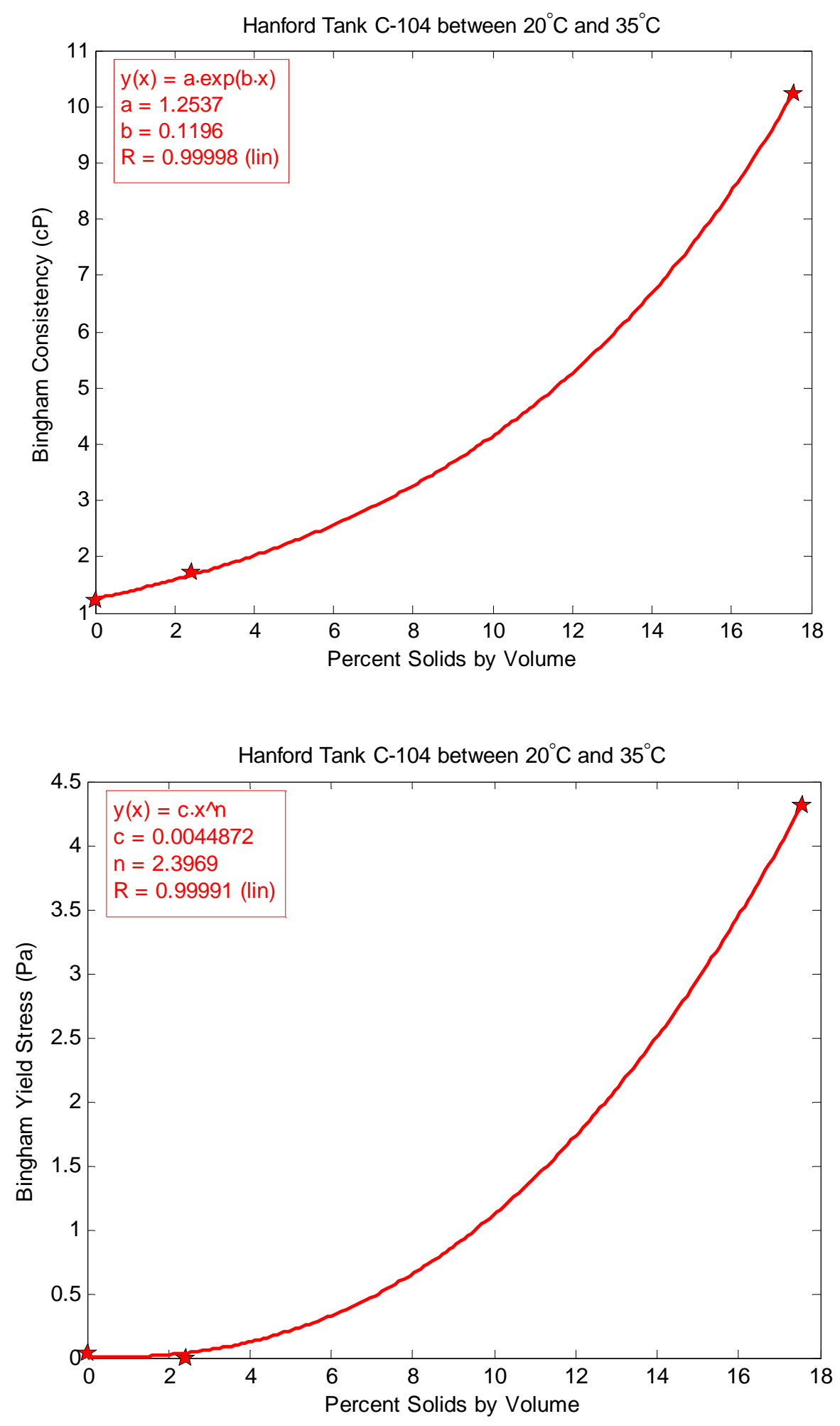

Stars $=$ as-received tank waste

Figure 12.6. Bingham Plastic Rheological Parameters as a Function of Solids Loading for Tank C-104 as an Example of an as-Received Hanford Core Sample 

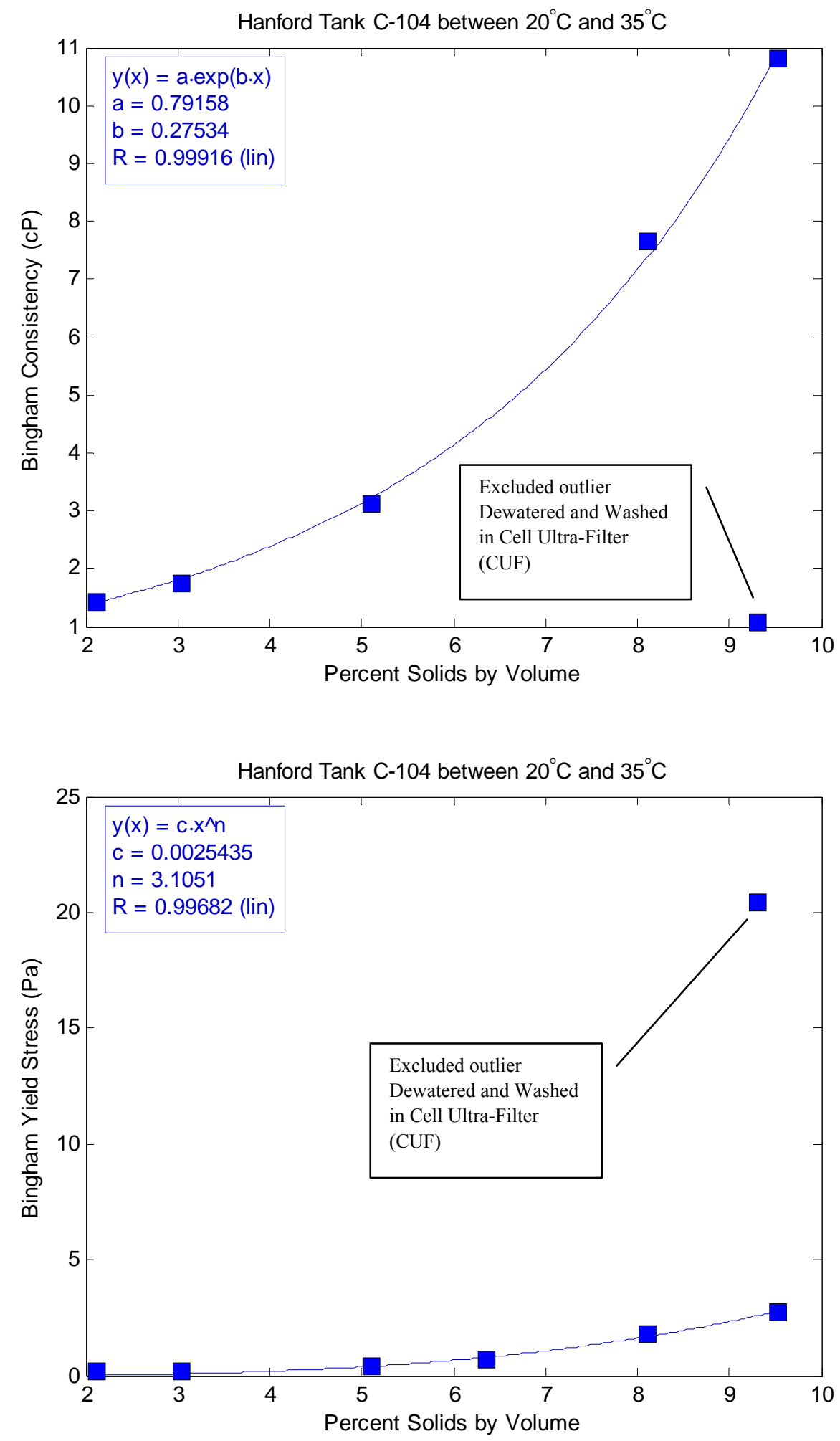

Squares represent water added as diluent

Figure 12.7. Bingham Plastic Rheological Parameters as a Function of Solids Loading for Tank C-104 as an Example of a Hanford Core Sample Diluted with Water 
This peak shows the import of sample process history as it corresponds to a sample that was processed in the cell ultra-filter. The sample underwent similar chemical changes as it was dewatered and water washed to a certain solids loading. However, the use of the filtration equipment may have altered the slurry physical properties through dissolution of salt cake particles, a lowered ionic strength (zeta potential), and potential particle attrition from the pump/filter loop. For this reason, the point was discarded from the correlation analysis but kept in the above analysis on maximum rheological values as sample process history will vary widely from tank retrieval through vitrification. A more detailed look at the affect of WTP pretreatment process operations on rheological properties is needed to gain confidence in the current process design.

Example plots from Appendix B have been selected to illustrate two important observations made while preparing this report. These plots are shown in Figures 12.8 and 12.9. Figure 12.8 shows the expected "mechanically dominated" dilution behavior where rheological properties exponentially decrease with water dilution due to the simple reduction of particle/particle interactions.

In reality, the parameters $a, b, c$, and $d$ from Eq. (12.2) and (12.3) can vary significantly with slurry chemical and physical properties such as zeta potential, particle size, particle chemistry, and aqueous chemistry. As dilution with water occurs, all of these properties are likely to vary as salt particles dissolve changing average particle size and chemistry, aqueous species are diluted while salt species are added, and solution equilibrium is achieved. The fact that the expected "mechanical" trend is maintained during this process indicates that the effect of these changes is minimal and the slurry rheology is dominated by mechanical forces.

On the other hand, Figure 12.9 shows "chemically dominated" dilution behavior where rheological properties actually increase to a maximum value with water dilution. In this scenario, as the sample is diluted with water, chemical properties vary as salt particles dissolve. This changes average particle size, particle chemistry, aqueous speciation, and interaction potentials as a new solution equilibrium is achieved. Saltcake solids are typically on the order of several tens or hundreds of micrometers, while sludge particles are on the order of ten micrometers or smaller (Wells et al. 2007). The shift to smaller particle size, $d$, alone will result in a peak with regards to yield stress as the parameter, $c \propto d^{-2}$ (Zhou et al. 2001). Additionally, dilution will ultimately result in a reduction in solution ionic strength and zeta potential. The parameter $c$ will reach a maximum value when the zeta potential is zero (Zhou et al. 2001). Consequently, in some cases, dilution with water will result in a rheological maximum due to these surface chemistry effects. Several additional examples of "mechanically dominated" and "chemically dominated" dilution phenomena can be seen in Appendix B. 

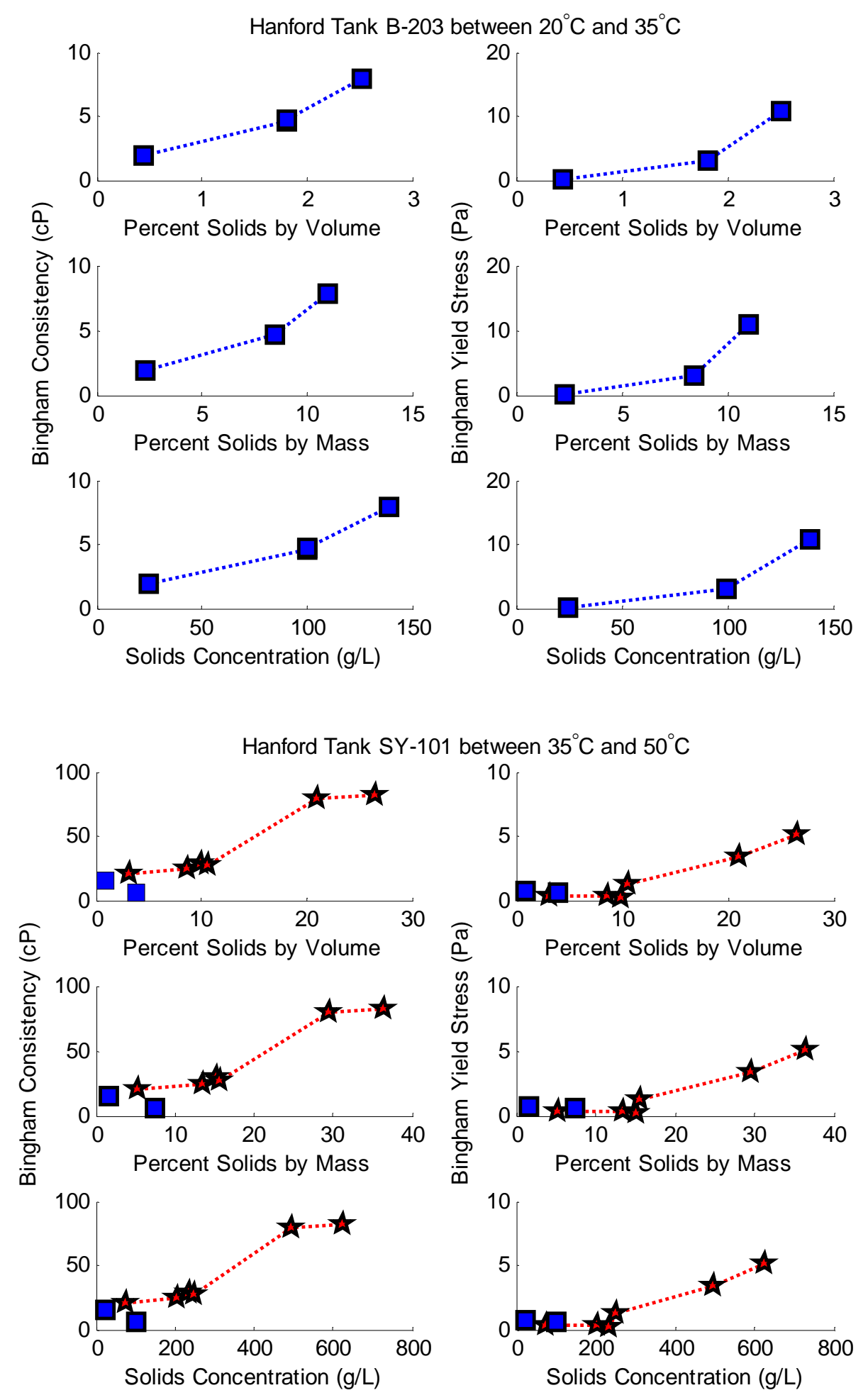

Stars $=$ as-received tank waste; squares $=$ water added as diluent

Figure 12.8. Bingham Plastic Rheological Parameters as a Function of Solids Loading for a WaterDiluted Hanford Core Sample Showing Decreasing Rheology as Dilution Occurs 

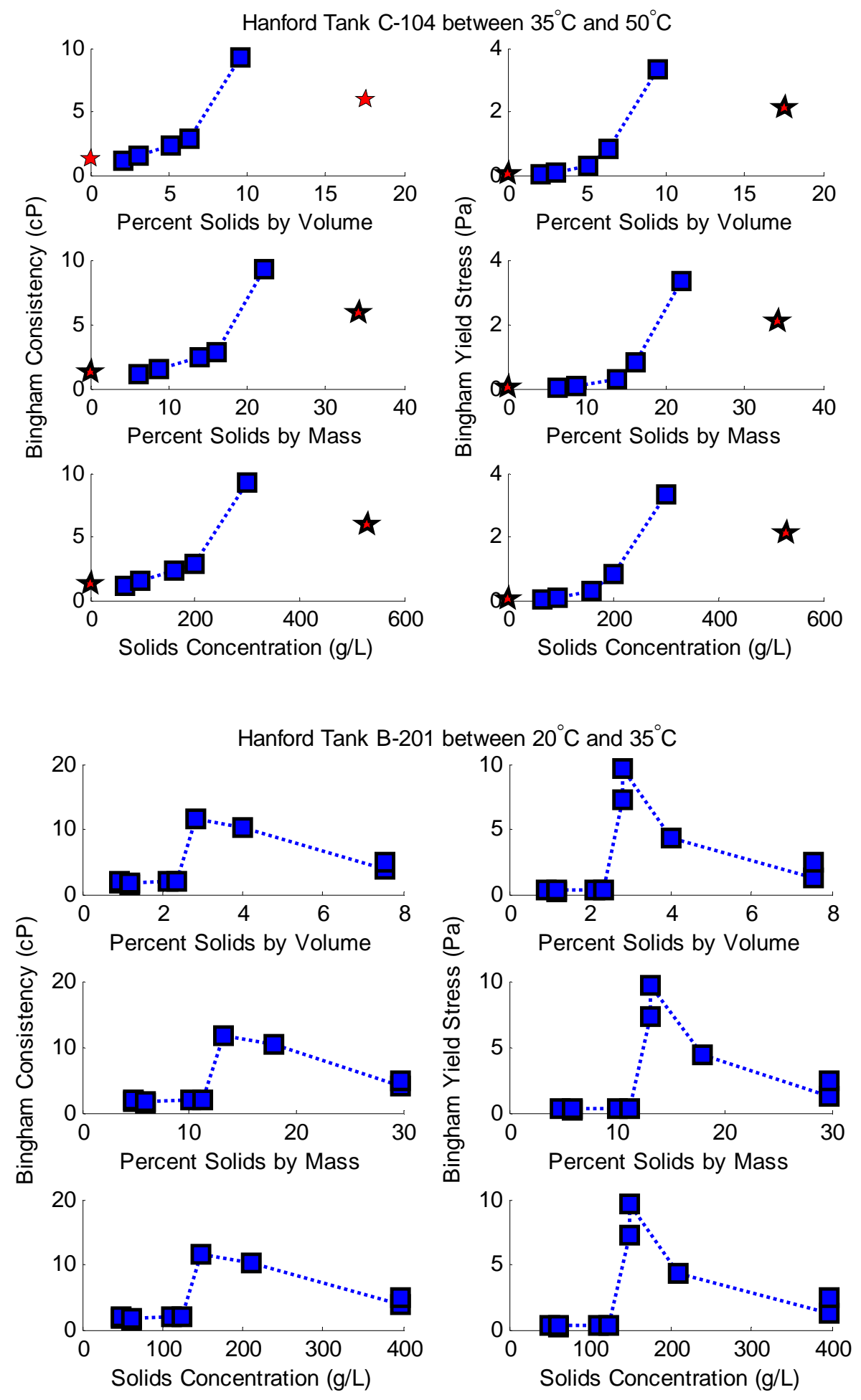

Stars $=$ as-received tank waste; squares $=$ water added as diluent

Figure 12.9. Bingham Plastic Rheological Parameters as a Function of Solids Loading for a WaterDiluted Hanford Core Sample Showing a Rheological Peak as Dilution Occurs 


\subsection{Transient Modeling}

During off-normal events, the WTP processing system may lose power and become inoperable. In these situations, the slurries will remain quiescent for some time until backup/emergency systems are used or the primary system is restored. Understanding how rheological properties vary with time under quiescent conditions is an important design consideration. From the information presented above, an estimation of this transient rheological behavior can be obtained. The solids interface height of a sediment bed as a function of time (Renko 1996) in a sedimentation column was described in the sedimentation section of this report and shown as:

$$
z\left(t, z_{0}\right)=\frac{C \phi_{0} z_{0}}{\alpha}+\left(z_{0}-\frac{C \phi_{0} z_{0}}{\alpha}\right) e^{\frac{-\alpha}{\phi_{0} z_{0}} t}
$$

where

$\mathrm{Z} \quad=$ the interface height $(\mathrm{m})$ at time $t$ in hours

$z_{0} \quad=$ the initial interface height $(\mathrm{m})$

$\phi_{0} \quad=$ the initial solids concentration of the suspended sample (volume fraction)

$\alpha$ and $C=$ fitting parameters in $\mathrm{m} / \mathrm{hr}^{-1}$.

The solids concentration profile as a function of time was also presented by Renko (1998) and is shown as Eq. (13.2).

$$
\phi(\mathrm{t}, \mathrm{z})=\frac{\phi_{0}}{\frac{\mathrm{C} \phi_{0}}{\alpha}+\left(1-\frac{\mathrm{C} \phi_{0}}{\alpha}\right)\left(1-\frac{\mathrm{t} \alpha}{\phi_{0} \mathrm{z}_{0}}\right) \mathrm{e}^{\frac{-\alpha}{\phi_{0} \mathrm{z}_{0}} \mathrm{t}}}
$$

As sedimentation proceeds, the local Bingham consistency and yield parameter vary as a function of solids concentration. These equations were described in the Bingham plastic modeling section of this report and are shown as Eq. (13.3) and (13.4):

$$
\begin{gathered}
\eta_{P}=a e^{b \phi} \\
\tau_{O}^{B}=c \phi^{d}
\end{gathered}
$$

where

$$
\begin{array}{ll}
\eta_{P} & =\text { Bingham consistency } \\
\tau_{O}^{B} & =\text { Bingham yield stress } \\
\mathrm{b}, c, \text { and } d & =\text { fitting parameters }
\end{array}
$$

Shear strength as a function of time, for cohesive sludge layers that exclude frictional forces generated by coarse particles, was described in the shear strength modeling section of this report and is shown as 


$$
G_{t}=\left(\tau_{O}^{B}-\tau_{\infty}\right) e^{-k t_{g}}+\tau_{\infty}
$$

where

$$
\begin{aligned}
G_{t} & =\text { shear strength at any gel time } \\
\tau_{\infty} & =\text { single point equilibrium shear strength measurement } \\
\tau_{O}^{B} & =\text { Bingham yield stress } \\
t_{g} & =\text { gel time } \\
k & =\text { gel time constant }\left(\mathrm{hr}^{-1}\right) .
\end{aligned}
$$

The equilibrium shear strength, $\tau_{\infty}$, (see Eq. 13.6) is assumed to have the same form and fitting parameter, $d$, as the correlation for Bingham yield stress (see Eq. 13.4). Consequently, if an equilibrium shear strength has been measured (due to the limited amount of Hanford waste data, a single point rather than an average is used for this calculation) at a given solids concentration, $\phi$, the fitting parameter $C_{\infty}$ can be solved.

$$
\tau_{\infty}=c_{\infty} \phi^{d}
$$

Combining Eq. (13.1) through (13.6) allows one to predict solids concentration, Bingham consistency, Bingham yield stress, and shear strength as a function of time. However, Eq. (13.5) is only valid for a slurry in which the solids concentration remains constant (i.e., a fully settled slurry). Eq. (13.5) cannot be applied directly to an actively sedimenting slurry because both the gel structure and solids concentration are changing simultaneously. Additionally, if coarse particles are present in the sludge, frictional forces will be generated in addition to cohesive, interparticle forces. The frictional force contribution would need to be accounted as an additional term in Eq. 13.5. A discussion on the affects of frictional forces on shear strength was discussed by Poloski et al. (2006b).

To use this Eq. 13.5 during sedimentation, the gel time for shear strength calculation must be adjusted to maintain a constant shear strength due to the sedimentation process while adjusting to the transient shear strength curve that matches the settled solids concentration. This is done through a numerical simulation constructed by considering two periods of time during the sedimentation process, $t_{0}$ and $t_{1}$, where $t_{0}<t_{1}$. As sedimentation proceeds, solids concentration increases from an initial value at time $t_{0}$. The transient shear strength curve for the new solids concentration shifts due to the new solids concentration at time $t_{1}$. Therefore, a correction to the gel time is made to keep the shear strength constant due to sedimentation but reflects the new transient shear strength curve at the more fully settled solids concentrations. The correction for this shear strength gel time can be mathematically found as Eq. (13.7). The new gel time is calculated as Eq. (13.8).

$$
\Delta t_{g}=\frac{1}{k} \ln \left[\frac{\left(\tau_{O, 0}^{B}-\tau_{\infty, 0}\right)+\left(\tau_{\infty, 0}-\tau_{\infty, 1}\right) e^{-k\left(t_{0}-t_{g, 0}\right)}}{\left(\tau_{O, 1}^{B}-\tau_{\infty, 1}\right)}\right]
$$




$$
t_{g, 1}=t_{g, 0}+\Delta t_{g}
$$

From these equations, the rheological properties are observed to grow exponentially with solids concentration. Fully settled slurries typically exhibit large rheological properties, and a level of dilution is required for retrieval and processing. In this section, a $50 \%$ by volume dilution from the fully settled configuration is assumed as a realistic tank retrieval condition (the actual amount of diluant used will vary with retrieval technology and engineering parameters). When fully settled, the sludge interface level would then be at $75 \%$ of the total vessel height. Several process scales should be considered in this assessment. A summary of several significant height scales are shown in Table 13.1.

Table 13.1. Typical Process Heights

\begin{tabular}{||l|c|c||}
\hline \multicolumn{1}{|c|}{$\begin{array}{c}\text { Process } \\
\text { System }\end{array}$} & \multicolumn{2}{c|}{ Length Scale } \\
SI & \multicolumn{1}{c|}{ Imperial } \\
\hline Piping & $0.1 \mathrm{~m}$ & $3.9 \mathrm{in}$ \\
\hline Small Vessel & $1 \mathrm{~m}$ & $3.3 \mathrm{ft}$ \\
\hline Large Vessel & $10 \mathrm{~m}$ & $33 \mathrm{ft}$ \\
\hline
\end{tabular}

Since the sedimentation experiments typically occurred under ambient temperature conditions, modeling was limited to rheological data in the $20^{\circ}-35^{\circ} \mathrm{C}$ temperature range. The average sedimentation parameters from Section 11, $\alpha$ and $C$, for each tank were used in the simulation. Bingham consistency and yield stress modeling parameters from Section 12 were also applied. Table 13.2 shows the values used for each of the variables in these equations. Only limited data were available for this analysis, and the relative volume of each waste type analyzed compared with the total volume of the sludge waste types is shown in Figure 13.1.

With these values, estimates on the transient growth of rheological parameters as a function of depth were performed. The results at the three length scales are shown in Appendix E. Figures 13.2-13.4 show typical examples of these results. The figures indicate that sedimentation will occur faster in the smallscale systems such as pipes than in the larger systems such as plant vessels, reaching fully settled configurations on the order of tens, hundreds, and thousands of hours for each of the process scales from smallest to largest, respectively.

However, as sedimentation proceeds, the solids concentration at the bottom of the layer is significantly higher than at the top. This result indicates that rheological properties significantly increase from the top of the sediment bed to the bottom. A thick sludge heel begins to forms at the bottom of the sediment bed. The sludge heel then reaches the maximum rheological properties due to gravitational forces that increase solids concentration at the bottom of the sediment bed and interparticle bonds that develop as the particles remain quiescent. The thickness of this heel then appears to expand, as these forces are maximized from the bottom of the bed to the top, until the entire sediment bed has the same rheological properties as the heel. Also note that the heel will also contain coarse and dense particles that will slowly settle to the bottom of the sediment layer if the required agitation is not sufficient to suspend the particles during normal operation or if the rheological properties of the sludge are not sufficient to suspend the particles during the sedimentation period. The thick sludge heel may be difficult to retrieve and reaches a substantial portion of the overall sediment bed on the order of 10, 100, and 1000 hours for each process scale considered from smallest to largest. This process is shown graphically in Figure 13.5. 
Table 13.2. Variable Values used in Transient Modeling

\begin{tabular}{|c|c|c|c|c|c|c|c|c|c|c|c|}
\hline $\begin{array}{l}\text { Waste } \\
\text { Type }\end{array}$ & $\begin{array}{c}\text { Waste } \\
\text { Tank }\end{array}$ & $\begin{array}{l}\text { Waste } \\
\text { Status }\end{array}$ & $\begin{array}{c}\text { Temperatur } \\
\text { e Category } \\
\left({ }^{\circ} \mathrm{C}\right)\end{array}$ & $\begin{array}{c}\text { Consistency } \\
\text { Parameter, } \\
a(c P) \\
\end{array}$ & $\begin{array}{c}\text { Consistency } \\
\text { Parameter, } \\
\text { b (-) }\end{array}$ & \begin{tabular}{|c|} 
Yield Stress \\
Parameter, \\
$c$ \\
$(\mathrm{~Pa})$
\end{tabular} & $\begin{array}{c}\text { Yield Stress } \\
\text { Parameter, } d \\
(-)\end{array}$ & $\begin{array}{c}\text { Maximum } \\
\text { Measure Shear } \\
\text { Strength } \\
(\mathrm{Pa})\end{array}$ & $\begin{array}{c}\text { Shear Strength } \\
\text { Parameter, } \\
c_{\infty},(\mathrm{Pa})\end{array}$ & $\begin{array}{c}C \\
(\mathbf{m m} / \mathbf{h r})\end{array}$ & $\begin{array}{c}\alpha \\
(\mathrm{mm} / \mathrm{hr})\end{array}$ \\
\hline \multicolumn{4}{|c|}{ Source $\rightarrow$} & \multicolumn{4}{|c|}{\begin{tabular}{|c|} 
Table 12.1 \\
\end{tabular}} & Figure 11.3 & Eq. (13.6) & \multicolumn{2}{|c|}{ Table 10.1 } \\
\hline 224 & B-202 & Washed & $20-35$ & 1.51 & 36 & $7.21 \mathrm{E}+04$ & 2.94 & 750 & $5.25 \mathrm{E}+07$ & 16.9 & 0.38 \\
\hline 224 & B-203 & Washed & $20-35$ & 1.30 & 72 & $1.31 \mathrm{E}+07$ & 3.80 & 2,280 & $4.03 \mathrm{E}+10$ & 21.8 & 0.27 \\
\hline 224 & $\mathrm{~T}-203$ & Washed & $20-35$ & 1.08 & 79 & $2.50 \mathrm{E}+05$ & 2.60 & 3,770 & $5.79 \mathrm{E}+08$ & 16.8 & 0.17 \\
\hline $2 \mathrm{C}$ & $\mathrm{T}-110$ & Washed & $20-35$ & 1.16 & 46 & $1.21 \mathrm{E}+04$ & 2.68 & 70 & $2.39 \mathrm{E}+06$ & 11.3 & 0.23 \\
\hline CWP & C-104 & Salt Cake & $20-35$ & 1.25 & 12 & $2.79 \mathrm{E}+02$ & 2.40 & 7,077 & $2.12 \mathrm{E}+06$ & 4.3 & 0.40 \\
\hline CWP & C-104 & Washed & $20-35$ & 0.78 & 28 & $4.13 \mathrm{E}+03$ & 3.11 & 7,077 & $1.14 \mathrm{E}+07$ & 4.3 & 0.40 \\
\hline
\end{tabular}




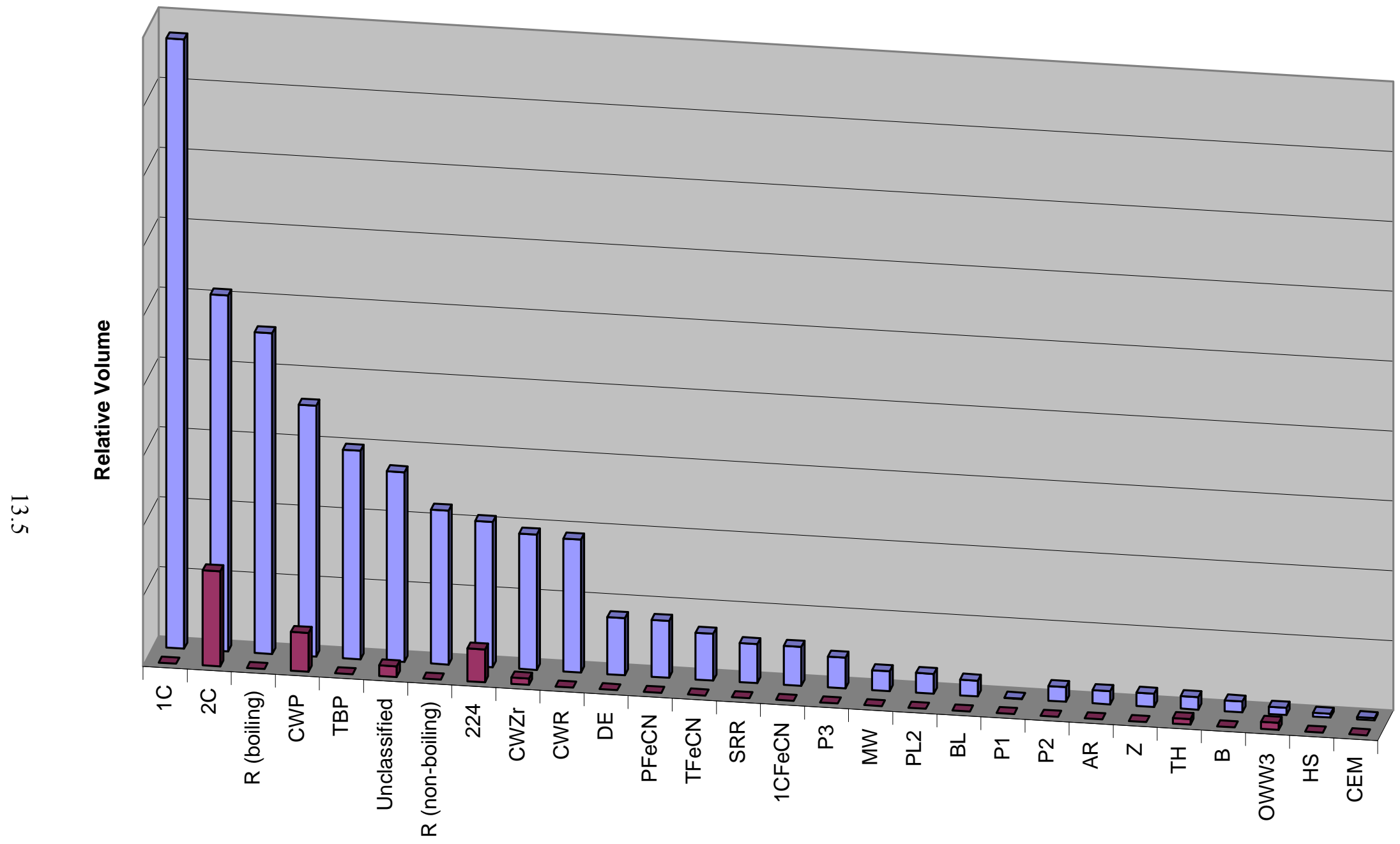

Waste Type

Figure 13.1. Relative Volume of Waste Types Analyzed based on Waste Tank Data Available for Transient Modeling 


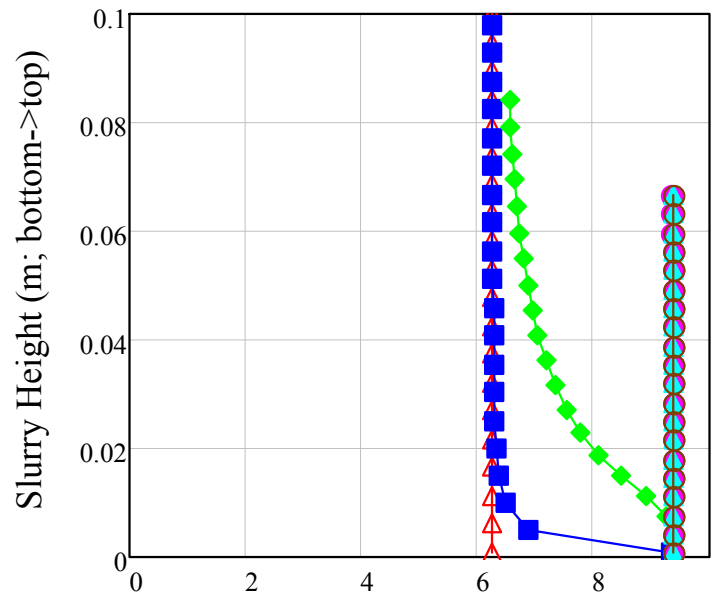

Solids Concentration (Volume Percent) $\triangle \triangle 0 \mathrm{hr}$

- $1 \mathrm{hr}$

$\leftrightarrow 10 \mathrm{hr}$

- $100 \mathrm{hr}$

$\triangle 200 \mathrm{hr}$

$\ominus \ominus 1000 \mathrm{hr}$

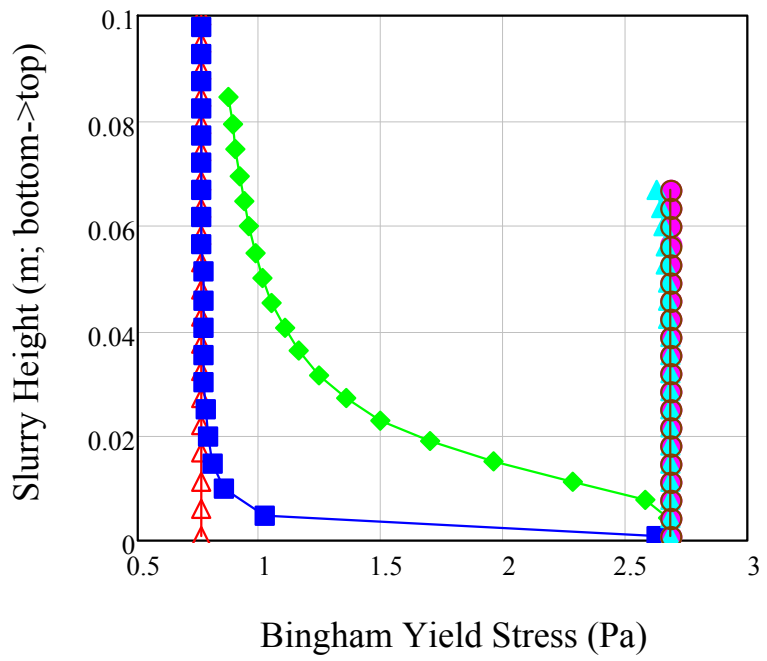

$\triangle \triangle 0 \mathrm{hr}$

- $1 \mathrm{hr}$

$\leftrightarrow 10 \mathrm{hr}$

- $100 \mathrm{hr}$

$\triangle 200 \mathrm{hr}$

$\ominus \ominus 1000 \mathrm{hr}$

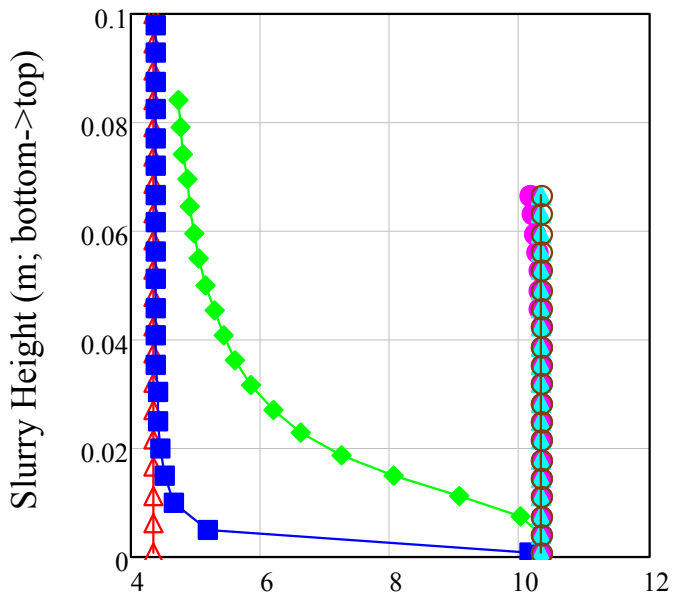

Bingham Consistency (cP)

$\triangle \triangle 0 \mathrm{hr}$

- $1 \mathrm{hr}$

$\leftrightarrow 10 \mathrm{hr}$

- $100 \mathrm{hr}$

$\triangle 200 \mathrm{hr}$

$\ominus \ominus 1000 \mathrm{hr}$

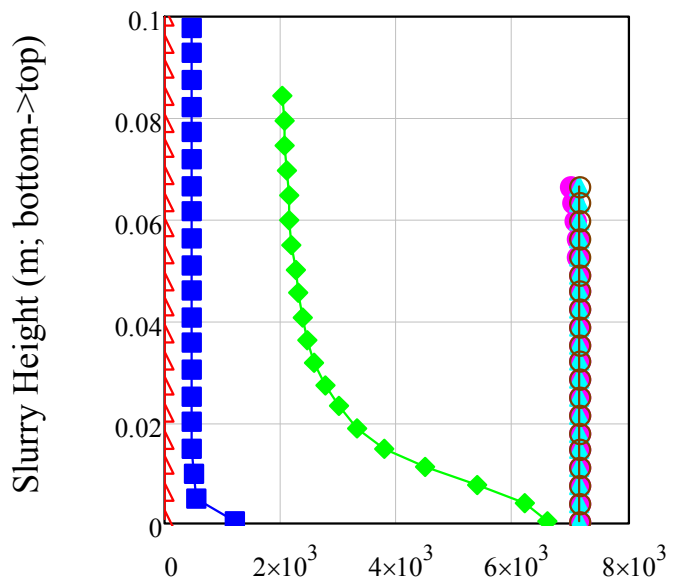

Shear Strength $(\mathrm{Pa})$

$\triangle \triangle 0 \mathrm{hr}$

- $1 \mathrm{hr}$

$\leftrightarrow 10 \mathrm{hr}$

$\rightarrow 100 \mathrm{hr}$

$\triangle 200 \mathrm{hr}$

$\ominus \ominus 1000 \mathrm{hr}$

Figure 13.2. Predicted Sludge Properties from Hanford Tank C-104 with Water Dilution at a Starting Slurry Height of $0.1 \mathrm{~m}$ with 33\% Volume Excess Supernatant from Fully Settled Configuration; Rheological Properties Taken at a Temperature Range of $20^{\circ}$ $35^{\circ} \mathrm{C}$. 


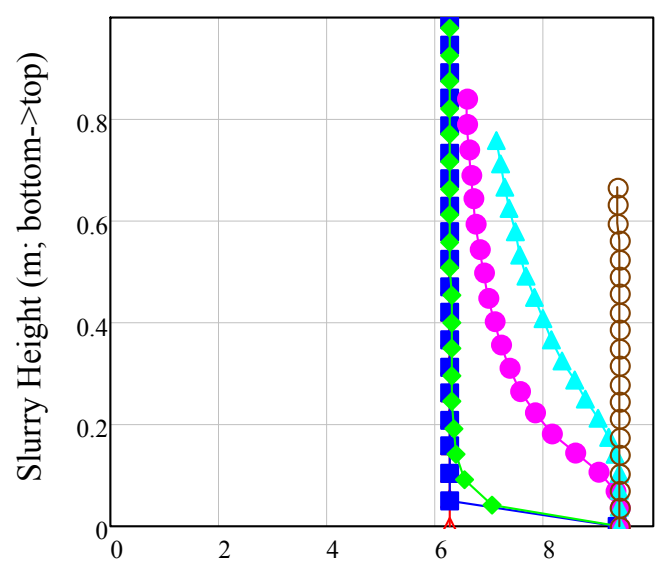

Solids Concentration (Volume Percent)

$\triangle \triangle 0 \mathrm{hr}$

- $1 \mathrm{hr}$

$\leftrightarrow 10 \mathrm{hr}$

- $100 \mathrm{hr}$

$\triangle 200 \mathrm{hr}$

$\ominus \ominus 1000 \mathrm{hr}$

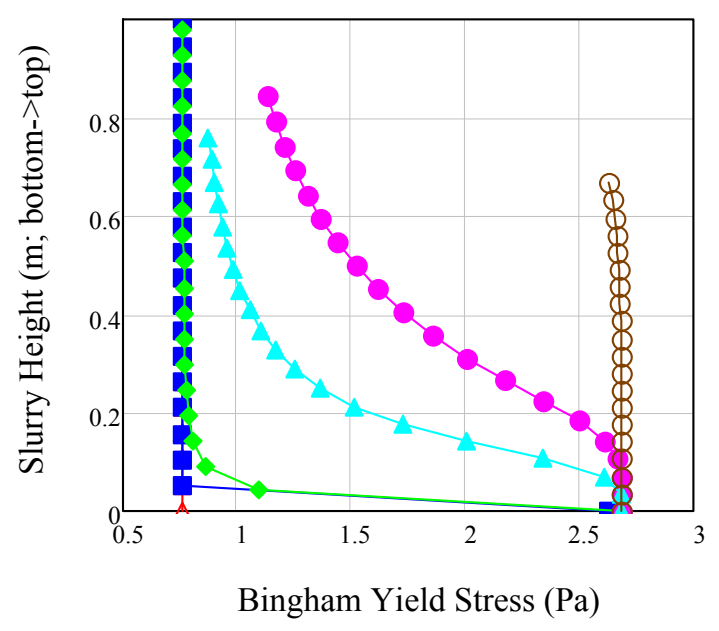

$\triangle \triangle 0 \mathrm{hr}$

- $1 \mathrm{hr}$

$\leftrightarrow 10 \mathrm{hr}$

- $100 \mathrm{hr}$

$\triangle 200 \mathrm{hr}$

$\ominus \ominus 1000 \mathrm{hr}$

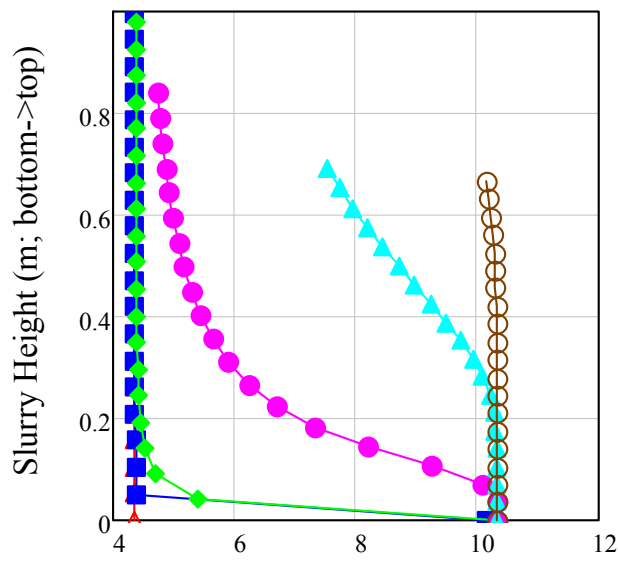

Bingham Consistency (cP)

$\triangle \triangle 0 \mathrm{hr}$

- $1 \mathrm{hr}$

$\leftrightarrow 10 \mathrm{hr}$

- $100 \mathrm{hr}$

$\triangle 200 \mathrm{hr}$

$\ominus \ominus 1000 \mathrm{hr}$

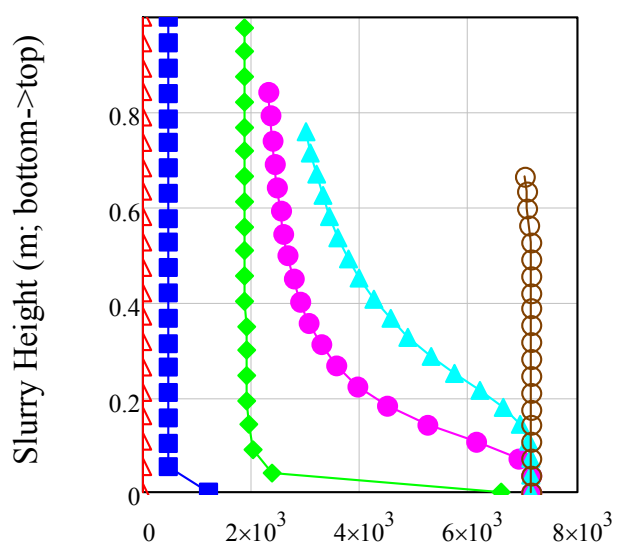

Shear Strength $(\mathrm{Pa})$

$\triangle \triangle 0 \mathrm{hr}$

- $1 \mathrm{hr}$

$\leftrightarrow 10 \mathrm{hr}$

- $100 \mathrm{hr}$

$\triangle 200 \mathrm{hr}$

$\ominus \ominus 1000 \mathrm{hr}$

Figure 13.3. Predicted Sludge Properties from Hanford Tank C-104 with Water Dilution at Starting Slurry Height of $1 \mathrm{M}$ with 33\% Volume Excess Supernatant from Fully Settled Configuration; Rheological Properties Taken at a Temperature Range of $20^{\circ}-35^{\circ} \mathrm{C}$ 


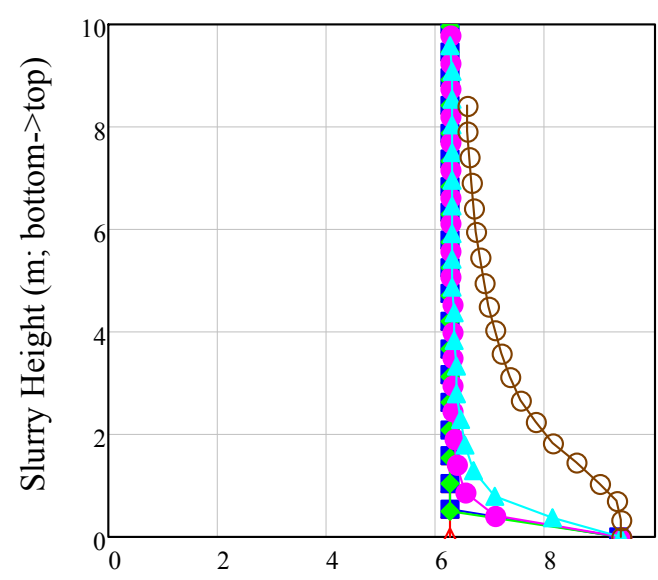

Solids Concentration (Volume Percent) $\triangle \triangle 0 \mathrm{hr}$

- $1 \mathrm{hr}$

$\leadsto 10 \mathrm{hr}$

$100 \mathrm{hr}$

$-200 \mathrm{hr}$

$\ominus \ominus 1000 \mathrm{hr}$

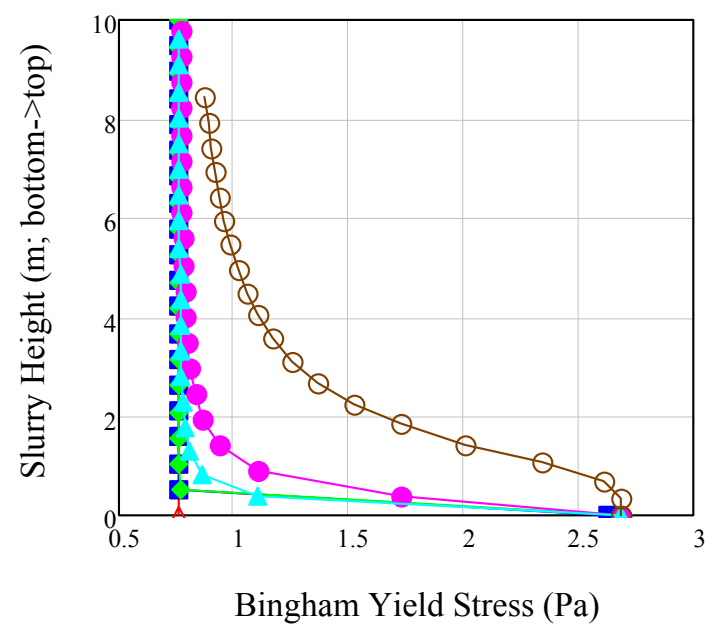

$\triangle \triangle 0 \mathrm{hr}$

- $1 \mathrm{hr}$

$\leftrightarrow 10 \mathrm{hr}$

- $100 \mathrm{hr}$

$\triangle 200 \mathrm{hr}$

$\ominus \ominus 1000 \mathrm{hr}$

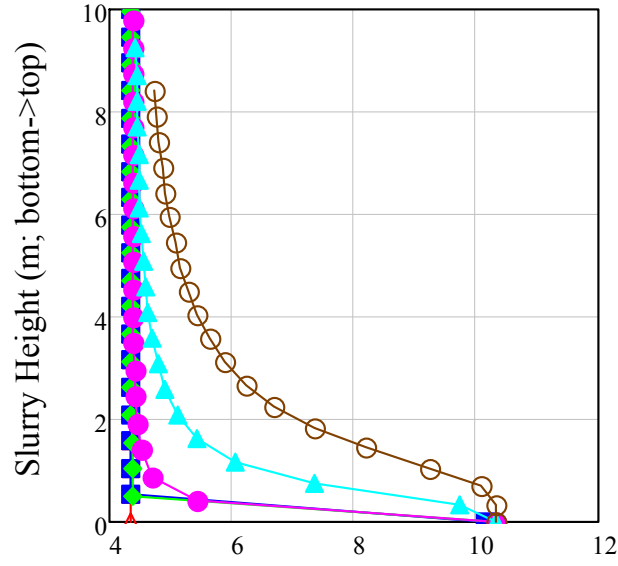

Bingham Consistency (cP)

$\triangle \triangle 0 \mathrm{hr}$

- $1 \mathrm{hr}$

$\leftrightarrow 10 \mathrm{hr}$

$100 \mathrm{hr}$

$\triangle 200 \mathrm{hr}$

$\ominus \ominus 1000 \mathrm{hr}$

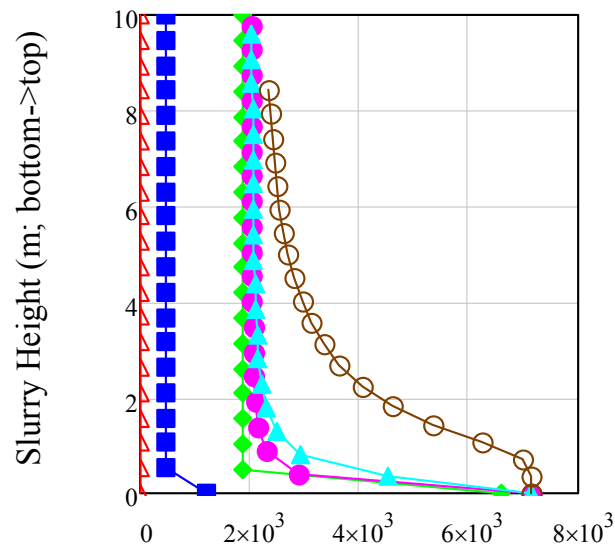

Shear Strength $(\mathrm{Pa})$

$\triangle \triangle 0 \mathrm{hr}$

- $1 \mathrm{hr}$

$\leftrightarrow 10 \mathrm{hr}$

- $100 \mathrm{hr}$

$\triangle 200 \mathrm{hr}$

$\ominus \ominus 1000 \mathrm{hr}$

Figure 13.4. Predicted Sludge Properties from Hanford Tank C-104 with Water Dilution at Starting Slurry Height of $10 \mathrm{M}$ with 33\% Volume Excess Supernatant from Fully Settled Configuration; Rheological Properties Taken at a Temperature Range of $20^{\circ}-35^{\circ} \mathrm{C}$ 


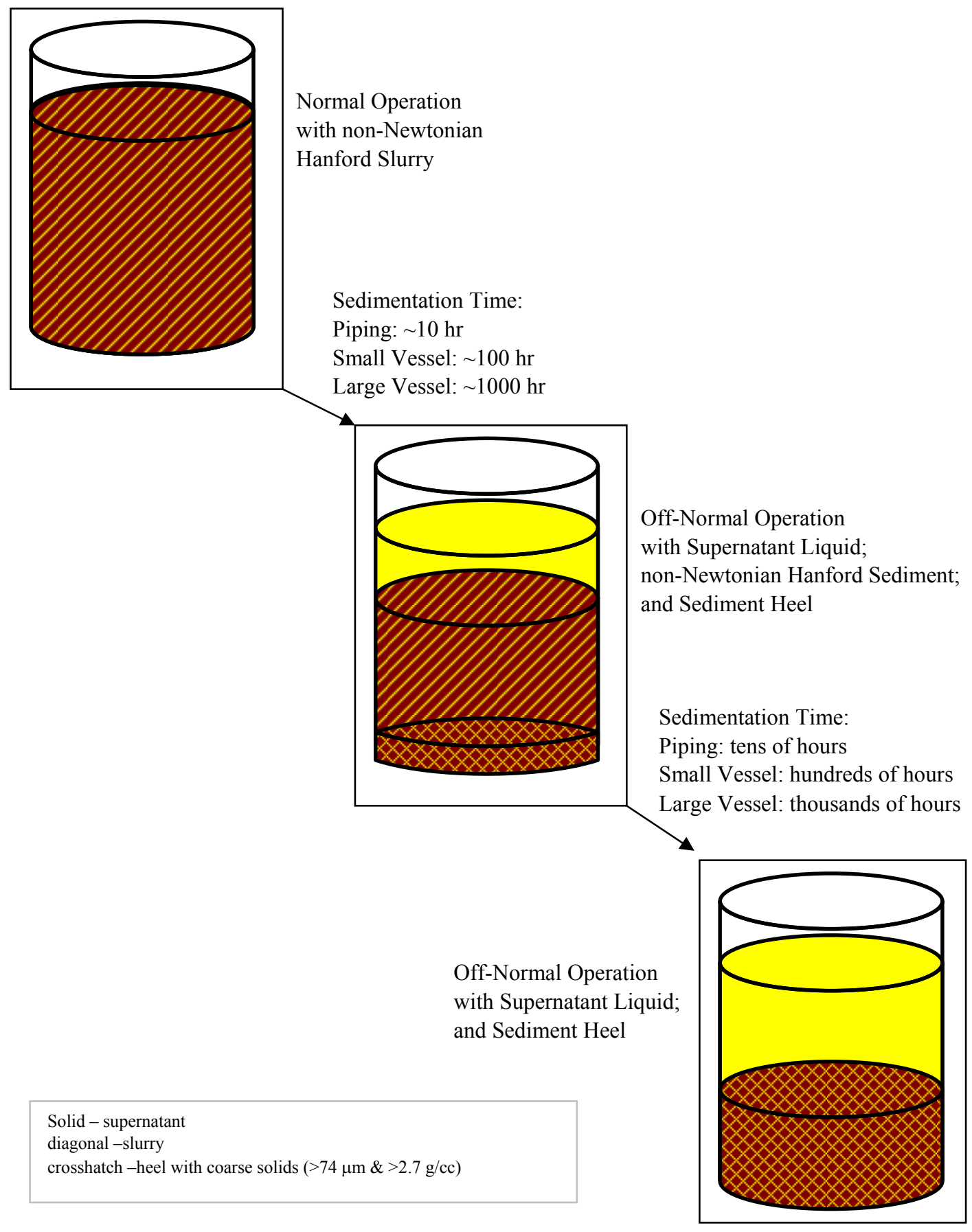

Figure 13.5. Illustration of Development of Sludge Process Heel and Fully Settled Configuration at Various Process Scales 
Recovery from these slurry configurations will require the application of all of the parameters identified in this table. Initially the sediment will be gelled to some extent, due to the development of interparticle bonds as the particles remain quiescent and in close proximity, and will possess a shear strength threshold that must be overcome. After the sediment is mobilized, the Bingham consistency and yield stress parameters will be elevated due to the large concentration of undissolved solids in the sediment bed. As mixing proceeds and supernatant liquid is blended into the sediment, the rheological properties will drop to the normal operation range when the slurry is fully suspended. This is shown graphically in Figure 13.6.

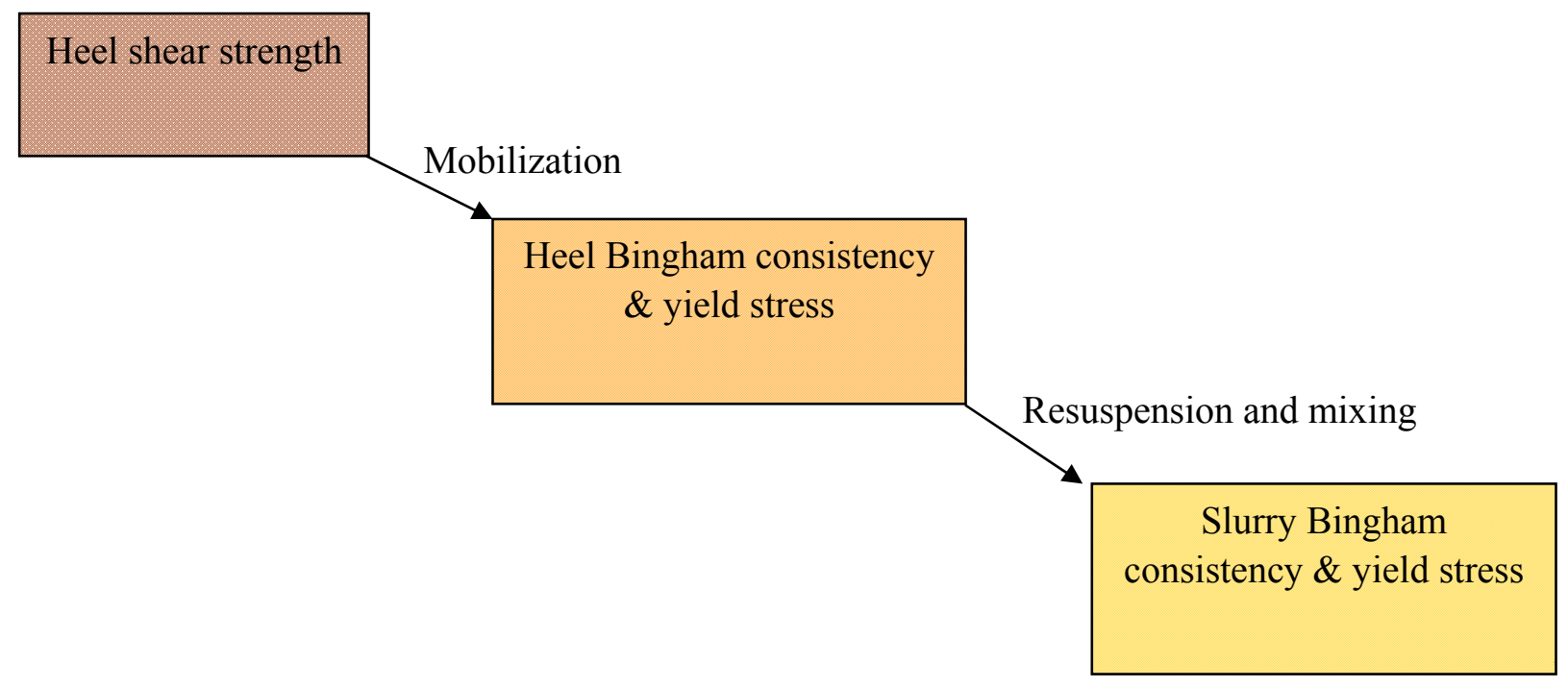

Figure 13.6. Rheological Properties Encountered During Recovery from Process Upset Conditions 


\subsection{Rheology Summary and Processing Scenarios}

The data discussed in this report can be applied to a wide variety of process conditions. Three major process systems where these data apply are as flow in process vessels, vertical piping, and horizontal piping. In these process systems, several sludge configurations can be identified as potential operational scenarios. These scenarios and sludge configurations are shown in Figures 14.1 through 14.3 for process vessels (e.g. WTP EFRT issue M3), vertical piping, and horizontal piping (e.g. WTP EFRT issue M1), respectively.

Table 14.1 provides a summary of the rheological parameters for each of the fluid layers shown in the associated process scenario figures. This table was compiled from the previous sections on sedimentation, shear strength, Bingham rheological parameters, and supernatant viscosity. In addition, the section discussing transient rheological modeling was summarized to provide an indication of the time scales for rheology regrowth at various process length scales. Recovery from these slurry configurations will require the application of all of the parameters identified in Table 14.1 through the process illustrated in Figure 13.6.

Table 14.1. Range of Rheological Parameters and Regrowth Times at Typical Process Scales

\begin{tabular}{|c|c|c|c|c|}
\hline Category & $\begin{array}{l}\text { Heel Shear } \\
\text { strength }\end{array}$ & $\begin{array}{c}\text { Slurry/Sediment } \\
\text { Heel Bingham } \\
\text { Yield Stress } \\
\end{array}$ & $\begin{array}{c}\text { Slurry/Sediment } \\
\text { Heel Bingham } \\
\text { Consistency } \\
\end{array}$ & $\begin{array}{l}\text { Supernatant } \\
\text { Viscosity }\end{array}$ \\
\hline $\operatorname{Min}^{(a)}$ & $40 \mathrm{~Pa}$ & $0 \mathrm{~Pa}$ & $1 \mathrm{cP}$ & $1 \mathrm{cP}$ \\
\hline Median $^{(\mathrm{a})}$ & $700 \mathrm{~Pa}$ & $1.5 \mathrm{~Pa}$ & $8 \mathrm{cP}$ & $8 \mathrm{cP}$ \\
\hline $\operatorname{Max}^{(a)}$ & $25,000 \mathrm{~Pa}$ & $40 \mathrm{~Pa}$ & $110 \mathrm{cP}$ & $30 \mathrm{cP}$ \\
\hline $\begin{array}{l}\text { Tank heel property after } 10 \text { hours of } \\
\text { sedimentation in process piping } \\
(0.1 \mathrm{~m} \text { sludge height })\end{array}$ & $25,000 \mathrm{~Pa}$ & $40 \mathrm{~Pa}$ & $110 \mathrm{cP}$ & $\mathrm{n} / \mathrm{a}$ \\
\hline $\begin{array}{l}\text { Tank heel property after } 100 \text { hours of } \\
\text { sedimentation in a medium-scale vessel } \\
(1 \mathrm{~m} \text { sludge height) }\end{array}$ & $25,000 \mathrm{~Pa}$ & $40 \mathrm{~Pa}$ & $110 \mathrm{cP}$ & $\mathrm{n} / \mathrm{a}$ \\
\hline $\begin{array}{l}\text { Tank heel property after } 1000 \text { hours of } \\
\text { sedimentation in a large-scale vessel } \\
(10 \text { m sludge height) }\end{array}$ & $25,000 \mathrm{~Pa}$ & $40 \mathrm{~Pa}$ & $110 \mathrm{cP}$ & $\mathrm{n} / \mathrm{a}$ \\
\hline
\end{tabular}


Normal operation with Newtonian fluid

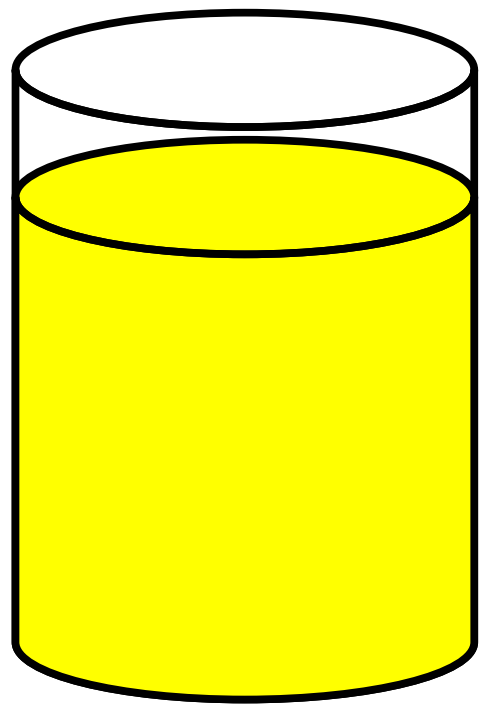

Process impacts:

- cloud height

- blend times

Off-normal operation with Newtonian fluid

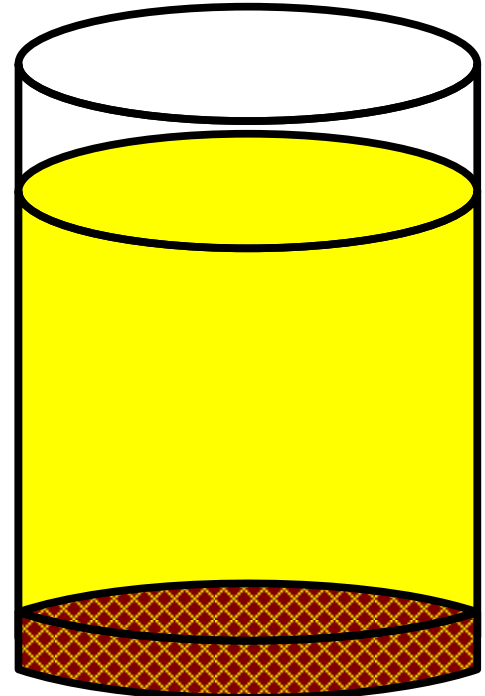

Process impacts:

- off-bottom resuspension of solids

- blend time of solids layer
Normal operation with non-Newtonian fluid

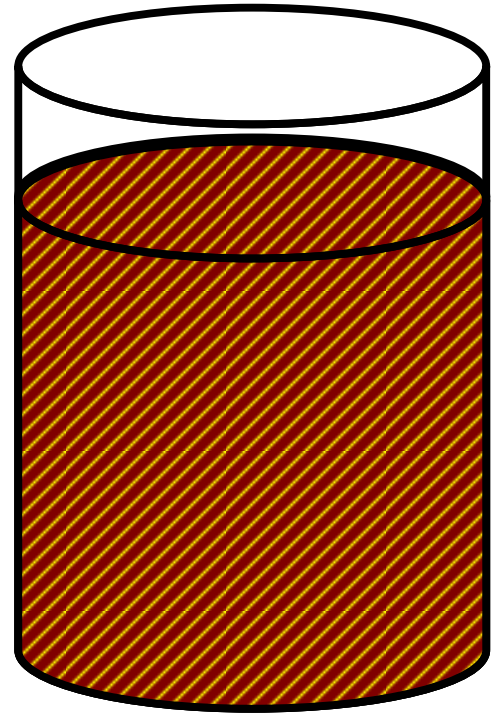

Process impacts:

- mixing performance

- gas retention \& release

Off-Normal operation with non-Newtonian fluid

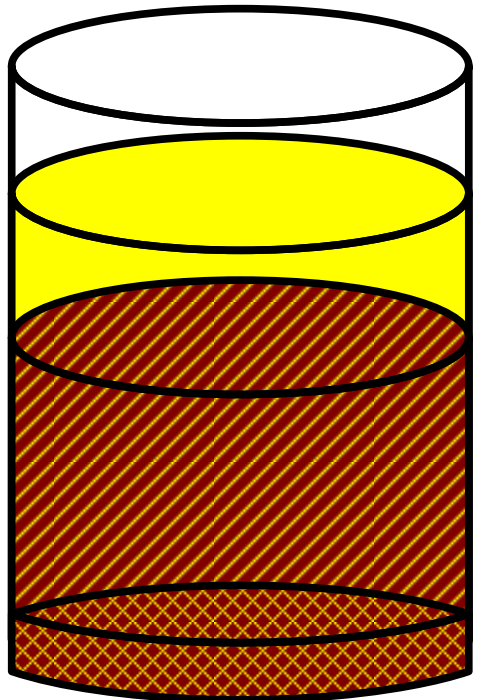

Process impacts:

- restart of mechanical agitators/PJMs

- off-bottom resuspension of solids

- blend time liquid/solids layer

- gas retention \& release

Solid - supernatant; diagonal - slurry; crosshatch - heel with coarse solids $(>74 \mu \mathrm{m} \&>2.7 \mathrm{~g} / \mathrm{cc})$

Figure 14.1. Example Operational Scenarios for Process Vessels 
Normal operation with

Newtonian fluid

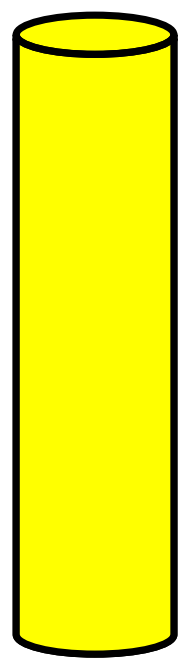

Process impacts:

- critical flow velocity
Normal operation with non-Newtonian fluid

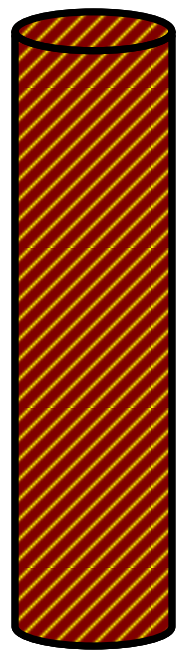

Process impacts:

- flow velocity and pressure drop

- flow regime
Off-normal operation with non-Newtonian fluid

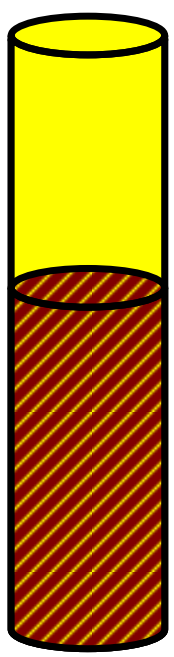

Process impacts:

- restart

- flushing effectiveness

Solid - supernatant; diagonal - slurry; crosshatch - heel with coarse solids $(>74 \mu \mathrm{m} \mathrm{\&}>2.7 \mathrm{~g} / \mathrm{cc})$

Figure 14.2. Example Operational Scenarios for Vertical Process Piping

Normal operation with Newtonian fluid

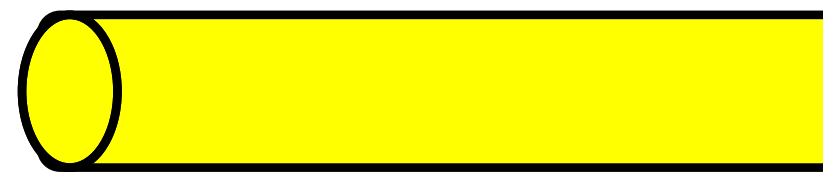

Process impacts:

- critical flow velocity

Off-normal operation with Newtonian fluid

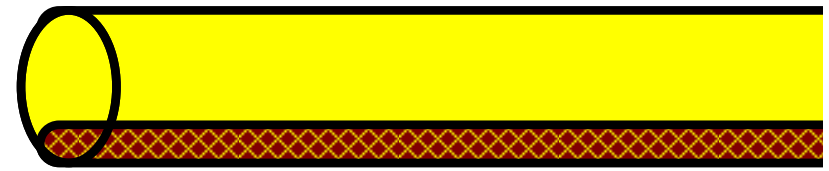

Process impacts:

- restart

- flushing effectiveness
Normal operation with non-Newtonian fluid

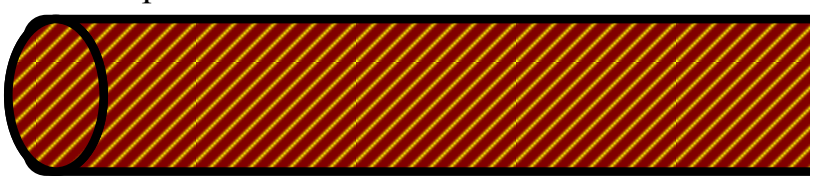

Process impacts:

- flow velocity and pressure drop

- flow regime

Off-Normal operation with non-Newtonian fluid

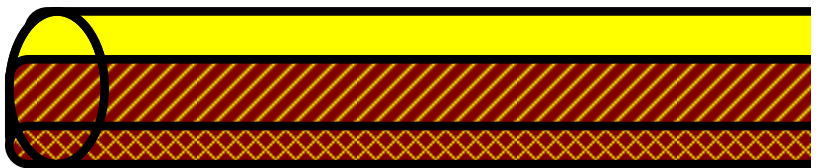

Process impacts:

- restart

- flushing effectiveness

Solid - supernatant; diagonal - slurry; crosshatch - heel with coarse solids $(>74 \mu \mathrm{m} \&>2.7 \mathrm{~g} / \mathrm{cc})$

Figure 14.3. Example Operational Scenarios for Horizontal Process Piping 
Rheology data and associated physical properties for Hanford tank wastes were compiled from all the readily available reports, but many gaps were observed when analyzing the data. These data include in situ as well as laboratory analysis of samples removed from the tanks. The gaps in the waste types analyzed are reported in each section of the report. Figure 14.4 provides a summary of these gaps. The relative volume of wastes modeled for liquid viscosity, sedimentation, shear strength, rheological parameters (Bingham plastic model), and rheological parameters as a function of settling are plotted as a function of waste type.

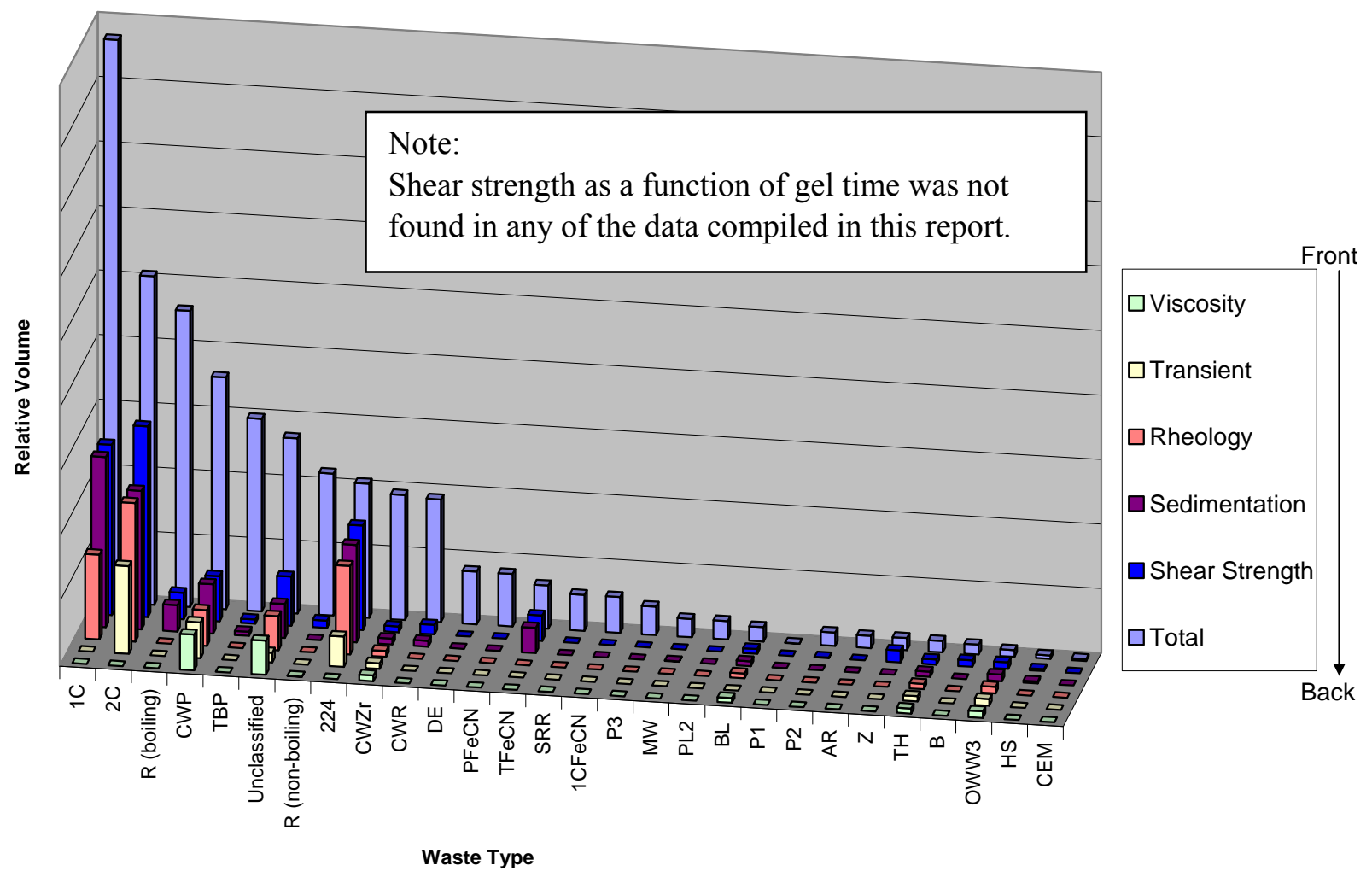

Figure 14.4. Relative Volume of Waste Types Modeled Based on Waste Tank Data Available for Liquid Viscosity, Sedimentation, Shear Strength, Rheology, and Transient Modeling Compared to the Total Volume of Each Sludge Waste Type 


\subsection{References}

Bechtel National, Inc. July 2004. WTP Integrated Processing Strategy Description. 24590-WTP-3YD50-00002 Rev 0, BNI, Richland, Washington.

Bechtel National, Inc. 2006. Issue Response Plan for Implementation of External Flowsheet Review Team (EFRT) Recommendations - M3, Inadequate Mixing System Design. 24590-WTP-PL-ENG-060013, Rev 000, Bechtel National, Inc., Richland, Washington.

Bechtel National, Inc. December 2006. Integrated Sampling and Analysis Requirements Document. 24590-WTP-PL-PR-04-0001 Rev 1, BNI, Richland, Washington.

CRC. 1975. CRC Handbook of Chemistry and Physics, 56 ${ }^{\text {th }}$ Ed. RC Weast (Ed). CRC Press, Cleveland, Ohio.

Ferraris F and N Martys. 2001. "Fresh Concrete Rheology - Recent Developments." Materials Science of Concrete VI, S Mindess and J Skalny, eds., pp. 215-241.

Gauglitz PA and JT Aikin. 1997. Waste Behavior During Horizontal Extrusion: Effect of Waste Strength for Bentonite and Kaolin/Ludox Simulants and Strength Estimates for Wastes from Hanford Tanks 241-SY-103, AW-101, AN-103, and S-102. PNNL-11706, Pacific Northwest National Laboratory, Richland, Washington.

Higley BA and DE Place. February 2004. Hanford Defined Waste Model, Rev. 5.0. RPP-19822, CH2M Hill Hanford Group, Richland, Washington.

Meacham JE. 2003. Aluminum Wash and Leach Factors. RPP-11079, CH2M HILL Hanford Group, Inc., Richland, Washington.

Morrey EV and JM Tingey. 1996. Comparison of Simulants to Actual Neutralized Current Acid Waste: Process and Product Testing of Three NCAW Core Samples from Tanks 101-AZ and 102-AZ. PNNL-11098, Pacific Northwest National Laboratory, Richland, Washington.

Poloski AP, PA Meyer, LK Jagoda, and PR Hrma. 2003a. Technical Basis for LAW Vitrification Stream Physical and Rheological Property Bounding Conditions. WTP-RPT-098 Rev. 0, Battelle - Pacific Northwest Division, Richland, Washington.

Poloski AP, PR Bredt, JW Chenault, and RG Swoboda. 2003b. Rheological and Physical Properties of AZ-101 HLW Pretreated Sludge and Melter Feed. WTP-RPT-096 Rev. 0, Battelle - Pacific Northwest Division, Richland, Washington.

Poloski AP, PA Meyer, LK Jagoda, and PR Hrma. 2004. Non-Newtonian Slurry Simulant Development and Selection for Pulse Jet Mixer Testing. PNWD-3495 (WTP-RPT-111 Rev. 0), Battelle - Pacific Northwest Division, Richland, Washington. 
Poloski AP, ST Arm, OP Bredt, TB Calloway, Y Onishi, RA Peterson, GL Smith, and HD Smith. 2006a. Final Report: Technical Basis for HLW Vitrification Stream Physical and Rheological Property Bounding Conditions. PNWD-3675 (WTP-RPT-112 Rev. 0), Battelle - Pacific Northwest Division, Richland, Washington.

Poloski AP, PR Bredt, RC Daniel, and AE Saez. 2006b. "The Contribution of Frictional Contacts to the Shear Strength of Coarse Glass Bead Powders and Slurries." Rheologica Acta 46(2):249-259.

Rassat SD, LA Mahoney, BE Wells, DP Mendoza, DD Caldwell. 2003. Assessment of Physical Properties of Transuranic Waste in Hanford Single-Shell Tanks. PNNL-14221, Pacific Northwest National Laboratory, Richland, Washington.

Renko EK. 1996. “A model for batch settling curve.” Water SA. 22(4)339-344.

Renko EK. 1998. "Modeling hindered batch settling Part II: A model for computing solids profile of calcium carbonate slurry." Water SA, 24(4)331-336.

Shephard CL, JB Colson, KO Pasamehmetoglu, C Unal, JN Edwards, and J Abbott. 1994. Theory of Operation for the Ball Rheometer. PNL-10240, Pacific Northwest National Laboratory, Richland, Washington.

Speers RA, KR Holme, MA Tung, and WT Williamson. 1987. "Drilling fluid shear stress overshoot behavior." Rheologica. Acta, 26:447-452

Stewart CW, JM Alzheimer, ME Brewster, G Chen, RE Mendoza, HC Reid, CL Shephard, and G Terrones. 1996. In Situ Rheology and Gas Volume in Hanford Double-Shell Waste Tanks. PNNL-11296, Pacific Northwest National Laboratory, Richland, Washington.

Wells BE, MA Knight, EC Buck, RC Daniel, SK Cooley, LA Mahoney, PA Meyer, AP Poloski, JM Tingey, WS Callaway, III, GA Cooke, ME Johnson, MG Thien, DJ Washenfelder, JJ Davis, MN Hall, G Smith, SL Thomson, and Y Onishi. 2007. Estimate of Hanford Waste Insoluble Solid Particle Size and Density Distribution. PNWD-3824 (WTP-RPT-153 Rev. 0) Battelle - Pacific Northwest Division, Richland, Washington.

Zhou Z, PJ Scales, and DV Boger. 2001. "Chemical and physical control of the rheology of concentrated metal oxide suspensions.” Chemical Engineering Science, 56: 2901-2920. 
Appendix A

Tabulation of Available Rheology Data and Associated Physical and Chemical Data for Hanford Tank Wastes 


\section{Appendix A - Tabulation of Available Rheology Data and Associated Physical and Chemical Data for Hanford Tank Wastes}


Appendix B

Raw Data Used in Evaluating Correlations of the Bingham Plastic Model Parameters with Solids Content 


\section{Appendix B - Raw Data Used in Evaluating Correlations of the Bingham Plastic Model Parameters with Solids Content}

In the figures in this appendix, the stars = as-received tank waste; squares = water added as diluent; and circles $=$ post washing and caustic leaching.

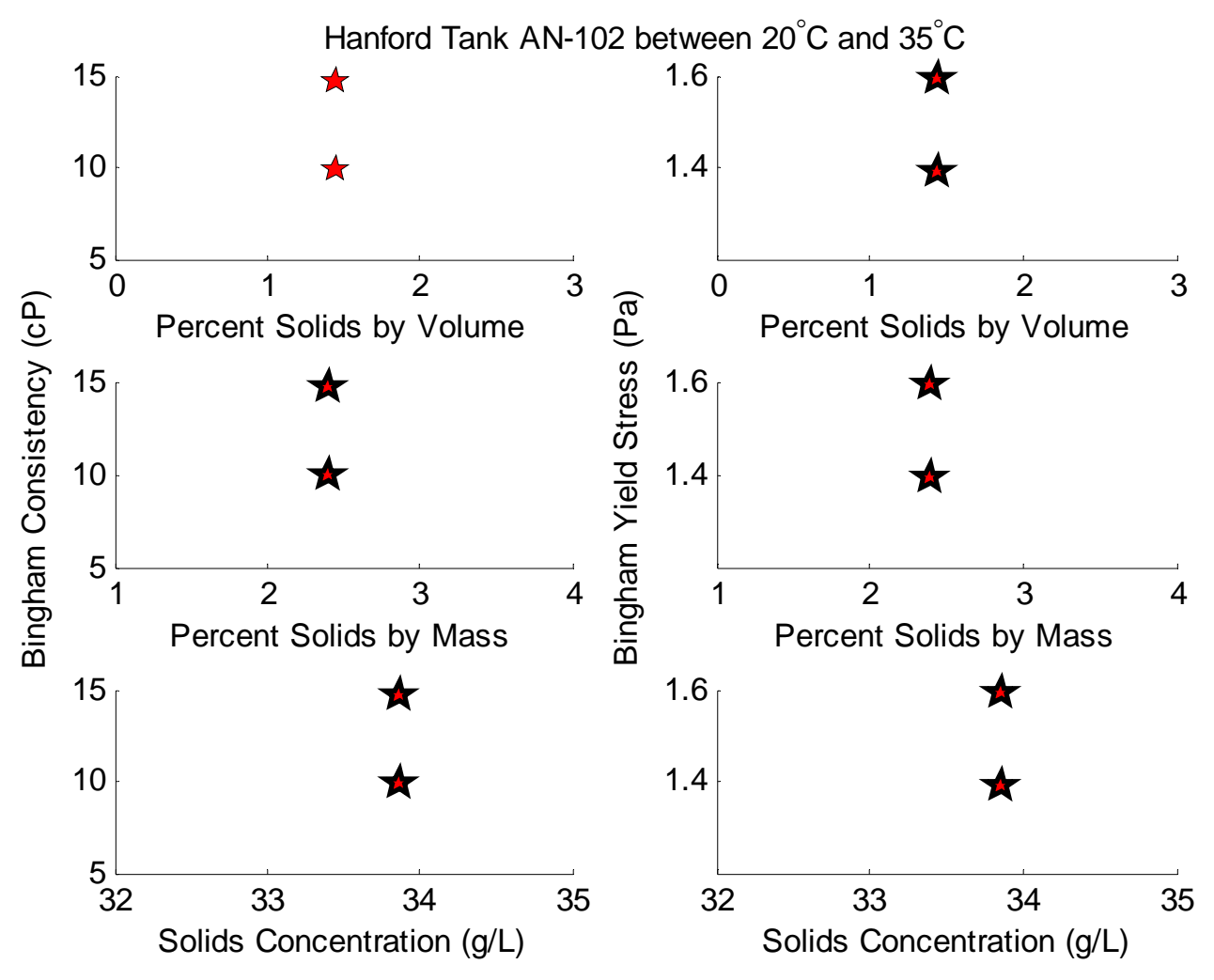

B. 1 

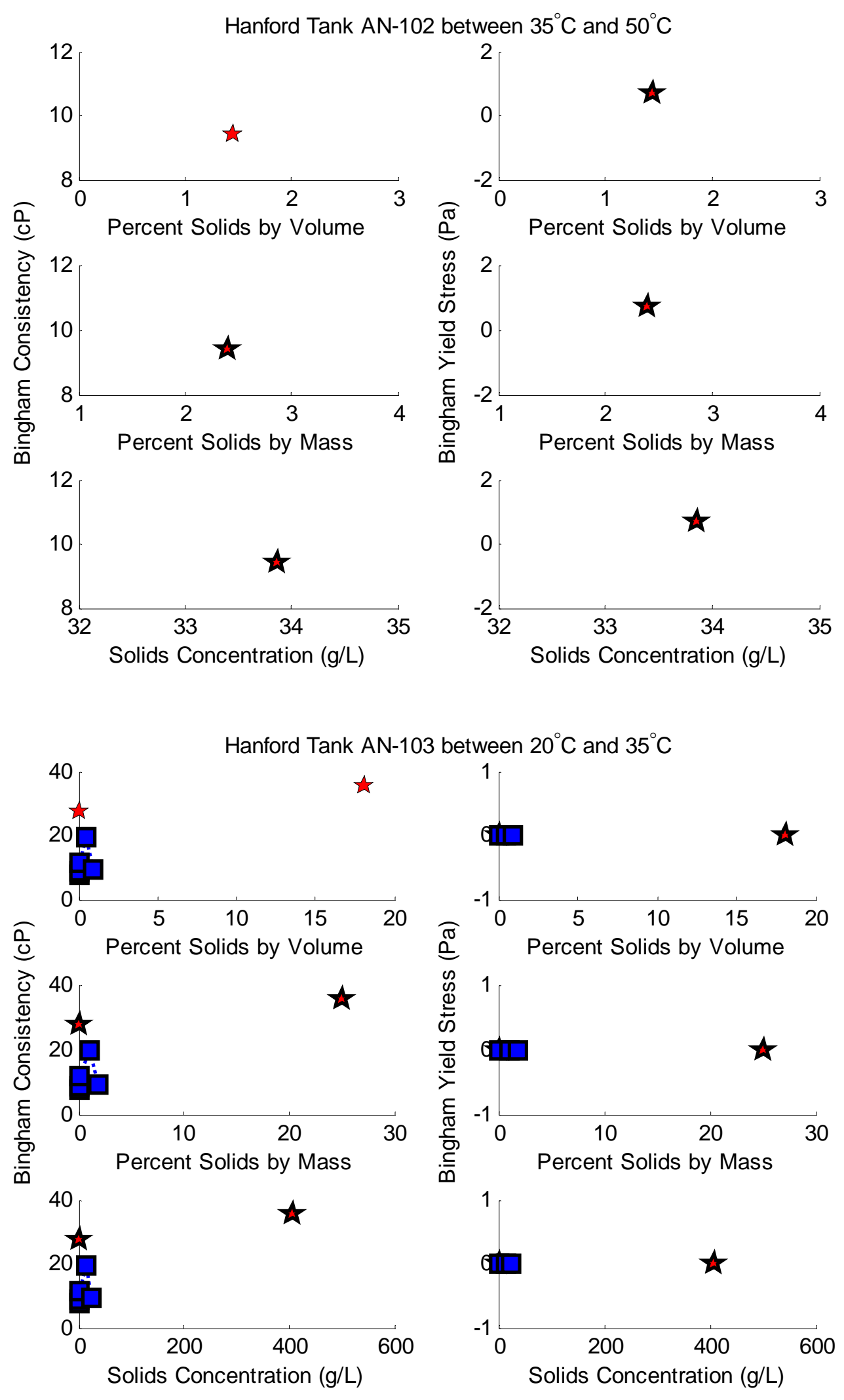

B. 2 

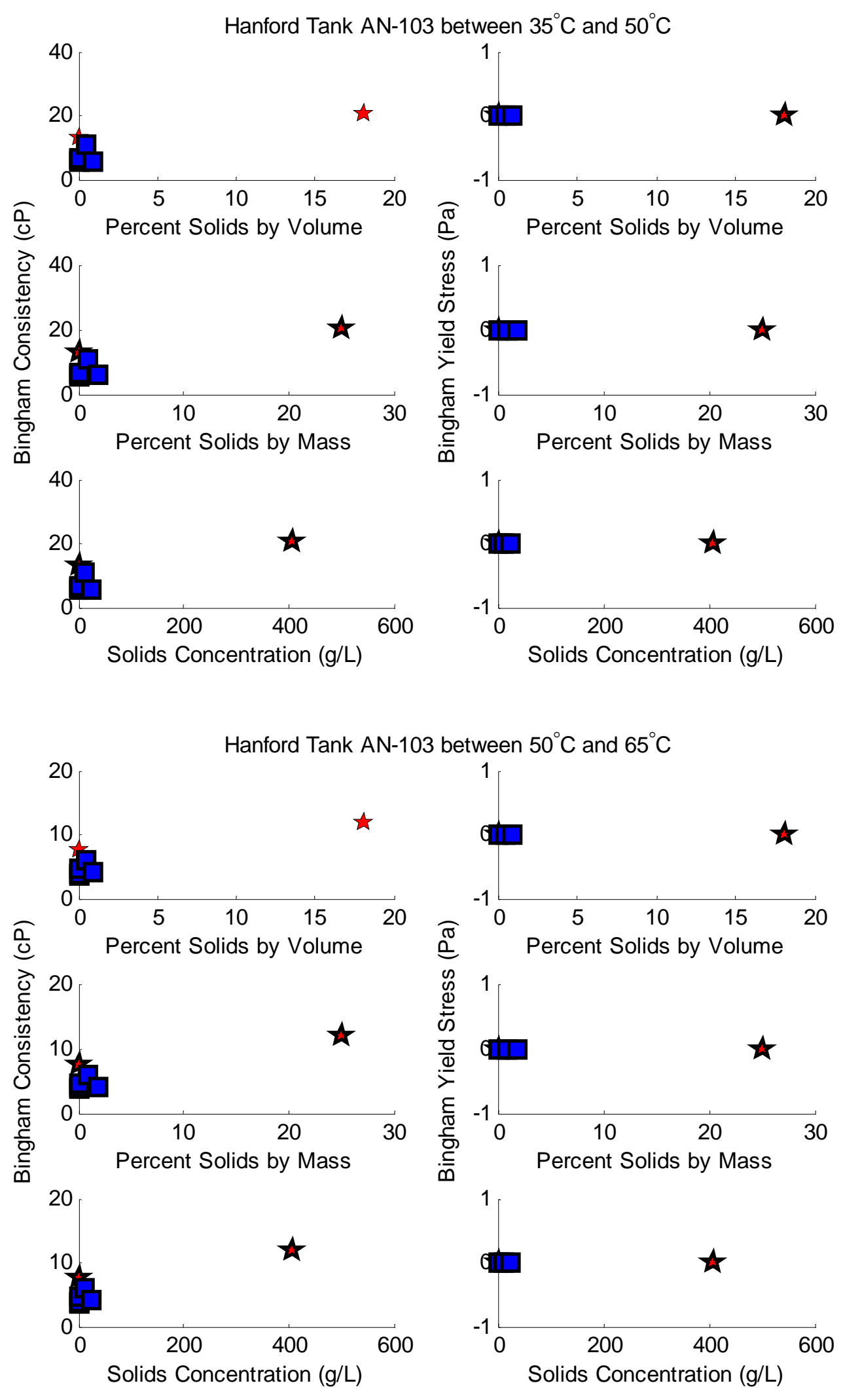

B. 3 

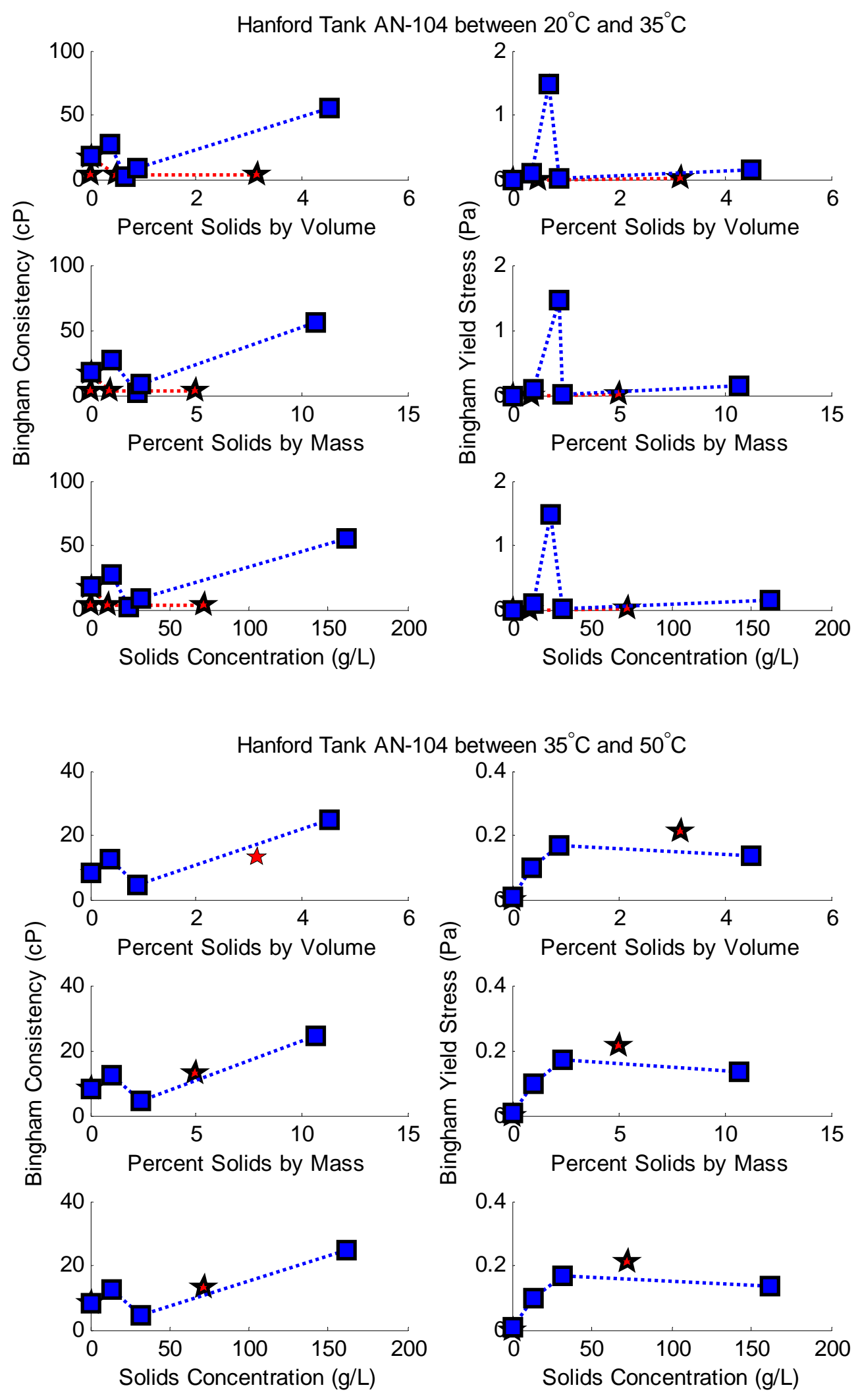

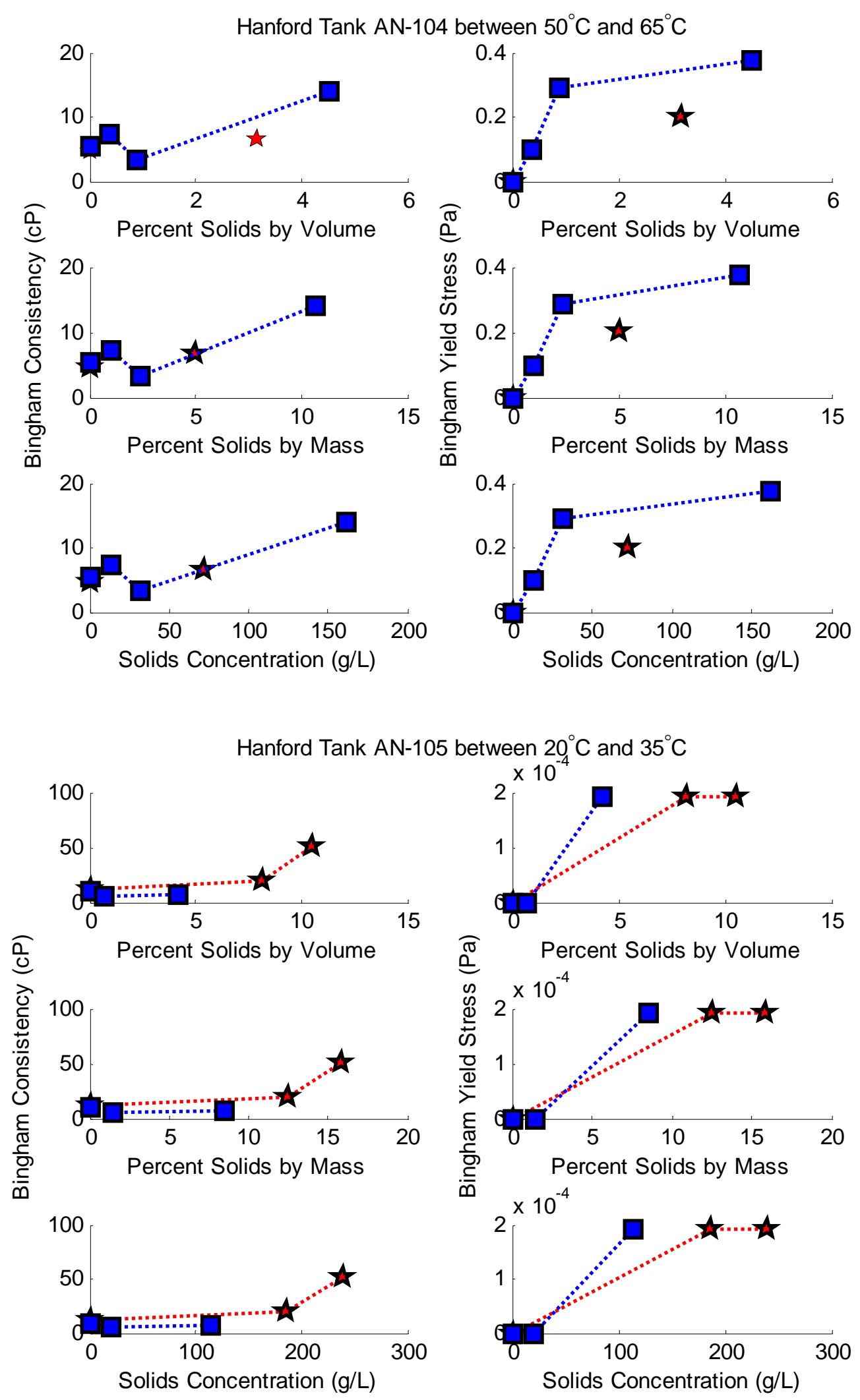

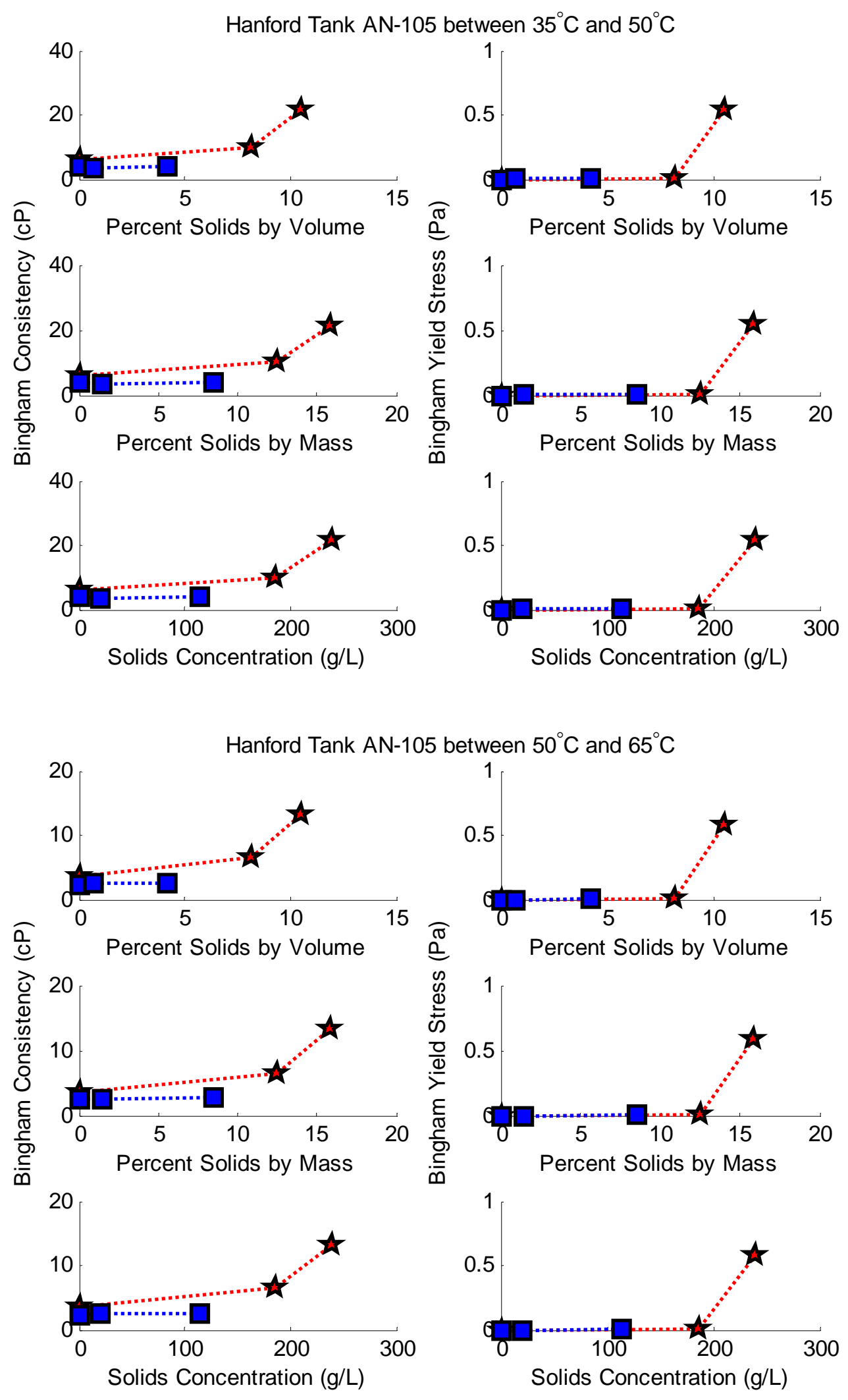

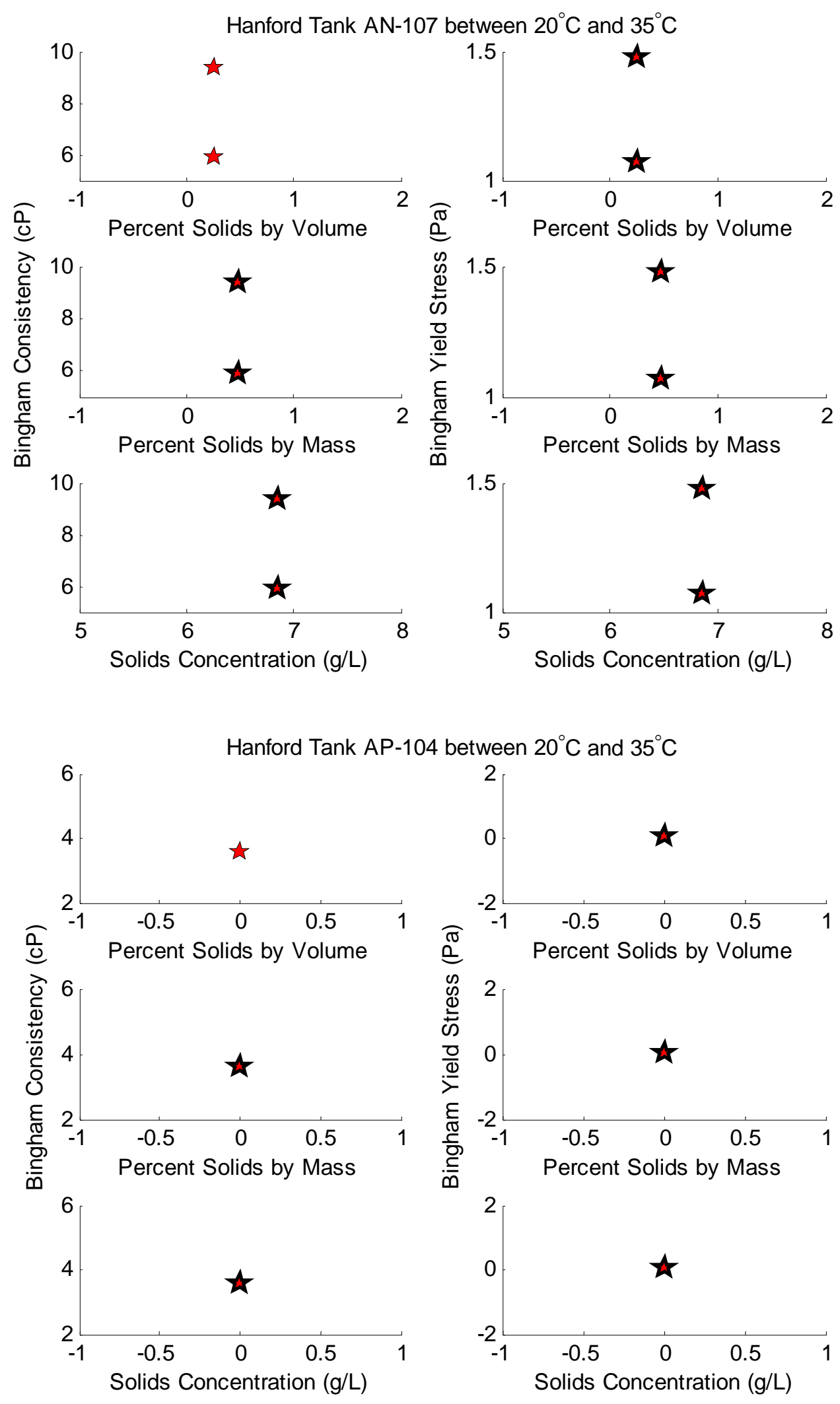

B. 7 

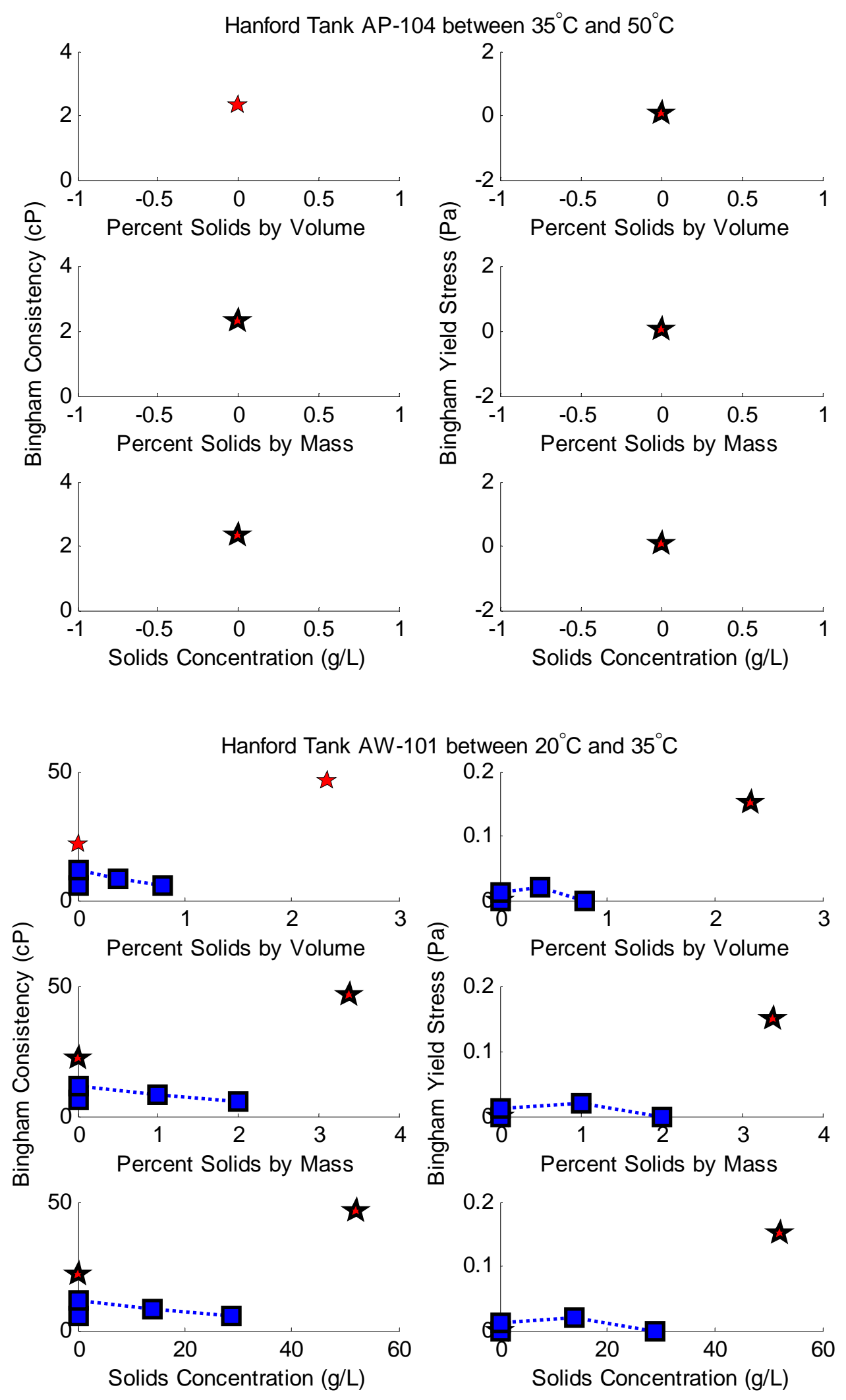

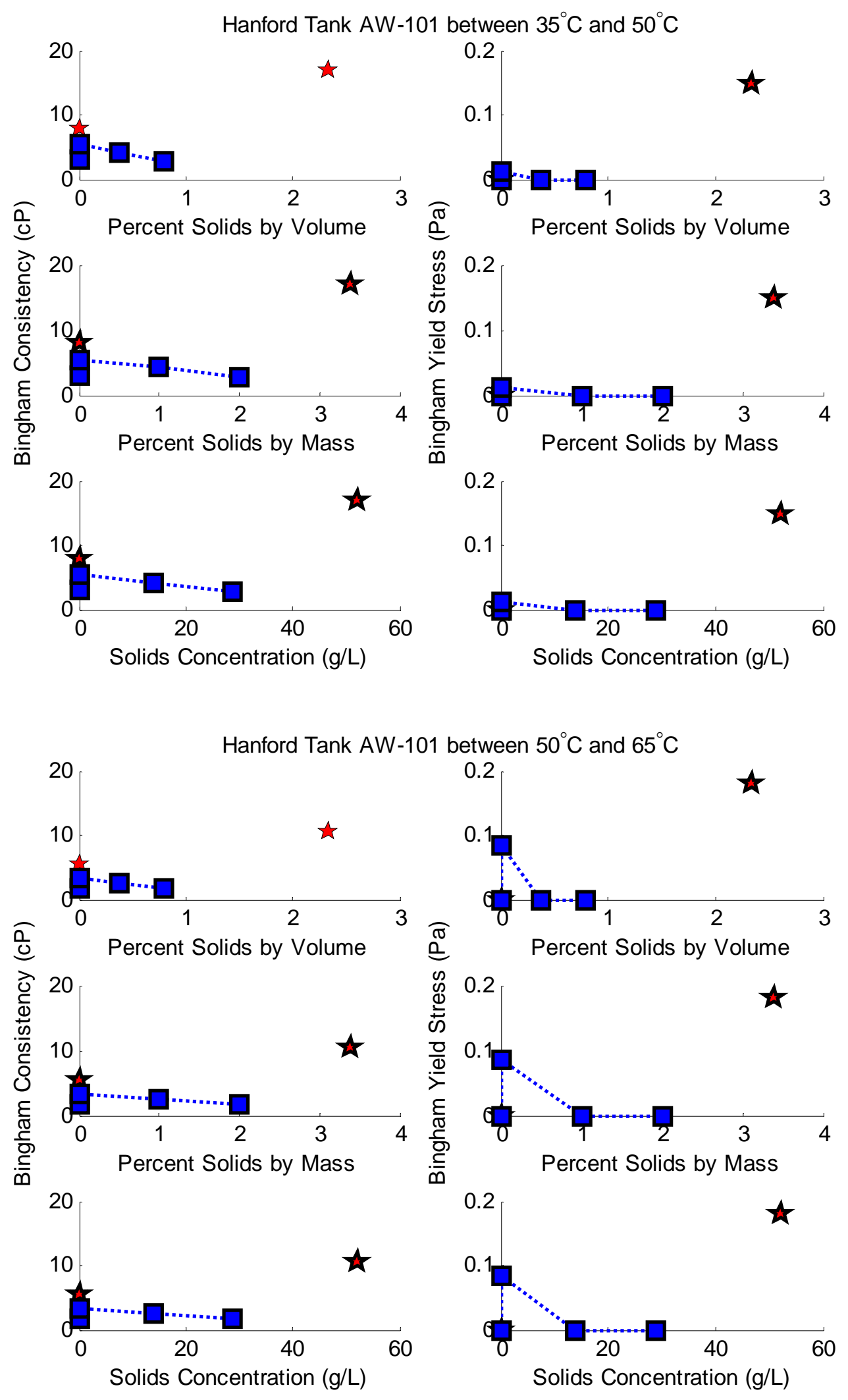

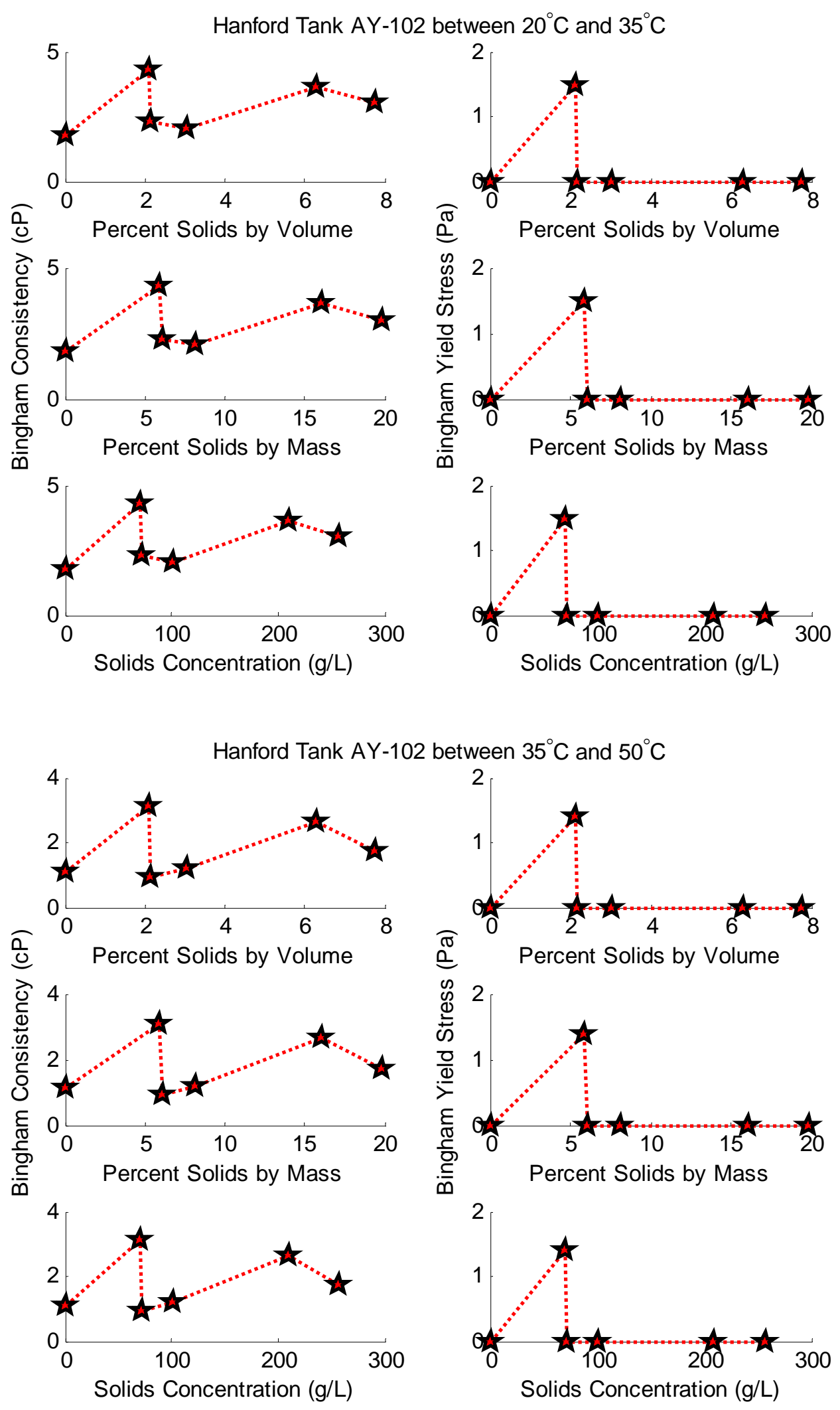

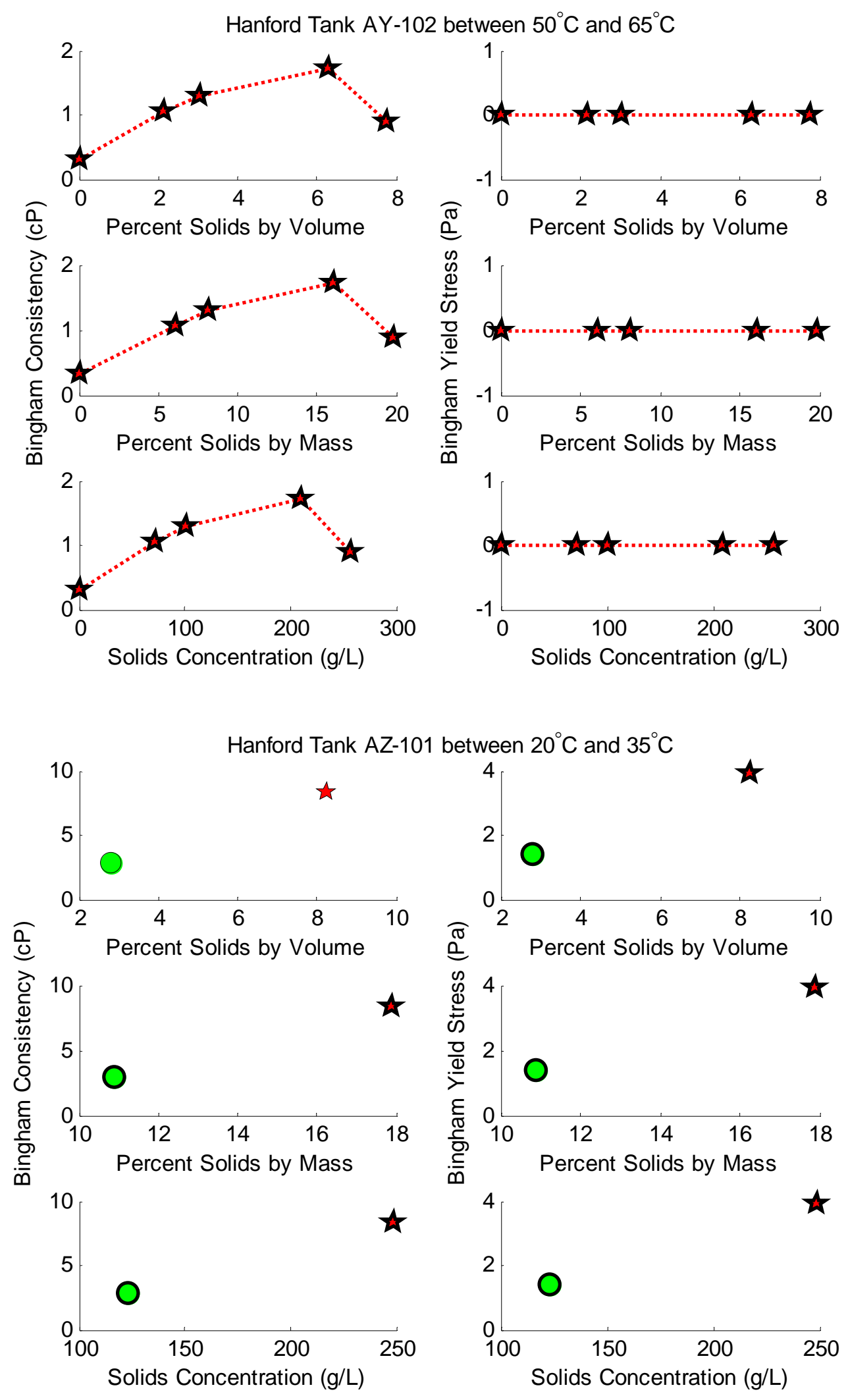

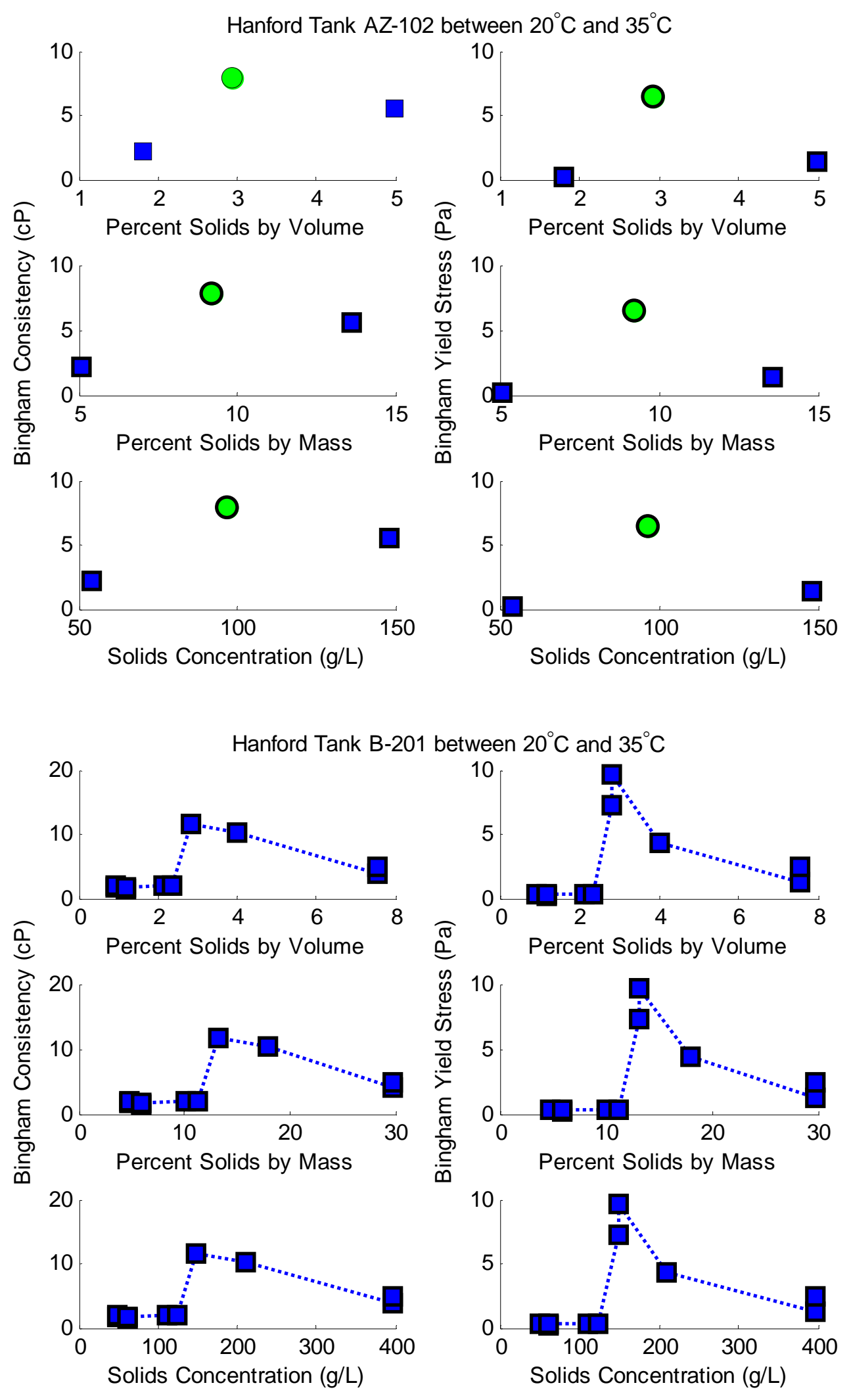

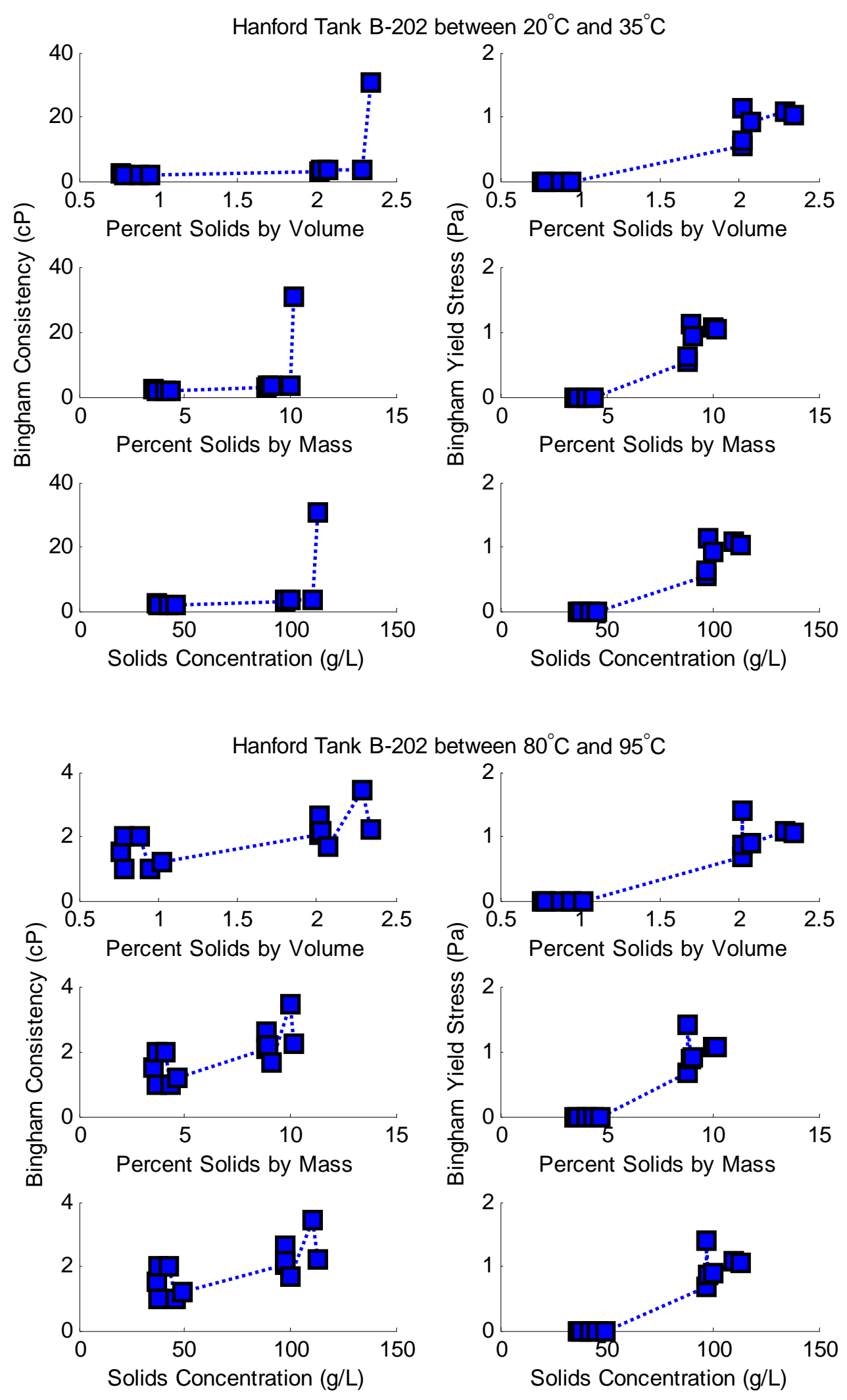

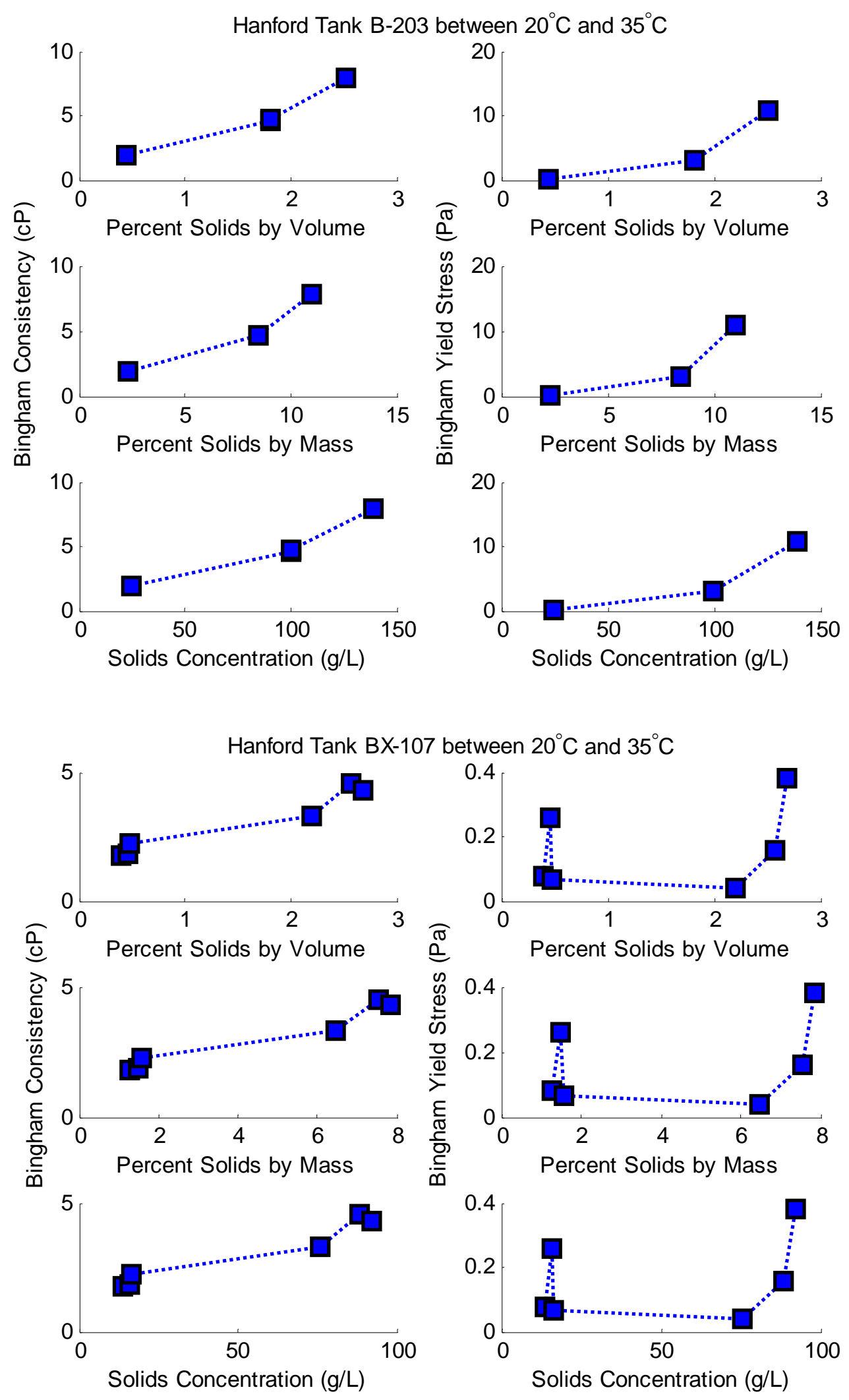

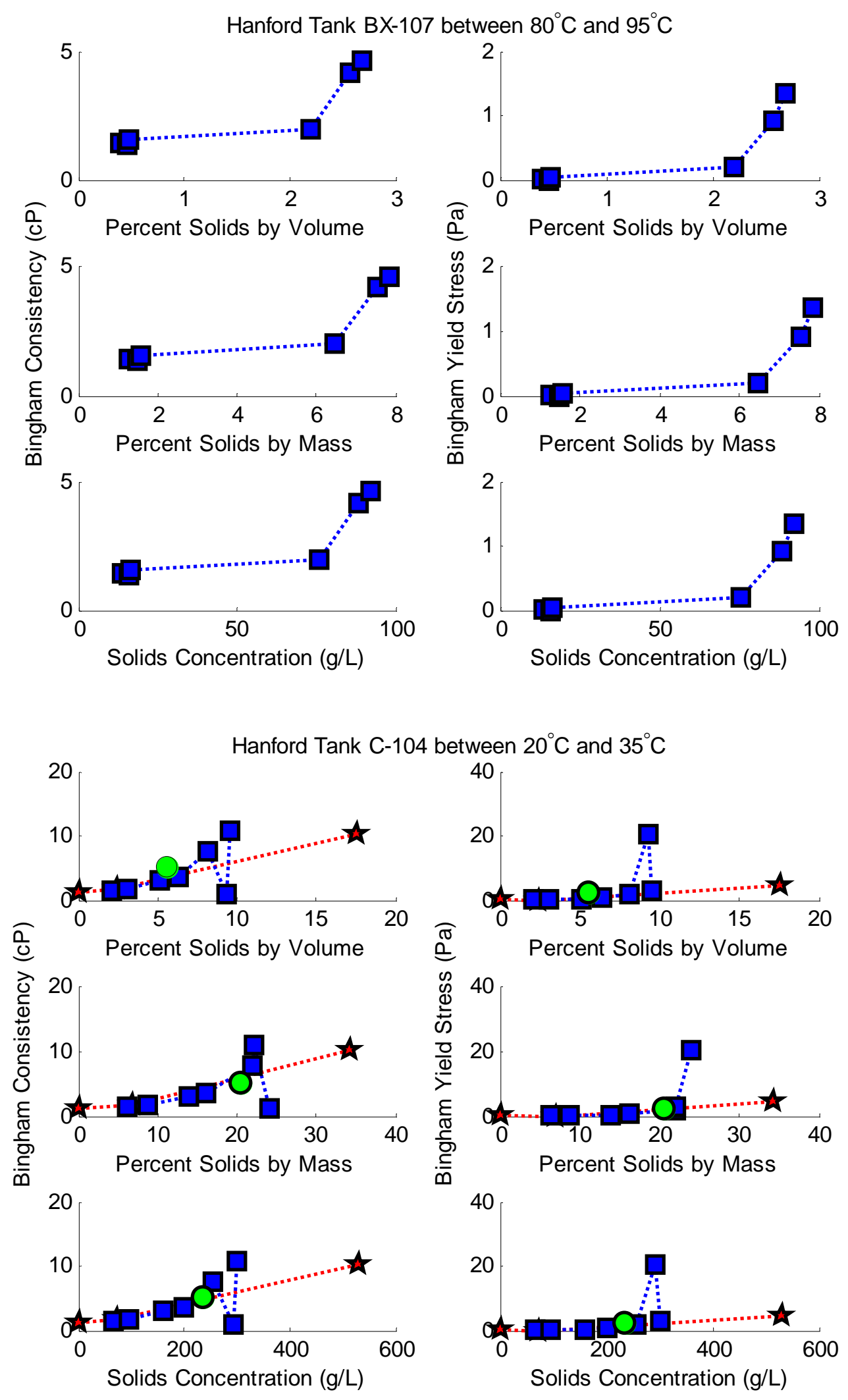

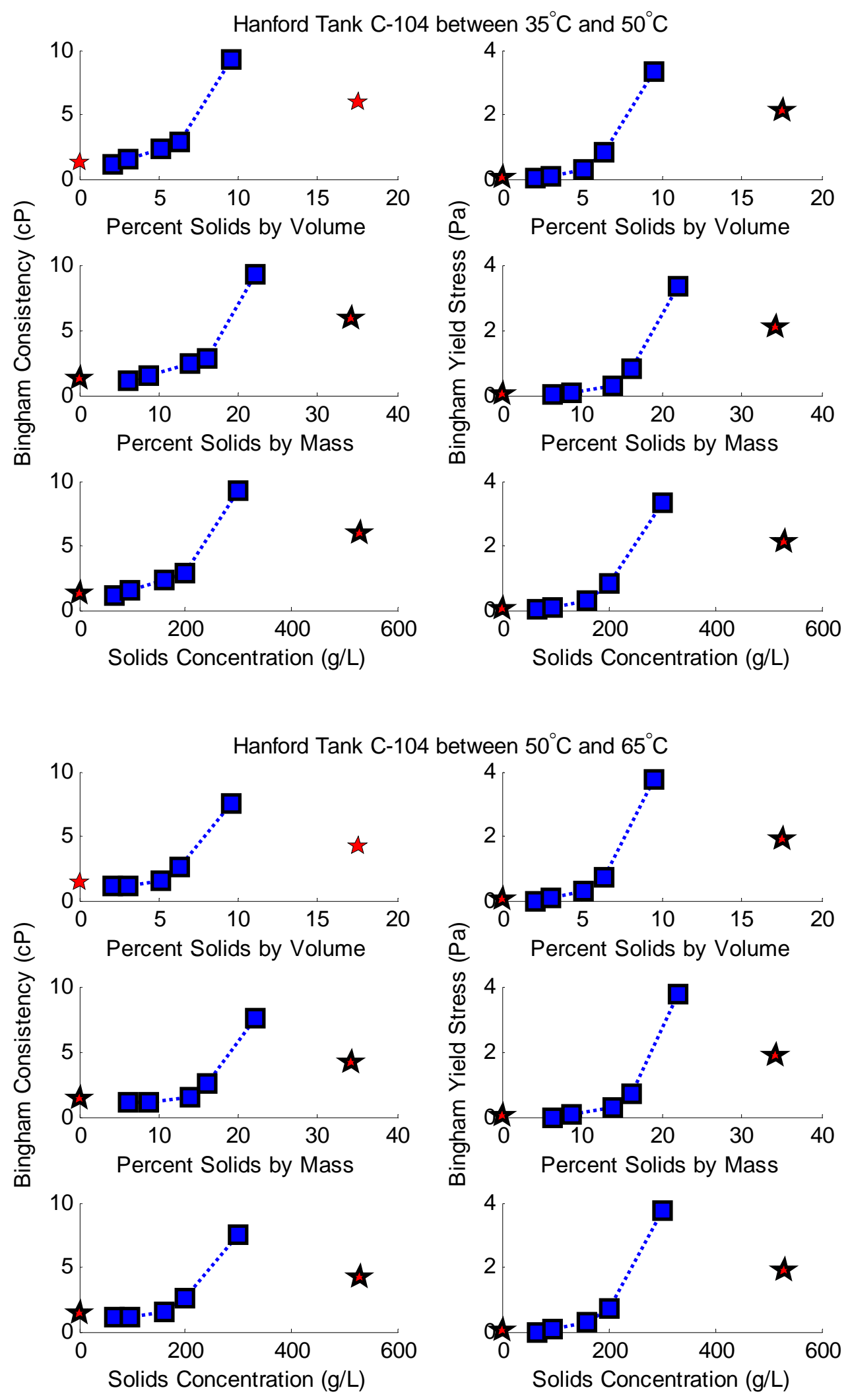

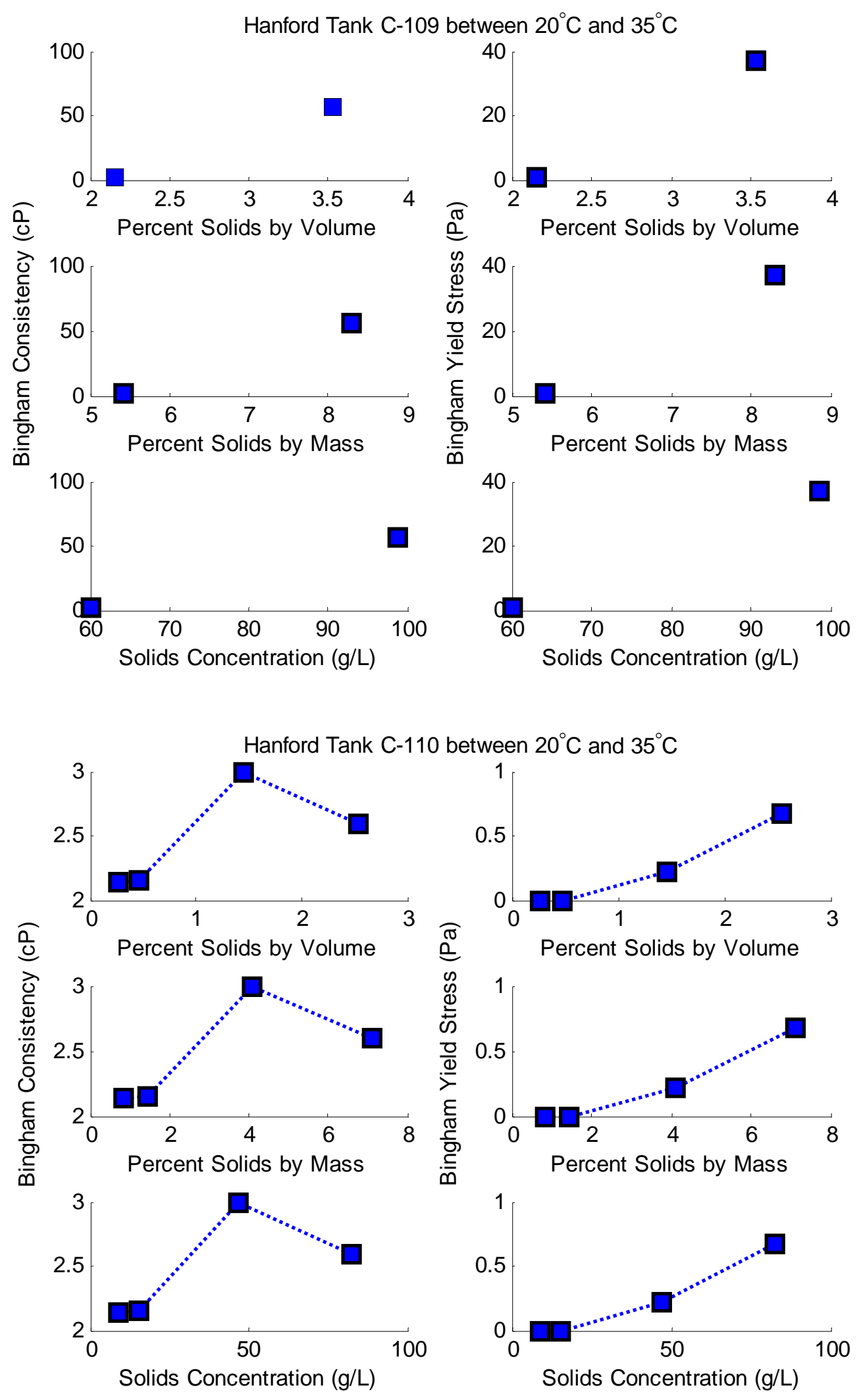

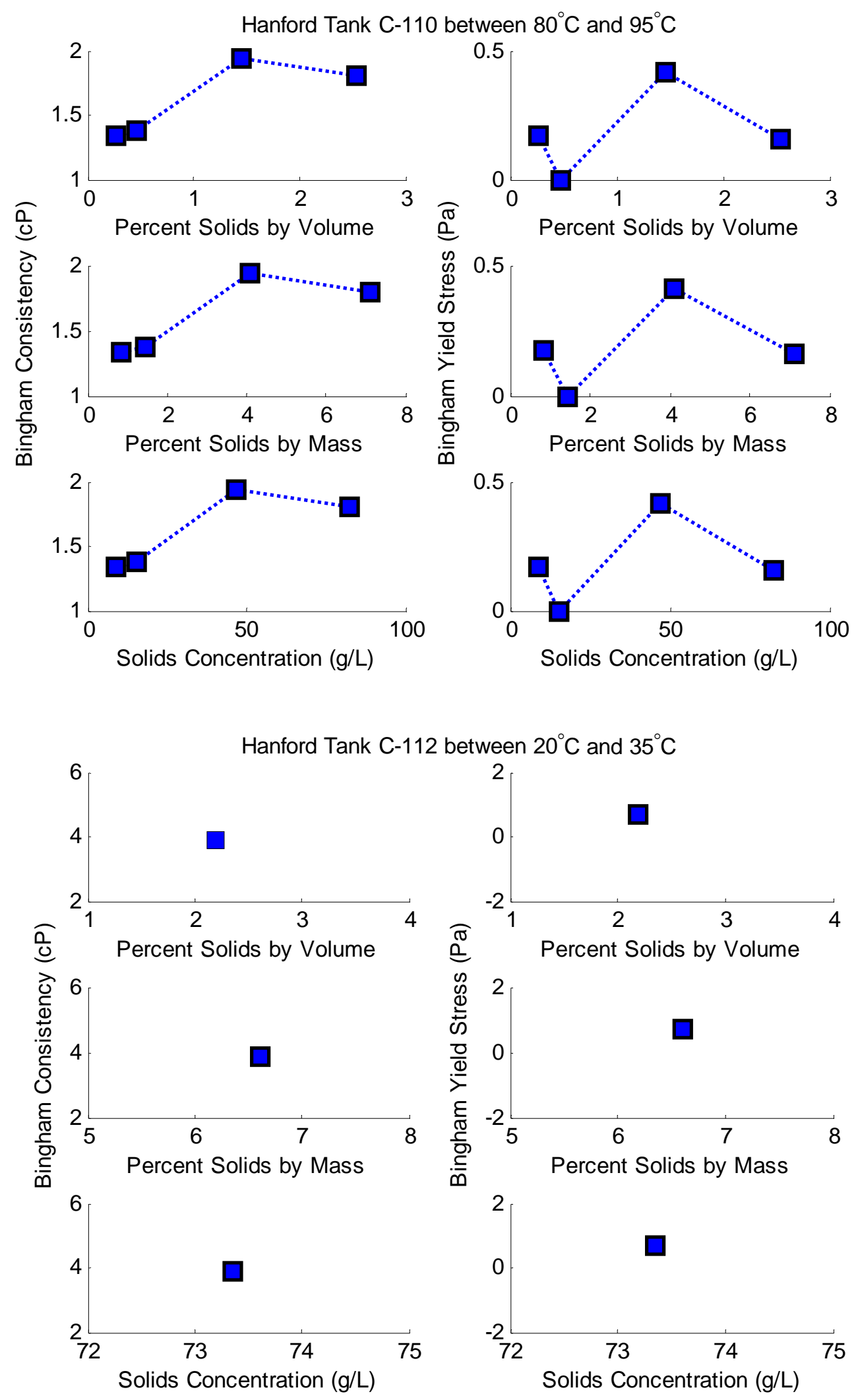

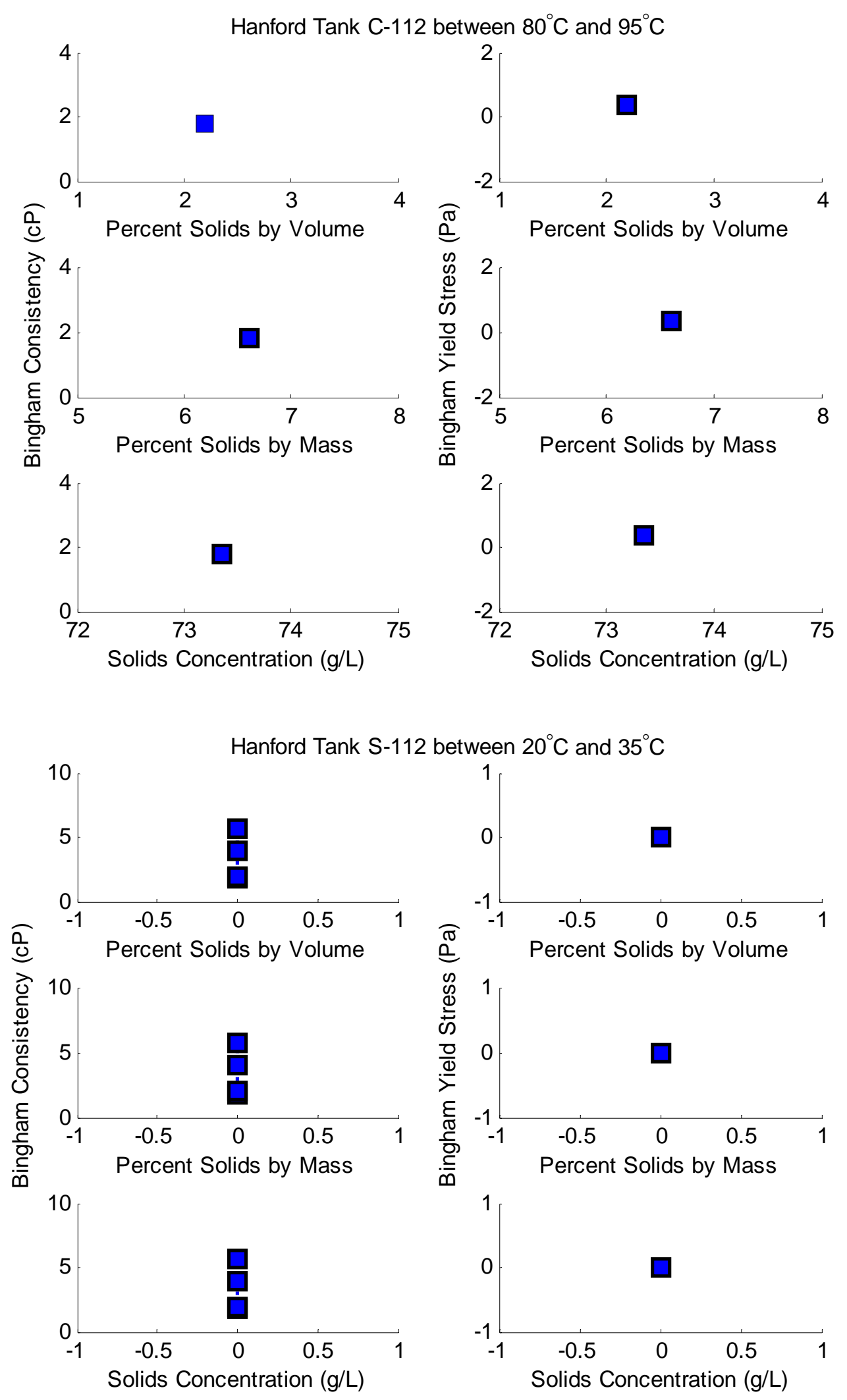

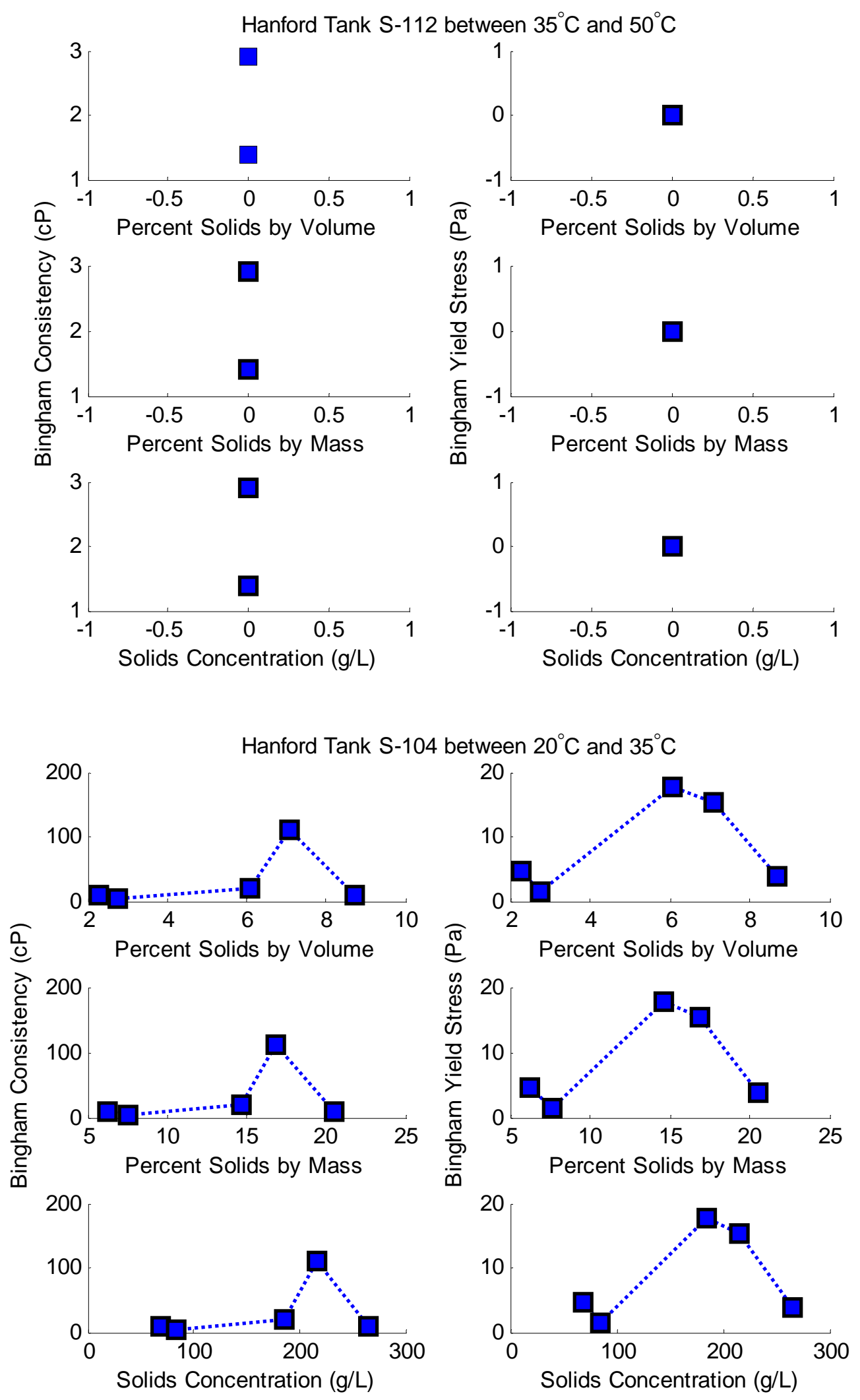

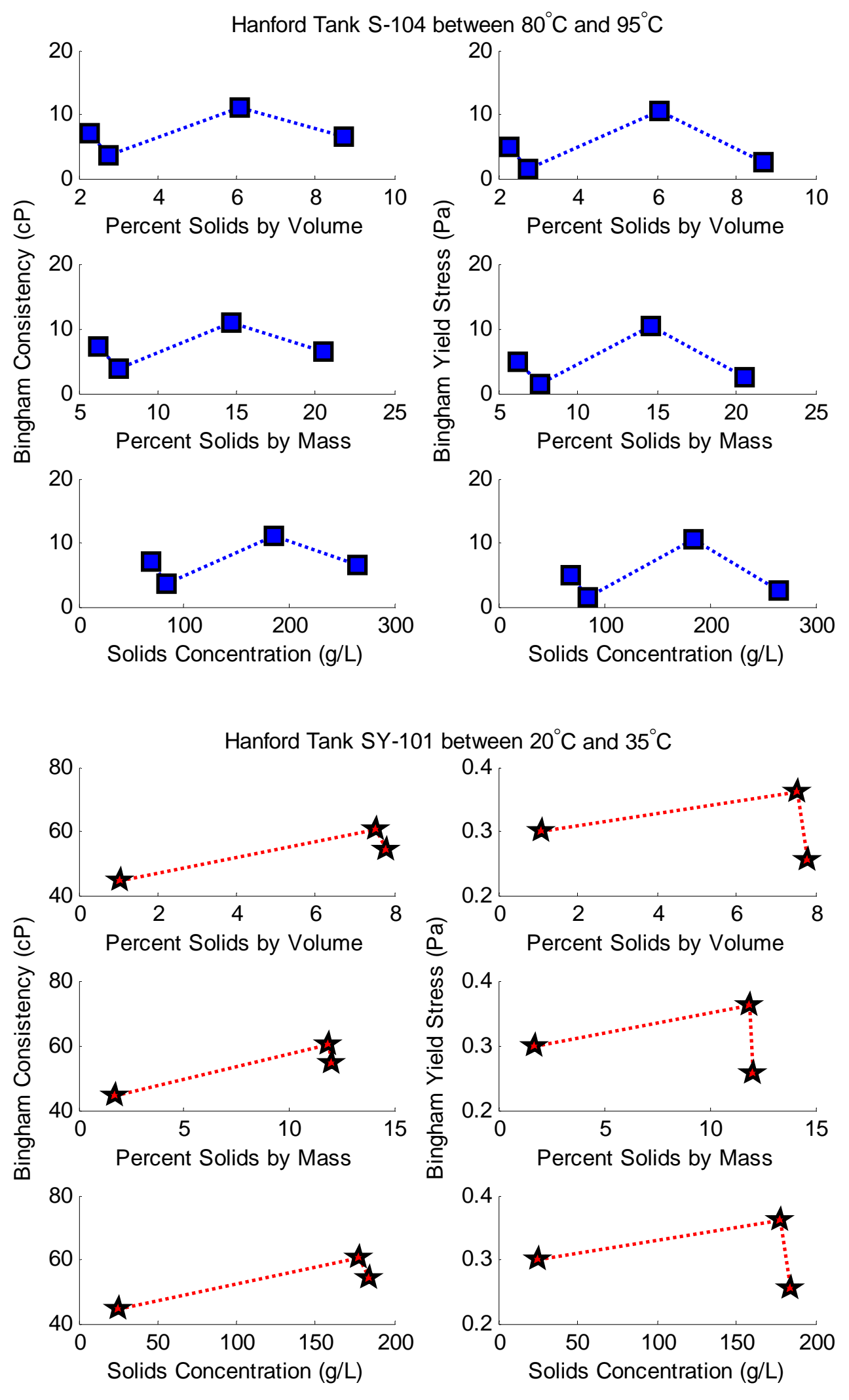

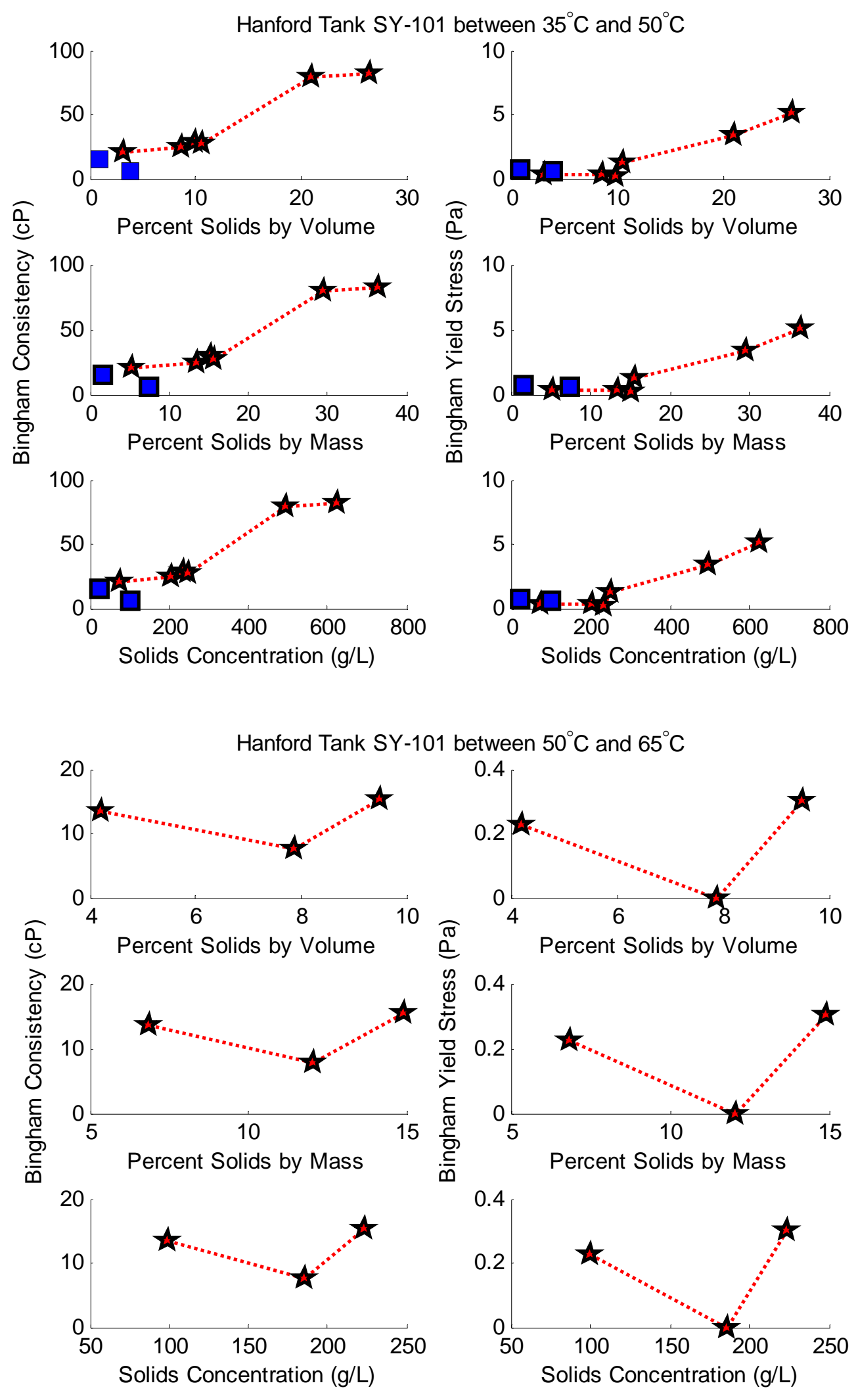

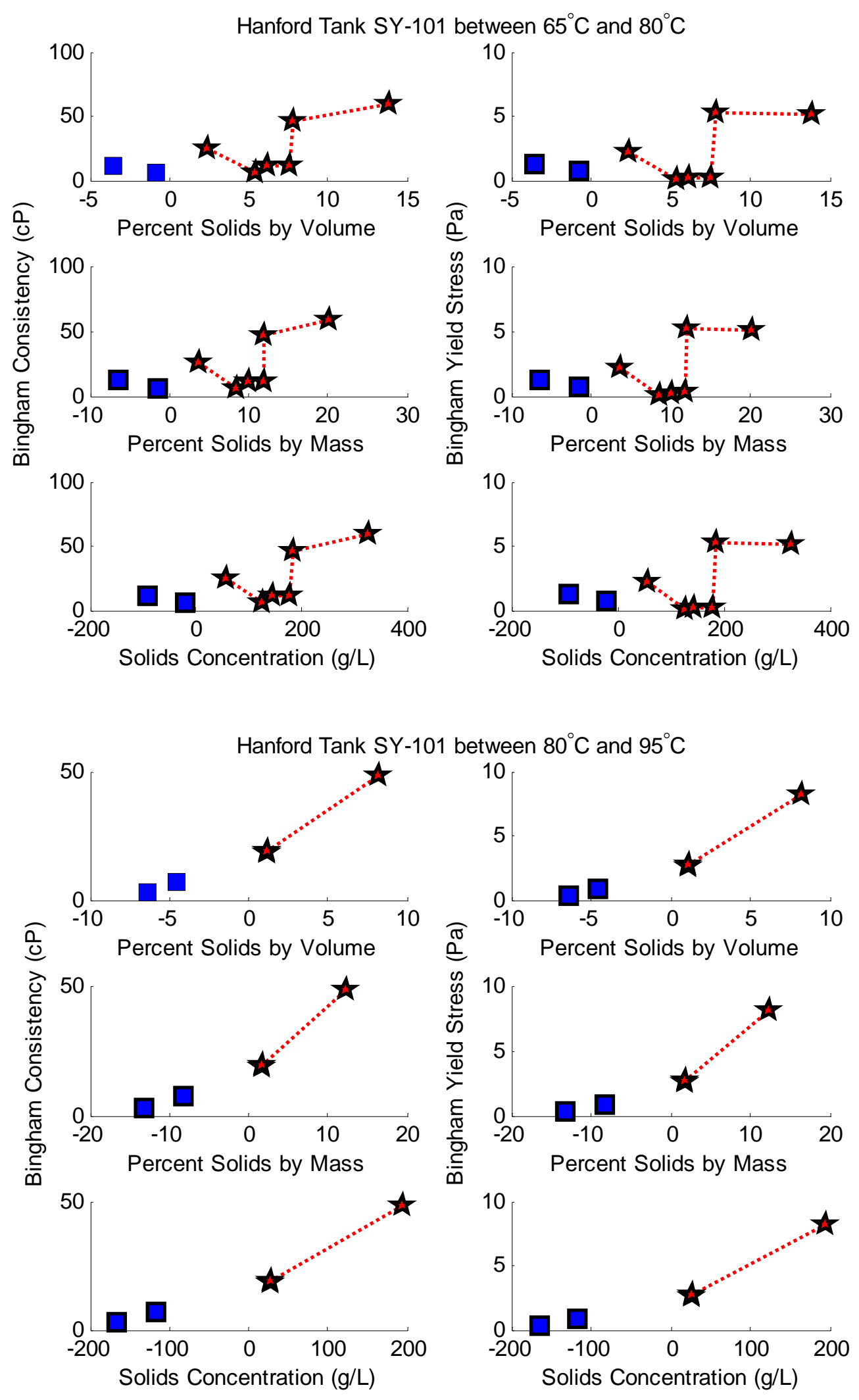

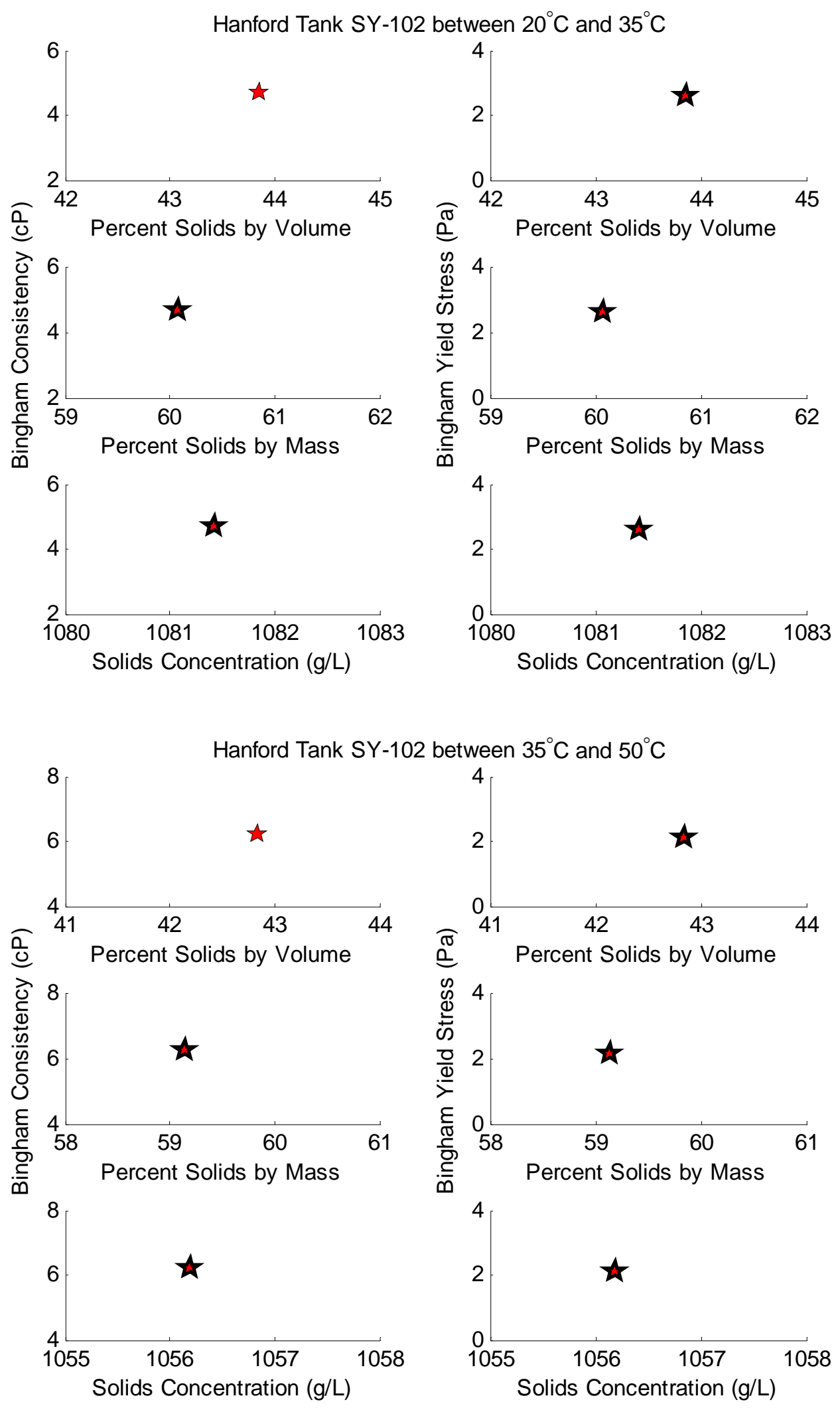


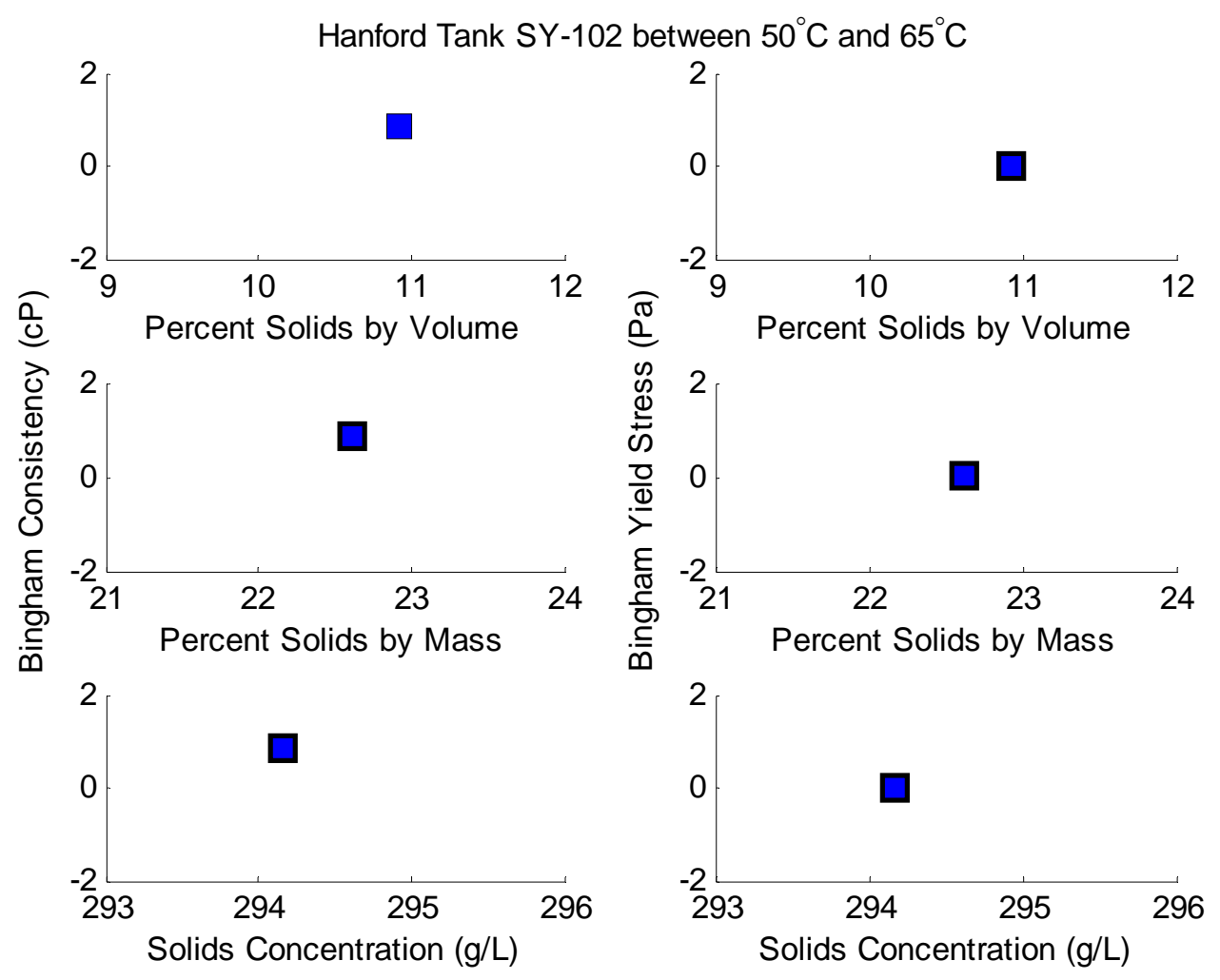

Hanford Tank T-102 between $20^{\circ} \mathrm{C}$ and $35^{\circ} \mathrm{C}$
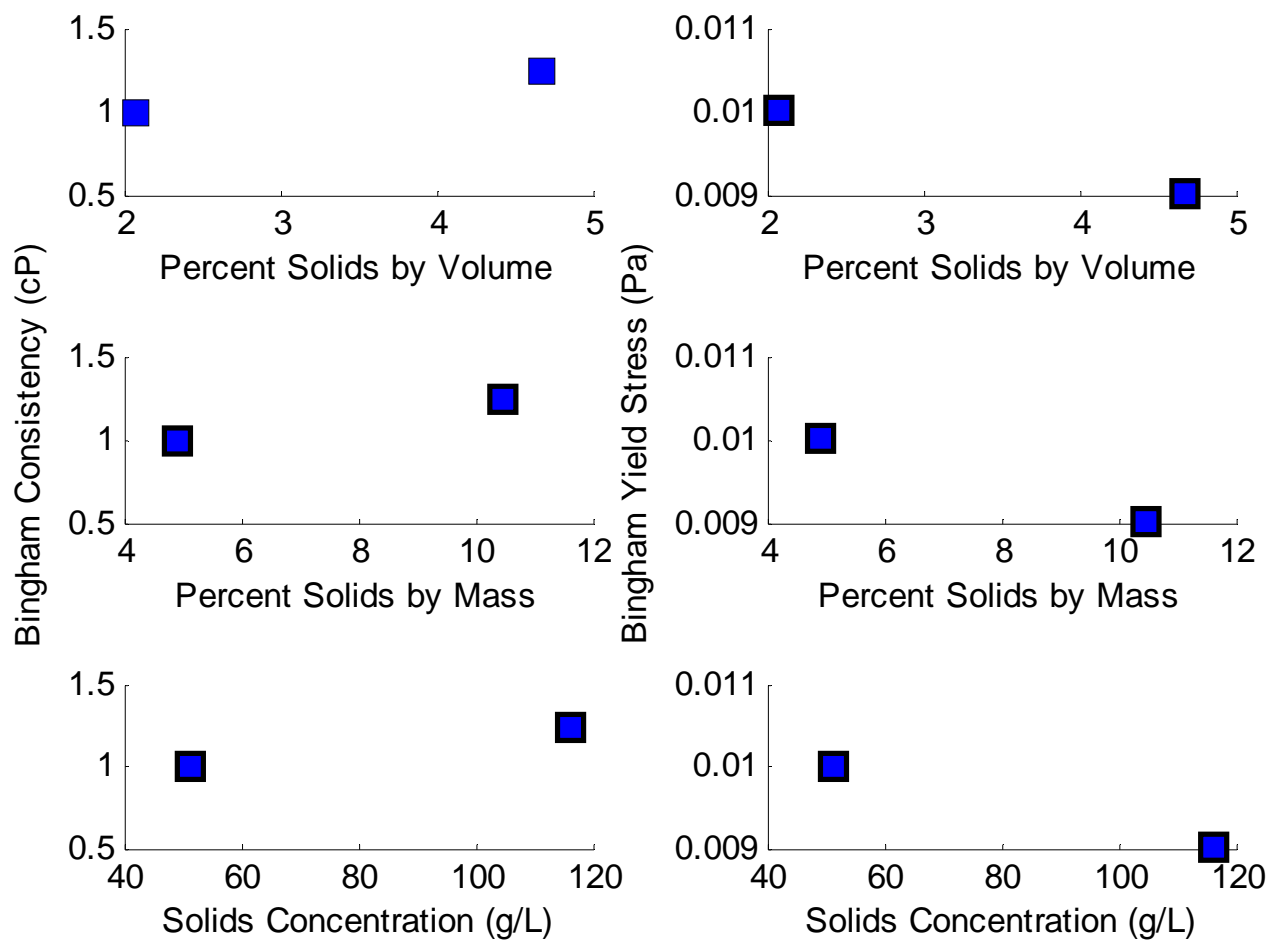

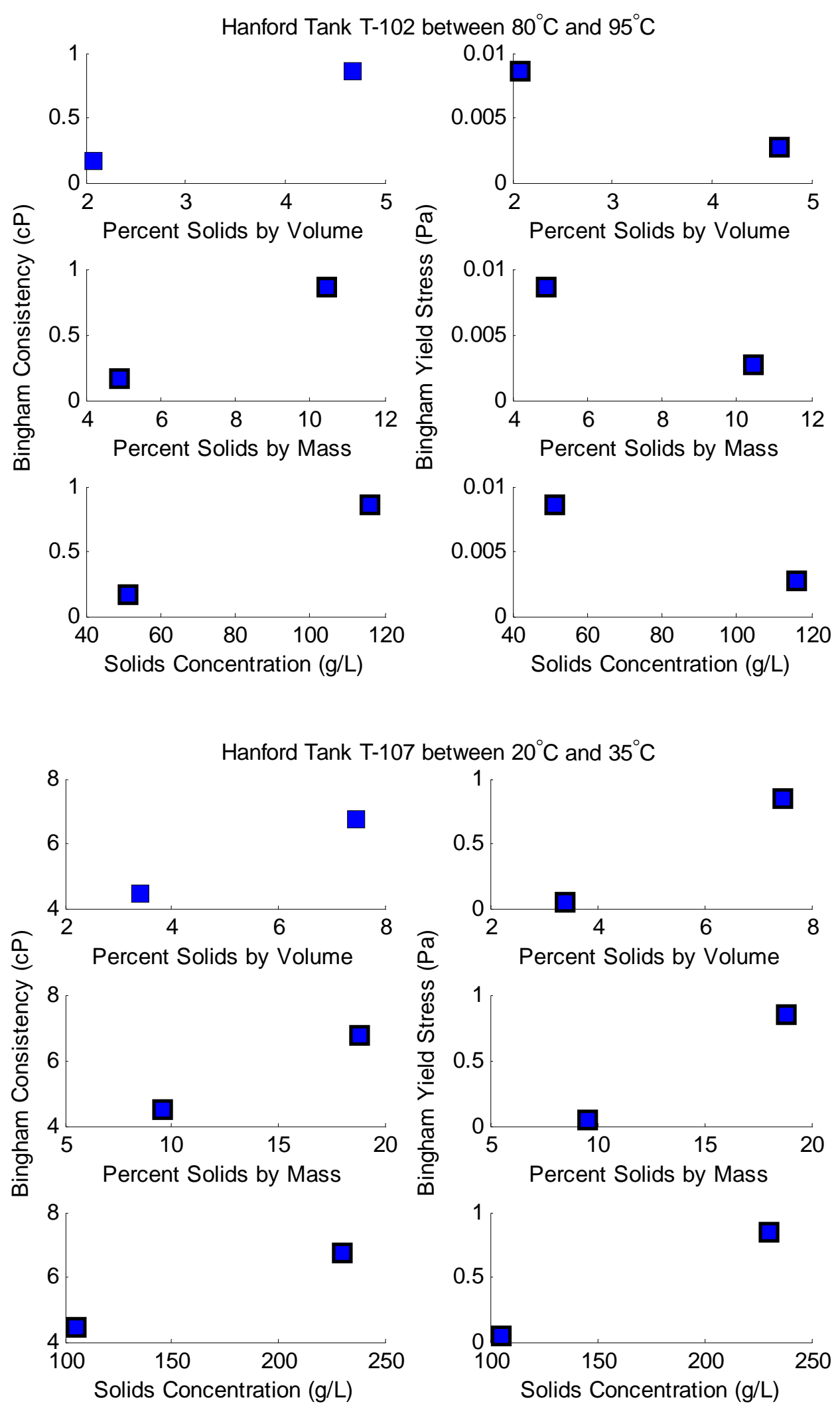

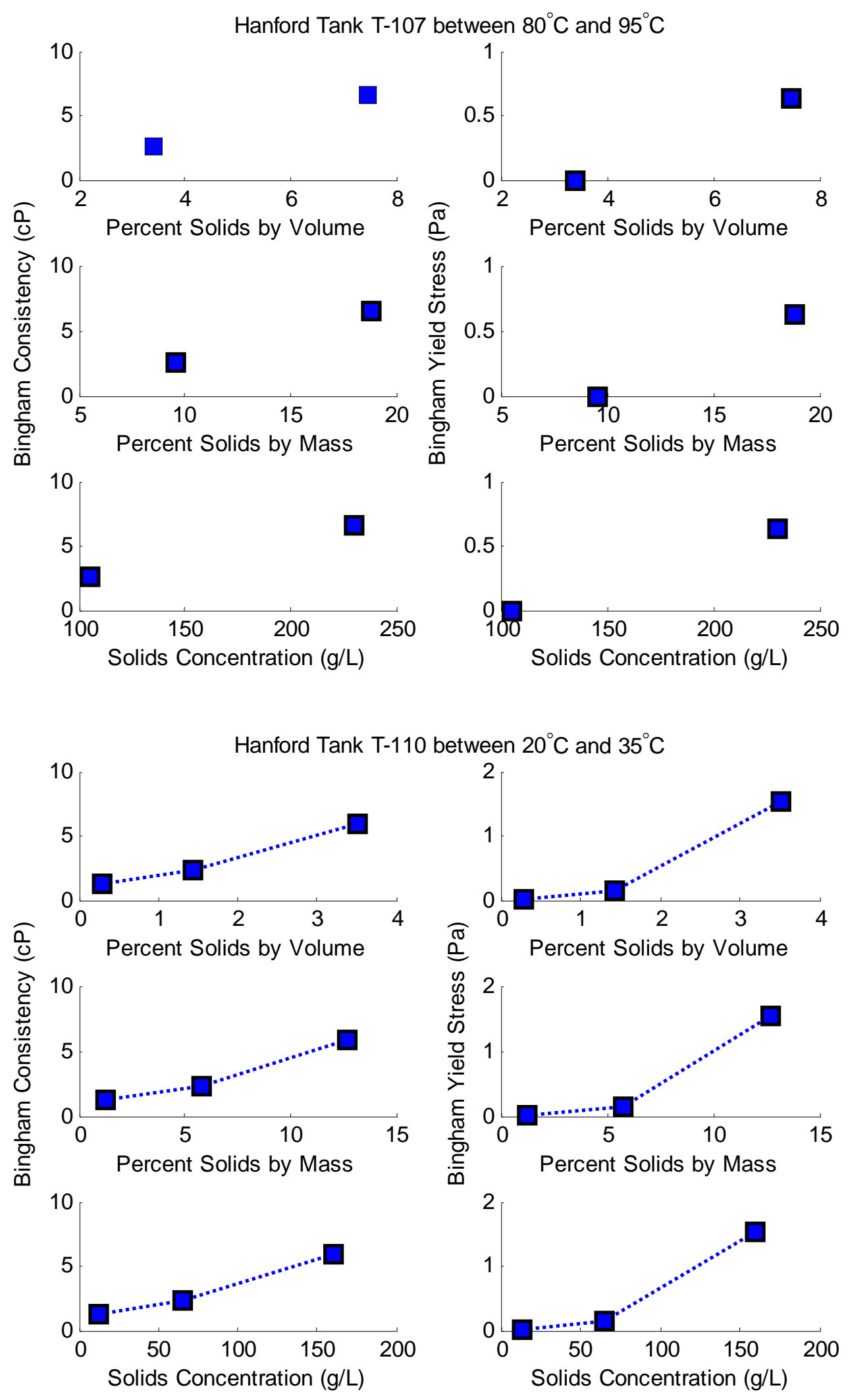

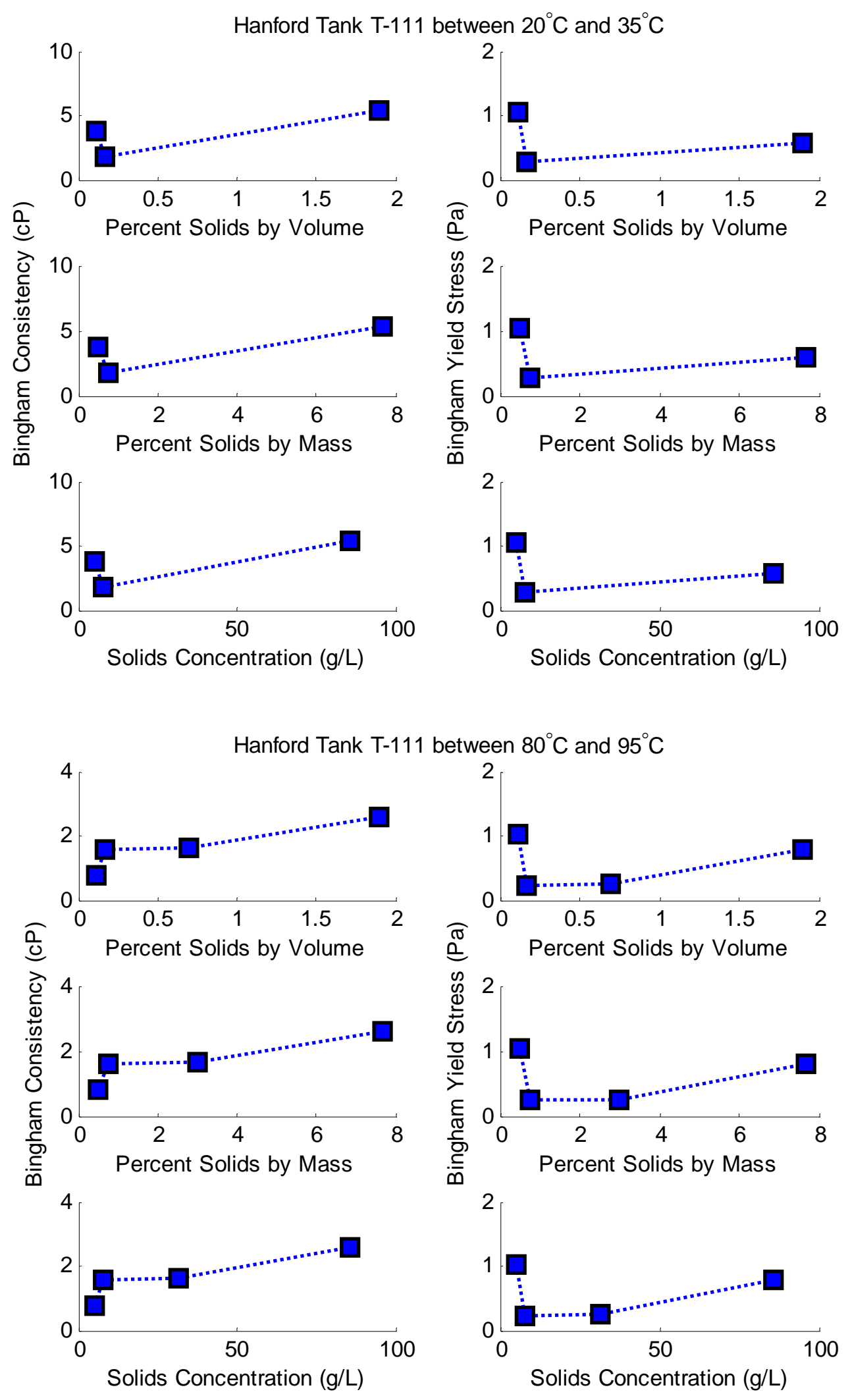

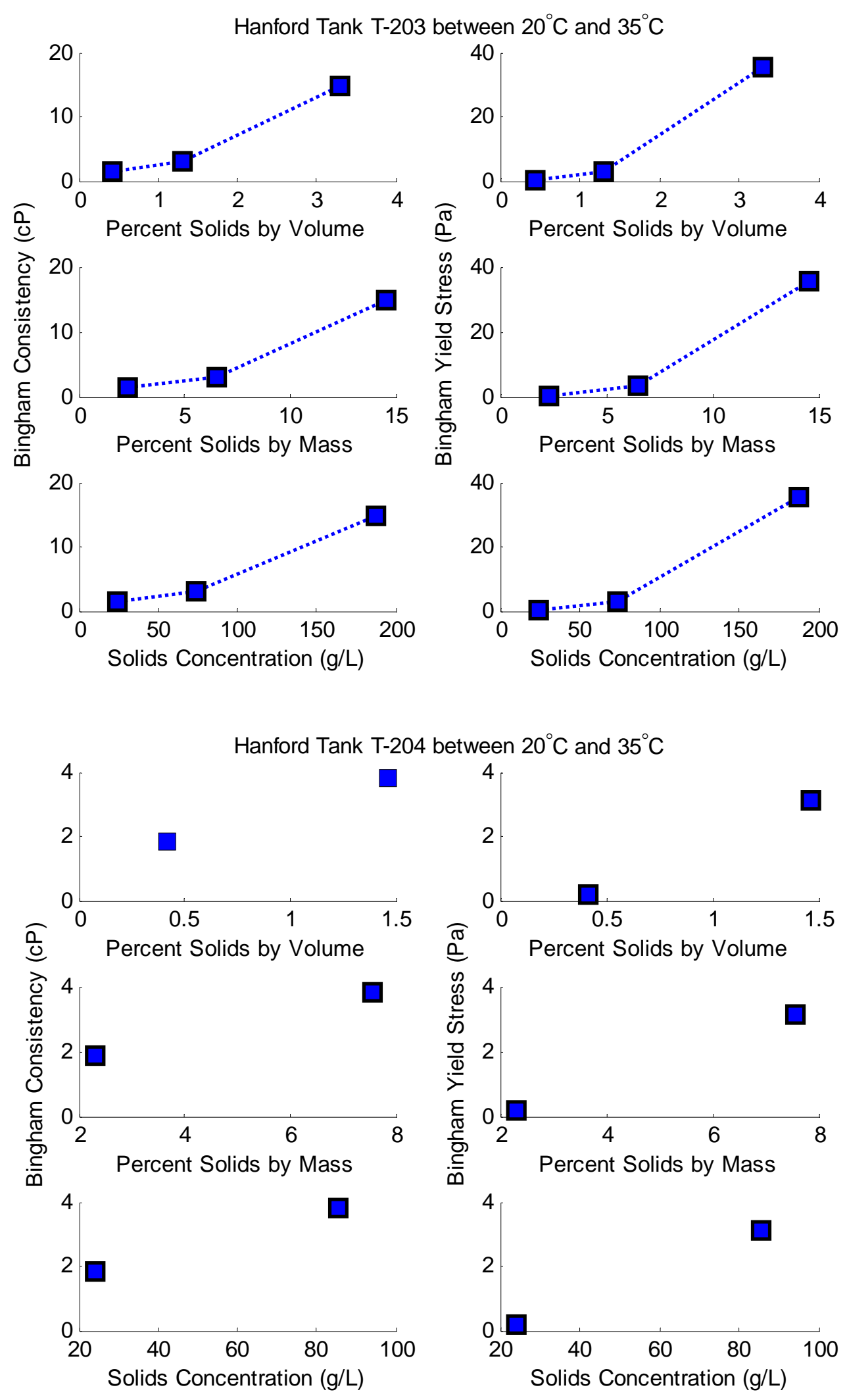


\section{Appendix C}

Correlations of the Bingham Plastic Model Parameters with Solids Content 


\section{Appendix C - Correlations of the Bingham Plastic Model Parameters with Solids Content}

In the figures in this appendix, the stars = as-received tank waste; squares = water added as diluent; and circles $=$ post washing and caustic.
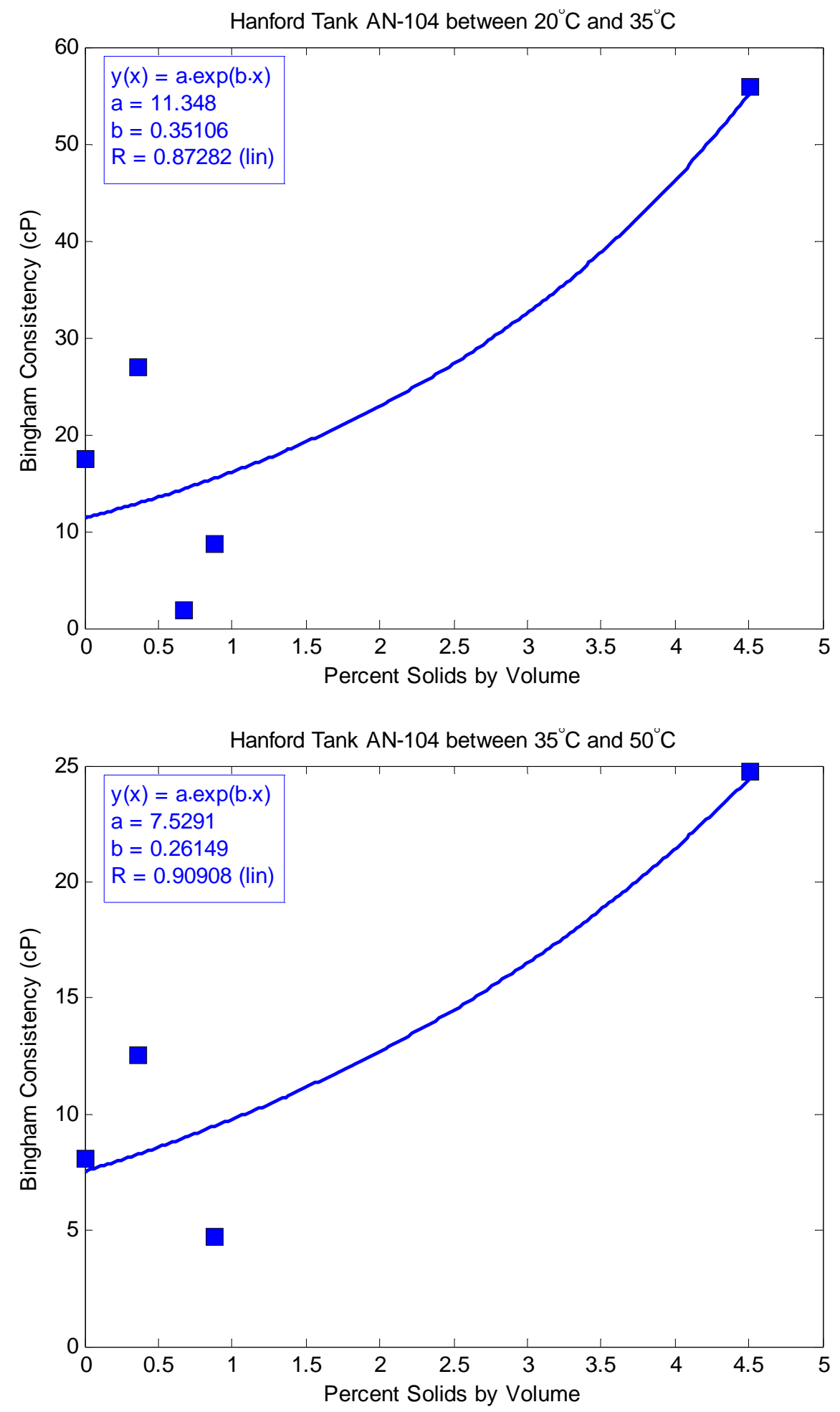

C.1 


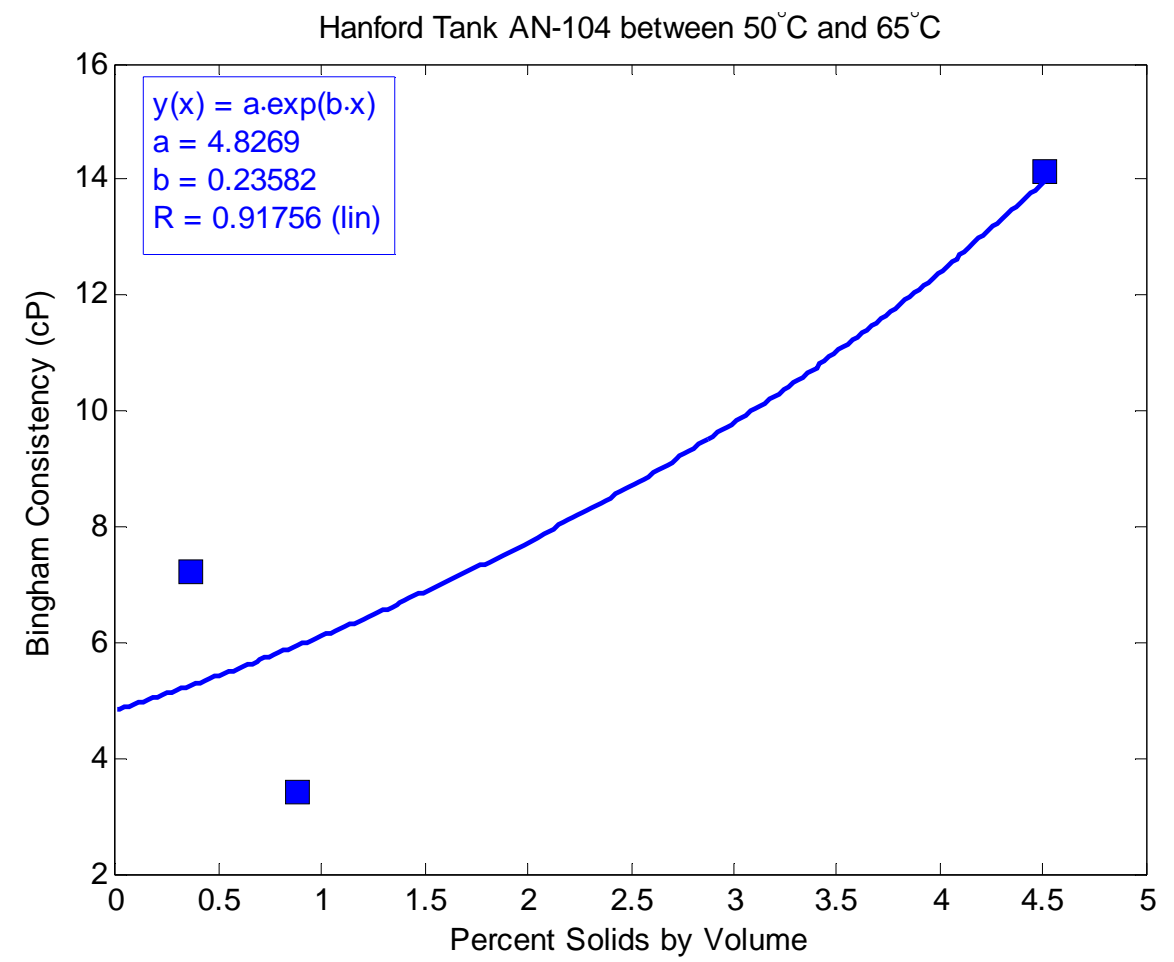



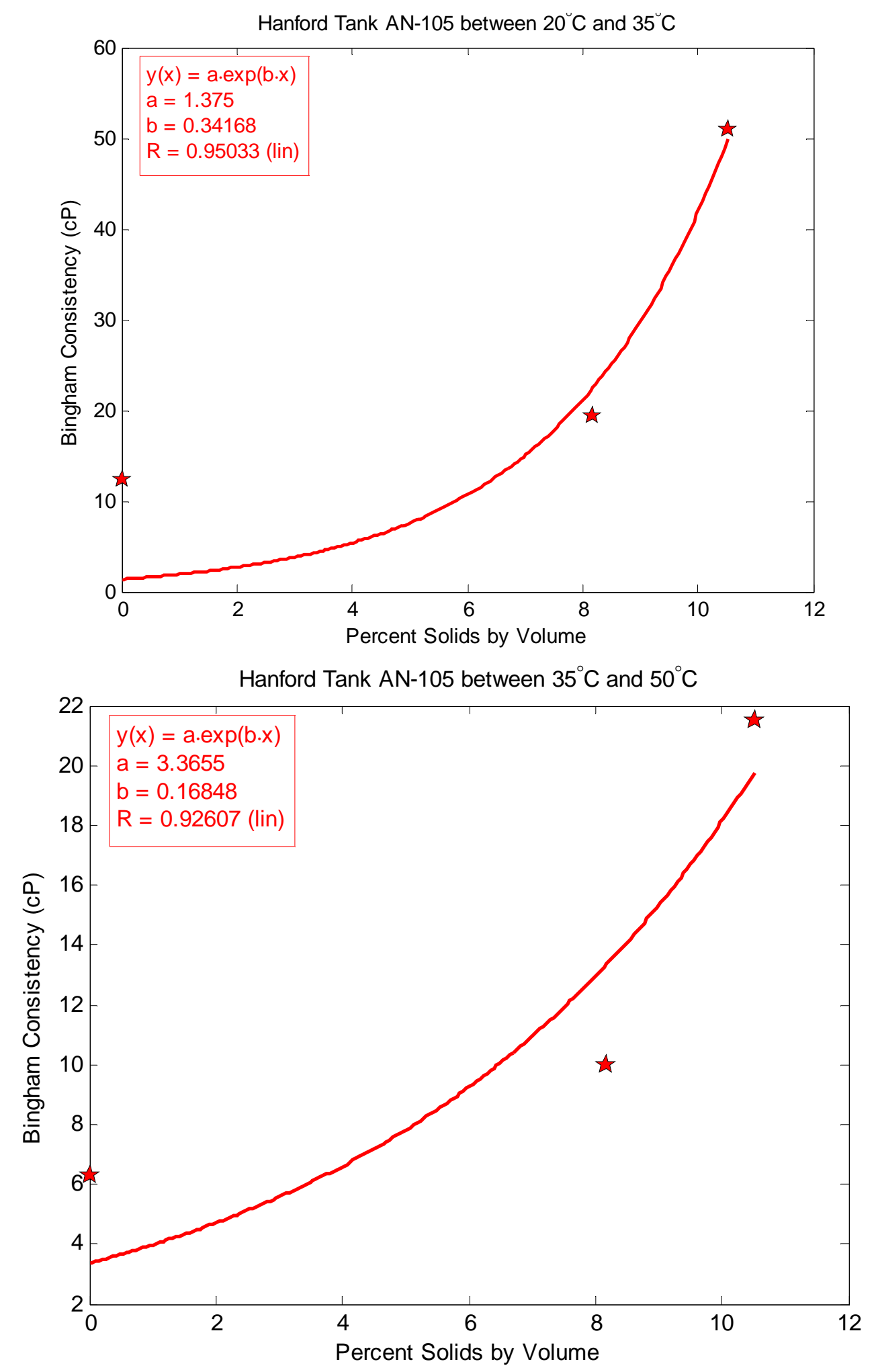

C. 3 
Hanford Tank AN-105 between $50^{\circ} \mathrm{C}$ and $65^{\circ} \mathrm{C}$

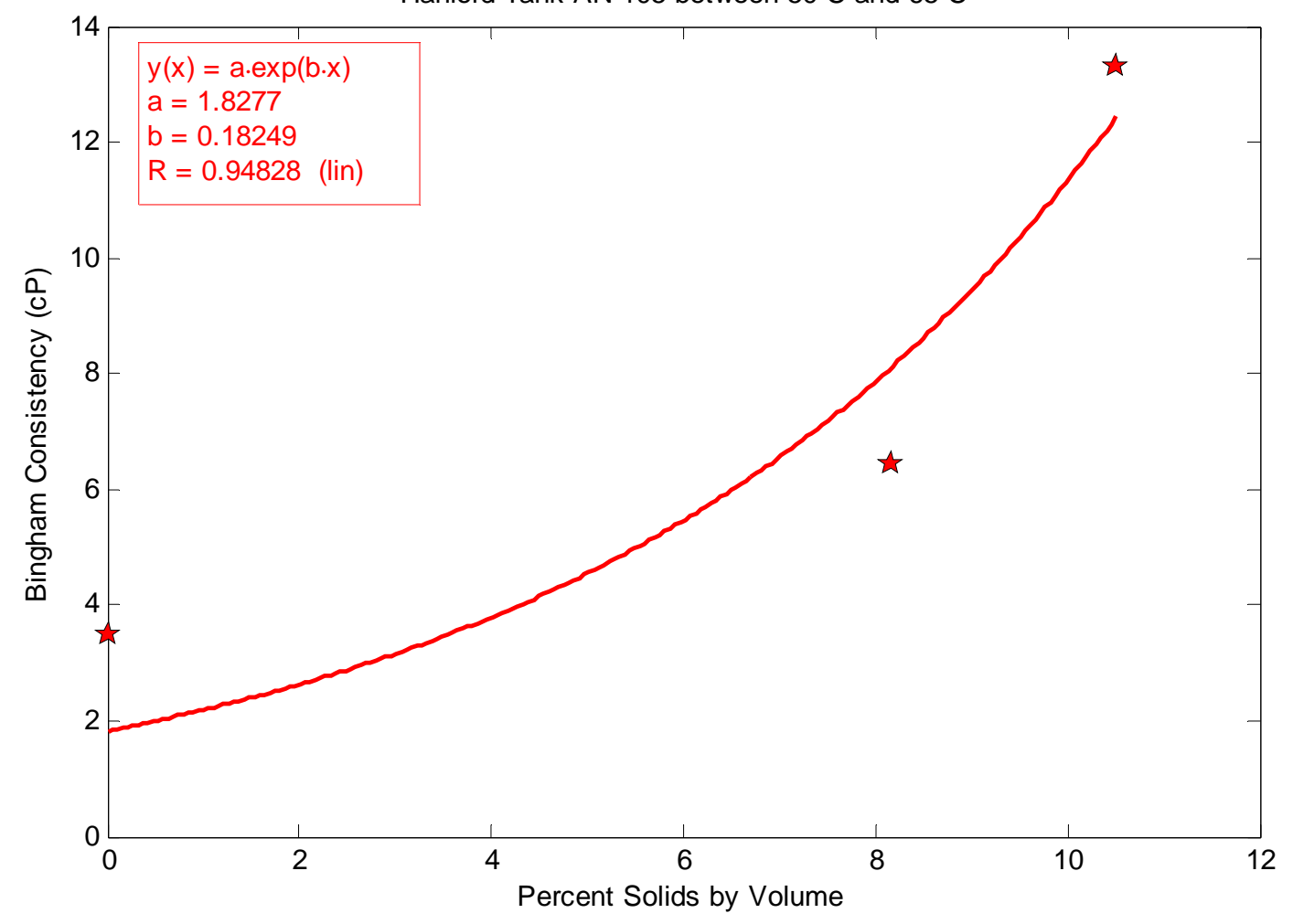




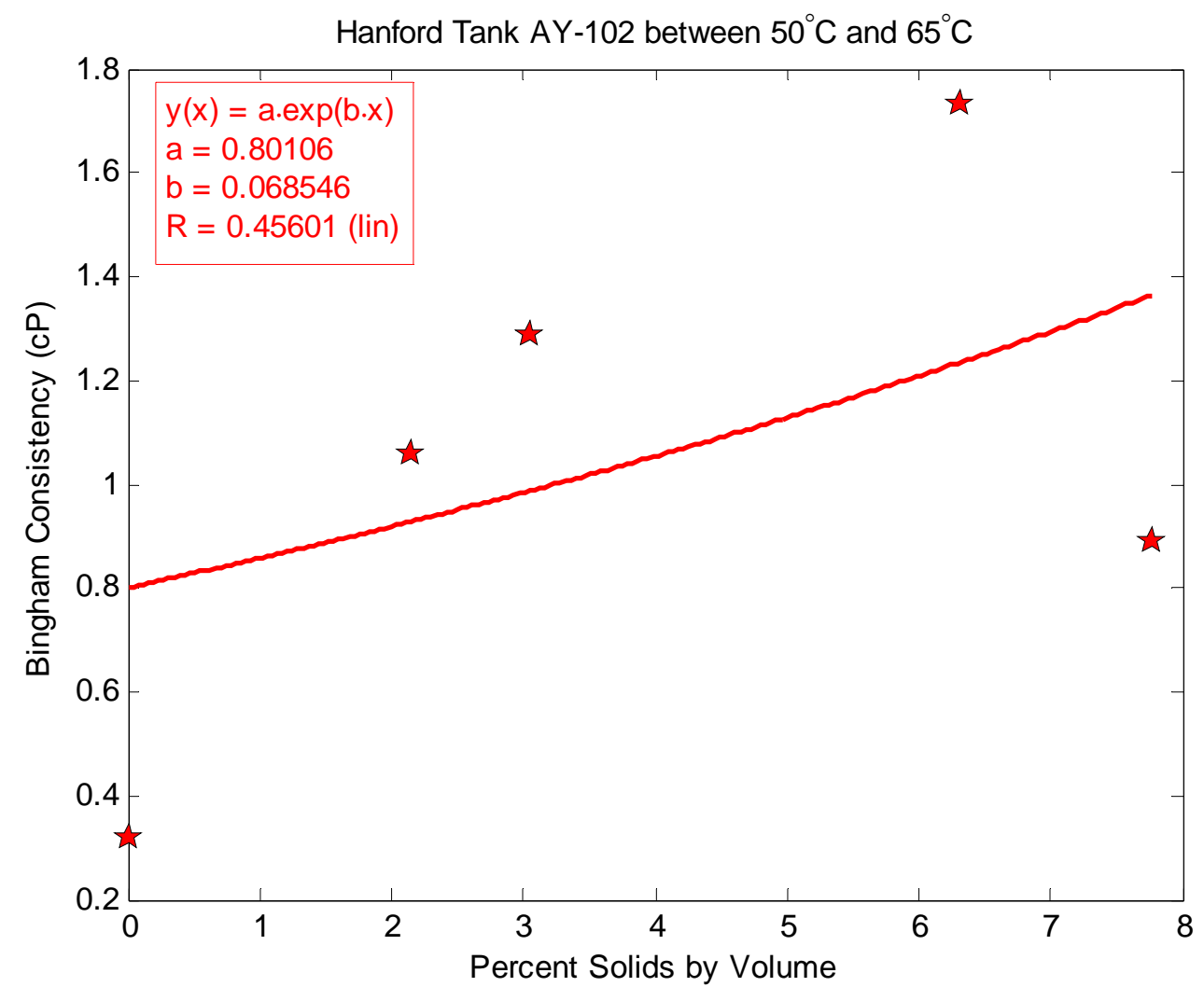

C. 5 

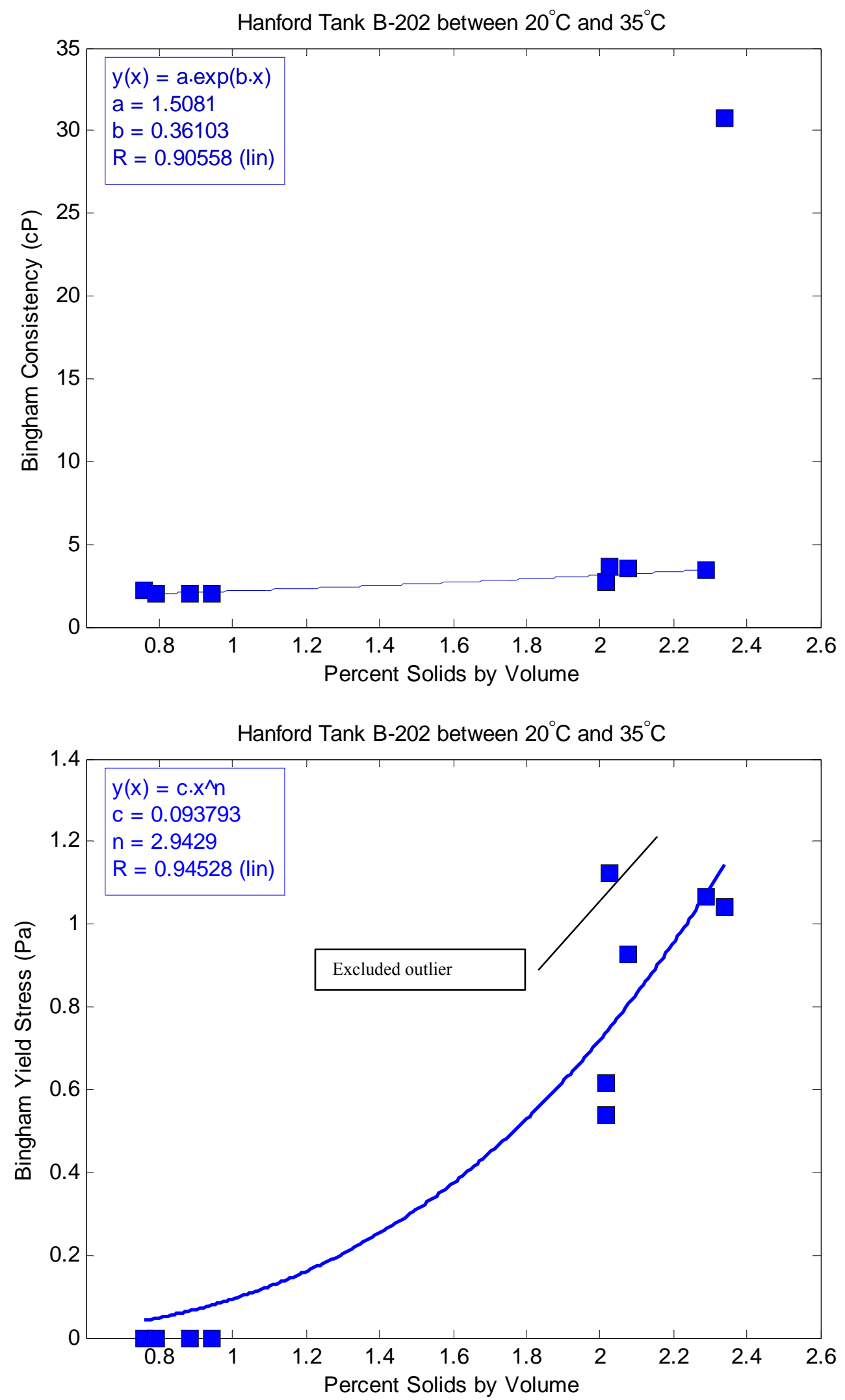

C. 6 

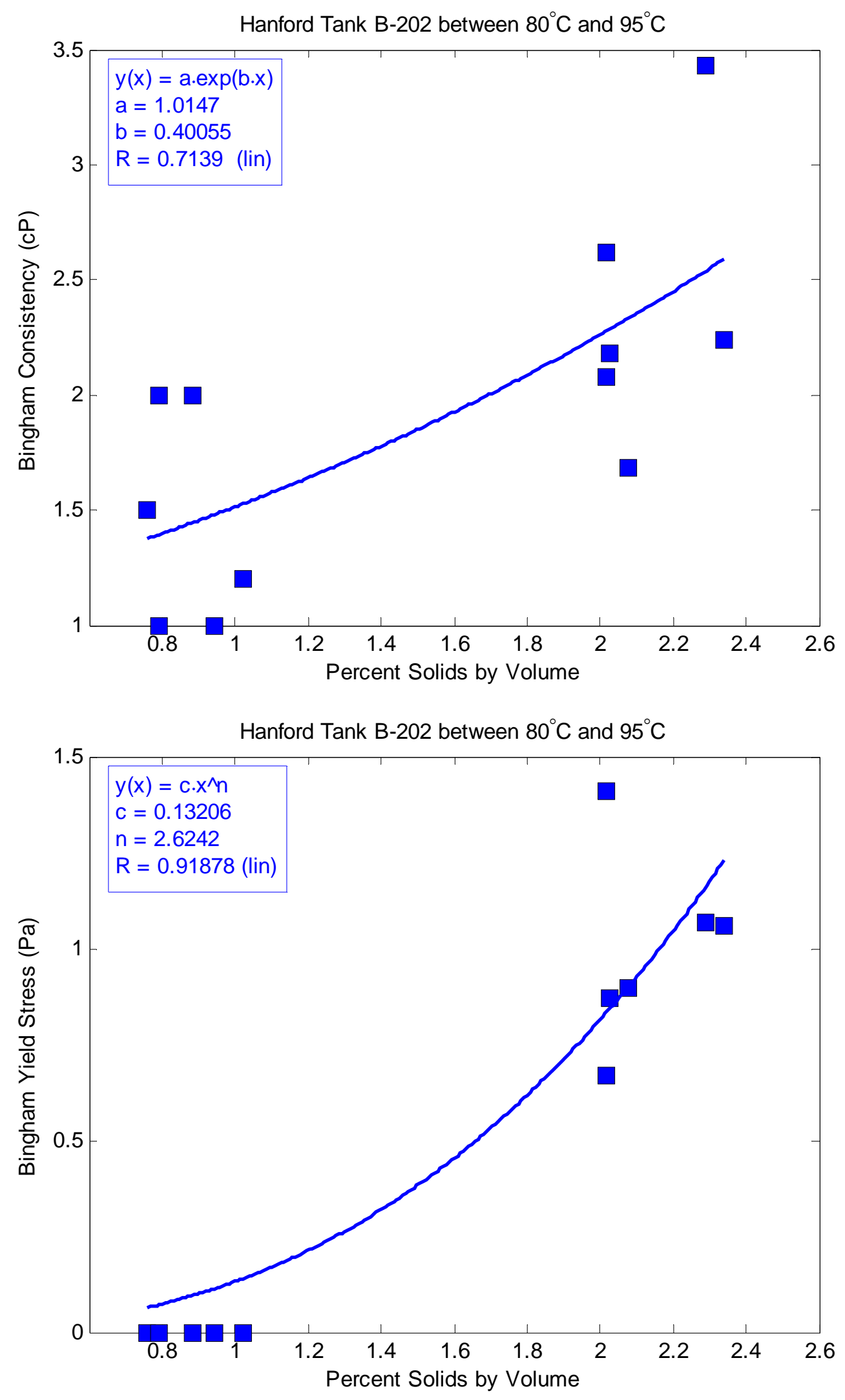

C. 7 


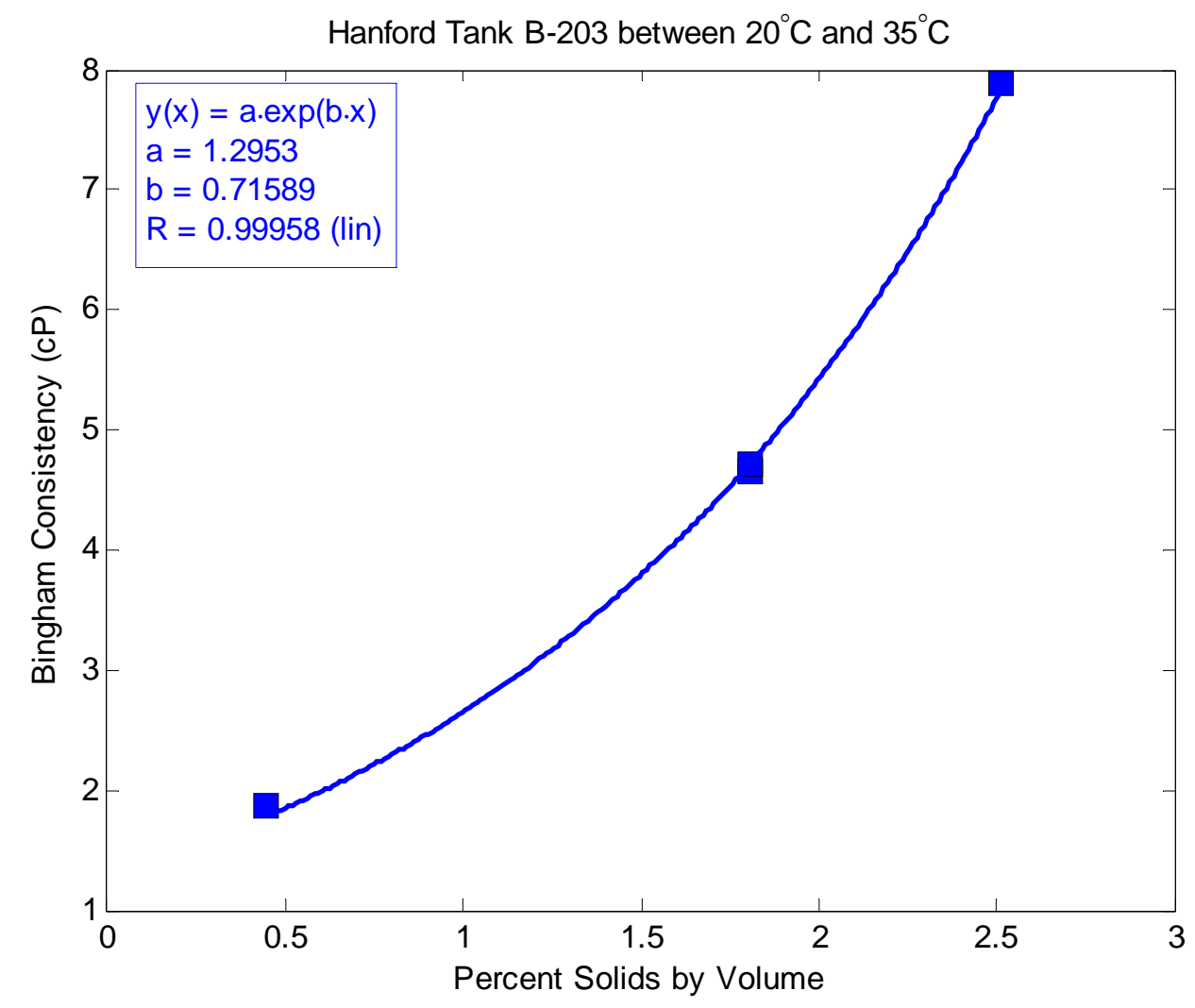



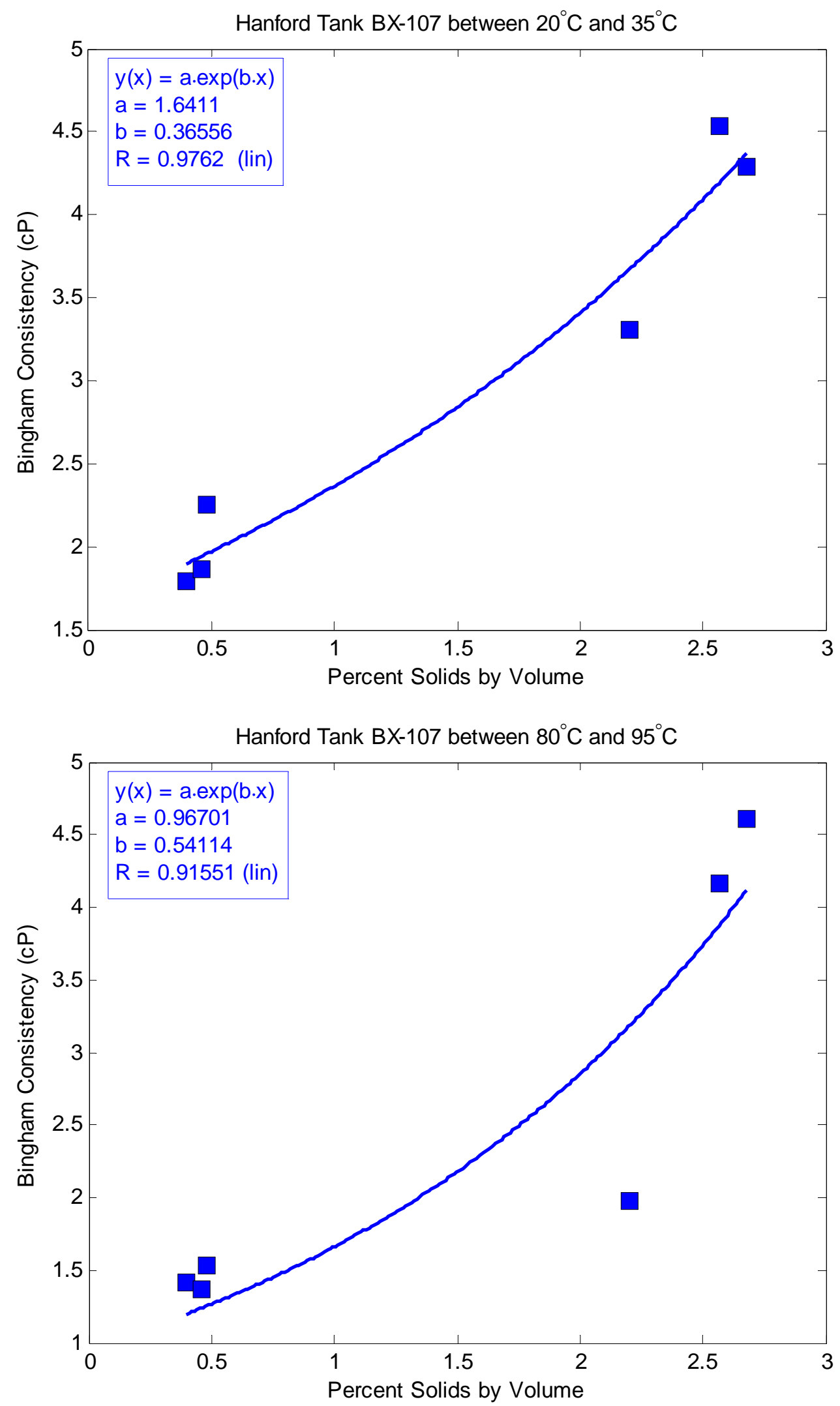
Hanford Tank BX-107 between $80^{\circ} \mathrm{C}$ and $95^{\circ} \mathrm{C}$

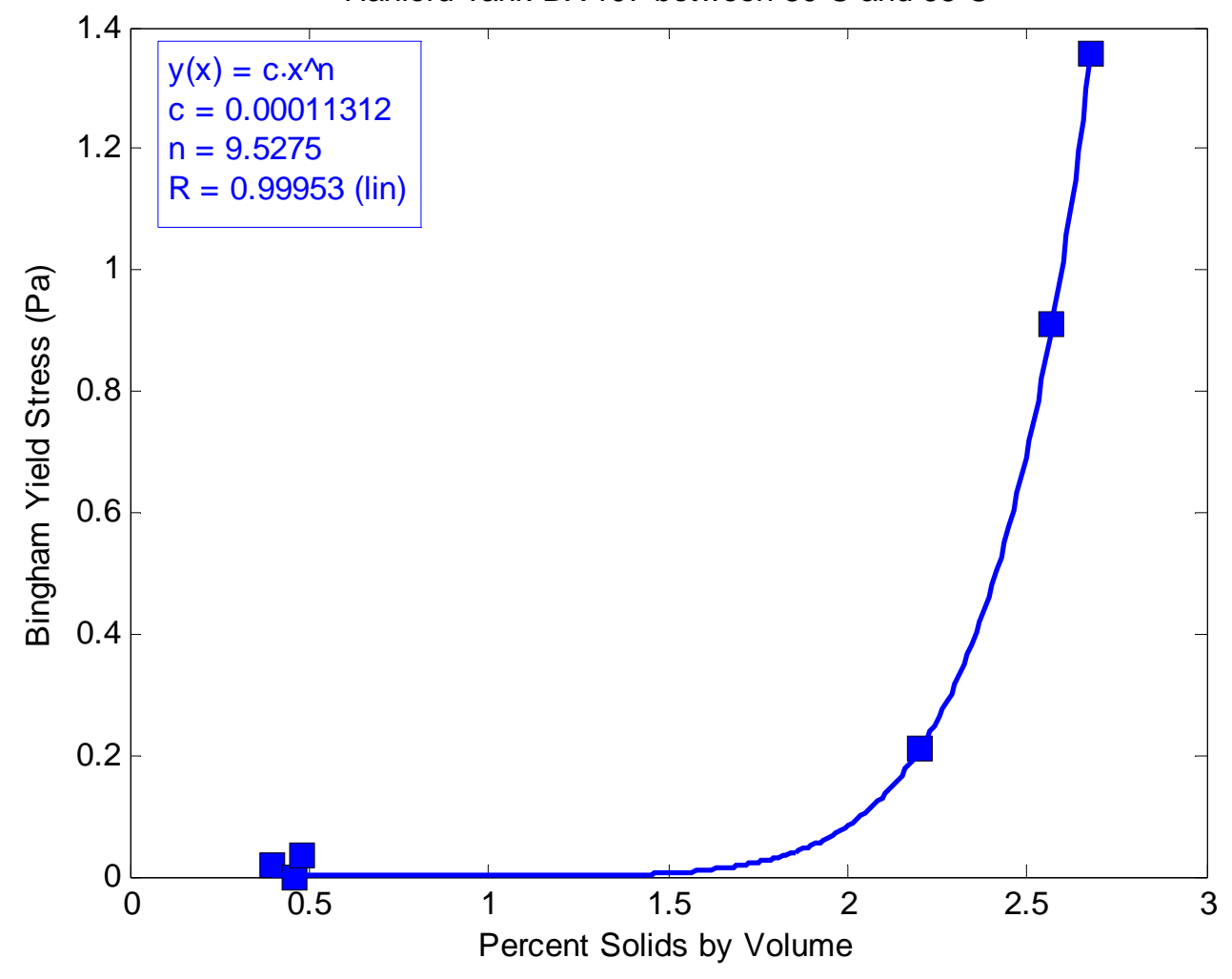

C. 10 

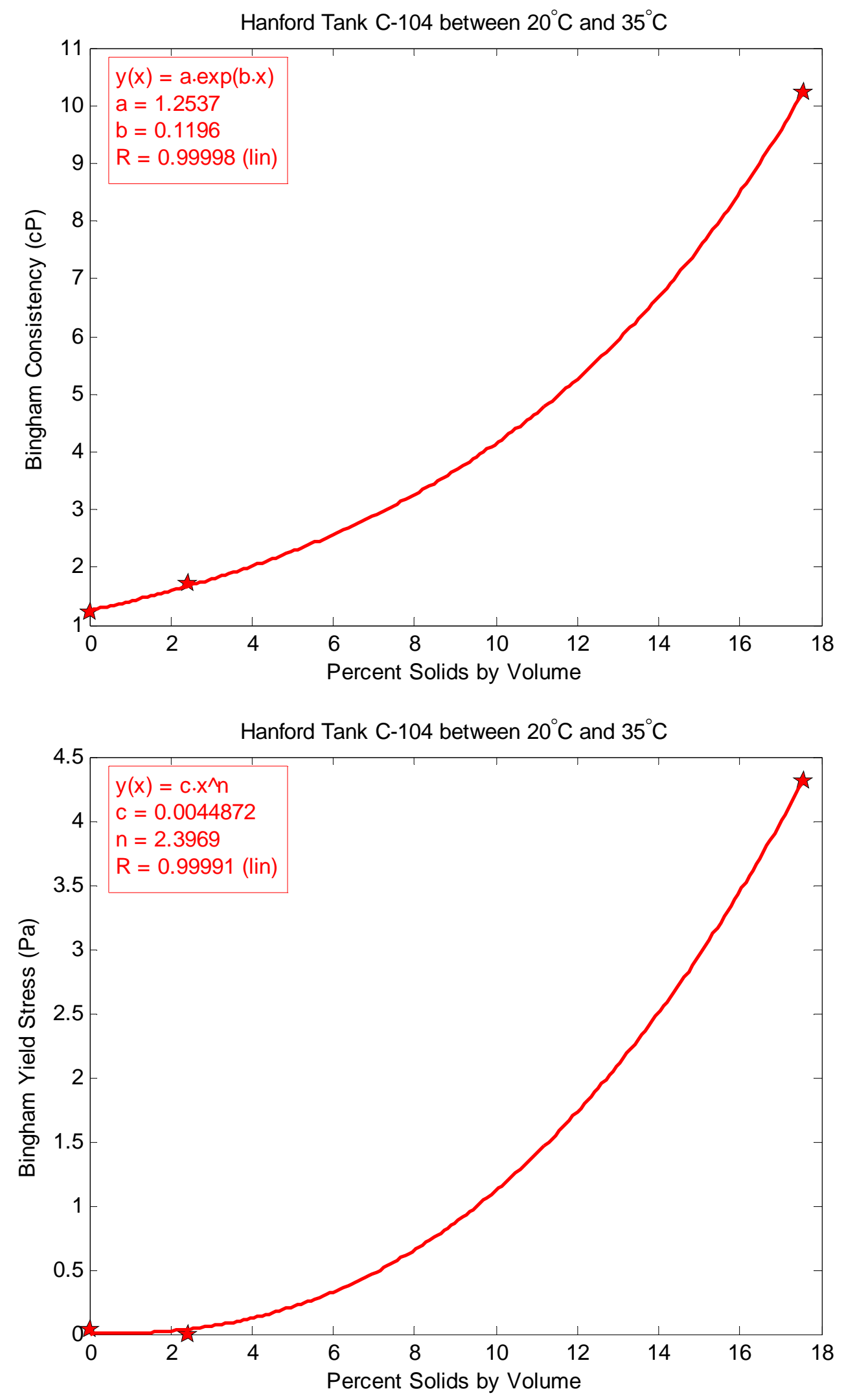

C. 1 

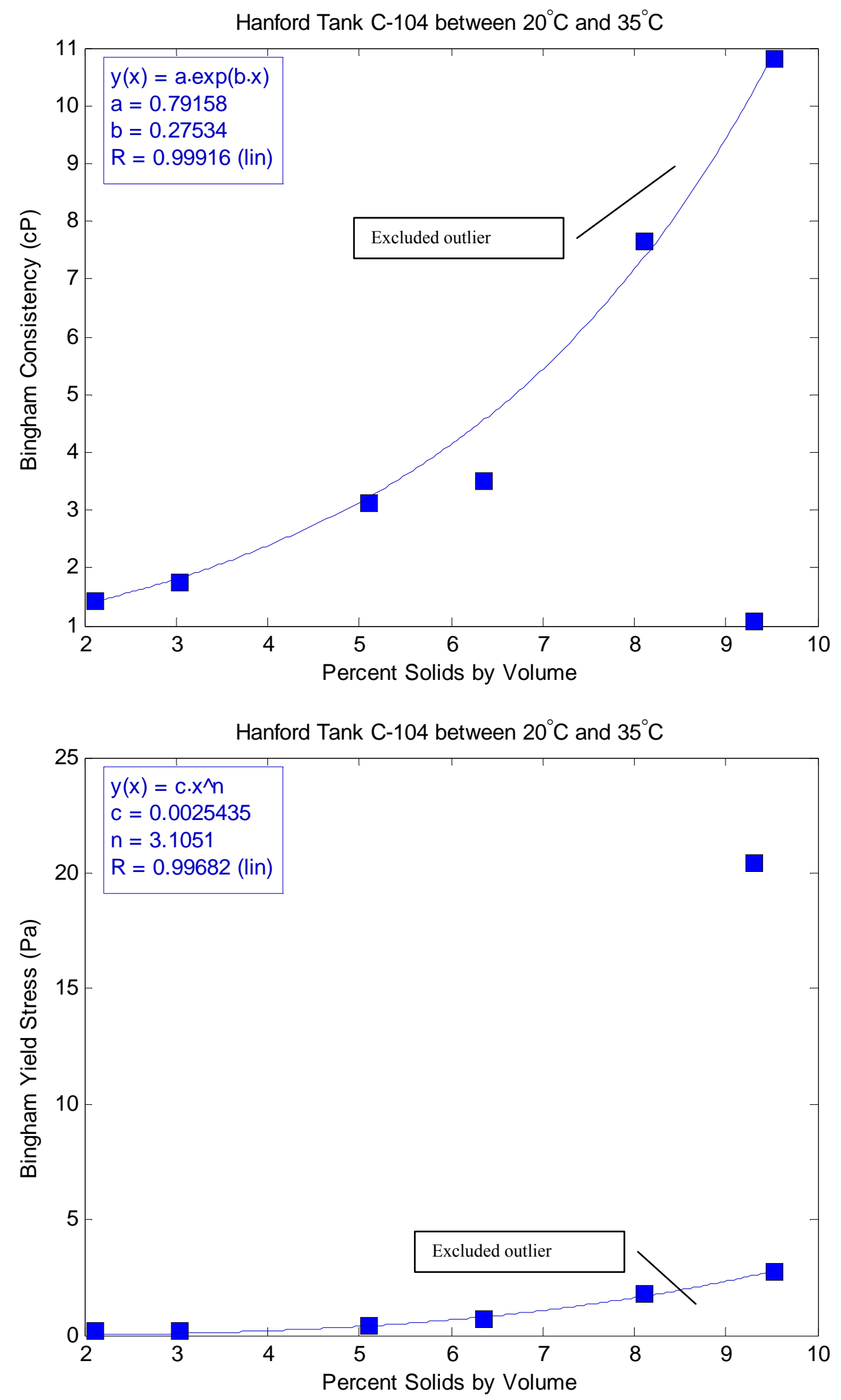

C. 2 

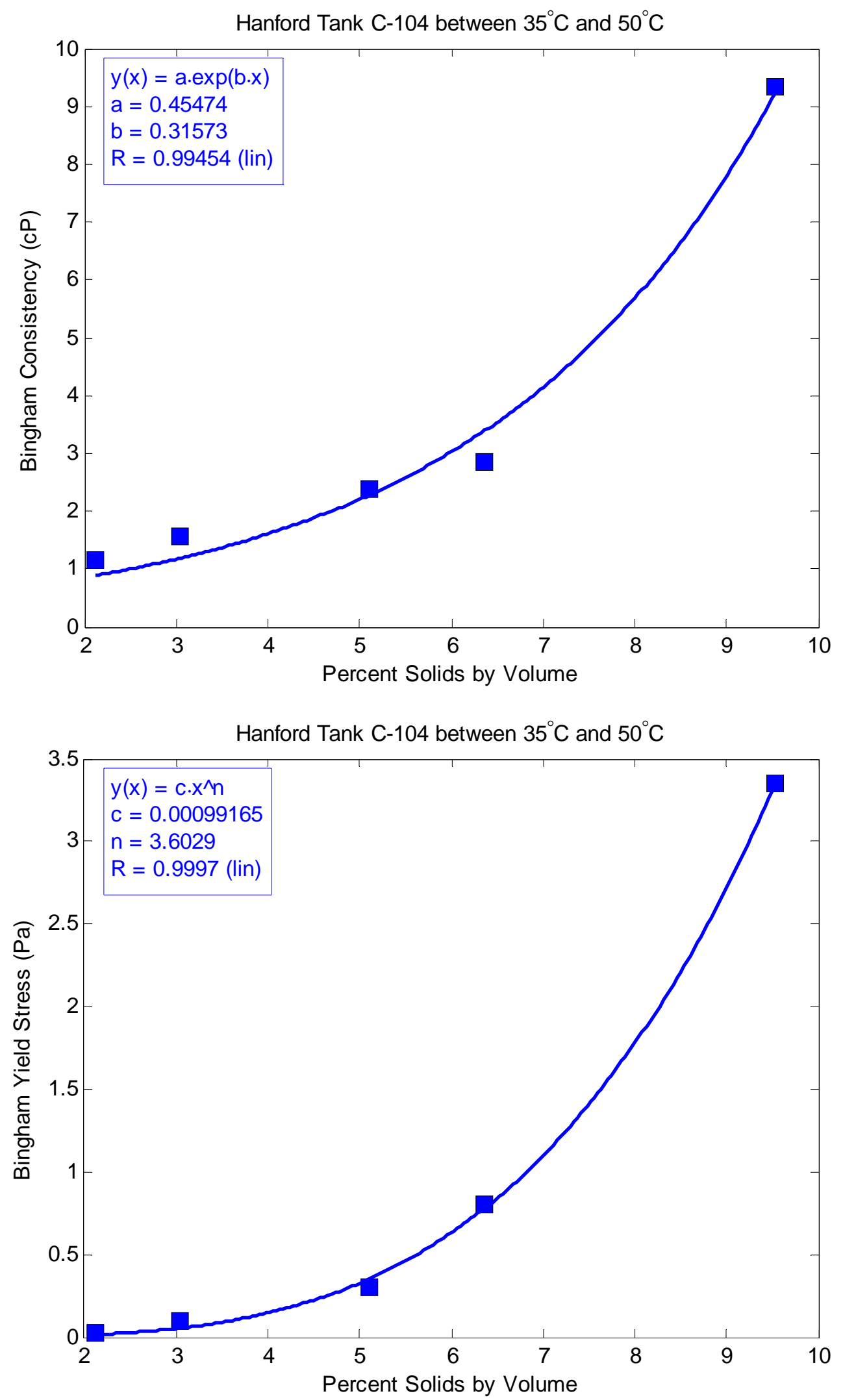

C. 3 

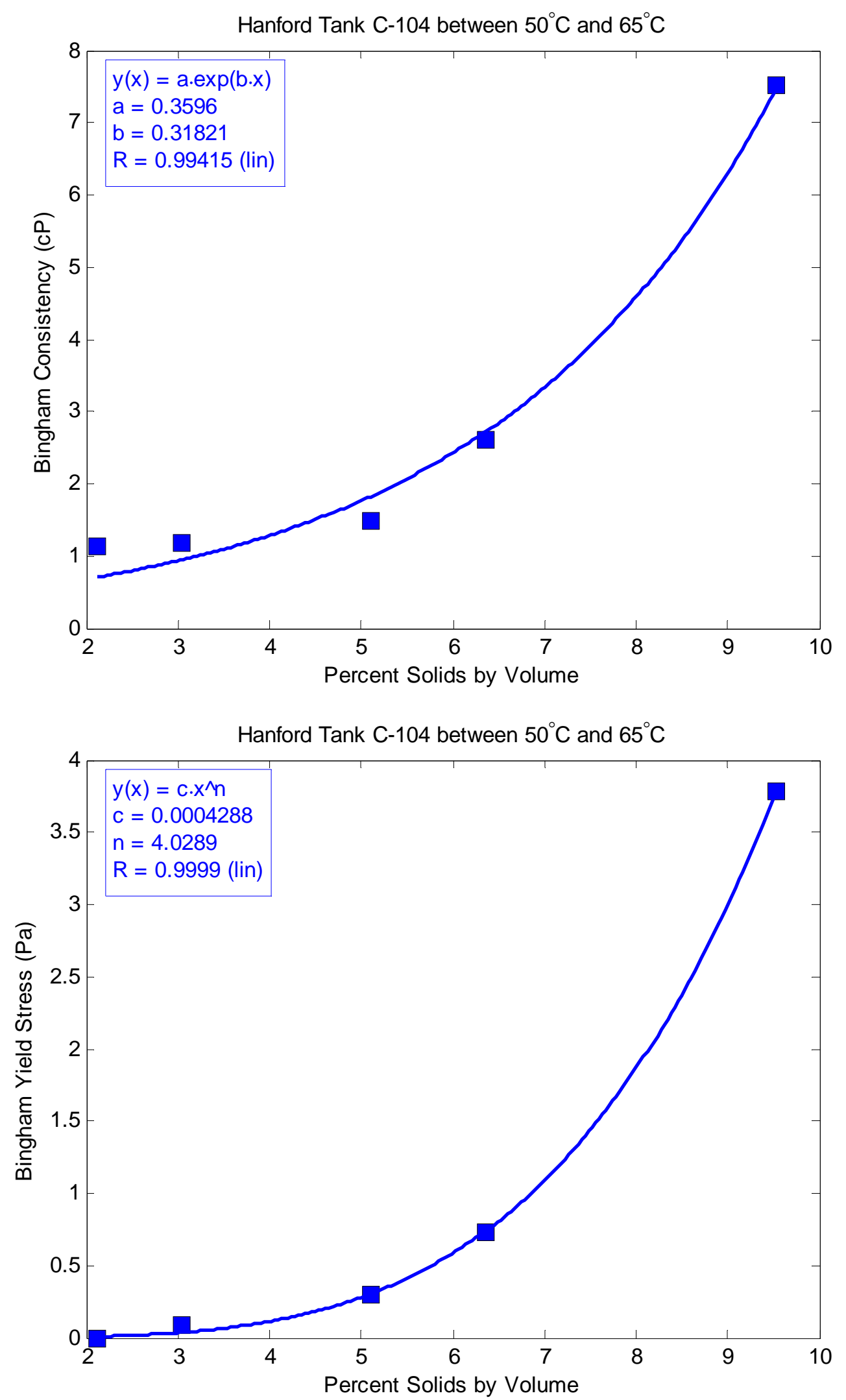

C. 4 

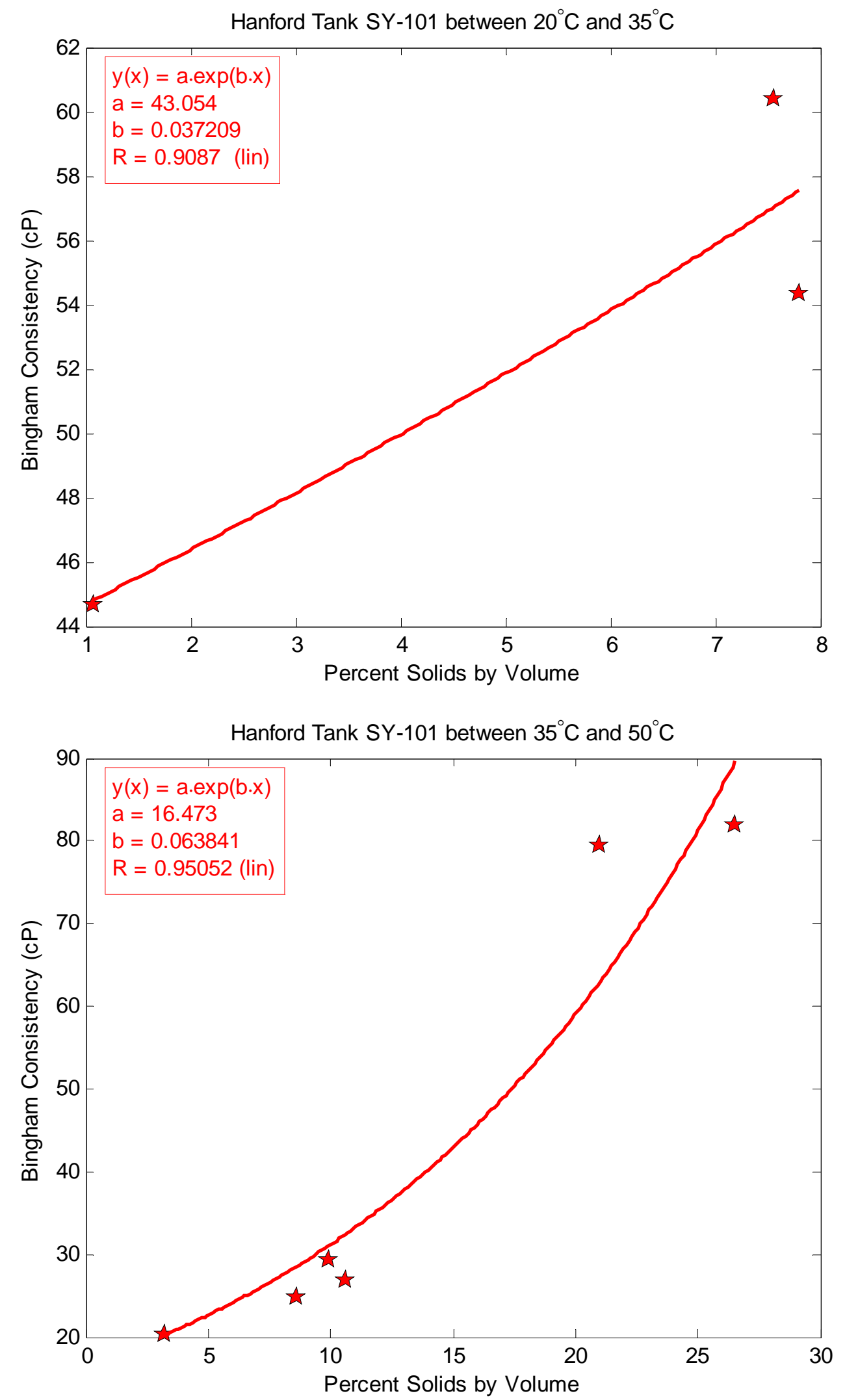
Hanford Tank SY-101 between $35^{\circ} \mathrm{C}$ and $50^{\circ} \mathrm{C}$
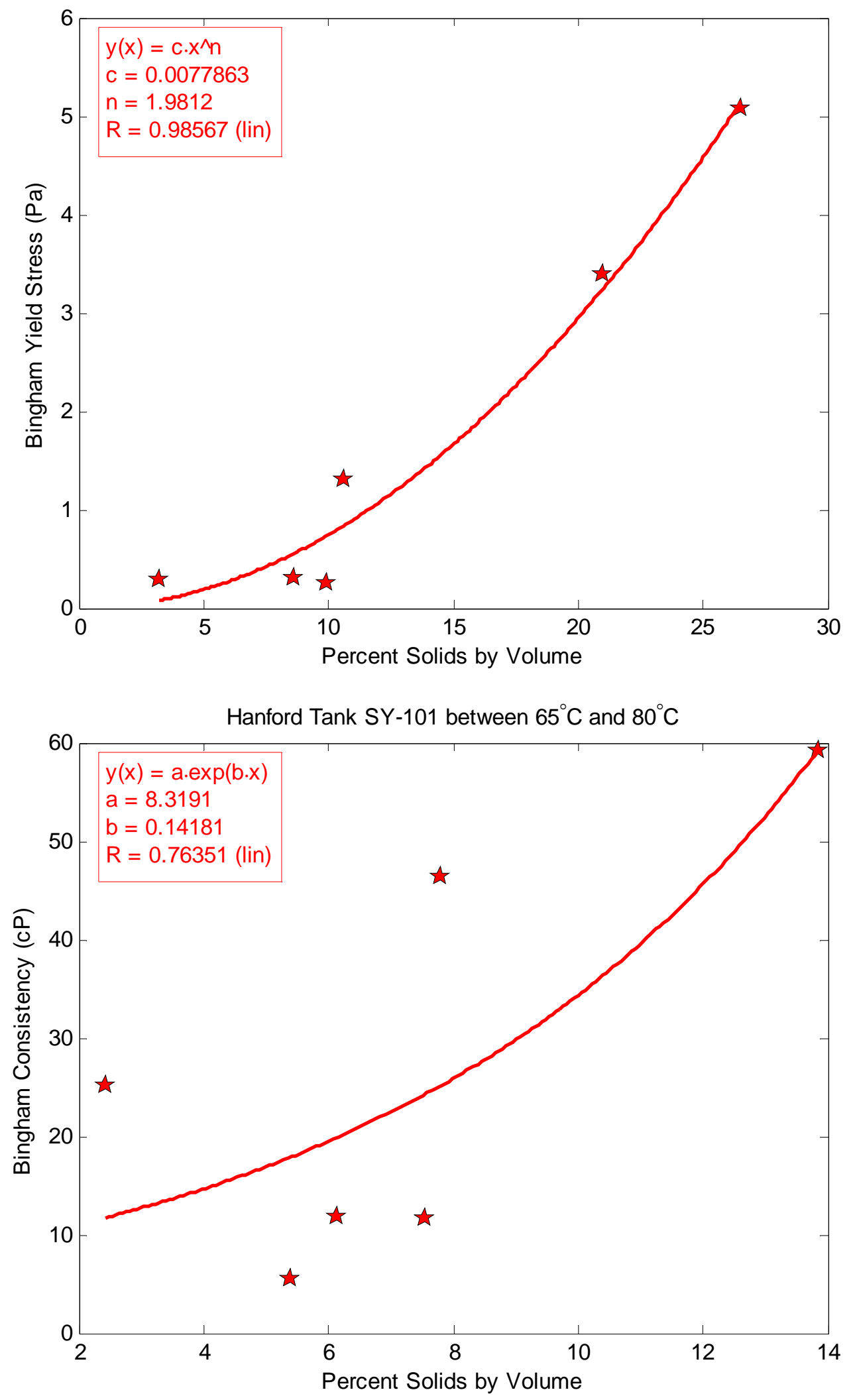

C.6 
Hanford Tank SY-101 between $65^{\circ} \mathrm{C}$ and $80^{\circ} \mathrm{C}$
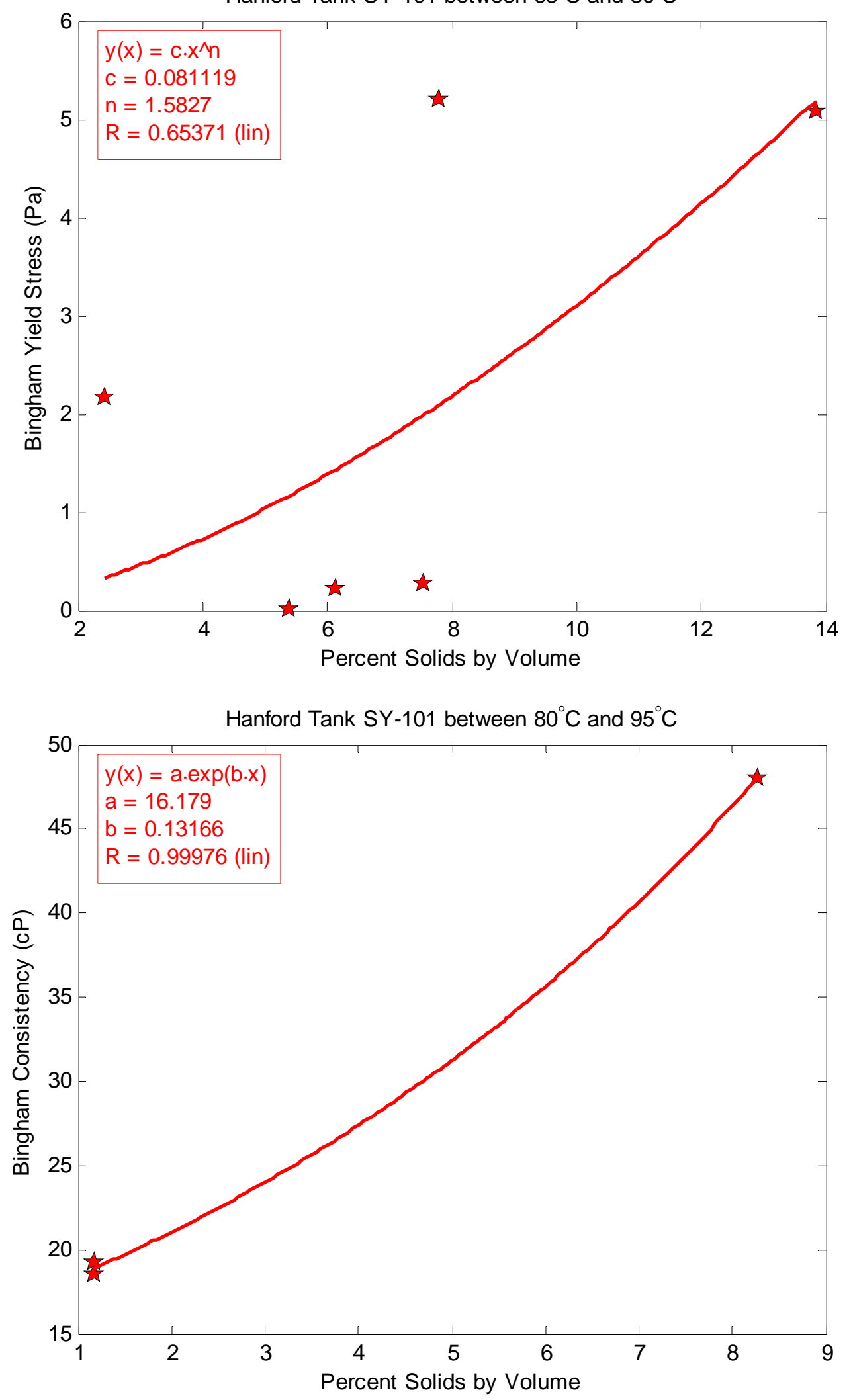

C. 7 

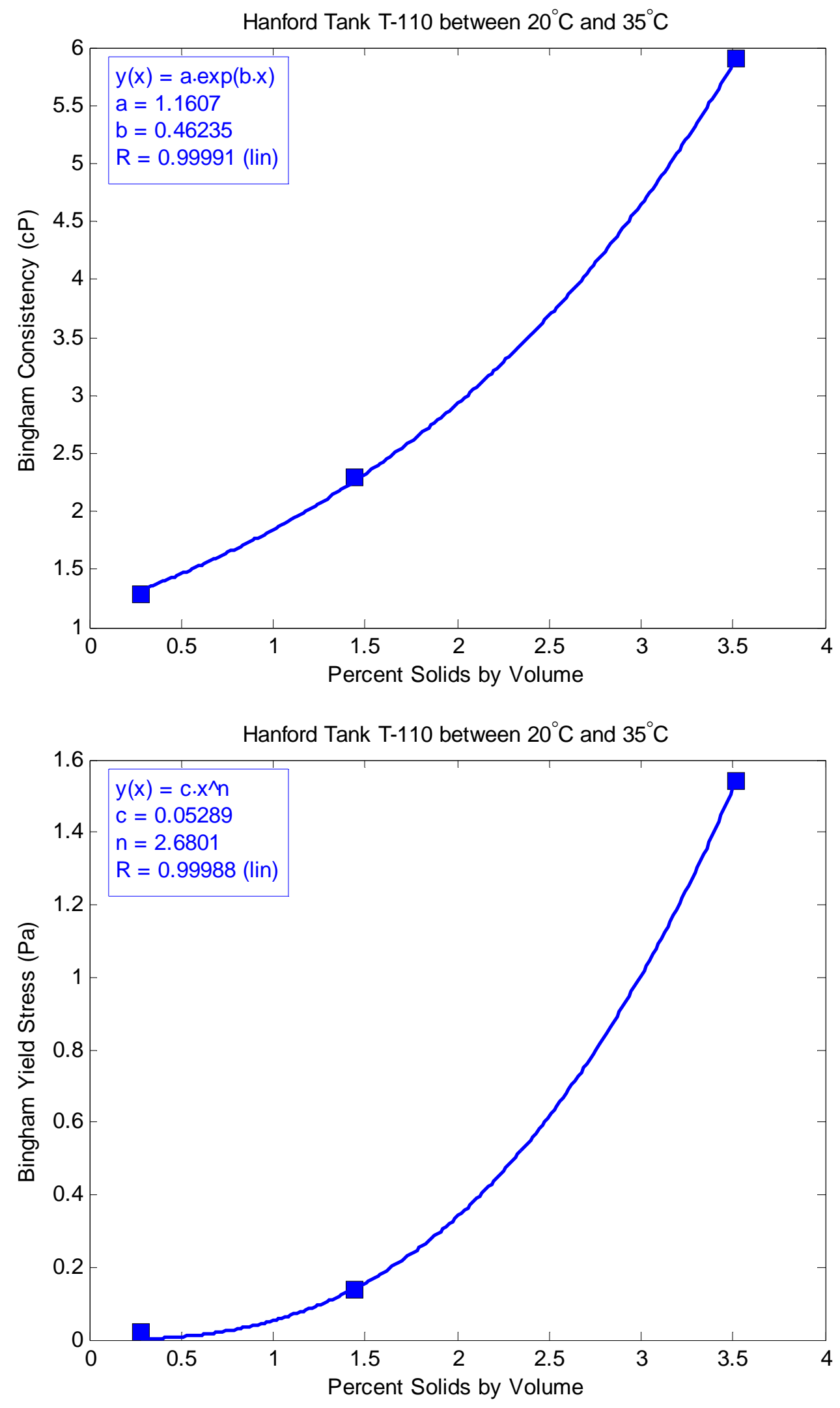

C. 8 

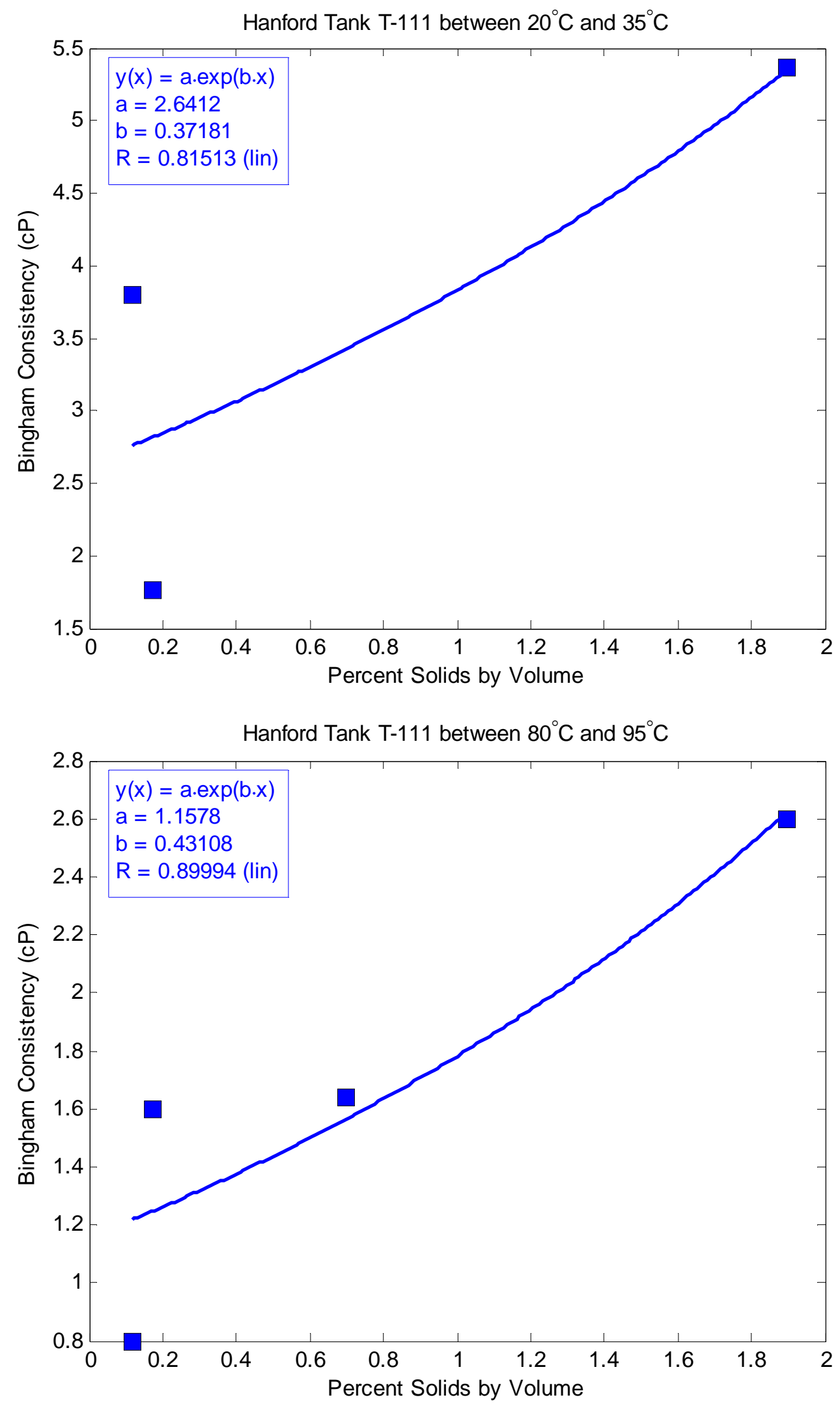

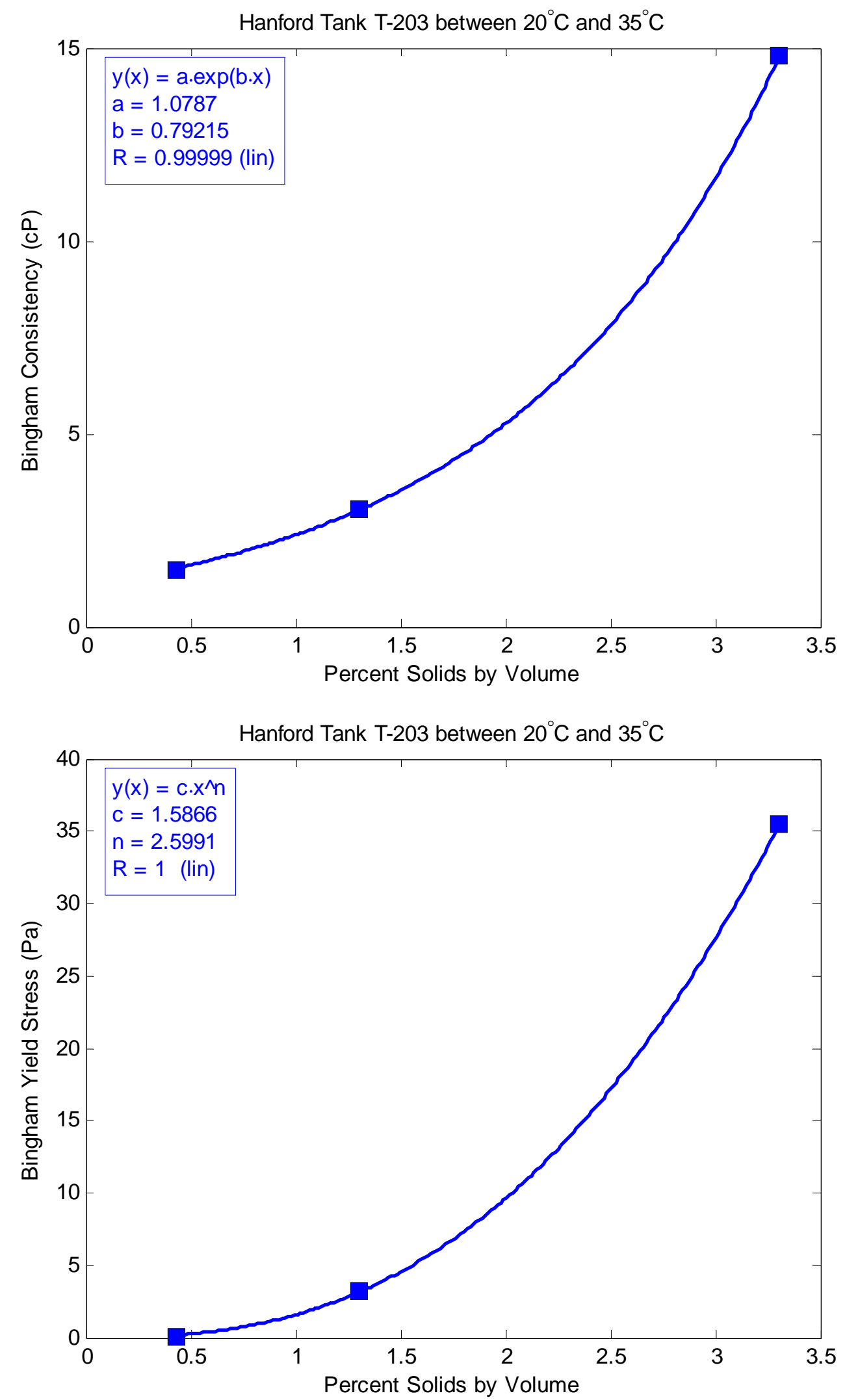

C. 10 
Appendix D

Sedimentation Layer Properties as Sludge Settles Under Different Starting Heights 


\section{Appendix D - Sedimentation Layer Properties as Sludge Settles Under Different Starting Heights}

Predicted sludge properties from Hanford Tank B-202 with water dilution at a starting slurry height of $0.1 \mathrm{~m}$ with $33 \%$ volume excess supernatant from fully settled configuration; rheological properties taken at a temperature range of $20^{\circ}-35^{\circ} \mathrm{C}$.

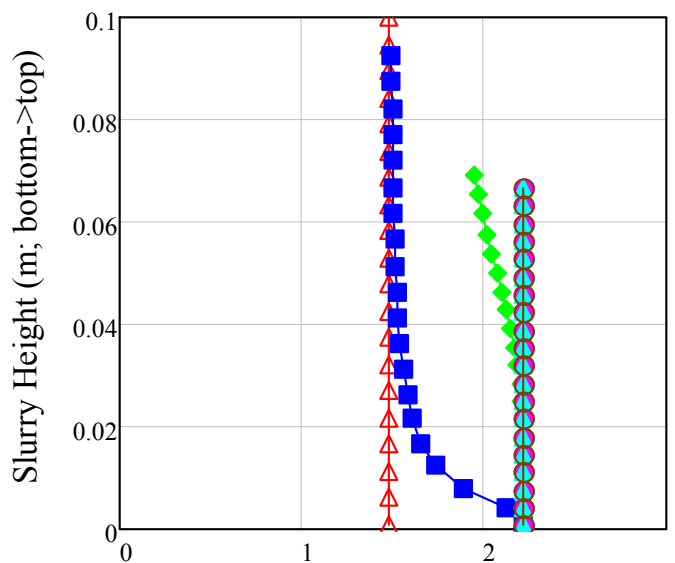

Solids Concentration (Volume Percent)

$\triangle \triangle 0 \mathrm{hr}$

- $1 \mathrm{hr}$

$\leftrightarrow 10 \mathrm{hr}$

- $100 \mathrm{hr}$

$\triangle 200 \mathrm{hr}$

$\ominus \odot 1000 \mathrm{hr}$

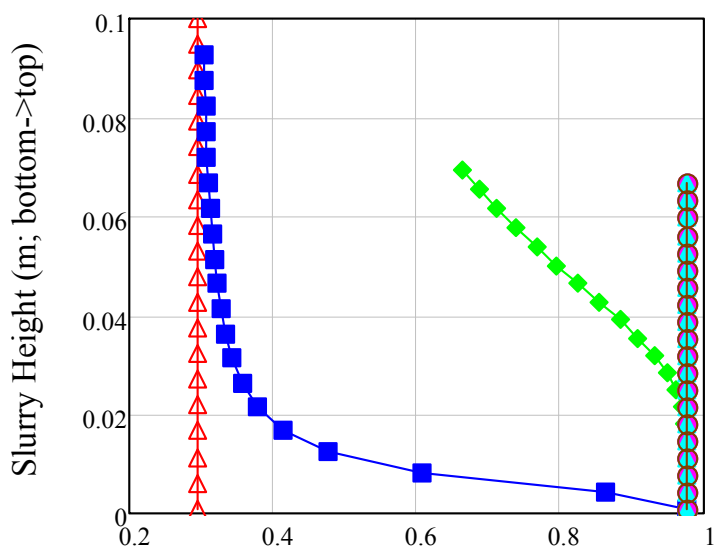

Bingham Yield Stress (Pa)

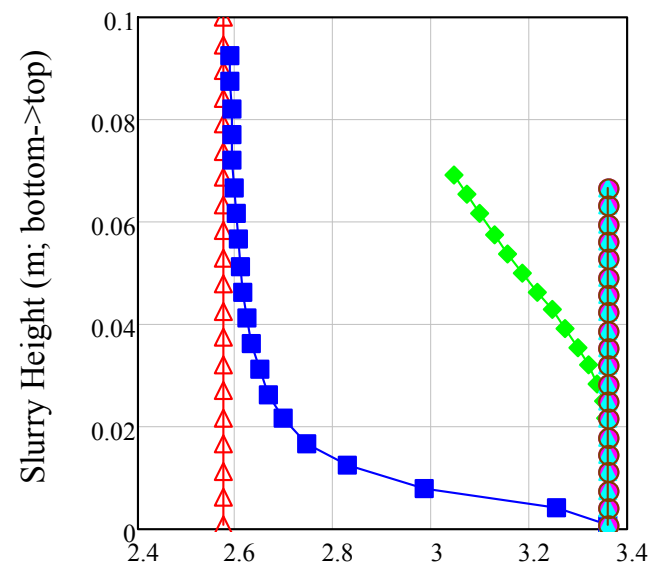

Bingham Consistency (cP)

$\triangle \triangle 0 \mathrm{hr}$

- $1 \mathrm{hr}$

$\leftrightarrow 10 \mathrm{hr}$

- $100 \mathrm{hr}$

$\triangle 200 \mathrm{hr}$

$\ominus \ominus 1000 \mathrm{hr}$

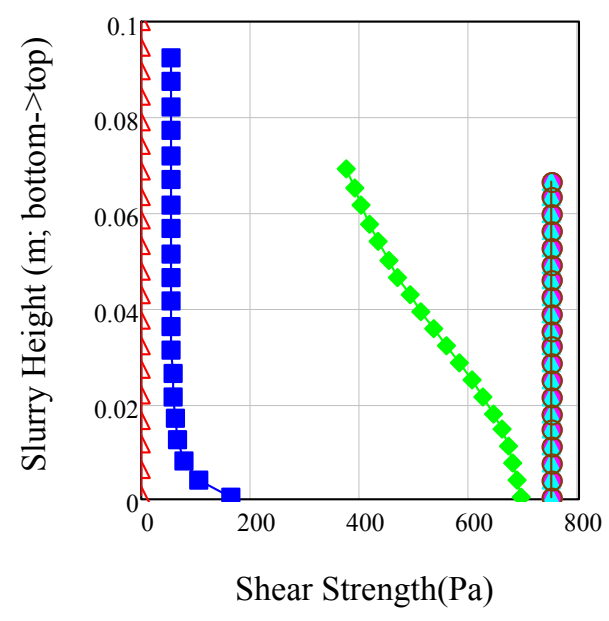

$\triangle \triangle 0 \mathrm{hr}$

- $1 \mathrm{hr}$

$\leftrightarrow 10 \mathrm{hr}$

- $100 \mathrm{hr}$

$\triangle 200 \mathrm{hr}$

$\ominus \ominus 1000 \mathrm{hr}$ 
Predicted sludge properties from Hanford Tank B-202 with water dilution at a starting slurry height of $1 \mathrm{~m}$ with $33 \%$ volume excess supernatant from fully settled configuration; rheological properties taken at a temperature range of $20^{\circ}-35^{\circ} \mathrm{C}$.

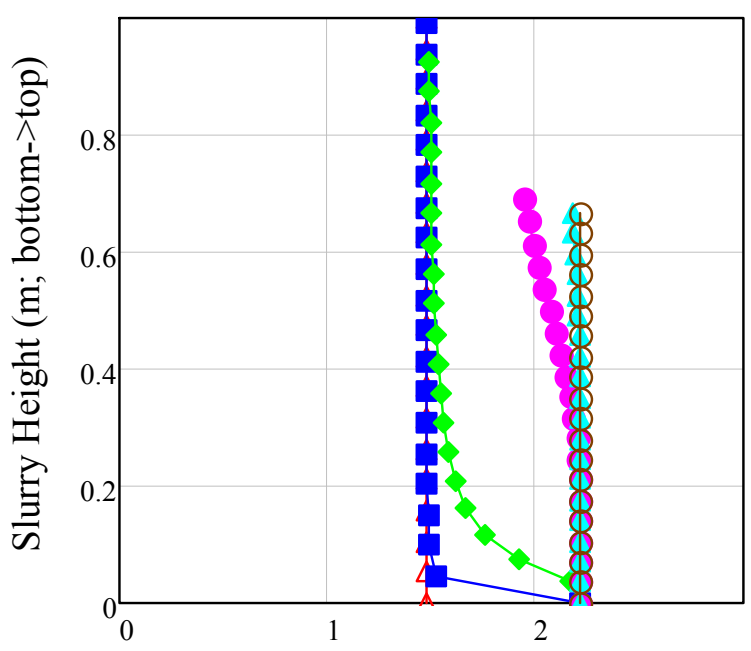

Solids Concentration (Volume Percent) $\triangle \triangle 0 \mathrm{hr}$

- $1 \mathrm{hr}$

$\leftrightarrow 10 \mathrm{hr}$

- $100 \mathrm{hr}$

$\triangle 200 \mathrm{hr}$

$\ominus \ominus 1000 \mathrm{hr}$

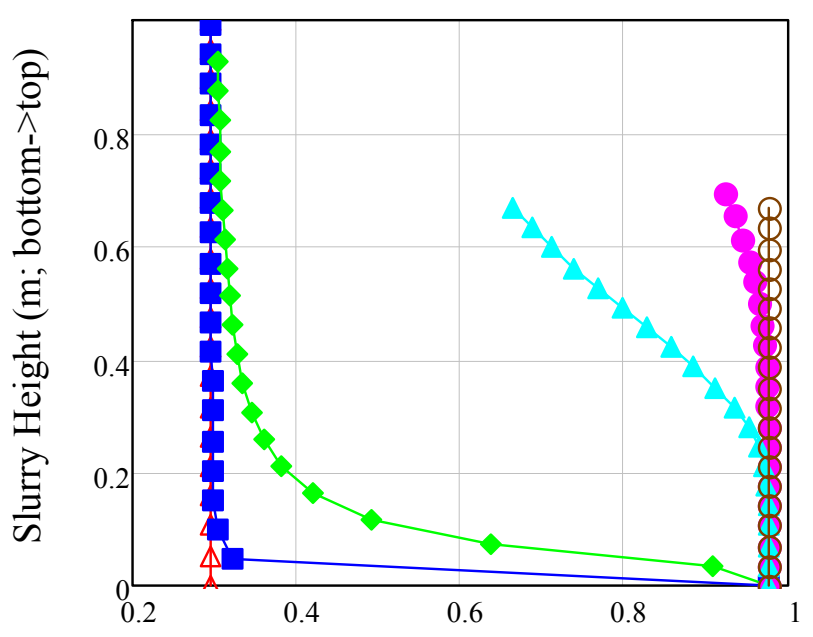

Bingham Yield Stress $(\mathrm{Pa})$

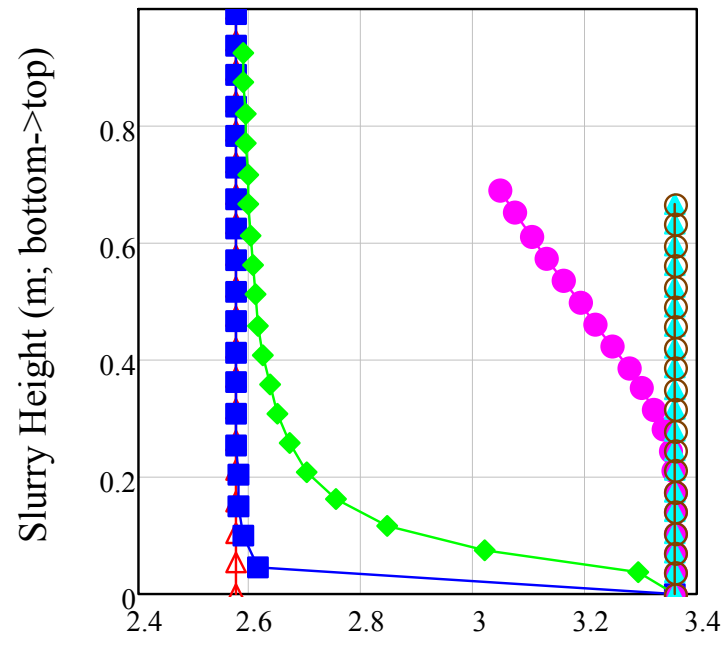

Bingham Consistency (cP)

$\triangle \triangle 0 \mathrm{hr}$

- $1 \mathrm{hr}$

$\leftrightarrow 10 \mathrm{hr}$

$\rightarrow 100 \mathrm{hr}$

$\triangle 200 \mathrm{hr}$

$\ominus \ominus 1000 \mathrm{hr}$

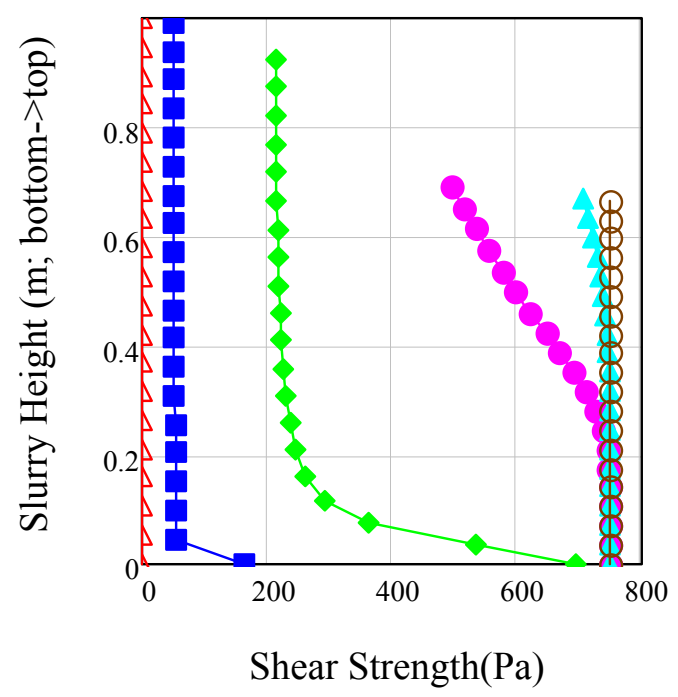

$\triangle \triangle 0 \mathrm{hr}$

- $1 \mathrm{hr}$

$\leadsto 10 \mathrm{hr}$

- $100 \mathrm{hr}$

$\triangle 200 \mathrm{hr}$

$\ominus \ominus 1000 \mathrm{hr}$ 
Predicted sludge properties from Hanford Tank B-202 with water dilution at a starting slurry height of $10 \mathrm{~m}$ with $33 \%$ volume excess supernatant from fully settled configuration; rheological properties taken at a temperature range of $20^{\circ}-35^{\circ} \mathrm{C}$.

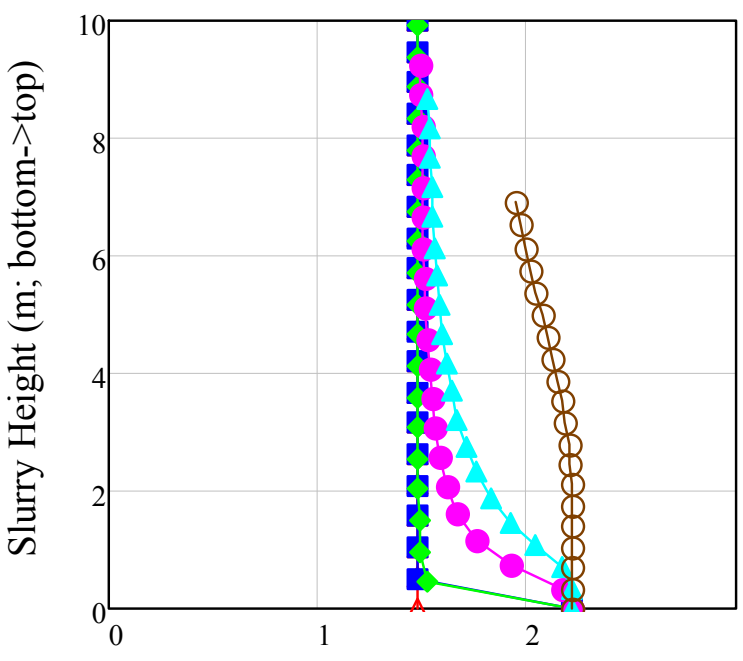

Solids Concentration (Volume Percent) $\triangle \triangle 0 \mathrm{hr}$

- $1 \mathrm{hr}$

$\leftrightarrow 10 \mathrm{hr}$

$100 \mathrm{hr}$

$\pm 200 \mathrm{hr}$

$\ominus \ominus 1000 \mathrm{hr}$

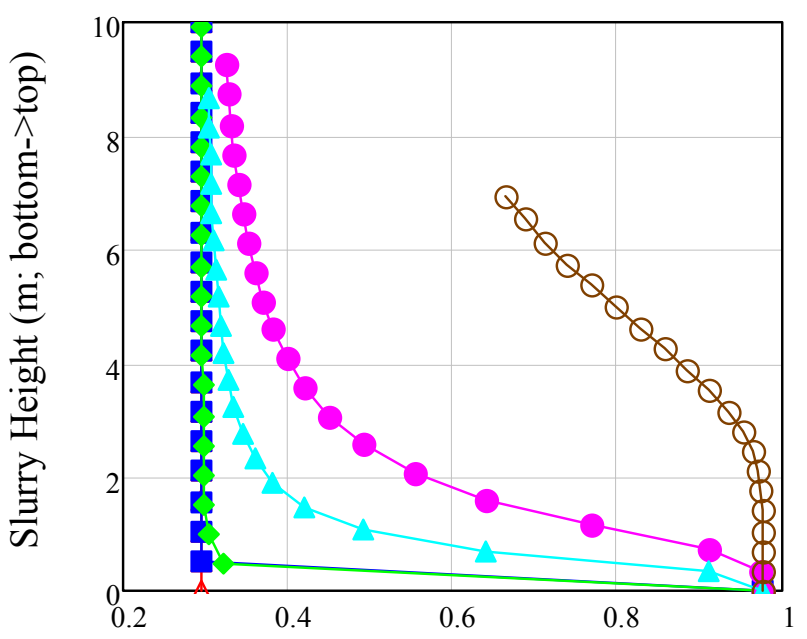

Bingham Yield Stress (Pa)

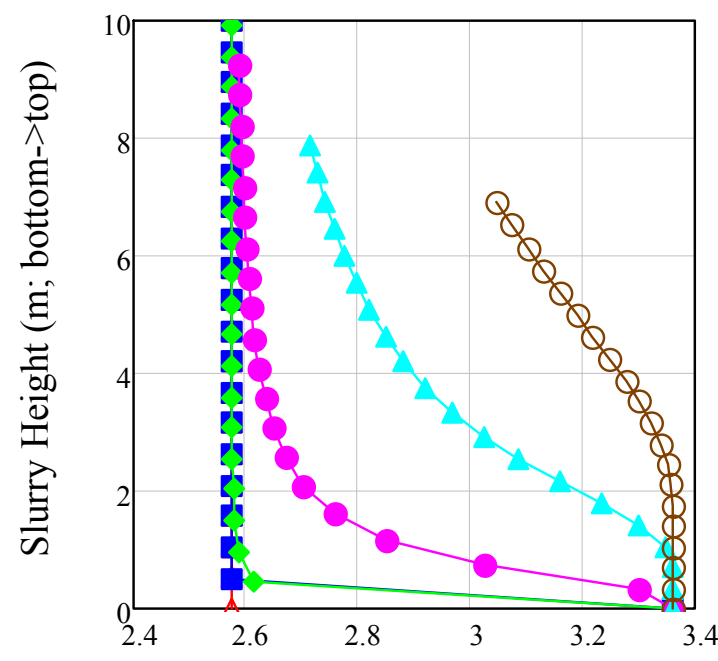

Bingham Consistency (cP)

$\triangle \triangle 0 \mathrm{hr}$

- $1 \mathrm{hr}$

$\leftrightarrow 10 \mathrm{hr}$

- $100 \mathrm{hr}$

$\pm 200 \mathrm{hr}$

$\ominus \ominus 1000 \mathrm{hr}$

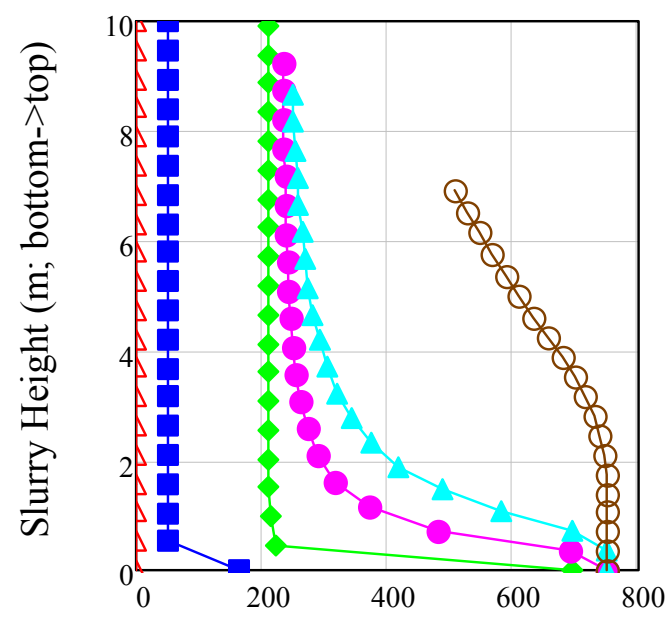

Shear Strength $(\mathrm{Pa})$

$\triangle \triangle 0 \mathrm{hr}$

- $1 \mathrm{hr}$

$\leadsto 10 \mathrm{hr}$

- $100 \mathrm{hr}$

$\pm 200 \mathrm{hr}$

$\ominus \ominus 1000 \mathrm{hr}$ 
Predicted sludge properties from Hanford Tank B-203 with water dilution at a starting slurry height of $0.1 \mathrm{~m}$ with $33 \%$ volume excess supernatant from fully settled configuration; rheological properties taken at a temperature range of $20^{\circ}-35^{\circ} \mathrm{C}$.

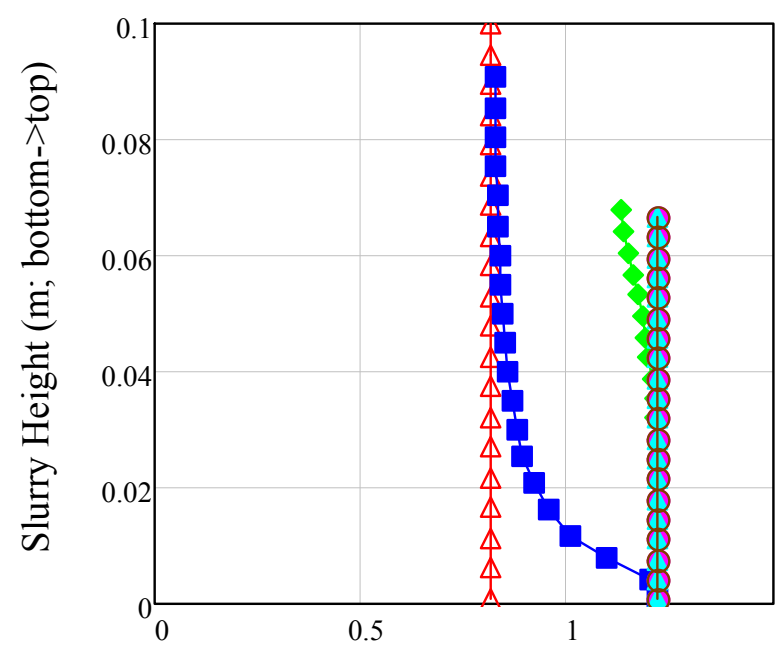

Solids Concentration (Volume Percent) $\triangle \triangle 0 \mathrm{hr}$

- $1 \mathrm{hr}$

$\leftrightarrow 10 \mathrm{hr}$

$0-100 \mathrm{hr}$

$\triangle 200 \mathrm{hr}$

$\ominus \ominus 1000 \mathrm{hr}$

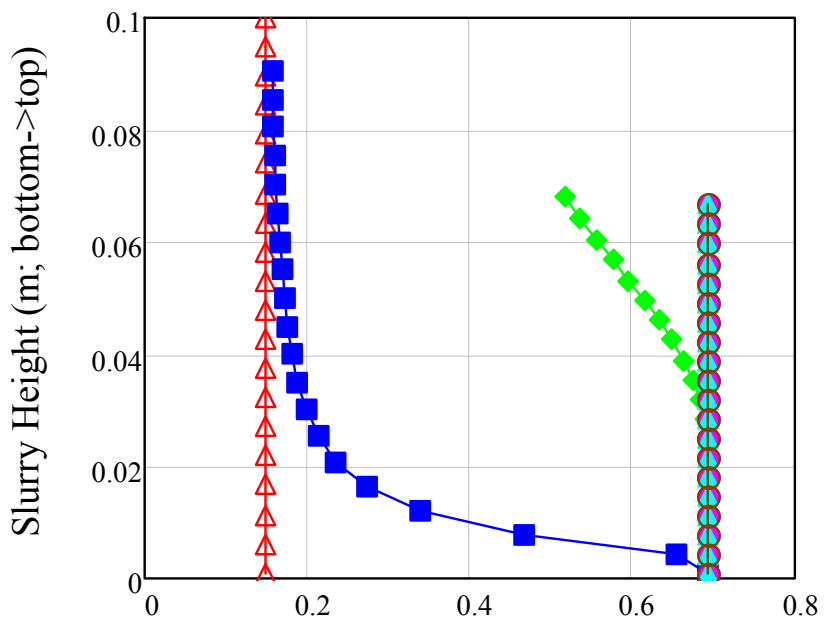

Bingham Yield Stress (Pa)

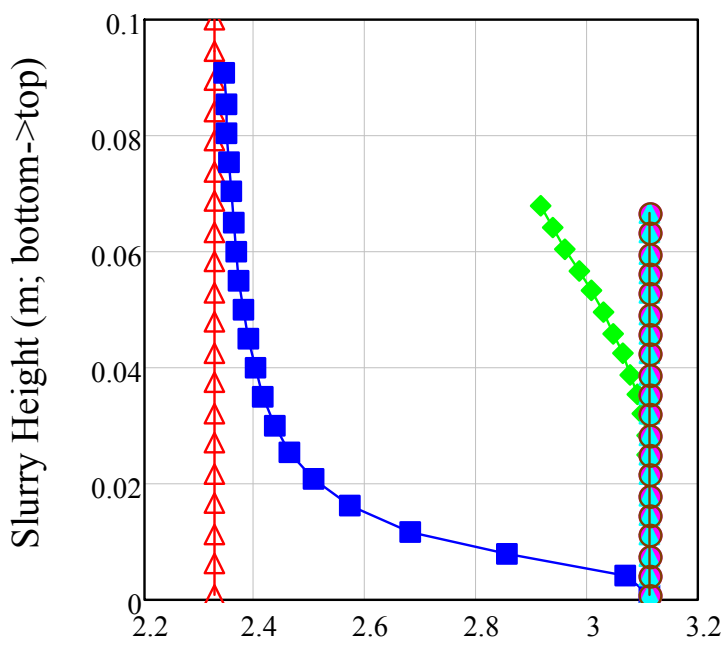

Bingham Consistency (cP)

$\triangle \triangle 0 \mathrm{hr}$

- $1 \mathrm{hr}$

$\leftrightarrow 10 \mathrm{hr}$

- $100 \mathrm{hr}$

$\triangle 200 \mathrm{hr}$

$\ominus \ominus 1000 \mathrm{hr}$

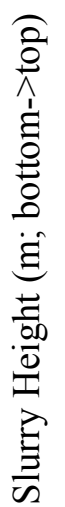

$\triangle \triangle 0 \mathrm{hr}$

Shear Strength $(\mathrm{Pa})$

$\triangle \triangle 0 \mathrm{hr}$

- $1 \mathrm{hr}$

$\leftrightarrow 10 \mathrm{hr}$

- $100 \mathrm{hr}$

$\triangle 200 \mathrm{hr}$

$\ominus \ominus 1000 \mathrm{hr}$ 
Predicted sludge properties from Hanford Tank B-203 with water dilution at a starting slurry height of $1 \mathrm{~m}$ with $33 \%$ volume excess supernatant from fully settled configuration; rheological properties taken at a temperature range of $20^{\circ}-35^{\circ} \mathrm{C}$.

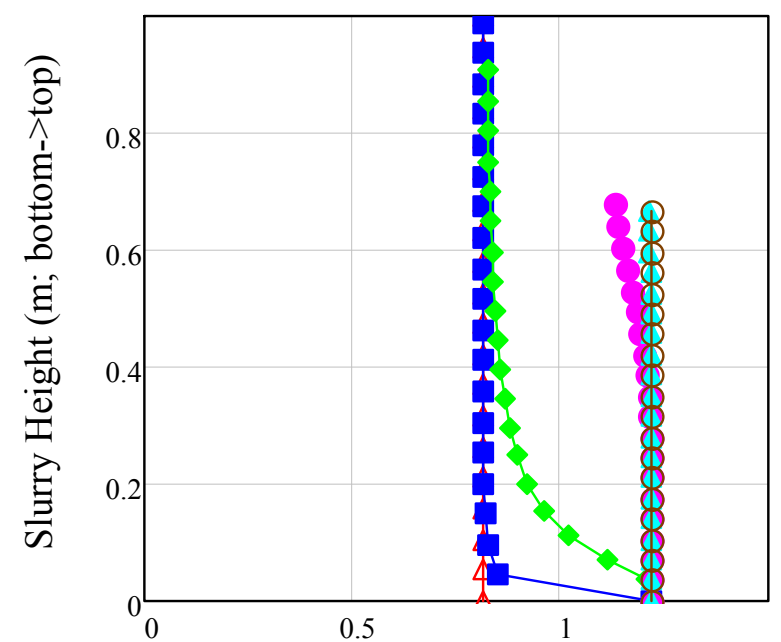

Solids Concentration (Volume Percent) $\triangle \triangle 0 \mathrm{hr}$

- $1 \mathrm{hr}$

$\leftrightarrow 10 \mathrm{hr}$

- $100 \mathrm{hr}$

$\triangle 200 \mathrm{hr}$

$\ominus \ominus 1000 \mathrm{hr}$

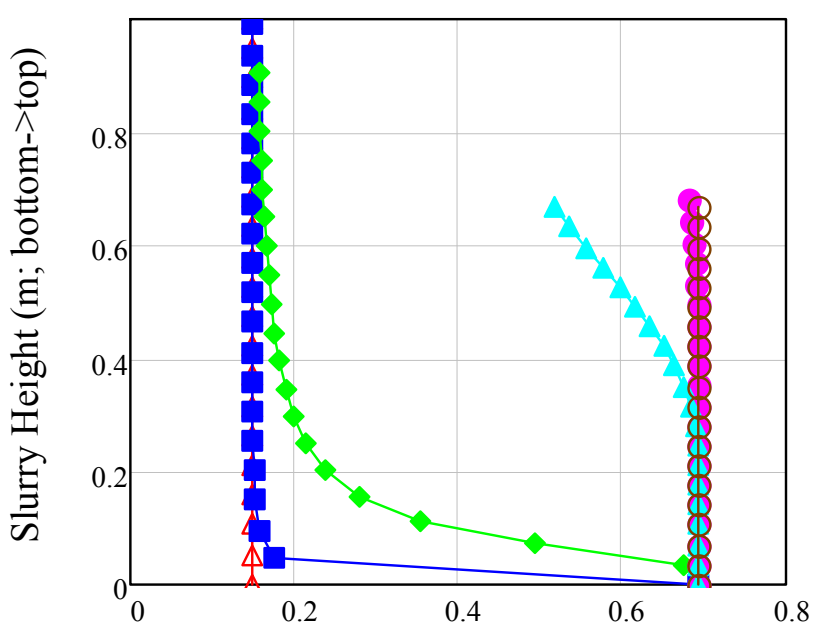

Bingham Yield Stress $(\mathrm{Pa})$

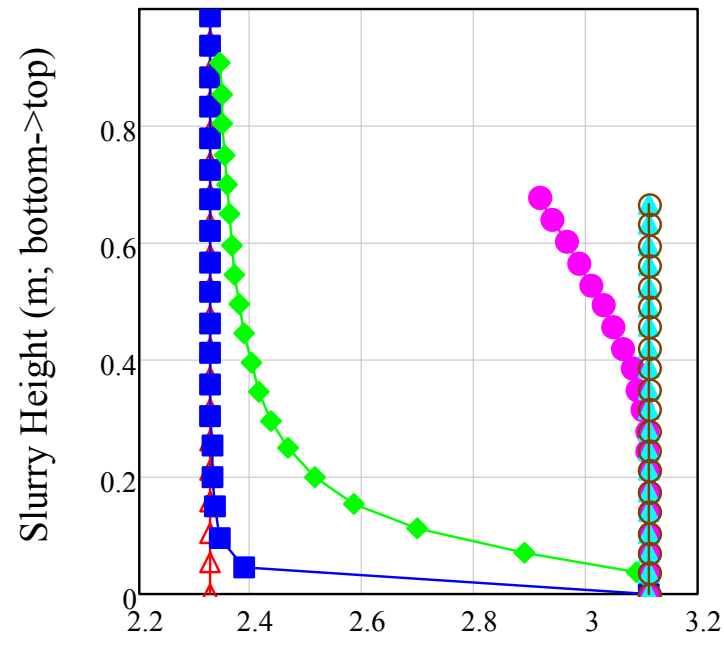

Bingham Consistency (cP)

$\triangle \triangle 0 \mathrm{hr}$

- $1 \mathrm{hr}$

$\leftrightarrow 10 \mathrm{hr}$

- $100 \mathrm{hr}$

$\pm 200 \mathrm{hr}$

$\ominus \ominus 1000 \mathrm{hr}$

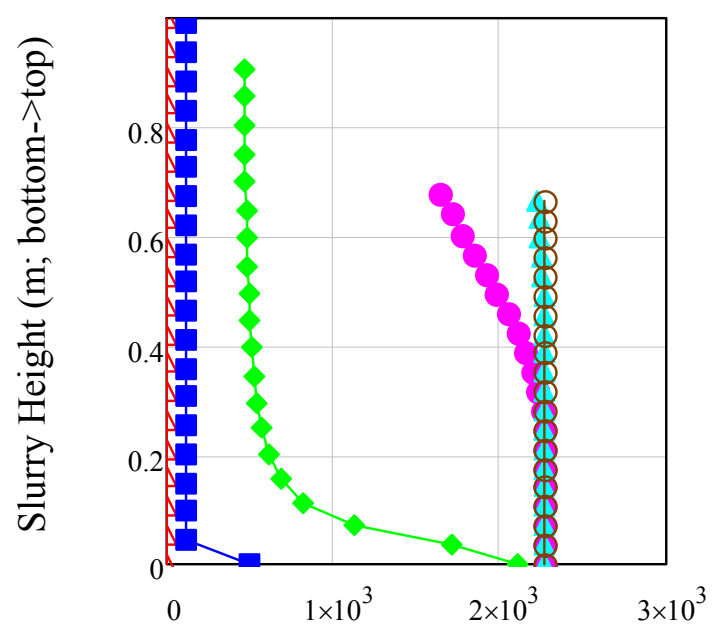

Shear Strength $(\mathrm{Pa})$

$\triangle \triangle 0 \mathrm{hr}$

- $1 \mathrm{hr}$

$\leftrightarrow 10 \mathrm{hr}$

- $100 \mathrm{hr}$

$\triangle 200 \mathrm{hr}$

$\ominus \ominus 1000 \mathrm{hr}$ 
Predicted sludge properties from Hanford Tank B-203 with water dilution at a starting slurry height of $10 \mathrm{~m}$ with $33 \%$ volume excess supernatant from fully settled configuration; rheological properties taken at a temperature range of $20^{\circ}-35^{\circ} \mathrm{C}$.

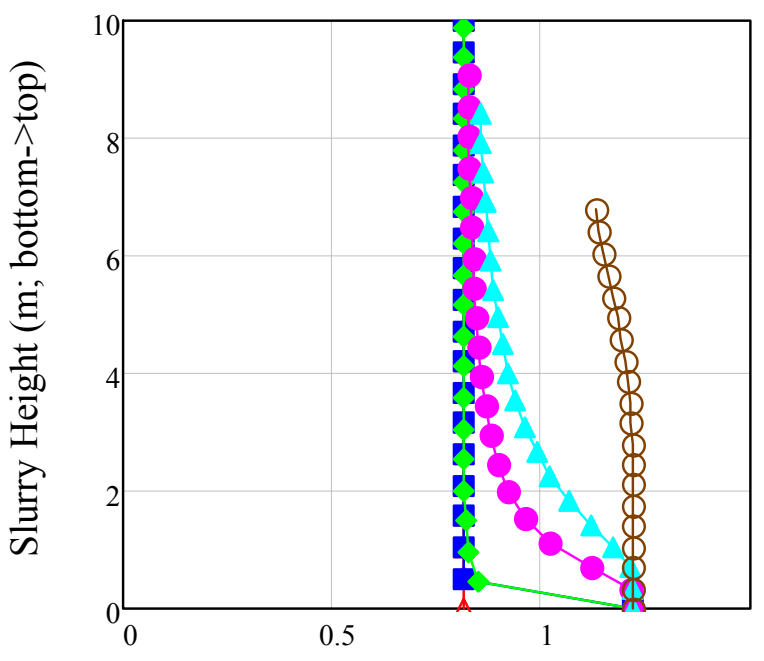

Solids Concentration (Volume Percent)

$\triangle \triangle 0 \mathrm{hr}$

- $1 \mathrm{hr}$

$\leftrightarrow 10 \mathrm{hr}$

$0-100 \mathrm{hr}$

$\triangle 200 \mathrm{hr}$

$\ominus \ominus 1000 \mathrm{hr}$

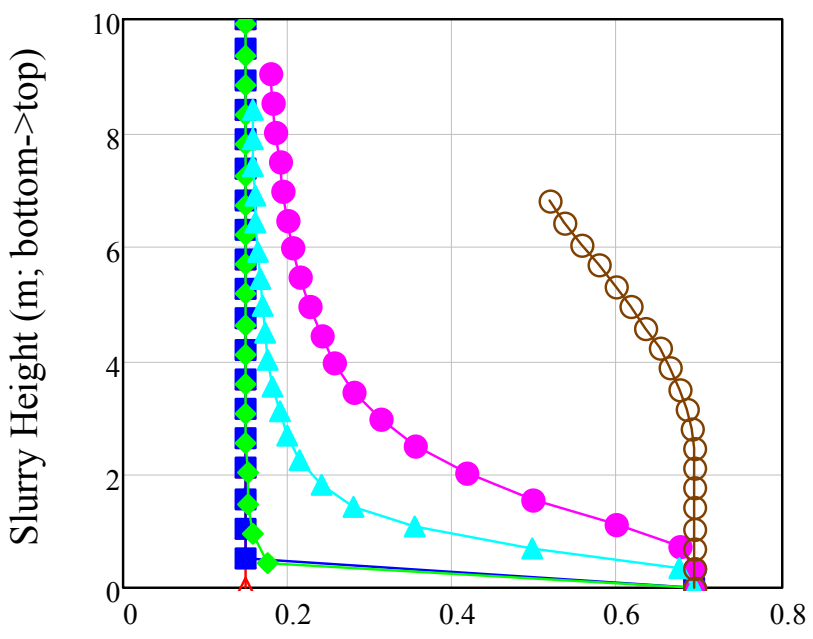

Bingham Yield Stress $(\mathrm{Pa})$

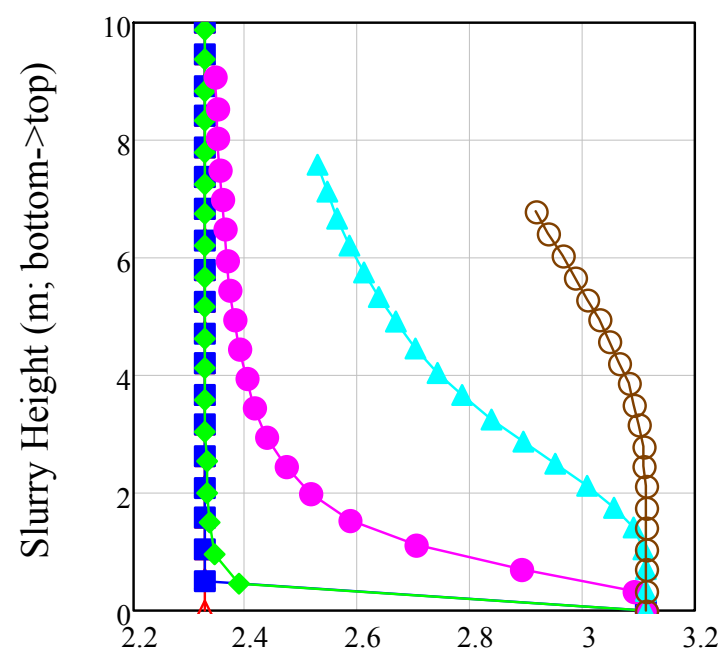

Bingham Consistency (cP)

$\triangle \triangle 0 \mathrm{hr}$

- $1 \mathrm{hr}$

$\leftrightarrow 10 \mathrm{hr}$

- $100 \mathrm{hr}$

$\triangle 200 \mathrm{hr}$

$\ominus \ominus 1000 \mathrm{hr}$

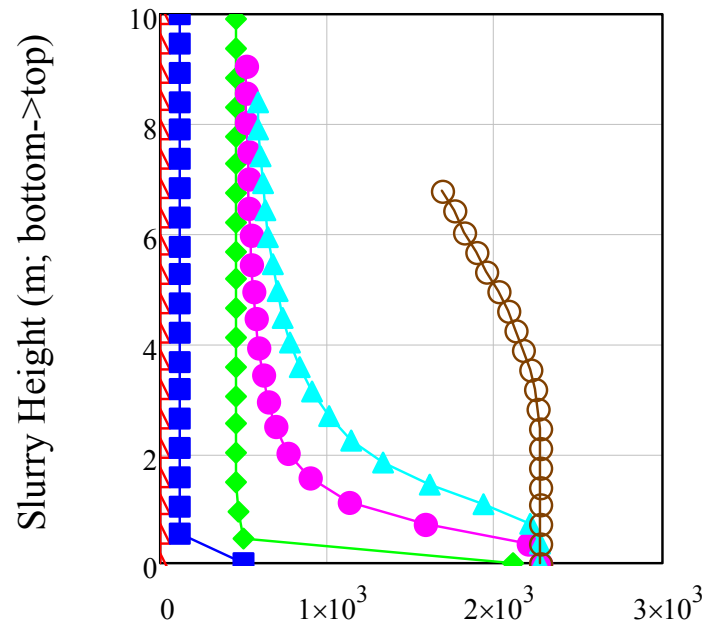

Shear Strength(Pa)

$\triangle \triangle 0 \mathrm{hr}$

- $1 \mathrm{hr}$

$\leftrightarrow 10 \mathrm{hr}$

- $100 \mathrm{hr}$

$\triangle 200 \mathrm{hr}$

$\ominus \ominus 1000 \mathrm{hr}$ 
Predicted sludge properties from Hanford Tank T-203 with water dilution at a starting slurry height of $0.1 \mathrm{~m}$ with $33 \%$ volume excess supernatant from fully settled configuration; rheological properties taken at a temperature range of $20^{\circ}-5^{\circ} \mathrm{C}$.

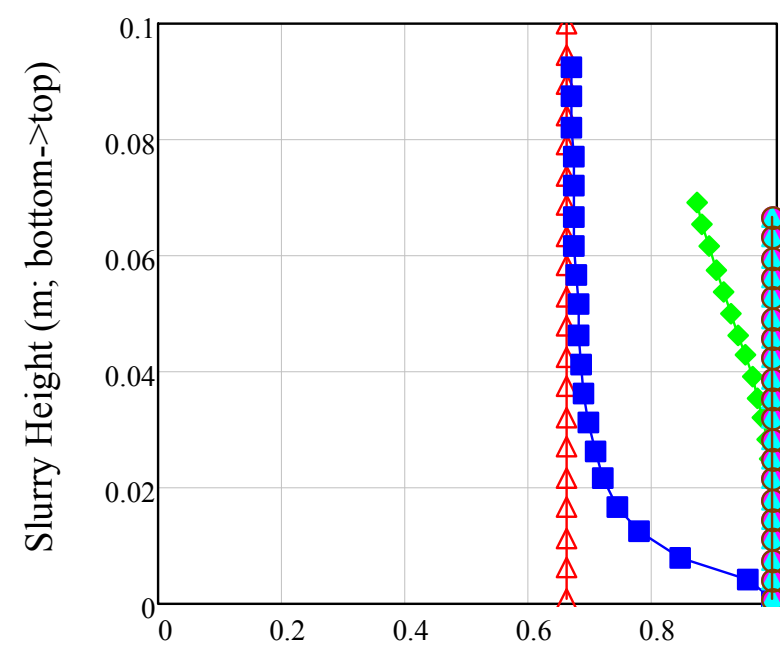

Solids Concentration (Volume Percent) $\triangle \triangle 0 \mathrm{hr}$

- $1 \mathrm{hr}$

$\leftrightarrow 10 \mathrm{hr}$

$0-100 \mathrm{hr}$

$\triangle 200 \mathrm{hr}$

$\ominus \ominus 1000 \mathrm{hr}$

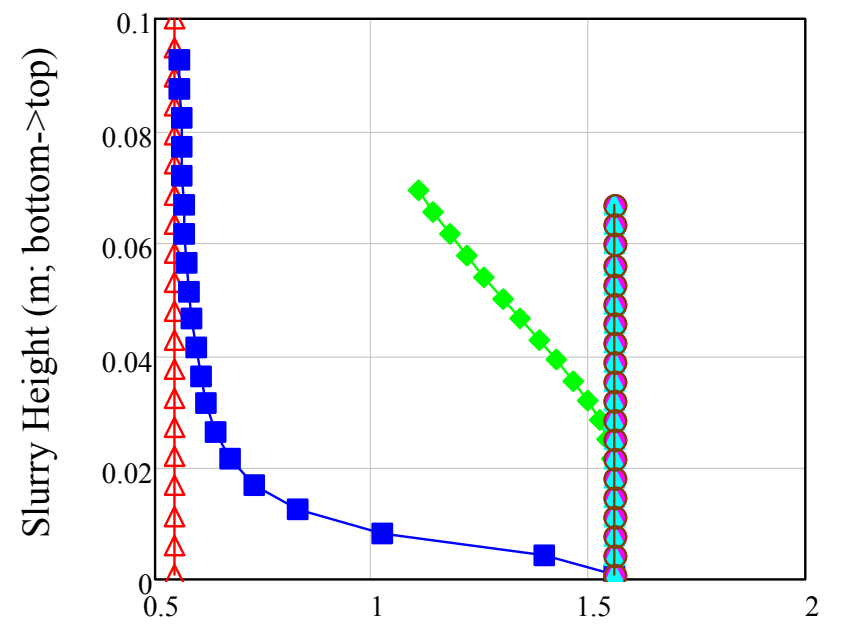

Bingham Yield Stress $(\mathrm{Pa})$

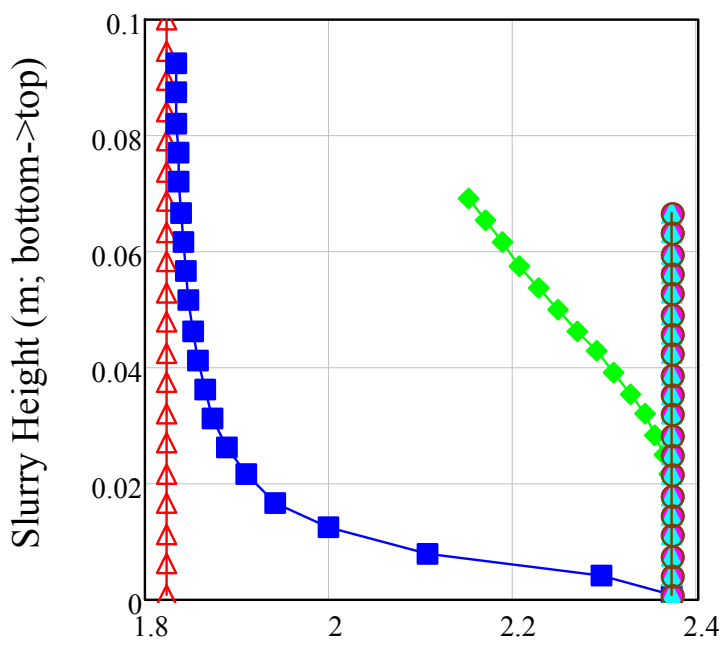

Bingham Consistency $(\mathrm{cP})$

$\triangle \triangle 0 \mathrm{hr}$

- $1 \mathrm{hr}$

$\leadsto 10 \mathrm{hr}$

- $100 \mathrm{hr}$

$\triangle 200 \mathrm{hr}$

$\ominus \ominus 1000 \mathrm{hr}$

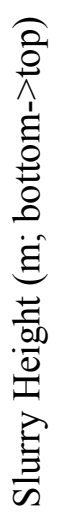

Shear Strength $(\mathrm{Pa})$

$\triangle \triangle 0 \mathrm{hr}$

- $1 \mathrm{hr}$

$\leftrightarrow 10 \mathrm{hr}$

- $100 \mathrm{hr}$

$\triangle 200 \mathrm{hr}$

$\ominus \ominus 1000 \mathrm{hr}$ 
Predicted sludge properties from Hanford Tank T-203 with water dilution at a starting slurry height of $1 \mathrm{~m}$ with $33 \%$ volume excess supernatant from fully settled configuration; rheological properties taken at a temperature range of $20^{\circ}-35^{\circ} \mathrm{C}$.

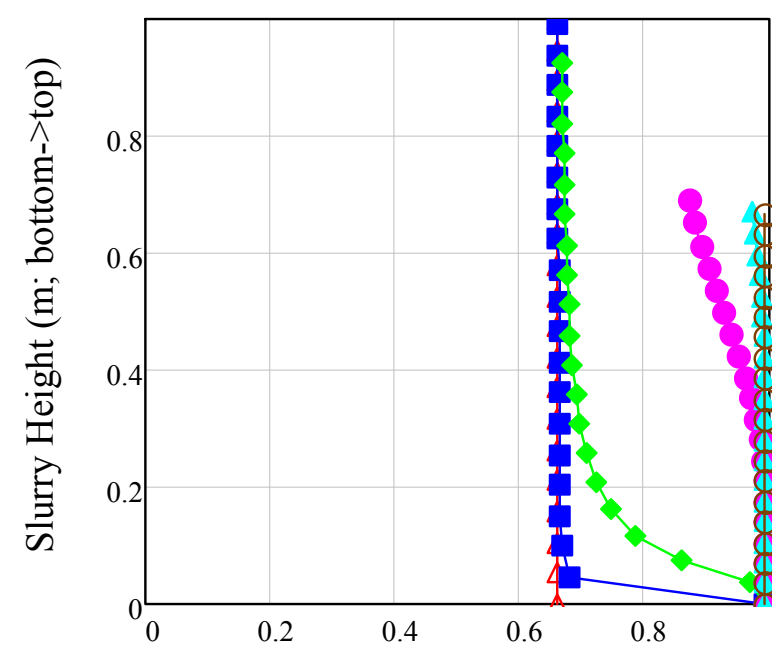

Solids Concentration (Volume Percent) $\triangle \triangle 0 \mathrm{hr}$

- $1 \mathrm{hr}$

$\leftrightarrow 10 \mathrm{hr}$

- $100 \mathrm{hr}$

$\triangle 200 \mathrm{hr}$

$\ominus \ominus 1000 \mathrm{hr}$

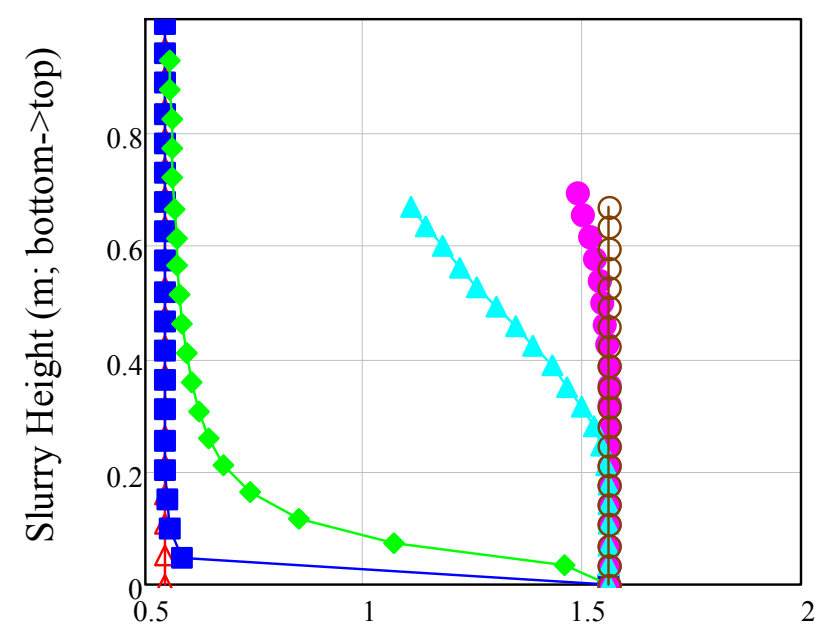

Bingham Yield Stress $(\mathrm{Pa})$

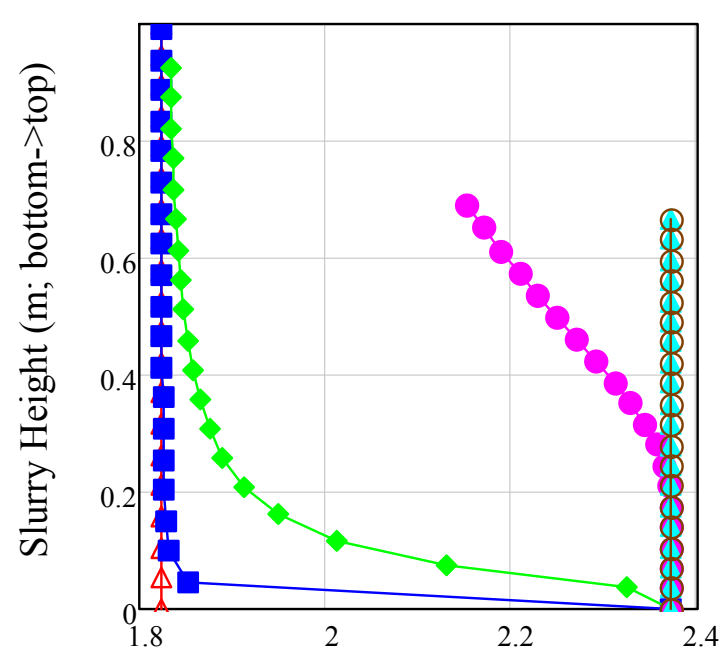

Bingham Consistency (cP)

$\triangle \triangle 0 \mathrm{hr}$

- $1 \mathrm{hr}$

$\leftrightarrow 10 \mathrm{hr}$

$100 \mathrm{hr}$

$\pm 200 \mathrm{hr}$

$\ominus \ominus 1000 \mathrm{hr}$

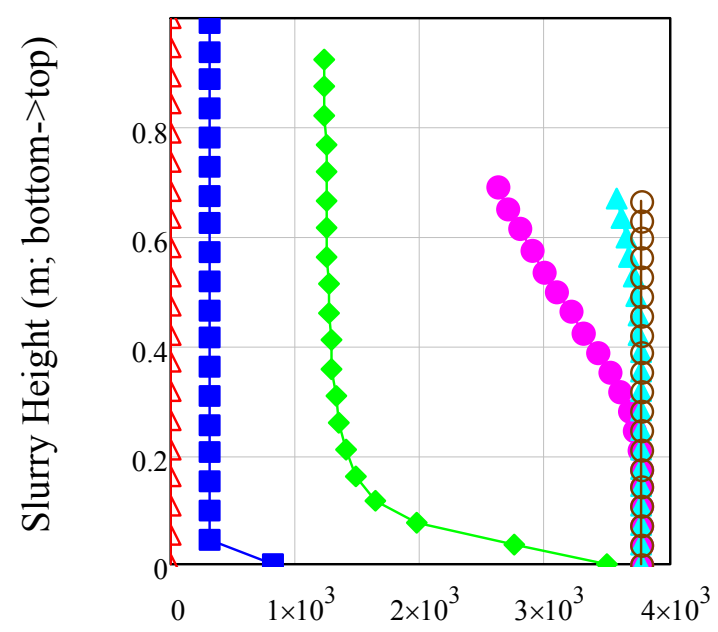

Shear Strength $(\mathrm{Pa})$

$\triangle \triangle 0 \mathrm{hr}$

- $1 \mathrm{hr}$

$\leftrightarrow 10 \mathrm{hr}$

- $100 \mathrm{hr}$

$\triangle 200 \mathrm{hr}$

$\ominus \ominus 1000 \mathrm{hr}$ 
Predicted sludge properties from Hanford Tank T-203 with water dilution at a starting slurry height of $10 \mathrm{~m}$ with $33 \%$ volume excess supernatant from fully settled configuration; rheological properties taken at a temperature range of $20^{\circ}-35^{\circ} \mathrm{C}$.

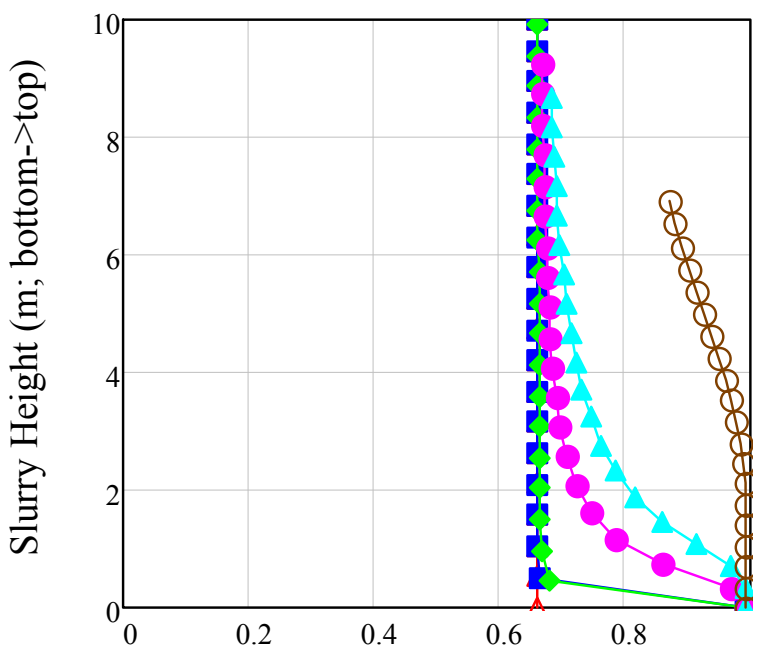

Solids Concentration (Volume Percent)

$\triangle \triangle 0 \mathrm{hr}$

- $1 \mathrm{hr}$

$\leftrightarrow 10 \mathrm{hr}$

- $100 \mathrm{hr}$

$\triangle 200 \mathrm{hr}$

$\ominus \ominus 1000 \mathrm{hr}$

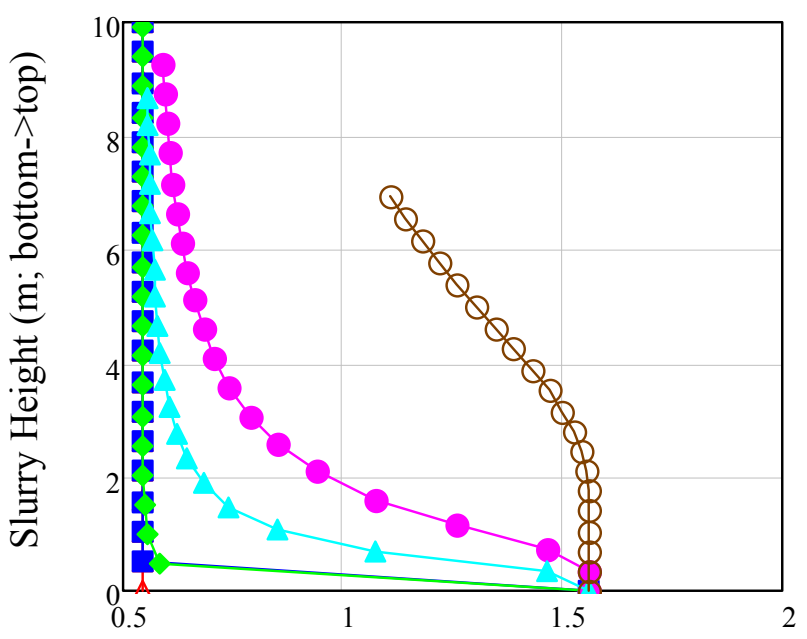

Bingham Yield Stress $(\mathrm{Pa})$

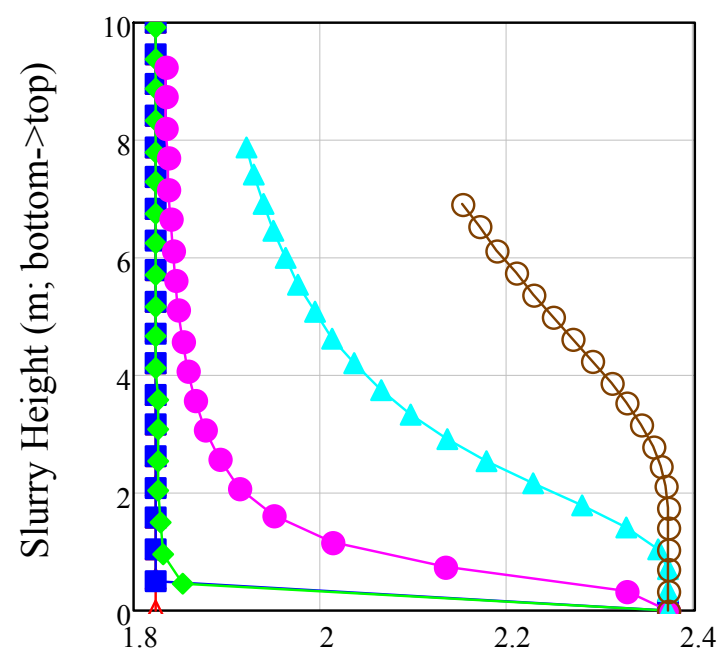

Bingham Consistency (cP)

$\triangle \triangle 0 \mathrm{hr}$

- $1 \mathrm{hr}$

$\leftrightarrow 10 \mathrm{hr}$

- $100 \mathrm{hr}$

$\triangle 200 \mathrm{hr}$

$\ominus \ominus 1000 \mathrm{hr}$

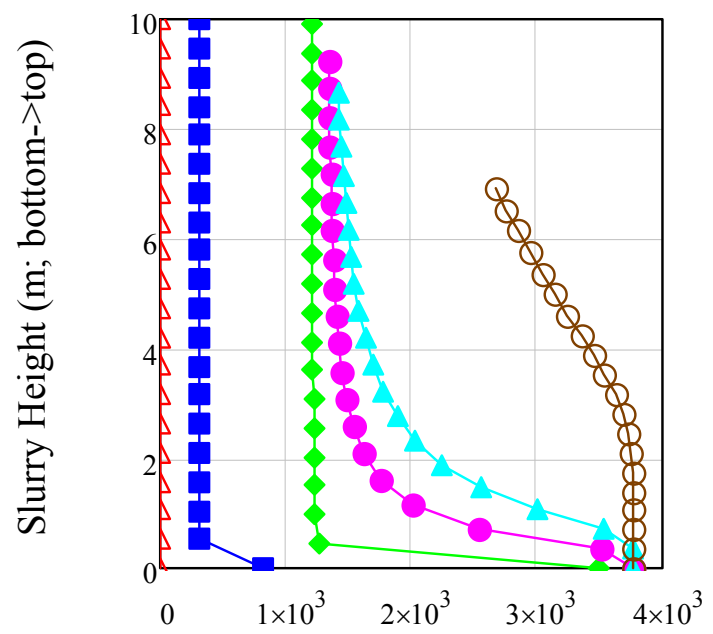

Shear Strength $(\mathrm{Pa})$

$\triangle \triangle 0 \mathrm{hr}$

- $1 \mathrm{hr}$

$\leftrightarrow 10 \mathrm{hr}$

- $100 \mathrm{hr}$

$\triangle 200 \mathrm{hr}$

$\ominus \ominus 1000 \mathrm{hr}$ 
Predicted sludge properties from Hanford Tank T-110 with water dilution at a starting slurry height of $0.1 \mathrm{~m}$ with $33 \%$ volume excess supernatant from fully settled configuration; rheological properties taken at a temperature range of $20^{\circ}-35^{\circ} \mathrm{C}$.

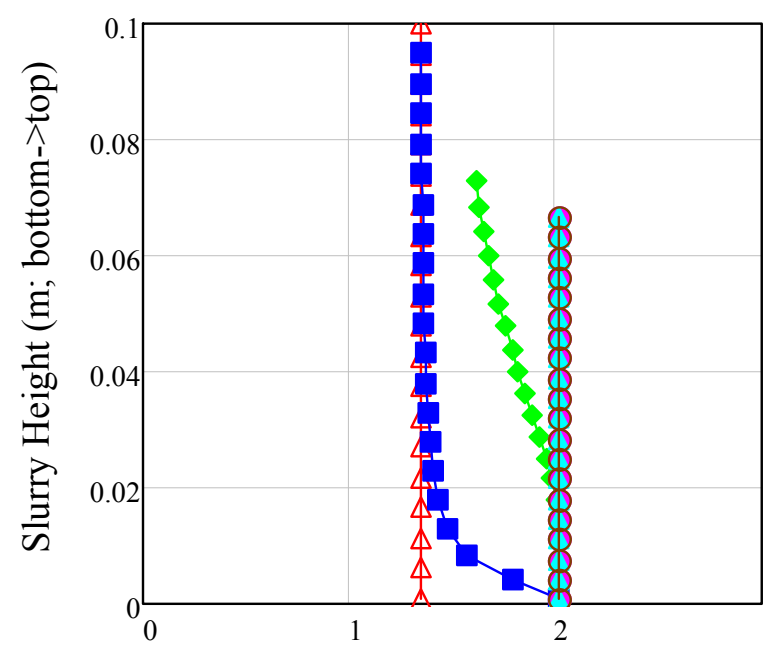

Solids Concentration (Volume Percent) $\triangle \triangle 0 \mathrm{hr}$

a- $1 \mathrm{hr}$

$\leftrightarrow 10 \mathrm{hr}$

$100 \mathrm{hr}$

$\triangle 200 \mathrm{hr}$

$\ominus \ominus 1000 \mathrm{hr}$

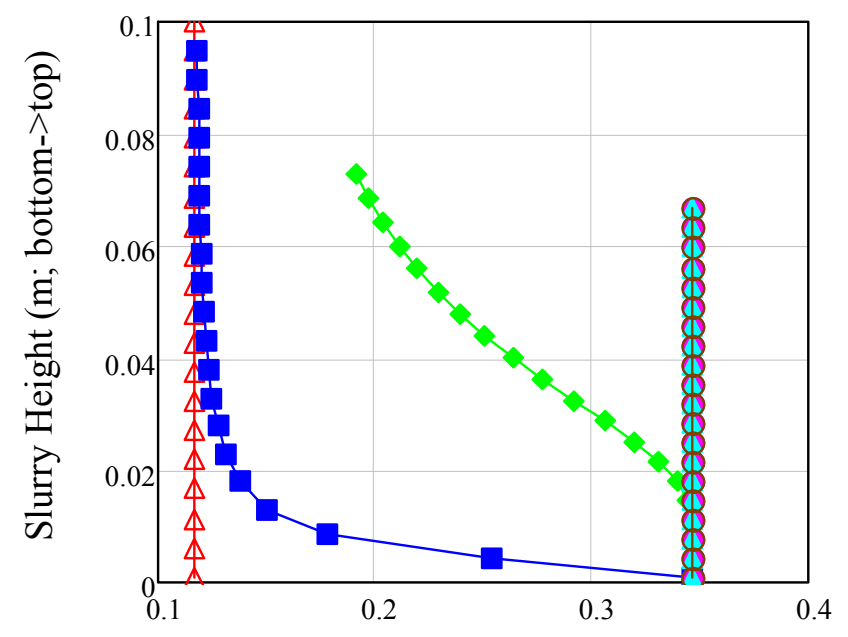

Bingham Yield Stress $(\mathrm{Pa})$

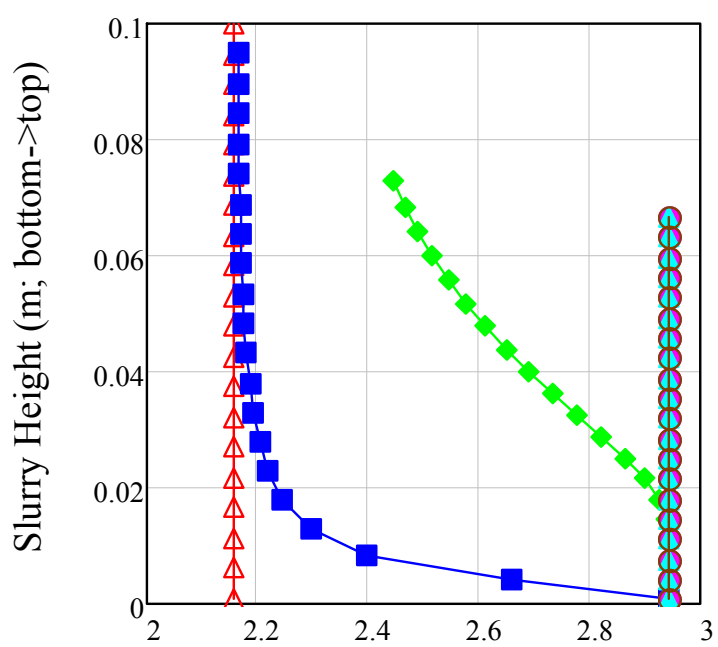

Bingham Consistency (cP)

$\triangle \triangle 0 \mathrm{hr}$

- $1 \mathrm{hr}$

$\leftrightarrow 10 \mathrm{hr}$

- $100 \mathrm{hr}$

$\triangle 200 \mathrm{hr}$

$\ominus \ominus 1000 \mathrm{hr}$

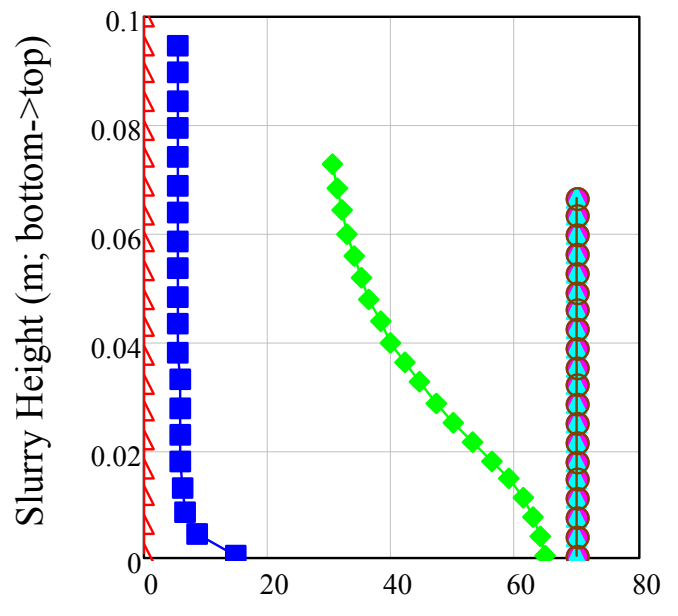

Shear Strength $(\mathrm{Pa})$

$\triangle \triangle 0 \mathrm{hr}$

- $1 \mathrm{hr}$

$\leftrightarrow 10 \mathrm{hr}$

- $100 \mathrm{hr}$

$\triangle 200 \mathrm{hr}$

$\ominus \odot 1000 \mathrm{hr}$ 
Predicted sludge properties from Hanford Tank T-110 with water dilution at a starting slurry height of $1 \mathrm{~m}$ with $33 \%$ volume excess supernatant from fully settled configuration; rheological properties taken at a temperature range of $20^{\circ}-35^{\circ} \mathrm{C}$.

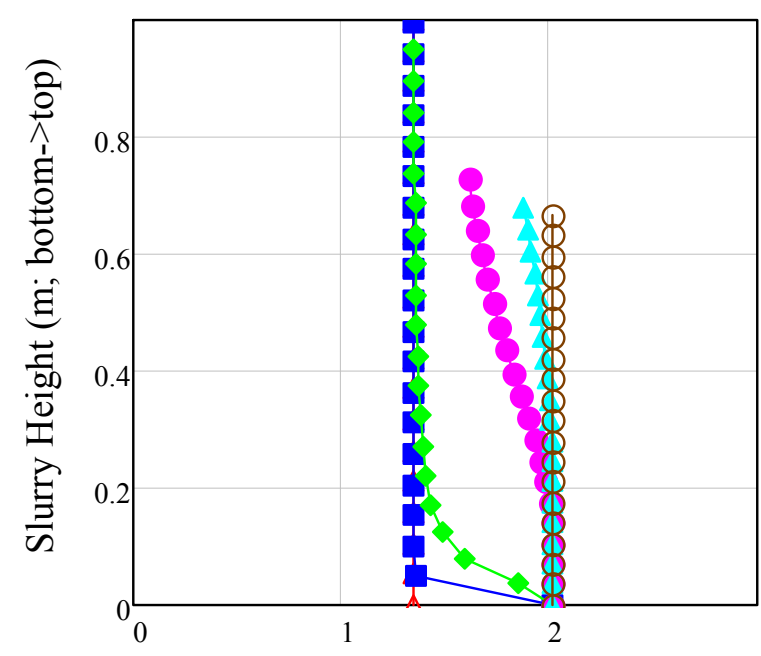

Solids Concentration (Volume Percent) $\triangle \triangle 0 \mathrm{hr}$

a $1 \mathrm{hr}$

$\leftrightarrow 10 \mathrm{hr}$

- $100 \mathrm{hr}$

$\triangle 200 \mathrm{hr}$

$\ominus \ominus 1000 \mathrm{hr}$

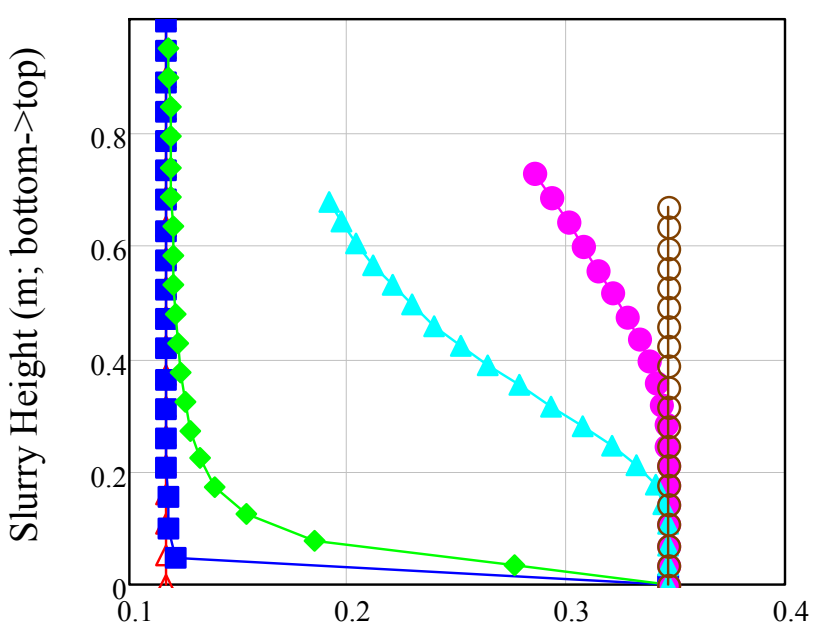

Bingham Yield Stress $(\mathrm{Pa})$

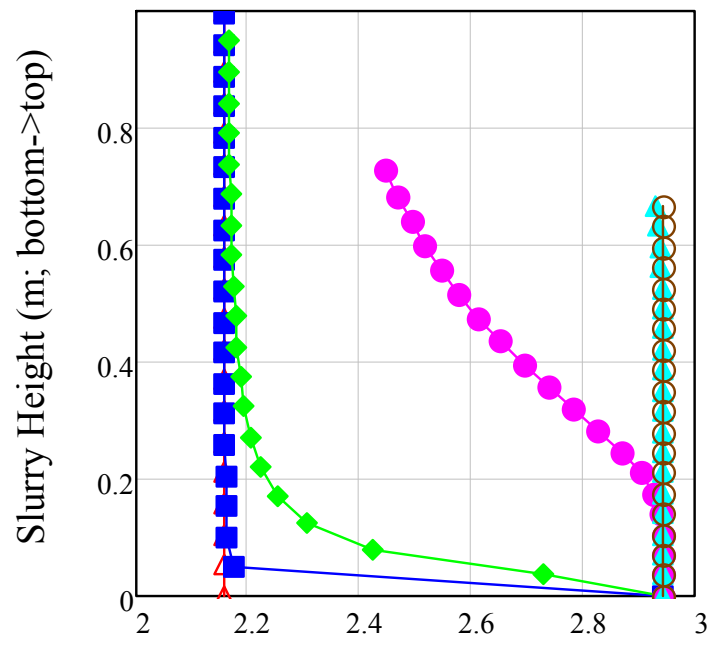

Bingham Consistency (cP)

$\triangle \triangle 0 \mathrm{hr}$

- $1 \mathrm{hr}$

$\leftrightarrow 10 \mathrm{hr}$

- $100 \mathrm{hr}$

$\pm 200 \mathrm{hr}$

$\ominus \ominus 1000 \mathrm{hr}$

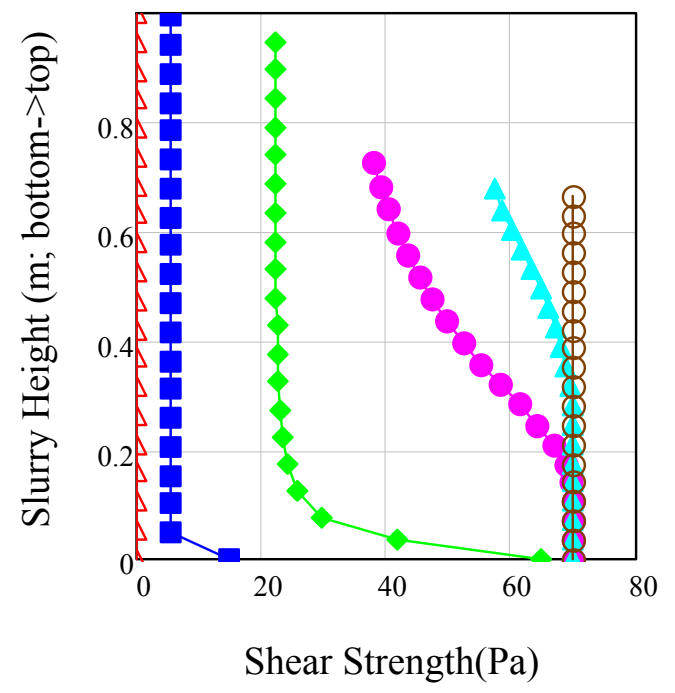

$\triangle \triangle 0 \mathrm{hr}$

- $1 \mathrm{hr}$

$\leftrightarrow 10 \mathrm{hr}$

- $100 \mathrm{hr}$

$\triangle 200 \mathrm{hr}$

$\ominus \ominus 1000 \mathrm{hr}$ 
Predicted sludge properties from Hanford Tank T-110 with water dilution at a starting slurry height of $10 \mathrm{~m}$ with $33 \%$ volume excess supernatant from fully settled configuration; rheological properties taken at a temperature range of $20^{\circ}-35^{\circ} \mathrm{C}$.

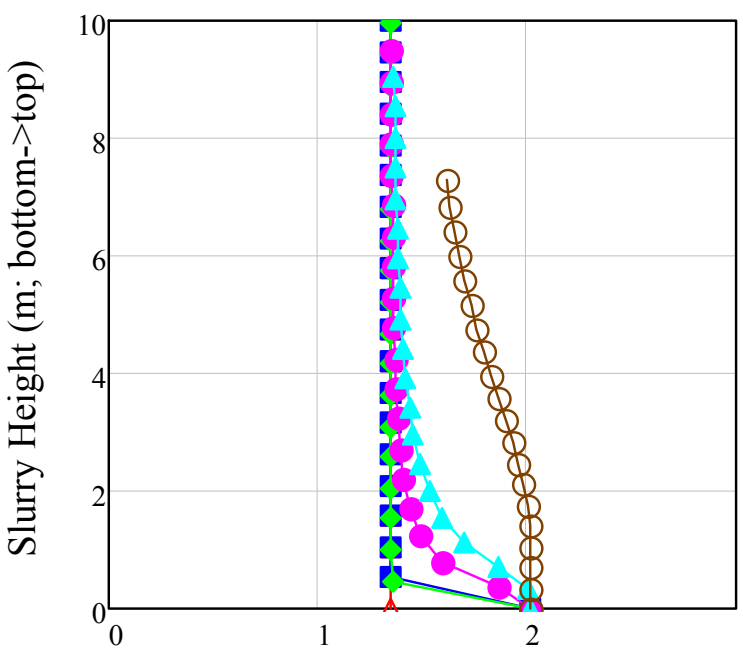

Solids Concentration (Volume Percent) $\triangle \triangle 0 \mathrm{hr}$

- $1 \mathrm{hr}$

$\leftrightarrow 10 \mathrm{hr}$

$100 \mathrm{hr}$

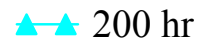

$\ominus \ominus 1000 \mathrm{hr}$

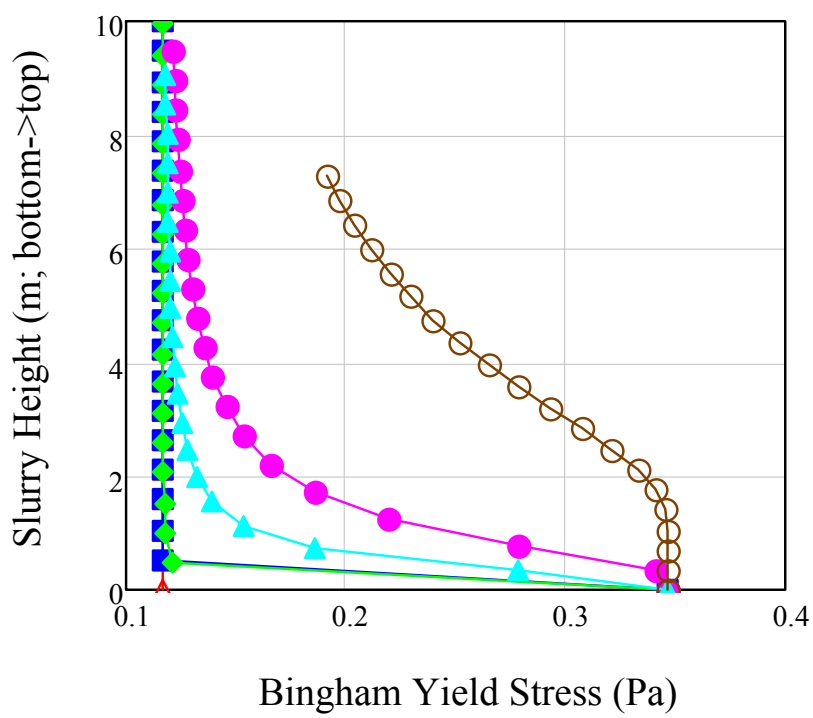

$\triangle \triangle 0 \mathrm{hr}$

- $1 \mathrm{hr}$

$\leftrightarrow 10 \mathrm{hr}$

- $100 \mathrm{hr}$

$\triangle 200 \mathrm{hr}$

$\ominus \ominus 1000 \mathrm{hr}$

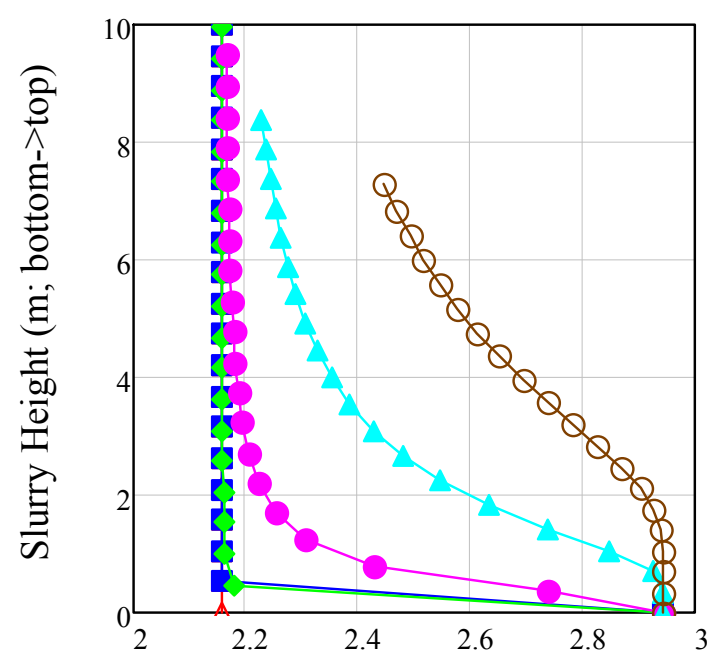

Bingham Consistency $(\mathrm{cP})$

$\triangle \triangle 0 \mathrm{hr}$

- $1 \mathrm{hr}$

$\leftrightarrow 10 \mathrm{hr}$

- $100 \mathrm{hr}$

$\triangle 200 \mathrm{hr}$

$\ominus \ominus 1000 \mathrm{hr}$

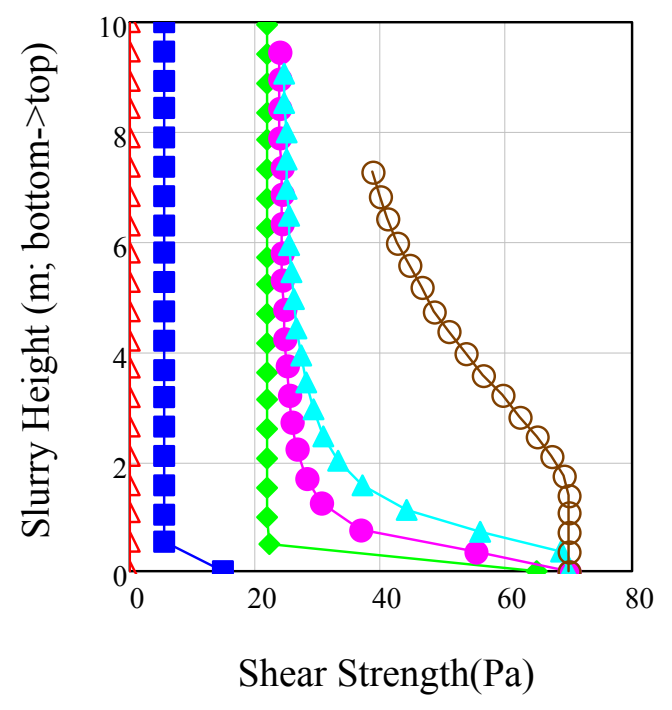

$\triangle \triangle 0 \mathrm{hr}$

- $1 \mathrm{hr}$

$\leadsto 10 \mathrm{hr}$

- $100 \mathrm{hr}$

$\triangle 200 \mathrm{hr}$

$\ominus \ominus 1000 \mathrm{hr}$ 
Predicted sludge properties from Hanford Tank C-104 without water dilution at a starting slurry height of $0.1 \mathrm{~m}$ with $33 \%$ volume excess supernatant from fully settled configuration; rheological properties taken at a temperature range of $20^{\circ}-35^{\circ} \mathrm{C}$.

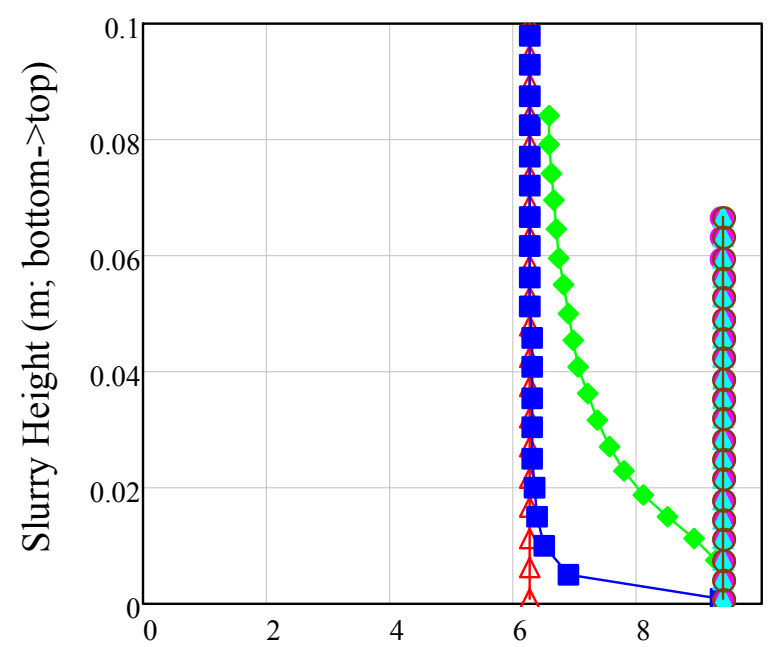

Solids Concentration (Volume Percent) $\triangle \triangle 0 \mathrm{hr}$

- $1 \mathrm{hr}$

$\leftrightarrow 10 \mathrm{hr}$

$100 \mathrm{hr}$

$\triangle 200 \mathrm{hr}$

$\ominus \ominus 1000 \mathrm{hr}$

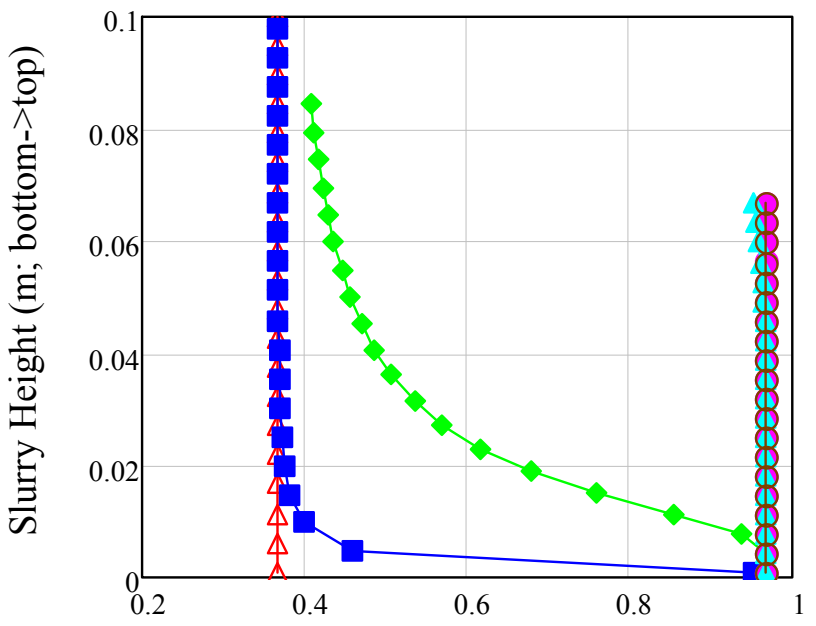

Bingham Yield Stress (Pa)

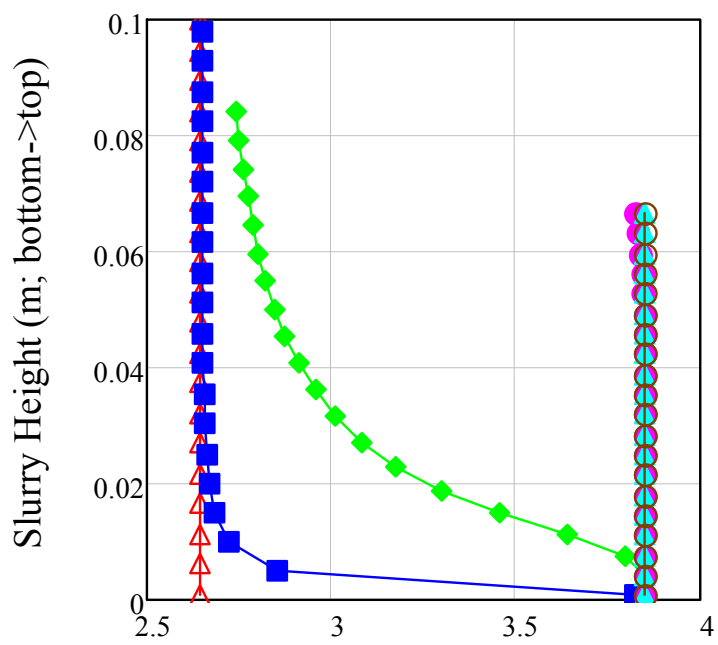

Bingham Consistency (cP)

$\triangle \triangle 0 \mathrm{hr}$

- $1 \mathrm{hr}$

$\leftrightarrow 10 \mathrm{hr}$

- $100 \mathrm{hr}$

$\triangle 200 \mathrm{hr}$

$\ominus \ominus 1000 \mathrm{hr}$

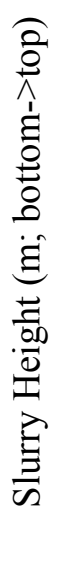

Shear Strength $(\mathrm{Pa})$

$\triangle \triangle 0 \mathrm{hr}$

- $1 \mathrm{hr}$

$\leftrightarrow 10 \mathrm{hr}$

- $100 \mathrm{hr}$

$\triangle 200 \mathrm{hr}$

$\ominus \ominus 1000 \mathrm{hr}$ 
Predicted sludge properties from Hanford Tank C-104 without water dilution at a starting slurry height of $1 \mathrm{~m}$ with $33 \%$ volume excess supernatant from fully settled configuration; rheological properties taken at a temperature range of $20^{\circ}-35^{\circ} \mathrm{C}$.

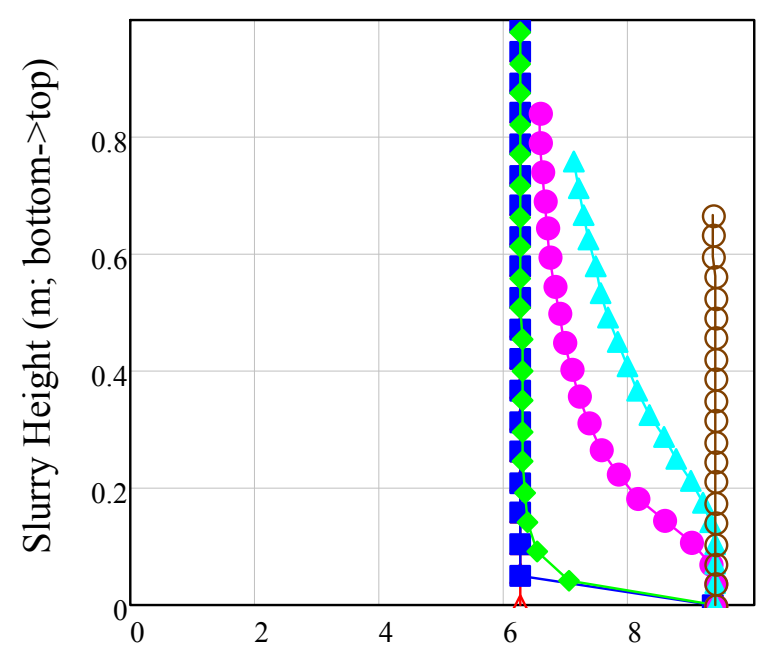

Solids Concentration (Volume Percent) $\triangle \triangle 0 \mathrm{hr}$

- $1 \mathrm{hr}$

$\leftrightarrow 10 \mathrm{hr}$

- $100 \mathrm{hr}$

$\triangle 200 \mathrm{hr}$

$\ominus \ominus 1000 \mathrm{hr}$

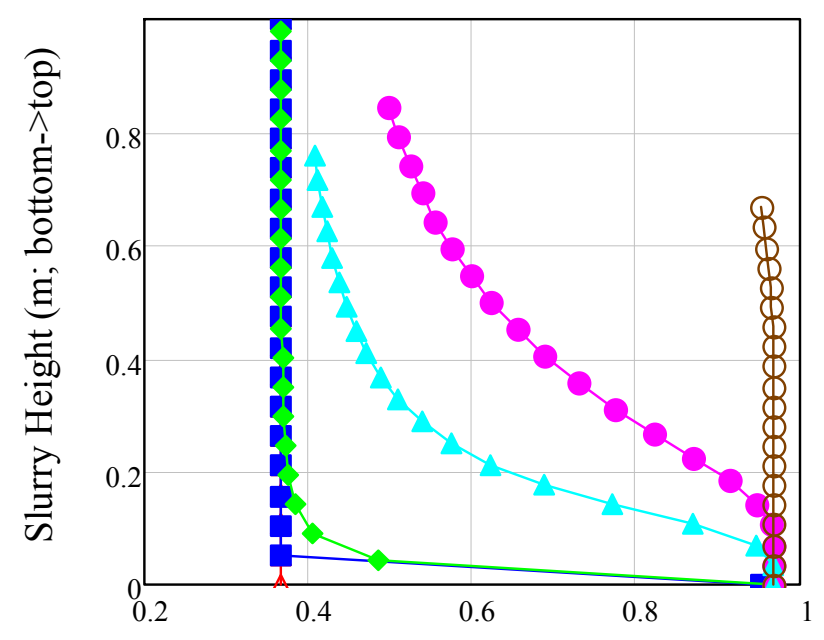

Bingham Yield Stress $(\mathrm{Pa})$

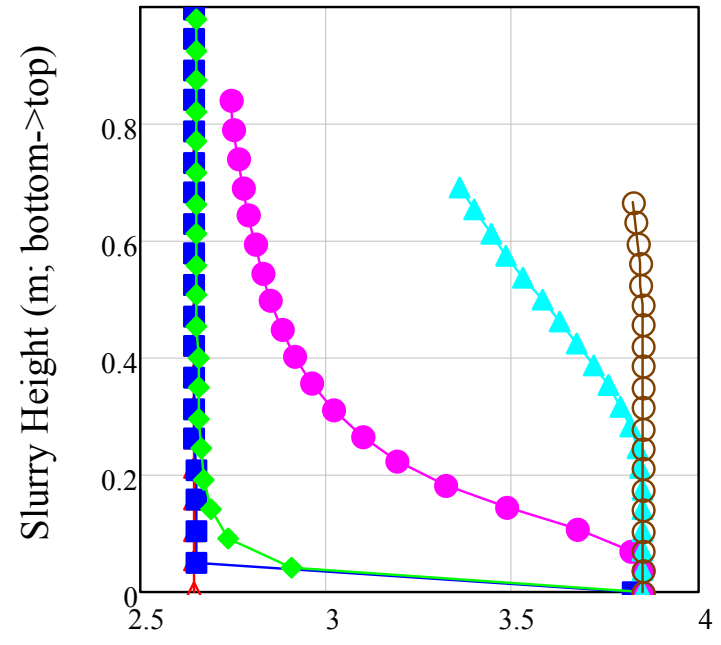

Bingham Consistency (cP)

$\triangle \triangle 0 \mathrm{hr}$

- $1 \mathrm{hr}$

$\leftrightarrow 10 \mathrm{hr}$

- $100 \mathrm{hr}$

$\triangle 200 \mathrm{hr}$

$\ominus \ominus 1000 \mathrm{hr}$

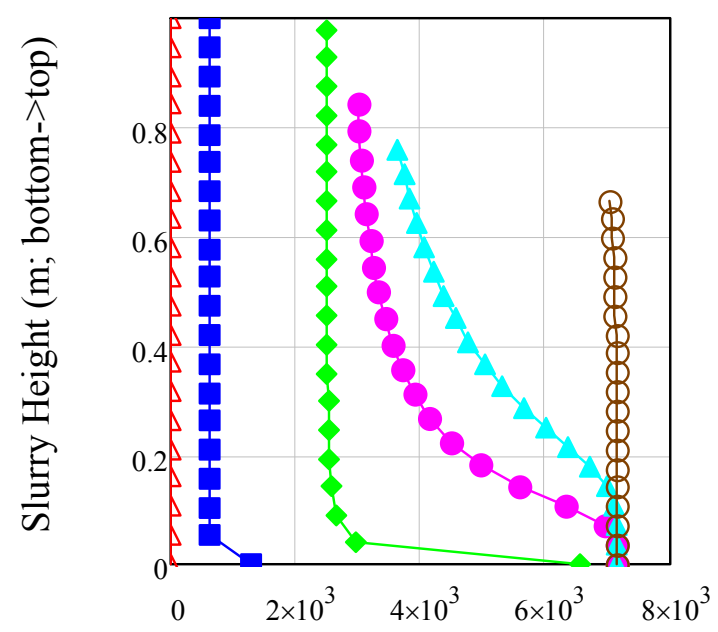

Shear Strength $(\mathrm{Pa})$

$\triangle \triangle 0 \mathrm{hr}$

- $1 \mathrm{hr}$

$\leftrightarrow 10 \mathrm{hr}$

- $100 \mathrm{hr}$

$\triangle 200 \mathrm{hr}$

$\ominus \ominus 1000 \mathrm{hr}$ 
Predicted sludge properties from Hanford Tank C-104 without water dilution at a starting slurry height of $10 \mathrm{~m}$ with $33 \%$ volume excess supernatant from fully settled configuration; rheological properties taken at a temperature range of $20^{\circ}-35^{\circ} \mathrm{C}$.

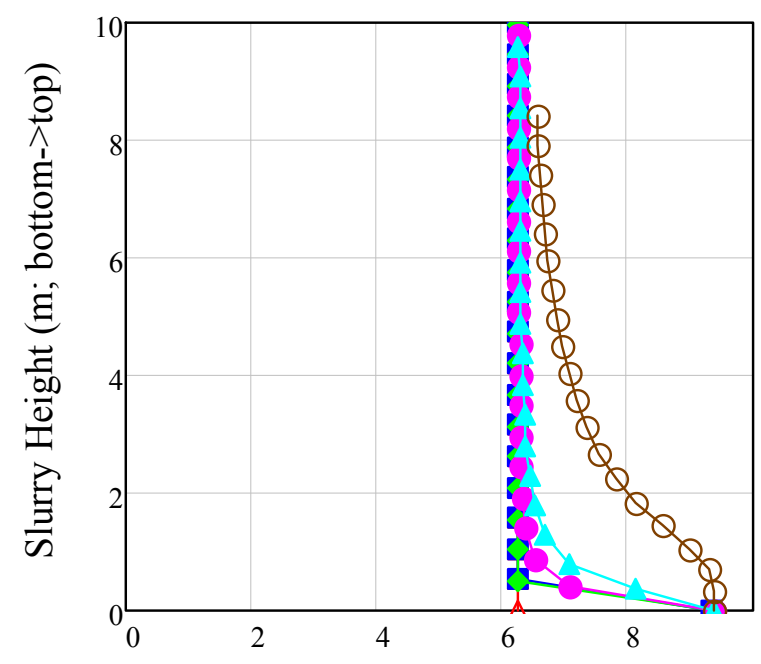

Solids Concentration (Volume Percent) $\triangle \triangle 0 \mathrm{hr}$

- $1 \mathrm{hr}$

$\leftrightarrow 10 \mathrm{hr}$

$100 \mathrm{hr}$

$\pm 200 \mathrm{hr}$

$\ominus \ominus 1000 \mathrm{hr}$

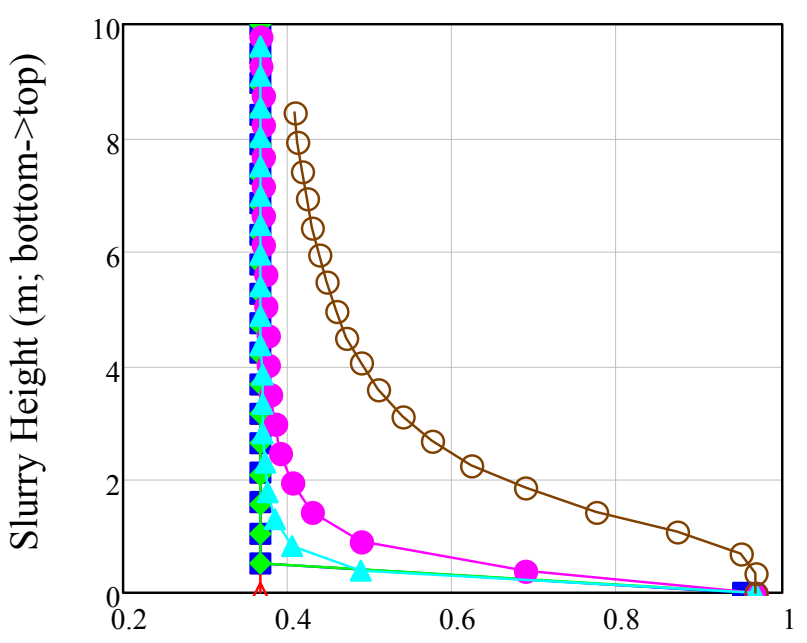

Bingham Yield Stress (Pa)

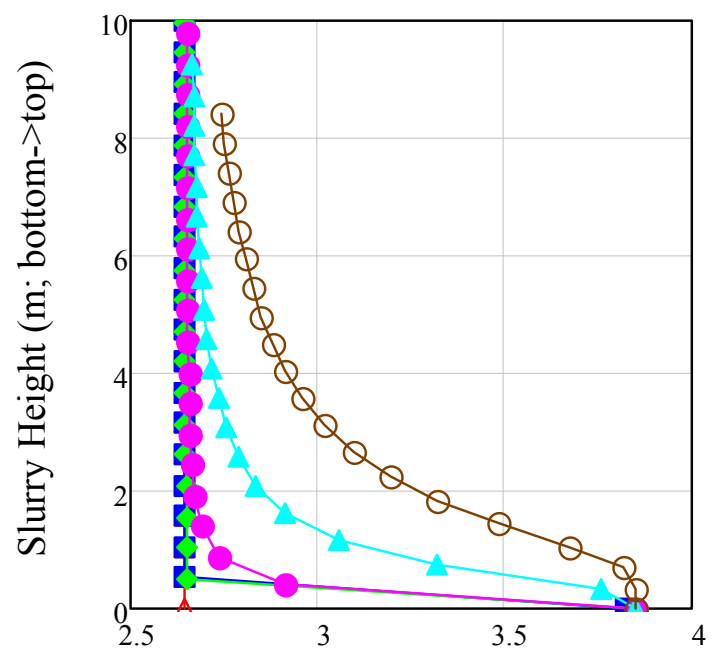

Bingham Consistency (cP)

$\triangle \triangle 0 \mathrm{hr}$

- $1 \mathrm{hr}$

$\leftrightarrow 10 \mathrm{hr}$

- $100 \mathrm{hr}$

$\triangle 200 \mathrm{hr}$

$\ominus \ominus 1000 \mathrm{hr}$

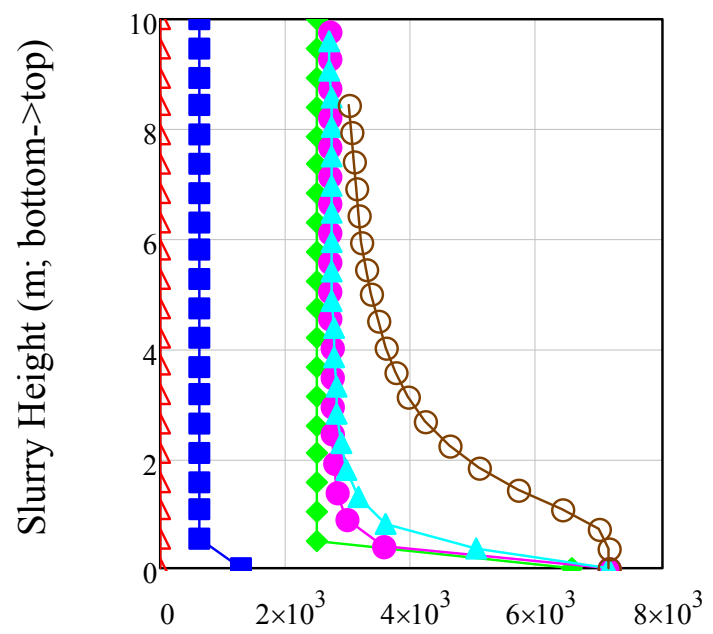

Shear Strength $(\mathrm{Pa})$

$\triangle \triangle 0 \mathrm{hr}$

- $1 \mathrm{hr}$

$\leftrightarrow 10 \mathrm{hr}$

- $100 \mathrm{hr}$

$\triangle 200 \mathrm{hr}$

$\ominus \ominus 1000 \mathrm{hr}$ 
Predicted sludge properties from Hanford Tank C-104 with water dilution at a starting slurry height of $0.1 \mathrm{~m}$ with $33 \%$ volume excess supernatant from fully settled configuration; rheological properties taken at a temperature range of $20^{\circ}-35^{\circ} \mathrm{C}$.

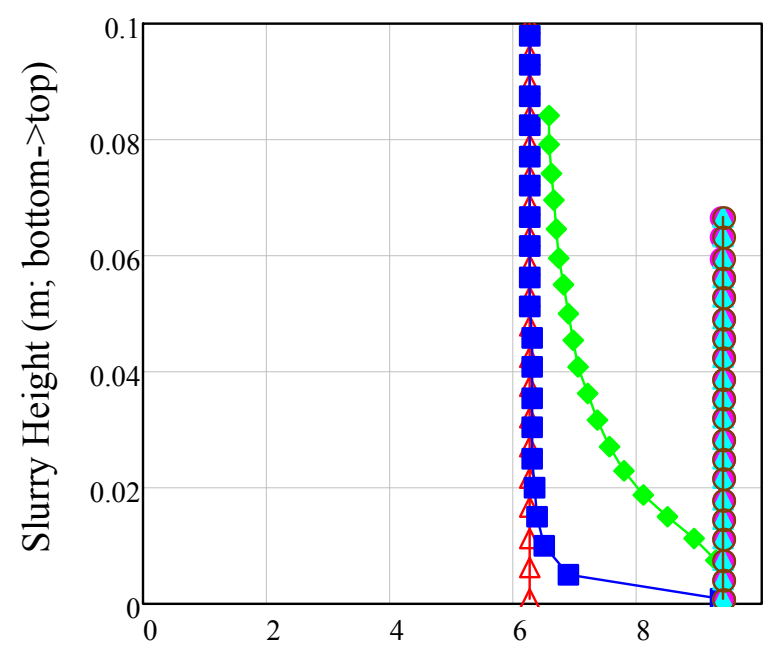

Solids Concentration (Volume Percent) $\triangle \triangle 0 \mathrm{hr}$

a- $1 \mathrm{hr}$

$\leftrightarrow 10 \mathrm{hr}$

$100 \mathrm{hr}$

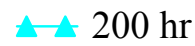

$\ominus \ominus 1000 \mathrm{hr}$

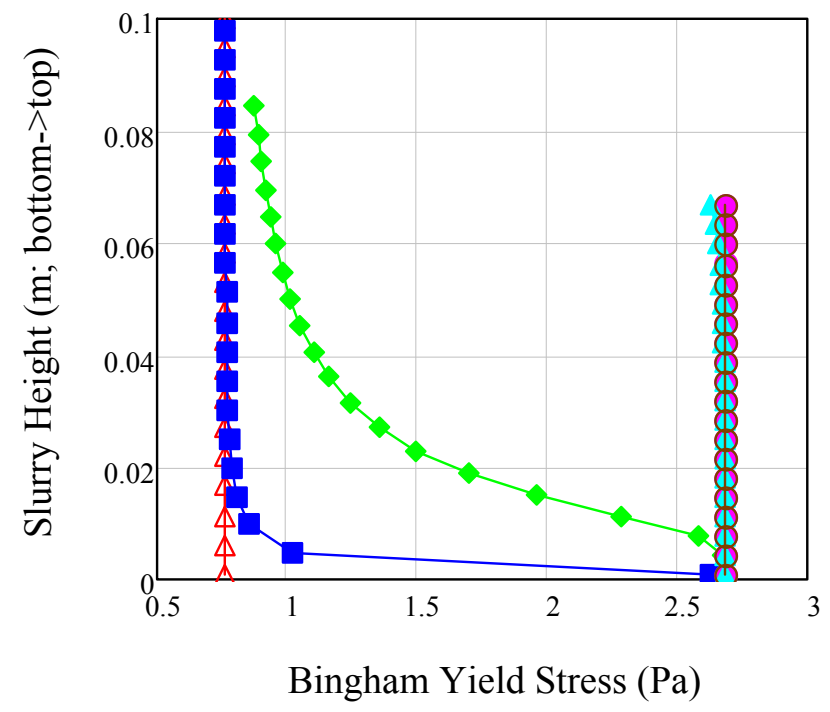

$\triangle \triangle 0 \mathrm{hr}$

- $1 \mathrm{hr}$

$\leftrightarrow 10 \mathrm{hr}$

- $100 \mathrm{hr}$

$\triangle 200 \mathrm{hr}$

$\ominus \ominus 1000 \mathrm{hr}$

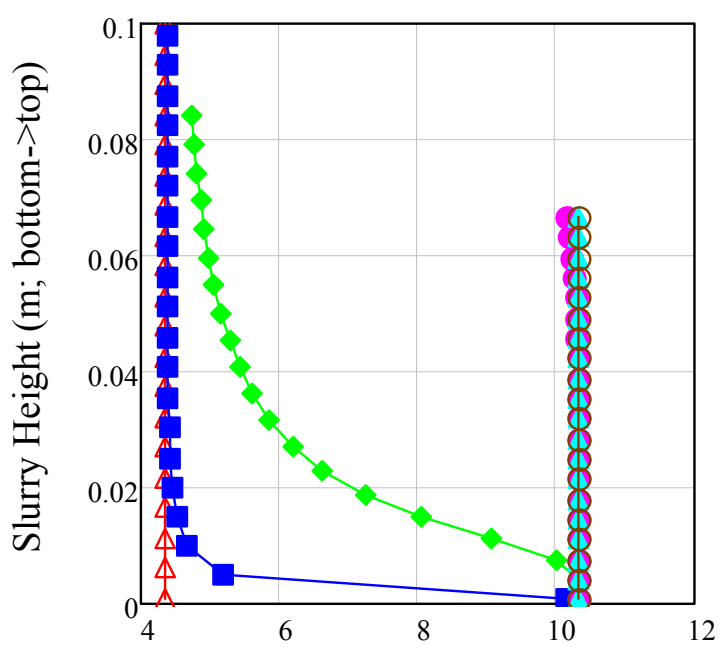

Bingham Consistency (cP) $\triangle \triangle 0 \mathrm{hr}$
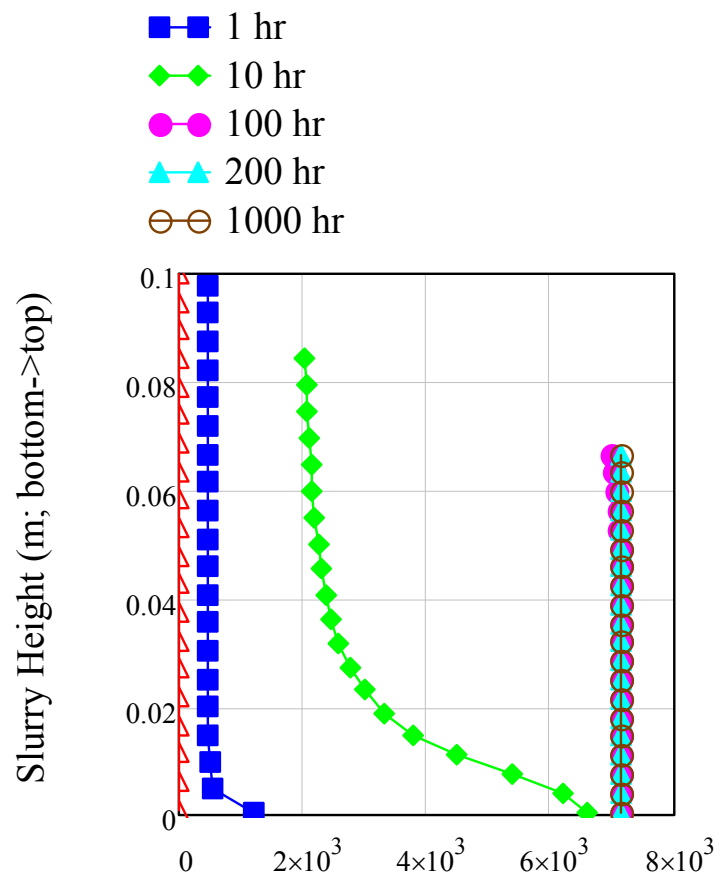

Shear Strength $(\mathrm{Pa})$

$\triangle \triangle 0 \mathrm{hr}$

- $1 \mathrm{hr}$

$\leftrightarrow 10 \mathrm{hr}$

$\rightarrow 100 \mathrm{hr}$

$\triangle 200 \mathrm{hr}$

$\ominus \ominus 1000 \mathrm{hr}$ 
Predicted sludge properties from Hanford Tank C-104 with water dilution at a starting slurry height of $1 \mathrm{~m}$ with $33 \%$ volume excess supernatant from fully settled configuration; rheological properties taken at a temperature range of $20^{\circ}-35^{\circ} \mathrm{C}$.

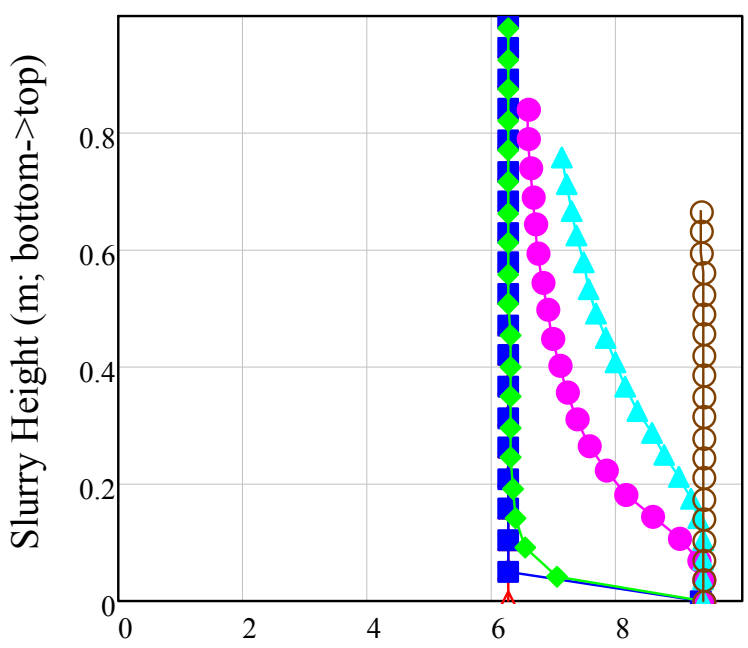

Solids Concentration (Volume Percent) $\triangle \triangle 0 \mathrm{hr}$

a $1 \mathrm{hr}$

$\leftrightarrow 10 \mathrm{hr}$

- $100 \mathrm{hr}$

$\triangle 200 \mathrm{hr}$

$\ominus \ominus 1000 \mathrm{hr}$

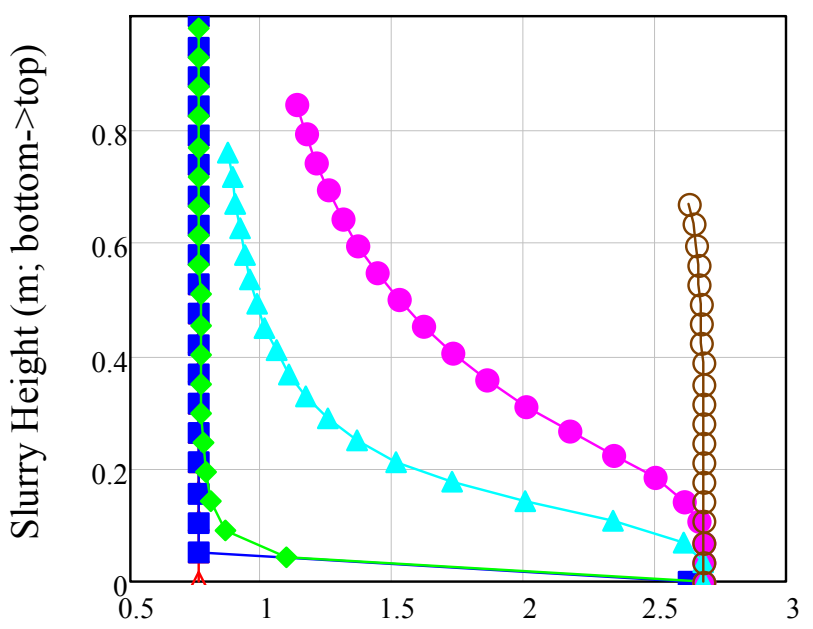

Bingham Yield Stress $(\mathrm{Pa})$

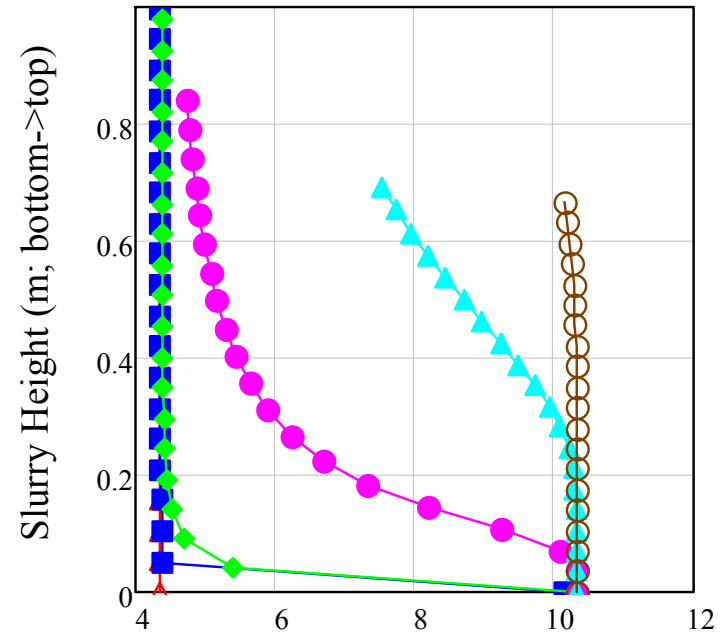

Bingham Consistency (cP)

$\triangle \triangle 0 \mathrm{hr}$

- $1 \mathrm{hr}$

$\leftrightarrow 10 \mathrm{hr}$

- $100 \mathrm{hr}$

$\triangle 200 \mathrm{hr}$

$\ominus \ominus 1000 \mathrm{hr}$

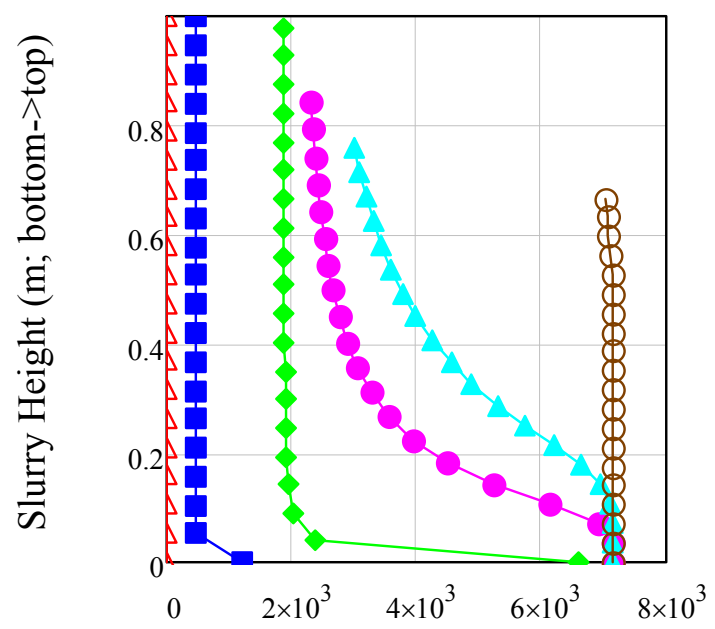

Shear Strength $(\mathrm{Pa})$

$\triangle \triangle 0 \mathrm{hr}$

- $1 \mathrm{hr}$

$\leftrightarrow 10 \mathrm{hr}$

- $100 \mathrm{hr}$

$\triangle 200 \mathrm{hr}$

$\ominus \ominus 1000 \mathrm{hr}$ 
Predicted sludge properties from Hanford Tank C-104 with water dilution at a starting slurry height of $10 \mathrm{~m}$ with $33 \%$ volume excess supernatant from fully settled configuration; rheological properties taken at a temperature range of $20^{\circ}-35^{\circ} \mathrm{C}$.

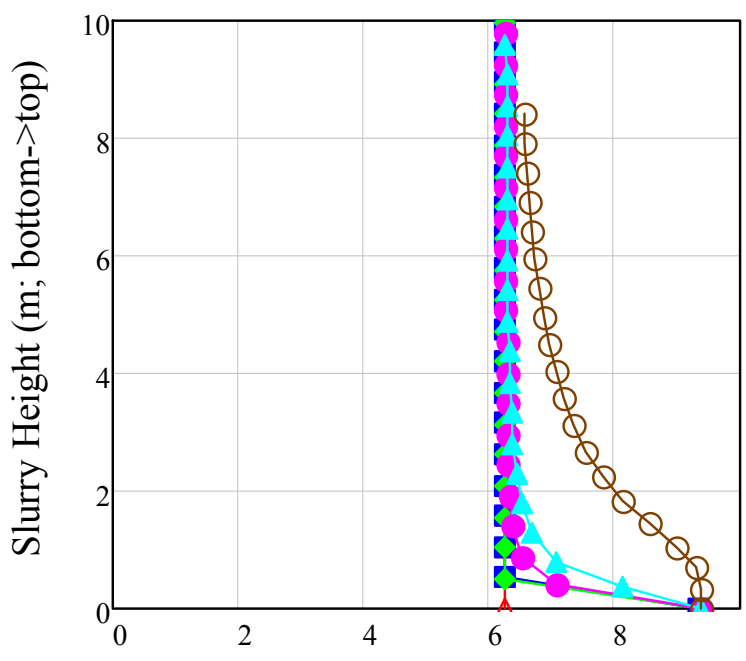

Solids Concentration (Volume Percent) $\triangle \triangle 0 \mathrm{hr}$

- $1 \mathrm{hr}$

$\leftrightarrow 10 \mathrm{hr}$

$100 \mathrm{hr}$

$\pm 200 \mathrm{hr}$

$\ominus \ominus 1000 \mathrm{hr}$

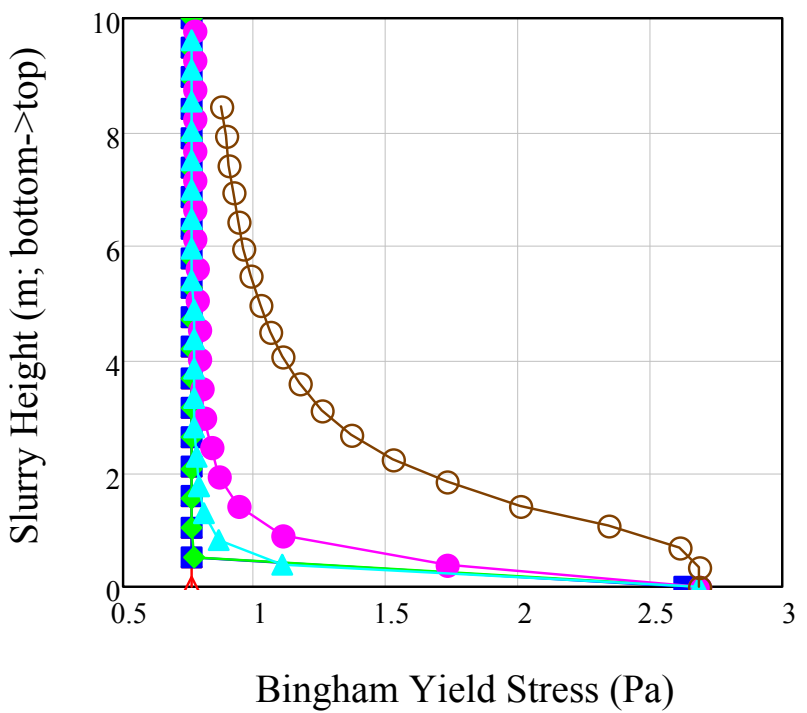

$\triangle \triangle 0 \mathrm{hr}$

- $1 \mathrm{hr}$

$\leftrightarrow 10 \mathrm{hr}$

- $100 \mathrm{hr}$

$\triangle 200 \mathrm{hr}$

$\ominus \ominus 1000 \mathrm{hr}$

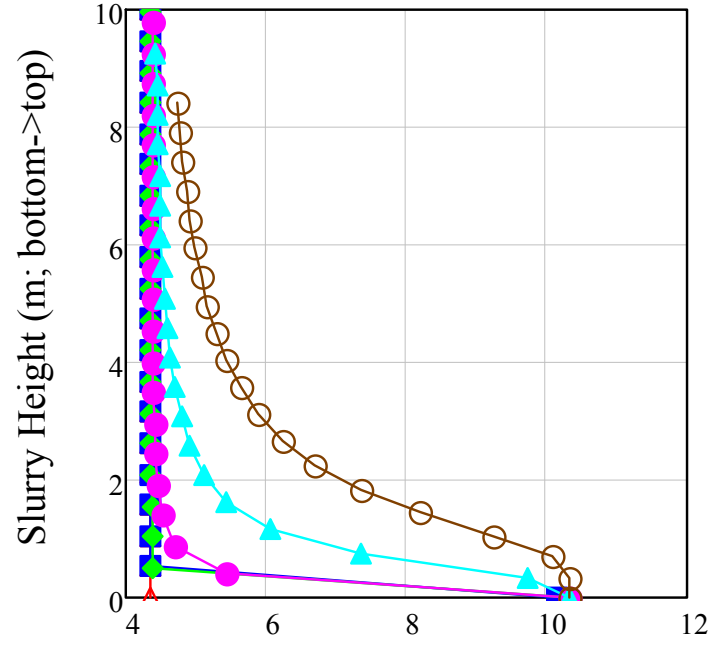

Bingham Consistency (cP)

$\triangle \triangle 0 \mathrm{hr}$

- $1 \mathrm{hr}$

$\leftrightarrow 10 \mathrm{hr}$

$100 \mathrm{hr}$

$\pm 200 \mathrm{hr}$

$\ominus \ominus 1000 \mathrm{hr}$

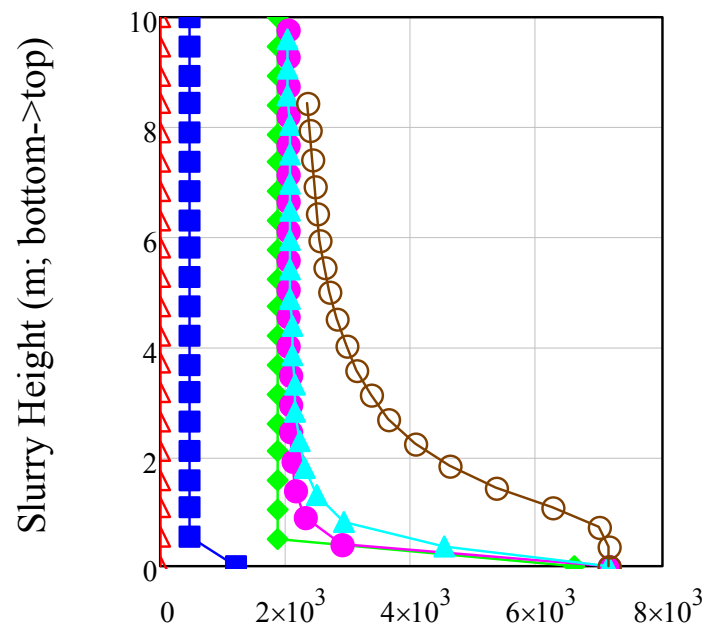

Shear Strength $(\mathrm{Pa})$

$\triangle \triangle 0 \mathrm{hr}$

a $1 \mathrm{hr}$

$\leftrightarrow 10 \mathrm{hr}$

- $100 \mathrm{hr}$

$\triangle 200 \mathrm{hr}$

$\ominus \ominus 1000 \mathrm{hr}$ 


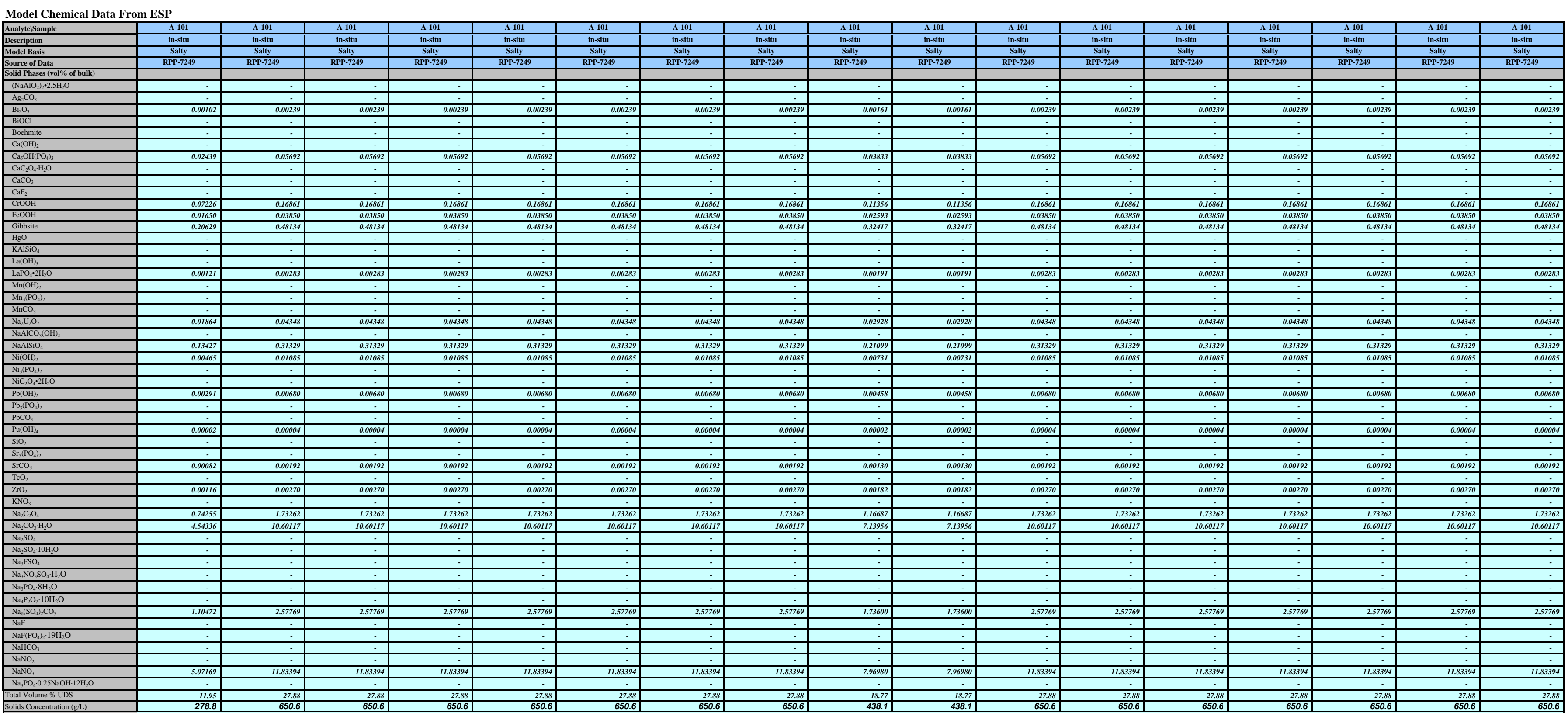




\begin{tabular}{|c|c|c|c|c|c|c|c|c|c|c|c|c|c|c|c|c|c|}
\hline $\begin{array}{l}\text { Physical Property Data } \\
\text { Physical Property }\end{array}$ & $\bar{T}^{A-101}$ & $\frac{A-101}{A-10}$ & $\frac{A-101}{A-1}$ & $\overline{A-101}$ & $\frac{A \cdot 01}{A-101}$ & A-101 & $\overline{A-101}$ & A-101 & A-101 & $\begin{array}{c}A-101 \\
\end{array}$ & A-101 & $\begin{array}{c}A-101 \\
\end{array}$ & $\frac{A-101}{A^{-1}}$ & $\frac{A-101}{A-101}$ & A-101 & $\frac{A-101}{T^{2}-1}$ & $\begin{array}{c}A-101 \\
\end{array}$ \\
\hline \multicolumn{18}{|l|}{ Source of nata } \\
\hline Temperature (C) & $32-37$ & $32-37$ & $32-37$ & $32-37$ & $32-37$ & $32-37$ & $32-37$ & $32-37$ & $32-37$ & $32-37$ & $32-37$ & $32-37$ & $32-37$ & $32-37$ & $32-37$ & $32-37$ & $32-37$ \\
\hline 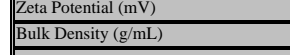 & 1.56 & 1.70 & $\frac{1.70}{1.0}$ & $\frac{1.70}{1.70}$ & 1,70 & $\frac{1.70}{1.70}$ & $\frac{1.70}{1.0}$ & $\frac{1.70}{1.70}$ & $\frac{1.62}{1.62}$ & $\frac{1.62}{1.2}$ & $\frac{1.70}{1.00}$ & $\frac{1.70}{1.70}$ & $\frac{1.70}{1.0}$ & $\frac{1.70}{1.70}$ & $\frac{1.70}{1.70}$ & $\frac{1.70}{1.70}$ & $\frac{1.70}{1.70}$ \\
\hline \multicolumn{18}{|l|}{ 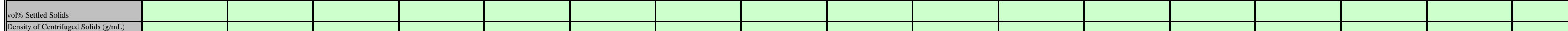 } \\
\hline \multicolumn{18}{|l|}{ vol\% Cennififged Solids } \\
\hline \multicolumn{18}{|l|}{ were Centifituged solids } \\
\hline 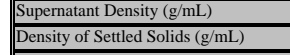 & 1.46 & 1.46 & 1.46 & & 1.46 & 1.46 & 1.46 & 1.46 & 1.46 & 1.46 & 1.46 & 1.46 & 1.46 & 1.46 & 1.46 & 1.46 & 1.46 \\
\hline \multicolumn{18}{|l|}{ wo\% Sertled Supermatant } \\
\hline \multicolumn{18}{|l|}{\begin{tabular}{|l} 
wo\% dissolved solids in supenatant \\
\end{tabular}} \\
\hline \multirow{2}{*}{\multicolumn{18}{|c|}{ wo\% toat solids in Centrifuged Sludge }} \\
\hline \multicolumn{15}{|l|}{ we\% Toal Solids } & & & \\
\hline wwo uDs & $\begin{array}{ll}17.87 \\
\end{array}$ & $\begin{array}{ll}38.27 \\
\end{array}$ & 38.27 & 38.27 & 38.27 & 38.27 & 38.27 & 38.27 & 27.05 & 27.05 & 38.27 & 38.27 & 38.27 & 38.27 & 38.27 & 38.27 & 38.27 \\
\hline 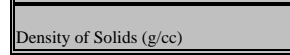 & 2.33 & $2,2.33$ & 2.33 & ${ }^{2.33}$ & 2,33 & 2.33 & 2.33 & 2.33 & $2,2.33$ & 2.33 & 2.33 & 2.33 & 2.33 & 2,33 & 2.33 & 2.33 & 2.33 \\
\hline
\end{tabular}




\begin{tabular}{|c|c|c|c|c|c|c|c|c|c|c|c|c|c|c|c|c|c|}
\hline 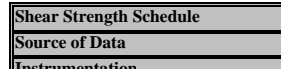 & 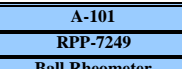 & $\frac{A-101}{R \cdot P-2.249}$ & 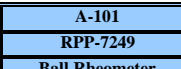 & 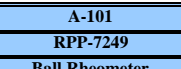 & $\frac{A-101}{R \cdot 1-1249}$ & $\frac{A-101}{R \cdot P-2.249}$ & $\begin{array}{c}\text { A-101 } \\
\text { RPP-2749 } \\
\end{array}$ & $\begin{array}{c}\text { A-101 } \\
\text { RP-2-249 } \\
\end{array}$ & 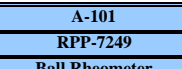 & 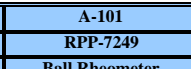 & 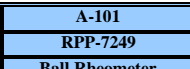 & 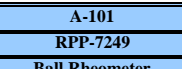 & 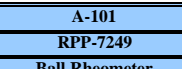 & 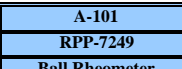 & $\frac{A-101}{R-P \cdot 249}$ & $\frac{A-1.10}{R \cdot P P-249}$ & 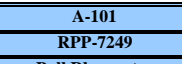 \\
\hline 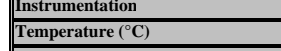 & $\begin{array}{l}\text { Ball Rheometer } \\
63\end{array}$ & \begin{tabular}{|l} 
Ball RReomet \\
63 \\
\end{tabular} & & $\begin{array}{l}\text { Ball Rheom } \\
62 \\
\end{array}$ & & 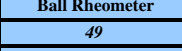 & $\begin{array}{l}\text { Ball Rheo } \\
47 \\
\end{array}$ & $\begin{array}{l}\text { Ball Rheor } \\
44 \\
\end{array}$ & & & & & & & & & \\
\hline & $\begin{array}{l}\text { Riser } 15 \\
\text { Depth from bottom of }\end{array}$ & $\begin{array}{c}\text { Riser } 15 \\
\text { Depth from bottom of }\end{array}$ & $\begin{array}{c}\text { Riser } 15 \\
\text { Flantity golids }\end{array}$ & $\begin{array}{l}\text { Riser } 15 \\
\text { Fllaating golids } \\
\end{array}$ & $\begin{array}{l}\text { Riser } 15 \\
\text { Floatin Solids } \\
\end{array}$ & 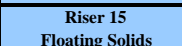 & 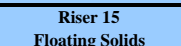 & $\begin{array}{ll}\text { Riser } 15 \\
\text { Fllating Solids } \\
\end{array}$ & 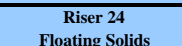 & 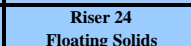 & $\begin{array}{c}\text { Riser } 24 \\
\text { Fllaating Solids } \\
\end{array}$ & $\begin{array}{ll}\text { Riser } 24 \\
\text { Fing }\end{array}$ & $\begin{array}{l}\text { Riser } 24 \\
\text { Pl }\end{array}$ & 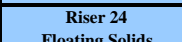 & 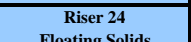 & & \\
\hline & 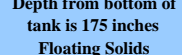 & 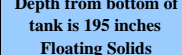 & 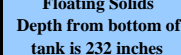 & 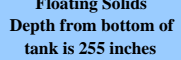 & 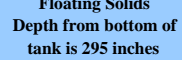 & 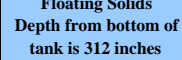 & 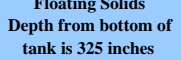 & 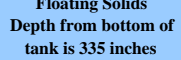 & 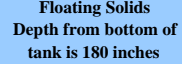 & 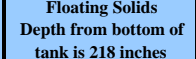 & 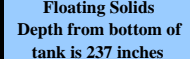 & 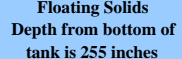 & 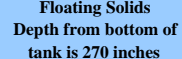 & 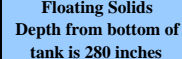 & 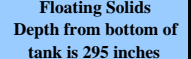 & 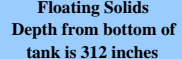 & 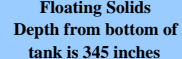 \\
\hline 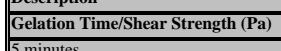 & & & & & & & & & & & & & & & & & \\
\hline 1immeses & & & & & & & & & & & & & & & & & \\
\hline 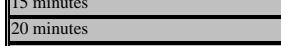 & & & & & & & & & & & & & & & & & \\
\hline 40 & & & & & & & & & & & & & & & & & \\
\hline thour & & & & & & & & & & & & & & & & & \\
\hline 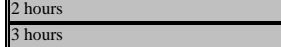 & & & & & & & & & & & & & 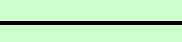 & & & & \\
\hline 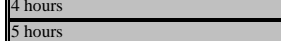 & & & & & & & & & & & & & & & & & \\
\hline$\frac{6 \text { houns }}{2 \text { 2h hours }}$ & & & & & & & & & & & & & & & & & \\
\hline hours & & & & & & & & & & & & & & & & & \\
\hline hours & & & & & & & & & & & & & & & & & \\
\hline 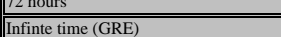 & $\begin{array}{l}110 \\
10\end{array}$ & 700 & 440 & $\frac{200}{200}$ & 2 & 440 & $\begin{array}{l}110 \\
\end{array}$ & 400 & & & & & & & & & \\
\hline
\end{tabular}


Model Chemical Data From ESP

\begin{tabular}{|c|c|c|c|}
\hline AnalytelSample & AN-102 & AN-102 & AN-102 \\
\hline Description & as received & as received & as received \\
\hline Model Basis & Salty & Salty & Salty \\
\hline Report No/Source of Data & $\begin{array}{l}\text { WTP-RPT-021 } \\
\text { Rev. } 1\end{array}$ & $\begin{array}{l}\text { WTP-RPT-021 } \\
\text { Rev. } 1\end{array}$ & $\begin{array}{l}\text { WTP-RPT-021 } \\
\text { Rev. } 1\end{array}$ \\
\hline \multicolumn{4}{|l|}{ Solid Phases (vol\% of bulk) } \\
\hline$\left(\mathrm{NaAlO}_{2}\right)_{2} \cdot 2.5 \mathrm{H}_{2} \mathrm{O}$ & - & - & - \\
\hline $\mathrm{Ag}_{2} \mathrm{CO}_{3}$ & - & - & - \\
\hline $\mathrm{Bi}_{2} \mathrm{O}_{3}$ & 0.00010 & 0.00010 & 0.00010 \\
\hline \multicolumn{4}{|l|}{$\mathrm{BiOCl}$} \\
\hline Boehmite & - & - & - \\
\hline $\mathrm{Ca}(\mathrm{OH})_{2}$ & - & - & - \\
\hline $\mathrm{Ca}_{5} \mathrm{OH}\left(\mathrm{PO}_{4}\right)_{3}$ & 0.00898 & 0.00898 & 0.00898 \\
\hline $\mathrm{CaC}_{2} \mathrm{O}_{4} \cdot \mathrm{H}_{2} \mathrm{O}$ & - & - & - \\
\hline $\mathrm{CaCO}_{3}$ & - & - & - \\
\hline $\mathrm{CaF}_{2}$ & - & - & - \\
\hline$\overline{\mathrm{CrOOH}}$ & 0.00116 & 0.00116 & 0.00116 \\
\hline $\mathrm{FeOOH}$ & 0.00187 & 0.00187 & 0.00187 \\
\hline Gibbsite & 0.22125 & 0.22125 & 0.22125 \\
\hline $\mathrm{HgO}$ & - & - & - \\
\hline $\mathrm{KAISiO}_{4}$ & - & - & - \\
\hline $\mathrm{La}(\mathrm{OH})_{3}$ & - & - & - \\
\hline $\mathrm{LaPO}_{4} \cdot 2 \mathrm{H}_{2} \mathrm{O}$ & 0.00003 & 0.00003 & 0.00003 \\
\hline $\mathrm{Mn}(\mathrm{OH})_{2}$ & 0.00008 & 0.00008 & 0.00008 \\
\hline $\mathrm{Mn}_{3}\left(\mathrm{PO}_{4}\right)_{2}$ & - & - & - \\
\hline $\mathrm{MnCO}_{3}$ & - & - & - \\
\hline $\mathrm{Na}_{2} \mathrm{U}_{2} \mathrm{O}_{7}$ & 0.00085 & 0.00085 & 0.00085 \\
\hline $\mathrm{NaAlCO}_{3}(\mathrm{OH})_{2}$ & - & - & - \\
\hline $\mathrm{NaAlSiO}_{4}$ & - & - & - \\
\hline$\overline{\mathrm{Ni}(\mathrm{OH})_{2}}$ & 0.00267 & 0.00267 & 0.00267 \\
\hline $\mathrm{Ni}_{3}\left(\mathrm{PO}_{4}\right)_{2}$ & - & - & - \\
\hline \multicolumn{4}{|l|}{$\mathrm{NiC}_{2} \mathrm{O}_{4} \cdot 2 \mathrm{H}_{2} \mathrm{O}$} \\
\hline $\mathrm{Pb}(\mathrm{OH})_{2}$ & 0.00043 & 0.00043 & 0.00043 \\
\hline $\mathrm{Pb}_{3}\left(\mathrm{PO}_{4}\right)_{2}$ & - & - & - \\
\hline $\mathrm{PbCO}_{3}$ & - & - & - \\
\hline $\mathrm{Pu}(\mathrm{OH})_{4}$ & 0.00000 & 0.00000 & 0.00000 \\
\hline $\mathrm{SiO}_{2}$ & - & - & - \\
\hline $\mathrm{Sr}_{3}\left(\mathrm{PO}_{4}\right)_{2}$ & - & - & - \\
\hline $\mathrm{SrCO}_{3}$ & 0.00006 & 0.00006 & 0.00006 \\
\hline $\mathrm{TcO}_{2}$ & 0.00000 & 0.00000 & 0.00000 \\
\hline $\mathrm{ZrO}_{2}$ & 0.00026 & 0.00026 & 0.00026 \\
\hline $\mathrm{KNO}_{3}$ & - & - & - \\
\hline $\mathrm{Na}_{2} \mathrm{C}_{2} \mathrm{O}_{4}$ & 0.11439 & 0.11439 & 0.11439 \\
\hline $\mathrm{Na}_{2} \mathrm{CO}_{3} \cdot \mathrm{H}_{2} \mathrm{O}$ & 0.30599 & 0.30599 & 0.30599 \\
\hline \multicolumn{4}{|l|}{$\mathrm{Na}_{2} \mathrm{SO}_{4}$} \\
\hline $\mathrm{Na}_{2} \mathrm{SO}_{4} \cdot 10 \mathrm{H}_{2} \mathrm{O}$ & - & - & - \\
\hline $\mathrm{Na}_{3} \mathrm{FSO}_{4}$ & 0.08019 & 0.08019 & 0.08019 \\
\hline $\mathrm{Na}_{3} \mathrm{NO}_{3} \mathrm{SO}_{4} \cdot \mathrm{H}_{2} \mathrm{O}$ & - & $\overline{-}$ & - \\
\hline $\mathrm{Na}_{3} \mathrm{PO}_{4} \cdot 8 \mathrm{H}_{2} \mathrm{O}$ & - & - & - \\
\hline $\mathrm{Na}_{4} \mathrm{P}_{2} \mathrm{O}_{7} \cdot 10 \mathrm{H}_{2} \mathrm{O}$ & - & - & - \\
\hline $\mathrm{Na}_{6}\left(\mathrm{SO}_{4}\right)_{2} \mathrm{CO}_{3}$ & 0.04604 & 0.04604 & 0.04604 \\
\hline $\mathrm{NaF}$ & - & - & - \\
\hline $\mathrm{NaF}\left(\mathrm{PO}_{4}\right)_{2} \cdot 19 \mathrm{H}_{2} \mathrm{O}$ & - & $\overline{-}$ & - \\
\hline $\mathrm{NaHCO}_{3}$ & - & - & - \\
\hline $\mathrm{NaNO}_{2}$ & - & - & - \\
\hline $\mathrm{NaNO}_{3}$ & 0.66221 & 0.66221 & 0.66221 \\
\hline $\mathrm{Na}_{3} \mathrm{PO}_{4} \cdot 0.25 \mathrm{NaOH} \cdot 12 \mathrm{H}_{2} \mathrm{O}$ & - & $\overline{-}$ & - \\
\hline Total Volume \% UDS & 1.4 & 1.4 & 1.4 \\
\hline Solids Concentration $(\mathrm{g} / \mathrm{L})$ & 33.9 & 33.9 & 33.9 \\
\hline
\end{tabular}


Rheological Data

\begin{tabular}{|c|c|c|c|}
\hline$\longdiv { \text { Model/model Parameter } }$ & $\overline{A N-102}$ & $\overline{A A N-102}$ & $\overline{A N N-102}$ \\
\hline Temperature $\left({ }^{\circ} \mathrm{C}\right)$ & 25 & 35 & 50 \\
\hline Source of Data & $\begin{array}{l}\text { WTP-RPT-021 } \\
\text { Rev. } 1\end{array}$ & \begin{tabular}{|l} 
WTP-RPT-021 \\
Rev. 1
\end{tabular} & $\begin{array}{l}\text { WTP-RPT-021 } \\
\text { Rev. } 1\end{array}$ \\
\hline \multicolumn{4}{|l|}{ Notes } \\
\hline \multicolumn{4}{|l|}{ Newtonian: } \\
\hline$\eta$ - Newtonian viscosity (cP) & $\mathrm{n} / \mathrm{m}$ & $\mathrm{n} / \mathrm{m}$ & $\mathrm{n} / \mathrm{m}$ \\
\hline $\mathrm{r}$ - correlation coefficient & $\mathrm{n} / \mathrm{m}$ & $n / m$ & $n / m$ \\
\hline \multicolumn{4}{|l|}{\begin{tabular}{|l} 
Ostwald (or Power Law): \\
\end{tabular}} \\
\hline $\mathrm{m}-$ the consistency coefficient $\left(\mathrm{Pa} \cdot \mathrm{s}^{-\mathrm{n}}\right)$ & 0.05943 & 0.08108 & 0.05002 \\
\hline n-the power-law exponent & 0.8135 & 0.7081 & 0.7581 \\
\hline $\mid \mathrm{r}$ - correlation coefficient & 1 & 0.97 & 0.98 \\
\hline \multicolumn{4}{|l|}{ Bingham Plastic: } \\
\hline$\tau_{\mathrm{B}}$ - the Bingham yield stress $(\mathrm{Pa})$ & 1.592 & 1.39 & 0.7177 \\
\hline$\eta \eta_{p}$ - the plastic viscosity (Pa-s) & 0.0147 & 0.0099 & 0.0094 \\
\hline $\mid \mathrm{r}$ - correlation coefficient & 0.98 & 0.98 & 0.99 \\
\hline \multicolumn{4}{|l|}{ Herschel-Bulkley: } \\
\hline$\tau_{\mathrm{H}}$ - the yield stress $(\mathrm{Pa})$ & 0.84 & 0.2102 & 0.2362 \\
\hline $\begin{array}{l}\text { k - the Herschel-Bulkely consistency } \\
\text { coefficient }\left(\text { Pas }{ }^{-b}\right)\end{array}$ & 0.04164 & 0.03547 & 0.0158 \\
\hline $\begin{array}{l}\text { b - the Hershel-Bulkely power-law } \\
\text { exponent }\end{array}$ & 0.8666 & 0.8358 & 0.9362 \\
\hline $\mid \begin{array}{r}\mathrm{r} \text { - correlation coefficient } \\
\text {. }\end{array}$ & 1 & 0.91 & 1 \\
\hline
\end{tabular}

\section{Physical Property Data}

\begin{tabular}{|c|c|c|c|}
\hline Physical Property & $\overline{A N-102}$ & AN-102 & AN-102 \\
\hline Notes & $\begin{array}{c}\text { WTP-RPT-021 } \\
\text { Table } 4.4\end{array}$ & $\begin{array}{c}\text { WTP-RPT-021 } \\
\text { Table } 4.4\end{array}$ & $\begin{array}{c}\text { WTP-RPT-021 } \\
\text { Table } 4.4\end{array}$ \\
\hline \multicolumn{4}{|l|}{ Temperature $\left({ }^{\circ} \mathrm{C}\right)$} \\
\hline \multicolumn{4}{|l|}{ Zeta Potential (mV) } \\
\hline Bulk Density $(\mathrm{g} / \mathrm{mL})$ & 1.411 & 1.411 & 1.411 \\
\hline vol\% Settled Solids & 22 & 22 & 22 \\
\hline Density of Centrifuged Solids ( $\mathrm{g} / \mathrm{mL}$ ) & 1.49 & 1.49 & 1.49 \\
\hline vol\% Centrifuged Solids & 13.4 & 13.4 & 13.4 \\
\hline wt\% Centrifuged Solids & 14.1 & 14.1 & 14.1 \\
\hline Supernatant Density $(\mathrm{g} / \mathrm{mL})$ & 1.40 & 1.40 & 1.40 \\
\hline Density of Settled Solids $(\mathrm{g} / \mathrm{mL})$ & $\mathrm{n} / \mathrm{m}$ & $\mathrm{n} / \mathrm{m}$ & $\mathrm{n} / \mathrm{m}$ \\
\hline wt $\%$ Settled Supernatant & $\mathrm{n} / \mathrm{m}$ & $\mathrm{n} / \mathrm{m}$ & $\mathrm{n} / \mathrm{m}$ \\
\hline wt\% dissolved solids in supernatant & 51.5 & 51.5 & 51.5 \\
\hline wt $\%$ total solids in Centrifuged Sludge & 52.9 & 52.9 & 52.9 \\
\hline $\mathrm{wt} \%$ Total Solids & & & \\
\hline wt\% UDS & 2.4 & 2.4 & 2.4 \\
\hline Density of Solids $(\mathrm{g} / \mathrm{cc})$ & 2.34 & 2.34 & 2.34 \\
\hline
\end{tabular}









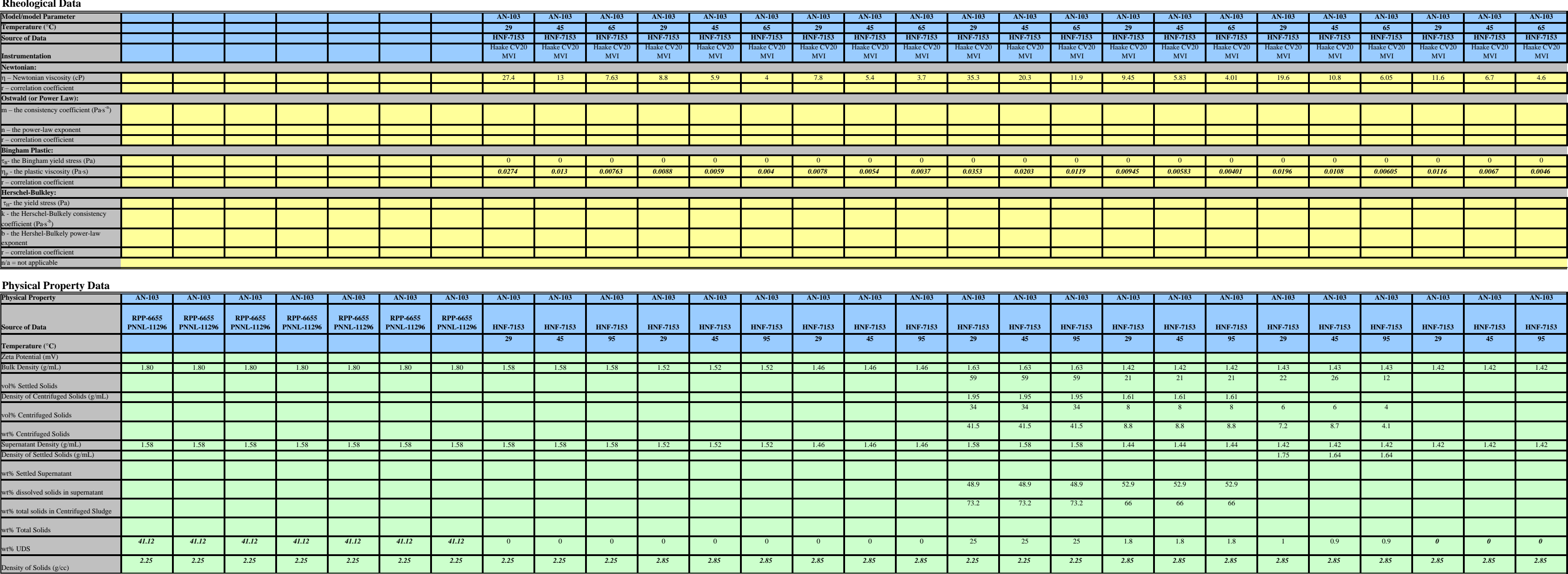




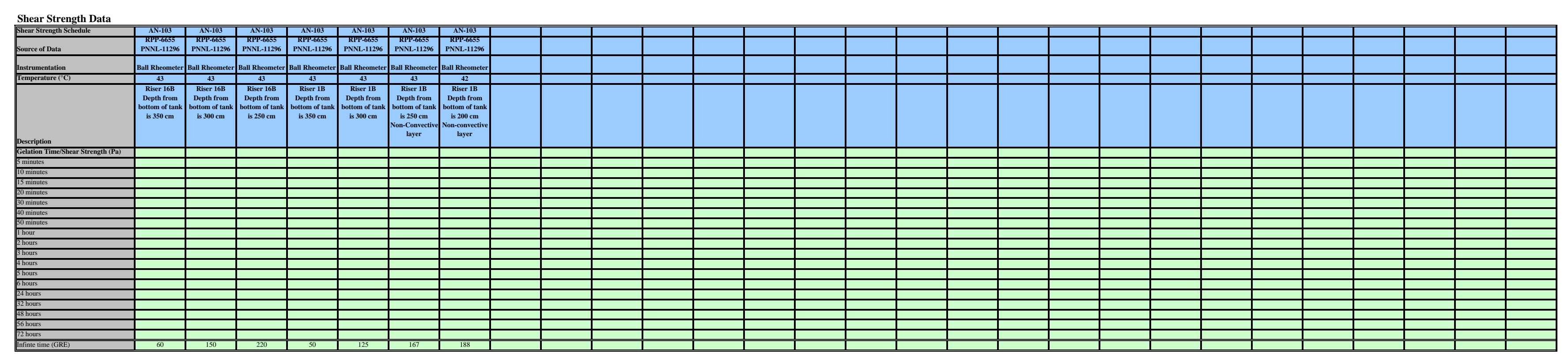




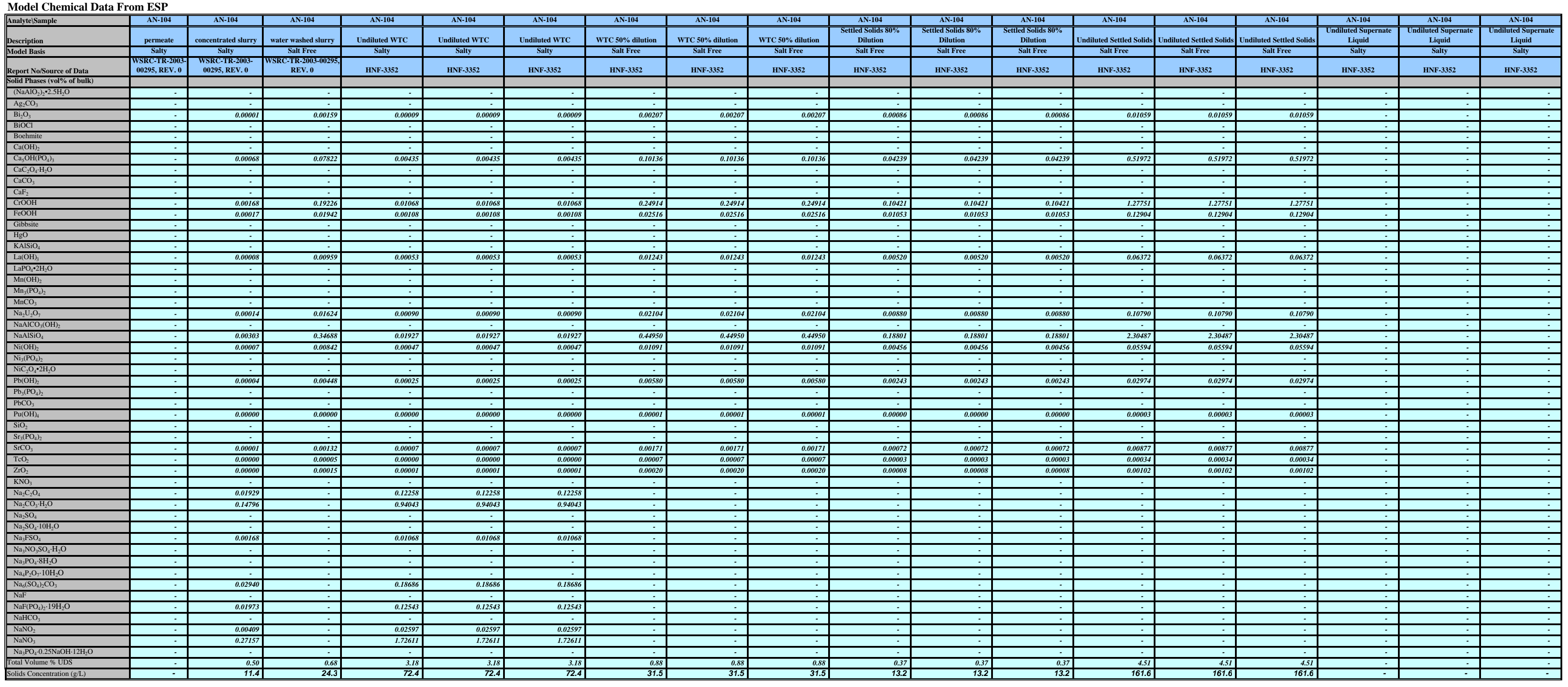




\begin{tabular}{|c|c|c|c|c|c|c|c|c|c|c|c|c|c|c|c|c|c|c|}
\hline & $\frac{10 \times 124}{25}$ & ${ }_{A N-104}^{-25}$ & $\frac{A^{A N}-104}{25}$ & $\frac{1 \mathrm{AN}-104}{25}$ & $\begin{array}{ll}A^{4 N-104} \\
45\end{array}$ & $\begin{array}{ll}A N-104 \\
65\end{array}$ & $\begin{array}{ll}A^{2}-104 \\
25\end{array}$ & $\frac{A N-194}{45}$ & $\frac{4 N-104}{65}$ & $\begin{array}{ll}\text { AN-104 } \\
25 \\
25\end{array}$ & $\frac{A N-104}{45}$ & $\begin{array}{ll}4 \mathrm{AN}-124 \\
65\end{array}$ & $\frac{A^{4-104}}{25}$ & $\frac{A N=124}{45}$ & $\frac{4 N-104}{65}$ & $\frac{A N=124}{25}$ & $\begin{array}{ll}4 A^{4 N-124} \\
45\end{array}$ & $\begin{array}{ll}A N-124 \\
65\end{array}$ \\
\hline 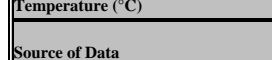 & 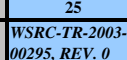 & 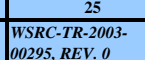 & 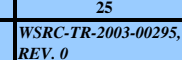 & 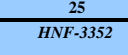 & $\frac{4532}{H N F-3352}$ & $\frac{65}{H N=3352}$ & $\frac{2532}{H N F \cdot 332}$ & $\begin{aligned} 4 N E-332 \\
H\end{aligned}$ & 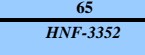 & $\frac{2532}{H N F-3352}$ & $\frac{4532}{H N F \cdot 332}$ & $\frac{65}{H N E-332}$ & $\frac{25352}{H N N-332}$ & $\frac{4532}{H N F \cdot 332}$ & $\frac{65}{H N F-352}$ & $\frac{2.3532}{H N F-332}$ & $\frac{4032}{H N F-332}$ & $\begin{array}{l}65 \\
N F-332 \\
\end{array}$ \\
\hline 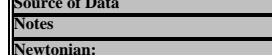 & & & & Page B7 & Page B14 & Page 221 & Page B28 & Page B35 & Page Bat2 & Page Bat9 & Page B B56 & Page Bas & Page B 70 & Page B78 & Page Be 35 & Page B92 & Page B99 & Page Bloc \\
\hline 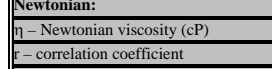 & $\frac{35}{\mathrm{~nm}}$ & $\frac{3.85}{\frac{N m}{n m}}$ & $\frac{\mathrm{n} / \mathrm{m}}{\mathrm{n} / \mathrm{m}}$ & $\frac{2879}{1.000}$ & 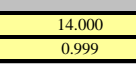 & $\frac{7.51}{0.997}$ & $\frac{\frac{8776}{0.0999}}{0.096}$ & $\frac{5,48}{0.997}$ & $\frac{4.500}{0.997}$ & $\frac{27,466}{0.9999}$ & $\frac{12.890}{0.999}$ & $\frac{7.694}{-0.992}$ & $\frac{56465}{0.999}$ & $\frac{2350}{0.0965}$ & $\frac{15.80}{0.979}$ & $\frac{1.620}{1.000}$ & $\frac{8.071}{0.995}$ & $\frac{4.57}{0.99}$ \\
\hline 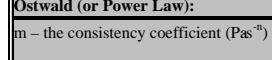 & ${ }^{\mathrm{nm}} \mathrm{m}$ & $\mathrm{n}_{\mathrm{m}}^{\mathrm{m}}$ & $\mathrm{n}^{\mathrm{m} m}$ & 0.003 & 0.024 & 0.023 & 0.012 & 0.010 & 0.020 & 0.000 & 0.039 & 0.020 & 0.064 & 0.062 & 0.088 & 0.017 & 0.008 & 0.005 \\
\hline 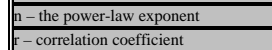 & $\frac{n \mathrm{~mm}}{\mathrm{~nm}}$ & $\frac{n \mathrm{n} / \mathrm{m}}{\mathrm{n} / \mathrm{m}}$ & $\frac{n \mathrm{~nm}}{\mathrm{n} n \mathrm{~m}}$ & $\frac{0.990}{1.000}$ & $\frac{0.905}{0.098}$ & 0.0909 & $\frac{0.933}{1000}$ & $\frac{0,097}{0.990}$ & $\frac{0.738}{0.976}$ & $\frac{0.929}{1.1000}$ & $\begin{array}{ll}0.798 \\
0.996\end{array}$ & $\frac{0.823}{1.000}$ & $\frac{0.978}{0.0999}$ & $\frac{0.836}{0.098}$ & $\frac{0.685}{0.097}$ & $\frac{1.000}{1000}$ & $\frac{1.000}{0.995}$ & $\frac{1000}{0.997}$ \\
\hline 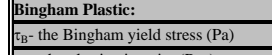 & $\frac{0}{10}$ & 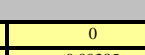 & & 0.0022 & 0.211 & 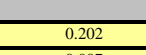 & - & 0.170 & 0.289 & 0.0 .099 & 0.0988 & 0.098 & 0.1 .166 & 0.135 & 0.3 .376 & 0.00 & 0.000 & 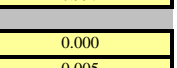 \\
\hline 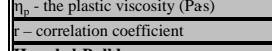 & $\frac{0.0053}{n \mathrm{~mm}}$ & $\frac{0.0335}{\frac{0 . m 5}{n}}$ & $\frac{0.019}{n+m}$ & $\frac{0.03}{1.000}$ & $\frac{0.13}{0.999}$ & 00.072 & 0.0099 & $\frac{0.003}{0.992}$ & 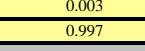 & 00.027 & 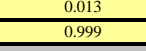 & 0.0072 & 0.0.969 & . 0.056 & $\frac{0.14}{0.979}$ & $\frac{0.07}{1.000}$ & 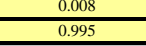 & $\frac{0.00}{0.0}$ \\
\hline 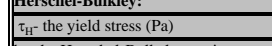 & $n_{\mathrm{mm}}$ & n & nin & 0.000 & 0.198 & 0.194 & 0.010 & 0.169 & 0.2911 & 0.000 & 0.0000 & 0.003 & 0.0011 & 0.001 & 0.001 & 0.000 & 0.000 & 0.000 \\
\hline $\begin{array}{l}\text { k-the Herschel-Bulkely consistency } \\
\text { coefficicunt (Pasas) }\end{array}$ & & $\frac{n \pi m}{n / m}$ & ${ }^{\mathrm{nm} m}$ & & & & & & & & & & & & 0.0083 & & & \\
\hline \begin{tabular}{|l|} 
e- he Hershel-Bulkely power-alaw \\
exponent
\end{tabular} & $\mathrm{nm}^{\mathrm{nm}}$ & $\mathrm{nm}$ & $2 \mathrm{~nm}$ & 0.980 & 0.980 & 0.867 & 0.966 & 1.000 & 0.986 & 0.930 & 0.799 & 0.822 & 0.978 & 0.386 & 0.685 & 1.000 & 1.000 & 1.000 \\
\hline 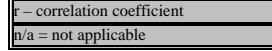 & nim & - & & 1.00 & - & ( > & 1.000 & - & 00996 & (1000 & 0.966 & sow & 0.9999 & (1098 & -7 & 1.000 & 0.995 & \\
\hline \multicolumn{19}{|l|}{$\begin{array}{l}\text { Physical Property Data } \\
\text { |rhysical Property }\end{array}$} \\
\hline Notes & & & & & & & & & & & & & & & & & & \\
\hline Temperature (C) & 25 & 25 & 25 & 25 & 45 & 65 & 25 & 45 & 65 & 25 & 45 & 65 & 25 & $\frac{45}{45}$ & 65 & 25 & 45 & 65 \\
\hline 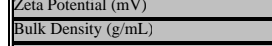 & $\frac{1.26}{1.26}$ & $\frac{1.27}{1.27}$ & 1.11 & 1.45 & $\frac{1.45}{1.45}$ & 1.45 & $\frac{1.33}{1.33}$ & 1.33 & 1.33 & $\frac{1.32}{1.32}$ & $\frac{1.32}{1.32}$ & $\frac{1.32}{1.32}$ & 1.52 & $\frac{1.52}{1.5}$ & $\frac{1.52}{1.52}$ & 1.42 & 1.42 & 1.42 \\
\hline 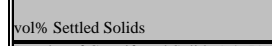 & & & & & & & & & & & & & & & & & & nim \\
\hline I & $\frac{o}{n \mathrm{~m}}$ & $\frac{0}{n / m}$ & $\frac{o}{n / m}$ & $\frac{1.52}{27}$ & $\frac{1.52}{27}$ & $\frac{1.52}{27}$ & $\frac{1.98}{3}$ & $\frac{1.98}{3}$ & $\frac{1.98}{3}$ & $\frac{1.45}{6}$ & $\frac{1.45}{6}$ & $\frac{1.45}{6}$ & $\frac{1.62}{50}$ & $\frac{1.62}{50}$ & $\frac{1.52}{50}$ & $\frac{0}{0}$ & $\frac{0}{0}$ & $\frac{0}{0}$ \\
\hline 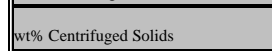 & $\frac{\mathrm{nm}}{\mathrm{nm}}$ & $\mathrm{n} / \mathrm{m}$ & $\frac{n \mathrm{~nm}}{\mathrm{~nm}}$ & $\frac{\mathrm{nm}}{\mathrm{m} m}$ & $\frac{\mathrm{nm}}{\mathrm{nm}}$ & $\frac{n+m}{n m}$ & $n^{\prime \prime m}$ & $n^{\prime \prime m}$ & ${ }_{\mathrm{n} / \mathrm{m}}$ & $\mathrm{nm}$ & $\mathrm{n} / \mathrm{m}$ & $n$ & $\mathrm{n}^{\mathrm{n} m}$ & ${ }_{\mathrm{nm} m}$ & $\mathrm{~nm}_{\mathrm{m}}$ & $\mathrm{n} / \mathrm{m}$ & $n^{\prime \prime m}$ & nim \\
\hline 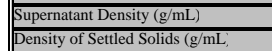 & $\frac{1.26}{n+m}$ & $\frac{1.26}{n+m}$ & $\frac{1.09}{n+09}$ & $\frac{1.42}{10 m}$ & $\frac{1.42}{n+m}$ & $\frac{1.42}{1 / m}$ & $\frac{1.31}{n \text { ngm }}$ & $\frac{1.31}{1 \mathrm{vm}}$ & $\frac{1.31}{10 m}$ & $\frac{1.31}{1 \mathrm{~nm}}$ & 1.31 & 1.31 & $\frac{1.42}{1 \mathrm{vm}}$ & $\frac{1.42}{\mathrm{~N} / \mathrm{m}}$ & $\frac{1.42}{\mathrm{~N}_{\mathrm{m}}}$ & $\frac{1.42}{1 / n}$ & $\frac{1.42}{10 \mathrm{~m}}$ & $\frac{1.42}{n ! m}$ \\
\hline w\%o\% Setled Supenatant & $\mathrm{nm}^{\mathrm{nm}}$ & n/m & $\mathrm{nm}^{\mathrm{nm}}$ & $\frac{\mathrm{nm}}{\mathrm{mm}}$ & $\frac{\mathrm{nm}}{\mathrm{nm}}$ & $\mathrm{n}_{\mathrm{m} m}^{\mathrm{m}}$ & $\mathrm{nm}$ & $\mathrm{nm}_{\mathrm{mm}}$ & $\mathrm{nm}^{\mathrm{mm}}$ & $\frac{\mathrm{mm}}{\mathrm{nm}}$ & $\frac{\mathrm{n} / \mathrm{m}}{\mathrm{n}}$ & $\mathrm{nm}_{\mathrm{m}}$ & $\mathrm{n}^{\mathrm{m} m}$ & $\frac{\mathrm{mm}}{\mathrm{nm}}$ & $n^{n / m}$ & n/m & $\frac{1}{\mathrm{n} / \mathrm{m}}$ & nim \\
\hline w\%o dissolved solids in superatant & 29.3 & 29.1 & 11.6 & $\mathrm{~nm}$ & $\frac{\mathrm{nm}}{\mathrm{nm}}$ & $\frac{n}{n / m}$ & $\frac{\mathrm{nm}}{\mathrm{nm}}$ & $\frac{\mathrm{nm}}{\mathrm{nm}}$ & ${ }^{n m}$ & $\mathrm{~nm}_{\mathrm{m}}$ & $\frac{\mathrm{n} / \mathrm{m}}{\mathrm{n}}$ & $\frac{\mathrm{nm}}{\mathrm{nm}}$ & ${ }^{n m}$ & ${ }^{\mathrm{n} m \mathrm{~m}}$ & ${ }^{\mathrm{n} m \mathrm{~m}}$ & $\frac{\mathrm{nm}}{\mathrm{nm}}$ & $\frac{\mathrm{n} / \mathrm{m}}{\mathrm{n}}$ & $n$ \\
\hline w\%\% total solids in Centrifuged Sludge & $\mathrm{n}^{\mathrm{n} / \mathrm{m}}$ & $\mathrm{n}^{\mathrm{n} m}$ & $\mathrm{n}^{\mathrm{nm}}$ & $\mathrm{nm}^{\mathrm{nm}}$ & $\mathrm{n}^{\mathrm{nm}}$ & $\mathrm{n}^{\mathrm{n} / \mathrm{m}}$ & $\mathrm{n}^{\mathrm{n} / \mathrm{m}}$ & $\mathrm{n}^{\mathrm{n} m \mathrm{~m}}$ & $\mathrm{n}^{\mathrm{n} / \mathrm{m}}$ & $\mathrm{nm}$ & 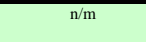 & $\mathrm{n} / \mathrm{m}$ & $\mathrm{n}^{\mathrm{m} m}$ & $\mathrm{n}^{\mathrm{m} m \mathrm{~m}}$ & $\mathrm{n}^{\mathrm{m} m}$ & $\frac{\mathrm{n} m \mathrm{~m}}{\mathrm{n}}$ & $\frac{n}{n / m}$ & $\mathrm{n} / \mathrm{m}$ \\
\hline wo\% Toal Solids & 203 & ${ }^{30}$ & $\frac{11.6}{11}$ & $\frac{\mathrm{nm}}{\mathrm{nm}}$ & $\mathrm{n} / \mathrm{m}$ & $\mathrm{n} / \mathrm{m}$ & $\mathrm{n} / \mathrm{m}$ & $\frac{\mathrm{nm}}{\mathrm{nm}}$ & $\mathrm{n} / \mathrm{m}$ & $\frac{\mathrm{nm}}{\mathrm{n} m}$ & $\mathrm{n} / \mathrm{m}$ & $\mathrm{n} / \mathrm{m}$ & $\mathrm{n}^{\mathrm{n} / \mathrm{m}}$ & $\mathrm{nm}^{\mathrm{m} m}$ & ${ }^{\mathrm{n} m \mathrm{~m}}$ & $\mathrm{n} / \mathrm{m}$ & $\mathrm{n} / \mathrm{m}$ & $\mathrm{n} / \mathrm{m}$ \\
\hline wor uns & $0^{\circ}$ & 0.9 & 2.2 & ${ }^{5}$ & & 等 & 237 & 237 & 237 & 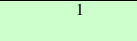 & 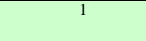 & +5 & 10.65 & 10.05 & ${ }^{10.65}$ & $0^{\circ}$ & $0^{\circ}$ & 0 \\
\hline$s \operatorname{cosec}^{2}$ & 2.28 & 2.28 & 3.58 & 228 & 2.28 & 2.28 & 3.58 & 3.58 & $\frac{3.58}{3.58}$ & 3.58 & 3.58 & 3.58 & $\begin{array}{l}3.58 \\
\end{array}$ & 3.58 & 3.58 & 228 & 2.28 & $2.28>-28$ \\
\hline
\end{tabular}



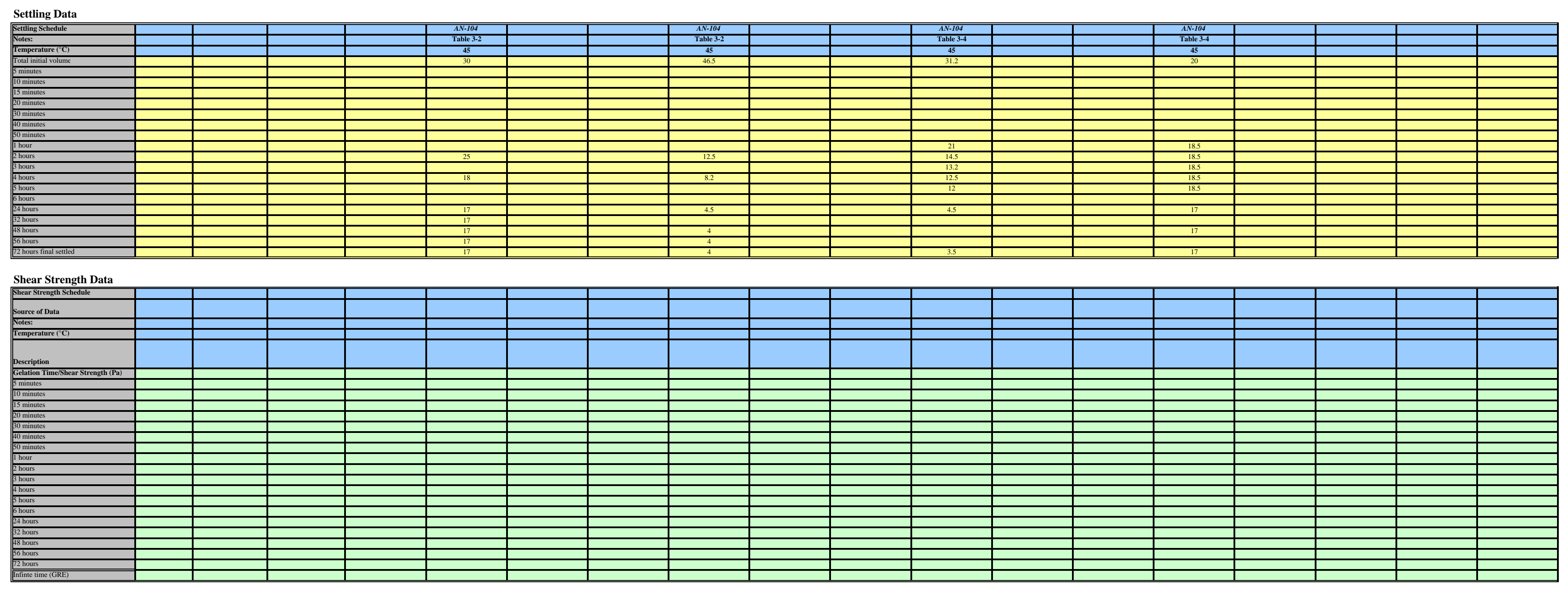


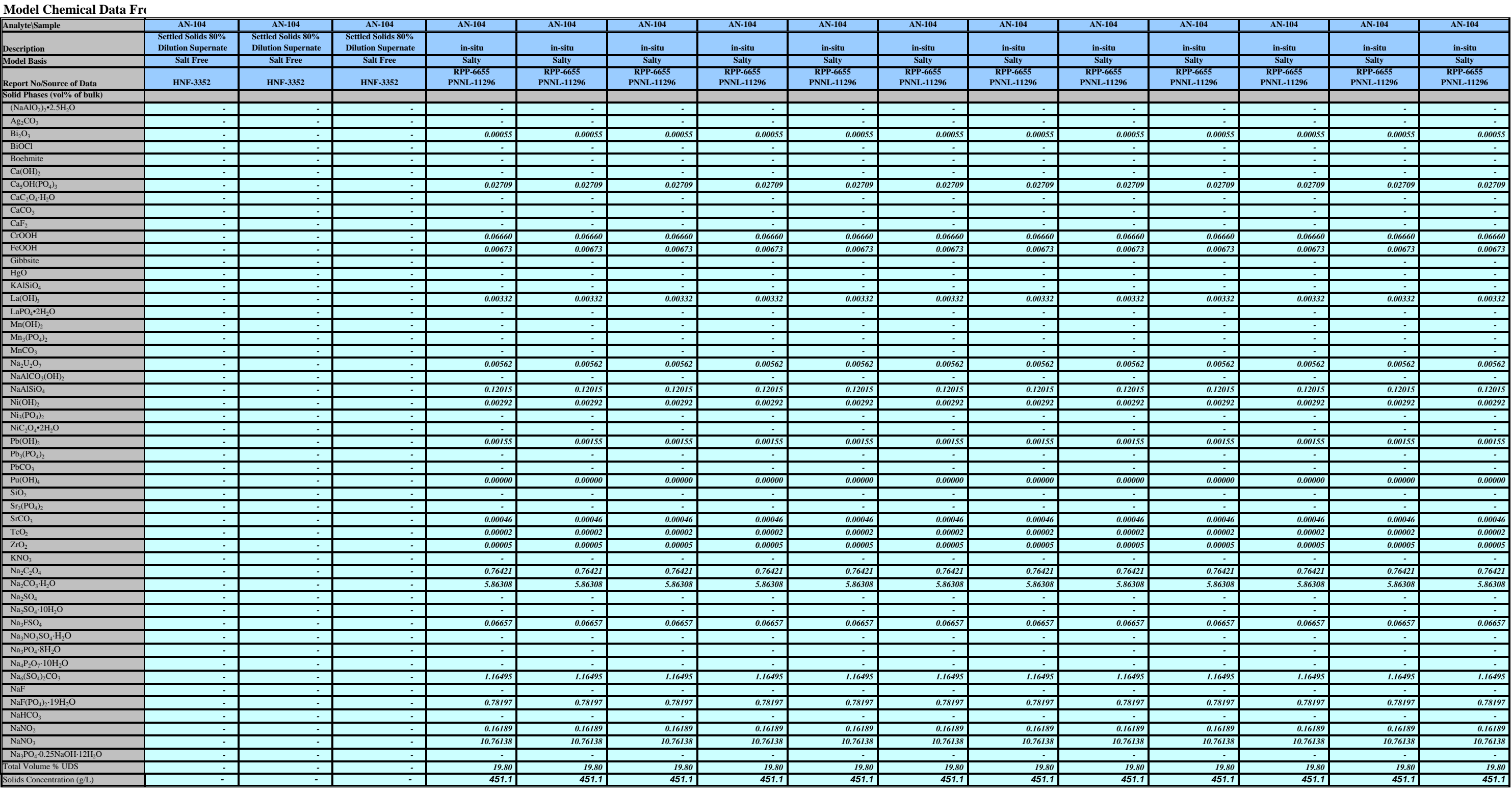




\section{Rheological Data}

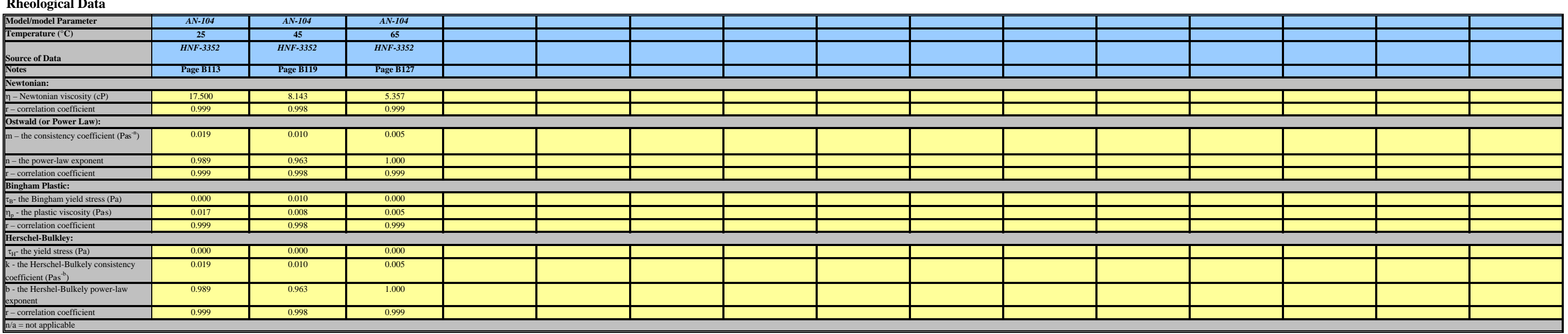

\begin{tabular}{|c|c|c|c|c|c|c|c|c|c|c|c|c|c|c|c|}
\hline $\begin{array}{l}\text { Physical Property Data } \\
\text { Phylycial }\end{array}$ & $\mid \overline{A N-104}$ & $\frac{\overline{N N-104}}{N^{2}}$ & $4 \overline{A N-104}$ & AN:104 & AN-104 & AN-104 & AN & AN & ${ }^{A N-100}$ & ANN-104 & AN-104 & AN & ANN-104 & ${ }^{A N-104}$ & 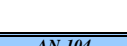 \\
\hline Nots & & & & & & & & & & & & & & & \\
\hline Temperature (C) & 25 & ${ }_{45}$ & 65 & & & & & & & & & & & & \\
\hline 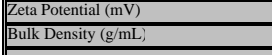 & 1.31 & $\frac{1.31}{1.31}$ & . & $\frac{1.59}{1.59}$ & $\frac{1.59}{1.59}$ & 1.59 & $\frac{1.59}{9}$ & 1.59 & 1.59 & 1.59 & 1.59 & 1.59 & $\frac{1.59}{1.59}$ & $\frac{1.59}{1.59}$ & $\frac{1.59}{1.59}$ \\
\hline 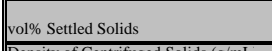 & ${ }^{n / m}$ & $\frac{\mathrm{mm}}{\mathrm{mm}}$ & ${ }^{n / m}$ & & & & & & & & & & & & \\
\hline 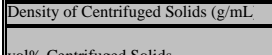 & $\frac{0}{0}$ & $\frac{0}{0}$ & 0 & & & & & & & & & & & & \\
\hline w\%\% Cenrifinged Solids & ${ }^{n / m}$ & $\mathrm{~nm}_{\mathrm{m}}$ & ${ }^{n / m}$ & & & & & & & & & & & & \\
\hline 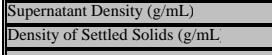 & $\frac{1.31}{\mathrm{nim}}$ & $\frac{131}{\mathrm{nim}}$ & $\frac{1.31}{\mathrm{~N} n \mathrm{~m}}$ & 1.42 & 1.42 & 1.42 & 1.42 & 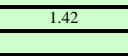 & $\frac{1.42}{4-1}$ & 1.42 & 1.42 & 1.42 & 1,42 & 1.42 & 1.42 \\
\hline wi\%s Settled Supematant & $\mathrm{n} / \mathrm{m}$ & 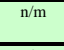 & & & & & & & & & & & & & \\
\hline \begin{tabular}{|l} 
wo dissolve solids in supematant \\
\end{tabular} & ${ }^{n / m}$ & $\mathrm{~nm}$ & ${ }^{n \mathrm{~m}}$ & 50 & 50 & 50 & 50 & 50 & 50 & 50 & 50 & 50 & 50 & 50 & 50 \\
\hline w1\%\% total solids in Centrifuged Sludge & ${ }^{n / m}$ & $\frac{\mathrm{nm}}{\mathrm{mm}}$ & ${ }^{n / m}$ & & & & & & & & & & & & \\
\hline \begin{tabular}{|l|l|l|l}
$w_{6}$ Toal Solids \\
\end{tabular} & $n^{n / m}$ & $\mathrm{~nm}_{\mathrm{mm}}$ & n/m & 55 & ${ }^{55}$ & ${ }^{55}$ & 55 & ${ }^{55}$ & 55 & ${ }^{55}$ & 55 & 55 & 55 & 55 & ${ }^{55}$ \\
\hline wot uns & $0^{0}$ & 0 & 0 & 28337065002 & 283.37450502 & 20337450502 & 203.374050502 & 20377460502 & 20337405002 & 2033706050202 & 203374050502 & 203373050502 & 20373705002 & 28337065002 & 20337405002 \\
\hline 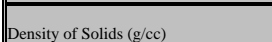 & 3.58 & 3.58 & 3.58 & 2.28 & 228 & 2.28 & 2288 & 2.28 & 2.28 & ${ }_{228}^{228}$ & 2.28 & 228 & 228 & 2.28 & 2.28 \\
\hline
\end{tabular}



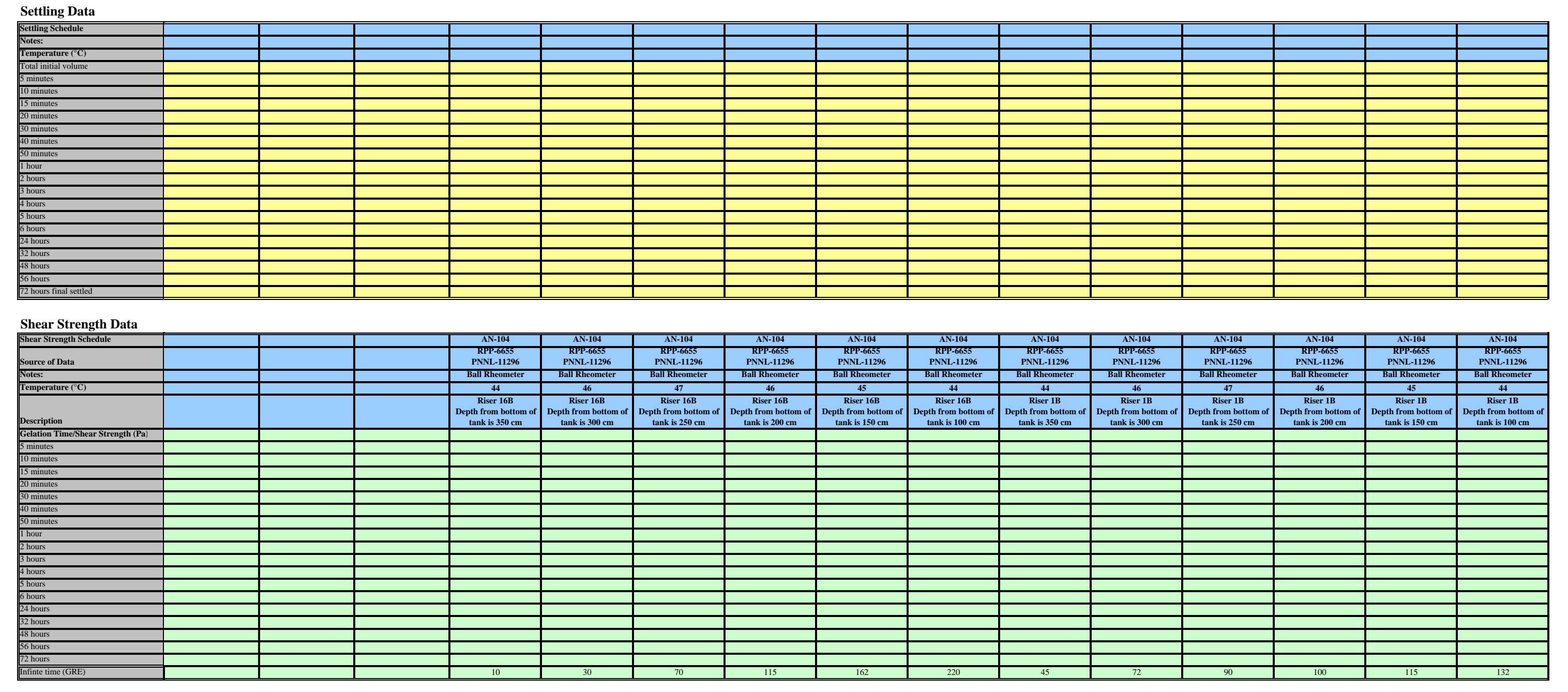
Model Chemical Data From ESP

\begin{tabular}{|c|c|c|c|c|c|c|c|c|c|c|c|c|c|c|c|}
\hline 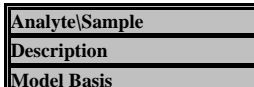 & 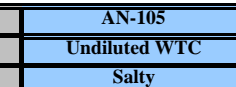 & 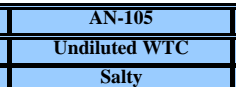 & $\begin{array}{l}\text { AN-105 } \\
\text { Undiluted WTC } \\
\text { Sally }\end{array}$ & 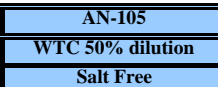 & 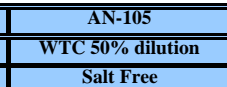 & 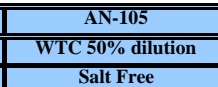 & $\begin{array}{l}\text { AN-105 } \\
\text { Dilution } \\
\text { Salt free }\end{array}$ & \begin{tabular}{c|c} 
AN-105 \\
Dilution \\
Salt Free \\
\end{tabular} & $\begin{array}{c}\mathrm{AN}-105 \\
\text { Dilitition } \\
\text { Salt Free }\end{array}$ & 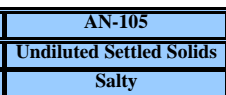 & \begin{tabular}{|l|} 
AN-105 \\
Undiluted Setter Solids \\
Salty
\end{tabular} & 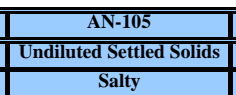 & $\begin{array}{c}\text { AN-105 } \\
\text { Liquidi } \\
\text { Sally }\end{array}$ & \begin{tabular}{|c|} 
AN-105 \\
Lifimid \\
Sally \\
\end{tabular} & \begin{tabular}{|c|} 
AN-105 \\
Liquiid \\
Salty
\end{tabular} \\
\hline $\begin{array}{l}\text { Model Basis } \\
\text { Report No Source of Data }\end{array}$ & 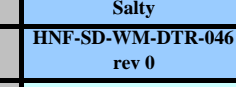 & 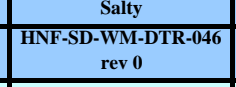 & 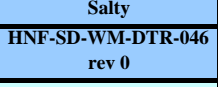 & 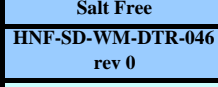 & 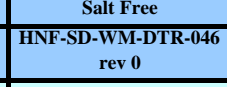 & 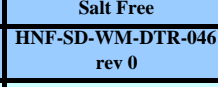 & \begin{tabular}{|c} 
Salt Free \\
$\begin{array}{c}\text { HNF-SD-WM-DTR-046 } \\
\text { rev 0 }\end{array}$ \\
\end{tabular} & 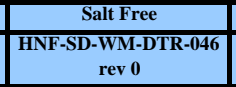 & 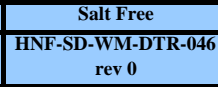 & $\begin{array}{c}\text { Sally } \\
\text { HNN-SD-WM-DTR-046 } \\
\text { revo }\end{array}$ & \begin{tabular}{|c|} 
Saly \\
HNN-SD-WM-DTR-046 \\
rev0
\end{tabular} & $\begin{array}{l}\text { Saly } \\
\text { HNN-SD-WM-DTR-046 } \\
\text { revo }\end{array}$ & $\begin{array}{c}\text { Saly } \\
\substack{\text { HNN-SD-WM-DTR-246 } \\
\text { rev }}\end{array}$ & 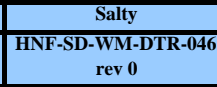 & 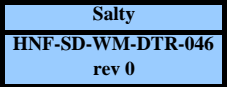 \\
\hline 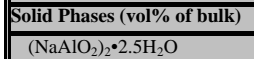 & & & & & & & & & & & & & & & \\
\hline \begin{tabular}{|l|l|}
$\mathrm{AB}_{\mathrm{B} C \mathrm{O}_{3}}$ \\
$\mathrm{~B}_{\mathrm{O}}$
\end{tabular} & 8000327 & 8000327 & 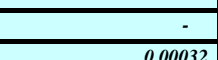 & (800025 & 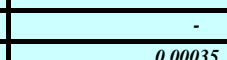 & 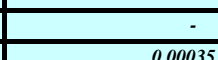 & 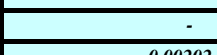 & 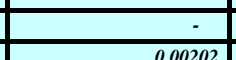 & ${ }^{2002020}$ & 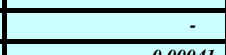 & sement & sencent & & & \\
\hline & 0.00032 & 0.00323 & 0.00032 & 0.00035 & 0.00035 & 0.00035 & 0.00222 & 0.002024 & 0.002022 & 0.00041 & 0.00041 & 0.00041 & & & \\
\hline \begin{tabular}{|l|l|} 
Boehmite \\
Carloth
\end{tabular} & & & & & & & & & & & & & & & \\
\hline 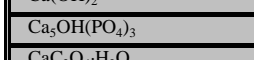 & 0.03600 & 0.03600 & 0.03600 & 0.03950 & 0.03550 & 0.03950 & 0.23032 & 0.23032 & 0.23032 & 0.04629 & 0.04629 & 0.0429 & & & \\
\hline $\mid$\begin{tabular}{|l|l|}
$\mathrm{CaC}_{2} \mathrm{O}_{4} \mathrm{H}_{2} \mathrm{O}$ \\
$\mathrm{CaCO}_{3}$
\end{tabular} & & & & & & & & & & & & & & & \\
\hline \begin{tabular}{|l|l}
$\mathrm{CaF}_{2}$ \\
$\mathrm{Car}_{2}$
\end{tabular} & & & & & & & & & & & & & & & \\
\hline 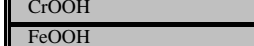 & $\frac{0.04971}{0.028020}$ & $\begin{array}{ll}0.04971 \\
\end{array}$ & 0.09971 & 0.05453 & 0.05453 & 0.054533 & 0.31801 & 0.31801 & 0.31801 & 0.06391 & 0.06391 & 0.06391 & & & \\
\hline \begin{tabular}{|l|l} 
Gibbsite \\
\end{tabular} & 0.0 .035934 & $\begin{array}{l}0.03649 \\
0.5634 \\
\end{array}$ & 0.0.65394 & o. 0.0320233 & o. 0.06353 & 0.003033 & $\begin{array}{ll}0.02233 \\
3.61681\end{array}$ & $\begin{array}{l}0.02334 \\
3.6681 \\
\end{array}$ & 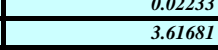 & $\begin{array}{l}0.00449 \\
0.72687 \\
\end{array}$ & $\begin{array}{l}0.042497 \\
0.7267\end{array}$ & $\begin{array}{l}0.04249 \\
0.72687 \\
0\end{array}$ & & & \\
\hline \begin{tabular}{|l|l|}
$\mathrm{HgO}^{2}$ \\
$\mathrm{KAlSO}_{4}$
\end{tabular} & 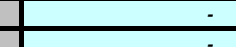 & & & & & & & & & & & & & & \\
\hline 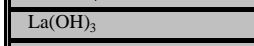 & & & & & & & & & & & & & & & \\
\hline 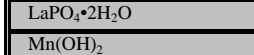 & 0.00042 & $\begin{array}{ll}0.00042 \\
-2\end{array}$ & $\begin{array}{ll}0.00042 \\
-\end{array}$ & 0.00046 & 0.00046 & 0.00046 & 0.02269 & 0.0026 & 0.02269 & 0.00054 & 0.00054 & 0.00054 & & & \\
\hline \begin{tabular}{|l|l}
$\mathrm{Mn}_{3}\left(\mathrm{PO}_{0}\right)_{2}$ \\
\end{tabular} & & & & & & & & & & & & & & & \\
\hline$\frac{\mathrm{MnCO}_{3}}{\mathrm{Na}_{\mathrm{Na}} \mathrm{U}_{0} \mathrm{O}}$ & 0.00143 & . 0.00143 & 0.00143 & 0.00157 & 0.00157 & 0.00157 & 0.00917 & .0.091 & 0.00917 & $\overline{0.00184}$ & D.00184 & D.00184 & & & \\
\hline $\mathrm{NaACO}_{3}(\mathrm{OH})_{2}$ & & & & & & & & & & & 0.00164 & & & & \\
\hline \begin{tabular}{|l|l|} 
NaAlsio, \\
Nifothe
\end{tabular} & 0.000011 & $\begin{array}{ll}0.00011 \\
0.00139 \\
\end{array}$ & $\begin{array}{l}0.00011 \\
0.00103\end{array}$ & $\begin{array}{l}0.00013 \\
0.0013\end{array}$ & $\begin{array}{l}0.00013 \\
0.0013 \\
0.013\end{array}$ & 0.00013 & $\begin{array}{l}0.00073 \\
0.00695\end{array}$ & $\begin{array}{ll}0.00073 \\
0.00559 \\
\end{array}$ & $\begin{array}{ll}0.00073 \\
0.00695\end{array}$ & $\begin{array}{l}0.00015 \\
0.00132 \\
\end{array}$ & $\begin{array}{ll}0.00015 \\
0.0137\end{array}$ & $\begin{array}{l}0.00015 \\
0.00132 \\
\end{array}$ & & & \\
\hline 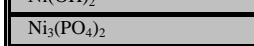 & 0 & & & & & & & & & & & & & & \\
\hline \begin{tabular}{|l|l|l}
$\mathrm{NiC}_{2} \mathrm{O}_{4} \cdot 2 \mathrm{H}_{2} \mathrm{O}$ \\
$\mathrm{Pb}\left(\mathrm{OH} \mathrm{H}_{2}\right.$
\end{tabular} & 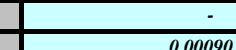 & 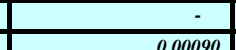 & 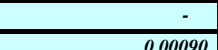 & 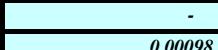 & 000099 & 0000098 & 800574 & 80.0574 & 800574 & (00015 & nonct & nomet & & & \\
\hline \begin{tabular}{|l}
$\mathrm{Pb}_{3}\left(\mathrm{PO}_{2} / 2 / 2\right.$ \\
\end{tabular} & & & & & & & & & $0.0055 / 4$ & 0.0015 & 0.00113 & 0.00113 & & & \\
\hline$\frac{\mathrm{PBCO}_{3}}{\mathrm{PBCO}_{3}}$ & 0,0000 & & 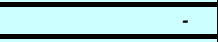 & & $\bar{F}$ & 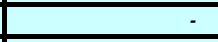 & 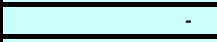 & 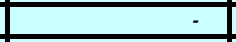 & 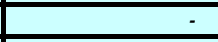 & & 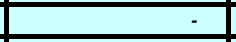 & & & & \\
\hline 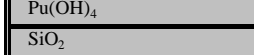 & 0.00000 & 0.00000 & 0.00000 & 0.00000 & 0.00000 & 0.00000 & 0.00001 & 0.00001 & 0.00001 & 0.00000 & 0.00000 & 0.00000 & & & \\
\hline 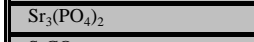 & & & & & & & & & & & & & & & \\
\hline 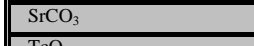 & 0.00024 & D. 0.00244 & 0.00024 & $\frac{0.0026}{0.026}$ & 0.00026 & $\begin{array}{l}0.00026 \\
0\end{array}$ & 0.00153 & . & $\frac{0.00153}{0.03}$ & 0.00031 & |0.00031 & 0.000311 & & & \\
\hline$\frac{\mathrm{T}_{\mathrm{CO}} \mathrm{CO}_{2}}{\mathrm{Z}_{\mathrm{T}} \mathrm{r}_{2}}$ & 0.00001 & 0.00001 & 0.00001 & 0.00001 & 0.00001 & 0.00001 & 0.00005 & 0.00005 & 0.00005 & 0.00001 & 0.00001 & 2.000001 & & & \\
\hline $\mathrm{KNO}_{3}$ & & & & & & & & & & & & & & & \\
\hline 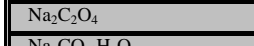 & $\frac{0.32204}{0.2007}$ & $\begin{array}{ll}0.32204 \\
02007 \\
\end{array}$ & 0.032204 & & & & & & & 0.41405 & 0.41405 & 0.44145 & & & \\
\hline $\mathrm{Na}_{2} \mathrm{Na}_{3} \mathrm{C}_{3} \mathrm{H}_{2}$ & 2.98997 & 2.98997 & 208997 & & & & & & & 3.84423 & 3.84423 & 3.84425 & & & \\
\hline $\mathrm{N}_{2} \mathrm{SO}_{4}^{4} \mathrm{OH}_{2} \mathrm{O}$ & & & & & & & & & & & & & & & \\
\hline \begin{tabular}{|l|l|}
$\mathrm{Na}_{3} \mathrm{FSO}_{2}$ \\
$\mathrm{~N}_{3}$
\end{tabular} & 0.30222 & $\begin{array}{ll}0.30222 \\
0\end{array}$ & $\begin{array}{ll}0.30222 \\
\end{array}$ & & & & & & & 0.38855 & 0.38856 & 0.38856 & & & \\
\hline 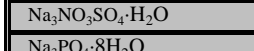 & & 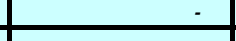 & & & & & & & & & & & & & \\
\hline 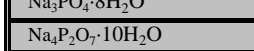 & $\ldots$ & & & & & & & & & & & & & & \\
\hline 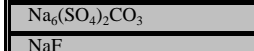 & 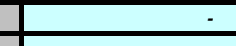 & - & & & & & & & & & & & & & \\
\hline $\mid$\begin{tabular}{|l}
$\mathrm{NaF}\left(\mathrm{PO}_{4}\right)_{2} \cdot 19 \mathrm{H}_{2} \mathrm{O}$ \\
\end{tabular} & 0.53076 & 0.53076 & $\begin{array}{l}0.53376 \\
\end{array}$ & & & & & & & 0.68240 & 0.68240 & 0.68240 & & & \\
\hline & & & & & & & & & & & & & & & \\
\hline $\mathrm{NaNO}_{3}$ & 3.36654 & $\begin{array}{ll}3.36854 \\
\end{array}$ & 3.36654 & & & & & & & 4.33098 & 4.33098 & 4.33098 & & & \\
\hline 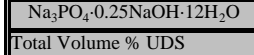 & 8.1 & ${ }_{8.17}$ & & & & & & & & & 10.51 & 10.5 & & & \\
\hline & 186.0 & $\begin{array}{l}186.0 \\
\end{array}$ & $\begin{array}{l}186.0 \\
\end{array}$ & $\frac{1.25}{19.5}$ & $\frac{1.25}{19.5}$ & 19.5 & 214.0 & 114.0 & 114.0 & 239.2 & $\frac{239.2}{239.2}$ & 239.2 & & & \\
\hline
\end{tabular}


Rheological Data

\begin{tabular}{|c|c|c|c|c|c|c|c|c|c|c|c|c|c|c|c|}
\hline 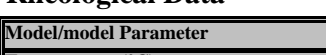 & ${ }_{A N-105}$ & ${ }_{A N-105}$ & ${ }_{A N-105}$ & ${ }_{A N-105}$ & ${ }_{A N-105}$ & ${ }_{A N-105}$ & ${ }_{A N-105}$ & ${ }_{A N-105}$ & ${ }_{A N-105}$ & ${ }_{A N-105}$ & $\overline{A N-105}$ & ${ }_{A N-105}$ & ${ }_{A N-105}$ & ${ }_{A N-105}$ & ${ }^{A N-105}$ \\
\hline $\begin{array}{l}\text { Iemperature }(\mathrm{C}) \\
\text { Source of Data } \\
\text { Note }\end{array}$ & 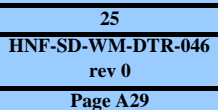 & \begin{tabular}{|c|}
5 \\
HNF-SD-WM-DTR-246 \\
rev \\
Page A37
\end{tabular} & 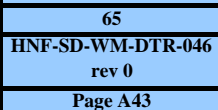 & $\begin{array}{c}25 \\
\text { HNF-SD-WM-DTR-046 } \\
\text { rev } \\
\text { Page A6 }\end{array}$ & \begin{tabular}{|c|}
5 \\
HNF-SD-WM-DTR-046 \\
rev \\
Page Als
\end{tabular} & 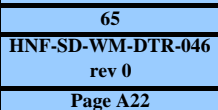 & 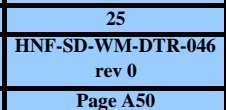 & \begin{tabular}{|c|}
5 \\
HNE-SD-WM-DTR-046 \\
rev \\
Pega A57
\end{tabular} & 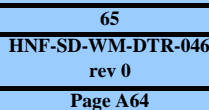 & 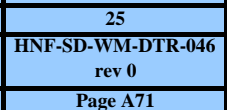 & 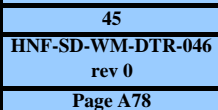 & 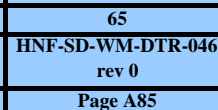 & \begin{tabular}{|c|}
25 \\
HNN-SD-WM-DTR-046 \\
revo \\
Thale 2.3 .34
\end{tabular} & 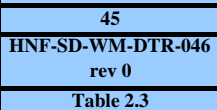 & 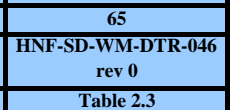 \\
\hline 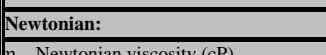 & & & & & & & & & & & & & & & \\
\hline 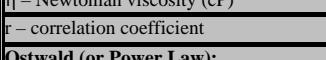 & 1.000 & $\frac{3.000}{1.000}$ & $\frac{0.998}{0.998}$ & $\frac{0.000}{1.000}$ & 0.997 & 0.994 & 0.997 & . & $\frac{2.024}{0.994}$ & 0.994 & 0.0999 & 0.997 & 10.00 & .00 & \\
\hline 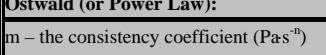 & 0.020 & 0.017 & 0.011 & 0.006 & 0.006 & 0.003 & 0.009 & 0.004 & 0.003 & 0.068 & 0.057 & 0.065 & & & \\
\hline n-the power-law exponent & 0.996 & 0.899 & 0.095 & 0.995 & 0.907 & 1.000 & 0.961 & 1.000 & 0.953 & 0.947 & 0.841 & 0.742 & & & \\
\hline 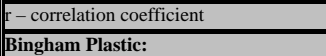 & & & & & & & & & & & & & & & \\
\hline $\mathrm{T}_{\mathrm{B}-\text { the Bingham yield stress (Pa) }}$ & 0.000 & 0.000 & 0.000 & 0.000 & 0.002 & 0.000 & 0.000 & 0.000 & 0.000 & 0.000 & 0.543 & 0.581 & 0.000 & 0.000 & 0.000 \\
\hline 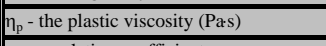 & 0.019 & 0.010 & 0.006 & 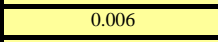 & 0.004 & 8002 & 4 & $\overline{0.004}$ & 0.003 & 0.0511 & $\sqrt{2 n^{2}}$ & $\overline{0.013}$ & $\overline{0.012}$ & 0.006 & 0.004 \\
\hline 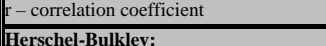 & 1.000 & 1.000 & 0.998 & 1.000 & 0.997 & 0.994 & 0.997 & 0.991 & 0.994 & 0.994 & 0.999 & 0.997 & & & \\
\hline 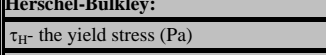 & 0.028 & 0.028 & 0.041 & 0.000 & 0.000 & 0.000 & 0.035 & 0.028 & 0.017 & 0.025 & 0.512 & 0.496 & & & \\
\hline 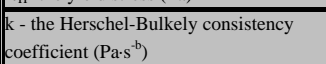 & 0,020 & 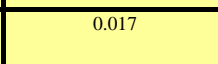 & 0.010 & $\mathrm{C}^{\circ}$ & ${ }^{2}$ & 0.003 & 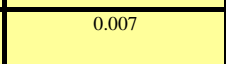 & ${ }^{2}$ & & 4005 & 0,026 & 40025 & & & \\
\hline 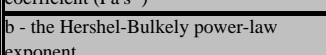 & 0.997 & 0.905 & 0.912 & 1.000 & 0.913 & 1.000 & 0.990 & 1.000 & 0.993 & $\overline{998}$ & 0.966 & 888 & & & \\
\hline conereation coefficicie & 1.000 & 0.998 & 0.993 & 1000 & 0.996 & & 0.997 & 0.991 & $\overline{0.99}$ & 0.99 & 1.000 & 1.000 & & & \\
\hline
\end{tabular}

Physical Property Data

\begin{tabular}{|c|c|c|c|c|c|c|c|c|c|c|c|c|c|c|c|}
\hline Physical Property & AN-105 & $\mathrm{AN}-105^{-105}$ & $\mathrm{AN}-105$ & ${ }_{\mathrm{AN}-105}$ & ${ }_{A N-105}$ & ${ }_{\mathrm{AN}-105}$ & $\frac{A N-105}{A N}$ & $\mathrm{AN}-105^{\mathrm{A}}$ & $\begin{array}{l}\mathrm{AN}-105 \\
\end{array}$ & $\mathrm{AN}-105^{\mathrm{A}}$ & ${ }_{A N-105}$ & ${ }^{\mathrm{AN}-105}$ & ${ }_{\mathrm{AN}-105}$ & $\mathrm{AN}-105^{\mathrm{AN}}$ & $\mathrm{AN-105}$ \\
\hline Notes & & & & & & & & & & & & & & & \\
\hline Temperature $\left({ }^{\circ} C\right)$ & 25 & 45 & 65 & 25 & 45 & 65 & 25 & 45 & 65 & 25 & 45 & 65 & 25 & 45 & 65 \\
\hline \begin{tabular}{|l} 
Zeta Potental (mV) \\
Bulk Densiy (g/mL)
\end{tabular} & 1.49 & 1.49 & 1.49 & 1.33 & $\overline{1.33}$ & $\overline{1.33}$ & $\overline{1.34}$ & $\overline{1.34}$ & $\overline{1.34}$ & $\overline{1.51}$ & 4.51 & $\left.\frac{1.51}{1.5}+2\right)$ & 1.42 & $\frac{1.42}{142}$ & $\frac{1.42}{1.2}+2>$ \\
\hline volos Settled Solids & $\mathrm{n} / \mathrm{m}$ & $\mathrm{n} / \mathrm{m}$ & $\mathrm{n} / \mathrm{m}$ & $\mathrm{n} / \mathrm{m}$ & $\mathrm{n} / \mathrm{m}$ & $n^{\prime / m}$ & $\mathrm{n}^{\mathrm{m}}$ & $\mathrm{n} / \mathrm{m}$ & $\mathrm{n} / \mathrm{m}$ & ${ }^{n / m}$ & $\mathrm{n}^{\mathrm{m}}$ & $\mathrm{n} / \mathrm{m}$ & $\mathrm{n} / \mathrm{m}$ & $\mathrm{n}^{\mathrm{m} m}$ & $\mathrm{n} / \mathrm{m}$ \\
\hline Densily of Centrifiged Solids (g/mL) & 1.63 & 1.63 & 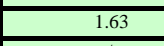 & 1.49 & 1.49 & $\frac{1.49}{1.9}$ & 1.44 & $\frac{1.44}{1.4}$ & 1.44 & 1.79 & 1.79 & 1.79 & 0 & 0 & 0 \\
\hline vol\% Centrifuged Solids & $\mathrm{n/m}$ & $\mathrm{n} / \mathrm{m}$ & $\overline{n / m}$ & $\mathrm{n} / \mathrm{m}$ & $\overline{n / m}$ & $\overline{n / m}$ & $\overline{n / m}$ & $\mathrm{~nm}$ & $\mathrm{n/m}$ & $\mathrm{n} / \mathrm{m}$ & $\overline{n / m}$ & nn/m & & & \\
\hline w\% Centrifuged Solids & $\mathrm{n}^{\mathrm{m}}$ & $\mathrm{n}^{\mathrm{m} m}$ & $\mathrm{n}^{\mathrm{m} m}$ & $\mathrm{n}^{\mathrm{m} m}$ & $\mathrm{n}^{\mathrm{m}}$ & $\frac{n}{n / m}$ & $\mathrm{n}^{\mathrm{m} m}$ & 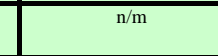 & $\mathrm{n}^{\mathrm{m}}$ & $\mathrm{n}^{\mathrm{m} m}$ & $\mathrm{n} / \mathrm{m}$ & $n$ & $\mathrm{n} / \mathrm{m}$ & $\mathrm{n} / \mathrm{m}$ & $\mathrm{n}^{\mathrm{m}}$ \\
\hline 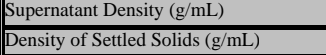 & $\frac{1.42}{n \mathrm{~nm}}$ & 1.42 & $\frac{1.42}{n \mathrm{~nm}}$ & $\frac{1.32}{n \mathrm{~nm}}$ & $\frac{1.32}{\mathrm{~nm} / \mathrm{m}}$ & $\frac{1.32}{n ! m}$ & $\frac{1.28}{n ! m}$ & 1.28 & 1.28 & $\frac{1.42}{n \mathrm{~nm}}$ & $\frac{1.42}{\mathrm{n} / \mathrm{m}}$ & $\frac{1.42}{n n_{m}}$ & $\frac{1.42}{n ! m}$ & $\frac{1.42}{n ! m}$ & $\frac{1.42}{n ! m}$ \\
\hline W\%\% Settled Supernatant & $\mathrm{n} / \mathrm{m}$ & $\mathrm{n} / \mathrm{m}$ & $\mathrm{n} / \mathrm{m}$ & $\mathrm{n} / \mathrm{m}$ & $\mathrm{n} / \mathrm{m}$ & $\mathrm{n} / \mathrm{m}$ & $n^{\prime m}$ & $n^{\prime \prime m}$ & $\frac{n}{n / m}$ & $\mathrm{n} / \mathrm{m}$ & $n^{\prime m}$ & $\frac{n+m}{n / m}$ & $\overline{n / m}$ & $\mathrm{n}^{\mathrm{m} m}$ & $\frac{n^{\prime m}}{1 m}$ \\
\hline |wW\%o dissolved solids in superatant & 47.85 & 47.85 & 47.85 & 44.48 & 44.48 & 44.48 & 43.13 & $\overline{43.13}$ & 43.13 & 47.85 & 47.85 & 47.85 & 47.85 & 47.85 & 7.85 \\
\hline wwo toal solids in Centrifiged Sludge & $\mathrm{n} / \mathrm{m}$ & $\mathrm{n} / \mathrm{m}$ & $\mathrm{n} / \mathrm{m}$ & $\mathrm{n} / \mathrm{m}$ & $\mathrm{n} / \mathrm{m}$ & $\mathrm{n}^{\mathrm{m} m}$ & $\mathrm{n}^{\mathrm{m}}$ & $\mathrm{nm}$ & $\mathrm{n} / \mathrm{m}$ & $\overline{n / m}$ & $\mathrm{n} / \mathrm{m}$ & $n$ & $\overline{n / m}$ & $\mathrm{n}^{\prime / m}$ & $\mathrm{n} / \mathrm{m}$ \\
\hline Ww\% Total Solids & n/m & $n$ n/m & $n$ & n/m & $\mathrm{n} / \mathrm{m}$ & $\mathrm{nm}$ & $\frac{n d m}{n / m}$ & n/m & $\mathrm{nm}$ & $\overline{n / m}$ & $\mathrm{n} / \mathrm{m}$ & n/m & $\overline{n / m}$ & $\overline{n / m}$ & $\mathrm{n} / \mathrm{m}$ \\
\hline W\% UDS & 12.49 & 12.49 & 12.49 & $\overline{1.47}$ & 1.47 & 1.47 & 8.50 & 8.50 & 8.50 & 15.84 & $\overline{15.84}$ & 15.84 & o & 0 & ${ }_{0}$ \\
\hline & 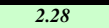 & 2.28 & $\overline{2.28}>>28$ & 2.70 & 2.70 & 2.70 & 2.70 & 2.70 & 2.70 & 2.28 & 2.28 & 2.28 & 2.28 & 2.28 & \\
\hline
\end{tabular}



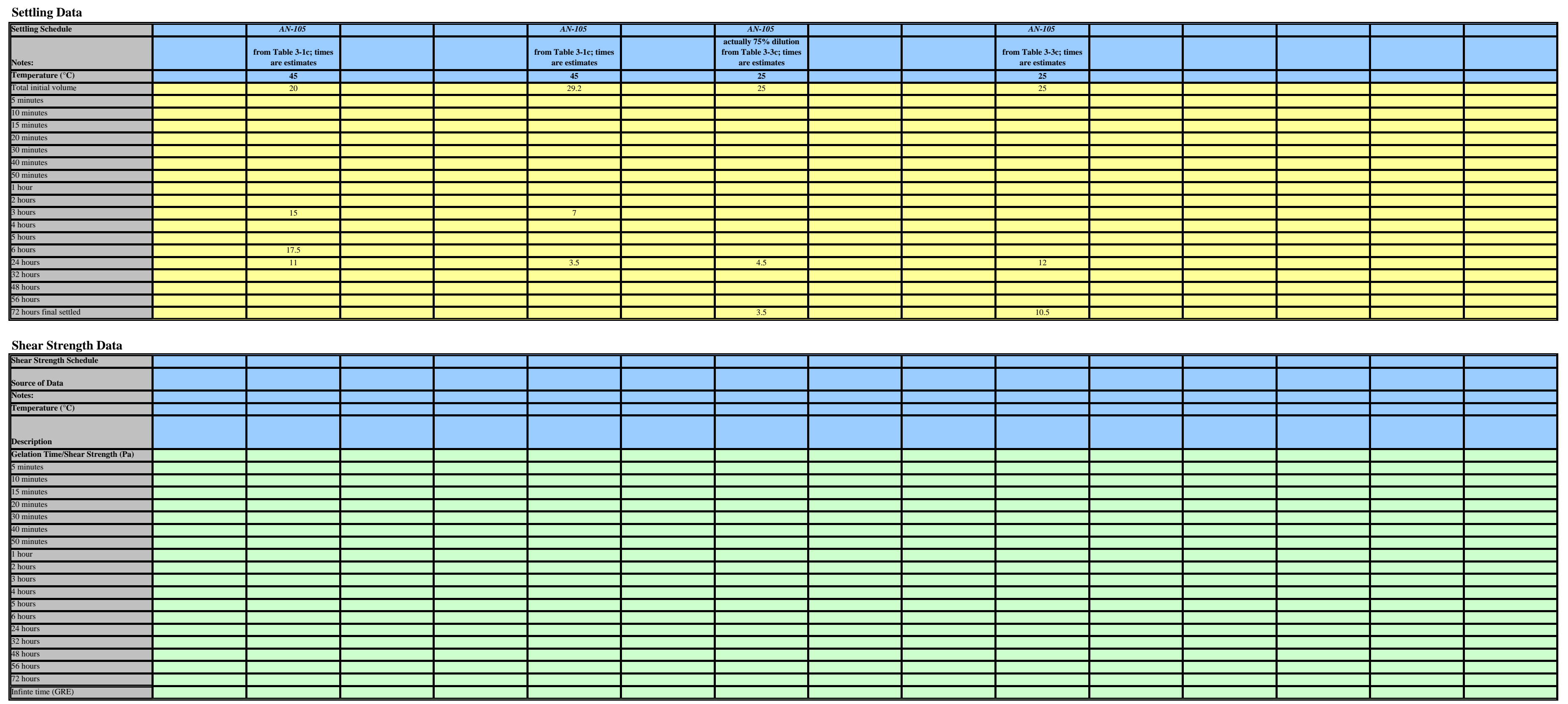
Model Chemical Data Frol

\begin{tabular}{|c|c|c|c|c|c|c|c|c|c|c|c|c|c|c|c|c|}
\hline 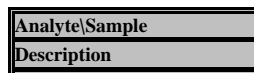 & \begin{tabular}{|l|l} 
AN-105 \\
dillution by volume
\end{tabular} & \begin{tabular}{|l|l} 
AN-105 \\
dillution by volume \\
\end{tabular} & \begin{tabular}{|l|l|l|l} 
AN-105 \\
dillution by volume \\
\end{tabular} & 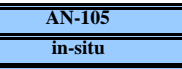 & $\begin{array}{ll}\frac{\mathrm{AN}-105}{\mathrm{in}-\mathrm{itit}} \\
\end{array}$ & $\begin{array}{ll}A \mathrm{AN}-105 \\
\text { in-situ }\end{array}$ & $\begin{array}{l}A N-105 \\
\text { in-situ }\end{array}$ & 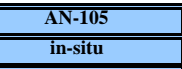 & $\begin{array}{l}\text { AN-105 } \\
\text { in-situ }\end{array}$ & 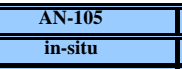 & 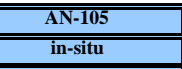 & $\begin{array}{l}A N-105 \\
\text { in-situ }\end{array}$ & 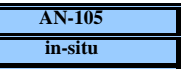 & $\begin{array}{l}\text { AN-105 } \\
\text { inistiut }\end{array}$ & $\begin{array}{l}A N-105 \\
\text { in-situ }\end{array}$ & 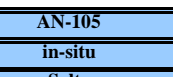 \\
\hline 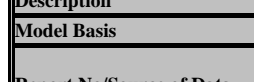 & 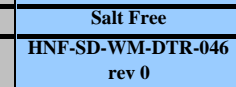 & $\begin{array}{c}\text { Salt Free } \\
\text { HNN-SD-WMNDTR-046 } \\
\text { rev }\end{array}$ & 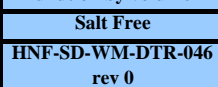 & 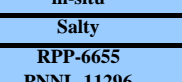 & 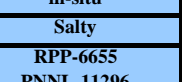 & 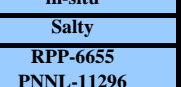 & $\begin{array}{c}\frac{\text { Saly }}{\text { Raly-655 }} \\
\text { pyp-6509 }\end{array}$ & 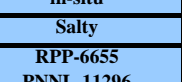 & 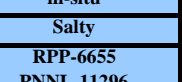 & $\begin{array}{l}\frac{\text { Saly }}{\text { SRPP.655 }} \\
\text { pyp-6509 }\end{array}$ & $\frac{\text { Sally }}{\text { RPP-6655 }}$ & $\frac{\text { Saly }}{\text { RPP-6655 }}$ & 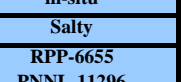 & $\begin{array}{c}\frac{\text { Saly }}{\text { Raly-655 }} \\
\text { pyp-6509 }\end{array}$ & $\frac{\text { Saly }}{\frac{\text { Saly }}{\text { RPP-6655 }}}$ & 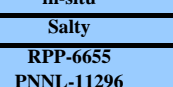 \\
\hline 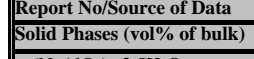 & & & & $\begin{array}{l}\text { PNNL-11296 } \\
\end{array}$ & & PNNL-11296 & & PNNL-11296 & PNNL-11296 & & PNNL-11296 & & & & & \\
\hline 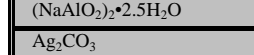 & & & & & & & & & & & & & & & & \\
\hline \begin{tabular}{|l|l|}
$\mathrm{Bi}_{2} \mathrm{O}_{3}$ \\
\end{tabular} & & & & 0.00077 & 0.00077 & 0.00077 & 0.00077 & 0.00077 & 0.00077 & 0.00077 & 0.00077 & 0.00077 & 0.0007 & 0.00077 & 0.00077 & 0.00077 \\
\hline \begin{tabular}{|l|} 
Biocl \\
Boelmite \\
\end{tabular} & & & & & & & & & & & & & & & & \\
\hline 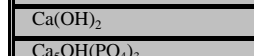 & & & & (0.097712 & (0.097213 & (0.09743 & (0.09773 & 0.087712 & $\ln ^{20712}$ & $\operatorname{lng}^{207012}$ & 5007107 & 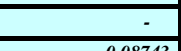 & (1) & 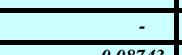 & (30072) & $\div$ \\
\hline 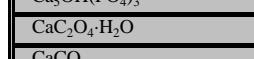 & & & & 0.08843 & .0.08743 & 0.068735 & 0.068743 & .0.08743 & .0.08743 & .0.06743 & .0.08743 & 0.06743 & 0.068743 & 0.087435 & & 0.08 \\
\hline \begin{tabular}{|l}
$\mathrm{CaCO}_{3}$ \\
$\mathrm{Car}_{2}$ \\
\end{tabular} & & & & & & & & & & & & & & & & \\
\hline \begin{tabular}{|l|} 
CroOH \\
FeCOH
\end{tabular} & & & & 0.12072 & 0.12072 & 0.12072 & 0.12072 & 0.12072 & 0.12072 & 0.12072 & 0.12072 & 0.12072 & 0.12072 & 0.12072 & 0.12072 & $\frac{0.12072}{.02070}$ \\
\hline 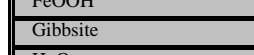 & & & & $\begin{array}{l}0.00489 \\
1.37297 \\
\end{array}$ & $\begin{array}{l}0.0 .0487 \\
.137297 \\
\end{array}$ & $\begin{array}{l}0.00848 \\
1.37297 \\
\end{array}$ & $\begin{array}{l}0.0 .048 \\
.137297 \\
\end{array}$ & $\begin{array}{l}0.0 .04897 \\
.1 .37297 \\
\end{array}$ & $\begin{array}{l}0.00848 \\
1.37297 \\
\end{array}$ & $\begin{array}{l}0.00848 \\
1.37297 \\
\end{array}$ & 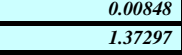 & 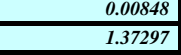 & $\begin{array}{l}0.00848 \\
1.37297 \\
\end{array}$ & 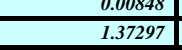 & $\begin{array}{l}0.004867 \\
1.37297 \\
\end{array}$ & $\begin{array}{l}0.00486 \\
1.37297 \\
\end{array}$ \\
\hline \begin{tabular}{|l|l}
$\mathrm{HgO}^{2}$ \\
$\mathrm{KAISOO}_{4}$
\end{tabular} & & & & & & & & & & & & & & & & \\
\hline $\mathrm{La}(\mathrm{OH})_{3}$ & & & & & & & & & & & & & & & & \\
\hline 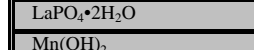 & & & & 0.00102 & 0.00102 & 0.00102 & 0.00102 & 0.00102 & 0.00102 & 0.00102 & 0.000102 & 0.00102 & 0.00102 & 0.00102 & 0.00102 & 0.00 \\
\hline 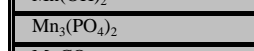 & & & & & & & & & & & & & & & & \\
\hline \begin{tabular}{|l|l}
$\mathrm{MnCO}_{3}$ \\
$\mathrm{Na}_{2} \mathrm{U}_{0} \mathrm{O}^{2}$
\end{tabular} & & & & 0.00348 & 0.00238 & .0.03238 & ,0.0348 & $\overline{0.0034}$ & .0.0348 & (0.00238 & 0.00348 & 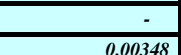 & .0.0378 & (0.00348 & .00348 & $\overline{0.00348}$ \\
\hline 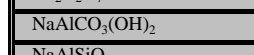 & & & & 0.00 & $\cdots$ & T & - & - & - & - & ( & - & 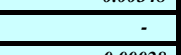 & - & 保 & 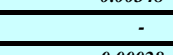 \\
\hline \begin{tabular}{|l}
$\mathrm{NaASIO}_{4}$ \\
$\mathrm{~N}(\mathrm{OOH})_{2}$ \\
\end{tabular} & & & & 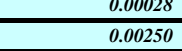 & $\frac{0.0028}{0.00250}$ & $\frac{0.00028}{0.0250}$ & $\frac{0.0028}{0.00250}$ & 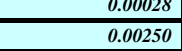 & $\frac{0.0028}{0.00250}$ & 0.0 .00250 & $\frac{0.0028}{0.0250}$ & $\frac{0.0028}{0.0250}$ & .0.0028 & .0.00025 & $\begin{array}{ll}0.002028 \\
.00250\end{array}$ & $\frac{0.0028}{0.00250}$ \\
\hline 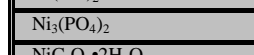 & & & & & & & & & & & & & & & & \\
\hline 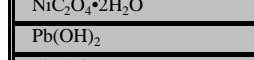 & & & & 0.00218 & 0.00218 & $\begin{array}{ll}0.00218 \\
\end{array}$ & $\frac{0.0218}{1.0218}$ & $\frac{0.0218}{1.0218}$ & 0.00218 & 0.00218 & 0.00218 & 0.00218 & 0.00218 & 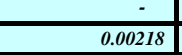 & 0.00218 & 0.00218 \\
\hline $\mid$\begin{tabular}{|ll}
$\mid \mathrm{Pb}_{3}\left(\mathrm{PO}_{4}\right)_{2}$ \\
$\mathrm{PCCO}_{3}$
\end{tabular} & & & & & & & & & & & & & & & & \\
\hline \begin{tabular}{|l}
$\mathrm{Pu}(\mathrm{OH})_{4}$ \\
\end{tabular} & & & & 0.00000 & 0.00000 & 0.00000 & 0.00000 & 0.00000 & 0.00000 & 0.00000 & 0.00000 & 0.00000 & 0.00000 & 0.00000 & 0.00000 & 0.00000 \\
\hline 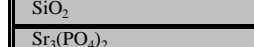 & & & & & & & & & & & & & & & & \\
\hline \begin{tabular}{|l|l}
$\mathrm{S}_{\mathrm{r}} \mathrm{CO}_{3}$ \\
\end{tabular} & - & & & 0.00058 & 0.00058 & 0.00058 & 0.00058 & 0.00058 & 0.00058 & 0.00058 & 0.00058 & 0.00058 & 0.00058 & 0.00058 & 0.00058 & 0.00058 \\
\hline \begin{tabular}{|l|l|}
$\mathrm{TC}_{2} \mathrm{C}_{2}$ \\
$\mathrm{Z} \mathrm{O}_{2}$
\end{tabular} & & & & 0.00002 & 0.00002 & 0.00002 & 0.00002 & 0.00002 & 0.00002 & 0.00002 & 0.00002 & 0.00002 & 0.00002 & 0.00002 & 0.00002 & 0.00002 \\
\hline tone & & & & & & & & & & & & & & & & \\
\hline \begin{tabular}{|l|l|}
$\mathrm{N}_{2} \mathrm{C}_{2} \mathrm{O}_{4}$ \\
\end{tabular} & & & & 0.78210 & 0.78210 & 0.78210 & 0.78210 & $\frac{0.78210}{.08105}$ & 0.78210 & 0.78210 & $\frac{0.78210}{.0705}$ & 0.78210 & 0.78210 & $\begin{array}{ll}0.78210 \\
0.710+\end{array}$ & 0.78210 & $\frac{0.72210}{.07210}$ \\
\hline \begin{tabular}{||l|l|}
$\mathrm{Na}_{2} \mathrm{C}_{3} \mathrm{O}_{3} \mathrm{H}_{2} \mathrm{O}$ \\
$\mathrm{Na}_{2} \mathrm{~S}_{4}$
\end{tabular} & & & & 7.26135 & 7.26135 & 7.26135 & 7.26135 & 7.26135 & 7.26135 & 7.26135 & 7.26135 & 7.26135 & 7.26135 & 7.26135 & 7.26135 & \\
\hline 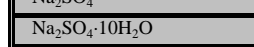 & & & & & & & & & & & & & & & & \\
\hline & & & & 0.03395 & 0.73395 & 0.73395 & 0.73395 & 0.73395 & 0.73395 & $\begin{array}{ll}0.73395 \\
\end{array}$ & 0.73395 & 0.73395 & 0.73395 & $\begin{array}{lll}0.73395 \\
0\end{array}$ & 0.73395 & 0.03395 \\
\hline 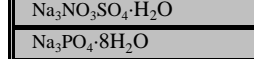 & & & & & - & & & & & & & & & & & \\
\hline 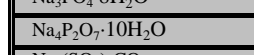 & & & & & & & & & & & & & & & & \\
\hline $\begin{array}{lll}\mathrm{Na}_{6}\left(\mathrm{SO}_{2}\right]_{2} \mathrm{CO}_{3} \\
\mathrm{NaF}\end{array}$ & & & & & & & & & & & & & & & & \\
\hline 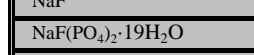 & & & & 1.28899 & 1.28899 & 1.28899 & 1.28899 & 1.2.8899 & 1.28399 & 1.28899 & 1.28899 & 1.28899 & 1.28899 & 1.28899 & 1.28899 & 1.28899 \\
\hline & & & & & & & & & & & & & & & & \\
\hline & & & & 8.18075 & 8.18075 & 8.18075 & 8.18075 & 8.18075 & 8.18075 & 8.18075 & 8.18075 & 8.18075 & 8.18075 & 8.18075 & 8.18075 & 8.18075 \\
\hline$\frac{1}{10}$ & & & & & & & & & & 19.85 & & & & 19.85 & 19.85 & 19.85 \\
\hline Solid & & & & 451.8 & 451.8 & 451.8 & 451.8 & 451.8 & 451.8 & 451.8 & & 451.8 & & $\frac{451.8}{451.8}$ & 451.8 & $\frac{19.85}{451.8}$ \\
\hline
\end{tabular}




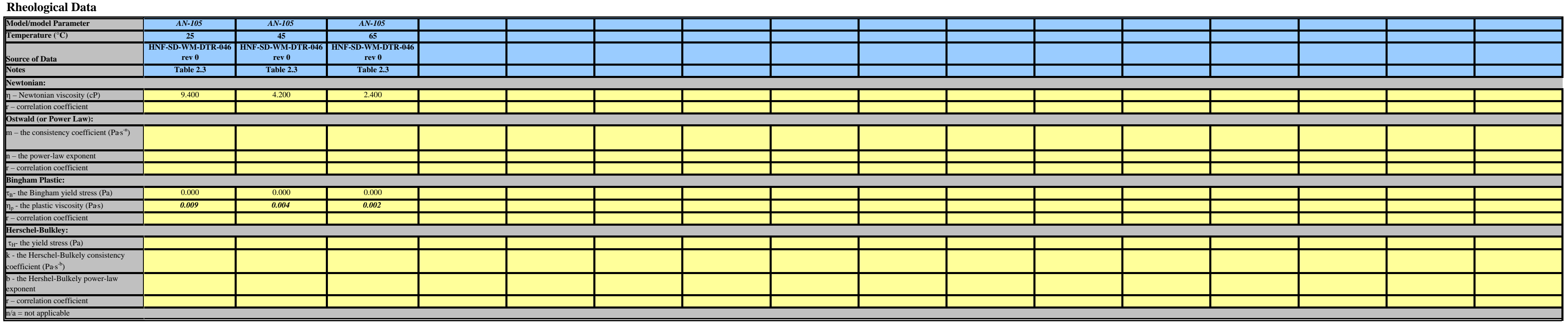

Physical Property Data

\begin{tabular}{|c|c|c|c|c|c|c|c|c|c|c|c|c|c|c|c|c|}
\hline \begin{tabular}{|l|l|l|l|l} 
Physical Property \\
\end{tabular} & $\begin{array}{lll}\mathrm{AN}-105 \\
\end{array}$ & $\overline{A N-105}$ & $A \mathrm{AN}-105$ & \begin{tabular}{c|c|}
$\mathrm{AN}-105$ \\
\end{tabular} & AN-105 & $\mathrm{AN}^{\mathrm{AN}-105}$ & AN-105 & ${ }_{A N-105}$ & $\begin{array}{l}\mathrm{AN}-105 \\
\end{array}$ & $\mathrm{AN}-105^{-10}$ & $\begin{array}{l}\mathrm{AN}-105 \\
\end{array}$ & $\begin{array}{l}\mathrm{AN}-105 \\
\end{array}$ & $\begin{array}{cc}A N-105 \\
\end{array}$ & $\begin{array}{l}\mathrm{AN}-105 \\
\end{array}$ & $\begin{array}{l}\mathrm{AN}-105 \\
\end{array}$ & $\begin{array}{ll}\text { AN-105 } \\
\end{array}$ \\
\hline Notes & & & & & & & & & & & & & & & & \\
\hline Temperature $(\mathrm{C})$ & 25 & 45 & 65 & & & & & & & & & & & & & \\
\hline 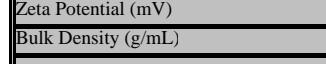 & 1.35 & 1.35 & 1.35 & 1.59 & 1.59 & $\begin{array}{r}1.59 \\
\end{array}$ & 1.59 & 1.59 & 1.59 & 1.59 & 1.59 & 1.59 & 1.59 & 1.59 & 1.59 & $\frac{1.59}{1.59}$ \\
\hline 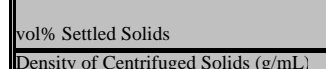 & $\mathrm{n} / \mathrm{m}$ & ${ }^{n / m}$ & $\mathrm{n} / \mathrm{m}$ & & & & & & & & & & & & & \\
\hline Density of Centrifiged Solid (gmL) & $\frac{0}{0}$ & $\frac{0}{0}$ & $\frac{0}{0}$ & 1.66 & 1.66 & 1.66 & 1.66 & 1.66 & 1.66 & 1.66 & 1.66 & 1.66 & 1.66 & 1.66 & 1.66 & 1.66 \\
\hline |vol\% Centifituged Solids & s & 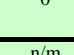 & & & & & & & & & & & & & & \\
\hline 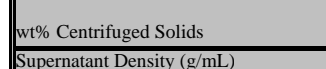 & ${ }^{\mathrm{n} / \mathrm{m}}$ & $\mathrm{n} / \mathrm{m}$ & $\mathrm{n} / \mathrm{m}$ & & & & & & & & & & & & & \\
\hline 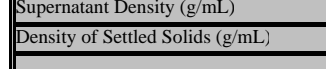 & $\frac{1.35}{n ! m}$ & $\frac{1.35}{\mathrm{nmm}}$ & $\frac{\frac{1.35}{n(m}}{\frac{n m}{n}}$ & 1.42 & $\frac{1.42}{1.25}$ & 1.42 & 1.42 & 1.42 & 1.42 & 1.42 & 1.42 & $\frac{1.42}{1.25}$ & 1.42 & 1.42 & 1.42 & 1.42 \\
\hline ww\% Settled Supernatant & ${ }^{n / m}$ & $\mathrm{n} / \mathrm{m}$ & $\mathrm{n} / \mathrm{m}$ & & & & & & & & & & & & & \\
\hline wwerodissoved solids in supernatant & 45.49 & 45.49 & 45.49 & ${ }^{50}$ & ${ }^{50}$ & ${ }^{50}$ & ${ }^{50}$ & ${ }^{50}$ & ${ }^{50}$ & 50 & ${ }^{50}$ & 50 & 50 & 50 & 50 & 50 \\
\hline |wo\% total solids in Centrifuged Sludge & $n^{n / m}$ & ${ }^{n / m}$ & $\mathrm{n} / \mathrm{m}$ & 59 & 59 & 59 & 59 & ${ }^{59}$ & 59 & 59 & 59 & 59 & 59 & 59 & 59 & 59 \\
\hline wiot Total Solids & $\mathrm{n} / \mathrm{m}$ & $\mathrm{n} / \mathrm{m}$ & $\mathrm{n} / \mathrm{m}$ & & & & & & & & & & & & & \\
\hline wor UDS & ${ }^{\circ}$ & 0 & ${ }^{0}$ & 28.42 & 28.42 & 28.42 & 28.42 & 28.42 & 28.42 & 28.42 & 28.42 & 28.42 & 28.42 & 28.42 & 28.42 & 8.42 \\
\hline Density of Solids g(g/c) & 2.70 & 2.70 & 2.70 & 2.28 & 2.28 & 2.28 & 2.28 & 2.28 & 2.28 & 2.28 & 2.28 & & & & & \\
\hline
\end{tabular}




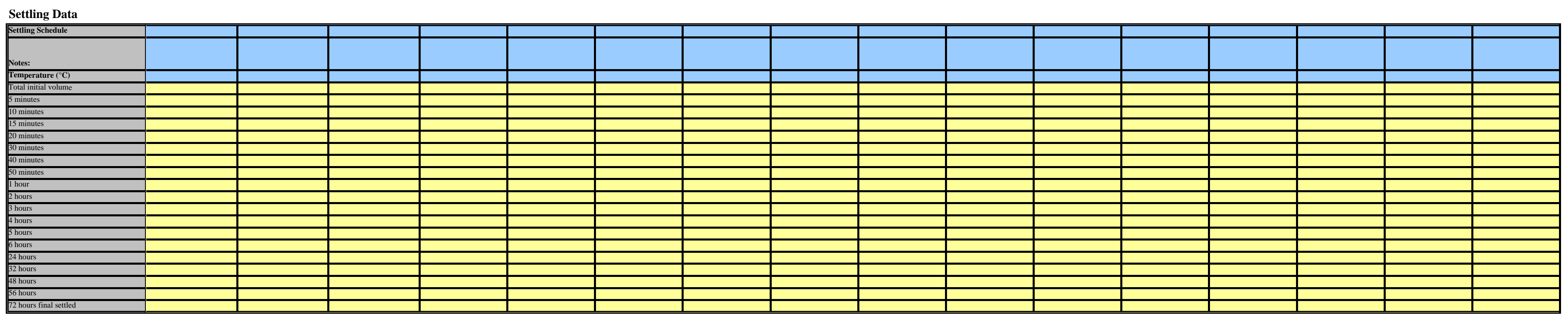

\begin{tabular}{|c|c|c|c|c|c|c|c|c|c|c|c|c|c|c|c|c|}
\hline $\begin{array}{l}\text { Shear Strength Data } \\
\text { Shear Strength Schedule }\end{array}$ & & & & AN-105 & ${ }_{\mathrm{AN}-105}$ & AN-105 & AN-105 & $\begin{array}{ll}A \mathrm{AN}-105 \\
\end{array}$ & $\begin{array}{ll}A \mathrm{AN}-105 \\
\end{array}$ & $\mathrm{AN}^{\mathrm{AN}-105}$ & $\begin{array}{ll}\mathrm{AN}-105 \\
\end{array}$ & AN-105 & AN-10: & $\mathrm{AN}-10$. & AN-105 & \begin{tabular}{|c|}
$\mathrm{AN}-10:$ \\
\end{tabular} \\
\hline Source of Data & & & & $\begin{array}{c}\text { RPP-6655 } \\
\text { PNLL11296 }\end{array}$ & $\begin{array}{l}\text { RPP-6655 } \\
\text { PNNL11296 }\end{array}$ & 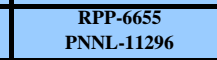 & $\begin{array}{l}\text { RPP-6655 } \\
\text { PNNL11296 }\end{array}$ & $\begin{array}{c}\text { RPP-6655 } \\
\text { PNNL-11296 }\end{array}$ & $\begin{array}{c}\text { RPP. } 6655 \\
\text { PNNL1-1296 }\end{array}$ & $\begin{array}{l}\text { RPP.-655 } \\
\text { PNNL-1296 }\end{array}$ & $\begin{array}{c}\text { RPP.-655 } \\
\text { PNNL11929 }\end{array}$ & $\begin{array}{c}\text { RPP.-655 } \\
\text { PNNL-1296 }\end{array}$ & $\begin{array}{c}\text { RPP-6655 } \\
\text { PNNL-12126 }\end{array}$ & $\begin{array}{c}\text { RPP.6655 } \\
\text { PNNL1-1296 }\end{array}$ & $\begin{array}{l}\text { RPP-6655 } \\
\text { PNNL-1296 }\end{array}$ & $\begin{array}{c}\text { RPP.6655 } \\
\text { PNNL119296 }\end{array}$ \\
\hline Notes: & & & & & & Ball Rhe & & \begin{tabular}{|l|l|l|l|l} 
Ball Rheom \\
\end{tabular} & \begin{tabular}{|l|l|l|l|l|l} 
Ball Rhee \\
\end{tabular} & & & & & & Ball Rheo & $\begin{array}{l}\text { Ball Rheometer } \\
\end{array}$ \\
\hline Temperature ( $(\mathrm{C})$ & & & & 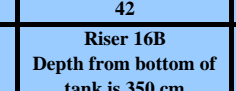 & \begin{tabular}{c|c}
43 \\
Riser 16B \\
Depth hrom botom of \\
trfk
\end{tabular} & \begin{tabular}{c|}
42 \\
Riser 16B \\
Depth from bottom of \\
th of
\end{tabular} & \begin{tabular}{c|} 
Riser 16B \\
Depht from bottom of \\
treve
\end{tabular} & \begin{tabular}{|c} 
Riser 16B \\
Depth from bottom of
\end{tabular} & $\begin{array}{c}\text { Riser 16B } \\
\text { Depht from bottom of } \\
\end{array}$ & $\begin{array}{c}\text { Riser } \\
\text { Depe } \\
\text { Deph from bottom of }\end{array}$ & \begin{tabular}{|c|} 
Riser 1B \\
Depth from bottom of \\
\end{tabular} & \begin{tabular}{|c|} 
Riser $1 \mathrm{IB}$ \\
Depth from bottom of
\end{tabular} & 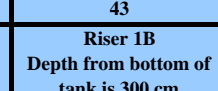 & \begin{tabular}{|c|} 
Riser $1 \mathrm{1B}$ \\
Depth from bottom of \\
thry
\end{tabular} & \begin{tabular}{|c|} 
Riser $1 \mathrm{IB}$ \\
Depth from bottom of
\end{tabular} & \begin{tabular}{|c|}
40 \\
Riser $1 \mathrm{~B}$ \\
Depth from bottom of
\end{tabular} \\
\hline $\begin{array}{ll}\text { Gelation TimeS Shear Strenght (Pa) } \\
\end{array}$ & & & & & & & & & & & & & & & & \\
\hline $\begin{array}{l}\frac{5 \text { minimes }}{10 \text { minutes }} \\
\end{array}$ & & & & & & & & & & & & & & & & \\
\hline 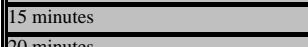 & & & & & & & & & & & & & & & & \\
\hline $\begin{array}{ll}30 \text { mimutues } \\
0 \text { minuts }\end{array}$ & & & & & & & & & & & & & & & & \\
\hline 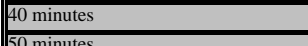 & & & & & & & & & & & & & & & & \\
\hline thour & & & & & & & & & & & & & & & & \\
\hline 2 hours & & & & & & & & & & & & & & & & \\
\hline 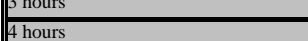 & & & & & & & & & & & & & & & & \\
\hline Shours & & & & & & & & & & & & & & & & \\
\hline$\frac{21 \text { uns }}{24 \text { hours }}$ & & & & & & & & & & & & & & & & \\
\hline 48 hours & & & & & & & & & & & & & & & & \\
\hline | & & & & & & & & & & & & & & & & \\
\hline Inffite fime (GRR & & & & 35 & 70 & 100 & $\frac{125}{125}$ & 145 & 160 & 173 & 50 & 90 & 133 & 165 & 188 & 205 \\
\hline
\end{tabular}


241-AN-107

Model Chemical Data From ESP

\begin{tabular}{|c|c|c|}
\hline AnalytelSample & AN-107 & AN-107 \\
\hline Description & as received & as received \\
\hline Model Basis & Salty & Salty \\
\hline Report No/Source of Data & $\begin{array}{c}\text { WSRC-TR-2003-00210, REV } \\
0\end{array}$ & $\begin{array}{c}\text { WSRC-TR-2003-00210, REV } \\
0\end{array}$ \\
\hline \multicolumn{3}{|l|}{ Solid Phases (vol\% of bulk) } \\
\hline$\left(\mathrm{NaAlO}_{2}\right)_{2} \cdot 2.5 \mathrm{H}_{2} \mathrm{O}$ & - & $\overline{-}$ \\
\hline $\mathrm{Ag}_{2} \mathrm{CO}_{3}$ & 0.00000 & 0.00000 \\
\hline $\mathrm{Bi}_{2} \mathrm{O}_{3}$ & 0.00001 & 0.00001 \\
\hline $\mathrm{BiOCl}$ & - & - \\
\hline Boehmite & - & $\overline{-}$ \\
\hline $\mathrm{Ca}(\mathrm{OH})_{2}$ & - & - \\
\hline $\mathrm{Ca}_{5} \mathrm{OH}\left(\mathrm{PO}_{4}\right)_{3}$ & - & - \\
\hline $\mathrm{CaC}_{2} \mathrm{O}_{4} \cdot \mathrm{H}_{2} \mathrm{O}$ & - & - \\
\hline $\mathrm{CaCO}_{3}$ & - & $\overline{-}$ \\
\hline$\overline{\mathrm{CaF}_{2}}$ & 0.00359 & 0.00359 \\
\hline $\mathrm{CrOOH}$ & 0.00051 & 0.00051 \\
\hline$\overline{\mathrm{FeOOH}}$ & 0.01104 & 0.01104 \\
\hline Gibbsite & - & - \\
\hline $\mathrm{HgO}$ & - & - \\
\hline $\mathrm{KAISiO}_{4}$ & - & - \\
\hline $\mathrm{La}(\mathrm{OH})_{3}$ & - & - \\
\hline $\mathrm{LaPO}_{4} \cdot 2 \mathrm{H}_{2} \mathrm{O}$ & 0.00011 & 0.00011 \\
\hline$\overline{\mathrm{Mn}(\mathrm{OH})_{2}}$ & - & $\overline{-}$ \\
\hline $\mathrm{Mn}_{3}\left(\mathrm{PO}_{4}\right)_{2}$ & $\overline{-}$ & $\overline{-}$ \\
\hline $\mathrm{MnCO}_{3}$ & 0.00406 & 0.00406 \\
\hline $\mathrm{Na}_{2} \mathrm{U}_{2} \mathrm{O}_{7}$ & - & $\overline{-}$ \\
\hline $\mathrm{NaAlCO}_{3}(\mathrm{OH})_{2}$ & 0.16510 & 0.16510 \\
\hline $\mathrm{NaAlSiO}_{4}$ & - & - \\
\hline $\mathrm{Ni}(\mathrm{OH})_{2}$ & 0.00197 & 0.00197 \\
\hline $\mathrm{Ni}_{3}\left(\mathrm{PO}_{4}\right)_{2}$ & - & - \\
\hline $\mathrm{NiC}_{2} \mathrm{O}_{4} \cdot 2 \mathrm{H}_{2} \mathrm{O}$ & - & $\overline{-}$ \\
\hline$\overline{\mathrm{Pb}(\mathrm{OH})_{2}}$ & 0.00062 & 0.00062 \\
\hline $\mathrm{Pb}_{3}\left(\mathrm{PO}_{4}\right)_{2}$ & - & - \\
\hline $\mathrm{PbCO}_{3}$ & - & - \\
\hline$\frac{\mathrm{Pu}(\mathrm{OH})_{4}}{4}$ & 0.00000 & 0.00000 \\
\hline $\mathrm{SiO}_{2}$ & - & - \\
\hline$\overline{\mathrm{Sr}_{3}\left(\mathrm{PO}_{4}\right)_{2}}$ & - & $\overline{-}$ \\
\hline $\mathrm{SrCO}_{3}$ & 0.00002 & 0.00002 \\
\hline $\mathrm{TcO}_{2}$ & 0.00000 & 0.00000 \\
\hline $\mathrm{ZrO}_{2}$ & 0.00023 & 0.00023 \\
\hline $\mathrm{KNO}_{3}$ & - & - \\
\hline $\mathrm{Na}_{2} \mathrm{C}_{2} \mathrm{O}_{4}$ & - & - \\
\hline $\mathrm{Na}_{2} \mathrm{CO}_{3} \cdot \mathrm{H}_{2} \mathrm{O}$ & - & - \\
\hline $\mathrm{Na}_{2} \mathrm{SO}_{4}$ & - & $\overline{-}$ \\
\hline $\mathrm{Na}_{2} \mathrm{SO}_{4} \cdot 10 \mathrm{H}_{2} \mathrm{O}$ & - & - \\
\hline $\mathrm{Na}_{3} \mathrm{FSO}_{4}$ & 0.04934 & 0.04934 \\
\hline $\mathrm{Na}_{3} \mathrm{NO}_{3} \mathrm{SO}_{4} \cdot \mathrm{H}_{2} \mathrm{O}$ & - & - \\
\hline $\mathrm{Na}_{3} \mathrm{PO}_{4} \cdot 8 \mathrm{H}_{2} \mathrm{O}$ & - & - \\
\hline $\mathrm{Na}_{4} \mathrm{P}_{2} \mathrm{O}_{7} \cdot 10 \mathrm{H}_{2} \mathrm{O}$ & - & - \\
\hline $\mathrm{Na}_{6}\left(\mathrm{SO}_{4}\right)_{2} \mathrm{CO}_{3}$ & - & - \\
\hline $\mathrm{NaF}$ & 0.02402 & 0.02402 \\
\hline $\mathrm{NaF}\left(\mathrm{PO}_{4}\right)_{2} \cdot 19 \mathrm{H}_{2} \mathrm{O}$ & - & - \\
\hline $\mathrm{NaHCO}_{3}$ & - & - \\
\hline $\mathrm{NaNO}_{2}$ & - & - \\
\hline $\mathrm{NaNO}_{3}$ & - & - \\
\hline $\mathrm{Na}_{3} \mathrm{PO}_{4} \cdot 0.25 \mathrm{NaOH} \cdot 12 \mathrm{H}_{2} \mathrm{O}$ & - & - \\
\hline Total Volume \% UDS & 0.26 & 0.26 \\
\hline Solids Concentration (g/L) & 6.9 & 6.9 \\
\hline
\end{tabular}

Note: Numbers in italics are calculated values. Numbers in normal font are reported data. 
241-AN-107

Rheological Data

\begin{tabular}{|c|c|c|}
\hline Model/model Parameter & AN-107 & AN-107 \\
\hline Temperature $\left({ }^{\circ} \mathrm{C}\right)$ & 25 & 40 \\
\hline Source of Data & $\begin{array}{c}\text { WSRC-TR-2003-00210, REV } \\
0\end{array}$ & $\begin{array}{c}\text { WSRC-TR-2003-00210, REV } \\
0\end{array}$ \\
\hline \multicolumn{3}{|l|}{ Notes } \\
\hline \multicolumn{3}{|l|}{ Newtonian: } \\
\hline$\eta$ - Newtonian viscosity (cP) & $\mathrm{n} / \mathrm{m}$ & $\mathrm{n} / \mathrm{m}$ \\
\hline $\mathrm{r}$ - correlation coefficient & $\mathrm{n} / \mathrm{m}$ & $\mathrm{n} / \mathrm{m}$ \\
\hline \multicolumn{3}{|l|}{ Ostwald (or Power Law): } \\
\hline $\mathrm{m}$ - the consistency coefficient $\left(\mathrm{Pa} \cdot \mathrm{s}^{-\mathrm{n}}\right)$ & $\mathrm{n} / \mathrm{m}$ & $\mathrm{n} / \mathrm{m}$ \\
\hline $\mathrm{n}$ - the power-law exponent & $\mathrm{n} / \mathrm{m}$ & $\mathrm{n} / \mathrm{m}$ \\
\hline $\mathrm{r}$ - correlation coefficient & $\mathrm{n} / \mathrm{m}$ & $\mathrm{n} / \mathrm{m}$ \\
\hline \multicolumn{3}{|l|}{ Bingham Plastic: } \\
\hline$\tau_{\mathrm{B}^{-}}$the Bingham yield stress $(\mathrm{Pa})$ & 1.48 & 1.07 \\
\hline$\eta_{p}$ - the plastic viscosity (Pa·s) & 0.00936 & 0.00587 \\
\hline $\mathrm{r}$ - correlation coefficient & 0.997 & 0.993 \\
\hline \multicolumn{3}{|l|}{ Herschel-Bulkley: } \\
\hline$\tau_{\mathrm{H}^{-}}$the yield stress $(\mathrm{Pa})$ & $\mathrm{n} / \mathrm{m}$ & $\mathrm{n} / \mathrm{m}$ \\
\hline $\begin{array}{l}\text { k - the Herschel-Bulkely consistency } \\
\text { coefficient }\left(\mathrm{Pa} \cdot \mathrm{s}^{\text {-b }}\right)\end{array}$ & $\mathrm{n} / \mathrm{m}$ & $\mathrm{n} / \mathrm{m}$ \\
\hline $\begin{array}{l}\text { b - the Hershel-Bulkely power-law } \\
\text { exponent }\end{array}$ & $\mathrm{n} / \mathrm{m}$ & $\mathrm{n} / \mathrm{m}$ \\
\hline $\mathrm{r}$ - correlation coefficient & $\mathrm{n} / \mathrm{m}$ & $\mathrm{n} / \mathrm{m}$ \\
\hline
\end{tabular}

Physical Property Data

\begin{tabular}{||l|c|c|}
\hline Physical Property & AN-107 & AN-107 \\
\hline Notes & & \\
\hline Temperature ( ${ }^{\circ}$ C) & 25 & $\mathbf{4 0}$ \\
\hline Zeta Potential (mV) & 1.42 & $\mathbf{1 . 4 2}$ \\
\hline Bulk Density (g/mL) & $\mathrm{n} / \mathrm{m}$ & $\mathrm{n} / \mathrm{m}$ \\
\hline vol\% Settled Solids & $\mathbf{1 . 4 8}$ & $\mathbf{1 . 4 6}$ \\
\hline Density of Centrifuged Solids (g/mL) & 8 & 8 \\
\hline vol\% Centrifuged Solids & 6.88 & 6.88 \\
\hline wt\% Centrifuged Solids & 1.42 & $\mathbf{1 . 4 2}$ \\
\hline Supernatant Density (g/mL) & $\mathrm{n} / \mathrm{m}$ & $\mathrm{n} / \mathrm{m}$ \\
\hline Density of Settled Solids (g/mL) & $\mathrm{n} / \mathrm{m}$ & 49.25 \\
\hline wt\% Settled Supernatant & 49.25 & $\mathrm{n} / \mathrm{m}$ \\
\hline wt\% dissolved solids in supernatant & $\mathrm{n} / \mathrm{m}$ & 49.5 \\
\hline wt\% total solids in Centrifuged Sludge & 49.5 & 0.483 \\
\hline wt\% Total Solids & & 2.63 \\
\hline wt\% UDS & & \\
\hline Density of Solids (g/cc) & & \\
\hline \hline
\end{tabular}

Note: Numbers in italics are calculated values. Numbers in normal font are reported data. 
241-AP-104

Model Chemical Data From ESP

\begin{tabular}{|c|c|c|}
\hline AnalytelSample & AP-104 & AP-104 \\
\hline Description & as received & - as received \\
\hline Model Basis & Salty & Salty \\
\hline Report No/Source of Data & $\begin{array}{c}\text { WTP-RPT-069, Rev. } 0 \\
\text { (PNWD-3334) }\end{array}$ & $\begin{array}{l}\text { WTP-RPT-069, Rev. } 0 \\
\text { (PNWD-3334) }\end{array}$ \\
\hline \multicolumn{3}{|l|}{ Solid Phases (vol\% of bulk) } \\
\hline$\left(\mathrm{NaAlO}_{2}\right)_{2} \cdot 2.5 \mathrm{H}_{2} \mathrm{O}$ & - & - \\
\hline $\mathrm{Ag}_{2} \mathrm{CO}_{3}$ & - & - \\
\hline $\mathrm{Bi}_{2} \mathrm{O}_{3}$ & - & - \\
\hline $\mathrm{BiOCl}$ & - & - \\
\hline Boehmite & - & - \\
\hline $\mathrm{Ca}(\mathrm{OH})_{2}$ & - & - \\
\hline $\mathrm{Ca}_{5} \mathrm{OH}\left(\mathrm{PO}_{4}\right)_{3}$ & - & - \\
\hline $\mathrm{CaC}_{2} \mathrm{O}_{4} \cdot \mathrm{H}_{2} \mathrm{O}$ & - & - \\
\hline $\mathrm{CaCO}_{3}$ & - & - \\
\hline $\mathrm{CaF}_{2}$ & - & - \\
\hline $\mathrm{CrOOH}$ & - & $\overline{-}$ \\
\hline $\mathrm{FeOOH}$ & - & - \\
\hline Gibbsite & - & - \\
\hline$\overline{\mathrm{HgO}}$ & - & - \\
\hline $\mathrm{KAlSiO}_{4}$ & - & - \\
\hline $\mathrm{La}(\mathrm{OH})_{3}$ & - & - \\
\hline $\mathrm{LaPO}_{4} \cdot 2 \mathrm{H}_{2} \mathrm{O}$ & - & - \\
\hline $\mathrm{Mn}(\mathrm{OH})_{2}$ & - & - \\
\hline $\mathrm{Mn}_{3}\left(\mathrm{PO}_{4}\right)_{2}$ & - & - \\
\hline $\mathrm{MnCO}_{3}$ & - & - \\
\hline $\mathrm{Na}_{2} \mathrm{U}_{2} \mathrm{O}_{7}$ & - & - \\
\hline $\mathrm{NaAlCO}_{3}(\mathrm{OH})_{2}$ & - & - \\
\hline $\mathrm{NaAlSiO}_{4}$ & - & - \\
\hline $\mathrm{Ni}(\mathrm{OH})_{2}$ & - & - \\
\hline $\mathrm{Ni}_{3}\left(\mathrm{PO}_{4}\right)_{2}$ & - & - \\
\hline $\mathrm{NiC}_{2} \mathrm{O}_{4} \cdot 2 \mathrm{H}_{2} \mathrm{O}$ & - & - \\
\hline $\mathrm{Pb}(\mathrm{OH})_{2}$ & - & - \\
\hline $\mathrm{Pb}_{3}\left(\mathrm{PO}_{4}\right)_{2}$ & - & - \\
\hline $\mathrm{PbCO}_{3}$ & - & - \\
\hline $\mathrm{Pu}(\mathrm{OH})_{4}$ & - & - \\
\hline $\mathrm{SiO}_{2}$ & - & - \\
\hline $\mathrm{Sr}_{3}\left(\mathrm{PO}_{4}\right)_{2}$ & - & - \\
\hline $\mathrm{SrCO}_{3}$ & - & - \\
\hline $\mathrm{TcO}_{2}$ & - & - \\
\hline $\mathrm{ZrO}_{2}$ & - & - \\
\hline $\mathrm{KNO}_{3}$ & - & - \\
\hline $\mathrm{Na}_{2} \mathrm{C}_{2} \mathrm{O}_{4}$ & - & - \\
\hline $\mathrm{Na}_{2} \mathrm{CO}_{3} \cdot \mathrm{H}_{2} \mathrm{O}$ & - & - \\
\hline $\mathrm{Na}_{2} \mathrm{SO}_{4}$ & - & - \\
\hline $\mathrm{Na}_{2} \mathrm{SO}_{4} \cdot 10 \mathrm{H}_{2} \mathrm{O}$ & - & - \\
\hline $\mathrm{Na}_{3} \mathrm{FSO}_{4}$ & - & - \\
\hline $\mathrm{Na}_{3} \mathrm{NO}_{3} \mathrm{SO}_{4} \cdot \mathrm{H}_{2} \mathrm{O}$ & - & - \\
\hline $\mathrm{Na}_{3} \mathrm{PO}_{4} \cdot 8 \mathrm{H}_{2} \mathrm{O}$ & - & - \\
\hline $\mathrm{Na}_{4} \mathrm{P}_{2} \mathrm{O}_{7} \cdot 10 \mathrm{H}_{2} \mathrm{O}$ & - & - \\
\hline $\mathrm{Na}_{6}\left(\mathrm{SO}_{4}\right)_{2} \mathrm{CO}_{3}$ & - & - \\
\hline $\mathrm{NaF}$ & - & - \\
\hline $\mathrm{NaF}\left(\mathrm{PO}_{4}\right)_{2} \cdot 19 \mathrm{H}_{2} \mathrm{O}$ & - & - \\
\hline $\mathrm{NaHCO}_{3}$ & - & - \\
\hline $\mathrm{NaNO}_{2}$ & - & - \\
\hline $\mathrm{NaNO}_{3}$ & - & - \\
\hline $\mathrm{Na}_{3} \mathrm{PO}_{4} \cdot 0.25 \mathrm{NaOH} \cdot 12 \mathrm{H}_{2} \mathrm{O}$ & - & - \\
\hline Total Volume \% UDS & - & - \\
\hline Solids Concentration (g/L) & - & - \\
\hline
\end{tabular}

Note: Numbers in italics are calculated values. Numbers in normal font are reported data. 
241-AP-104

Rheological Data

\begin{tabular}{|c|c|c|}
\hline Model/model Parameter & AP-104 & AP-104 \\
\hline Temperature $\left({ }^{\circ} \mathrm{C}\right)$ & 25 & 40 \\
\hline Source of Data & $\begin{array}{l}\text { WTP-RPT-069, Rev. } 0 \\
\text { (PNWD-3334) }\end{array}$ & $\begin{array}{l}\text { WTP-RPT-069, Rev. } 0 \\
\text { (PNWD-3334) }\end{array}$ \\
\hline \multicolumn{3}{|l|}{ Notes } \\
\hline \multicolumn{3}{|l|}{ Newtonian: } \\
\hline$\eta$ - Newtonian viscosity (cP) & 3.68 & 2.37 \\
\hline $\mathrm{r}$ - correlation coefficient & 0.9982 & 0.9976 \\
\hline \multicolumn{3}{|l|}{ Ostwald (or Power Law): } \\
\hline $\mathrm{m}$ - the consistency coefficient $\left(\mathrm{Pa} \cdot \mathrm{s}^{-\mathrm{n}}\right)$ & 0.0048 & 0.00324 \\
\hline $\mathrm{n}$ - the power-law exponent & 0.959 & 0.9525 \\
\hline $\mathrm{r}$ - correlation coefficient & 0.999 & 0.9988 \\
\hline \multicolumn{3}{|l|}{ Bingham Plastic: } \\
\hline$\tau_{\mathrm{B}^{-}}$the Bingham yield stress $(\mathrm{Pa})$ & 0.049 & 0.03798 \\
\hline$\eta_{\mathrm{p}}$ - the plastic viscosity (Pa-s) & 0.0036 & 0.00231 \\
\hline $\mathrm{r}-$ correlation coefficient & 0.9988 & 0.9984 \\
\hline \multicolumn{3}{|l|}{ Herschel-Bulkley: } \\
\hline$\tau_{\mathrm{H}^{-}}$the yield stress $(\mathrm{Pa})$ & 0 & 0 \\
\hline $\begin{array}{l}\mathrm{k} \text { - the Herschel-Bulkely consistency } \\
\text { coefficient }\left(\mathrm{Pa} \cdot \mathrm{s}^{-\mathrm{b}}\right)\end{array}$ & 0.0048 & 0.00299 \\
\hline $\begin{array}{l}\text { b - the Hershel-Bulkely power-law } \\
\text { exponent }\end{array}$ & 0.959 & 0.9635 \\
\hline $\mathrm{r}-$ correlation coefficient & 0.999 & 0.9986 \\
\hline
\end{tabular}

Physical Property Data

\begin{tabular}{||l|c|c|}
\hline Physical Property & AP-104 & AP-104 \\
\hline Notes & & \\
\hline Temperature $\left({ }^{\circ} \mathbf{C}\right)$ & 25 & $\mathbf{4 0}$ \\
\hline Zeta Potential $(\mathrm{mV})$ & & $\mathbf{1 . 2 6}$ \\
\hline Bulk Density (g/mL) & $\mathrm{n} / \mathrm{m}$ & $\mathrm{n} / \mathrm{m}$ \\
\hline vol\% Settled Solids & $\mathrm{n} / \mathrm{m}$ & $\mathrm{n} / \mathrm{m}$ \\
\hline Density of Centrifuged Solids (g/mL) & $\mathrm{n} / \mathrm{m}$ & $\mathrm{n} / \mathrm{m}$ \\
\hline vol\% Centrifuged Solids & $\mathrm{n} / \mathrm{m}$ & $\mathrm{n} / \mathrm{m}$ \\
\hline wt\% Centrifuged Solids & 1.30 & 1.30 \\
\hline Supernatant Density (g/mL) & $\mathrm{n} / \mathrm{m}$ & $\mathrm{n} / \mathrm{m}$ \\
\hline Density of Settled Solids (g/mL) & $\mathrm{n} / \mathrm{m}$ & \\
\hline wt $\%$ Settled Supernatant & 32 & $\mathrm{n} / \mathrm{m}$ \\
\hline wt $\%$ dissolved solids in supernatant & $\mathrm{n} / \mathrm{m}$ & 31.2 \\
\hline wt\% total solids in Centrifuged Sludge & 31.2 & 0.00 \\
\hline wt\% Total Solids & & 2.30 \\
\hline wt\% UDS & & \\
\hline Density of Solids (g/cc) & & \\
\hline \hline
\end{tabular}

Note: Numbers in italics are calculated values. Numbers in normal font are reported data. 


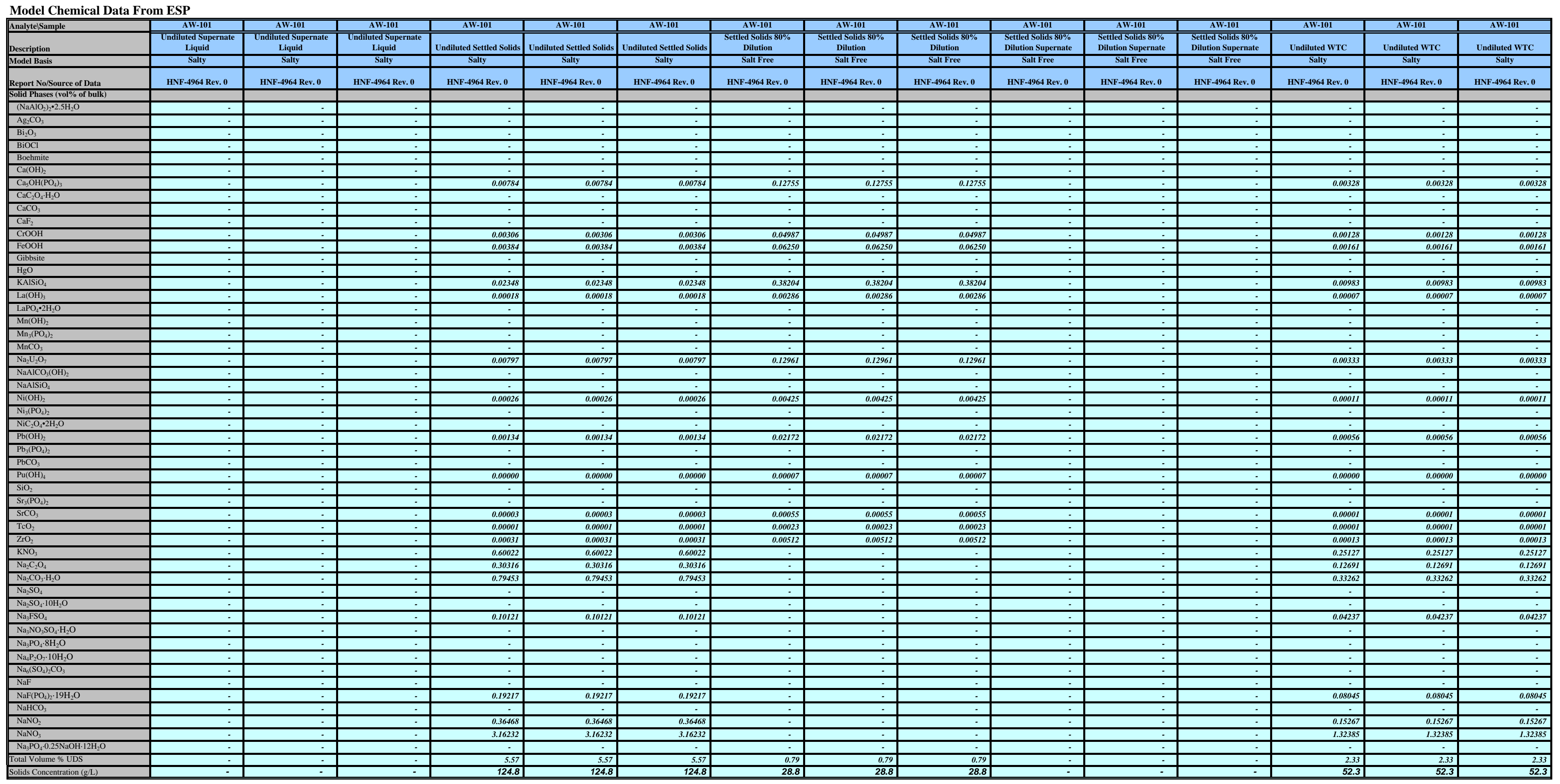


Rheological Data

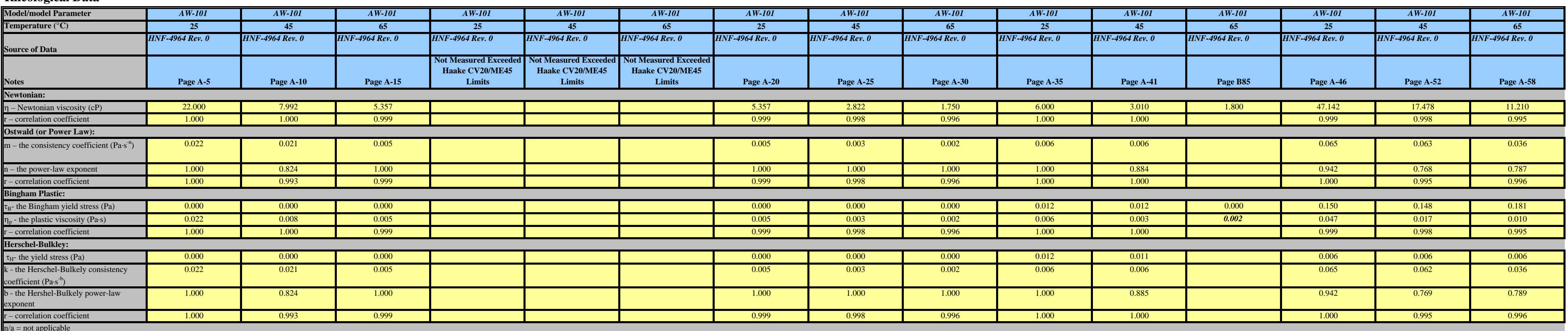

Physical Property Data

\begin{tabular}{|c|c|c|c|c|c|c|c|c|c|c|c|c|c|c|c|}
\hline |hinsical Property & $\overline{A W-101}$ & $\overline{A W-101}$ & $\overline{A W-10}$ & $\overline{A W-10}$ & $\overline{A W W-10}$ & $\overline{A W-101}$ & $\overline{A W-10}$ & $\overline{A W-10}$ & $\overline{A W-10}$ & $\overline{A W-10}$ & $\overline{A W-10}$ & $\overline{A W-10}$ & $\overline{A W-10}$ & $\overline{A W-101}$ & $\overline{A W-10}$ \\
\hline \multicolumn{16}{|l|}{ Notes } \\
\hline $\mid$ Temperature $\left({ }^{\circ} \mathrm{C}\right)$ & 25 & 45 & 65 & 25 & 45 & 65 & 25 & 45 & 65 & 25 & 45 & 65 & 25 & 45 & 65 \\
\hline 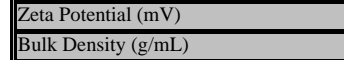 & $\frac{1.52}{1.5}$ & 1.52 & 1.52 & 1.56 & 1.56 & 1.56 & 1.44 & 1.44 & 1.44 & 1.42 & 1.4 & 1.42 & 1.54 & 7.54 & 1.54 \\
\hline $\mid \begin{array}{l}\text { vol\% Settled Solids } \\
\text { | }\end{array}$ & 0 & 0 & 0 & $\mathrm{n} / \mathrm{m}$ & $n$ n/m & $n / m$ & $n$ n/m & $\mathrm{n}^{\mathrm{n} / \mathrm{m}}$ & $n$ n/m & $\mathrm{n}^{\mathrm{n} / \mathrm{m}}$ & $n^{n / m}$ & $\mathrm{n} / \mathrm{m}$ & $n / m$ & $\mathrm{n} / \mathrm{m}$ & $n / m$ \\
\hline Density of Centrifituged Solids $(\mathrm{g} / \mathrm{mL})$ & n/a & n/a & n/a & 1.68 & 1.68 & 1.68 & 1.42 & 1.42 & 1.42 & 1.42 & 1.42 & $\frac{1.42}{1}$ & 1.68 & 1.68 & 1.68 \\
\hline \begin{tabular}{|l|l} 
vol\% Centrifuged Solids \\
\end{tabular} & $\mathrm{n}^{\prime \mathrm{m}}$ & $\mathrm{n} / \mathrm{m}$ & n/m & $\mathrm{n} / \mathrm{m}$ & ${ }^{\mathrm{n} / \mathrm{m}}$ & $\mathrm{n}^{\prime \mathrm{m}}$ & $\mathrm{n}^{\mathrm{n} m}$ & $\mathrm{n} / \mathrm{m}$ & $\mathrm{n}^{\mathrm{m} m}$ & $\mathrm{n} / \mathrm{m}$ & $\mathrm{n}^{\mathrm{n} m}$ & $\mathrm{n} / \mathrm{m}$ & $n^{n / m}$ & ${ }^{\mathrm{n} / \mathrm{m}}$ & $n^{n / m}$ \\
\hline $\mid$\begin{tabular}{|l|l} 
w\% Centifiuged Solids \\
\end{tabular} & $\frac{n / m}{n / m}$ & $\frac{n / m}{n / m}$ & $n^{n / m}$ & $\mathrm{n} / \mathrm{m}$ & ${ }^{n / m}$ & $\mathrm{n}^{\mathrm{m} m}$ & ${ }^{n / m}$ & $\mathrm{n} / \mathrm{m}$ & $n / m$ & $\mathrm{n} / \mathrm{m}$ & $n / m$ & $\mathrm{n} / \mathrm{m}$ & $n / m$ & ${ }^{n / m}$ & $\mathrm{n}^{\mathrm{m} m}$ \\
\hline$\frac{g(m L)}{g(n)}$ & 1.52 & 1.52 & 1.52 & 1.52 & 1.52 & 1.52 & 1.42 & 1.42 & 1.42 & 1.42 & 1.42 & 1.42 & 1.52 & 1.52 & 1.52 \\
\hline Density of Sertled Solids (ggmL) & $\frac{\text { na }}{n+2}$ & $\frac{n a}{n / 2}$ & $\frac{n / a}{n / a}$ & $\frac{n / m}{n}$ & $\mathrm{r}^{\mathrm{nm}}$ & $\frac{n / m}{n / m}$ & $\frac{n / m}{n / m}$ & ${ }^{n / m}$ & & $\mathrm{n} / \mathrm{m}$ & $\frac{n / m}{n / m}$ & $\frac{\mathrm{n} / \mathrm{m}}{\mathrm{n} / \mathrm{m}}$ & $\frac{n / m}{n / m}$ & 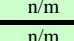 & $\frac{m i n}{n}$ \\
\hline w1\% Settled Supernatant & 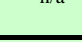 & wat & wid & & we & & & $\mathrm{n} / \mathrm{m}$ & ${ }^{n / m}$ & & & & & & $\mathrm{n} / \mathrm{m}$ \\
\hline 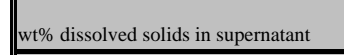 & $\mathrm{n} / \mathrm{m}$ & $\mathrm{n} / \mathrm{m}$ & n/m & $\mathrm{n} / \mathrm{m}$ & ${ }^{n / m}$ & $\mathrm{n}^{\prime \mathrm{m}}$ & ${ }^{n / m}$ & $\mathrm{n} / \mathrm{m}$ & ${ }^{n / m}$ & $\mathrm{n} / \mathrm{m}$ & ${ }^{n / m}$ & $\mathrm{n} / \mathrm{m}$ & ${ }^{n / m}$ & ${ }^{\mathrm{n} / \mathrm{m}}$ & $\mathrm{n} / \mathrm{m}$ \\
\hline \begin{tabular}{|l|l} 
wo\% toat solids in Centrifinged Sludge \\
\end{tabular} & n/a & n/a & n/a & $\mathrm{n} / \mathrm{m}$ & ${ }^{\mathrm{n} / \mathrm{m}}$ & $\mathrm{n}^{\prime \mathrm{m}}$ & $\mathrm{n}^{\mathrm{n} m}$ & $\mathrm{n}^{\mathrm{n} / \mathrm{m}}$ & $\mathrm{n}^{\mathrm{m} m}$ & $\mathrm{n} / \mathrm{m}$ & $\mathrm{n}^{\mathrm{n} m}$ & $\mathrm{n} / \mathrm{m}$ & ${ }^{\mathrm{n} / \mathrm{m}}$ & $\mathrm{n}^{\mathrm{n} / \mathrm{m}}$ & $\mathrm{n}^{\prime / m}$ \\
\hline \begin{tabular}{|l|l} 
wo\% Toal Solids \\
\end{tabular} & $n^{\prime \prime m}$ & n/m & ${ }^{n / m}$ & $\mathrm{n} / \mathrm{m}$ & ${ }^{n / m}$ & $\mathrm{n}^{\prime / m}$ & ${ }^{n / m}$ & $n^{\prime / m}$ & ${ }^{n / m}$ & $\mathrm{n} / \mathrm{m}$ & ${ }^{n / m}$ & $n^{\prime / m}$ & ${ }^{n / m}$ & ${ }^{n / m}$ & $\mathrm{n}^{\mathrm{m} m}$ \\
\hline w1\% UDS & 0.00 & 0.00 & 0.00 & 8.00 & 8.00 & 8.00 & 2.00 & 2.00 & 2.00 & 0.00 & ${ }^{0.00}$ & 0.00 & 3.40 & 3.40 & 3.40 \\
\hline Density of Solids (g/cc) & 2.24 & 2.24 & 2.24 & 2.24 & ${ }^{2.24}$ & 2.24 & 3.66 & 3.66 & 3.66 & 3.66 & 3.66 & 3.66 & ${ }^{2.24}$ & 2.24 & 2.24 \\
\hline
\end{tabular}




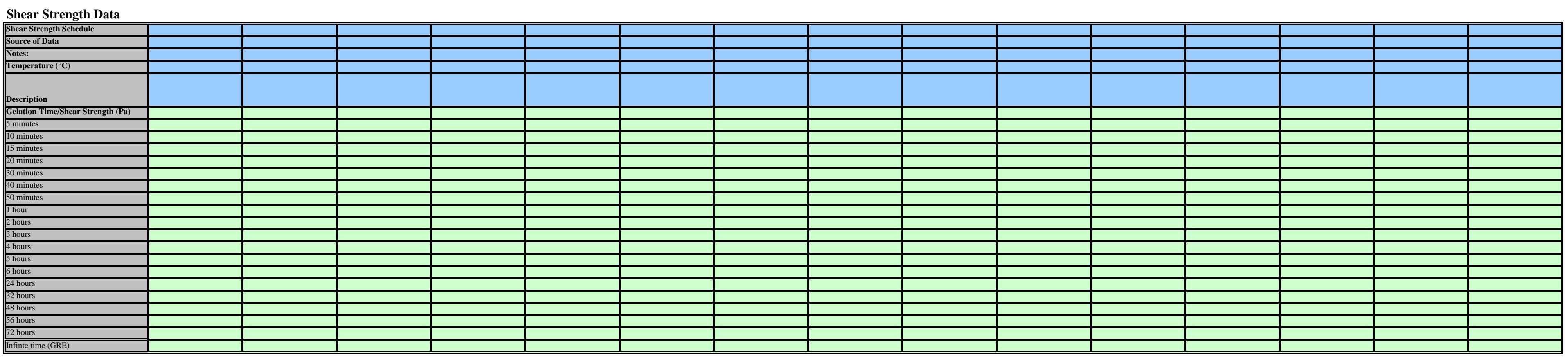


Model Chemical Data Fro




Rheological Data

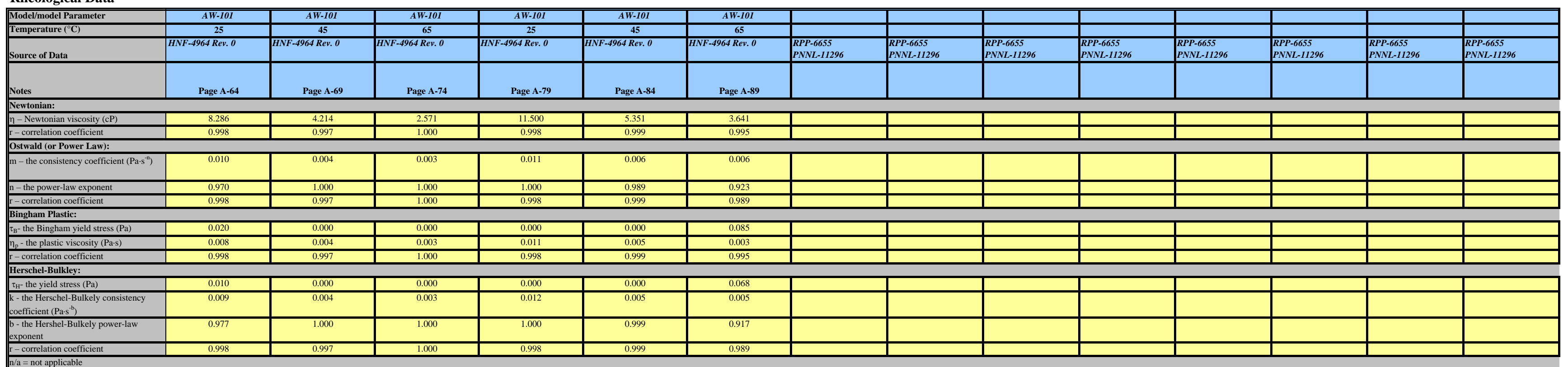

\begin{tabular}{|c|c|c|c|c|c|c|c|c|c|c|c|c|c|c|}
\hline $\begin{array}{l}\text { Physical Property Data } \\
\text { Physical Property }\end{array}$ & $\overline{A W W-101}$ & $\overline{A W W-101}$ & 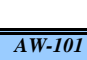 & $\overline{\overline{A W-101}}$ & $\overline{A W W-101}$ & $\overline{\overline{A W-101}}$ & & & & & & & & \\
\hline \multicolumn{15}{|l|}{ Notes } \\
\hline Temperature $\left({ }^{\circ} \mathrm{C}\right)$ & 25 & 45 & 65 & 25 & 45 & 65 & & & & & & & & \\
\hline $\begin{array}{l}\text { eetat Potetialal (mV) } \\
\text { Bulk Density }(\mathrm{g} / \mathrm{mL})\end{array}$ & 1.39 & 1.39 & 1.39 & 1.38 & 1.38 & 1.38 & 1.52 & 1.52 & 1.52 & 1.52 & 152 & 152 & 152 & 152 \\
\hline Wvilo Sertled Solids & $\frac{n / m}{n / m}$ & $\mathrm{n} / \mathrm{m}$ & $n / m$ & $\frac{1.00}{n / m}$ & $\mathrm{n} / \mathrm{m}$ & $\frac{1.00}{n / m}$ & 32.2 & 32.22 & 32.2 & 32.2 & 32.2 & 32.2 & 32.2 & 32.2 \\
\hline 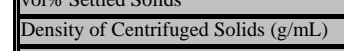 & 1.006 & 1.006 & 1.006 & 1.006 & 1.006 & 1.006 & $\overline{1.68}$ & 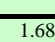 & 7.68 & 1.68 & $\overline{1.68}$ & 1.68 & $\overline{1.68}$ & 1.68 \\
\hline \begin{tabular}{|l|l} 
vol\% Centrifuged Solids \\
\end{tabular} & $\frac{n / m}{n / m}$ & $\mathrm{n} / \mathrm{m}$ & ${ }^{n / m}$ & $n$ n/m & $\mathrm{n}^{\prime / m}$ & $n / m$ & 15.3 & 15.3 & 15.3 & 15.3 & 15.3 & 15.3 & 15.3 & 15.3 \\
\hline Ww\% Centrifiuged Solids & $\mathrm{n}^{\mathrm{n} / \mathrm{m}}$ & $\mathrm{n} / \mathrm{m}$ & $n^{n / m}$ & $n / m$ & $n$ n/m & $n$ n/m & 16.9 & 16.9 & 16.9 & 16.9 & 16.9 & 16.9 & 16.9 & 16.9 \\
\hline Supenatant Density $(\mathrm{g} / \mathrm{mL})$ & 1.38 & 1.38 & 1.38 & 1.38 & 1.38 & 1.38 & 1.52 & 1.52 & 1.52 & 1.52 & 1.52 & 1.52 & 1.52 & 1.52 \\
\hline \begin{tabular}{|l|l|l|l} 
Density of Settled Solids $(\mathrm{g} / \mathrm{mL})$ \\
\end{tabular} & & & $\frac{\mathrm{n} / \mathrm{m}}{n / \mathrm{m}}$ & & & $n / m$ & & & & & & & & \\
\hline ww\% Setlled Supematant & $\mathrm{n} / \mathrm{m}$ & ${ }^{\mathrm{n} / \mathrm{m}}$ & $\mathrm{n}^{\mathrm{m}}$ & $\mathrm{n} / \mathrm{m}$ & $\mathrm{n} / \mathrm{m}$ & n/m & & & & & & & & \\
\hline |w\% dissolved solids in supernatant & $\mathrm{n} / \mathrm{m}$ & $\mathrm{n} / \mathrm{m}$ & $\mathrm{n} / \mathrm{m}$ & ${ }^{n / m}$ & $\mathrm{n} / \mathrm{m}$ & ${ }^{n / m}$ & ${ }^{55}$ & ${ }^{55}$ & ${ }^{55}$ & ${ }^{55}$ & ${ }^{55}$ & ${ }^{55}$ & ${ }^{55}$ & ${ }^{55}$ \\
\hline W\%\% toal solids in Centrifuged Sludge & $\mathrm{n} / \mathrm{m}$ & $\mathrm{n} / \mathrm{m}$ & $\frac{\mathrm{n} / \mathrm{m}}{\mathrm{n}}$ & ${ }^{n / m}$ & $\mathrm{n} / \mathrm{m}$ & $\mathrm{n} / \mathrm{m}$ & ${ }^{64}$ & ${ }^{64}$ & 64 & 64 & 64 & 64 & 64 & 64 \\
\hline w\%\% Total Solids & $\mathrm{n} / \mathrm{m}$ & $\mathrm{n} / \mathrm{m}$ & $n$ & $\mathrm{n} / \mathrm{m}$ & n/m & $\mathrm{n} / \mathrm{m}$ & & & & & & & & \\
\hline$\sqrt{\text { w\% UDS }}$ & 1.00 & 1.00 & $\overline{1.00}$ & 0.00 & 0.00 & 0.00 & 7.47 & 7.47 & $\begin{array}{l}7.47 \\
\end{array}$ & 7.47 & 7.47 & 7.47 & 7.47 & $\frac{47}{47}$ \\
\hline Densiviv o Solidis of & 3.66 & 3.66 & 3.66 & 3.66 & 3.66 & 3.66 & 2.2 & 2.24 & 2.24 & 2.24 & 2.24 & 2.24 & 2.24 & 2.24 \\
\hline
\end{tabular}




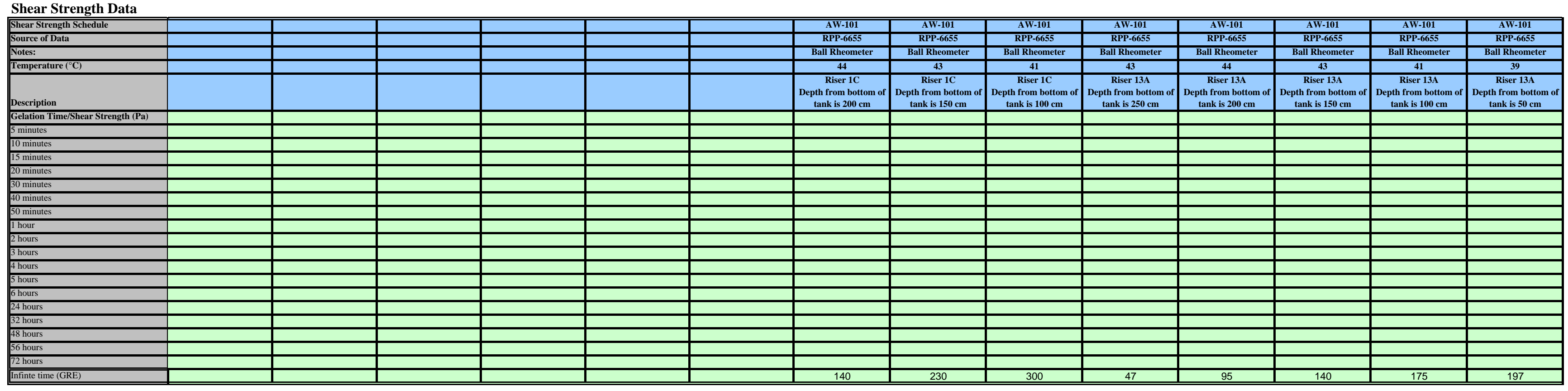




\section{Model Chemical Data From ESP}

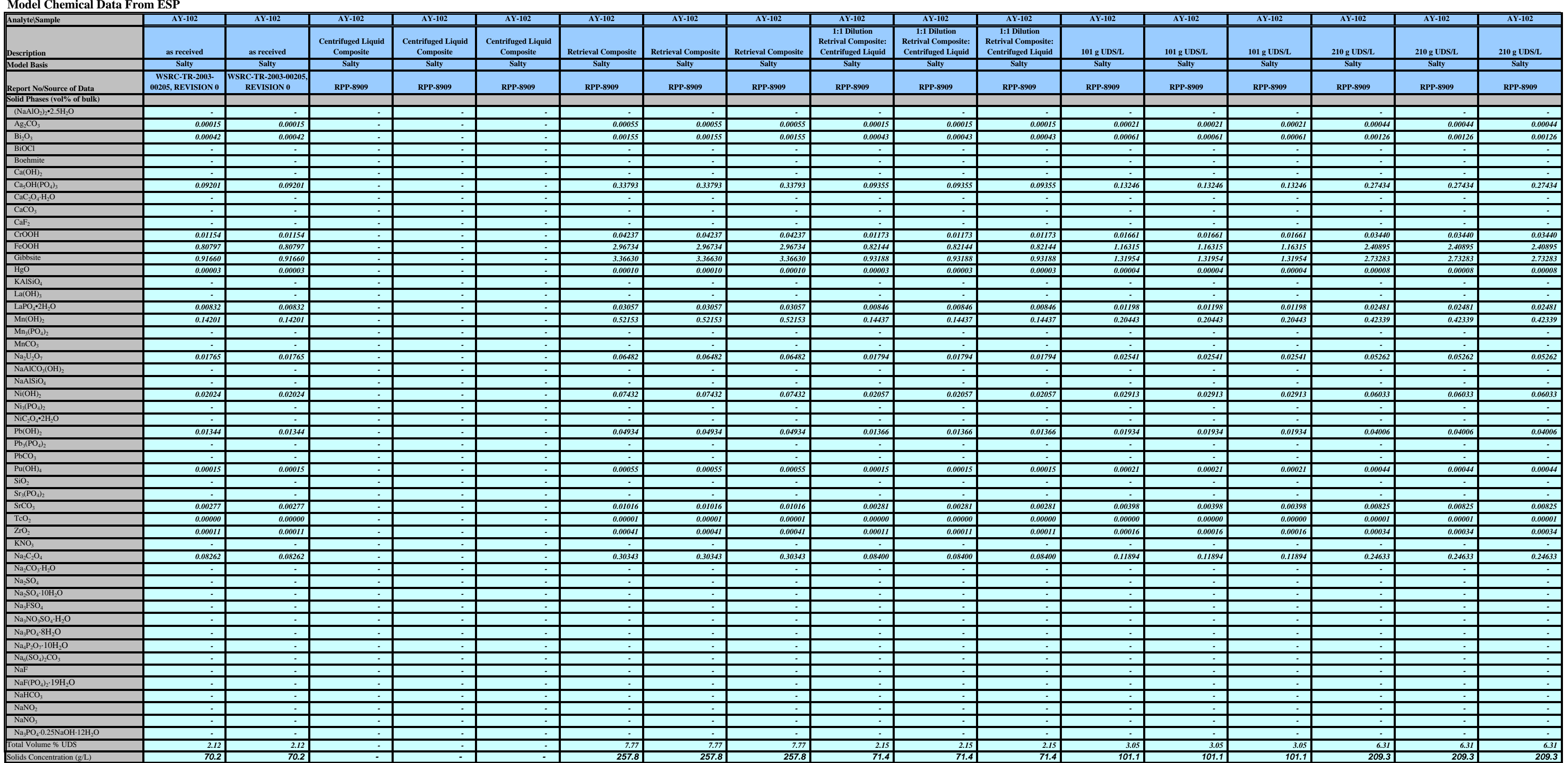


Rheological Data

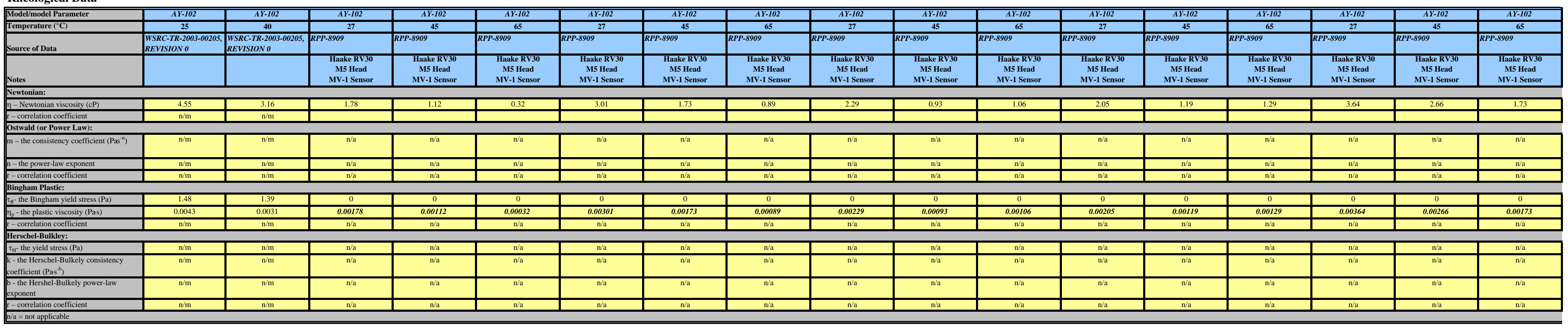

\begin{tabular}{|c|c|c|c|c|c|c|c|c|c|c|c|c|c|c|c|c|c|}
\hline \multirow{2}{*}{\multicolumn{18}{|c|}{ 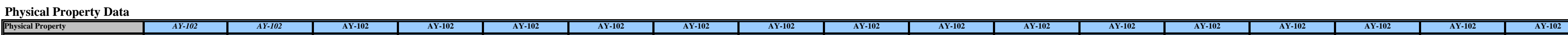 }} \\
\hline & $\overline{A Y Y-102}$ & $\overline{A Y Y-102}$ & $\begin{array}{l}\mathrm{AY}-102 \\
\end{array}$ & $\begin{array}{ll}A Y-102 \\
A\end{array}$ & $\begin{array}{ll}\mathrm{AY}-102 \\
\end{array}$ & $\begin{array}{ll}\mathrm{AY}-102 \\
\end{array}$ & $\begin{array}{ll}\mathrm{AY}-102 \\
\end{array}$ & $\begin{array}{ll}\mathrm{AY}-102 \\
\end{array}$ & $\begin{array}{ll}\mathrm{AY}-102 \\
\end{array}$ & $\begin{array}{l}\text { AY-102 } \\
\end{array}$ & $\begin{array}{l}\text { AY-102 } \\
\end{array}$ & $\begin{array}{l}\text { AY-102 } \\
\end{array}$ & $\begin{array}{ll}\mathrm{AY}-102 \\
\end{array}$ & $\begin{array}{ll}\mathrm{AY}-102 \\
\end{array}$ & $\begin{array}{ll}\mathrm{AXY}-102 \\
\end{array}$ & $\begin{array}{ll}\mathrm{AYY}-102 \\
\end{array}$ & $\begin{array}{ll}A Y-102 \\
\end{array}$ \\
\hline Notes & & & RPP-9909 & RPP-9909 & RPP-8909 & RPP-9899 & RPP-9909 & RPP.9909 & RPP.9909 & RPP-8909 & RPP-8909 & RPP-8909 & RPP-8909 & RPP-g909 & RPP.8999 & RPP.8999 & RPP-9899 \\
\hline Temperature $(\mathrm{C})$ & 25 & 40 & Ambient & Ambient & Ambient & Ambient & Ambient & Ambient & Ambient & Ambient & Ambient & Ambient & Ambient & Ambient & Ambiert & Ambiert & Ambient \\
\hline 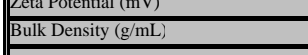 & 1.2 & 1.2 & 1.13 & 0.13 & 1.13 & 1.3 & 1.3 & 1.3 & $\frac{1.17}{1.17}$ & $\frac{1.17}{1.17}$ & $\frac{1.17}{1.17}$ & 1.24 & $\frac{1.24}{1.24}$ & 1.24 & 1.3 & 1.3 & 1.3 \\
\hline \begin{tabular}{|l} 
Wolo\% Sertiled Solids \\
\end{tabular} & ${ }^{n / m}$ & ${ }^{n / m}$ & ${ }^{0}$ & ${ }^{0}$ & & 52 & 52 & 52 & & & & & & & & & \\
\hline Density of Cennrifituged Solids $(\mathrm{g} m \mathrm{~mL}$ & 1.37 & 1.37 & $\mathrm{~nm}$ & $\mathrm{n} / \mathrm{m}$ & $\mathrm{n} / \mathrm{m}$ & $\mathrm{nm}$ & $\mathrm{nm}$ & $\mathrm{nm}$ & & & & & 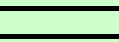 & - & E & E & \\
\hline \multicolumn{18}{|l|}{ wol\% Cennifituged Solids } \\
\hline w1\% Cenrifituged Solids & 20.4 & 20.4 & ${ }^{0}$ & ${ }^{0}$ & 0 & & & & & & & & & & & & \\
\hline 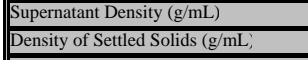 & $\frac{1.15}{\mathrm{nmm}}$ & $\frac{1.15}{\mathrm{nim}}$ & $\frac{1.13}{n \cdot m}$ & $\frac{1.13}{n \cdot \mathrm{m}}$ & $\frac{1.13}{n \cdot 1 / m}$ & $\frac{1.13}{1.47}$ & 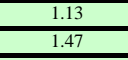 & $\frac{1.13}{1.47}$ & 1.13 & $\begin{array}{l}1.13 \\
.13\end{array}$ & & 1.13 & & & & 1.13 & 1.13 \\
\hline we\% Setrled Superatant & ${ }^{\mathrm{n} / \mathrm{m}}$ & ${ }^{n / m}$ & 100 & 100 & 100 & ${ }^{41}$ & ${ }^{41}$ & 41 & & & & & & & & & \\
\hline w1\% dissolved solids in supematant & ${ }^{16.3}$ & ${ }^{16.3}$ & 15.5 & 15.5 & 15.5 & ${ }^{16}$ & ${ }^{16}$ & ${ }^{16}$ & & & & & & & & & \\
\hline |w1\% total solids in Centrifuged Sludge & 45.5 & 45.5 & $\frac{\mathrm{nm}}{\mathrm{nm}}$ & $\frac{\mathrm{nm}}{\mathrm{nm}}$ & $\frac{\mathrm{nm}}{\mathrm{mm}}$ & ${ }^{46}$ & ${ }^{46}$ & 46 & & & & & & & & & \\
\hline $\mid$\begin{tabular}{|l|l} 
wo\% Total Solids \\
\end{tabular} & 21.2 & 21.2 & $\overline{15.5}$ & 15.5 & 15.5 & & & & & & & & & & & & \\
\hline wos UDS & 5.55 & ${ }^{5.85}$ & 0.00 & 0.00 & 0.00 & 19.83 & 19.83 & 19.83 & 6.10 & 6.10 & 6.10 & 8.15 & 8.15 & 8.15 & 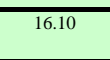 & 16.10 & 16.10 \\
\hline nsity of Solids gagct & 3.32 & 3.32 & 3.32 & 3.32 & 3.32 & 3.32 & 3.32 & 3.32 & 3.32 & 3.32 & 3.32 & 3.32 & 3,32 & 3.32 & 3.32 & 3.32 & 3.32 \\
\hline
\end{tabular}



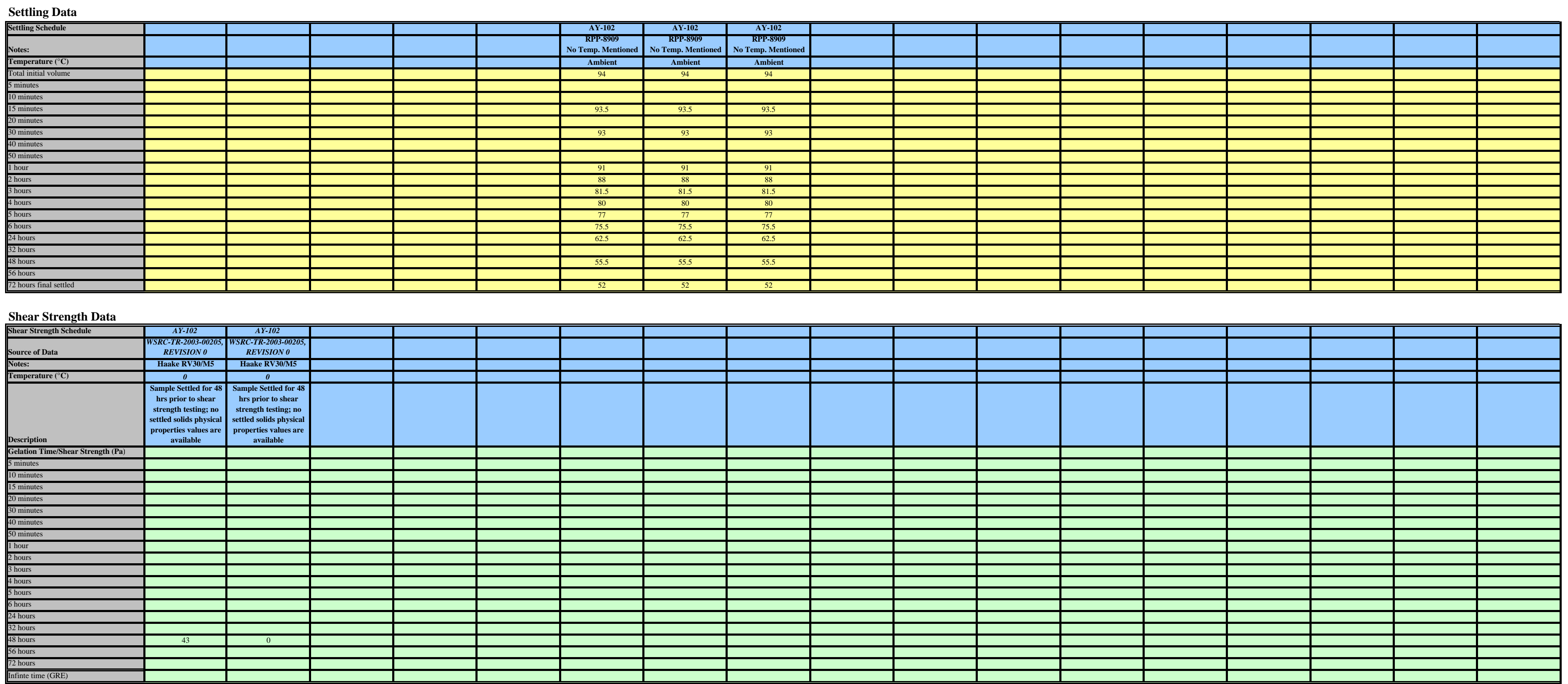
Model Chemical Data From ESP

\begin{tabular}{|c|c|c|}
\hline AnalytelSample & AZ-101 & AZ-101 \\
\hline Description & as received & $\begin{array}{c}\text { Caustic Washed and } \\
\text { Leached }\end{array}$ \\
\hline Model Basis & Salty & Leached \\
\hline Report No/Source of Data & WTP-RPT-043, Rev 1 & WTP-RPT-043, Rev 1 \\
\hline \multicolumn{3}{|l|}{ Solid Phases (vol\% of bulk) } \\
\hline$\left(\mathrm{NaAlO}_{2}\right)_{2} \cdot 2.5 \mathrm{H}_{2} \mathrm{O}$ & - & - \\
\hline $\mathrm{Ag}_{2} \mathrm{CO}_{3}$ & 0.00293 & 0.00328 \\
\hline $\mathrm{Bi}_{2} \mathrm{O}_{3}$ & - & - \\
\hline $\mathrm{BiOCl}$ & - & - \\
\hline Boehmite & - & - \\
\hline $\mathrm{Ca}(\mathrm{OH})_{2}$ & - & - \\
\hline $\mathrm{Ca}_{5} \mathrm{OH}\left(\mathrm{PO}_{4}\right)_{3}$ & 0.10549 & 0.11795 \\
\hline $\mathrm{CaC}_{2} \mathrm{O}_{4} \cdot \mathrm{H}_{2} \mathrm{O}$ & - & - \\
\hline $\mathrm{CaCO}_{3}$ & - & - \\
\hline $\mathrm{CaF}_{2}$ & - & - \\
\hline $\mathrm{CrOOH}$ & 0.00304 & 0.00340 \\
\hline $\mathrm{FeOOH}$ & 1.60010 & 1.78914 \\
\hline Gibbsite & 5.72952 & - \\
\hline $\mathrm{HgO}$ & - & - \\
\hline $\mathrm{KAlSiO}_{4}$ & - & - \\
\hline $\mathrm{La}(\mathrm{OH})_{3}$ & - & - \\
\hline $\mathrm{LaPO}_{4} \cdot 2 \mathrm{H}_{2} \mathrm{O}$ & 0.05674 & 0.06344 \\
\hline $\mathrm{Mn}(\mathrm{OH})_{2}$ & 0.02700 & 0.03019 \\
\hline $\mathrm{Mn}_{3}\left(\mathrm{PO}_{4}\right)_{2}$ & - & - \\
\hline $\mathrm{MnCO}_{3}$ & - & - \\
\hline $\mathrm{Na}_{2} \mathrm{U}_{2} \mathrm{O}_{7}$ & 0.07502 & 0.08388 \\
\hline $\mathrm{NaAlCO}_{3}(\mathrm{OH})_{2}$ & - & - \\
\hline $\mathrm{NaAlSiO}_{4}$ & 0.16550 & 0.18505 \\
\hline $\mathrm{Ni}(\mathrm{OH})_{2}$ & 0.10189 & 0.11393 \\
\hline $\mathrm{Ni}_{3}\left(\mathrm{PO}_{4}\right)_{2}$ & - & - \\
\hline $\mathrm{NiC}_{2} \mathrm{O}_{4} \cdot 2 \mathrm{H}_{2} \mathrm{O}$ & - & - \\
\hline $\mathrm{Pb}(\mathrm{OH})_{2}$ & 0.00253 & 0.00283 \\
\hline $\mathrm{Pb}_{3}\left(\mathrm{PO}_{4}\right)_{2}$ & - & - \\
\hline $\mathrm{PbCO}_{3}$ & - & - \\
\hline $\mathrm{Pu}(\mathrm{OH})_{4}$ & 0.00083 & 0.00093 \\
\hline $\mathrm{SiO}_{2}$ & - & - \\
\hline $\mathrm{Sr}_{3}\left(\mathrm{PO}_{4}\right)_{2}$ & - & - \\
\hline $\mathrm{SrCO}_{3}$ & 0.00964 & 0.01078 \\
\hline $\mathrm{TcO}_{2}$ & 0.00001 & 0.00001 \\
\hline $\mathrm{ZrO}_{2}$ & 0.36110 & 0.40376 \\
\hline $\mathrm{KNO}_{3}$ & - & - \\
\hline $\mathrm{Na}_{2} \mathrm{C}_{2} \mathrm{O}_{4}$ & - & - \\
\hline $\mathrm{Na}_{2} \mathrm{CO}_{3} \cdot \mathrm{H}_{2} \mathrm{O}$ & - & - \\
\hline $\mathrm{Na}_{2} \mathrm{SO}_{4}$ & - & - \\
\hline $\mathrm{Na}_{2} \mathrm{SO}_{4} \cdot 10 \mathrm{H}_{2} \mathrm{O}$ & - & - \\
\hline $\mathrm{Na}_{3} \mathrm{FSO}_{4}$ & - & - \\
\hline $\mathrm{Na}_{3} \mathrm{NO}_{3} \mathrm{SO}_{4} \cdot \mathrm{H}_{2} \mathrm{O}$ & - & - \\
\hline $\mathrm{Na}_{3} \mathrm{PO}_{4} \cdot 8 \mathrm{H}_{2} \mathrm{O}$ & - & - \\
\hline $\mathrm{Na}_{4} \mathrm{P}_{2} \mathrm{O}_{7} \cdot 10 \mathrm{H}_{2} \mathrm{O}$ & - & - \\
\hline $\mathrm{Na}_{6}\left(\mathrm{SO}_{4}\right)_{2} \mathrm{CO}_{3}$ & - & - \\
\hline $\mathrm{NaF}$ & - & - \\
\hline $\mathrm{NaF}\left(\mathrm{PO}_{4}\right)_{2} \cdot 19 \mathrm{H}_{2} \mathrm{O}$ & - & - \\
\hline $\mathrm{NaHCO}_{3}$ & - & - \\
\hline $\mathrm{NaNO}_{2}$ & - & - \\
\hline $\mathrm{NaNO}_{3}$ & - & - \\
\hline $\mathrm{Na}_{3} \mathrm{PO}_{4} \cdot 0.25 \mathrm{NaOH} \cdot 12 \mathrm{H}_{2} \mathrm{O}$ & - & - \\
\hline Total Volume \% UDS & 8.24 & 2.81 \\
\hline Solids Concentration $(\mathrm{g} / \mathrm{L})$ & 248.8 & 123.2 \\
\hline
\end{tabular}

Note: Numbers in italics are calculated values. Numbers in normal font are reported data. 


\section{Rheological Data}

\begin{tabular}{|c|c|c|}
\hline Model/model Parameter & $\overline{\text { AZ-101 }}$ & $\overline{\mathrm{AZZ-101}}$ \\
\hline Temperature $\left({ }^{\circ} \mathrm{C}\right)$ & 25 & 25 \\
\hline Source of Data & WTP-RPT-043, Rev 1 & WTP-RPT-043, Rev 1 \\
\hline \multicolumn{3}{|l|}{ Notes } \\
\hline \multicolumn{3}{|l|}{ Newtonian: } \\
\hline$\eta$ - Newtonian viscosity (cP) & $\mathrm{n} / \mathrm{m}$ & $\mathrm{n} / \mathrm{m}$ \\
\hline $\mathrm{r}$ - correlation coefficient & $\mathrm{n} / \mathrm{m}$ & $\mathrm{n} / \mathrm{m}$ \\
\hline \multicolumn{3}{|l|}{ Ostwald (or Power Law): } \\
\hline $\mathrm{m}$ - the consistency coefficient $\left(\mathrm{Pa} \cdot \mathrm{s}^{-\mathrm{n}}\right)$ & $\mathrm{n} / \mathrm{m}$ & $\mathrm{n} / \mathrm{m}$ \\
\hline $\mathrm{n}$ - the power-law exponent & $\mathrm{n} / \mathrm{m}$ & $\mathrm{n} / \mathrm{m}$ \\
\hline $\mathrm{r}$ - correlation coefficient & $\mathrm{n} / \mathrm{m}$ & $\mathrm{n} / \mathrm{m}$ \\
\hline \multicolumn{3}{|l|}{ Bingham Plastic: } \\
\hline$\tau_{\mathrm{B}}$ - the Bingham yield stress $(\mathrm{Pa})$ & 3.9 & 1.4 \\
\hline$\eta_{p}$ - the plastic viscosity (Pa.s) & 0.0083 & 0.0029 \\
\hline $\mathrm{r}$-correlation coefficient & 0.99 & 0.98 \\
\hline \multicolumn{3}{|l|}{ Herschel-Bulkley: } \\
\hline$\tau_{\mathrm{H}^{-}}$the yield stress $(\mathrm{Pa})$ & 2.4 & 0.873 \\
\hline $\begin{array}{l}\mathrm{k} \text { - the Herschel-Bulkely consistency } \\
\text { coefficient }\left(\mathrm{Pa} \cdot \mathrm{s}^{-\mathrm{b}}\right)\end{array}$ & 0.0592 & 0.0346 \\
\hline $\begin{array}{l}\mathrm{b} \text { - the Hershel-Bulkely power-law } \\
\text { exponent }\end{array}$ & 0.5 & 0.5 \\
\hline $\mathrm{r}$ - correlation coefficient & 1 & 0.98 \\
\hline $\mathrm{n} / \mathrm{a}=$ not applicable & & \\
\hline
\end{tabular}

\section{Physical Property Data}

\begin{tabular}{||l|c|c|}
\hline Physical Property & AZ-101 & AZ-101 \\
\hline Notes & & \\
\hline Temperature ( ${ }^{\circ}$ C) & 25 & 25 \\
\hline Zeta Potential (mV) & & 1.13 \\
\hline Bulk Density (g/mL) & 1.39 & $\mathrm{n} / \mathrm{m}$ \\
\hline vol\% Settled Solids & $\mathrm{n} / \mathrm{m}$ & 1.48 \\
\hline Density of Centrifuged Solids (g/mL) & 1.61 & 20.5 \\
\hline vol\% Centrifuged Solids & 36.4 & 27 \\
\hline wt $\%$ Centrifuged Solids & 43.9 & $\mathrm{n} / \mathrm{m}$ \\
\hline Supernatant Density (g/mL) & 1.19 & $\mathrm{n} / \mathrm{m}$ \\
\hline Density of Settled Solids (g/mL) & $\mathrm{n} / \mathrm{m}$ & 3.43 \\
\hline wt\% Settled Supernatant & $\mathrm{n} / \mathrm{m}$ & $\mathrm{n} / \mathrm{m}$ \\
\hline wt\% dissolved solids in supernatant & 26.2 & 13.7 \\
\hline wt\% total solids in Centrifuged Sludge & $\mathrm{n} / \mathrm{m}$ & 10.90 \\
\hline wt\% Total Solids & 3.02 & \\
\hline wt\% UDS & & \\
\hline Density of Solids (g/cc) & & \\
\hline \hline
\end{tabular}

Note: Numbers in italics are calculated values. Numbers in normal font are reported data. 


\section{1-AZ-102}

Model Chemical Data From ESP

\begin{tabular}{|c|c|c|c|}
\hline AnalytelSample & AZ-102 & $\overline{\mathrm{AZ-102}}$ & AZ-102 \\
\hline Description & $\begin{array}{c}\text { as received with } 6: 1 \mathrm{wt} \\
0.01 \mathrm{NaOH} \text { added }\end{array}$ & $\begin{array}{c}\text { concentrated slurry as } \\
\text { received with 6:1 wt } 0.01 \\
\mathrm{NaOH} \text { added }\end{array}$ & $\begin{array}{c}\text { Caustic Washed and } \\
\text { Leached }\end{array}$ \\
\hline Model Basis & Salt Free & Salt Free & Leached \\
\hline Report No/Source of Data & BNFL-RPT-038 Rev. 0 & BNFL-RPT-038 Rev. 0 & BNFL-RPT-038 Rev. 0 \\
\hline \multicolumn{4}{|l|}{ Solid Phases (vol\% of bulk) } \\
\hline$\left(\mathrm{NaAlO}_{2}\right)_{2} \cdot 2.5 \mathrm{H}_{2} \mathrm{O}$ & - & - & - \\
\hline $\mathrm{Ag}_{2} \mathrm{CO}_{3}$ & 0.00207 & 0.00571 & 0.00532 \\
\hline $\mathrm{Bi}_{2} \mathrm{O}_{3}$ & 0.00013 & 0.00035 & 0.00033 \\
\hline $\mathrm{BiOCl}$ & - & - & - \\
\hline Boehmite & - & - & - \\
\hline $\mathrm{Ca}(\mathrm{OH})_{2}$ & - & - & - \\
\hline $\mathrm{Ca}_{5} \mathrm{OH}\left(\mathrm{PO}_{4}\right)_{3}$ & 0.02282 & 0.06286 & 0.05856 \\
\hline $\mathrm{CaC}_{2} \mathrm{O}_{4} \cdot \mathrm{H}_{2} \mathrm{O}$ & - & - & - \\
\hline $\mathrm{CaCO}_{3}$ & - & - & - \\
\hline $\mathrm{CaF}_{2}$ & - & - & - \\
\hline $\mathrm{CrOOH}$ & 0.00304 & 0.00838 & 0.00781 \\
\hline $\mathrm{FeOOH}$ & 0.36541 & 1.00640 & 0.93763 \\
\hline Gibbsite & 0.66849 & 1.84113 & - \\
\hline $\mathrm{HgO}$ & - & - & - \\
\hline $\mathrm{KAlSiO}_{4}$ & - & - & - \\
\hline $\mathrm{La}(\mathrm{OH})_{3}$ & - & - & - \\
\hline $\mathrm{LaPO}_{4} \cdot 2 \mathrm{H}_{2} \mathrm{O}$ & 0.00933 & 0.02569 & 0.02394 \\
\hline $\mathrm{Mn}(\mathrm{OH})_{2}$ & 0.01000 & 0.02753 & 0.02565 \\
\hline $\mathrm{Mn}_{3}\left(\mathrm{PO}_{4}\right)_{2}$ & - & - & - \\
\hline $\mathrm{MnCO}_{3}$ & - & - & - \\
\hline $\mathrm{Na}_{2} \mathrm{U}_{2} \mathrm{O}_{7}$ & 0.02076 & 0.05718 & 0.05328 \\
\hline $\mathrm{NaAlCO}_{3}(\mathrm{OH})_{2}$ & 0.48125 & 1.32544 & 1.23487 \\
\hline $\mathrm{NaAlSiO}_{4}$ & 0.16669 & 0.45908 & 0.42771 \\
\hline $\mathrm{Ni}(\mathrm{OH})_{2}$ & 0.02233 & 0.06150 & 0.05730 \\
\hline $\mathrm{Ni}_{3}\left(\mathrm{PO}_{4}\right)_{2}$ & - & - & - \\
\hline $\mathrm{NiC}_{2} \mathrm{O}_{4} \cdot 2 \mathrm{H}_{2} \mathrm{O}$ & - & - & - \\
\hline $\mathrm{Pb}(\mathrm{OH})_{2}$ & 0.00184 & 0.00508 & 0.00473 \\
\hline $\mathrm{Pb}_{3}\left(\mathrm{PO}_{4}\right)_{2}$ & - & - & - \\
\hline $\mathrm{PbCO}_{3}$ & - & - & - \\
\hline $\mathrm{Pu}(\mathrm{OH})_{4}$ & 0.00023 & 0.00063 & 0.00059 \\
\hline $\mathrm{SiO}_{2}$ & - & - & - \\
\hline $\mathrm{Sr}_{3}\left(\mathrm{PO}_{4}\right)_{2}$ & - & - & - \\
\hline $\mathrm{SrCO}_{3}$ & 0.00097 & 0.00267 & 0.00249 \\
\hline $\mathrm{TcO}_{2}$ & 0.00001 & 0.00002 & 0.00002 \\
\hline $\mathrm{ZrO}_{2}$ & 0.03346 & 0.09216 & 0.08586 \\
\hline $\mathrm{KNO}_{3}$ & - & - & - \\
\hline $\mathrm{Na}_{2} \mathrm{C}_{2} \mathrm{O}_{4}$ & - & - & - \\
\hline $\mathrm{Na}_{2} \mathrm{CO}_{3} \cdot \mathrm{H}_{2} \mathrm{O}$ & - & - & - \\
\hline $\mathrm{Na}_{2} \mathrm{SO}_{4}$ & - & - & - \\
\hline $\mathrm{Na}_{2} \mathrm{SO}_{4} \cdot 10 \mathrm{H}_{2} \mathrm{O}$ & - & - & - \\
\hline $\mathrm{Na}_{3} \mathrm{FSO}_{4}$ & - & - & - \\
\hline $\mathrm{Na}_{3} \mathrm{NO}_{3} \mathrm{SO}_{4} \cdot \mathrm{H}_{2} \mathrm{O}$ & - & - & - \\
\hline $\mathrm{Na}_{3} \mathrm{PO}_{4} \cdot 8 \mathrm{H}_{2} \mathrm{O}$ & - & - & - \\
\hline $\mathrm{Na}_{4} \mathrm{P}_{2} \mathrm{O}_{7} \cdot 10 \mathrm{H}_{2} \mathrm{O}$ & - & - & - \\
\hline $\mathrm{Na}_{6}\left(\mathrm{SO}_{4}\right)_{2} \mathrm{CO}_{3}$ & - & - & - \\
\hline $\mathrm{NaF}$ & - & - & - \\
\hline $\mathrm{NaF}\left(\mathrm{PO}_{4}\right)_{2} \cdot 19 \mathrm{H}_{2} \mathrm{O}$ & - & - & - \\
\hline $\mathrm{NaHCO}_{3}$ & - & - & - \\
\hline $\mathrm{NaNO}_{2}$ & - & - & - \\
\hline $\mathrm{NaNO}_{3}$ & - & - & - \\
\hline $\mathrm{Na}_{3} \mathrm{PO}_{4} \cdot 0.25 \mathrm{NaOH} \cdot 12 \mathrm{H}_{2} \mathrm{O}$ & - & - & - \\
\hline Total Volume \% UDS & 1.81 & 4.98 & 2.93 \\
\hline Solids Concentration (g/L) & 53.8 & 148.2 & 96.6 \\
\hline
\end{tabular}

Note: Numbers in italics are calculated values. Numbers in normal font are reported data. 


\section{Rheological Data}

\begin{tabular}{|c|c|c|c|}
\hline Model/model Parameter & $\overline{A Z-102}$ & $\overline{A Z-102}$ & $\overline{A Z-102}$ \\
\hline Temperature $\left({ }^{\circ} \mathrm{C}\right)$ & 25 & 25 & 25 \\
\hline \multicolumn{4}{|c|}{ BNFL-RPT-038 Rev. 0} \\
\hline Notes & Appendix F; Figure 4 & Appendix F; Figure 9 & Appendix F; Figure 15 \\
\hline \multicolumn{4}{|l|}{ Newtonian: } \\
\hline$\eta$ - Newtonian viscosity (cP) & 2.589 & 9.908 & 17.74 \\
\hline $\mathrm{r}$ - correlation coefficient & 0.993 & 0.962 & 0.84 \\
\hline \multicolumn{4}{|l|}{ Ostwald (or Power Law): } \\
\hline $\mathrm{m}$ - the consistency coefficient $\left(\mathrm{Pa} \cdot \mathrm{s}^{-\mathrm{n}}\right)$ & 0.022 & 0.177 & 2.45 \\
\hline $\mathrm{n}$ - the power-law exponent & 0.631 & 0.504 & 0.25 \\
\hline $\mathrm{r}$ - correlation coefficient & 0.986 & 0.982 & 0.99 \\
\hline \multicolumn{4}{|l|}{ Bingham Plastic: } \\
\hline$\tau_{\mathrm{B}}$ - the Bingham yield stress $(\mathrm{Pa})$ & 0.134 & 1.377 & 6.53 \\
\hline$\eta_{p}$ - the plastic viscosity (Pa.s) & 0.002 & 0.006 & 0.01 \\
\hline $\mathrm{r}$ - correlation coefficient & 0.993 & 0.962 & 0.84 \\
\hline \multicolumn{4}{|l|}{ Herschel-Bulkley: } \\
\hline$\tau_{\mathrm{H}^{-}}$the yield stress $(\mathrm{Pa})$ & 0.000 & 0.939 & 4.55 \\
\hline $\begin{array}{l}\text { k - the Herschel-Bulkely consistency } \\
\text { coefficient }\left(\mathrm{Pa} \cdot \mathrm{s}^{-\mathrm{b}}\right)\end{array}$ & 0.027 & 0.047 & 0.26 \\
\hline $\begin{array}{l}\text { b - the Hershel-Bulkely power-law } \\
\text { exponent }\end{array}$ & 0.593 & 0.671 & 0.52 \\
\hline $\mathrm{r}$ - correlation coefficient & 0.983 & 0.983 & 0.95 \\
\hline
\end{tabular}

\section{Physical Property Data}

\begin{tabular}{|c|c|c|c|}
\hline$\sqrt{\text { Physical Property }}$ & $\overline{\overline{A Z-102}}$ & $\overline{A Z A-102}$ & 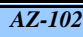 \\
\hline \multicolumn{4}{|l|}{ Notes } \\
\hline |Temperature $\left({ }^{\circ} \mathrm{C}\right)$ & 25 & 25 & 25 \\
\hline \multicolumn{4}{|l|}{ Zeta Potential (mV) } \\
\hline Bulk Density (g/mL) & 1.06 & 1.09 & 1.05 \\
\hline ||vol\% Settled Solids & $\mathrm{n} / \mathrm{m}$ & 96 & 93 \\
\hline Density of Centrifuged Solids (g/mL) & $\mathrm{n} / \mathrm{m}$ & 1.36 & 1.2 \\
\hline |vol\% Centrifuged Solids & $\mathrm{n} / \mathrm{m}$ & 26 & 26 \\
\hline |wt\% Centrifuged Solids & $\mathrm{n} / \mathrm{m}$ & 33 & 30 \\
\hline Supernatant Density $(\mathrm{g} / \mathrm{mL})$ & 1.02 & 1.02 & 1.04 \\
\hline Density of Settled Solids (g/mL) & $\mathrm{n} / \mathrm{m}$ & 1.07 & 1.07 \\
\hline |wt\% Settled Supernatant & $\mathrm{n} / \mathrm{m}$ & 95 & 94 \\
\hline wt\% dissolved solids in supernatant & $\mathrm{n} / \mathrm{m}$ & $\mathrm{n} / \mathrm{m}$ & $\mathrm{n} / \mathrm{m}$ \\
\hline wt $\%$ total solids in Centrifuged Sludge & $\mathrm{n} / \mathrm{m}$ & $\mathrm{n} / \mathrm{m}$ & $\mathrm{n} / \mathrm{m}$ \\
\hline wt\% Total Solids & $\mathrm{n} / \mathrm{m}$ & $\mathrm{n} / \mathrm{m}$ & $\mathrm{n} / \mathrm{m}$ \\
\hline wt $\%$ UDS & 5.10 & 13.60 & 9.20 \\
\hline |Density of Solids (g/cc) & 2.98 & 2.98 & 3.30 \\
\hline
\end{tabular}

Note: Numbers in italics are calculated values. Numbers in normal font are reported data. 
241-B-111

Model Chemical Data From ESP

\begin{tabular}{|c|c|c|}
\hline AnalytelSample & B-111 & B-111 \\
\hline Description & no dilution & no dilution \\
\hline Model Basis & Salty & Salty \\
\hline Report No/Source of Data & $\begin{array}{l}\text { Segment 3; WHC-SD- } \\
\text { WM-ER-382, rev } 0\end{array}$ & $\begin{array}{l}\text { Segment 5; WHC-SD- } \\
\text { WM-ER-382, rev } 0\end{array}$ \\
\hline \multicolumn{3}{|l|}{ Solid Phases (vol\% of bulk) } \\
\hline$\left(\mathrm{NaAlO}_{2}\right)_{2} \cdot 2.5 \mathrm{H}_{2} \mathrm{O}$ & - & - \\
\hline $\mathrm{Ag}_{2} \mathrm{CO}_{3}$ & 0.00014 & 0.00021 \\
\hline $\mathrm{Bi}_{2} \mathrm{O}_{3}$ & 0.25830 & 0.40743 \\
\hline $\mathrm{BiOCl}$ & 0.08115 & 0.12801 \\
\hline Boehmite & - & - \\
\hline $\mathrm{Ca}(\mathrm{OH})_{2}$ & - & - \\
\hline $\mathrm{Ca}_{5} \mathrm{OH}\left(\mathrm{PO}_{4}\right)_{3}$ & - & - \\
\hline $\mathrm{CaC}_{2} \mathrm{O}_{4} \cdot \mathrm{H}_{2} \mathrm{O}$ & - & - \\
\hline $\mathrm{CaCO}_{3}$ & - & - \\
\hline $\mathrm{CaF}_{2}$ & 0.05600 & 0.08834 \\
\hline $\mathrm{CrOOH}$ & 0.03960 & 0.06247 \\
\hline $\mathrm{FeOOH}$ & 0.81578 & 1.28677 \\
\hline Gibbsite & - & - \\
\hline $\mathrm{HgO}$ & - & - \\
\hline $\mathrm{KAlSiO}_{4}$ & - & - \\
\hline $\mathrm{La}(\mathrm{OH})_{3}$ & - & - \\
\hline $\mathrm{LaPO}_{4} \cdot 2 \mathrm{H}_{2} \mathrm{O}$ & 0.00027 & 0.00043 \\
\hline $\mathrm{Mn}(\mathrm{OH})_{2}$ & - & - \\
\hline $\mathrm{Mn}_{3}\left(\mathrm{PO}_{4}\right)_{2}$ & - & - \\
\hline $\mathrm{MnCO}_{3}$ & 0.00699 & 0.01102 \\
\hline $\mathrm{Na}_{2} \mathrm{U}_{2} \mathrm{O}_{7}$ & - & - \\
\hline $\mathrm{NaAlCO}_{3}(\mathrm{OH})_{2}$ & 0.26333 & 0.41536 \\
\hline $\mathrm{NaAlSiO}_{4}$ & - & - \\
\hline $\mathrm{Ni}(\mathrm{OH})_{2}$ & - & - \\
\hline $\mathrm{Ni}_{3}\left(\mathrm{PO}_{4}\right)_{2}$ & - & - \\
\hline $\mathrm{NiC}_{2} \mathrm{O}_{4} \cdot 2 \mathrm{H}_{2} \mathrm{O}$ & 0.00284 & 0.00448 \\
\hline $\mathrm{Pb}(\mathrm{OH})_{2}$ & - & - \\
\hline $\mathrm{Pb}_{3}\left(\mathrm{PO}_{4}\right)_{2}$ & - & - \\
\hline $\mathrm{PbCO}_{3}$ & 0.04051 & 0.06390 \\
\hline $\mathrm{Pu}(\mathrm{OH})_{4}$ & 0.00004 & 0.00006 \\
\hline $\mathrm{SiO}_{2}$ & 1.02591 & 1.61821 \\
\hline $\mathrm{Sr}_{3}\left(\mathrm{PO}_{4}\right)_{2}$ & - & - \\
\hline $\mathrm{SrCO}_{3}$ & 0.01394 & 0.02198 \\
\hline $\mathrm{TcO}_{2}$ & 0.00010 & 0.00016 \\
\hline $\mathrm{ZrO}_{2}$ & 0.00045 & 0.00071 \\
\hline $\mathrm{KNO}_{3}$ & - & - \\
\hline $\mathrm{Na}_{2} \mathrm{C}_{2} \mathrm{O}_{4}$ & 0.26828 & 0.42317 \\
\hline $\mathrm{Na}_{2} \mathrm{CO}_{3} \cdot \mathrm{H}_{2} \mathrm{O}$ & - & - \\
\hline $\mathrm{Na}_{2} \mathrm{SO}_{4}$ & - & - \\
\hline $\mathrm{Na}_{2} \mathrm{SO}_{4} \cdot 10 \mathrm{H}_{2} \mathrm{O}$ & - & - \\
\hline $\mathrm{Na}_{3} \mathrm{FSO}_{4}$ & - & - \\
\hline $\mathrm{Na}_{3} \mathrm{NO}_{3} \mathrm{SO}_{4} \cdot \mathrm{H}_{2} \mathrm{O}$ & - & - \\
\hline $\mathrm{Na}_{3} \mathrm{PO}_{4} \cdot 8 \mathrm{H}_{2} \mathrm{O}$ & - & - \\
\hline $\mathrm{Na}_{4} \mathrm{P}_{2} \mathrm{O}_{7} \cdot 10 \mathrm{H}_{2} \mathrm{O}$ & 6.86039 & 10.82119 \\
\hline $\mathrm{Na}_{6}\left(\mathrm{SO}_{4}\right)_{2} \mathrm{CO}_{3}$ & - & - \\
\hline $\mathrm{NaF}$ & - & - \\
\hline $\mathrm{NaF}\left(\mathrm{PO}_{4}\right)_{2} \cdot 19 \mathrm{H}_{2} \mathrm{O}$ & - & - \\
\hline $\mathrm{NaHCO}_{3}$ & - & - \\
\hline $\mathrm{NaNO}_{2}$ & - & - \\
\hline $\mathrm{NaNO}_{3}$ & - & - \\
\hline $\mathrm{Na}_{3} \mathrm{PO}_{4} \cdot 0.25 \mathrm{NaOH} \cdot 12 \mathrm{H}_{2} \mathrm{O}$ & - & - \\
\hline Total Volume \% UDS & 9.73 & 15.35 \\
\hline Solids Concentration (g/L) & 236.2 & 372.6 \\
\hline
\end{tabular}

Note: Numbers in italics are calculated values. Numbers in normal font are reported data. 
241-B-111

Physical Property Data

\begin{tabular}{|l|c|c|}
\hline Physical Property & $\mathbf{B}-111$ & $\mathbf{B}-111$ \\
\hline Notes & & $\mathbf{3 0}$ \\
\hline Temperature $\left({ }^{\circ} \mathrm{C}\right)$ & $\mathbf{3 0}$ & 1.35 \\
\hline Zeta Potential $(\mathrm{mV})$ & 1.27 & 100 \\
\hline Bulk Density (g/mL) & 100 & 1.45 \\
\hline vol\% Settled Solids & 1.38 & 63 \\
\hline Density of Centrifuged Solids (g/mL) & 57 & 67 \\
\hline vol\% Centrifuged Solids & 55 & 1.17 \\
\hline wt\% Centrifuged Solids & 1.15 & $\mathrm{n} / \mathrm{m}$ \\
\hline Supernatant Density (g/mL) & $\mathrm{n} / \mathrm{m}$ & $\mathrm{n} / \mathrm{m}$ \\
\hline Density of Settled Solids (g/mL) & $\mathrm{n} / \mathrm{m}$ & $\mathrm{n} / \mathrm{m}$ \\
\hline wt\% Settled Supernatant & 11.60 & 37.2 \\
\hline wt\% dissolved solids in supernatant & $\mathrm{n} / \mathrm{m}$ & 27.60 \\
\hline wt\% total solids in Centrifuged Sludge & 30.2 & 2.43 \\
\hline wt\% Total Solids & 18.60 & \\
\hline wt\% UDS & $\mathbf{2 . 4 3}$ & \\
\hline Density of Solids (g/cc) & & \\
\hline \hline
\end{tabular}

Note: Numbers in italics are calculated values. Numbers in normal font are reported data. 
241-B-111

\section{Shear Strength Data}

\begin{tabular}{||l|c|c|}
\hline Shear Strength Schedule & B-111 & B-111 \\
\hline Source of Data & $\begin{array}{c}\text { Segment 3; WHC-SD-WM } \\
\text { ER-382, rev 0 }\end{array}$ & $\begin{array}{c}\text { Segment 5; WHC-SD-WM- } \\
\text { ER-382, rev 0 }\end{array}$ \\
\hline Notes: & Page 5-14 & Page 5-14 \\
\hline Temperature ( ${ }^{\circ}$ C) & Unspecified & Unspecified \\
\hline Description & $\begin{array}{c}\text { Shear Vane at unspecified } \\
\text { gel time or temperature }\end{array}$ & $\begin{array}{c}\text { Shear Vane at unspecified } \\
\text { gel time or temperature }\end{array}$ \\
\hline Gelation Time/Shear Strength (Pa) & & \\
\hline 5 minutes & & \\
\hline 10 minutes & & \\
\hline 15 minutes & & \\
\hline 20 minutes & & \\
\hline 30 minutes & & \\
\hline 40 minutes & & \\
\hline 50 minutes & & \\
\hline 1 hour & & \\
\hline 2 hours & & \\
\hline 3 hours & & \\
\hline 4 hours & & \\
\hline 5 hours & & \\
\hline 6 hours & & \\
\hline 24 hours & & \\
\hline 32 hours & & \\
\hline 48 hours & & \\
\hline 56 hours & & \\
\hline 72 hours & & \\
\hline \hline Infinte time (GRE) & & \\
\hline & & \\
\hline
\end{tabular}

Note: Numbers in italics are calculated values. Numbers in normal font are reported data. 


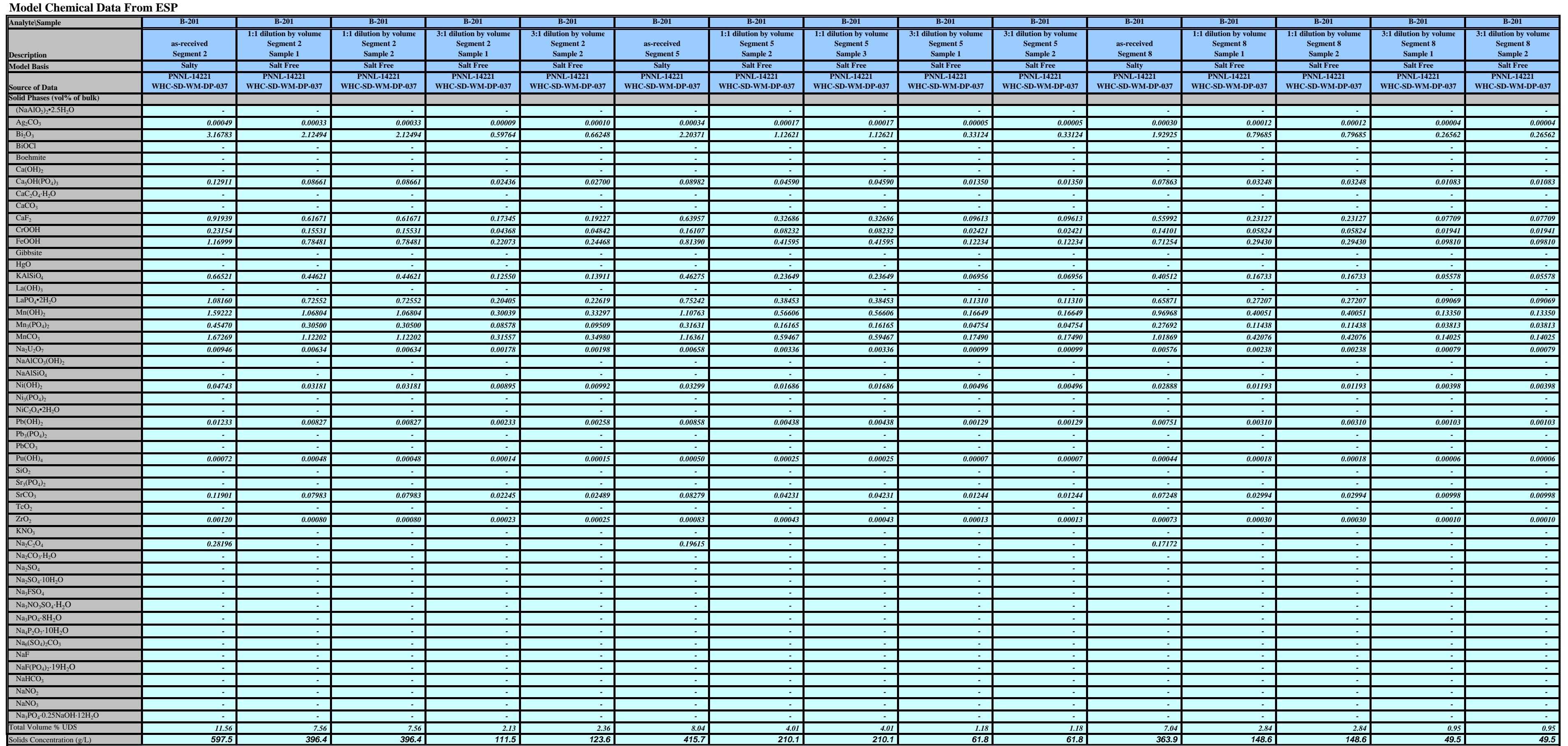


Rheological Data

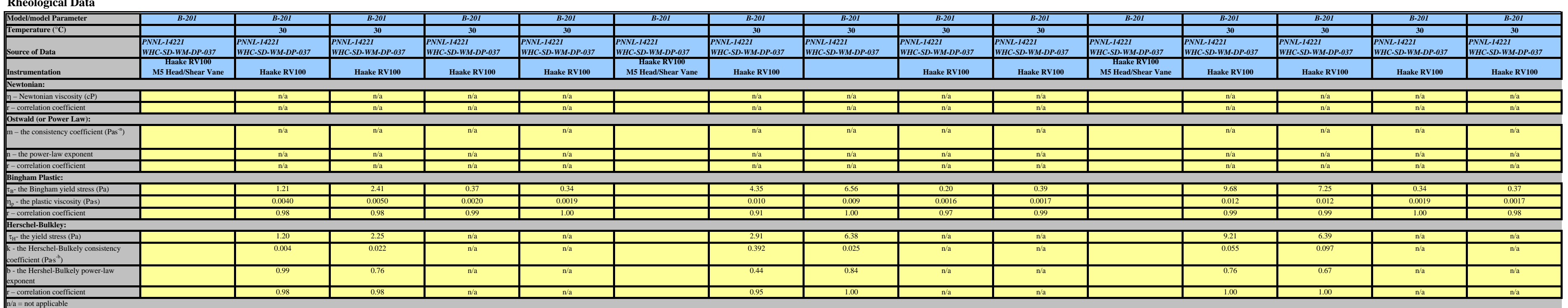

Physical Property Data

\begin{tabular}{|c|c|c|c|c|c|c|c|c|c|c|c|c|c|c|c|}
\hline Physical Property & $\overline{B-201}$ & $\overline{B-201}$ & $\overline{B-201}$ & $\overline{B-201}$ & $\overline{B-201}$ & B-201 & B-201 & B-201 & B-201 & B-201 & ${ }^{B-201}$ & $B^{B}-201$ & $\overline{\overline{B-201}}$ & $\overline{\overline{B-201}}$ & $\overline{B-201}$ \\
\hline Source of Data & $\begin{array}{c}\text { PNNL-14221 } \\
\text { WHC-SD-WM-DP-037 }\end{array}$ & $\begin{array}{c}\text { PNNL-14221 } \\
\text { WHC-SD-WM-DP-037 }\end{array}$ & $\begin{array}{c}\text { PNNL-14221 } \\
\text { WHC-SD-WM-DP-037 }\end{array}$ & $\begin{array}{c}\text { PNNL-14221 } \\
\text { WHC-SD-WM-DP-037 }\end{array}$ & $\begin{array}{c}\text { PNNL-14221 } \\
\text { WHC-SD-WM-DP-037 }\end{array}$ & $\begin{array}{c}\text { PNNL-14221 } \\
\text { WHC-SD-WM-DP-037 }\end{array}$ & $\begin{array}{c}\text { PNNL-14221 } \\
\text { WHC-SD-WM-DP-037 }\end{array}$ & $\begin{array}{c}\text { PNNL-14221 } \\
\text { WHC-SD-WM-DP-037 }\end{array}$ & $\begin{array}{c}\text { PNNL-14221 } \\
\text { WHC-SD-WM-DP-037 }\end{array}$ & $\begin{array}{c}\text { PNNL-14221 } \\
\text { WHC-SD-WM-DP-037 }\end{array}$ & $\begin{array}{c}\text { PNNL-14221 } \\
\text { WHC-SD-WM-DP-037 }\end{array}$ & $\begin{array}{c}\text { PNNL-14221 } \\
\text { WHC-SD-WM-DP-037 }\end{array}$ & $\begin{array}{c}\text { PNNL-14221 } \\
\text { WHC-SD-WM-DP-037 }\end{array}$ & $\begin{array}{c}\text { PNNL-14221 } \\
\text { WHC-SD-WM-DP-037 }\end{array}$ & $\begin{array}{c}\text { PNNL-14221 } \\
\text { WHC-SD-WM-DP-037 }\end{array}$ \\
\hline Temperature $(\mathrm{C})$ & 30 & 30 & 30 & 30 & 30 & 30 & 30 & 30 & 30 & 30 & 30 & 30 & 30 & 30 & 30 \\
\hline 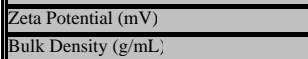 & 1.65 & 1.33 & 1.33 & 1.1 & 1.1 & $\frac{\mathrm{n}^{\prime m}}{1.51}$ & 1.17 & 1.17 & 1.05 & 1.05 & $\frac{n / m}{1.34}$ & $\frac{n / m}{1 / 13}$ & $\frac{n / m}{1.13}$ & $\frac{n^{\prime \prime m}}{1.05}$ & $\frac{n / m}{1.05}$ \\
\hline Vvol\% serted Solids & 100 & 82.7 & 82.9 & $\frac{42.5}{42.5}$ & 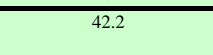 & 100 & 80 & 81.5 & 36.9 & 37.1 & 100 & 91.8 & 91.9 & 43.9 & 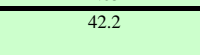 \\
\hline Densily of Centrifitged Solids (gemL & 1.66 & 1.59 & 1.59 & 1.48 & 1.48 & 1.52 & 1.4 & 1.4 & 1.36 & 1.36 & 1.37 & 1.33 & 1.33 & $\frac{1.24}{1.24}$ & 1.24 \\
\hline 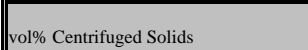 & 98 & 57.4 & 58.8 & 24 & 23.4 & 98 & 43.8 & 43.1 & 16.2 & 16.2 & 88 & 41.3 & 42.5 & 23.5 & 19.4 \\
\hline wor Cenrifinged Solids & 98 & 67.6 & 69.5 & 32.3 & 31.1 & 98 & 52.1 & 51.5 & 21.3 & 20.2 & 90 & 49.2 & 48.7 & 25 & 25.1 \\
\hline 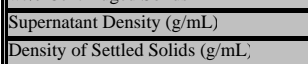 & $\frac{1.19}{1.65}$ & 1.01 & 1.01 & 1.01 & 1 & $\frac{1.19}{1.51}$ & 1.00 & $\frac{1.00}{1.0}$ & 1.00 & 1.00 & $\frac{1.05}{1.34}$ & 1.01 & 1.01 & $\frac{1.01}{1.1}$ & 1.01 \\
\hline \multicolumn{16}{|l|}{ 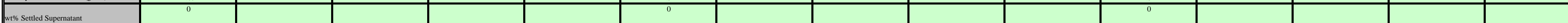 } \\
\hline w\%o dissolved solidis in supenatant & $\mathrm{n}^{\mathrm{m} m}$ & & & & & $\mathrm{n}^{\mathrm{n} / \mathrm{m}}$ & & & & & $n$ & & & & \\
\hline \multicolumn{16}{|l|}{\begin{tabular}{|l|l} 
wot toat solids in Centifiged Sludge \\
\end{tabular}} \\
\hline ww\% Total Solids & 53.6 & & & & & 31.6 & & & & & ${ }^{28}$ & & & & \\
\hline Ww\% UDS & 3621 & 29,90 & 29.80 & 10.13 & 11.23 & 27.53 & 17.96 & 17.96 & 5.88 & 5.88 & 27.16 & 13.15 & 13.15 & 4.72 & 4.72 \\
\hline Density of solids (g/cc) & 5.17 & 5.24 & 5.24 & 5.24 & 5.24 & 5.17 & 5.24 & 5.24 & 5.24 & 5.24 & 5.17 & 5.24 & 5.24 & 5.24 & 5.24 \\
\hline
\end{tabular}




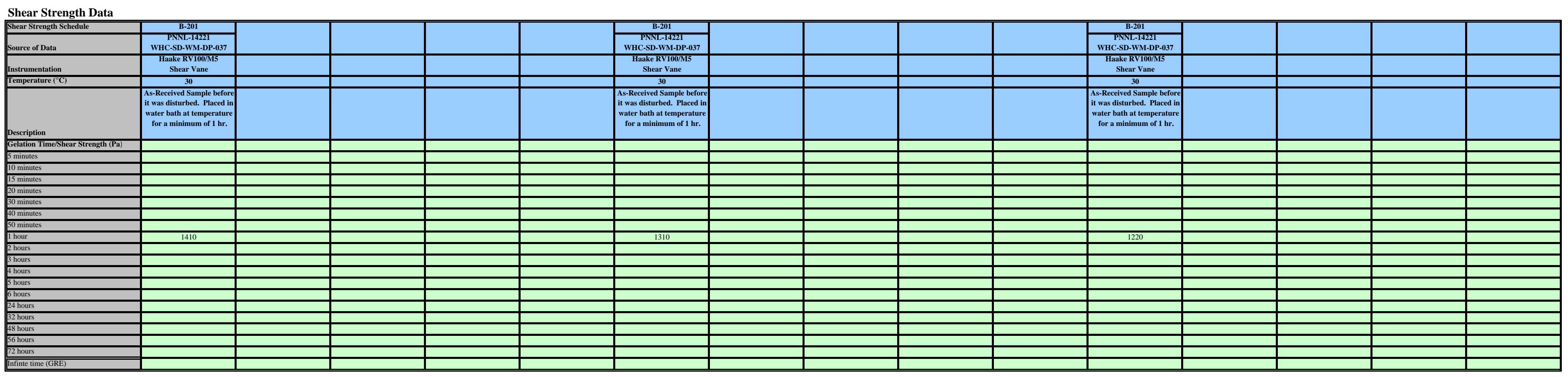




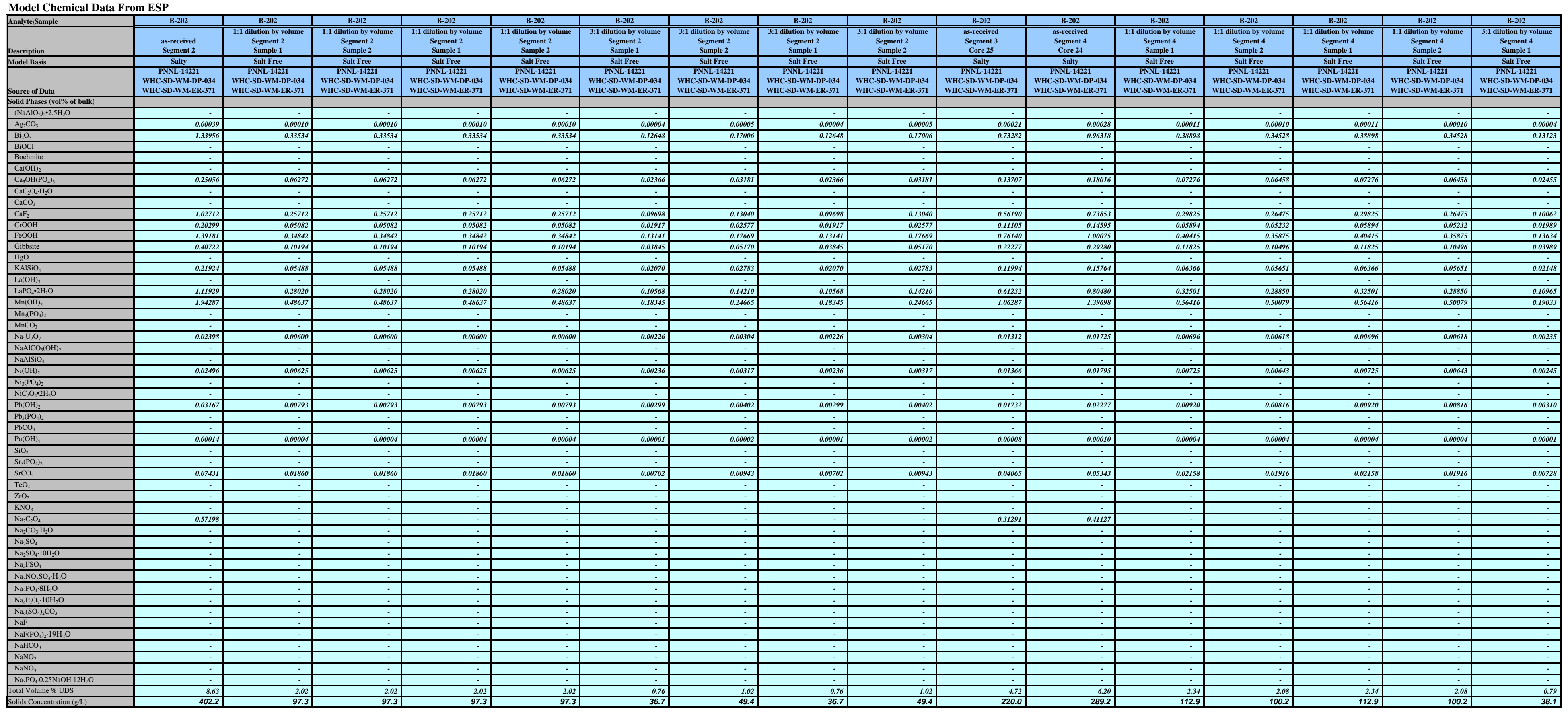




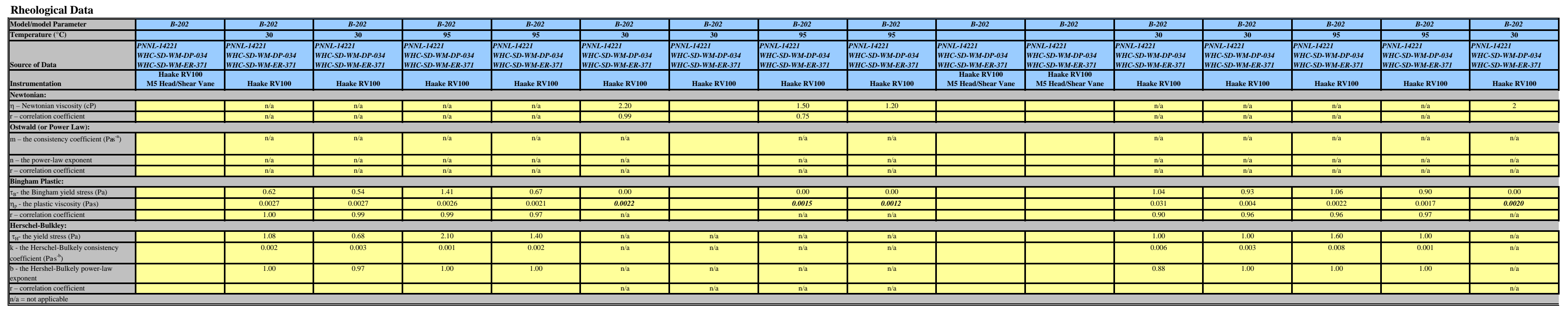

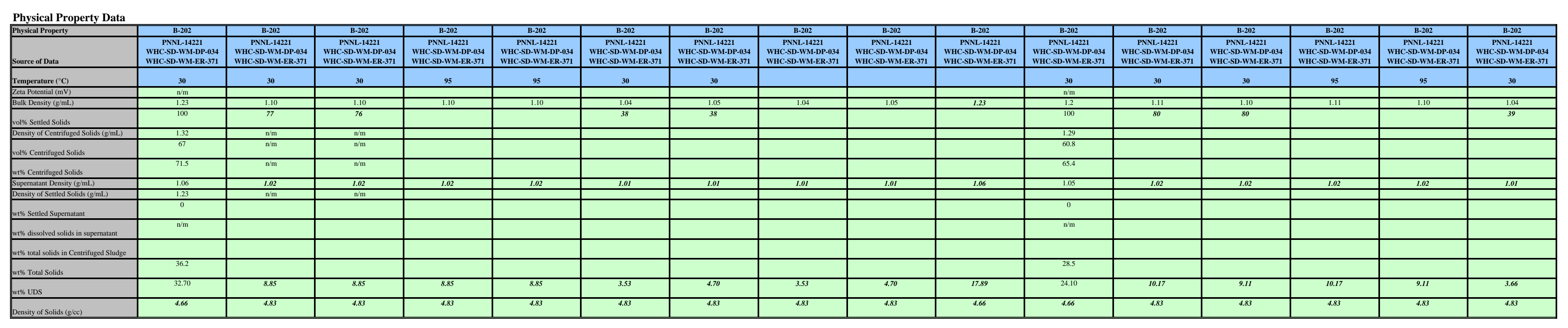



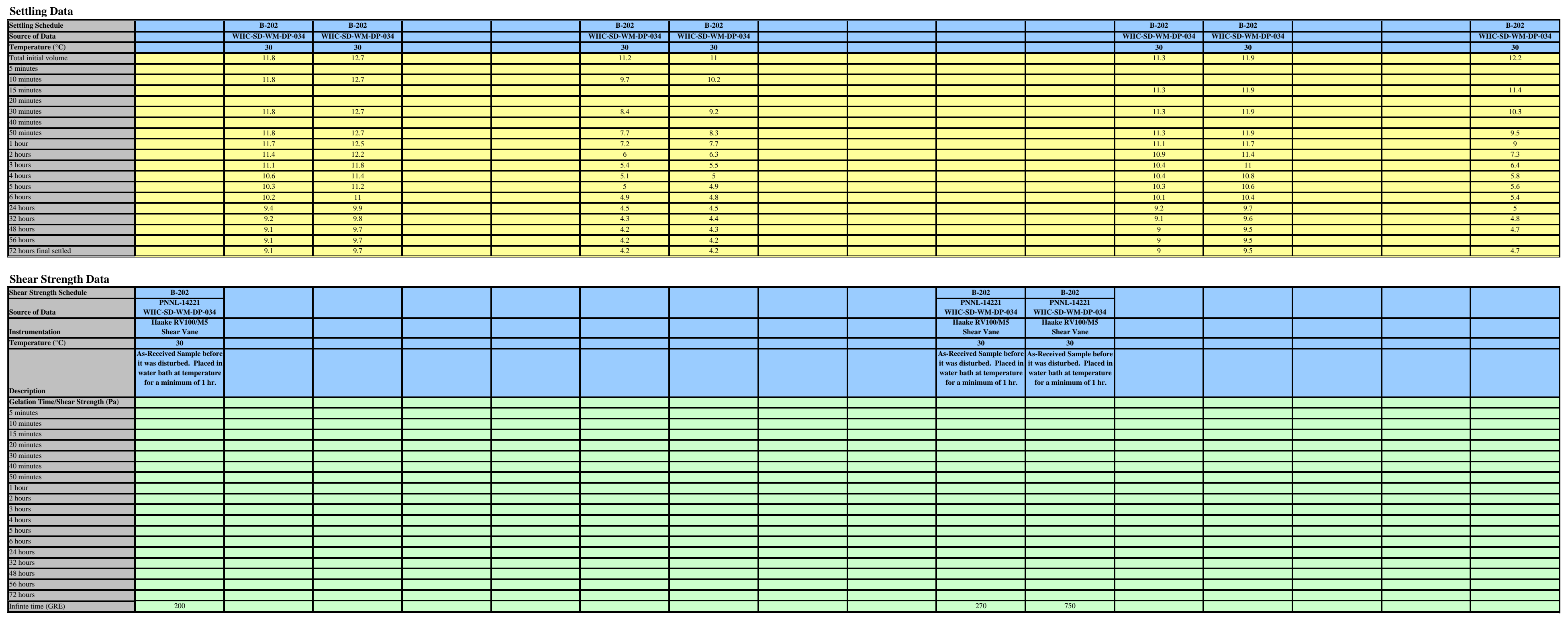


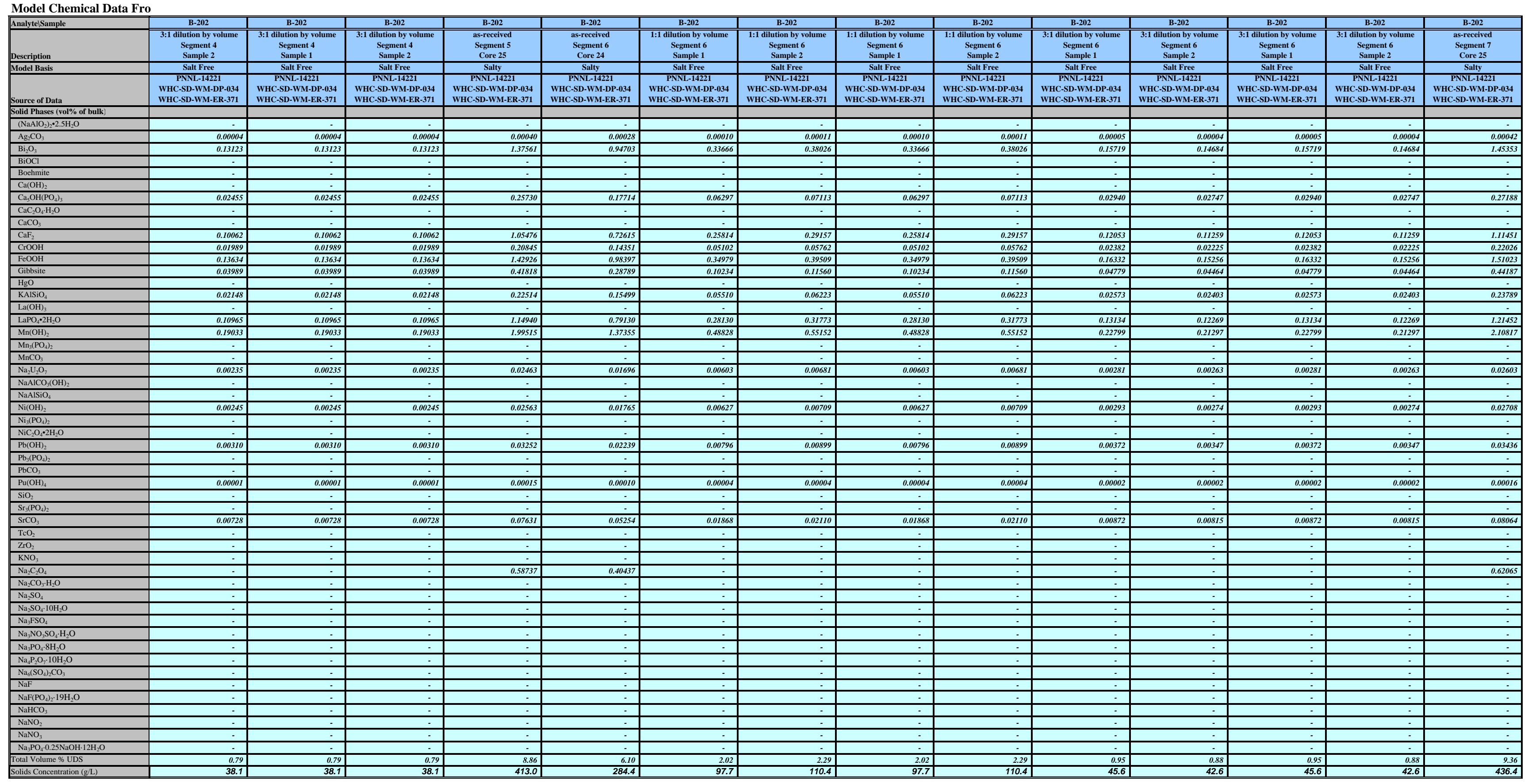




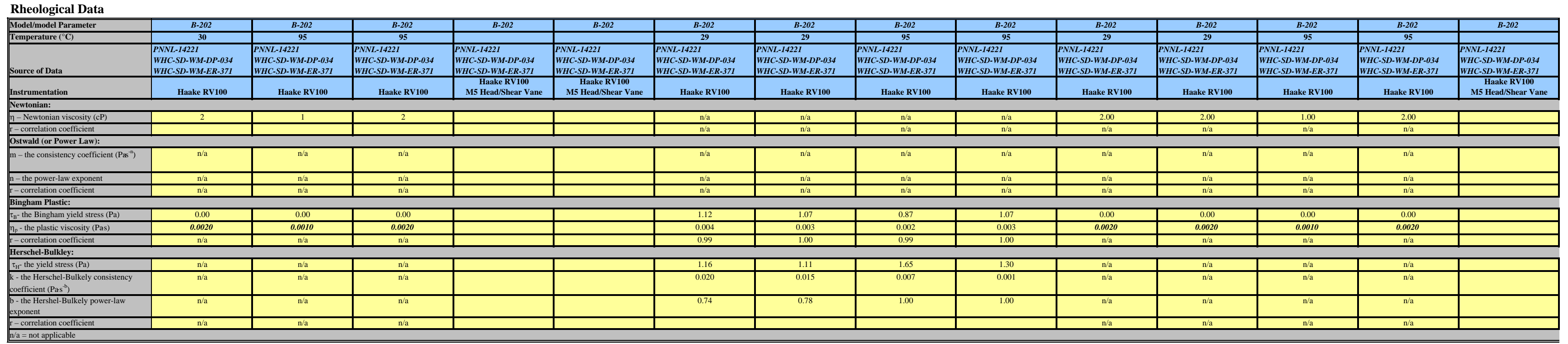

\begin{tabular}{|c|c|c|c|c|c|c|c|c|c|c|c|c|c|c|}
\hline $\begin{array}{l}\text { Physical Property Data } \\
\text { Physial Property } \\
\text { Source of Data }\end{array}$ & 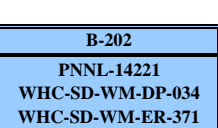 & 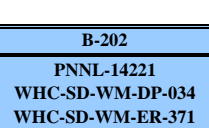 & 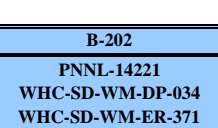 & 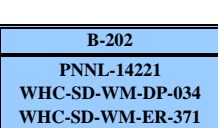 & 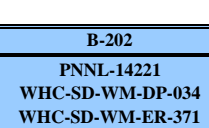 & 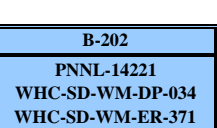 & 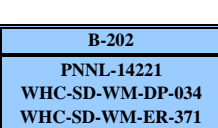 & 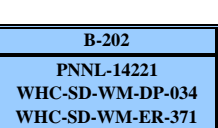 & 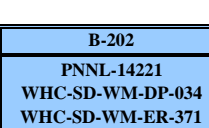 & 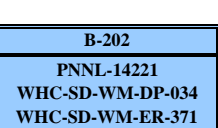 & 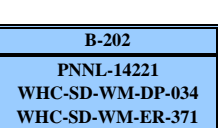 & 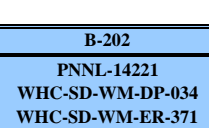 & 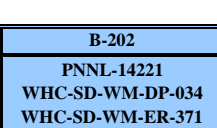 & 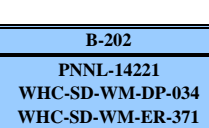 \\
\hline Temperature ( $(\mathrm{C})$ & 30 & & & & 30 & 29 & 29 & 95 & 95 & 29 & 29 & 95 & 95 & \\
\hline 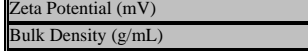 & 1.04 & 1.04 & 1.04 & $\overline{1.37}$ & $\frac{n ! m}{1.21}$ & $\frac{n / m}{1.09}$ & $\frac{n / m}{1.10}$ & 1.09 & $\overline{1.10}$ & $\frac{n / m}{1.04}$ & $\frac{n / m}{1.04}$ & $\frac{n / m}{1.04}$ & $\frac{n / m}{1.04}$ & 1.37 \\
\hline 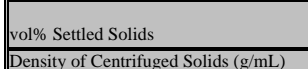 & ${ }^{39}$ & & & & ${ }^{100}$ & 86 & 86 & & & 48 & 47 & & & \\
\hline 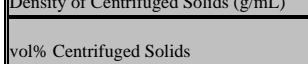 & & & & & $\frac{108}{686}$ & & & & & & & & & \\
\hline w\% Cenrifiuged Solids & & & & & 73.3 & & & & & & & & & \\
\hline 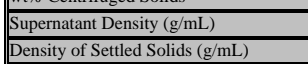 & 1.01 & 1.01 & 1.01 & 1.05 & $\frac{1.03}{1.21}$ & 1.01 & 1.01 & 1.01 & 1.01 & 1.01 & 1.01 & 1.01 & 1.01 & 1.03 \\
\hline \begin{tabular}{|l} 
wos Serted Superatant \\
\end{tabular} & & & & & 0 & & & & & & & & & \\
\hline 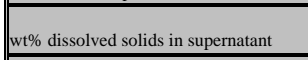 & & & & & $\mathrm{n}^{\prime \mathrm{m}}$ & & & & & & & & & \\
\hline w\%\% total solids in Centifituged Sludge & & & & & & & & & & & & & & \\
\hline ww\% Total Solids & & & & & ${ }^{27}$ & & & & & & & & & \\
\hline$\overline{\text { w\% uDS }}$ & 3.66 & 3.66 & 3.66 & 30.15 & 23.50 & 8.97 & 10.03 & 8.97 & 10.03 & 4.38 & 4.10 & 4.38 & 4.10 & 31.86 \\
\hline Density of solids (glcc) & 4.83 & 4.83 & 4.83 & 4.66 & 4.66 & 4.83 & 4.83 & 4.83 & 4.83 & 4.83 & 4.83 & 4.83 & 4.83 & 4.66 \\
\hline
\end{tabular}



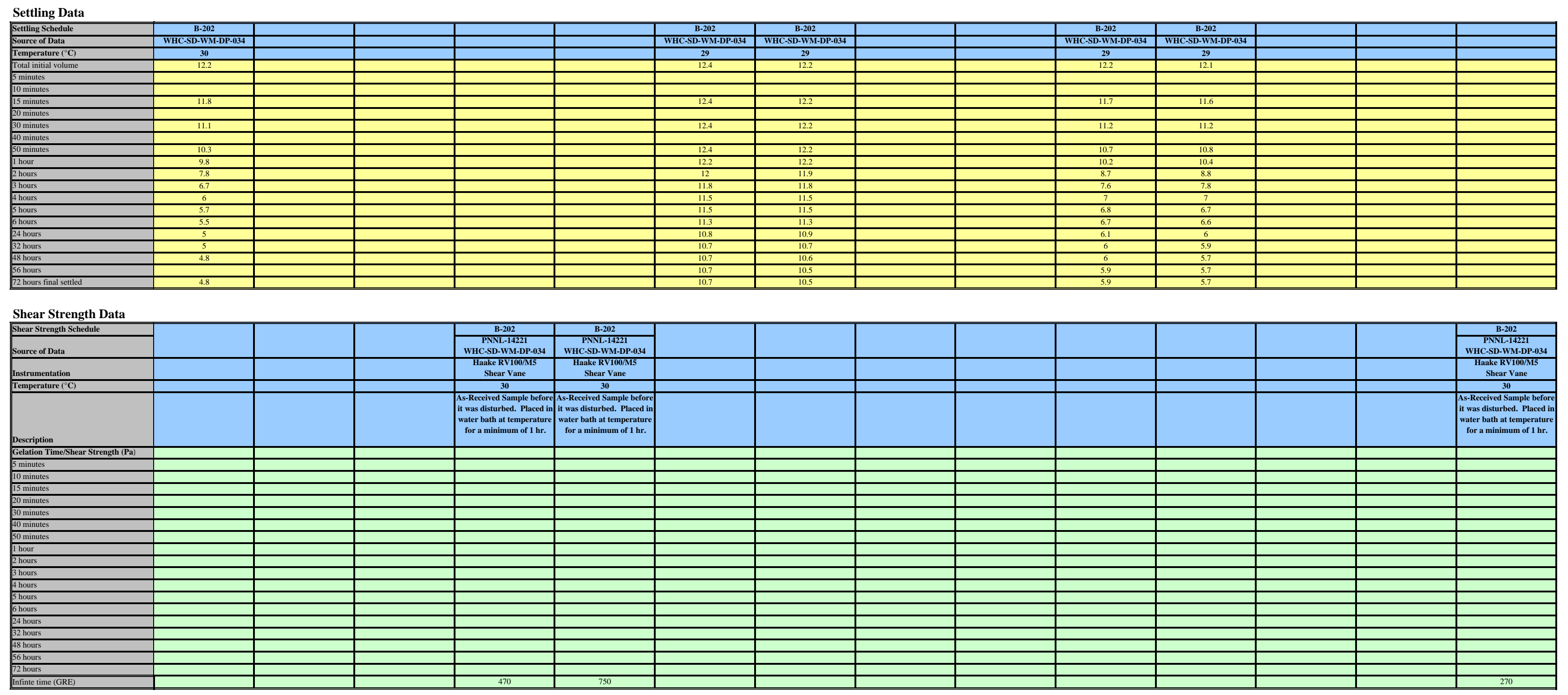
241-B-203

Model Chemical Data From ESP

\begin{tabular}{|c|c|c|c|c|c|}
\hline AnalytelSample & B-203 & B-203 & B-203 & B-203 & B-203 \\
\hline |Description & $\begin{array}{c}\text { Homogenized Tank } \\
\text { Composite }\end{array}$ & $\begin{array}{c}30 \text { wt } \% \text { dilution of a core } \\
\text { composite }\end{array}$ & $\begin{array}{c}\text { 1:1 Dilution of a core } \\
\text { composite }\end{array}$ & $\begin{array}{c}\text { 1:1 Dilution of a core } \\
\text { composite }\end{array}$ & $\begin{array}{c}\text { 4:1 Dilution of a core } \\
\text { composite }\end{array}$ \\
\hline Model Basis & Salty & Washed & Washed & Washed & Washed \\
\hline Source of Data & PNNL-14365 & PNNL-14365 & PNNL-14365 & PNNL-14365 & PNNL-14365 \\
\hline \multicolumn{6}{|l|}{ Solid Phases (vol\% of bulk) } \\
\hline$\left(\mathrm{NaAlO}_{2}\right)_{2} \cdot 2.5 \mathrm{H}_{2} \mathrm{O}$ & - & - & - & - & - \\
\hline $\mathrm{Ag}_{2} \mathrm{CO}_{3}$ & 0.00008 & 0.00012 & 0.00009 & 0.00009 & 0.00002 \\
\hline $\mathrm{Bi}_{2} \mathrm{O}_{3}$ & 0.45700 & 0.70830 & 0.50930 & 0.50930 & 0.12562 \\
\hline $\mathrm{BiOCl}$ & - & - & - & - & - \\
\hline Boehmite & - & - & - & - & - \\
\hline $\mathrm{Ca}(\mathrm{OH})_{2}$ & - & - & - & - & - \\
\hline $\mathrm{Ca}_{5} \mathrm{OH}\left(\mathrm{PO}_{4}\right)_{3}$ & - & - & - & - & - \\
\hline $\mathrm{CaC}_{2} \mathrm{O}_{4} \cdot \mathrm{H}_{2} \mathrm{O}$ & - & - & - & - & - \\
\hline $\mathrm{CaCO}_{3}$ & - & - & - & - & - \\
\hline $\mathrm{CaF}_{2}$ & 0.01200 & 0.01859 & 0.01337 & 0.01337 & 0.00330 \\
\hline $\mathrm{CrOOH}$ & 0.08346 & 0.12935 & 0.09301 & 0.09301 & 0.02294 \\
\hline $\mathrm{FeOOH}$ & 0.10857 & 0.16827 & 0.12100 & 0.12100 & 0.02984 \\
\hline Gibbsite & - & - & - & - & - \\
\hline $\mathrm{HgO}$ & - & - & - & - & - \\
\hline $\mathrm{KAlSiO}_{4}$ & 0.01013 & 0.01571 & 0.01129 & 0.01129 & 0.00279 \\
\hline $\mathrm{La}(\mathrm{OH})_{3}$ & 0.03248 & 0.05033 & 0.03619 & 0.03619 & 0.00893 \\
\hline $\mathrm{LaPO}_{4} \cdot 2 \mathrm{H}_{2} \mathrm{O}$ & 0.25651 & 0.39756 & 0.28587 & 0.28587 & 0.07051 \\
\hline $\mathrm{Mn}(\mathrm{OH})_{2}$ & 0.58814 & 0.91154 & 0.65544 & 0.65544 & 0.16167 \\
\hline $\mathrm{Mn}_{3}\left(\mathrm{PO}_{4}\right)_{2}$ & - & - & - & - & - \\
\hline $\mathrm{MnCO}_{3}$ & 0.04020 & 0.06230 & 0.04480 & 0.04480 & 0.01105 \\
\hline $\mathrm{Na}_{2} \mathrm{U}_{2} \mathrm{O}_{7}$ & 0.00368 & 0.00570 & 0.00410 & 0.00410 & 0.00101 \\
\hline $\mathrm{NaAlCO}_{3}(\mathrm{OH})_{2}$ & - & - & - & - & - \\
\hline $\mathrm{NaAlSiO}_{4}$ & - & - & - & - & - \\
\hline $\mathrm{Ni}(\mathrm{OH})_{2}$ & 0.00620 & 0.00961 & 0.00691 & 0.00691 & 0.00170 \\
\hline $\mathrm{Ni}_{3}\left(\mathrm{PO}_{4}\right)_{2}$ & - & - & - & - & - \\
\hline $\mathrm{NiC}_{2} \mathrm{O}_{4} \cdot 2 \mathrm{H}_{2} \mathrm{O}$ & - & - & - & - & - \\
\hline $\mathrm{Pb}(\mathrm{OH})_{2}$ & 0.00331 & 0.00513 & 0.00369 & 0.00369 & 0.00091 \\
\hline $\mathrm{Pb}_{3}\left(\mathrm{PO}_{4}\right)_{2}$ & - & - & - & - & - \\
\hline $\mathrm{PbCO}_{3}$ & - & - & - & - & - \\
\hline $\mathrm{Pu}(\mathrm{OH})_{4}$ & 0.00006 & 0.00009 & 0.00007 & 0.00007 & 0.00002 \\
\hline $\mathrm{SiO}_{2}$ & - & - & - & - & - \\
\hline $\mathrm{Sr}_{3}\left(\mathrm{PO}_{4}\right)_{2}$ & - & - & - & - & - \\
\hline $\mathrm{SrCO}_{3}$ & 0.02107 & 0.03266 & 0.02348 & 0.02348 & 0.00579 \\
\hline $\mathrm{TcO}_{2}$ & 0.00000 & 0.00000 & 0.00000 & 0.00000 & 0.00000 \\
\hline $\mathrm{ZrO}_{2}$ & 0.00006 & 0.00009 & 0.00006 & 0.00006 & 0.00002 \\
\hline $\mathrm{KNO}_{3}$ & - & - & - & - & - \\
\hline $\mathrm{Na}_{2} \mathrm{C}_{2} \mathrm{O}_{4}$ & - & - & - & - & - \\
\hline $\mathrm{Na}_{2} \mathrm{CO}_{3} \cdot \mathrm{H}_{2} \mathrm{O}$ & - & - & - & - & - \\
\hline $\mathrm{Na}_{2} \mathrm{SO}_{4}$ & - & - & - & - & - \\
\hline $\mathrm{Na}_{2} \mathrm{SO}_{4} \cdot 10 \mathrm{H}_{2} \mathrm{O}$ & - & - & - & - & - \\
\hline $\mathrm{Na}_{3} \mathrm{FSO}_{4}$ & - & - & - & - & - \\
\hline $\mathrm{Na}_{3} \mathrm{NO}_{3} \mathrm{SO}_{4} \cdot \mathrm{H}_{2} \mathrm{O}$ & - & - & - & - & - \\
\hline $\mathrm{Na}_{3} \mathrm{PO}_{4} \cdot 8 \mathrm{H}_{2} \mathrm{O}$ & - & - & - & - & - \\
\hline $\mathrm{Na}_{4} \mathrm{P}_{2} \mathrm{O}_{7} \cdot 10 \mathrm{H}_{2} \mathrm{O}$ & - & - & - & - & - \\
\hline $\mathrm{Na}_{6}\left(\mathrm{SO}_{4}\right)_{2} \mathrm{CO}_{3}$ & - & - & - & - & - \\
\hline $\mathrm{NaF}$ & - & - & - & - & - \\
\hline $\mathrm{NaF}\left(\mathrm{PO}_{4}\right)_{2} \cdot 19 \mathrm{H}_{2} \mathrm{O}$ & - & - & - & - & - \\
\hline $\mathrm{NaHCO}_{3}$ & - & - & - & - & - \\
\hline $\mathrm{NaNO}_{2}$ & - & - & - & - & - \\
\hline $\mathrm{NaNO}_{3}$ & - & - & - & - & - \\
\hline $\mathrm{Na}_{3} \mathrm{PO}_{4} \cdot 0.25 \mathrm{NaOH} \cdot 12 \mathrm{H}_{2} \mathrm{O}$ & - & - & - & - & - \\
\hline Total Volume \% UDS & 1.62 & 2.52 & 1.81 & 1.81 & 0.45 \\
\hline Solids Concentration $(\mathrm{g} / \mathrm{L})$ & 89.6 & 138.9 & 99.9 & 99.9 & 24.6 \\
\hline
\end{tabular}


Rheological Data

\begin{tabular}{|c|c|c|c|c|c|}
\hline Model/model Parameter & & B-203 & B-203 & B-203 & B-203 \\
\hline Temperature $\left({ }^{\circ} \mathrm{C}\right)$ & & 25 & 25 & 25 & 25 \\
\hline Source of Data & PNNL-14365 & PNNL-14365 & PNNL-14365 & PNNL-14365 & PNNL-14365 \\
\hline Instrumentation & & \begin{tabular}{|c|} 
Haake RS300 \\
Concentric Cylinders with \\
a $3 \mathrm{~mm}$ Gap
\end{tabular} & $\begin{array}{c}\text { Haake RS300 } \\
\text { Concentric Cylinders with } \\
\text { a } 3 \mathrm{~mm} \text { Gap }\end{array}$ & \begin{tabular}{|c|} 
Haake RS300 \\
Concentric Cylinders with \\
a 3 mm Gap
\end{tabular} & \begin{tabular}{|c|} 
Haake RS300 \\
Concentric Cylinders with \\
a $3 \mathrm{~mm}$ Gap
\end{tabular} \\
\hline \multicolumn{6}{|l|}{ Newtonian: } \\
\hline$\eta$ - Newtonian viscosity (cP) & & $\mathrm{n} / \mathrm{a}$ & $\mathrm{n} / \mathrm{a}$ & $\mathrm{n} / \mathrm{a}$ & $\mathrm{n} / \mathrm{a}$ \\
\hline $\mathrm{r}$ - correlation coefficient & & $\mathrm{n} / \mathrm{a}$ & $\mathrm{n} / \mathrm{a}$ & $\mathrm{n} / \mathrm{a}$ & $\mathrm{n} / \mathrm{a}$ \\
\hline \multicolumn{6}{|l|}{ Ostwald (or Power Law): } \\
\hline $\mathrm{m}$ - the consistency coefficient $\left(\mathrm{Pa} \cdot \mathrm{s}^{-\mathrm{n}}\right)$ & & $\mathrm{n} / \mathrm{a}$ & $\mathrm{n} / \mathrm{a}$ & $\mathrm{n} / \mathrm{a}$ & $\mathrm{n} / \mathrm{a}$ \\
\hline $\mathrm{n}$-the power-law exponent & & $\mathrm{n} / \mathrm{a}$ & $\mathrm{n} / \mathrm{a}$ & $n / a$ & $\mathrm{n} / \mathrm{a}$ \\
\hline $\mathrm{r}$ - correlation coefficient & & $\mathrm{n} / \mathrm{a}$ & $\mathrm{n} / \mathrm{a}$ & $\mathrm{n} / \mathrm{a}$ & $\mathrm{n} / \mathrm{a}$ \\
\hline \multicolumn{6}{|l|}{ Bingham Plastic: } \\
\hline$\tau_{\mathrm{B}}$ - the Bingham yield stress $(\mathrm{Pa})$ & & 10.90 & 3.07 & 3.15 & 0.13 \\
\hline$\eta_{p}$ - the plastic viscosity (Pa.s) & & 0.0079 & 0.0047 & 0.0047 & 0.0019 \\
\hline $\mathrm{r}$ - correlation coefficient & & 1.00 & 0.99 & 0.99 & 0.97 \\
\hline \multicolumn{6}{|l|}{ Herschel-Bulkley: } \\
\hline$\tau_{\mathrm{H}^{-}}$the yield stress $(\mathrm{Pa})$ & & $\mathrm{n} / \mathrm{a}$ & $\mathrm{n} / \mathrm{a}$ & $\mathrm{n} / \mathrm{a}$ & 0.01 \\
\hline $\begin{array}{l}\mathrm{k} \text { - the Herschel-Bulkely consistency } \\
\text { coefficient }\left(\mathrm{Pa} \cdot \mathrm{s}^{-\mathrm{b}}\right)\end{array}$ & & $\mathrm{n} / \mathrm{a}$ & $\mathrm{n} / \mathrm{a}$ & $\mathrm{n} / \mathrm{a}$ & 0.0049 \\
\hline \begin{tabular}{|l} 
b - the Hershel-Bulkely power-law \\
exponent
\end{tabular} & & $\mathrm{n} / \mathrm{a}$ & $\mathrm{n} / \mathrm{a}$ & $\mathrm{n} / \mathrm{a}$ & 0.84 \\
\hline $\mathrm{r}$-correlation coefficient & & $\mathrm{n} / \mathrm{a}$ & $\mathrm{n} / \mathrm{a}$ & $\mathrm{n} / \mathrm{a}$ & 0.98 \\
\hline
\end{tabular}

\section{Physical Property Data}

\begin{tabular}{|c|c|c|c|c|c|}
\hline Physical Property & $\overline{\mathrm{B}-203}$ & B-203 & B-203 & B-203 & B-203 \\
\hline Source of Data & PNNL-14365 & PNNL-14365 & PNNL-14365 & PNNL-14365 & PNNL-14365 \\
\hline \multicolumn{6}{|l|}{ Temperature $\left({ }^{\circ} \mathrm{C}\right)$} \\
\hline \multicolumn{6}{|l|}{ Zeta Potential (mV) } \\
\hline Bulk Density (g/mL) & 1.28 & 1.26 & 1.18 & 1.18 & 1.06 \\
\hline vol\% Settled Solids & 100 & 100 & 87.5 & 87.5 & 43.8 \\
\hline Density of Centrifuged Solids $(\mathrm{g} / \mathrm{mL})$ & 1.53 & 1.41 & 1.38 & 1.38 & 1.52 \\
\hline vol\% Centrifuged Solids & 83.7 & 54.5 & 38.5 & 38.5 & 13.9 \\
\hline wt\% Centrifuged Solids & 100 & 61 & 45 & 45 & 20 \\
\hline Supernatant Density $(\mathrm{g} / \mathrm{mL})$ & 1.21 & 1.15 & 1.10 & 1.10 & 1.04 \\
\hline Density of Settled Solids $(\mathrm{g} / \mathrm{mL})$ & & & & & \\
\hline wt \% Settled Supernatant & & & & & \\
\hline wt\% dissolved solids in supernatant & & & & & \\
\hline wt $\%$ total solids in Centrifuged Sludge & & & & & \\
\hline wt\% Total Solids & 40.5 & 28.6 & 19.7 & 19.7 & 8.5 \\
\hline wt\% UDS & 7.00 & 11.03 & 8.47 & 8.47 & 2.32 \\
\hline Density of Solids (g/cc) & 5.52 & 5.52 & 5.52 & 5.52 & 5.52 \\
\hline
\end{tabular}


Settling Data

\begin{tabular}{|c|c|c|c|c|}
\hline Settling Schedule & & & & \\
\hline Source of Data & PNNL-14365 & PNNL-14365 & PNNL-14365 & PNNL-14365 \\
\hline Temperature $\left({ }^{\circ} \mathrm{C}\right)$ & & & & \\
\hline Total initial volume & & 13.3 & 13.3 & 13 \\
\hline 5 minutes & & 13.3 & 13.3 & 12.6 \\
\hline 10 minutes & & 13.3 & 13.3 & 12.1 \\
\hline 15 minutes & & 13.3 & 13.3 & 11.4 \\
\hline 20 minutes & & 13.3 & 13.3 & 10.6 \\
\hline 30 minutes & & 13.3 & 13.3 & 9.5 \\
\hline 40 minutes & & & & \\
\hline 50 minutes & & & & \\
\hline 1 hour & & 13.3 & 13.3 & 7.3 \\
\hline 2 hours & & 13.05 & 13.05 & 6.2 \\
\hline 3 hours & & 13 & 13 & 6 \\
\hline 4 hours & & 12.95 & 12.95 & 5.9 \\
\hline 5 hours & & & & \\
\hline 6 hours & & 12.7 & 12.7 & 5.7 \\
\hline 24 hours & & 12 & 12 & 5.6 \\
\hline 32 hours & & & & 5.6 \\
\hline 48 hours & & 11.8 & 11.8 & 5.6 \\
\hline 56 hours & & 11.7 & 11.7 & 5.6 \\
\hline 72 hours final settled & & 11.7 & 11.7 & 5.6 \\
\hline
\end{tabular}

\section{Shear Strength Data}

\begin{tabular}{|c|c|c|c|c|c|}
\hline Shear Strength Schedule & B-203 & B-203 & & & \\
\hline Source of Data & PNNL-14365 & PNNL-14365 & & & \\
\hline Instrumentation & $\begin{array}{l}\text { Haake RS300 } \\
\text { Shear Vane }\end{array}$ & $\begin{array}{l}\text { Haake RS300 } \\
\text { Shear Vane }\end{array}$ & & & \\
\hline Temperature $\left({ }^{\circ} \mathrm{C}\right)$ & 25 & 25 & & & \\
\hline Description & $\begin{array}{c}\text { Homogenized Tank } \\
\text { Composite }\end{array}$ & $\begin{array}{l}30 \mathrm{wt} \% \text { dilution of the tank } \\
\text { composite }\end{array}$ & & & \\
\hline Gelation Time/Shear Stre & & & & & \\
\hline 5 minutes & & & & & \\
\hline 10 minutes & & & & & \\
\hline 15 minutes & & & & & \\
\hline 20 minutes & & & & & \\
\hline 30 minutes & & & & & \\
\hline 40 minutes & & & & & \\
\hline 50 minutes & & & & & \\
\hline 1 hour & & & & & \\
\hline 2 hours & & & & & \\
\hline 3 hours & & & & & \\
\hline 4 hours & & & & & \\
\hline 5 hours & & & & & \\
\hline 6 hours & & & & & \\
\hline 24 hours & & & & & \\
\hline 32 hours & & & & & \\
\hline 48 hours & & & & & \\
\hline 56 hours & & & & & \\
\hline 72 hours & 2280 & 60 & & & \\
\hline Infinte time (GRE) & & & & & \\
\hline
\end{tabular}




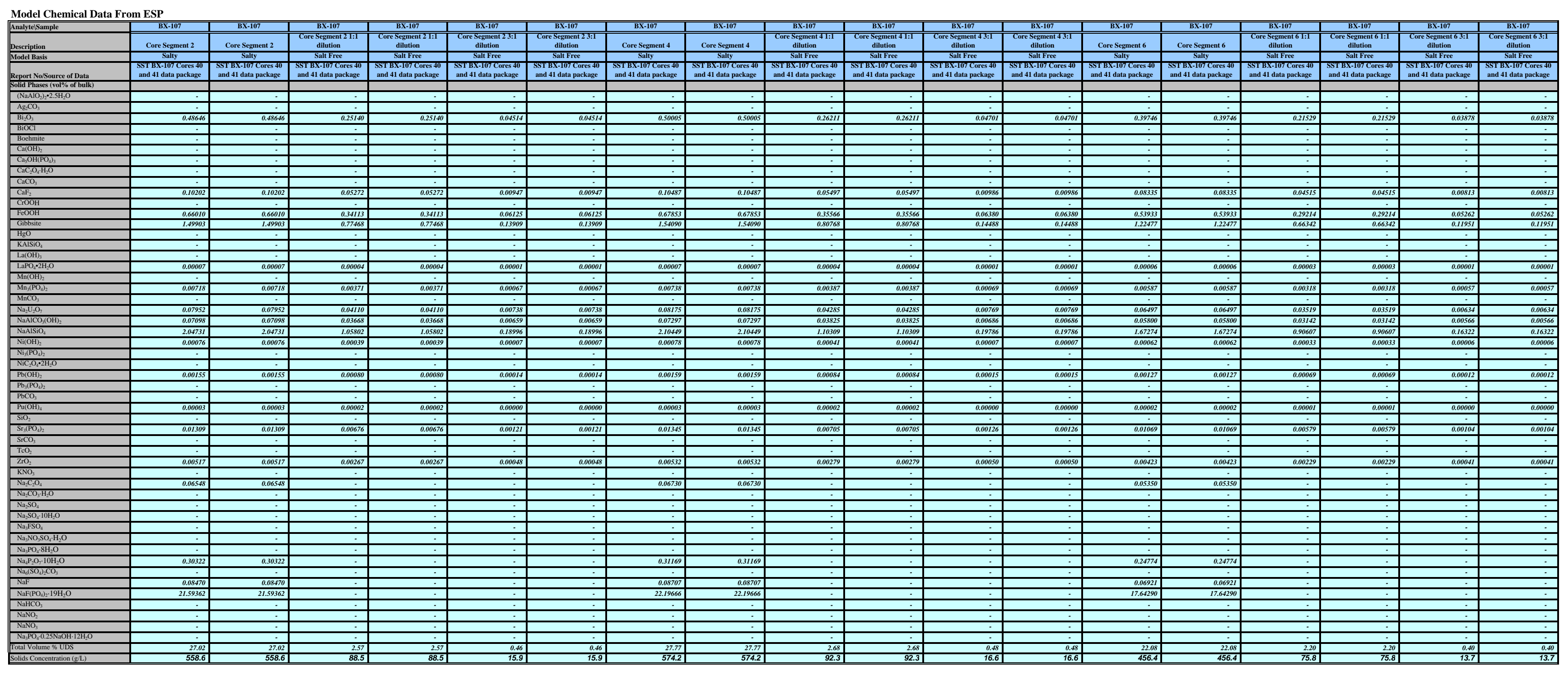

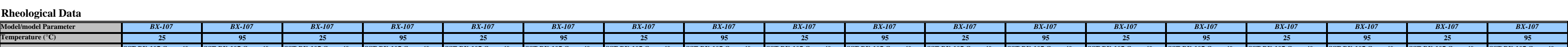

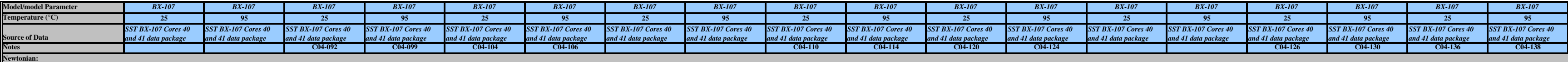

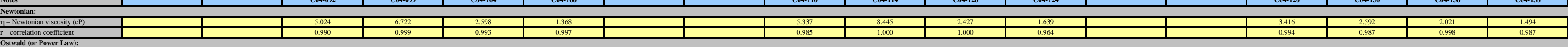

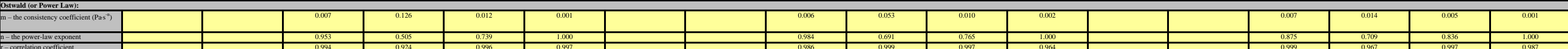

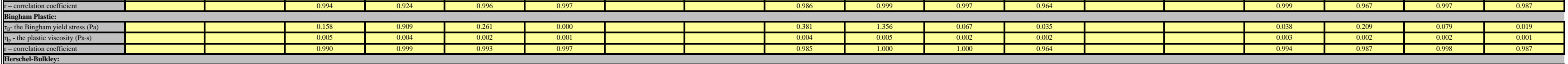

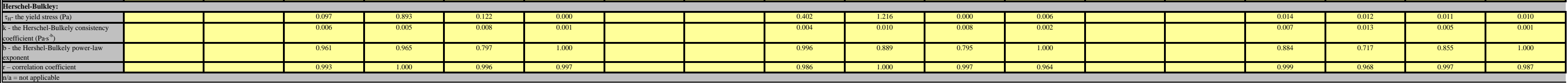




\begin{tabular}{|c|c|c|c|c|c|c|c|c|c|c|c|c|c|c|c|c|c|c|}
\hline 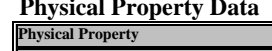 & $\frac{B \times-107}{B-10}$ & 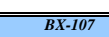 & 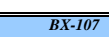 & $\mid \overline{B X-107}$ & $\overline{\mid B \times 107}$ & $\overline{B S \times 107}$ & $\overline{B S X-107}$ & 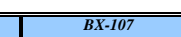 & $\frac{B \times 107}{B E}$ & $8 x-107$ & BX-107 & $\overline{B X \times 107}$ & $\mid \overline{B X X 107}$ & $\mid \overline{B X X-107}$ & $\frac{B \times 107}{B}$ & $\frac{B \times 107}{B}$ & $\frac{B \times-107}{2}$ & $\overline{\frac{D \times-107}{B \times 10}}$ \\
\hline Eates & & & & & & & & & & & & & & & & & & \\
\hline Temperature (C) & 25 & 25 & 25 & 25 & 25 & 25 & 25 & 25 & 25 & 25 & 25 & 25 & 25 & 25 & 25 & 25 & 25 & 25 \\
\hline 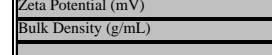 & $\frac{1.47}{\frac{1.40}{4}}$ & $\frac{1.47}{1.00}$ & $\frac{1.17}{1.177}$ & $\frac{1,17}{1,12}$ & $\frac{1.06}{1.06}$ & ${ }_{1.06}^{1,06}$ & $\frac{1.45}{1.140}$ & $\frac{1.45}{\frac{1.40}{10}}$ & 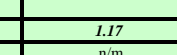 & $\frac{1.17}{1.172}$ & $\frac{1.06}{1.06}$ & $\frac{1.06}{1.05}$ & $\frac{1.4}{908}$ & $\frac{1.4}{908}$ & $\frac{1.17}{4,12}$ & $\frac{1.17}{1.17}$ & $\frac{1.06}{1.06}$ & ${ }_{1.06}^{1.06}$ \\
\hline 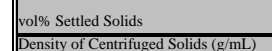 & & & & & & & & & & & & & & & & & & \\
\hline \begin{tabular}{|l} 
vol\% Cenrifiuged Solids \\
\end{tabular} & 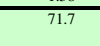 & & $\frac{n+m}{n / m}$ & nm & $\frac{n+m}{n / m}$ & 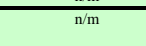 & & & & & $\frac{n m}{n n m}$ & & & & $\frac{n_{m}}{n+m}$ & 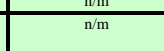 & $\frac{1 m}{n_{m}}$ & $n$ \\
\hline wero Cenurifiged Solids & 7.2 & 77.2 & ${ }^{\mathrm{nm} m}$ & $\mathrm{ntm}^{\mathrm{nm}}$ & $\mathrm{nm}^{\mathrm{nm}}$ & $\mathrm{ntm}^{\mathrm{nm}}$ & 80.6 & 80.6 & $\mathrm{ntm}^{\mathrm{nm}}$ & nim & $\mathrm{n}^{\mathrm{nm}}$ & $\mathrm{nm}$ & 70.4 & 70.4 & ${ }^{\mathrm{nm}}$ & $\mathrm{ntm}^{\mathrm{nm}}$ & $\mathrm{ntm}^{\mathrm{nm}}$ & $\mathrm{n}^{\mathrm{nm}}$ \\
\hline 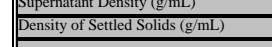 & $\frac{1.18}{n \mathrm{~nm}}$ & $\frac{1.18}{n m}$ & $\frac{1.11}{n+\mathrm{m}}$ & $\frac{1.1}{n \mathrm{~m}}$ & $\frac{1.05}{n m}$ & $\frac{1.05}{n \mathrm{~mm}}$ & $\frac{1.17}{n \cdot \mathrm{m}}$ & $\frac{1.17}{n ! m}$ & $\frac{1.11}{n \mathrm{~nm}}$ & $\frac{\frac{1.11}{n \mathrm{~m}}}{\mathrm{n}}$ & $\frac{1.05}{1 . \mathrm{m}}$ & $\frac{1.05}{\mathrm{~nm}}$ & $\frac{121}{\frac{121}{n}}$ & $\frac{1.21}{n \mathrm{~nm}}$ & 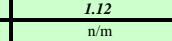 & $\frac{1.12}{n-1,2}$ & $\frac{1.06}{1,0 \mathrm{~m}}$ & $\frac{1.06}{n+n_{m}}$ \\
\hline 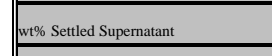 & $\mathrm{n}^{\mathrm{mm}}$ & $\mathrm{n}^{\mathrm{nm}}$ & ${ }^{n / m}$ & $\frac{1 \mathrm{~mm}}{\mathrm{~nm}}$ & $\frac{1 \mathrm{mim}}{\mathrm{nm}}$ & 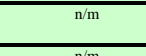 & $\frac{\pi \mathrm{m}}{\mathrm{nm}}$ & nim & $\mathrm{nim}^{\mathrm{nm}}$ & $\mathrm{n}^{\mathrm{nm} / \mathrm{m}}$ & ${ }^{\mathrm{nm} / \mathrm{m}}$ & 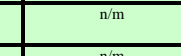 & n/m & n/m & $\mathrm{nm}$ & $\mathrm{nm}$ & $\mathrm{ntm}^{\mathrm{m}}$ & $\mathrm{nm}$ \\
\hline \%odisolved solids sin superatant & $\mathrm{nm}^{\mathrm{mm}}$ & nim & ${ }^{n / m}$ & ${ }^{\mathrm{nm}}$ & $\mathrm{nm}^{\mathrm{mm}}$ & ${ }^{\mathrm{nm} m}$ & ${ }^{\mathrm{nm}}$ & $\mathrm{nm}^{\mathrm{mm}}$ & ${ }^{\mathrm{nm} m}$ & $n^{n m}$ & ${ }^{n \mathrm{~nm}}$ & ${ }^{\mathrm{nm} m}$ & $\mathrm{~nm}^{\mathrm{nm}}$ & $\mathrm{nm}^{\mathrm{mm}}$ & $\mathrm{nm}^{\mathrm{nm}}$ & $\mathrm{nm}^{\mathrm{nm}}$ & $\mathrm{n}_{\mathrm{nm}}$ & $\mathrm{n}^{\mathrm{n} m}$ \\
\hline W\%o torat solids in Cenvifinged Sludge & $\mathrm{nm}$ & ${ }^{\mathrm{nm} m}$ & $\frac{\mathrm{nm}}{\mathrm{m}}$ & $n$ & ${ }^{\mathrm{nm}}$ & ${ }^{\mathrm{nm}}$ & ${ }^{\mathrm{nm}}$ & ${ }^{n \mathrm{~m}}$ & ${ }^{\mathrm{nm}}$ & ${ }^{\mathrm{nm}}$ & ${ }^{\mathrm{nm}}$ & $\mathrm{nm}^{\mathrm{mm}}$ & $n$ & $\mathrm{n}^{\mathrm{m} m}$ & ${ }^{\mathrm{nm}}$ & 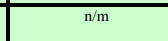 & $n$ & $n$ \\
\hline wo\% Toat Solids & - & 44.8 & $\mathrm{~nm}$ & ${ }^{\mathrm{nm}}$ & $\mathrm{n} / \mathrm{m}$ & $\mathrm{nm}$ & 45.6 & - & $\frac{\mathrm{n} m \mathrm{~m}}{3}$ & $\frac{\mathrm{nm}}{\mathrm{m}}$ & $\overline{n / m}$ & $\overline{\mathrm{n} / \mathrm{m}}$ & 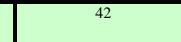 & - & ${ }^{\mathrm{nm}}$ & $\frac{\mathrm{nm}}{\mathrm{m}}$ & $\frac{\mathrm{nm}}{\mathrm{nm}}$ & $\mathrm{n} / \mathrm{m}$ \\
\hline wase ods & 38,00 & उв,10 & 7.54 & 2.54 & 1.50 & 1.50 & -3960 & 39,60 & 7.85 & 7.85 & 1.56 & 1.56 & 32,60 & 3260 & 6.47 & 6.47 & 1.28 & 1.28 \\
\hline & & 2,207 & 33 & 3.4 & 3.44 & $\frac{3.4}{3.4}$ & & & 3.4 & & & & & & & & & \\
\hline
\end{tabular}
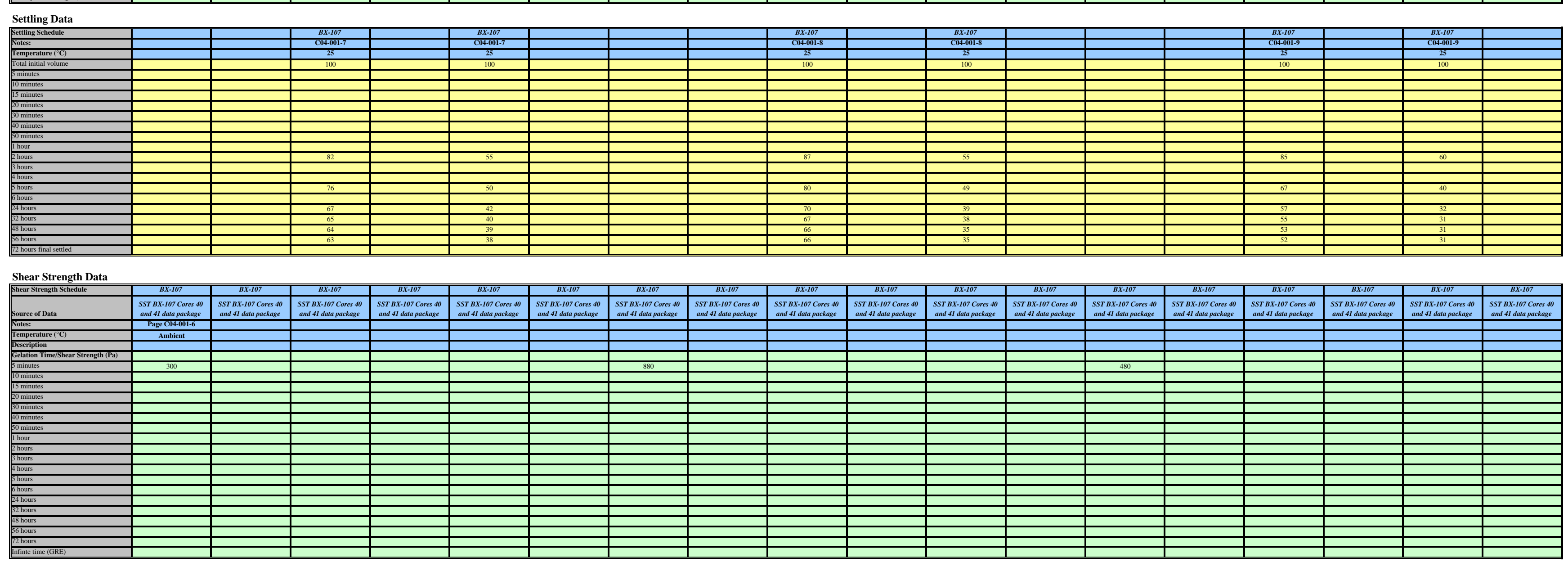


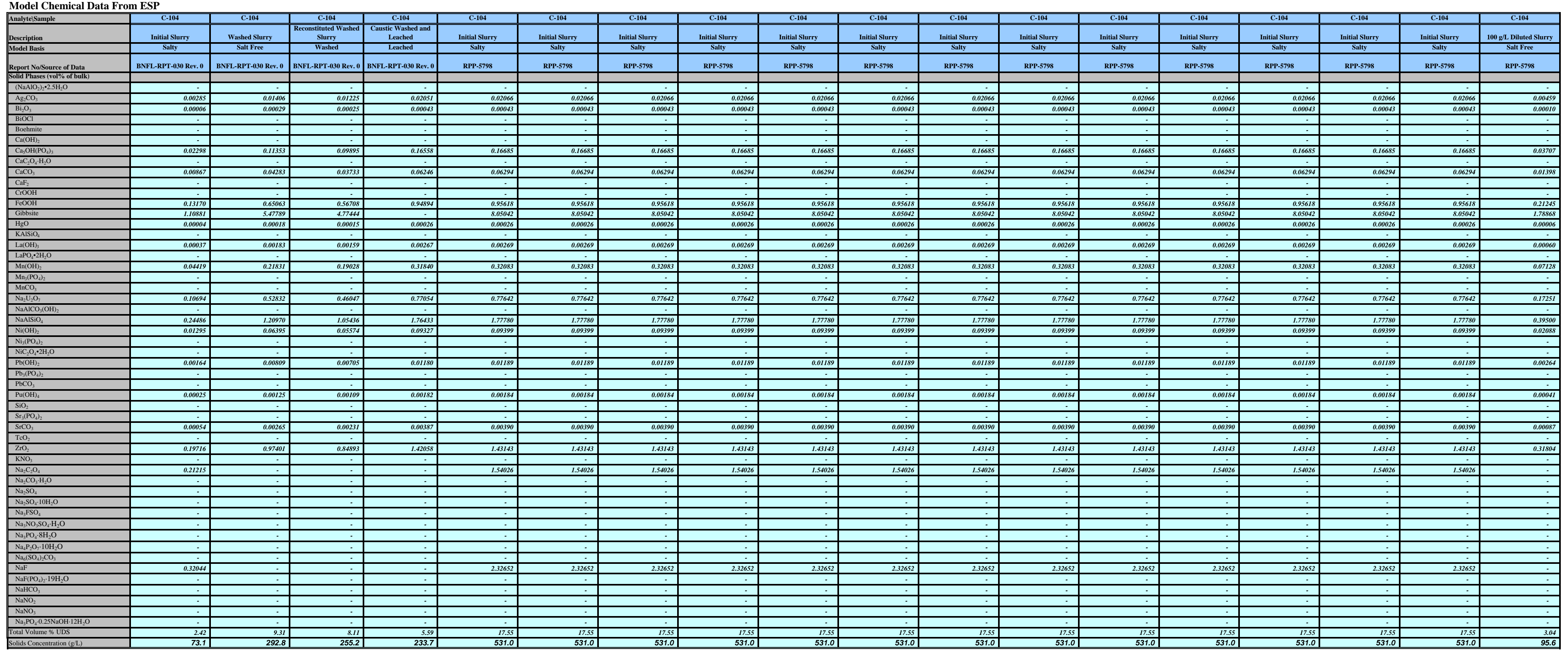




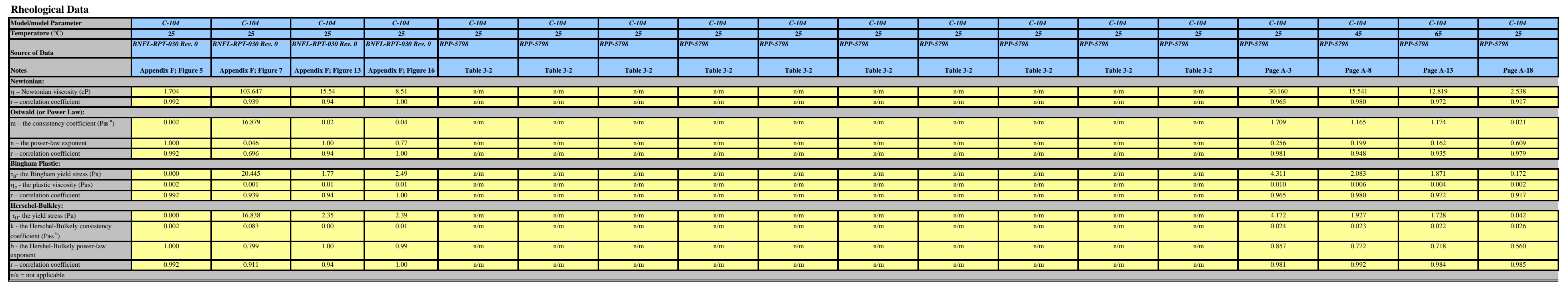

\begin{tabular}{|c|c|c|c|c|c|c|c|c|c|c|c|c|c|c|c|c|c|c|}
\hline \multicolumn{19}{|l|}{ Physical Property Data } \\
\hline voles & & & & & & & & & & & & & & & & & & \\
\hline Temperature (C) & 25 & 25 & 25 & 25 & 25 & 25 & 25 & 25 & 25 & 25 & 25 & 25 & 25 & 25 & 25 & 45 & 65 & 25 \\
\hline 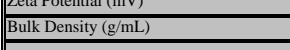 & $\frac{1.06}{1.06}$ & 1.22 & 1.16 & $\frac{1.14}{1.14}$ & 1.55 & 1.55 & 1.55 & 1.55 & $\frac{1.55}{1.51}$ & $\frac{1.55}{1.5}$ & 1.55 & 1.55 & 1.55 & $\frac{1.55}{1.5}$ & $\frac{1.55}{1.5}$ & $\frac{1.55}{1.5}$ & 1.55 & $\frac{1.10}{1.0}$ \\
\hline \begin{tabular}{|l}
$\mid$ volos Sentele Solits \\
\end{tabular} & 29.1 & 93.8 & & 95,2 & n/m & $\mathrm{n} / \mathrm{m}$ & $\mathrm{n} / \mathrm{m}$ & n/m & n/m & $\mathrm{n} / \mathrm{m}$ & n/m & n/m & n/m & $\mathrm{n} / \mathrm{m}$ & $\mathrm{n} / \mathrm{m}$ & $\mathrm{n} / \mathrm{m}$ & n/m & \\
\hline Density of Cenrifituged Solids (ggmL) & $\frac{n \pi m}{11}$ & $\frac{\mathrm{n} / \mathrm{m}}{46.2}$ & $\frac{\mathrm{nm} / \mathrm{m}}{50.6}$ & $\frac{n / m}{42.2}$ & $\frac{n}{n / m}$ & $\frac{\pi / m}{n / m}$ & $\frac{n / m}{n / m}$ & $\frac{n \mathrm{~mm}}{\mathrm{nnm}}$ & $\frac{n i m}{n n m}$ & $\frac{\mathrm{nm}}{\mathrm{nm}}$ & $\frac{\mathrm{n} / \mathrm{m}}{\mathrm{n} \mathrm{m} \mathrm{m}}$ & $\frac{n m}{n m m}$ & $\frac{n \mathrm{mim}}{\mathrm{nm}}$ & $\frac{n d m}{n / m}$ & $\frac{\mathrm{nm}}{\mathrm{nm}}$ & $\frac{n \mathrm{~mm}}{n \mathrm{~nm}}$ & $\frac{n \mathrm{~nm}}{\mathrm{~nm}}$ & $\frac{n \pi / m}{n / m}$ \\
\hline vol\% Cenrifiged Solids & 14.7 & 4.2 & & 50.9 & & & & & & & & & & & & & & \\
\hline 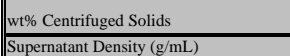 & $\frac{1.02}{1.2}$ & $\frac{5+2.2}{1.001}$ & $\frac{3.3011}{1.001}$ & $\frac{30.9}{1.001}$ & 1.24 & 1.24 & 1.24 & 1.24 & 1.24 & ${ }_{1.24}$ & $\frac{1.24}{1.24}$ & $\frac{1.24}{1.24}$ & $\frac{1.24}{1.24}$ & 1.24 & 1.24 & 1.24 & 1.24 & 1.04 \\
\hline 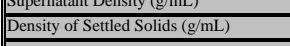 & $\frac{12}{1.2}$ & $\frac{1.282}{1.2}$ & 1.1 .18 & $\frac{1.18}{1.1}$ & $\frac{\mathrm{mm}}{\mathrm{nm}}$ & $\frac{\mathrm{nm}}{\mathrm{nm}}$ & $\frac{\pi}{n / m}$ & $\mathrm{~nm}$ & $\frac{\mathrm{mm}}{\mathrm{nm}}$ & $\frac{\mathrm{nm}}{\mathrm{nm}}$ & $\frac{n \mathrm{~m}}{\mathrm{~nm}}$ & $\frac{n}{n / m}$ & $\frac{n}{n / m}$ & $\frac{\mathrm{nm}}{\mathrm{nm}}$ & $\mathrm{nm}$ & $\mathrm{nm}$ & $\frac{n m}{n m}$ & 1.22 \\
\hline w\%\% Serted Supenanatant & nim & $\mathrm{n}^{\mathrm{n} / \mathrm{m}}$ & $\mathrm{n}^{\mathrm{n} \mathrm{m}}$ & $\mathrm{n}^{\mathrm{nm}}$ & nim & $\mathrm{n} / \mathrm{m}$ & $\mathrm{nm}$ & n/m & n/m & n/m & nim & n/m & nim & ${ }^{\mathrm{n} / \mathrm{m}}$ & $\mathrm{nm}$ & $\mathrm{n}^{\mathrm{m} m}$ & $n^{n / m}$ & $-n^{n / m}$ \\
\hline w\% dissolved solids in supernatant & n & $\mathrm{nm}$ & $\mathrm{nm}$ & $\mathrm{n}^{\mathrm{n} / \mathrm{m}}$ & $\mathrm{n} / \mathrm{m}$ & $\mathrm{n} / \mathrm{m}$ & $\mathrm{n} / \mathrm{m}$ & $\mathrm{n} / \mathrm{m}$ & $n^{\prime m m}$ & ${ }^{n / m}$ & $\mathrm{~nm}$ & n/m & $\mathrm{nm}_{\mathrm{m}}$ & $\mathrm{n} / \mathrm{m}$ & n/m & $\mathrm{n} / \mathrm{m}$ & $\mathrm{n} / \mathrm{m}$ & n/m \\
\hline wo\% total solids in Centrifiged Sludge & n/m & $\mathrm{n} / \mathrm{m}$ & $\mathrm{n} / \mathrm{m}$ & ${ }^{\mathrm{n} / \mathrm{m}}$ & $\mathrm{n} / \mathrm{m}$ & n/m & $\frac{n \mathrm{~nm}}{\mathrm{n}}$ & $\overline{n / m}$ & $\frac{n^{\prime \prime m}}{3}$ & $\overline{n / m}$ & $\overline{\mathrm{nm}}$ & n & $\overline{n m}$ & $\frac{n_{\mathrm{m}}}{}$ & $\frac{\mathrm{nm}}{\mathrm{n}}$ & n/m & ${ }_{n / m}$ & nt/m \\
\hline W\%\% Total solids & 9.7 & 24.9 & 22.1 & 21.2 & $\frac{\mathrm{n}^{\mathrm{m} m} \mathrm{~m}}{\mathrm{n}}$ & $\frac{n}{n / m}$ & 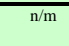 & n/m & n/m & $\frac{n^{\prime \prime m}}{n^{2}}$ & nim & nim & mim & $\frac{n^{\prime m}}{n^{2}}$ & n/m & $\frac{n^{n / m}}{n^{2}}$ & $\frac{n^{\prime / m}}{n^{2}}$ & $\sqrt{n / m}$ \\
\hline W\% UDS & 6.90 & 24,00 & 22.00 & 20.50 & 34.26 & 34.26 & $\begin{array}{ll}34.26 \\
\end{array}$ & 34.26 & $\begin{array}{l}3426 \\
43\end{array}$ & $\frac{34.26}{34}$ & $\begin{array}{ll}3426 \\
\end{array}$ & 3426 & 3426 & $\frac{3426}{3426}$ & $\begin{array}{l}3426 \\
4\end{array}$ & $\begin{array}{l}3426 \\
4\end{array}$ & 34.26 & 8.66 \\
\hline Donsily of solids (fyc) & $\begin{array}{l}3.03 \\
.03\end{array}$ & 3.15 & $\frac{3.15}{3.1}$ & 4.18 & 3.03 & 3.03 & 3.03 & 3.03 & 3.03 & 3.03 & 3.03 & 3.03 & 3.03 & 3.03 & 3.03 & 3.03 & 3.03 & 3.15 \\
\hline
\end{tabular}



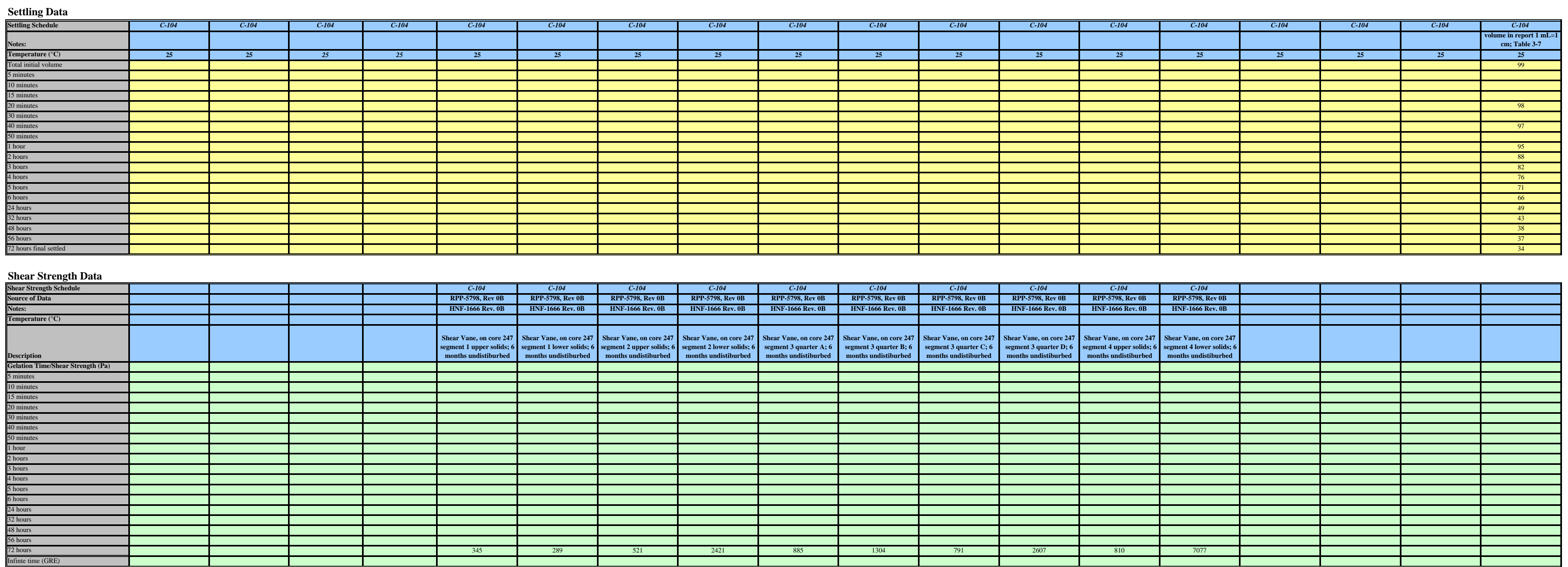


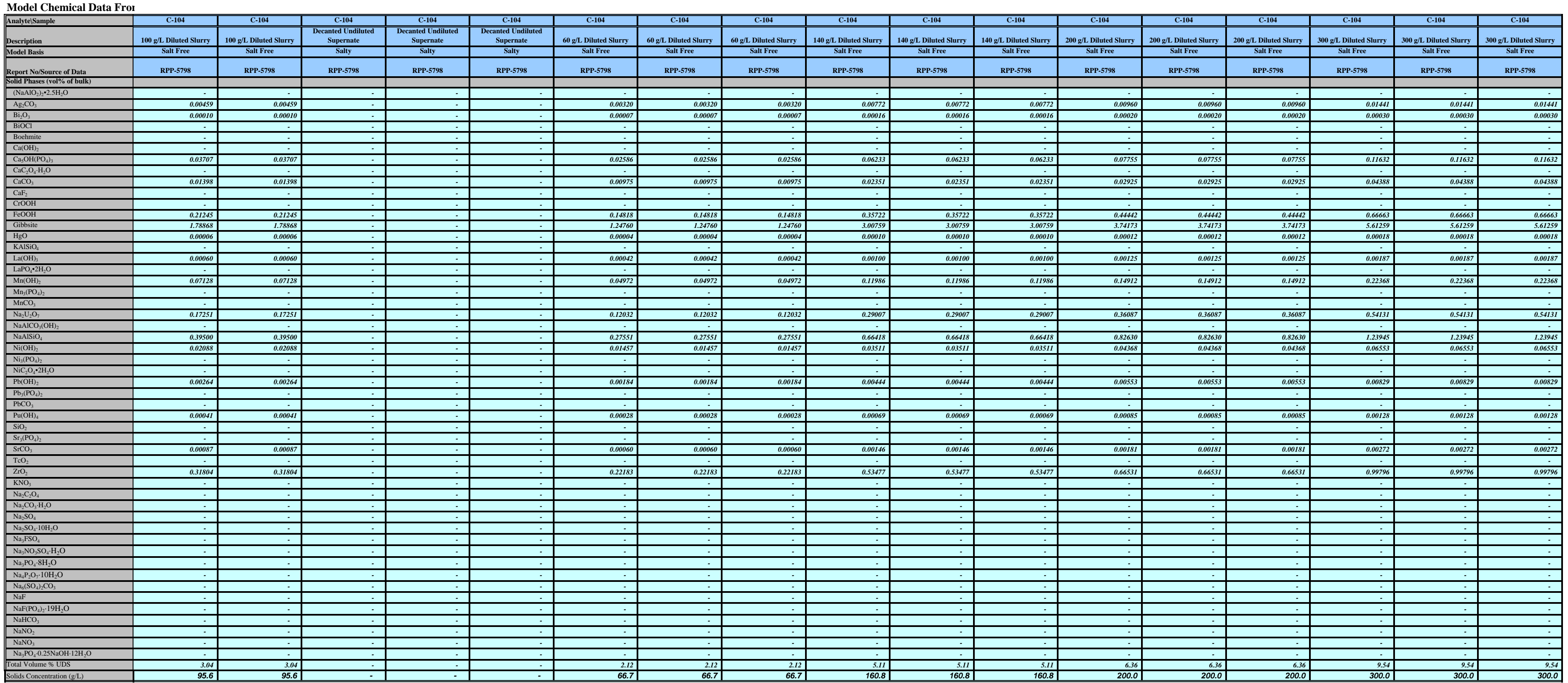


Rheological Data

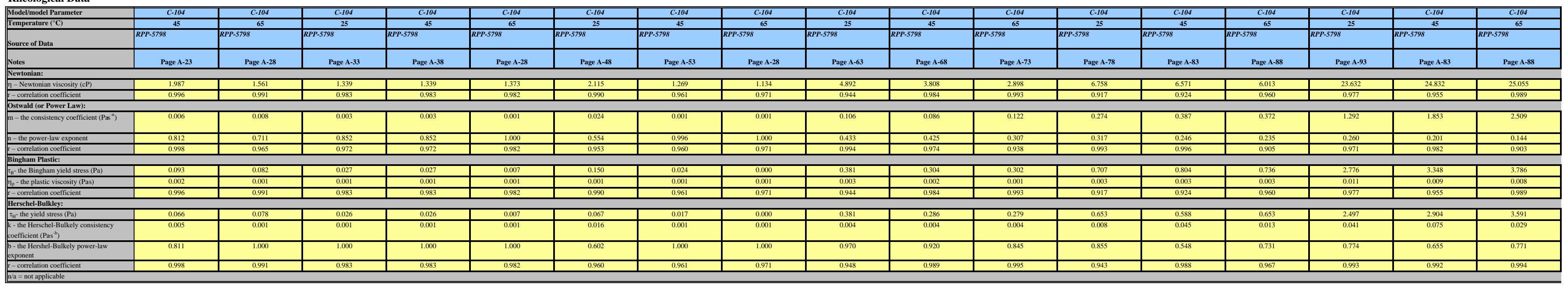

Physical Property Data

\begin{tabular}{|c|c|c|c|c|c|c|c|c|c|c|c|c|c|c|c|c|c|}
\hline $\begin{array}{l}\text { Plysical Insial Propery } \\
\text { Plyty Data }\end{array}$ & $C .-104$ & $\frac{c .104}{c-104}$ & $\frac{c .104}{c .104}$ & $\begin{array}{c}C-104 \\
\end{array}$ & $C-104$ & $\begin{array}{c}C-104 \\
\end{array}$ & $\begin{array}{c}C-104 \\
\end{array}$ & \begin{tabular}{cc|c|}
$C-104$ \\
\end{tabular} & $\begin{array}{c}c .104 \\
.104\end{array}$ & $c^{c .104}$ & $C_{-104}$ & $C-104$ & $C .104$ & 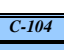 & $C-104$ & $C .104$ & $\frac{c-104}{c-104}$ \\
\hline \multicolumn{18}{|l|}{ Notes } \\
\hline Temperature ( $\mathrm{C}$ ) & 45 & 65 & 25 & 45 & 65 & 25 & 45 & 65 & 25 & 45 & 65 & 25 & 45 & 65 & 25 & 45 & 65 \\
\hline 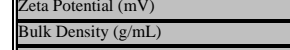 & $\frac{1.104}{1.04}$ & $\frac{1.104}{1.04}$ & ${ }_{1.24}^{1.24}$ & $\frac{1.24}{1.03}$ & 1.24 & 1.068 & 1.068 & 1.068 & $\frac{1.159}{1.59}$ & $\frac{1.159}{1.59}$ & 1.1 .59 & 1.24 & 1.24 & 1.24 & 1.36 & 1.36 & $\frac{1.36}{1.36}$ \\
\hline vologs sented Solits & n/m & $\mathrm{nm}^{\mathrm{nm}}$ & ${ }^{\mathrm{nm}}$ & $\mathrm{n} / \mathrm{m}$ & ${ }^{\mathrm{n} / \mathrm{m}}$ & ${ }^{\mathrm{n} / \mathrm{m}}$ & ${ }^{\mathrm{n} / \mathrm{m}}$ & ${ }^{n \mathrm{~mm}}$ & $\frac{\mathrm{nm}}{\mathrm{nm}}$ & $\frac{n \mathrm{~nm}}{\mathrm{~nm}}$ & ${ }^{n / m}$ & ${ }^{n / m}$ & $\frac{n^{\prime m}}{m}$ & $n^{n m}$ & ${ }^{\mathrm{nm}}$ & ${ }^{n / m}$ & $\frac{n}{n / m}$ \\
\hline Density of Cennifituged Soids (gmL) & $\frac{\mathrm{nm}}{\mathrm{nm} / \mathrm{m}}$ & $\frac{n \pi m}{n n m}$ & $\frac{n \mathrm{~m}}{\mathrm{~nm}}$ & $\frac{\mathrm{nm}}{\mathrm{nm}}$ & $\frac{\mathrm{nm}}{\mathrm{nm}}$ & $\frac{\mathrm{nm}}{\mathrm{nm}}$ & $\frac{\mathrm{nm}}{\mathrm{nmm}}$ & $\frac{n m}{n n m}$ & $\frac{n \mathrm{~nm}}{\mathrm{nmm}}$ & $\frac{n n^{n m}}{n+m}$ & $\frac{\mathrm{nm}}{\mathrm{nm}}$ & $\frac{\mathrm{nm}}{\mathrm{nmm}}$ & $\frac{\mathrm{nm}}{\mathrm{nm}}$ & $\frac{n m}{n m m}$ & $\frac{n m}{n m m}$ & $\frac{\mathrm{nm}}{\mathrm{nmm}}$ & $\frac{\mathrm{nm}}{\mathrm{nm}}$ \\
\hline vol\%o centifitued Solids & $\mathrm{nm}$ & $\mathrm{nm}_{\mathrm{m}}$ & $n_{\mathrm{nm}}$ & ${ }^{n / m}$ & $\overline{n / m}$ & $\mathrm{n}_{\mathrm{n} m}$ & $\mathrm{n} / \mathrm{m}$ & $n_{\mathrm{nm}}$ & $\mathrm{nm}_{\mathrm{m}}$ & $\mathrm{n}_{\mathrm{m}}$ & $n^{\prime / m}$ & $\overline{n / m}$ & $\overline{n / \mathrm{m}}$ & ${ }^{N m}$ & $\sqrt{\mathrm{mm}}$ & ${ }_{\mathrm{n} / \mathrm{m}}$ & $\overline{\mathrm{n} / \mathrm{m}}$ \\
\hline 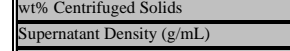 & 1.04 & $\frac{1.04}{1.04}$ & $\frac{1.24}{1.24}$ & 1.24 & 1.24 & $\frac{1.02}{1.2}$ & $\frac{1.02}{1.02}$ & $\frac{1.02}{1.02}$ & $\frac{1.05}{1.05}$ & $\frac{1.05}{1.05}$ & 1.05 & ${ }_{1.11}$ & 1.111 & t.1.1 & 1.17 & $\frac{1.17}{1.7}$ & ${ }_{1.17}^{1.17}$ \\
\hline 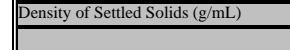 & $\frac{1.226}{\mathrm{n} / \mathrm{m}}$ & $\frac{1.226}{N m m}$ & & & & & & & $\frac{1.308}{n+m}$ & $\frac{1.308}{n+108}$ & $\frac{1.308}{1.006}$ & $\frac{\mathrm{nm}}{n+1}$ & $\frac{n m}{n ! m}$ & $\frac{n \mathrm{~m}}{1-2}$ & $\frac{n \mathrm{~m}}{-1}$ & $\frac{n m m}{n+m}$ & $\frac{n \mathrm{~mm}}{20 \mathrm{~m}}$ \\
\hline w\%\% Setled Superatant & ${ }^{n / m}$ & nim & $n^{n m}$ & ${ }^{n / m}$ & ${ }^{n / m}$ & ${ }^{n / m}$ & ${ }^{\mathrm{n} / \mathrm{m}}$ & $n^{n m}$ & ${ }^{\mathrm{nm} m}$ & $n^{\mathrm{nm}}$ & ${ }^{n / m}$ & ${ }^{n / m}$ & $\mathrm{n}^{\mathrm{m} m}$ & nim & ${ }^{\mathrm{nm}}$ & ${ }^{n / m}$ & ${ }^{n / m}$ \\
\hline w\%o dissolved solids in superanatant & ${ }^{\mathrm{n} / \mathrm{m}}$ & ${ }^{n / m}$ & ${ }^{n / m}$ & ${ }^{\mathrm{n} / \mathrm{m}}$ & ${ }^{n / m}$ & ${ }^{n / m}$ & ${ }^{n / m}$ & ${ }^{n m}$ & $\mathrm{n}_{\mathrm{m}}^{\mathrm{m}}$ & $\mathrm{n}^{\mathrm{nm}}$ & $\mathrm{n}^{\mathrm{n} m \mathrm{~m}}$ & ${ }^{n / m}$ & 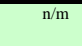 & ${ }_{n m}^{n m}$ & ${ }^{n m}$ & $n^{n / m}$ & $\overline{n / m}$ \\
\hline & n/m & ${ }^{n / m}$ & $n$ & ${ }^{n / m}$ & ${ }^{n / m}$ & ${ }^{n / m}$ & ${ }^{n / m}$ & $n$ & $\frac{n^{m} \mathrm{~m}}{n^{2}}$ & $\frac{n^{n m}}{n^{2}}$ & $\mathrm{n}^{\mathrm{n} m \mathrm{~m}}$ & ${ }^{n / m}$ & n/m & $n$ & $n$ & ${ }^{n / m}$ & $\overline{n / m}$ \\
\hline wwo" Toal solids & ${ }^{\mathrm{n} / \mathrm{m}}$ & ${ }^{n / m}$ & ${ }^{n / m}$ & ${ }^{n / m}$ & ${ }^{n / m}$ & ${ }^{n / m}$ & ${ }^{n / m}$ & ${ }^{\mathrm{nm}}$ & $\mathrm{n}^{\mathrm{nm}}$ & $\mathrm{n}^{\mathrm{nm}}$ & ${ }^{n \mathrm{~m} m}$ & ${ }^{n / m}$ & $\mathrm{n}^{\mathrm{n} m \mathrm{~m}}$ & ${ }^{n / m}$ & ${ }^{n / m}$ & ${ }^{n / m}$ & ${ }^{n / m}$ \\
\hline werovs & 8.66 & ${ }^{8.66}$ & 0.00 & 0.00 & 0.00 & ${ }^{6.24}$ & 0.24 & 6.24 & ${ }_{13.87}$ & ${ }_{13.87}$ & 13.87 & 6.13 & ${ }_{0.0 .13}$ & .13 & 2.206 & 22.06 & .06 \\
\hline & 3.15 & 3.15 & 3.03 & 3.03 & 3.03 & 3.15 & 3.15 & 3.15 & 3.15 & 3.15 & 3.15 & 3.15 & 3.15 & 3.15 & 3.15 & 3.15 & 3.15 \\
\hline
\end{tabular}



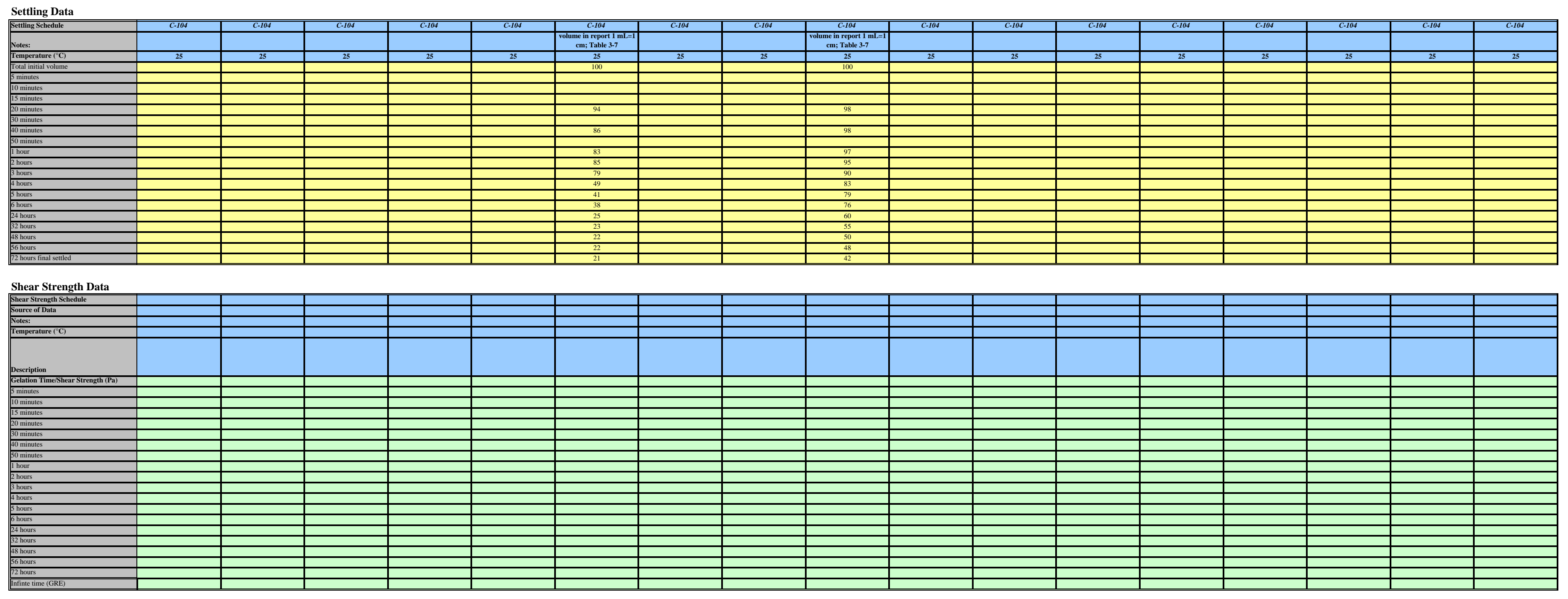
241-C-109

Model Chemical Data From ESP

\begin{tabular}{|c|c|c|c|}
\hline AnalytelSample & C-109 & C-109 & C-109 \\
\hline Description & no dilution & 1:1 dilution & 3:1 dilution \\
\hline Model Basis & Salty & Salt Free & Salt Free \\
\hline Report No/Source of Data & $\begin{array}{c}\text { WHC-SD-WM-ER-402, } \\
\text { rev 0; Core } 47\end{array}$ & $\begin{array}{c}\text { WHC-SD-WM-ER-402, } \\
\text { rev 0; Core } 47\end{array}$ & $\begin{array}{c}\text { WHC-SD-WM-ER-402, } \\
\text { rev 0; Core } 47\end{array}$ \\
\hline \multicolumn{4}{|l|}{ Solid Phases (vol\% of bulk) } \\
\hline$\left(\mathrm{NaAlO}_{2}\right)_{2} \cdot 2.5 \mathrm{H}_{2} \mathrm{O}$ & - & - & - \\
\hline $\mathrm{Ag}_{2} \mathrm{CO}_{3}$ & - & - & - \\
\hline $\mathrm{Bi}_{2} \mathrm{O}_{3}$ & 0.01311 & 0.01166 & 0.00711 \\
\hline $\mathrm{BiOCl}$ & - & - & - \\
\hline Boehmite & - & - & - \\
\hline $\mathrm{Ca}(\mathrm{OH})_{2}$ & - & - & - \\
\hline $\mathrm{Ca}_{5} \mathrm{OH}\left(\mathrm{PO}_{4}\right)_{3}$ & 0.41412 & 0.36843 & 0.22460 \\
\hline $\mathrm{CaC}_{2} \mathrm{O}_{4} \cdot \mathrm{H}_{2} \mathrm{O}$ & - & - & - \\
\hline $\mathrm{CaCO}_{3}$ & - & - & - \\
\hline $\mathrm{CaF}_{2}$ & - & - & - \\
\hline $\mathrm{CrOOH}$ & 0.00285 & 0.00254 & 0.00155 \\
\hline $\mathrm{FeOOH}$ & 0.22392 & 0.19921 & 0.12144 \\
\hline Gibbsite & 1.96610 & 1.74915 & 1.06632 \\
\hline $\mathrm{HgO}$ & - & - & - \\
\hline $\mathrm{KAlSiO}_{4}$ & - & - & - \\
\hline $\mathrm{La}(\mathrm{OH})_{3}$ & - & - & - \\
\hline $\mathrm{LaPO}_{4} \cdot 2 \mathrm{H}_{2} \mathrm{O}$ & 0.00007 & 0.00006 & 0.00004 \\
\hline $\mathrm{Mn}(\mathrm{OH})_{2}$ & - & - & - \\
\hline $\mathrm{Mn}_{3}\left(\mathrm{PO}_{4}\right)_{2}$ & 0.00459 & 0.00409 & 0.00249 \\
\hline $\mathrm{MnCO}_{3}$ & - & - & - \\
\hline $\mathrm{Na}_{2} \mathrm{U}_{2} \mathrm{O}_{7}$ & 0.08836 & 0.07861 & 0.04792 \\
\hline $\mathrm{NaAlCO}_{3}(\mathrm{OH})_{2}$ & 0.70261 & 0.62508 & 0.38106 \\
\hline $\mathrm{NaAlSiO}_{4}$ & 0.37371 & 0.33247 & 0.20268 \\
\hline $\mathrm{Ni}(\mathrm{OH})_{2}$ & 0.16172 & 0.14388 & 0.08771 \\
\hline $\mathrm{Ni}_{3}\left(\mathrm{PO}_{4}\right)_{2}$ & - & - & - \\
\hline $\mathrm{NiC}_{2} \mathrm{O}_{4} \cdot 2 \mathrm{H}_{2} \mathrm{O}$ & - & - & - \\
\hline $\mathrm{Pb}(\mathrm{OH})_{2}$ & 0.01165 & 0.01036 & 0.00632 \\
\hline $\mathrm{Pb}_{3}\left(\mathrm{PO}_{4}\right)_{2}$ & - & - & - \\
\hline $\mathrm{PbCO}_{3}$ & - & - & - \\
\hline $\mathrm{Pu}(\mathrm{OH})_{4}$ & 0.00002 & 0.00002 & 0.00001 \\
\hline $\mathrm{SiO}_{2}$ & - & - & - \\
\hline $\mathrm{Sr}_{3}\left(\mathrm{PO}_{4}\right)_{2}$ & - & - & - \\
\hline $\mathrm{SrCO}_{3}$ & 0.00548 & 0.00487 & 0.00297 \\
\hline $\mathrm{TcO}_{2}$ & - & - & - \\
\hline $\mathrm{ZrO}_{2}$ & 0.00022 & 0.00020 & 0.00012 \\
\hline $\mathrm{KNO}_{3}$ & - & - & - \\
\hline $\mathrm{Na}_{2} \mathrm{C}_{2} \mathrm{O}_{4}$ & 0.07715 & - & - \\
\hline $\mathrm{Na}_{2} \mathrm{CO}_{3} \cdot \mathrm{H}_{2} \mathrm{O}$ & - & - & - \\
\hline $\mathrm{Na}_{2} \mathrm{SO}_{4}$ & - & - & - \\
\hline $\mathrm{Na}_{2} \mathrm{SO}_{4} \cdot 10 \mathrm{H}_{2} \mathrm{O}$ & - & - & - \\
\hline $\mathrm{Na}_{3} \mathrm{FSO}_{4}$ & - & - & - \\
\hline $\mathrm{Na}_{3} \mathrm{NO}_{3} \mathrm{SO}_{4} \cdot \mathrm{H}_{2} \mathrm{O}$ & - & - & - \\
\hline $\mathrm{Na}_{3} \mathrm{PO}_{4} \cdot 8 \mathrm{H}_{2} \mathrm{O}$ & - & - & - \\
\hline $\mathrm{Na}_{4} \mathrm{P}_{2} \mathrm{O}_{7} \cdot 10 \mathrm{H}_{2} \mathrm{O}$ & - & - & - \\
\hline $\mathrm{Na}_{6}\left(\mathrm{SO}_{4}\right)_{2} \mathrm{CO}_{3}$ & - & - & - \\
\hline $\mathrm{NaF}$ & - & - & - \\
\hline $\mathrm{NaF}\left(\mathrm{PO}_{4}\right)_{2} \cdot 19 \mathrm{H}_{2} \mathrm{O}$ & 1.26441 & - & - \\
\hline $\mathrm{NaHCO}_{3}$ & - & - & - \\
\hline $\mathrm{NaNO}_{2}$ & - & - & - \\
\hline $\mathrm{NaNO}_{3}$ & - & - & - \\
\hline $\mathrm{Na}_{3} \mathrm{PO}_{4} \cdot 0.25 \mathrm{NaOH} \cdot 12 \mathrm{H}_{2} \mathrm{O}$ & 1.31565 & - & - \\
\hline Total Volume \% UDS & 6.63 & 3.53 & 2.15 \\
\hline Solids Concentration (g/L) & 156.2 & 98.7 & 60.2 \\
\hline
\end{tabular}

Note: Numbers in italics are calculated values. Numbers in normal font are reported data. 


\section{Rheological Data}

\begin{tabular}{|c|c|c|}
\hline Model/model Parameter & $\overline{C-109}$ & $\overline{C-109}$ \\
\hline Temperature $\left({ }^{\circ} \mathrm{C}\right)$ & 30 & 30 \\
\hline Source of Data & $\begin{array}{l}\text { WHC-SD-WM-ER-402, } \\
\text { rev 0; Core } 47\end{array}$ & $\begin{array}{l}\text { WHC-SD-WM-ER-402, } \\
\text { rev 0; Core } 47\end{array}$ \\
\hline Notes & $\begin{array}{l}\text { Entered from Joel } \\
\text { Tingey's Paper File }\end{array}$ & $\begin{array}{l}\text { Entered from Joel } \\
\text { Tingey's Paper File }\end{array}$ \\
\hline \multicolumn{3}{|l|}{ Newtonian: } \\
\hline$\eta$ - Newtonian viscosity (cP) & 164.527 & 5.490 \\
\hline $\mathrm{r}$ - correlation coefficient & 0.558 & 0.896 \\
\hline \multicolumn{3}{|l|}{ Ostwald (or Power Law): } \\
\hline $\mathrm{m}$ - the consistency coefficient $\left(\mathrm{Pa} \cdot \mathrm{s}^{-\mathrm{n}}\right)$ & 20.278 & 0.512 \\
\hline $\mathrm{n}$ - the power-law exponent & 0.186 & 0.215 \\
\hline $\mathrm{r}$ - correlation coefficient & 0.805 & 0.911 \\
\hline \multicolumn{3}{|l|}{ Bingham Plastic: } \\
\hline$\tau_{\mathrm{B}}$ - the Bingham yield stress $(\mathrm{Pa})$ & 37.203 & 0.929 \\
\hline$\eta_{p}$ - the plastic viscosity (Pa-s) & 0.057 & 0.003 \\
\hline $\mathrm{r}$ - correlation coefficient & 0.558 & 0.896 \\
\hline \multicolumn{3}{|l|}{ Herschel-Bulkley: } \\
\hline$\tau_{\mathrm{H}^{-}}$the yield stress $(\mathrm{Pa})$ & 16.131 & 0.906 \\
\hline $\begin{array}{l}\text { k - the Herschel-Bulkely consistency } \\
\text { coefficient }\left(\mathrm{Pa} \cdot \mathrm{s}^{-\mathrm{b}}\right)\end{array}$ & 7.214 & 0.004 \\
\hline $\begin{array}{l}\text { b - the Hershel-Bulkely power-law } \\
\text { exponent }\end{array}$ & 0.311 & 0.943 \\
\hline $\mathrm{r}$ - correlation coefficient & 0.761 & 0.902 \\
\hline $\mathrm{n} / \mathrm{a}=$ not applicable & & \\
\hline
\end{tabular}

\section{Physical Property Data}

\begin{tabular}{|c|c|c|c|}
\hline$\sqrt{\text { Physical Property }}$ & C-109 & $\overline{C-109}$ & $\overline{C-109}$ \\
\hline \multicolumn{4}{|l|}{ Notes } \\
\hline Temperature $\left({ }^{\circ} \mathrm{C}\right)$ & 30 & 30 & 30 \\
\hline \multicolumn{4}{|l|}{ Zeta Potential (mV) } \\
\hline Bulk Density (g/mL) & 1.23 & 1.19 & 1.11 \\
\hline |vol\% Settled Solids & 100 & 88 & $\overline{41}$ \\
\hline Density of Centrifuged Solids $(\mathrm{g} / \mathrm{mL})$ & 1.23 & $\mathrm{n} / \mathrm{m}$ & 1.39 \\
\hline ||vol\% Centrifuged Solids & 100 & $\mathrm{n} / \mathrm{m}$ & 21.1 \\
\hline wt\% Centrifuged Solids & 100 & $\mathrm{n} / \mathrm{m}$ & 27 \\
\hline Supernatant Density $(\mathrm{g} / \mathrm{mL})$ & 1.15 & 1.13 & 1.01 \\
\hline Density of Settled Solids $(\mathrm{g} / \mathrm{mL})$ & $\mathrm{n} / \mathrm{m}$ & $\mathrm{n} / \mathrm{m}$ & $\mathrm{n} / \mathrm{m}$ \\
\hline wt\% Settled Supernatant & $\mathrm{n} / \mathrm{m}$ & $\mathrm{n} / \mathrm{m}$ & $\mathrm{n} / \mathrm{m}$ \\
\hline |wt $\%$ dissolved solids in supernatant & 29.61 & 29.12 & 26.01 \\
\hline wt \% total solids in Centrifuged Sludge & $\mathrm{n} / \mathrm{m}$ & $\mathrm{n} / \mathrm{m}$ & $\mathrm{n} / \mathrm{m}$ \\
\hline wt\% Total Solids & $\mathrm{n} / \mathrm{m}$ & $\mathrm{n} / \mathrm{m}$ & $\mathrm{n} / \mathrm{m}$ \\
\hline wt\% UDS & 12.70 & 8.30 & 5.42 \\
\hline Density of Solids (g/cc) & 2.36 & 2.80 & 2.80 \\
\hline
\end{tabular}

Note: Numbers in italics are calculated values. Numbers in normal font are reported data. 
Settling Data

\begin{tabular}{|c|c|c|}
\hline Settling Schedule & $\overline{C-109}$ & $\overline{C-109}$ \\
\hline Notes: & $\begin{array}{c}\text { Page B-19; appears to be } \\
\text { fast settling }\end{array}$ & $\begin{array}{l}\text { Page B-19; appears to be } \\
\text { fast settling }\end{array}$ \\
\hline Temperature $\left({ }^{\circ} \mathrm{C}\right)$ & 30 & 30 \\
\hline Total initial volume & 100 & 100 \\
\hline \multicolumn{3}{|l|}{5 minutes } \\
\hline \multicolumn{3}{|l|}{10 minutes } \\
\hline \multicolumn{3}{|l|}{15 minutes } \\
\hline \multicolumn{3}{|l|}{20 minutes } \\
\hline \multicolumn{3}{|l|}{30 minutes } \\
\hline \multicolumn{3}{|l|}{40 minutes } \\
\hline \multicolumn{3}{|l|}{50 minutes } \\
\hline \multicolumn{3}{|l|}{1 hour } \\
\hline 2 hours & 97 & 63 \\
\hline \multicolumn{3}{|l|}{3 hours } \\
\hline \multicolumn{3}{|l|}{4 hours } \\
\hline 5 hours & 92 & 59 \\
\hline \multicolumn{3}{|l|}{6 hours } \\
\hline 24 hours & 88 & 45 \\
\hline \multicolumn{3}{|l|}{32 hours } \\
\hline 48 hours & 87 & 40 \\
\hline 56 hours & 86 & 39 \\
\hline 72 hours final settled & & \\
\hline
\end{tabular}

\section{Shear Strength Data}

\begin{tabular}{|c|c|c|c|}
\hline Shear Strength Schedule & $\overline{C E-109}$ & & \\
\hline Source of Data & $\begin{array}{c}\text { SG McKinley et al } \\
\text { Characterization Report } \\
\text { March 30, } 1993\end{array}$ & & \\
\hline \multicolumn{4}{|l|}{ Notes: } \\
\hline Temperature $\left({ }^{\circ} \mathrm{C}\right)$ & Unspecified & & \\
\hline Description & $\begin{array}{l}\text { Shear Vane at unspecified } \\
\text { gel time or temperature }\end{array}$ & & \\
\hline \multicolumn{4}{|c|}{ Gelation Time/Shear Strength (Pa) } \\
\hline \multicolumn{4}{|l|}{5 minutes } \\
\hline \multicolumn{4}{|l|}{10 minutes } \\
\hline \multicolumn{4}{|l|}{15 minutes } \\
\hline \multicolumn{4}{|l|}{20 minutes } \\
\hline \multicolumn{4}{|l|}{30 minutes } \\
\hline \multicolumn{4}{|l|}{40 minutes } \\
\hline \multicolumn{4}{|l|}{50 minutes } \\
\hline \multicolumn{4}{|l|}{1 hour } \\
\hline \multicolumn{4}{|l|}{2 hours } \\
\hline \multicolumn{4}{|l|}{3 hours } \\
\hline \multicolumn{4}{|l|}{4 hours } \\
\hline \multicolumn{4}{|l|}{5 hours } \\
\hline \multicolumn{4}{|l|}{6 hours } \\
\hline \multicolumn{4}{|l|}{24 hours } \\
\hline \multicolumn{4}{|l|}{32 hours } \\
\hline \multicolumn{4}{|l|}{48 hours } \\
\hline \multicolumn{4}{|l|}{56 hours } \\
\hline 72 hours & 1730 & & \\
\hline Infinte time (GRE) & & & \\
\hline
\end{tabular}

Note: Numbers in italics are calculated values. Numbers in normal font are reported data. 
Model Chemical Data From ESP

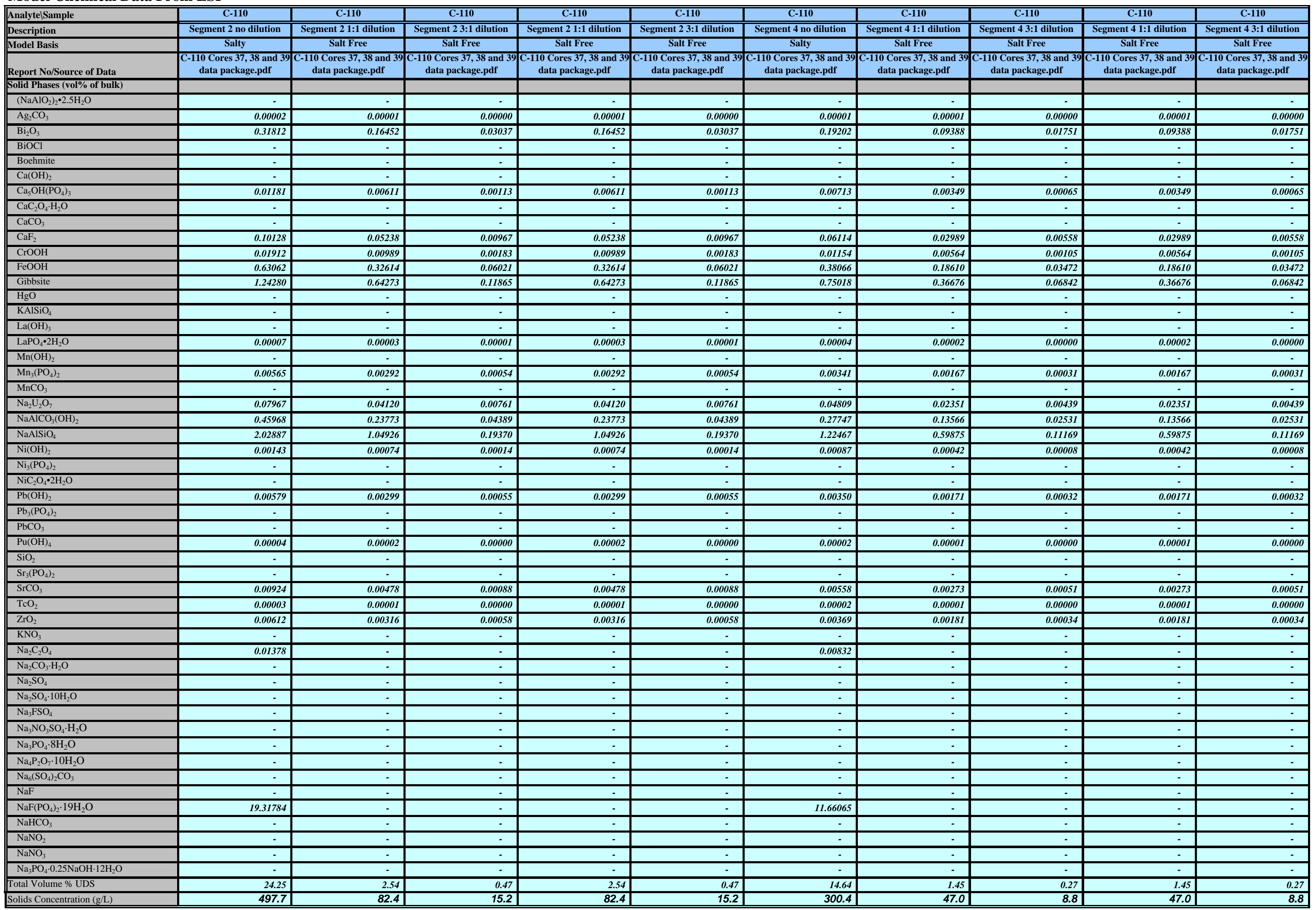


Rheological Data

\begin{tabular}{|c|c|c|c|c|c|c|c|c|c|c|}
\hline Model/model Parameter & $\overline{c-110}$ & 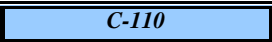 & $\overline{C-110}$ & $\overline{c-110}$ & $\overline{c c-110}$ & $\overline{c-110}$ & $\overline{c-110}$ & 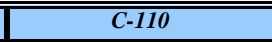 & $\overline{c-110}$ & $\overline{c-110}$ \\
\hline 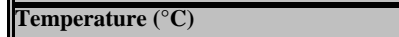 & 30 & 30 & 30 & 95 & 95 & 30 & 30 & 30 & 95 & 95 \\
\hline SSource of Data & C-110 Cores 37, 38 and 39 & C-110 Cores 37,38 and 39 & C-110 Cores 37,38 and 34 & $\begin{array}{l}-110 \text { Cores } 37,38 \text { and } 39 \\
\text { - }\end{array}$ & C-110 Cores 37,38 and $3: 4$ & C-110 Cores 37,38 and 39 & C-110 Cores 37,38 and 34 & C-110 Cores 37,38 and 39 & -110 Cores 37,38 an & 110 Cores 37,38 an \\
\hline Nortes un Dala & & & & data packagepdof & datat packagepdf & datat package.pdf & $\begin{array}{l}\text { data package.pdf } \\
\text { Co4-093 }\end{array}$ & data package.pdf & data package.pdf & data package.pdf \\
\hline \multicolumn{11}{|l|}{ Newtonian: } \\
\hline \begin{tabular}{|l} 
- Newtonian viscosity (cP) \\
\end{tabular} & & 4.937 & 2.160 & 2.251 & 1.376 & & 3.637 & 2.144 & 3.156 & 1.824 \\
\hline r-correlation coefficient & & 0.998 & 1.000 & 0.985 & 0.978 & & 0.997 & 1.000 & 0.998 & 0.983 \\
\hline \multicolumn{11}{|l|}{\begin{tabular}{|l} 
Sotwald (or Power Law): \\
\end{tabular}} \\
\hline m-the consistency coefficient (Pa-s" & & 0.011 & 0.003 & 0.011 & 0.001 & & 0.013 & 0.002 & 0.049 & 0.012 \\
\hline$\|$ n-the power-law exponent & & 0.858 & 0.924 & 0.733 & 1.000 & & 0.777 & 0.998 & 0.533 & 0.681 \\
\hline $\mid$ r-correlation coefficient & & 0.997 & 0.998 & 0.945 & 0.978 & & 0.991 & 1.000 & 0.940 & 0.927 \\
\hline \multicolumn{11}{|l|}{\begin{tabular}{|l} 
Bingham Plastic: \\
\end{tabular}} \\
\hline$\tau_{\mathrm{B} B}$ - the Bingham yield stress (Pa) & & 0.676 & 0.000 & 0.159 & 0.000 & & 0.218 & 0.000 & $\begin{array}{ll}0.414 \\
\end{array}$ & 0.173 \\
\hline$\| \eta_{p}$ - the plastic viscosity (Pa:s) & & 0.003 & 0.002 & 0.002 & 0.001 & & 0.003 & 0.002 & 0.002 & 0.001 \\
\hline rir-correlation coefficient & & 0.998 & 1.000 & 0.985 & 0.978 & & 0.997 & 1.000 & 0.998 & 0.983 \\
\hline \multicolumn{11}{|l|}{ Herschel-Bunteley: } \\
\hline$\tau_{\tau_{\mathrm{H}} \text { - the yied stress }(\mathrm{Pa})}$ & & 0.420 & 0.000 & 0.144 & 0.000 & & 0.200 & 0.000 & 0.403 & 0.174 \\
\hline 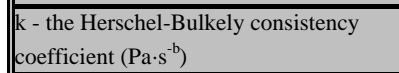 & & 0.004 & 0.003 & 0.004 & 0.001 & & 0.004 & 0.002 & 0.003 & 0.001 \\
\hline $\begin{array}{l}\text { b- the Hershel-Bulkely power-law } \\
\text { exxonent }\end{array}$ & & 0.979 & 0.971 & 0.876 & 1.000 & & 0.935 & 1.000 & 0.942 & 1.000 \\
\hline $\mid$\begin{tabular}{|l}
$\mid \mathrm{r}$-correlation coefficient \\
\end{tabular} & & 0.999 & 0.999 & 0.972 & 0.978 & & 0.998 & 1.000 & 0.999 & 0.983 \\
\hline N/a = not applicable & & & & & & & & & & \\
\hline \multicolumn{11}{|l|}{ Physical Property Data } \\
\hline \begin{tabular}{|l|} 
Physical Property \\
\end{tabular} & $\frac{C-110}{c}$ & $\frac{\mathrm{C}-110}{\mathrm{C}}$ & $\frac{C-110}{c}$ & $\overline{C-110}$ & $\overline{c-110}$ & $\overline{c-110}$ & $\frac{C-110}{c-10}$ & $\overline{c-110}$ & $\frac{\overline{C-110}}{\mathrm{C}-10}$ & $\overline{c-110}$ \\
\hline \multicolumn{11}{|l|}{ Notes } \\
\hline Temperature $\left({ }^{\circ} \mathrm{C}\right)$ & 30 & 30 & 30 & 30 & 30 & 30 & 30 & 30 & 30 & 30 \\
\hline 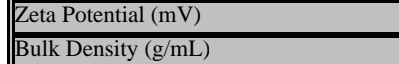 & 1.41 & $\overline{1.16}$ & $\overline{1.06} \mathrm{Y}>\mathrm{C}>\mathrm{C}$ & $\overline{1.16}$ & 106 & 1.48 & 115 & $\overline{106}$ & 115 & $\overline{1.06}$ \\
\hline $\mid \begin{array}{l}\mid \text { vol\% Setled Solids } \\
\text {. }\end{array}$ & 100 & $\mathrm{n} / \mathrm{m}$ & $\mathrm{n} / \mathrm{m}$ & $\mathrm{n} / \mathrm{m}$ & $\mathrm{n} / \mathrm{m}$ & 100 & $\mathrm{n} / \mathrm{m}$ & $\mathrm{n} / \mathrm{m}$ & $\mathrm{n} / \mathrm{m}$ & $n / m$ \\
\hline Density of Centrifuged Solids $(\mathrm{g} / \mathrm{mL})$ & 1.49 & $\overline{\mathrm{n} / \mathrm{m}}$ & $\mathrm{n} / \mathrm{m}$ & $\mathrm{n} / \mathrm{m}$ & $\mathrm{n} / \mathrm{m}$ & 1.59 & $\mathrm{n} / \mathrm{m}$ & $\overline{\mathrm{n} / \mathrm{m}}$ & $\overline{\mathrm{n} / \mathrm{m}}$ & $\frac{\mathrm{n} / \mathrm{m}}{2}$ \\
\hline 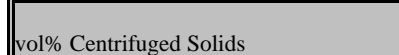 & 75.7 & $\mathrm{n} / \mathrm{m}$ & $\mathrm{n} / \mathrm{m}$ & $\mathrm{n} / \mathrm{m}$ & $\mathrm{n} / \mathrm{m}$ & 76.2 & $\frac{n}{n / m}$ & $\mathrm{n} / \mathrm{m}$ & $\mathrm{n} / \mathrm{m}$ & $\mathrm{n} / \mathrm{m}$ \\
\hline |w1\% Centrifuged Solids & 80 & $\mathrm{n} / \mathrm{m}$ & $\mathrm{n} / \mathrm{m}$ & $\mathrm{n} / \mathrm{m}$ & $\mathrm{n} / \mathrm{m}$ & 81.6 & $\mathrm{n} / \mathrm{m}$ & $\mathrm{n} / \mathrm{m}$ & $\overline{\mathrm{n} / \mathrm{m}}$ & $\frac{\mathrm{n} / \mathrm{m}}{\mathrm{m}}$ \\
\hline Supernatant Density (g/mL) & 1.15 & 1.10 & 1.05 & 1.10 & 1.05 & 1.13 & 1.12 & 1.05 & 1.12 & 1.05 \\
\hline Density of Settled Solids (g/mL) & $\frac{n / m}{n / m}$ & $\frac{n^{\prime m} \mathrm{~m}}{3}$ & $\frac{n / m}{n / m}$ & $\frac{n / m}{n / m}$ & $\frac{\mathrm{n} / \mathrm{m}}{2}$ & $\frac{\mathrm{n} / \mathrm{m}}{2}$ & $\overline{n / m}$ & $\mathrm{n} / \mathrm{m}$ & $\mathrm{n} / \mathrm{m}$ & $\frac{n / m}{n / m}$ \\
\hline |w1\% Settled Supermatant & $\mathrm{n} / \mathrm{m}$ & $\mathrm{n} / \mathrm{m}$ & $\mathrm{n} / \mathrm{m}$ & $\mathrm{n} / \mathrm{m}$ & $\mathrm{n} / \mathrm{m}$ & $\mathrm{n} / \mathrm{m}$ & $\mathrm{n} / \mathrm{m}$ & $\mathrm{n} / \mathrm{m}$ & $\mathrm{n} / \mathrm{m}$ & $\mathrm{n} / \mathrm{m}$ \\
\hline |wt\% dissolved solids in supernatant & 26.69 & 25.57 & 24.29 & 25.57 & 24.29 & 26.23 & 25.91 & 24.46 & 25.91 & 24.46 \\
\hline |w1\% total solids in Centrifuged Sludge & $\mathrm{n} / \mathrm{m}$ & $\mathrm{n} / \mathrm{m}$ & $\frac{\mathrm{n} / \mathrm{m}}{\mathrm{n}}$ & $\mathrm{n} / \mathrm{m}$ & $\frac{\mathrm{n} / \mathrm{m}}{\mathrm{n}}$ & $\mathrm{n} / \mathrm{m}$ & $\frac{\mathrm{n} / \mathrm{m}}{\mathrm{n}}$ & $\mathrm{n} / \mathrm{m}$ & $\mathrm{n} / \mathrm{m}$ & $\frac{\mathrm{n} / \mathrm{m}}{\mathrm{n}}$ \\
\hline ||w\%\% Total Solids & 40.3 & $\mathrm{n} / \mathrm{m}$ & $\mathrm{n} / \mathrm{m}$ & $\mathrm{n} / \mathrm{m}$ & $\mathrm{n} / \mathrm{m}$ & 28.4 & $\overline{n / m}$ & $\mathrm{n} / \mathrm{m}$ & $\overline{\mathrm{n} / \mathrm{m}}$ & $\overline{n / m}$ \\
\hline |ww\% UDS & 35.30 & 7.13 & 1.44 & 7.13 & 1.44 & 20.30 & 4.10 & 0.83 & 4.10 & 0.83 \\
\hline |Density of Solids (g/cc) & 2.05 & 3.24 & 3.24 & 3.24 & 3.24 & 2.05 & 3.24 & 3.24 & 3.24 & 3.24 \\
\hline
\end{tabular}




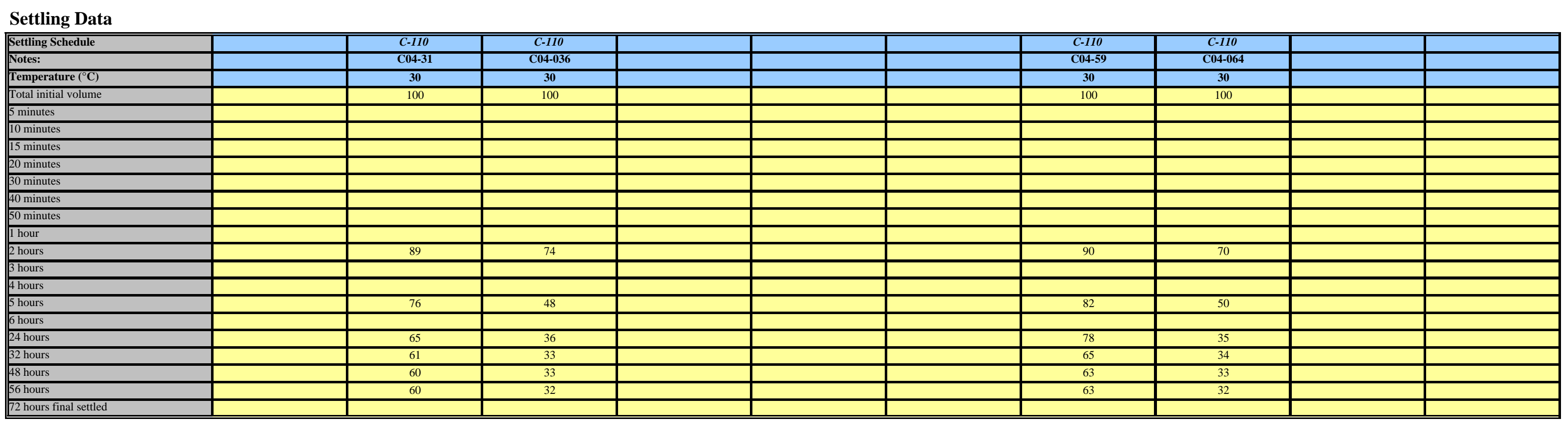

\begin{tabular}{|c|c|c|c|c|c|c|c|c|c|c|}
\hline Shear Strength Schedule & $\overline{C-110}$ & $\overline{C-110}$ & $\overline{c-110}$ & $\overline{C-110}$ & $\overline{C-110}$ & $C-110$ & $C-110$ & $\overline{C-110}$ & $\mathrm{C}-110$ & $\overline{C-110}$ \\
\hline Source of Data & $\begin{array}{l}\text { C-110 Cores } 37,38 \text { and } 39 \\
\text { data package.pdd }\end{array}$ & $\begin{array}{l}\text { C-110 Corese } 37,38 \text { and } 39 \\
\text { data packagepdf }\end{array}$ & $\begin{array}{l}\text { C-110 Coreses } 37,38 \text { and } 39 \\
\text { data package.pdf }\end{array}$ & $\begin{array}{l}\text { C-110 Cores } 37,38 \text { and } 39 \\
\text { data apackaepepdf }\end{array}$ & $\begin{array}{l}\text { C-110 Cores } 37,38 \text { and } 39 \\
\text { data package.pdd }\end{array}$ & $\begin{array}{l}\text { C-110 Cores } 37,38 \text { and } 39 \\
\text { data package.pdf }\end{array}$ & $\begin{array}{l}\text { C-110 Cores } 37,38 \text { and } 39 \\
\text { data package.pdf } 39\end{array}$ & $\begin{array}{r}\text { C-110 Cores } 37,38 \text { and } 39 \\
\text { data package.pdf } 39\end{array}$ & $\begin{array}{l}-110 \text { Cores } 37,38 \text { and } 39 \\
\text { data package.pdf } 39\end{array}$ & $\begin{array}{l}\text { C-110 Cores } 37,38 \text { and } 39 \\
\text { data packagepdf }\end{array}$ \\
\hline Notes: & Page C04-008 & & & & & Page C04-008 & & & & \\
\hline Temperature $\left({ }^{\circ} \mathrm{C}\right)$ & Unspecified & & & & & Unspecified & & & & \\
\hline Description & $\begin{array}{l}\text { Shear Vane at unspecifiec } \\
\text { gel time or temperature }\end{array}$ & & & & & $\begin{array}{l}\text { Shear Vane at unspecified } \\
\text { gel time or temperature }\end{array}$ & & & & \\
\hline \begin{tabular}{|l} 
Gelation Time/Shear Strength (Pa) \\
\end{tabular} & & & & & & & & & & \\
\hline 5 minutes & & & & & & & & & & \\
\hline 10 minutes & & & & & & & & & & \\
\hline 15 minutes & & & & & & & & & & \\
\hline $\begin{array}{l}20 \text { minutes } \\
3 \text { minutes }\end{array}$ & & & & & & & & & & \\
\hline 40 minutes & & & & & & & & & & \\
\hline 50 minutes & & & & & & & & & & \\
\hline i hour & & & & & & & & & & \\
\hline 2 hours & & & & & & & & & & \\
\hline 3 hours & & & & & & & & & & \\
\hline $4 \frac{4 \text { hours }}{5 \text { h hours }}$ & & & & & & & & & & \\
\hline 6 hours & & & & & & & & & & \\
\hline 24 hours & & & & & & & & & & \\
\hline 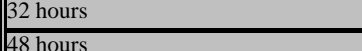 & & & & & & & & & & \\
\hline $\begin{array}{l}40 \text { oulums } \\
56 \text { hours }\end{array}$ & & & & & & & & & & \\
\hline 72 hours & 500 & & & & & 500 & & & & \\
\hline Infinte te (GRF & & & & & & & & & & \\
\hline
\end{tabular}


241-C-112

Model Chemical Data From ESP

\begin{tabular}{|c|c|c|c|c|}
\hline AnalytelSample & 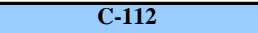 & $\overline{\mathrm{C}-112}$ & $\overline{\mathrm{C}-112}$ & $\overline{C-112}$ \\
\hline Description & Core 36 composite & $\begin{array}{c}\text { Core Composite 1:1 } \\
\text { dilution }\end{array}$ & $\begin{array}{c}\text { Core Composite 3:1 } \\
\text { dilution }\end{array}$ & $\begin{array}{c}\text { Core Composite 3:1 } \\
\text { dilution }\end{array}$ \\
\hline Model Basis & Salty & Salt Free & Salt Free & Salt Free \\
\hline Report No/Source of Data & $\begin{array}{c}\text { SST C-112 Core } 36 \text { data } \\
\text { package }\end{array}$ & $\begin{array}{c}\text { SST C-112 Core } 36 \text { data } \\
\text { package }\end{array}$ & $\begin{array}{c}\text { SST C-112 Core } 36 \text { data } \\
\text { package }\end{array}$ & $\begin{array}{c}\text { SST C-112 Core } 36 \text { data } \\
\text { package }\end{array}$ \\
\hline \multicolumn{5}{|l|}{ Solid Phases (vol\% of bulk) } \\
\hline$\left(\mathrm{NaAlO}_{2}\right)_{2} \cdot 2.5 \mathrm{H}_{2} \mathrm{O}$ & - & - & - & - \\
\hline $\mathrm{Ag}_{2} \mathrm{CO}_{3}$ & 0.00004 & 0.00001 & 0.00001 & 0.00001 \\
\hline $\mathrm{Bi}_{2} \mathrm{O}_{3}$ & 0.08264 & 0.03255 & 0.01208 & 0.01208 \\
\hline $\mathrm{BiOCl}$ & - & - & - & - \\
\hline Boehmite & - & - & - & - \\
\hline $\mathrm{Ca}(\mathrm{OH})_{2}$ & - & - & - & - \\
\hline $\mathrm{Ca}_{5} \mathrm{OH}\left(\mathrm{PO}_{4}\right)_{3}$ & 3.02056 & 1.18982 & 0.44146 & 0.44146 \\
\hline $\mathrm{CaC}_{2} \mathrm{O}_{4} \cdot \mathrm{H}_{2} \mathrm{O}$ & - & - & - & - \\
\hline $\mathrm{CaCO}_{3}$ & - & - & - & - \\
\hline $\mathrm{CaF}_{2}$ & - & - & - & - \\
\hline $\mathrm{CrOOH}$ & 0.00997 & 0.00393 & 0.00146 & 0.00146 \\
\hline $\mathrm{FeOOH}$ & 1.65469 & 0.65179 & 0.24184 & 0.24184 \\
\hline Gibbsite & 1.77648 & 0.69977 & 0.25964 & 0.25964 \\
\hline $\mathrm{HgO}$ & - & - & - & - \\
\hline $\mathrm{KAlSiO}_{4}$ & - & - & - & - \\
\hline $\mathrm{La}(\mathrm{OH})_{3}$ & - & - & - & - \\
\hline $\mathrm{LaPO}_{4} \cdot 2 \mathrm{H}_{2} \mathrm{O}$ & 0.00435 & 0.00171 & 0.00064 & 0.00064 \\
\hline $\mathrm{Mn}(\mathrm{OH})_{2}$ & - & - & - & - \\
\hline $\mathrm{Mn}_{3}\left(\mathrm{PO}_{4}\right)_{2}$ & 0.02757 & 0.01086 & 0.00403 & 0.00403 \\
\hline $\mathrm{MnCO}_{3}$ & - & - & - & - \\
\hline $\mathrm{Na}_{2} \mathrm{U}_{2} \mathrm{O}_{7}$ & 1.84757 & 0.72777 & 0.27003 & 0.27003 \\
\hline $\mathrm{NaAlCO}_{3}(\mathrm{OH})_{2}$ & 4.59954 & 1.81179 & 0.67224 & 0.67224 \\
\hline $\mathrm{NaAlSiO}_{4}$ & 0.88887 & 0.35013 & 0.12991 & 0.12991 \\
\hline $\mathrm{Ni}(\mathrm{OH})_{2}$ & 0.96973 & 0.38198 & 0.14173 & 0.14173 \\
\hline $\mathrm{Ni}_{3}\left(\mathrm{PO}_{4}\right)_{2}$ & - & - & - & - \\
\hline $\mathrm{NiC}_{2} \mathrm{O}_{4} \cdot 2 \mathrm{H}_{2} \mathrm{O}$ & - & - & - & - \\
\hline $\mathrm{Pb}(\mathrm{OH})_{2}$ & 0.07341 & 0.02892 & 0.01073 & 0.01073 \\
\hline $\mathrm{Pb}_{3}\left(\mathrm{PO}_{4}\right)_{2}$ & - & - & - & - \\
\hline $\mathrm{PbCO}_{3}$ & - & - & - & - \\
\hline $\mathrm{Pu}(\mathrm{OH})_{4}$ & 0.00009 & 0.00004 & 0.00001 & 0.00001 \\
\hline $\mathrm{SiO}_{2}$ & - & - & - & - \\
\hline $\mathrm{Sr}_{3}\left(\mathrm{PO}_{4}\right)_{2}$ & 0.03066 & 0.01208 & 0.00448 & 0.00448 \\
\hline $\mathrm{SrCO}_{3}$ & - & - & - & - \\
\hline $\mathrm{TcO}_{2}$ & - & - & - & - \\
\hline $\mathrm{ZrO}_{2}$ & 0.00074 & 0.00029 & 0.00011 & 0.00011 \\
\hline $\mathrm{KNO}_{3}$ & - & - & - & - \\
\hline $\mathrm{Na}_{2} \mathrm{C}_{2} \mathrm{O}_{4}$ & 0.84145 & - & - & - \\
\hline $\mathrm{Na}_{2} \mathrm{CO}_{3} \cdot \mathrm{H}_{2} \mathrm{O}$ & - & - & - & - \\
\hline $\mathrm{Na}_{2} \mathrm{SO}_{4}$ & - & - & - & - \\
\hline $\mathrm{Na}_{2} \mathrm{SO}_{4} \cdot 10 \mathrm{H}_{2} \mathrm{O}$ & - & - & - & - \\
\hline $\mathrm{Na}_{3} \mathrm{FSO}_{4}$ & - & - & - & - \\
\hline $\mathrm{Na}_{3} \mathrm{NO}_{3} \mathrm{SO}_{4} \cdot \mathrm{H}_{2} \mathrm{O}$ & - & - & - & - \\
\hline $\mathrm{Na}_{3} \mathrm{PO}_{4} \cdot 8 \mathrm{H}_{2} \mathrm{O}$ & - & - & - & - \\
\hline $\mathrm{Na}_{4} \mathrm{P}_{2} \mathrm{O}_{7} \cdot 10 \mathrm{H}_{2} \mathrm{O}$ & 7.23122 & - & - & - \\
\hline $\mathrm{Na}_{6}\left(\mathrm{SO}_{4}\right)_{2} \mathrm{CO}_{3}$ & - & - & - & - \\
\hline $\mathrm{NaF}$ & - & - & - & - \\
\hline $\mathrm{NaF}\left(\mathrm{PO}_{4}\right)_{2} \cdot 19 \mathrm{H}_{2} \mathrm{O}$ & - & - & - & - \\
\hline $\mathrm{NaHCO}_{3}$ & - & - & - & - \\
\hline $\mathrm{NaNO}_{2}$ & - & - & - & - \\
\hline $\mathrm{NaNO}_{3}$ & - & - & - & - \\
\hline $\mathrm{Na}_{3} \mathrm{PO}_{4} \cdot 0.25 \mathrm{NaOH} \cdot 12 \mathrm{H}_{2} \mathrm{O}$ & - & - & - & - \\
\hline Total Volume \% UDS & 23.06 & 5.90 & 2.19 & 2.19 \\
\hline Solids Concentration (g/L) & 653.9 & 197.7 & 73.4 & 73.4 \\
\hline
\end{tabular}

Note: Numbers in italics are calculated values. Numbers in normal font are reported data. 


\section{Rheological Data}

\begin{tabular}{|c|c|c|}
\hline Model/model Parameter & $\overline{C-112}$ & $\overline{C-112}$ \\
\hline Temperature $\left({ }^{\circ} \mathrm{C}\right)$ & 25 & 95 \\
\hline Source of Data & $\begin{array}{l}\text { SST C-112 Core } 36 \text { data } \\
\text { package }\end{array}$ & $\begin{array}{l}\text { SST C-112 Core } 36 \text { data } \\
\text { package }\end{array}$ \\
\hline Notes & Page C04-029 & Page C04-031 \\
\hline \multicolumn{3}{|l|}{ Newtonian: } \\
\hline$\eta$ - Newtonian viscosity (cP) & 5.880 & 2.872 \\
\hline $\mathrm{r}$ - correlation coefficient & 0.954 & 0.978 \\
\hline \multicolumn{3}{|l|}{ Ostwald (or Power Law): } \\
\hline $\mathrm{m}$ - the consistency coefficient $\left(\mathrm{Pa} \cdot \mathrm{s}^{-\mathrm{n}}\right)$ & 0.158 & 0.061 \\
\hline $\mathrm{n}$ - the power-law exponent & 0.440 & 0.480 \\
\hline $\mathrm{r}$ - correlation coefficient & 0.979 & 0.949 \\
\hline \multicolumn{3}{|l|}{ Bingham Plastic: } \\
\hline$\tau_{\mathrm{B}}$ - the Bingham yield stress $(\mathrm{Pa})$ & 0.697 & 0.368 \\
\hline$\eta_{p}$ - the plastic viscosity (Pas) & 0.004 & 0.002 \\
\hline $\mathrm{r}$ - correlation coefficient & 0.954 & 0.978 \\
\hline \multicolumn{3}{|l|}{ Herschel-Bulkley: } \\
\hline$\tau_{\mathrm{H}^{-}}$the yield stress $(\mathrm{Pa})$ & 0.497 & 0.271 \\
\hline $\begin{array}{l}\text { k - the Herschel-Bulkely consistency } \\
\text { coefficient }\left(\mathrm{Pa} \cdot \mathrm{s}^{\text {-b }}\right)\end{array}$ & 0.044 & 0.016 \\
\hline $\begin{array}{l}\text { b - the Hershel-Bulkely power-law } \\
\text { exponent }\end{array}$ & 0.612 & 0.650 \\
\hline $\mathrm{r}$ - correlation coefficient & 0.997 & 0.982 \\
\hline
\end{tabular}

\section{Physical Property Data}

\begin{tabular}{|c|c|c|c|c|}
\hline $\mid \overline{\text { Physical Property }}$ & $\overline{C-112}$ & $\overline{C-112}$ & $\overline{C-112}$ & $\overline{C-112}$ \\
\hline \multicolumn{5}{|l|}{ Notes } \\
\hline Temperature $\left({ }^{\circ} \mathrm{C}\right)$ & 32 & 32 & 32 & 32 \\
\hline \multicolumn{5}{|l|}{ Zeta Potential (mV) } \\
\hline Bulk Density (g/mL) & 1.70 & 1.24 & 1.11 & 1.11 \\
\hline ||vol\% Settled Solids & 100 & 74.4 & 42.4 & 42.4 \\
\hline Density of Centrifuged Solids (g/mL) & 1.95 & $\mathrm{n} / \mathrm{m}$ & 1.39 & 1.39 \\
\hline vol\% Centrifuged Solids & 83 & $\mathrm{n} / \mathrm{m}$ & 21.4 & 21.4 \\
\hline wt\% Centrifuged Solids & $\mathrm{n} / \mathrm{m}$ & $\mathrm{n} / \mathrm{m}$ & 27 & 27 \\
\hline Supernatant Density (g/mL) & 1.23 & 1.09 & 1.01 & 1.01 \\
\hline Density of Settled Solids (g/mL) & & $\mathrm{n} / \mathrm{m}$ & $\mathrm{n} / \mathrm{m}$ & $\mathrm{n} / \mathrm{m}$ \\
\hline |wt\% Settled Supernatant & $\mathrm{n} / \mathrm{m}$ & $\mathrm{n} / \mathrm{m}$ & $\mathrm{n} / \mathrm{m}$ & $\mathrm{n} / \mathrm{m}$ \\
\hline wt $\%$ dissolved solids in supernatant & 26.87 & 23.75 & 22.06 & 22.06 \\
\hline wt\% total solids in Centrifuged Sludge & $\mathrm{n} / \mathrm{m}$ & $\mathrm{n} / \mathrm{m}$ & $\mathrm{n} / \mathrm{m}$ & $\mathrm{n} / \mathrm{m}$ \\
\hline wt\% Total Solids & 55 & $\mathrm{n} / \mathrm{m}$ & $\mathrm{n} / \mathrm{m}$ & $\mathrm{n} / \mathrm{m}$ \\
\hline wt\% UDS & 38.47 & 15.94 & 6.61 & 6.61 \\
\hline |Density of Solids (g/cc) & 2.84 & 3.35 & 3.35 & 3.35 \\
\hline
\end{tabular}

Note: Numbers in italics are calculated values. Numbers in normal font are reported data. 
Settling Data

\begin{tabular}{|c|c|c|c|}
\hline Settling Schedule & $\overline{C-112}$ & $\overline{C C-112}$ & \\
\hline Notes: & C04-001-7 & $\begin{array}{l}\text { C04-001-7 } \\
\end{array}$ & \\
\hline Temperature $\left({ }^{\circ} \mathrm{C}\right)$ & 25 & 25 & \\
\hline Total initial volume & 100 & 100 & \\
\hline \multicolumn{4}{|l|}{5 minutes } \\
\hline \multicolumn{4}{|l|}{10 minutes } \\
\hline \multicolumn{4}{|l|}{15 minutes } \\
\hline \multicolumn{4}{|l|}{20 minutes } \\
\hline \multicolumn{4}{|l|}{30 minutes } \\
\hline \multicolumn{4}{|l|}{40 minutes } \\
\hline \multicolumn{4}{|l|}{50 minutes } \\
\hline \multicolumn{4}{|l|}{1 hour } \\
\hline 2 hours & 96 & 87 & \\
\hline \multicolumn{4}{|l|}{3 hours } \\
\hline \multicolumn{4}{|l|}{4 hours } \\
\hline 5 hours & 94 & 62 & \\
\hline \multicolumn{4}{|l|}{6 hours } \\
\hline 24 hours & 88 & 50 & \\
\hline 32 hours & 76 & 47 & \\
\hline 48 hours & 75 & 42 & \\
\hline 56 hours & 74 & 42 & \\
\hline 72 hours final settled & & & \\
\hline
\end{tabular}

\section{Shear Strength Data}

\begin{tabular}{|c|c|c|c|c|}
\hline Shear Strength Schedule & $\overline{C C-112}$ & & & \\
\hline Source of Data & $\begin{array}{c}\text { SST C-112 Core } 36 \text { data } \\
\text { package }\end{array}$ & & & \\
\hline Notes: & Page 1-14 & & & \\
\hline Temperature $\left({ }^{\circ} \mathrm{C}\right)$ & Unspecified & & & \\
\hline Description & $\begin{array}{l}\text { Page 1-13, 1-14: } 10 \text { weeks } \\
\text { undisturbed (1680 hrs), } 1 \\
\text { hour in water bath at } \\
\text { unspecified temperature }\end{array}$ & & & \\
\hline \multicolumn{5}{|c|}{ Gelation Time/Shear Strength (Pa) } \\
\hline \multicolumn{5}{|l|}{5 minutes } \\
\hline \multicolumn{5}{|l|}{10 minutes } \\
\hline \multicolumn{5}{|l|}{15 minutes } \\
\hline \multicolumn{5}{|l|}{20 minutes } \\
\hline \multicolumn{5}{|l|}{30 minutes } \\
\hline \multicolumn{5}{|l|}{40 minutes } \\
\hline \multicolumn{5}{|l|}{50 minutes } \\
\hline \multicolumn{5}{|l|}{1 hour } \\
\hline \multicolumn{5}{|l|}{2 hours } \\
\hline \multicolumn{5}{|l|}{3 hours } \\
\hline \multicolumn{5}{|l|}{4 hours } \\
\hline \multicolumn{5}{|l|}{5 hours } \\
\hline \multicolumn{5}{|l|}{6 hours } \\
\hline \multicolumn{5}{|l|}{24 hours } \\
\hline \multicolumn{5}{|l|}{32 hours } \\
\hline \multicolumn{5}{|l|}{48 hours } \\
\hline \multicolumn{5}{|l|}{56 hours } \\
\hline 72 hours & 1600 & & & \\
\hline Infinte time (GRE) & & & & \\
\hline
\end{tabular}




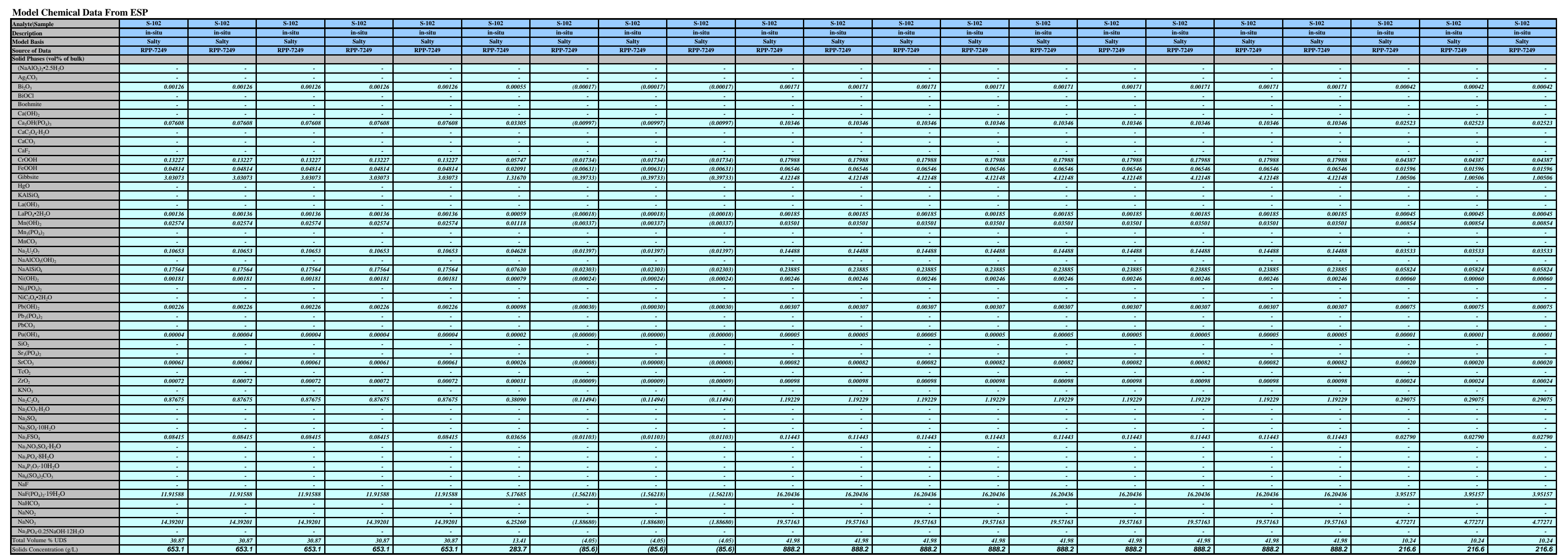

\begin{tabular}{|c|c|c|c|c|c|c|c|c|c|c|c|c|c|c|c|c|c|c|c|c|c|}
\hline 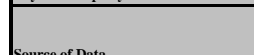 & & & & 3.02 & 3.06 & & & & 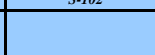 & 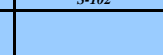 & 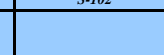 & 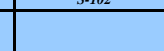 & 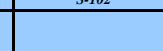 & 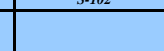 & 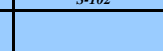 & 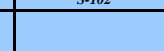 & 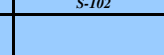 & 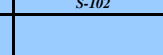 & 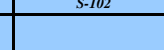 & 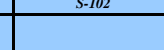 & \\
\hline 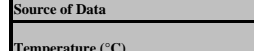 & & & & & & & & & & & & & & & & & & & & & \\
\hline 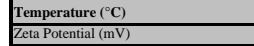 & & & & & & & & & & & & & & & & & & & & & \\
\hline 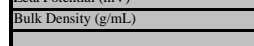 & 1.68 & 1.68 & 1.68 & 1.68 & 1.68 & ${ }_{157}^{157}$ & ${ }_{1.46}^{14}$ & 1.46 & 1.46 & $\frac{1.75}{1.5}$ & 1.55 & 1.75 & 1155 & $\frac{1.55}{1.5}$ & 1,75 & 1.75 & 1.75 & 1.75 & 1.55 & 1.55 & 1.55 \\
\hline 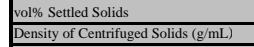 & -1 & 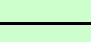 & - & - & - & - & - & - & - & - & 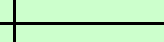 & - & - & 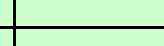 & - & 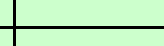 & - & & & & \\
\hline 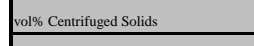 & & & & & & & & & & & & & & & & & & & & & \\
\hline 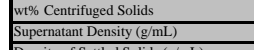 & 1.49 & 1.49 & $\begin{array}{ll}1,99 \\
\end{array}$ & $\begin{array}{l}1.1 .9 \\
\end{array}$ & ${ }_{1.96}$ & ${ }_{1.40}$ & ${ }_{1.49}$ & 1,49 & 1,99 & 1.49 & $\begin{array}{ll}1.49 \\
\end{array}$ & 1.49 & $\frac{1.49}{1.49}$ & 1.49 & 1.49 & 1.49 & $\overline{1.49}$ & 1,49 & $\overline{1.49}$ & 1,49 & 1.49 \\
\hline 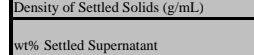 & & & & & & & & & & + & & + & & & - & 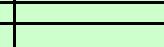 & 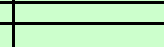 & - & 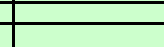 & - & 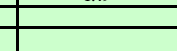 \\
\hline 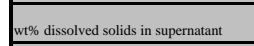 & & & & & & & & & & & & & & & & & & & & & \\
\hline 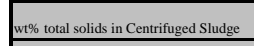 & & & & & & & & & & & & & & & & & & & & & \\
\hline Mas Toul Solits & 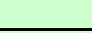 & 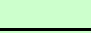 & $\square$ & 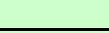 & 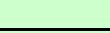 & 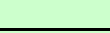 & 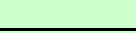 & 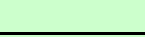 & 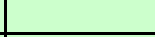 & 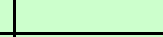 & 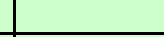 & 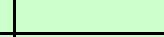 & 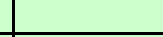 & & 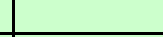 & 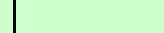 & 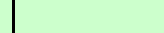 & 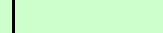 & 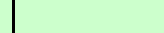 & 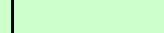 & \\
\hline 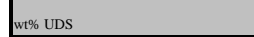 & 38.8B & satai & S3.8B & 38Bab & 3ase & 1807 & .566 & .356 & . 5866 & ${ }_{50075}^{0.75}$ & ${ }^{50,75}$ & 50.75 & 50.75 & 50,25 & 50.75 & $50,0.5$ & 50075 & 50,75 & 1397 & 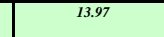 & 13.97 \\
\hline Irsolists & 2.12 & 212 & 2.12 & 2.12 & 211 & 2.1. & 2.1 & 2.12 & 2,12 & 2.12 & 2.12 & 2,12 & & & & & & & & & \\
\hline
\end{tabular}




\begin{tabular}{|c|c|c|c|c|c|c|c|c|c|c|c|c|c|c|c|c|c|c|c|c|c|}
\hline 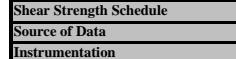 & 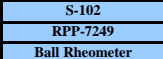 & 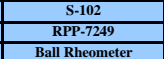 & 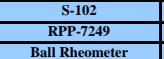 & 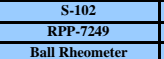 & 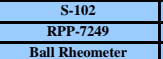 & 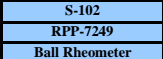 & 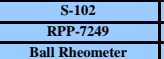 & 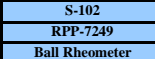 & 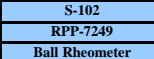 & 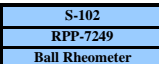 & 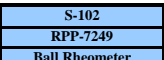 & 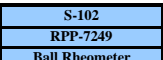 & 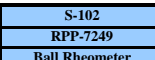 & 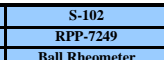 & 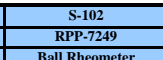 & 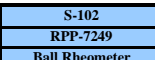 & 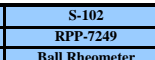 & 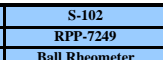 & 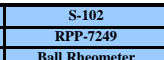 & 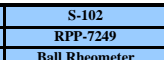 & 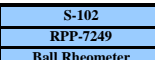 \\
\hline & 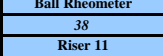 & 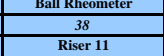 & 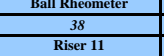 & 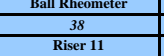 & 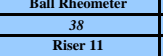 & 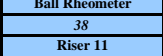 & 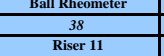 & 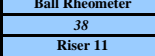 & 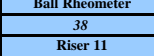 & 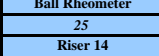 & 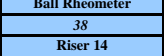 & 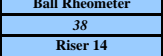 & 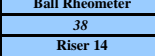 & 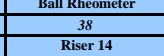 & 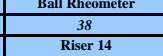 & 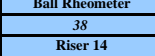 & 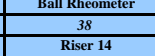 & 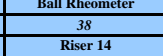 & 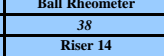 & 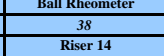 & 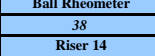 \\
\hline & 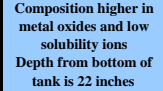 & 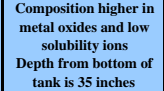 & 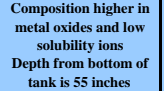 & 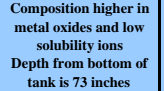 & 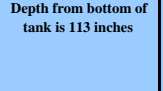 & 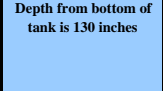 & 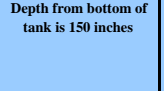 & 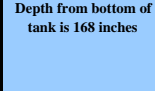 & 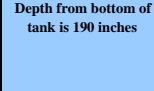 & 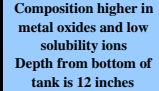 & 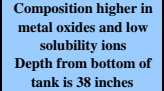 & 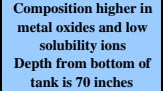 & 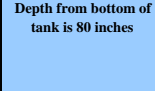 & 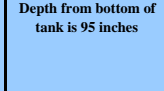 & 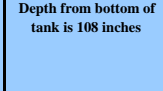 & 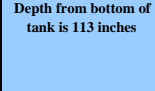 & 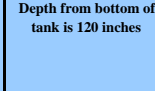 & 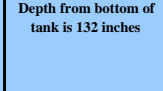 & 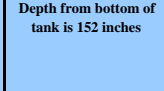 & 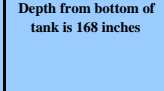 & 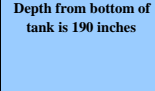 \\
\hline 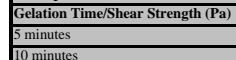 & & & & & & & & & & & & & & & & & & & & & \\
\hline nutus & & & & & & & & & & & & & & & & & & & & & \\
\hline & & & & & & & & & & & & & & & & & & & & & \\
\hline & & & & & & & & & & & & & & & & & & & & & \\
\hline & & & & & & & & & & & & & & & & & & & & & \\
\hline & & & & & & & & & & & & & & & & & & & & & \\
\hline & & & & & & & & & & & & & & & & & & & & & \\
\hline & $F$ & 1000 & 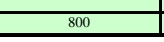 & 00 & & & & & & & & & & & & & & & & & \\
\hline
\end{tabular}




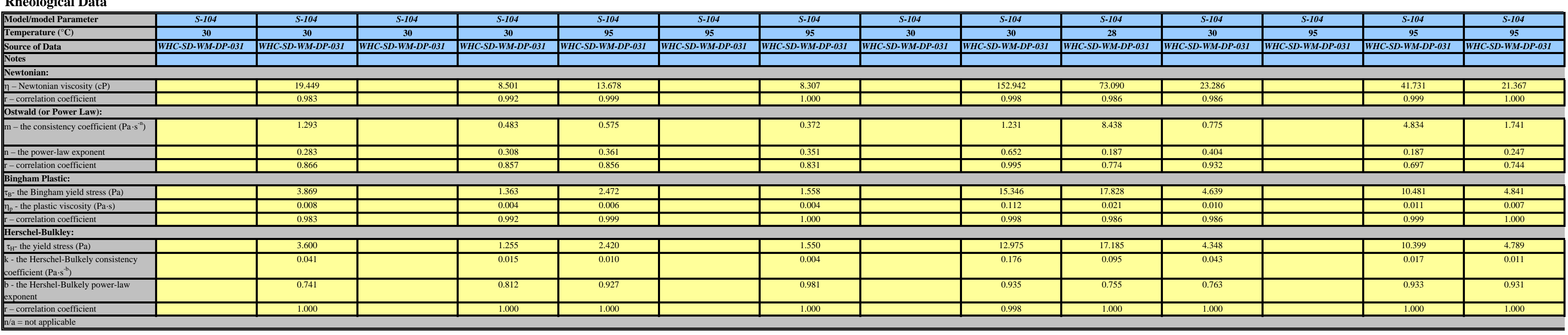

\begin{tabular}{|c|c|c|c|c|c|c|c|c|c|c|c|c|c|c|}
\hline \multicolumn{15}{|l|}{ Physical Property Data } \\
\hline $\begin{array}{l}\text { Physical Property } \\
\end{array}$ & $\begin{array}{c}\text { S-104 } \\
\end{array}$ & 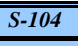 & 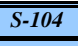 & 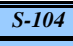 & 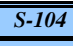 & 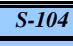 & \begin{tabular}{c|c|c|}
$s-104$ \\
\end{tabular} & 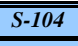 & 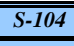 & 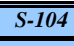 & 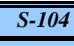 & \begin{tabular}{c|c|c|}
$s-104$ \\
\end{tabular} & 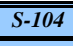 & 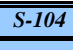 \\
\hline \multicolumn{15}{|l|}{ Notes } \\
\hline Temperature $\left({ }^{\circ} \mathrm{C}\right)$ & 30 & 30 & 30 & 30 & 30 & 30 & 30 & 30 & 30 & 30 & 30 & 30 & 30 & 30 \\
\hline $\begin{array}{l}\frac{\text { Zeta Potennial }(\mathrm{mV} V}{\text { Bulk Density }(\mathrm{gmL})} \\
\end{array}$ & 4.64 & 1.29 & 1.27 & 1.11 & 1.29 & 1.27 & 1.11 & 1.64 & 1.28 & 1.26 & 1.11 & 1.28 & 1.26 & 1.11 \\
\hline vol\% Settled Solids & 100 & $\mathrm{n} / \mathrm{m}$ & $\frac{n / m}{n / m}$ & $\mathrm{n} / \mathrm{m}$ & $\mathrm{n}^{\mathrm{n} m}$ & $\mathrm{n}^{\mathrm{m} m}$ & $\mathrm{n}^{\mathrm{n} / \mathrm{m}}$ & 100 & $\mathrm{n} / \mathrm{m}$ & $\mathrm{n} / \mathrm{m}$ & $\frac{\mathrm{n} / \mathrm{m}}{\mathrm{n} / \mathrm{s}}$ & $\mathrm{n} / \mathrm{m}$ & $\frac{n}{n / m}$ & $\frac{\mathrm{n} / \mathrm{m}}{\mathrm{n}}$ \\
\hline Density of Centrifuged Solids (g/mL) & 1.71 & $\mathrm{n} / \mathrm{m}$ & $\mathrm{n} / \mathrm{m}$ & $\mathrm{n} / \mathrm{m}$ & $\mathrm{n} / \mathrm{m}$ & $\mathrm{n} / \mathrm{m}$ & $\mathrm{n} / \mathrm{m}$ & 1.71 & $\mathrm{n} / \mathrm{m}$ & $\mathrm{n} / \mathrm{m}$ & $\mathrm{n} / \mathrm{m}$ & $\mathrm{n} / \mathrm{m}$ & $\mathrm{n} / \mathrm{m}$ & $\mathrm{n} / \mathrm{m}$ \\
\hline vol\% Centrifuged Solids & 82.5 & $\mathrm{n} / \mathrm{m}$ & $\mathrm{n} / \mathrm{m}$ & $\mathrm{n} / \mathrm{m}$ & $\mathrm{n} / \mathrm{m}$ & $\mathrm{n} / \mathrm{m}$ & $\mathrm{n}^{\mathrm{n} / \mathrm{m}}$ & 100 & $\mathrm{n} / \mathrm{m}$ & $\mathrm{n}^{\mathrm{n} / \mathrm{m}}$ & $n^{n / m}$ & $\mathrm{n} / \mathrm{m}$ & $\frac{\mathrm{n} / \mathrm{m}}{\mathrm{m}}$ & $\mathrm{n} / \mathrm{m}$ \\
\hline w1\% Centrifuged Solids & 86.1 & $\frac{\mathrm{n} / \mathrm{m}}{\mathrm{m}}$ & $\mathrm{n}^{\mathrm{n} / \mathrm{m}}$ & $\mathrm{n}^{\mathrm{n} / \mathrm{m}}$ & ${ }^{\mathrm{n} / \mathrm{m}}$ & ${ }^{n / m}$ & $\mathrm{n}^{\mathrm{n} / \mathrm{m}}$ & ${ }^{100}$ & $\mathrm{n}^{\mathrm{n} m}$ & $\mathrm{n}^{\mathrm{m} m}$ & $n / m$ & $\frac{\mathrm{n} / \mathrm{m}}{\mathrm{m}}$ & $\mathrm{n}^{\mathrm{n} / \mathrm{m}}$ & $\mathrm{n}^{\mathrm{n} m}$ \\
\hline Supermatant Density $(g / m L)$ & 1.28 & 1.12 & 1.12 & 1.05 & 1.12 & 1.12 & 1.05 & 1.28 & 1.14 & 1.14 & 1.06 & 1.14 & 1.14 & 1.06 \\
\hline Density of Settled Solids $(g / m L)$ & $\mathrm{n} / \mathrm{m}$ & $\mathrm{n} / \mathrm{m}$ & $\mathrm{n} / \mathrm{m}$ & $\mathrm{n} / \mathrm{m}$ & $\mathrm{n} / \mathrm{m}$ & $\mathrm{n} / \mathrm{m}$ & $\mathrm{n} / \mathrm{m}$ & & $\mathrm{n} / \mathrm{m}$ & $\mathrm{n} / \mathrm{m}$ & $\mathrm{n} / \mathrm{m}$ & $\mathrm{n} / \mathrm{m}$ & $\mathrm{n} / \mathrm{m}$ & $\mathrm{n} / \mathrm{m}$ \\
\hline w\%\% Settled Supernatant & $\mathrm{n} / \mathrm{m}$ & $\mathrm{n} / \mathrm{m}$ & $\mathrm{n} / \mathrm{m}$ & $\mathrm{n} / \mathrm{m}$ & $\mathrm{n} / \mathrm{m}$ & $\mathrm{n} / \mathrm{m}$ & $\mathrm{n} / \mathrm{m}$ & $\mathrm{n} / \mathrm{m}$ & $\mathrm{n} / \mathrm{m}$ & $\mathrm{n} / \mathrm{m}$ & $\mathrm{n} / \mathrm{m}$ & $\mathrm{n} / \mathrm{m}$ & $\mathrm{n} / \mathrm{m}$ & $\mathrm{n} / \mathrm{m}$ \\
\hline w\%\% dissolved solids in supernatant & 40.59 & 35.66 & 35.66 & 33.35 & 35.66 & 35.66 & 33.35 & 40.59 & 36.30 & 36.30 & 33.65 & 36.30 & 36.30 & 33.65 \\
\hline \begin{tabular}{|l} 
w\%\% total solid in Cenrifituged Sludge \\
\end{tabular} & ${ }^{n / m}$ & $\mathrm{n}^{\mathrm{n} / \mathrm{m}}$ & $\mathrm{n}^{\mathrm{n} / \mathrm{m}}$ & $\mathrm{n}^{\mathrm{n} m}$ & $\mathrm{n}^{\mathrm{n} / \mathrm{m}}$ & $n^{n / m}$ & $\mathrm{n}^{\mathrm{n} / \mathrm{m}}$ & $\mathrm{n}^{\mathrm{n} / \mathrm{m}}$ & $\mathrm{n}^{\mathrm{n} / \mathrm{m}}$ & $\mathrm{n} / \mathrm{m}$ & $\mathrm{n}^{\mathrm{m} m}$ & $\mathrm{n}^{\mathrm{n} / \mathrm{m}}$ & $\mathrm{n} / \mathrm{m}$ & $\mathrm{n}^{\mathrm{m} m}$ \\
\hline w\%\% Total Solids & 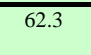 & $\mathrm{n}^{\mathrm{m} / \mathrm{m}}$ & $\mathrm{n}^{\mathrm{n} / \mathrm{m}}$ & $\mathrm{n}^{\mathrm{m} / \mathrm{m}}$ & $\mathrm{n} / \mathrm{m}$ & $\mathrm{n}^{\mathrm{m} m}$ & $\mathrm{n} / \mathrm{m}$ & 67.7 & $\frac{\mathrm{n} / \mathrm{m}}{\mathrm{m}}$ & $\frac{\mathrm{n} / \mathrm{m}}{\mathrm{n}}$ & $\mathrm{n}^{\mathrm{n} / \mathrm{m}}$ & $\mathrm{n}^{\mathrm{n} / \mathrm{m}}$ & $\frac{\mathrm{n} / \mathrm{m}}{\mathrm{m}}$ & $\frac{\mathrm{n} / \mathrm{m}}{\mathrm{m}}$ \\
\hline w\% UDS & 55.40 & 20.49 & 17.82 & 7.58 & 20.49 & 17.82 & 7.58 & 45.63 & 16.88 & 14.68 & 6.24 & 16.88 & 14.68 & 6.24 \\
\hline Density of Solids $(g / c)$ & 2.71 & 3.04 & 3.04 & 3.04 & 3.04 & 3.04 & 3.04 & 2.71 & 3.04 & 3.04 & 3.04 & 3.04 & 3.04 & 3.04 \\
\hline
\end{tabular}



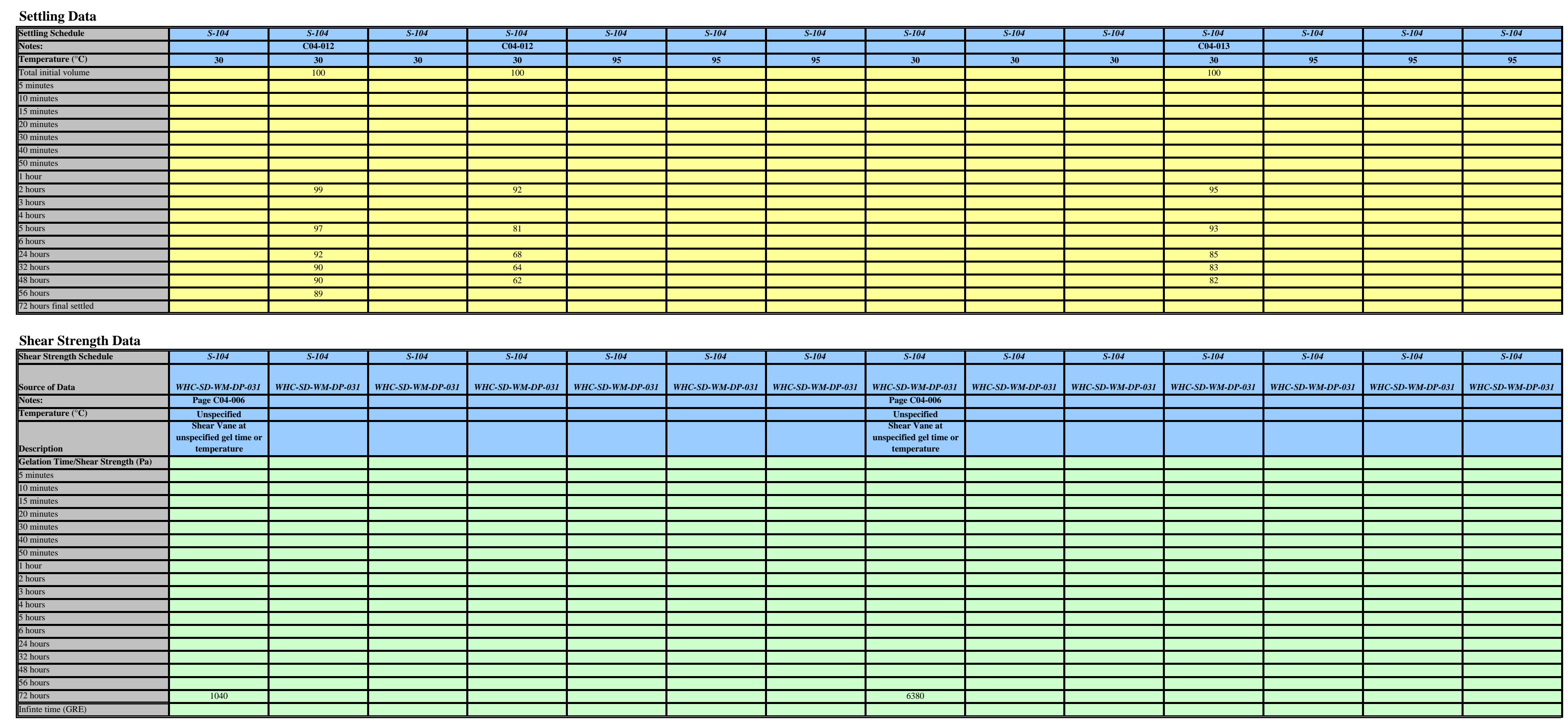
241-S-112

\begin{tabular}{|c|c|c|c|c|c|c|}
\hline AnalytelSample & S-112 & S-112 & S-112 & S-112 & S-112 & S-112 \\
\hline Description & $\begin{array}{c}\text { V1L/V2L; decanted } \\
\text { Saltcake supernate after } \\
\text { 1st contact with water at } \\
50 \mathrm{C}\end{array}$ & \begin{tabular}{|c|} 
V1L/V2L; decanted \\
Saltcake supernate after \\
1st contact with water at \\
$50 \mathrm{C}$
\end{tabular} & \begin{tabular}{|c|} 
V1L/V2L; decanted \\
Saltcake supernate after \\
1st contact with water at \\
$50 \mathrm{C}$
\end{tabular} & $\begin{array}{c}\text { V1SV2S; decanted } \\
\text { Saltcake supernate after } \\
\text { 2nd contact with water at } \\
\text { 50C }\end{array}$ & $\begin{array}{c}\text { V1SV2S; decanted } \\
\text { Saltcake supernate after } \\
\text { 2nd contact with water at } \\
50 \mathrm{C}\end{array}$ & \begin{tabular}{|c|} 
V1SV2S; decanted \\
Saltcake supernate after \\
2nd contact with water at \\
$50 \mathrm{C}$
\end{tabular} \\
\hline Model Basis & Salt Free & Salt Free & Salt Free & Salt Free & Salt Free & Salt Free \\
\hline Report No/Source of Data & RPP-10984 Rev. 0 & RPP-10984 Rev. 0 & RPP-10984 Rev. 0 & RPP-10984 Rev. 0 & RPP-10984 Rev. 0 & RPP-10984 Rev. 0 \\
\hline
\end{tabular}

\section{Rheological Data}

\begin{tabular}{|c|c|c|c|c|c|c|}
\hline Model/model Parameter & S-112 & S-112 & S-112 & S-112 & $S-112$ & S-112 \\
\hline Temperature $\left({ }^{\circ} \mathrm{C}\right)$ & 20 & 35 & 50 & 20 & 35 & 50 \\
\hline Source of Data & RPP-10984 Rev. 0 & RPP-10984 Rev. 0 & RPP-10984 Rev. 0 & RPP-10984 Rev. 0 & RPP-10984 Rev. 0 & RPP-10984 Rev. 0 \\
\hline Notes & Table 3-17 & Table 3-17 & Table 3-17 & Table 3-17 & Table 3-17 & Table 3-17 \\
\hline \multicolumn{7}{|l|}{ Newtonian: } \\
\hline$\eta$ - Newtonian viscosity (cP) & 5.700 & 4.000 & 2.900 & 2.000 & 1.800 & 1.400 \\
\hline \multicolumn{7}{|l|}{$\mathrm{r}$ - correlation coefficient } \\
\hline \multicolumn{7}{|l|}{ Ostwald (or Power Law): } \\
\hline \multicolumn{7}{|l|}{$\mathrm{m}$ - the consistency coefficient $\left(\mathrm{Pa} \cdot \mathrm{s}^{-\mathrm{n}}\right)$} \\
\hline \multicolumn{7}{|l|}{$\mathrm{n}$ - the power-law exponent } \\
\hline \multicolumn{7}{|l|}{$\mathrm{r}$ - correlation coefficient } \\
\hline \multicolumn{7}{|l|}{ Bingham Plastic: } \\
\hline$\tau_{\mathrm{B}^{-}}$the Bingham yield stress $(\mathrm{Pa})$ & 0.000 & 0.000 & 0.000 & 0.000 & 0.000 & 0.000 \\
\hline$\eta_{p}$ - the plastic viscosity (Pa-s) & 0.006 & 0.004 & 0.003 & 0.002 & 0.002 & 0.001 \\
\hline \multicolumn{7}{|l|}{$\mathrm{r}$ - correlation coefficient } \\
\hline \multicolumn{7}{|l|}{ Herschel-Bulkley: } \\
\hline \multicolumn{7}{|l|}{$\tau_{\mathrm{H}^{-}}$the yield stress $(\mathrm{Pa})$} \\
\hline \multicolumn{7}{|l|}{$\begin{array}{l}\mathrm{k} \text { - the Herschel-Bulkely consistency } \\
\text { coefficient }\left(\mathrm{Pa} \cdot \mathrm{s}^{-\mathrm{b}}\right)\end{array}$} \\
\hline \multicolumn{7}{|l|}{$\begin{array}{l}\mathrm{b} \text { - the Hershel-Bulkely power-law } \\
\text { exponent }\end{array}$} \\
\hline \multicolumn{7}{|l|}{$\mathrm{r}$ - correlation coefficient } \\
\hline $\mathrm{n} / \mathrm{a}=$ not applicable & & & & & & \\
\hline
\end{tabular}

\section{Physical Property Data}

\begin{tabular}{|c|c|c|c|c|c|c|}
\hline Physical Property & S-112 & S-112 & S-112 & S-112 & S-112 & S-112 \\
\hline \multicolumn{7}{|l|}{ Notes } \\
\hline Temperature $\left({ }^{\circ} \mathrm{C}\right)$ & 50 & 50 & 50 & 50 & 50 & 50 \\
\hline Zeta Potential (mV) & $\mathrm{n} / \mathrm{m}$ & $\mathrm{n} / \mathrm{m}$ & $\mathrm{n} / \mathrm{m}$ & $\mathrm{n} / \mathrm{m}$ & $\mathrm{n} / \mathrm{m}$ & $\mathrm{n} / \mathrm{m}$ \\
\hline Bulk Density (g/mL) & 1.36 & 1.36 & 1.36 & 1.15 & 1.15 & 1.15 \\
\hline vol\% Settled Solids & $\mathrm{n} / \mathrm{m}$ & $\mathrm{n} / \mathrm{m}$ & $\mathrm{n} / \mathrm{m}$ & $\mathrm{n} / \mathrm{m}$ & $\mathrm{n} / \mathrm{m}$ & $\mathrm{n} / \mathrm{m}$ \\
\hline Density of Centrifuged Solids ( $\mathrm{g} / \mathrm{mL}$ ) & $\mathrm{n} / \mathrm{m}$ & $\mathrm{n} / \mathrm{m}$ & $\mathrm{n} / \mathrm{m}$ & $\mathrm{n} / \mathrm{m}$ & $\mathrm{n} / \mathrm{m}$ & $\mathrm{n} / \mathrm{m}$ \\
\hline vol\% Centrifuged Solids & $\mathrm{n} / \mathrm{m}$ & $\mathrm{n} / \mathrm{m}$ & $\mathrm{n} / \mathrm{m}$ & $\mathrm{n} / \mathrm{m}$ & $\mathrm{n} / \mathrm{m}$ & $\mathrm{n} / \mathrm{m}$ \\
\hline wt\% Centrifuged Solids & $\mathrm{n} / \mathrm{m}$ & $\mathrm{n} / \mathrm{m}$ & $\mathrm{n} / \mathrm{m}$ & $\mathrm{n} / \mathrm{m}$ & $\mathrm{n} / \mathrm{m}$ & $\mathrm{n} / \mathrm{m}$ \\
\hline Supernatant Density $(\mathrm{g} / \mathrm{mL})$ & 1.36 & 1.36 & 1.36 & 1.16 & 1.16 & 1.16 \\
\hline Density of Settled Solids $(\mathrm{g} / \mathrm{mL})$ & $\mathrm{n} / \mathrm{m}$ & $\mathrm{n} / \mathrm{m}$ & $\mathrm{n} / \mathrm{m}$ & $\mathrm{n} / \mathrm{m}$ & $\mathrm{n} / \mathrm{m}$ & $\mathrm{n} / \mathrm{m}$ \\
\hline wt\% Settled Supernatant & $\mathrm{n} / \mathrm{m}$ & $\mathrm{n} / \mathrm{m}$ & $\mathrm{n} / \mathrm{m}$ & $\mathrm{n} / \mathrm{m}$ & $\mathrm{n} / \mathrm{m}$ & $\mathrm{n} / \mathrm{m}$ \\
\hline wt $\%$ dissolved solids in supernatant & $\mathrm{n} / \mathrm{m}$ & $\mathrm{n} / \mathrm{m}$ & $\mathrm{n} / \mathrm{m}$ & $\mathrm{n} / \mathrm{m}$ & $\mathrm{n} / \mathrm{m}$ & $\mathrm{n} / \mathrm{m}$ \\
\hline wt $\%$ total solids in Centrifuged Sludge & $\mathrm{n} / \mathrm{m}$ & $\mathrm{n} / \mathrm{m}$ & $\mathrm{n} / \mathrm{m}$ & $\mathrm{n} / \mathrm{m}$ & $\mathrm{n} / \mathrm{m}$ & $\mathrm{n} / \mathrm{m}$ \\
\hline wt $\%$ Total Solids & $\mathrm{n} / \mathrm{m}$ & $\mathrm{n} / \mathrm{m}$ & $\mathrm{n} / \mathrm{m}$ & $\mathrm{n} / \mathrm{m}$ & $\mathrm{n} / \mathrm{m}$ & $\mathrm{n} / \mathrm{m}$ \\
\hline wt $\%$ UDS & 0.00 & 0.00 & 0.00 & 0.00 & 0.00 & 0.00 \\
\hline Density of Solids (g/cc) & 3.02 & 3.02 & 3.02 & 3.02 & 3.02 & 3.02 \\
\hline
\end{tabular}


Model Chemical Data From ESP

\begin{tabular}{|c|c|c|c|c|c|c|c|c|c|c|c|c|c|c|c|}
\hline 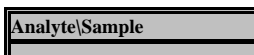 & SYY-101 & $\begin{array}{ll}\text { SY-101 } \\
\end{array}$ & $\begin{array}{ll}\mathrm{SY}-101 \\
\end{array}$ & $\begin{array}{ll}\mathrm{SY}-101 \\
\end{array}$ & $\begin{array}{ll}\text { SY-101 } \\
\end{array}$ & $\begin{array}{ll}\mathrm{SY}-101 \\
\end{array}$ & 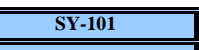 & $\begin{array}{ll}Y Y-101 \\
\end{array}$ & $\begin{array}{ll}\text { SY-101 } \\
\end{array}$ & $\begin{array}{ll}\mathrm{SY}-101 \\
\end{array}$ & $\begin{array}{ll}\text { SY-101 } \\
\end{array}$ & $\begin{array}{ll}5 \mathrm{Y}-101 \\
\end{array}$ & $\begin{array}{ll}\mathrm{SY}-101 \\
\end{array}$ & $\begin{array}{ll}\mathrm{SY}-101 \\
\end{array}$ & $\begin{array}{ll}\text { SY-101 } \\
\end{array}$ \\
\hline |Description & Initial Slury & $\begin{array}{c}\text { Window } \\
\text { Sigment } 4\end{array}$ & $\begin{array}{l}\text { Window } \\
\text { Segment } 4\end{array}$ & $\begin{array}{l}\text { Window } \\
\text { Segment } 4\end{array}$ & $\begin{array}{l}\text { Window } \mathrm{C} \\
\text { Segment } 4\end{array}$ & $\begin{array}{c}\text { Window C C } \\
\text { Segnent }\end{array}$ & $\begin{array}{l}\text { Window C } \\
\text { Segment } 8\end{array}$ & 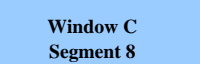 & 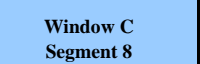 & 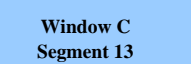 & 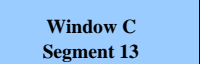 & 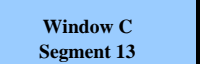 & 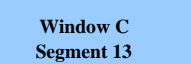 & $\begin{array}{l}\text { Window } C \\
\text { Segment } 15\end{array}$ & $\begin{array}{l}\text { Window C } \\
\text { Segment } 15\end{array}$ \\
\hline Model Basis & \begin{tabular}{|c|} 
Salty \\
\end{tabular} & 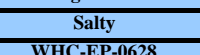 & 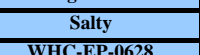 & 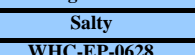 & 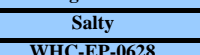 & 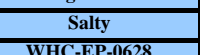 & 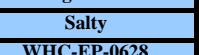 & 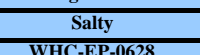 & 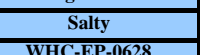 & 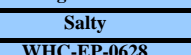 & Sally & 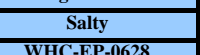 & $\begin{array}{l}\text { Salty } \\
\text { HCFP }\end{array}$ & 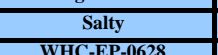 & 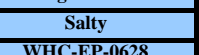 \\
\hline Repor Nol Sou & 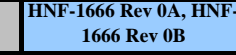 & 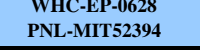 & $\begin{array}{l}\text { WHC-FP-628 } \\
\text { PNL-MIT52394 }\end{array}$ & $\begin{array}{l}\text { WHC-FF-0628 } \\
\text { PNL-MIT52394 }\end{array}$ & $\begin{array}{l}\text { WHC-FP-0.628 } \\
\text { PNLLMIT52394 }\end{array}$ & $\begin{array}{l}\text { WHC-FF-0.628 } \\
\text { PNLLMIT52394 }\end{array}$ & $\begin{array}{l}\text { WHE-CPF-628 } \\
\text { PNL-MIT52394 }\end{array}$ & $\begin{array}{l}\text { WHCCF-0.028 } \\
\text { PNL-MIT52394 }\end{array}$ & $\begin{array}{l}\text { WHC-FP-628 } \\
\text { PNL-MIT52394 }\end{array}$ & $\begin{array}{l}\text { WHC-FP-0.028 } \\
\text { PNLLMIT52394 }\end{array}$ & $\begin{array}{l}\text { WHC-FP-0628 } \\
\text { PNL-MIT52394 }\end{array}$ & $\begin{array}{l}\text { WHC-FP-0.028 } \\
\text { PNL-MIT52394 }\end{array}$ & $\begin{array}{l}\text { WHL-CP-0.628 } \\
\text { PNLLMT52394 }\end{array}$ & 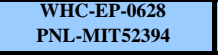 & 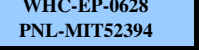 \\
\hline 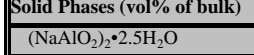 & & & & & & & & & & & & & & & \\
\hline & & & & & & & & & & & & & & & \\
\hline \begin{tabular}{|l|l}
$\mathrm{Bi}_{\mathrm{i}} \mathrm{O}_{3}$ \\
$\mathrm{~B}$
\end{tabular} & 0.00035 & 0.00045 & 0.00052 & 0.00057 & 0.00045 & $\begin{array}{ll}0.00047 \\
\end{array}$ & 0.00059 & 0.00047 & 0.00032 & 0.00006 & 0.00019 & $\begin{array}{l}0.0025 \\
0\end{array}$ & 0.00037 & 0.00129 & 0.00092 \\
\hline 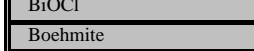 & & & & & & & & & & & & & & & \\
\hline & & & & & & & & & & & & & & & \\
\hline 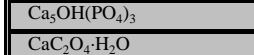 & 0.01535 & 0.01976 & 0.02258 & 0.02486 & 0.01976 & 0.02042 & 0.02596 & 0.02065 & $\begin{array}{ll}0.01411 \\
\end{array}$ & 0.00282 & 0.00038 & 0.01105 & 0.01607 & 0.05668 & \\
\hline $\mid$\begin{tabular}{|l|l|}
$\mathrm{CaCC}_{0} \mathrm{O}_{4} \mathrm{H}_{2} \mathrm{O}$ \\
$\mathrm{Ca}^{2}$
\end{tabular} & & & & & & & & & & & & & & & \\
\hline $\mathrm{CaF}_{2}$ & & & & & & & & & & & & & & & \\
\hline \begin{tabular}{|l|l|l|l} 
Croon \\
FoOH
\end{tabular} & 0.0 .15299 & 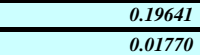 & $\frac{0.24247}{0.20233}$ & $\begin{array}{ll}0.24720 \\
0.0 .0228\end{array}$ & $\frac{0.9641}{0.06770}$ & $\frac{0.20298}{0.01829}$ & $\frac{0.25810}{0.0326}$ & $\begin{array}{l}0.20272 \\
0.0 .1550\end{array}$ & $\frac{0.14030}{0.01264}$ & $\frac{0.02865}{0.00233}$ & $\frac{0.0338}{0.00751}$ & 0.10987 & $\frac{0.1574}{0.01400}$ & 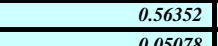 & $\frac{0.40771}{0.025201}$ \\
\hline \begin{tabular}{|l|l} 
Gibbsite \\
\end{tabular} & 20.01373 2.98106 & $\begin{array}{l}0.07710 \\
3.38716\end{array}$ & $\begin{array}{l}0.02023 \\
4.38532 \\
\end{array}$ & $\begin{array}{ll}0.02286 \\
4.82930\end{array}$ & $\begin{array}{l}0.0 .770 \\
3.38716 \\
\end{array}$ & $\begin{array}{l}0.01829 \\
3.96549 \\
\end{array}$ & $\begin{array}{l}0.02326 \\
5.04227 \\
\end{array}$ & $\frac{0.01850}{4.01019}$ & $\begin{array}{l}0.01264 \\
2.74083 \\
\end{array}$ & 0.0.0238 & . & $\frac{0.09090}{2.14636}$ & $\frac{0.01440}{3.12667}$ & 1..000893 & $\begin{array}{l}0.0 .02320 \\
7.84784\end{array}$ \\
\hline \begin{tabular}{|l|l} 
HgO \\
KAlSOO.
\end{tabular} & & & & & & & & & & & & & & & \\
\hline 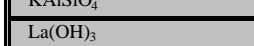 & & & & & & & & & & & & & & & \\
\hline \begin{tabular}{||l|l|}
$\mathrm{LaPO}_{9} \cdot 2 \mathrm{H}_{2} \mathrm{O}$ \\
\end{tabular} & 0.00034 & 0.00043 & 0.00049 & 0.00054 & 0.00043 & 0.00045 & 0.00057 & 0.00045 & 0.00031 & 0.00006 & 0.00018 & 0.00024 & 0.00035 & 0.00124 & 0.000 \\
\hline 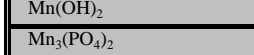 & & & & & & & & & & & & & & & - \\
\hline $\mathrm{MnCO}_{3}$ & & & & & & & & & & & & & & & \\
\hline 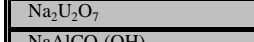 & 0.00205 & 0.00264 & 0.00302 & 0.00333 & 0.00264 & $\begin{array}{ll}0.00273 \\
\end{array}$ & $\begin{array}{l}0.00347 \\
0\end{array}$ & 0.00276 & 0.00189 & 0.00038 & 0.00112 & $\begin{array}{ll}0.00148 \\
\end{array}$ & 0.00215 & 0.00758 & 0.0054 \\
\hline 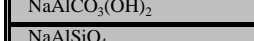 & & & & & & & & & & & & & & & \\
\hline $\mathrm{N}_{\mathrm{N}(\mathrm{OH})_{2}}$ & 0.00580 & 0.00746 & 0.00853 & 0.00939 & 0.00746 & 0.00771 & 0.00981 & 0.00780 & 0.00533 & 0.00107 & 0.00316 & 0.00417 & 0.00607 & 0.02141 & 0.0152 \\
\hline 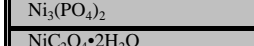 & & & & & & & & & & & & & & & \\
\hline$\frac{\mathrm{N}_{2} \mathrm{C}_{0} \mathrm{H}_{2}+2 \mathrm{H}_{2}}{\mathrm{~Pb}\left(\mathrm{O} \mathrm{H}_{2}\right.}$ & 0.00180 & 0.00032 & 0.00265 & 0.00292 & 0.00032 & $\begin{array}{l}0.00240 \\
\end{array}$ & 0.00305 & 0.00243 & $\begin{array}{l}0.00166 \\
\end{array}$ & 0.00033 & 0.00098 & 0.00130 & 0.00189 & 0.00666 & 0.00475 \\
\hline 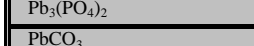 & & & & & & & & & & & & & & & \\
\hline 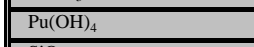 & 0.00000 & 0.00001 & 0.00001 & 0.00001 & 0.00001 & 0.00001 & 0.00001 & 0.00001 & 0.00000 & 0.00000 & 0.00000 & 0.00000 & 0.00000 & 0.00002 & 0.00001 \\
\hline 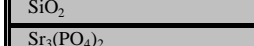 & 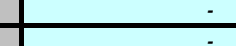 & & & & & & & 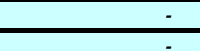 & - & & & 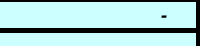 & & & \\
\hline \begin{tabular}{|l|l|l|}
$\mathrm{SrCO}_{3}$ \\
\end{tabular} & 0.00030 & 0.00038 & 0.00044 & 0.00048 & 0.00038 & 0.00039 & 0.00050 & 0.00040 & $\begin{array}{ll}0.00027 \\
.0027\end{array}$ & 0.00005 & 0.00016 & $\begin{array}{ll}0.00221 \\
\end{array}$ & 0.00031 & 0.00109 & 0.0007 \\
\hline 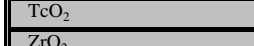 & 0.00002 & 0.00002 & 0.00002 & 0.00003 & 0.00002 & 0.00002 & 0.00003 & 0.00002 & 0.00001 & 0.00000 & 0.00001 & 0.00001 & 0.00002 & 0.00006 & \\
\hline \begin{tabular}{|l|l}
$4 \mathrm{KNO}_{2}$ \\
$\mathrm{KNO}_{3}$
\end{tabular} & & & & & & & & & & & 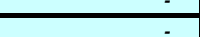 & & & 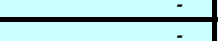 & \\
\hline $\mathrm{N}_{\mathrm{N}_{2} \mathrm{C}_{0} \mathrm{O}_{4}}$ & 1.76726 & 2.27477 & 2.59974 & 2.86294 & 2.27477 & 2.35005 & 2.98920 & 2.37736 & 1.62484 & 0.32497 & 0.96450 & 1.27242 & 1.95002 & 6.52640 & 4.6524 \\
\hline $\mathrm{Na}_{2} \mathrm{CO}_{3} \mathrm{H}_{2} \mathrm{O}$ & & & & & & & & & & & & & & & \\
\hline $\mid \frac{\mathrm{N}_{2} \mathrm{~N}_{2} \mathrm{~S}_{4}}{\mathrm{~N}_{2} \mathrm{O}_{4} 10 \mathrm{H}_{2}}$ & & & & & & & & & & & & & & & \\
\hline $\mathrm{Na}_{3} \mathrm{FOO}_{2}$ & & & & & & & & & & & & & & & \\
\hline \begin{tabular}{|l|l}
$\mathrm{N}_{2}, \mathrm{~N}_{0}, \mathrm{SO}_{4} \mathrm{H}_{2} \mathrm{O}$ \\
\end{tabular} & & & & & & & & & & & & & & & \\
\hline 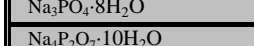 & & & & & & & & & & & & & & & \\
\hline 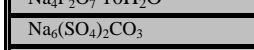 & & & & & & & & & & & & & & & \\
\hline & 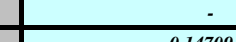 & & & , & 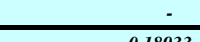 & (1) & M1070 & 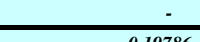 & पר: & $=0$ & 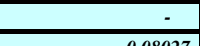 & 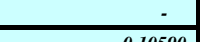 & 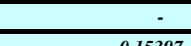 & 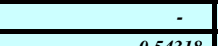 & \\
\hline 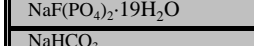 & 0.14709 & 0.18933 & 0.21637 & 0.23828 & 0.18933 & 0.19566 & 0.24879 & 0.19786 & 0.13523 & 0.02705 & 0.08027 & 0.10590 & 0.15397 & 0.54318 & 0.3877 \\
\hline \begin{tabular}{|l|l}
$\mathrm{NanO}_{3}$ \\
$\mathrm{NanO}_{2}$
\end{tabular} & 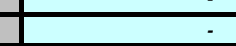 & & & & & & & & & & & & & & \\
\hline & & & YUSM & 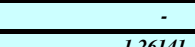 & & 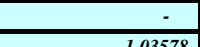 & & 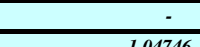 & (n)tron & 011 & (201006 & 5 & 0 & & \\
\hline 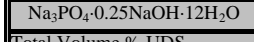 & 0.077865 & 1.00226 & 1.14544 & 1.26141 & 1.00226 & 1.03578 & 1.31704 & 1.04746 & 0.71590 & 0.14318 & 0.424966 & 0.56063 & 0.81512 & 2.87552 & 2.04985 \\
\hline 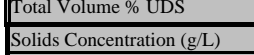 & $\frac{5.87}{138.3}$ & $\frac{7.55}{178.0}$ & $\frac{8.63}{203.4}$ & $\frac{9.50}{224.0}$ & $\frac{7.55}{178.0}$ & $\begin{aligned} 7.80 \\
183.9 \\
\end{aligned}$ & $\begin{aligned} & \frac{9.92}{233.9}-1 \\
&\end{aligned}$ & $\frac{7.89}{186.0}$ & $\frac{5.39}{127.1}$ & $\frac{1.08}{25.4}$ & $\frac{3.20}{75.5}$ & $\frac{4.22}{99.6}$ & $\frac{6.14}{144.7}$ & $\frac{21.66}{510.6}$ & $\frac{15.44}{364.0}$ \\
\hline
\end{tabular}


Rheological Data
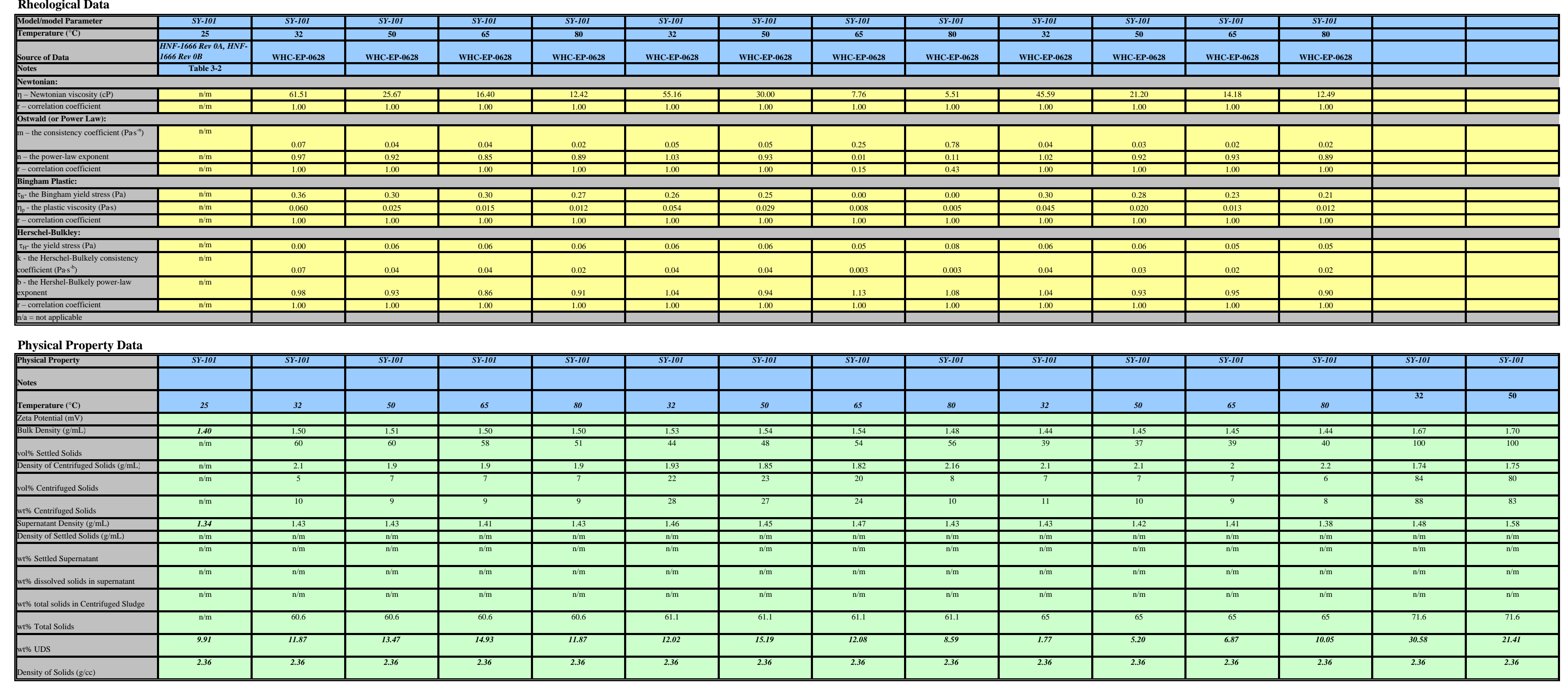

\begin{tabular}{|c|c|}
\hline 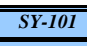 & $\overline{S Y Y-101}$ \\
\hline 32 & ${ }_{50}$ \\
\hline$\frac{1.50}{1.50}$ & 1.51 \\
\hline 60 & 60 \\
\hline$\frac{2.1}{5}$ & $\frac{1.9}{4.7}$ \\
\hline$\overline{10}$ & 9 \\
\hline$\frac{1.43}{n .4 m}$ & $\frac{1.43}{\mathrm{n} / \mathrm{m}}$ \\
\hline$\frac{n}{n / m}$ & ${ }^{n / m}$ \\
\hline$\frac{n / m}{n / m}$ & $\mathrm{n} / \mathrm{m}$ \\
\hline n/m & $\mathrm{n} / \mathrm{m}$ \\
\hline 60.6 & \\
\hline 1.87 & 13.4. \\
\hline
\end{tabular}
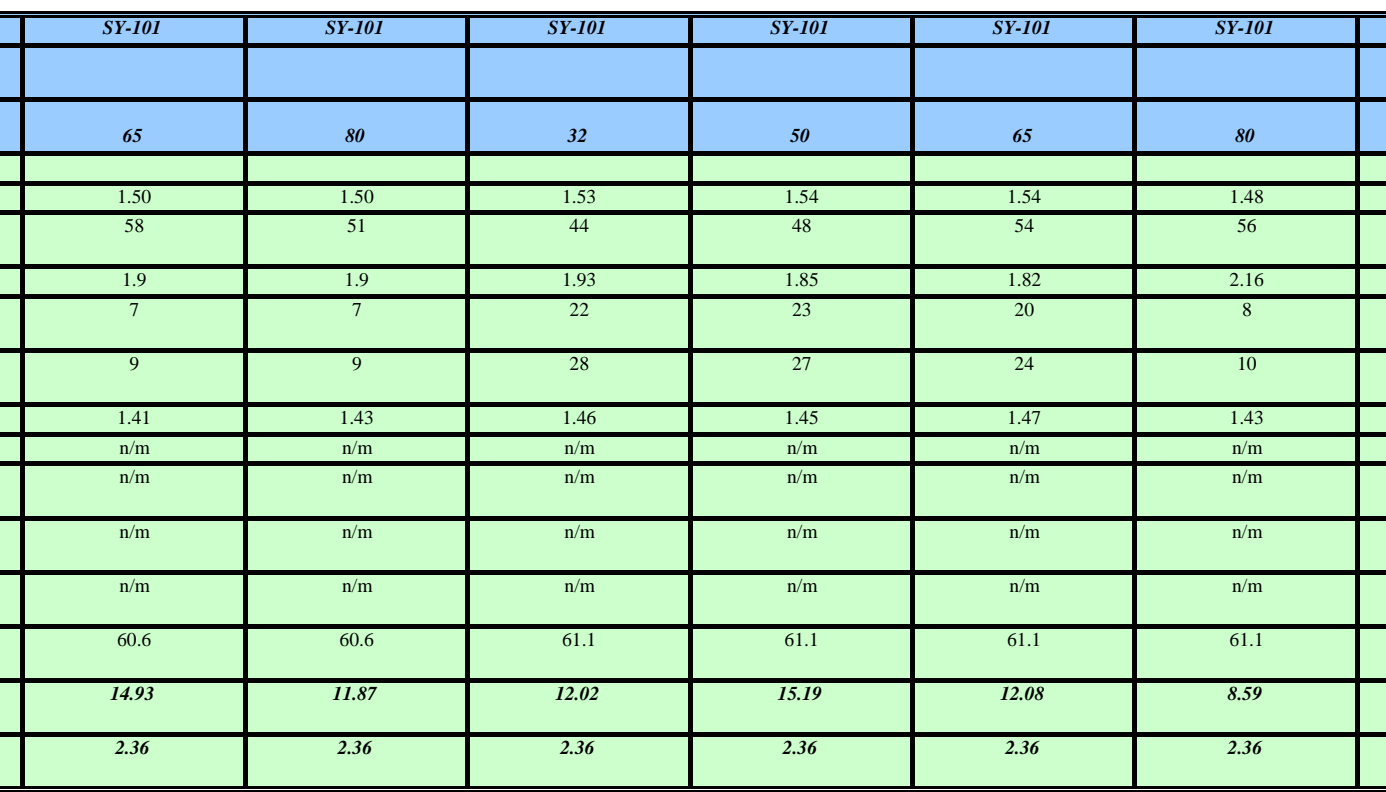

\begin{tabular}{|c|c|c|c|c|c|}
\hline $\begin{array}{l}\text { SY }-101 \\
\end{array}$ & $\begin{array}{ll}S_{Y}-101 \\
\end{array}$ & \begin{tabular}{|l|l|}
$S Y-101$ \\
\end{tabular} & $\begin{array}{l}\mathrm{SY}-101 \\
\end{array}$ & ${ }_{S Y-101}$ & SYY-101 \\
\hline 30 & 50 & $G$ & 80 & 32 & 50 \\
\hline & & & & & \\
\hline$\frac{1.44}{39}$ & $\frac{1.45}{37}$ & 1..45 39 & $\frac{1.44}{40}$ & $\frac{1.67}{100}$ & $\frac{1.70}{100}$ \\
\hline 2.1 & $\frac{2.1}{2.1}$ & 2 & 2.2 & 1.74 & 1.75 \\
\hline & & & 6 & 84 & \\
\hline 11 & 10 & 9 & 8 & 88 & 83 \\
\hline$\frac{1.43}{n \cdot m}$ & $\begin{array}{ll}1.42 \\
\mathrm{n} / \mathrm{m}\end{array}$ & $\frac{1.41}{\mathrm{n} \text { N/m }}$ & $\frac{1.38}{n_{\mathrm{m}}}$ & $\frac{1.48}{\mathrm{n} / \mathrm{m}}$ & $\frac{1.58}{1.58}$ \\
\hline$n$ & $\frac{1}{n / m}$ & n/m & $\frac{n}{n m m}$ & n/m & ntm \\
\hline n/m & $\frac{\pi / m}{n / m}$ & $n$ & 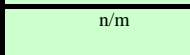 & $n$ & $\mathrm{n} / \mathrm{m}$ \\
\hline$n$ & 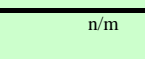 & n/m & $n$ & n/m & 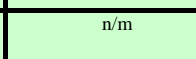 \\
\hline & ${ }^{50}$ & 65 & 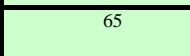 & 71.6 & $\overline{71.6}$ \\
\hline 1.77 & 5.20 & 6.87 & 10.05 & 30.58 & 21.41 \\
\hline 2.36 & 2.36 & 2.36 & 2.36 & 2.36 & 2.36 \\
\hline
\end{tabular}




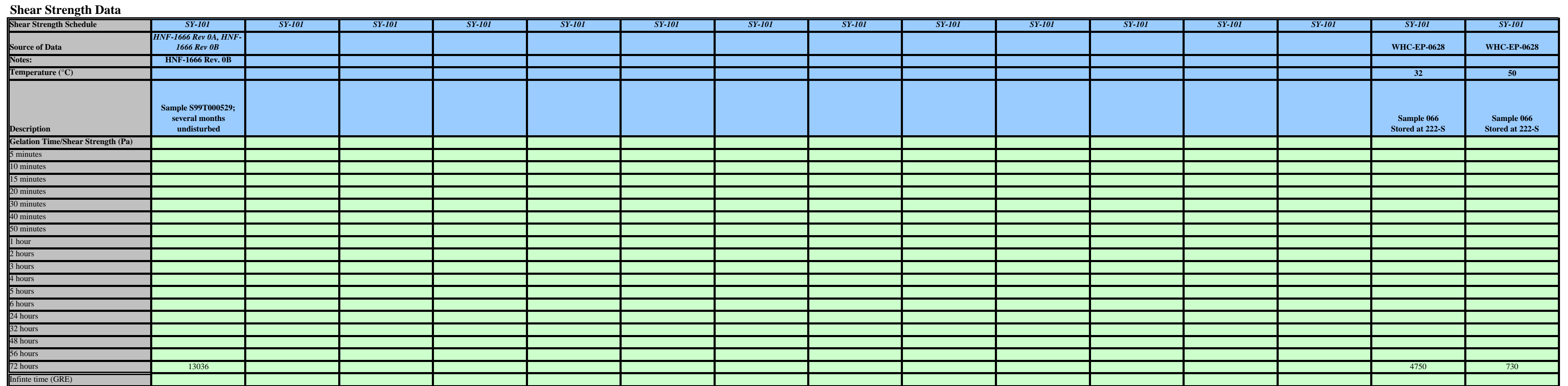




\section{Model Chemical Data Froı}

\begin{tabular}{|c|c|c|c|c|c|c|c|c|c|c|c|c|c|c|c|}
\hline 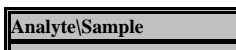 & $\begin{array}{ll}\mathrm{SY}-101 \\
\end{array}$ & $\begin{array}{ll}\text { SY-101 } \\
\end{array}$ & $\begin{array}{ll}\mathrm{SY}-101 \\
\end{array}$ & $\begin{array}{ll}\text { SY-101 } \\
\end{array}$ & $\begin{array}{ll}\text { SY-101 } \\
\end{array}$ & $\begin{array}{ll}\mathrm{SY}-101 \\
\end{array}$ & 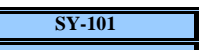 & $\begin{array}{ll}5 \mathrm{Y}-101 \\
\end{array}$ & $\begin{array}{l}\mathrm{SY}-101 \\
\end{array}$ & $\begin{array}{ll}\mathrm{SY}-101 \\
\end{array}$ & $\begin{array}{ll}\text { SY-101 } \\
\end{array}$ & $\begin{array}{ll}\text { SY-101 } \\
\end{array}$ & $\begin{array}{ll}\mathrm{SY}-101 \\
\end{array}$ & $\overline{S Y Y-101}$ & 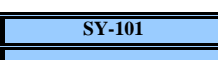 \\
\hline Descripition & 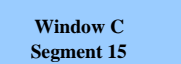 & 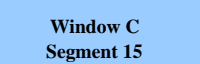 & $\begin{array}{l}\text { Window C } \\
\text { Segment } 1\end{array}$ & $\begin{array}{l}\text { Widdow C } \\
\text { Segment19 }\end{array}$ & 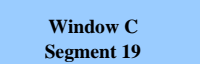 & 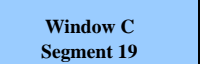 & 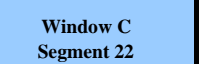 & 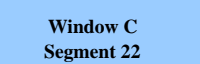 & 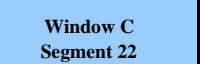 & 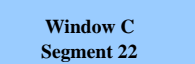 & $\begin{array}{l}\text { Auger R-9221 } \\
\text { Crust }\end{array}$ & $\begin{array}{l}\text { Auger R.9221 } \\
\text { Crust }\end{array}$ & $\begin{array}{l}\text { Auger R R.9221 } \\
\text { Cust }\end{array}$ & $\begin{array}{l}\text { Auger R.9221 } \\
\text { Crust }\end{array}$ & 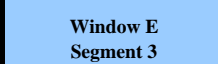 \\
\hline Model Basis & $\frac{\text { Salty }}{\text { WHC.028 }}$ & 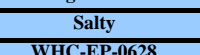 & & 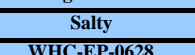 & 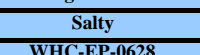 & 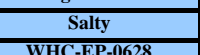 & 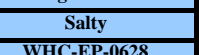 & 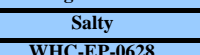 & 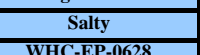 & 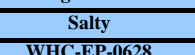 & 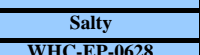 & & & 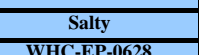 & $\begin{array}{c}\text { Salty } \\
\end{array}$ \\
\hline Report NolSource of Data & 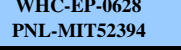 & 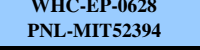 & 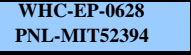 & $\begin{array}{l}\text { WHC-EP-6028 } \\
\text { PNL-MIT52394 }\end{array}$ & $\begin{array}{l}\text { WHC-FP-0.628 } \\
\text { PNLLMIT52394 }\end{array}$ & $\begin{array}{l}\text { WHCCF-0.028 } \\
\text { PNLLMIT52394 }\end{array}$ & $\begin{array}{l}\text { WHL-CPF-0.628 } \\
\text { PNL-MIT52394 }\end{array}$ & $\begin{array}{l}\text { WHCCFP-0.028 } \\
\text { PNLLMIT52394 }\end{array}$ & $\begin{array}{l}\text { WHC-FP-0.028 } \\
\text { PNLLMIT52394 }\end{array}$ & $\begin{array}{l}\text { WHCCF-0.028 } \\
\text { PNLLMIT52394 }\end{array}$ & $\begin{array}{l}\text { WHCCPP-0.028 } \\
\text { PNLLMIT52394 }\end{array}$ & $\begin{array}{l}\text { WHC-PF-0.628 } \\
\text { PNLLMITS2394 }\end{array}$ & $\begin{array}{l}\text { WHC-FP-0.028 } \\
\text { PNLLMITS2394 }\end{array}$ & 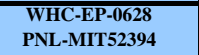 & WHC-SD-WM-DTR-026 \\
\hline 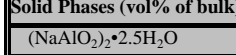 & & & & & & & & & & & & & & & \\
\hline 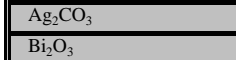 & 0.00129 & 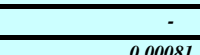 & 000125 & (2017323 & 000133 & 000195 & 000166 - & 800141 & S & $\operatorname{lng}^{0012}$ & הanpent & הenent & $\sqrt{\operatorname{sen} 20}$ & 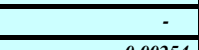 & $\overline{0}=0$ \\
\hline & & & & & & & & & & & & & & & 0.00076 \\
\hline \begin{tabular}{|l|l|} 
Bocelmitie \\
Coth
\end{tabular} & & & & & & & & & & & & & & & \\
\hline 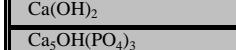 & 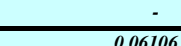 & nopreces & 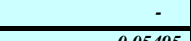 & 0.059323 & $\operatorname{lncos7}^{2}$ & - & ד & 0,06789 & - & 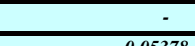 & S & nalogect & 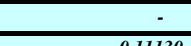 & rar & 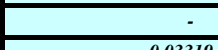 \\
\hline $\mathrm{CaC}_{2} \mathrm{O}_{4} \mathrm{H}_{2} \mathrm{O}$ & - & & 0.034935 & & & - 0.065559 & $0.0 / 292$ & 0.06176 & 0.059339 & 0.033786 & 0 & 0.13660 & 0.113130 & 0.11130 & 0.03 \\
\hline \begin{tabular}{|l|l|l}
$\mathrm{CaCO}_{3}$ \\
$\mathrm{CaF}_{3}$
\end{tabular} & & & & & & & & & & & & & & & \\
\hline $\mathrm{CrOOH}$ & 0.60702 & 0.35455 & 0.54632 & 0.57995 & 0.622013 & 0.85096 & 0.72494 & 0.61419 & 0.59045 & 0.53468 & 1.10655 & 1.13201 & 1.10655 & 1.10655 & 0.33001 \\
\hline 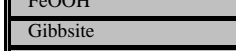 & 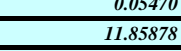 & 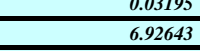 & $\begin{array}{l}\frac{0.04923}{10.67290} \\
\end{array}$ & $\begin{array}{l}0.02526 \\
11.32997 \\
\end{array}$ & $\begin{array}{l}\frac{0.06624}{12.1915} \\
215\end{array}$ & $\frac{0.07669}{16.62429}$ & $\begin{array}{l}0.06533 \\
14.16246 \\
\end{array}$ & $\begin{array}{l}\frac{0.05355}{11.99878} \\
\end{array}$ & $\begin{array}{l}0.05321 \\
1.53503 \\
\end{array}$ & $\frac{.04889}{10.4561}$ & $\begin{array}{l}\frac{0.09972}{21.61758} \\
\end{array}$ & $\begin{array}{l}0.10202 \\
22.1499 \\
\end{array}$ & $\frac{0.09972}{21.61758}$ & $\begin{array}{l}\frac{0.09972}{21.61758} \\
2\end{array}$ & $\frac{0.02974}{6.44710}$ \\
\hline & & & & & & & & & & & & & & & \\
\hline $\mathrm{IarOH}_{3}$ & & & & & & & & & & & & & & & \\
\hline \begin{tabular}{||l|l|}
$\mathrm{LaPO}_{0} \cdot{ }^{2} \mathrm{H}_{2} \mathrm{O}$ \\
\end{tabular} & 0.00133 & 0.00078 & 0.00120 & 0.00128 & 0.00137 & 0.00187 & 0.00159 & 0.00135 & 0.00130 & 0.00118 & 0.00243 & 0.00249 & 0.00243 & 0.00243 & 0.000 \\
\hline 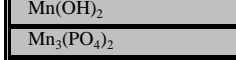 & & & & & & & & & & & & & & & \\
\hline 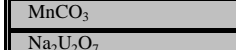 & 0.00817 & 0.00477 & 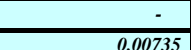 & 0.00780 & 0.00399 & 0.01145 & 0.00975 & 0.00276 & 0.00794 & 0.00719 & 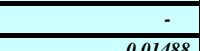 & 0.01523 & 0.01489 & nologe & 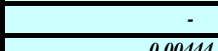 \\
\hline $\mathrm{NaAlCO}_{3}(\mathrm{OH})_{2}$ & & & & & & & & & & & & & . & & 0.0044 \\
\hline 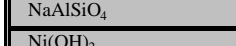 & 0.02306 & 0012177 & (202075 & 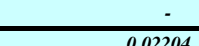 & 002371 & 0 & 0.02751 & 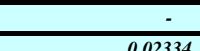 & (2) & 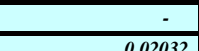 & (201001 & (2013011 & (2010204 & ( & 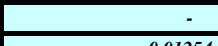 \\
\hline 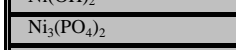 & ( > & & - & & (2) & & & ( & 0.02245 & . & . & (1) & 0.042044 & 0.042044 & 0.0123 \\
\hline 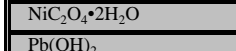 & 0.00718 & 0.00419 & 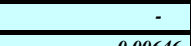 & O.006896 & 0.07738 & and & 000057 & 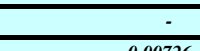 & nencon & 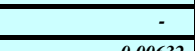 & ( & (201020 & (n) & 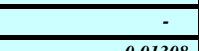 & 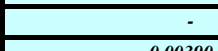 \\
\hline 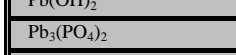 & $0.00 \pi 18$ & . & 0.0605060 & 0.000606 & 0.00358 & 0.010006 & - 0.00857 & 0.00126 & . & 0.00632 & 0.013068 & 0.01338 & 0.013088 & 0.0 .01308 & 0.00330 \\
\hline 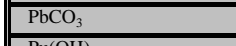 & & & & & & & & & & & & & & & \\
\hline \begin{tabular}{|l}
$\mathrm{Pu}(\mathrm{OH})_{4}$ \\
$\mathrm{NO}$
\end{tabular} & 0.00002 & 0.00001 & 0.00001 & 0.00002 & 0.00002 & 0.00002 & 0.00002 & 0.00002 & 0.00002 & 0.00001 & 0.00003 & 0.00003 & 0.00003 & 0.00003 & 0.00001 \\
\hline 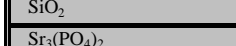 & & - & & & & & & & & & & 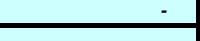 & & & \\
\hline \begin{tabular}{|l|l}
$\mathrm{SrCO}_{3}$ \\
$\mathrm{~T}^{2}$
\end{tabular} & 0.00118 & 0.00069 & 0.000106 & 0.00113 & 0.00121 & 0.00165 & $\begin{array}{ll}0.00141 \\
\end{array}$ & 0.0019 & 0.00115 & 0.000104 & 0.00215 & 0.00220 & 0.00215 & 0.00215 & 0.00064 \\
\hline 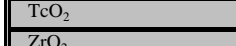 & 0.00006 & 0.00004 & .00006 & 0.00006 & .00007 & 0.00009 & 0.00008 & 0.00006 & 0.00006 & 0.00006 & 0.00012 & 0.00012 & 0.00012 & 0.00012 & 0.0000 \\
\hline \begin{tabular}{|l|l}
$4 \mathrm{KNO}_{2}$ \\
$\mathrm{KNO}_{3}$
\end{tabular} & & & & & & & & & & & & & & & \\
\hline $\mathrm{N}_{\mathrm{N}_{2} \mathrm{C}_{0} \mathrm{O}_{4}}$ & 7.03021 & 4.10618 & 6.32719 & 6.71672 & 7.22725 & 9.85535 & 8.39590 & 7.11321 & 6.83829 & 6.19245 & 12.81551 & 13.1038 & 12.81551 & 12.81551 & 3.82202 \\
\hline $\mathrm{Na}_{2} \mathrm{CO}_{3} \mathrm{H}_{2} \mathrm{O}$ & & & & & & & & & & & & & & & \\
\hline 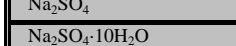 & & & & & & & & & & & & & & & \\
\hline \begin{tabular}{|l|l}
$\mathrm{Na}_{3} \mathrm{FSO}_{2}$ \\
$\mathrm{a}_{3}$
\end{tabular} & & & & & & & & & & & & & & & \\
\hline 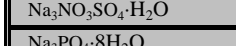 & & & & & & & & & & & & & & & \\
\hline 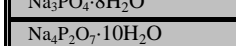 & & & & & & & & & & & & & & & \\
\hline 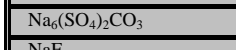 & & 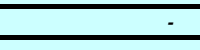 & & & & & & & & & & & & & \\
\hline $\mid$\begin{tabular}{|l|l}
$\mathrm{NaF}$ \\
$\mathrm{NaF}\left(\mathrm{PO}_{3}\right)_{2} 19 \mathrm{H}_{2} \mathrm{O}$
\end{tabular} & 0.58511 & 0.3475 & 0.52600 & 0.5502 & 0.60151 & $\frac{\dot{0}}{0.8224}$ & 0.69878 & 0.59202 & 0.56914 & 0.5159 & 1.06661 & 1.09116 & 1.06661 & 1.06661 & 0.31 \\
\hline$\|$ & & & & & & & & & & & & & & & . \\
\hline & & & & & & & & & & & & & & & \\
\hline 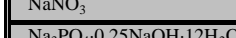 & 70750 & & & & & $\overline{124}+3$ & & 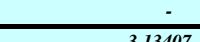 & 30120 & $\overline{2727}$ & 501010 & 5779 & 54040 & (1) & 160207 \\
\hline 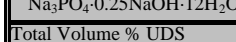 & $\frac{3.09750}{234}$ & 1.80917 & $\begin{array}{l}2.78775 \\
22.00 \\
\end{array}$ & 2.55937 & $\begin{array}{l}3.18341 \\
223.99 \\
\end{array}$ & $\begin{array}{l}4.34224 \\
32.71 \\
\end{array}$ & $\begin{array}{ll}3.69922 \\
27.87 \\
\end{array}$ & $\frac{3.5341}{20.31}$ & $\begin{array}{l}3.01293 \\
22.70 \\
\end{array}$ & 2.223836 & 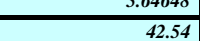 & $\frac{3.73 .35}{43.52}$ & 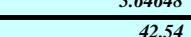 & .0.04068 & $\begin{array}{l}1.63397 \\
1269 \\
\end{array}$ \\
\hline \begin{tabular}{|l} 
Solids Concentration (g/ \\
\end{tabular} & $\frac{550.1}{550.1}$ & $\frac{321.3}{3213}$ & $\frac{495.0}{4950}$ & $\frac{525.5}{525}$ & $\frac{565.5}{565}$ & $\frac{771.1}{771}$ & 656.9 & $\frac{556.5}{565}$ & 535.0 & $\frac{484.5}{484.5}$ & $\frac{1,002.7}{1,027}$ & $\frac{1,025.8}{1,5}$ & $\frac{1,002.7}{1,027}$ & $\frac{1,002.7}{1,7}$ & $\frac{1.69}{299.0}$ \\
\hline
\end{tabular}




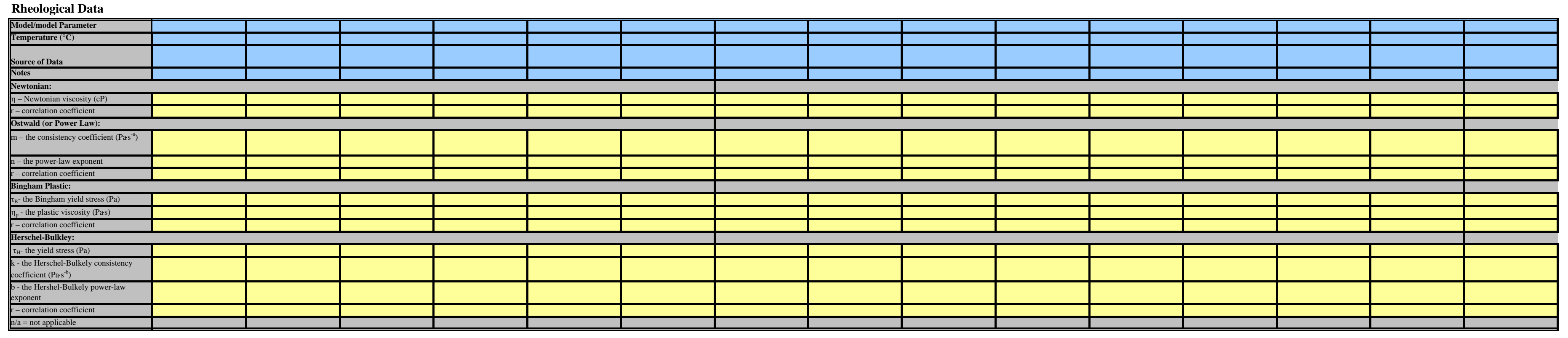

\begin{tabular}{|c|c|c|c|c|c|c|c|c|c|c|c|c|c|c|c|}
\hline \multicolumn{16}{|l|}{ Physical Property Data } \\
\hline Physical Property & SYY-101 & SYY-101 & 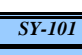 & SYY-101 & $\begin{array}{c}S Y-101 \\
\end{array}$ & $\begin{array}{l}5 Y-101 \\
\end{array}$ & $\begin{array}{l}S Y-101 \\
\end{array}$ & $\begin{array}{ll}S Y-101 \\
\end{array}$ & $\begin{array}{l}S Y-101 \\
\end{array}$ & SYY-101 & 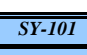 & SYY-101 & $\begin{array}{l}S Y-101 \\
\end{array}$ & 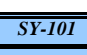 & 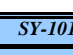 \\
\hline \multicolumn{16}{|l|}{ Notes } \\
\hline Temperature (C) & 65 & 80 & ${ }^{32}$ & 50 & 65 & ${ }^{80}$ & 32 & 50 & ${ }^{65}$ & 80 & 28 & 50 & 65 & ${ }^{80}$ & 32 \\
\hline \begin{tabular}{|l} 
Zeta Potennial (mV) \\
Bull Density $(\mathrm{gm})$
\end{tabular} & 1.70 & 1.66 & 1.68 & ${ }_{1.66}$ & 1.66 & 1.74 & $\frac{1.71}{1.71}$ & 1.71 & 1.71 & 1.70 & 1.77 & $\frac{1.78}{2}$ & 1.77 & 1.77 & 1.60 \\
\hline 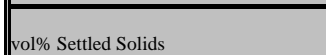 & 100 & 98 & 100 & 100 & 100 & 100 & ${ }^{100}$ & 100 & ${ }^{100}$ & 99 & ${ }^{100}$ & 100 & 100 & 100 & 71 \\
\hline Densily of Cenrifituged Solids (gmLL) & 1.76 & 1.79 & 1.75 & 1.75 & 1.73 & 1.9 & 1.78 & 1.82 & 1.79 & 1.79 & $\frac{1.82}{1.82}$ & 1.82 & 1.81 & 1.82 & 1.8 \\
\hline \multicolumn{16}{|l|}{\begin{tabular}{|l} 
wol\% Centrifiuged Solids \\
\end{tabular}} \\
\hline w\% Centrifuged Solids & ${ }^{78}$ & ${ }^{74}$ & 79 & ${ }^{76}$ & 67 & 68 & 83 & ${ }^{78}$ & 72 & 68 & 99 & 99 & 99 & 99 & 40 \\
\hline Supenatant Density (gemL) & $\frac{1.50}{\mathrm{nim}}$ & $\frac{1.55}{\mathrm{~nm}}$ & $\frac{1.50}{\mathrm{~nm}}$ & $\frac{1.46}{\mathrm{n} / \mathrm{m}}$ & $\begin{array}{ll}1.44 \\
1 / m\end{array}$ & 1.44 & $\begin{array}{c}1.46 \\
\end{array}$ & 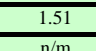 & $\frac{1.52}{10.72}$ & $\frac{1.53}{10.03}$ & $\begin{array}{ll}1.34 \\
n / m\end{array}$ & 1.34 & 1.34 & 1.34 & 1.49 \\
\hline 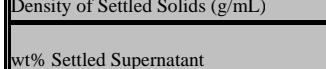 & nim & 罚m & nem & $\frac{\mathrm{nm}}{\mathrm{nm}}$ & $\frac{n \mathrm{~nm}}{\mathrm{n} / \mathrm{m}}$ & 罚m & $\frac{\mathrm{nm} / \mathrm{m}}{\mathrm{n} / \mathrm{m}}$ & $\frac{\mathrm{nm}}{\mathrm{n} / \mathrm{m}}$ & $\frac{\mathrm{n} m \mathrm{~m}}{\mathrm{n} / \mathrm{m}}$ & $\frac{n}{n / m}$ & $\frac{\mathrm{nm} m}{\mathrm{n}_{\mathrm{m}}^{\mathrm{m}}}$ & $\frac{\mathrm{nm}}{\mathrm{nm}}$ & nim & $\frac{\mathrm{nm}}{\mathrm{nm}}$ & $\frac{\mathrm{nm}}{\mathrm{nm}}$ \\
\hline |w1\% & $\mathrm{n} / \mathrm{m}$ & $\mathrm{nm}$ & $\mathrm{nm}$ & $\mathrm{n} / \mathrm{m}$ & n/m & $\mathrm{nm}$ & $\mathrm{n}^{\mathrm{n} / \mathrm{m}}$ & $\mathrm{n} / \mathrm{m}$ & $\mathrm{nm}$ & $\mathrm{n}_{\mathrm{m}}^{\mathrm{m}}$ & $\mathrm{n}^{\mathrm{n} m \mathrm{~m}}$ & $\mathrm{n} / \mathrm{m}$ & $\mathrm{n} / \mathrm{m}$ & $\mathrm{n} / \mathrm{m}$ & $\mathrm{n} / \mathrm{m}$ \\
\hline w\%\% total solids in Cenrifiuged Sludge & $\frac{\mathrm{n}^{\mathrm{m}} \mathrm{m}}{\mathrm{m}}$ & $\frac{n i m}{n / m}$ & $\frac{\mathrm{nm}}{\mathrm{nm}}$ & $\frac{\mathrm{n} / \mathrm{m}}{2}$ & 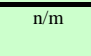 & $\mathrm{n}^{\mathrm{m} m}$ & $\mathrm{n}^{\mathrm{n} / \mathrm{m}}$ & $\mathrm{n}^{\mathrm{n} m}$ & $\frac{n}{n / m}$ & $\mathrm{n}^{\prime \mathrm{m}}$ & $\frac{\mathrm{nm}}{\mathrm{nm}}$ & $\mathrm{n}^{\prime / m}$ & n/m & $\frac{\mathrm{n} / \mathrm{m}}{2}$ & $\overline{n / m}$ \\
\hline ww\% Total solids & 71.6 & 71.6 & 68.6 & 68.6 & $\begin{array}{ll}68.6 \\
\end{array}$ & 68.6 & 71.5 & 71.5 & 71.5 & 71.5 & 85.7 & 85.7 & 85.7 & 85.7 & $\mathrm{n}^{\mathrm{n} / \mathrm{m}}$ \\
\hline w\% UDS & 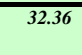 & 19.35 & 299.47 & 31.66 & 34.06 & 44,32 & $\begin{array}{l}38.42 \\
\end{array}$ & 32.55 & 31.29 & 20.50 & 56.65 & 57.63 & 56.65 & 56.65 & 18.69 \\
\hline 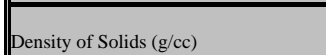 & 2.36 & 2.36 & 2.36 & 2.36 & 2.36 & 2.36 & 2.36 & 2.36 & 2.36 & 2.36 & 2.36 & 2.36 & 2.36 & 2.36 & 2.36 \\
\hline
\end{tabular}




\begin{tabular}{|c|c|c|c|c|c|c|c|c|c|c|c|c|c|c|c|}
\hline \multicolumn{16}{|l|}{$\begin{array}{l}\text { Shear Strength Data } \\
\text { Shear Strength Schedule }\end{array}$} \\
\hline \begin{tabular}{|l|l|} 
Shear Strenggth Schedule \\
\end{tabular} & $\begin{array}{lll}S Y-101 \\
\end{array}$ & $\begin{array}{lll}S Y-101 \\
\end{array}$ & $S Y-101$ & $\begin{array}{cl}S Y-101 \\
\end{array}$ & $\begin{array}{ll}S Y-101 \\
\end{array}$ & SY-101 & $\begin{array}{c}S Y-101 \\
\end{array}$ & $\begin{array}{cl}S Y-101 \\
\end{array}$ & $\begin{array}{c}S Y-101 \\
\end{array}$ & $\begin{array}{lll}S Y-101 \\
\end{array}$ & $\begin{array}{cl}\mathrm{SY}-101 \\
\end{array}$ & $\begin{array}{c}\mathrm{SY}-101 \\
\end{array}$ & $\begin{array}{lll}S Y-101 \\
\end{array}$ & $\begin{array}{cl}S Y-101 \\
\end{array}$ & $\begin{array}{lll}S Y-101 \\
\end{array}$ \\
\hline Source of Data & WHC-EP-0628 & WHC-FP-0628 & WHC-EP-0628 & WHC-EP-0628 & WHC-EP-0628 & พнС-EP-0628 & WHC-EP-0628 & WHC-PP-0628 & WHC-EP-0628 & WHC-EP-0628 & WHC-EP-0628 & WHC-EP-0628 & WHC-FP-0628 & шнС-EP-0628 & WHC-SD-WM-DRT-026 \\
\hline 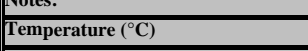 & 65 & 80 & 32 & 50 & 65 & 80 & 32 & 50 & 65 & 80 & 28 & 50 & $\frac{65}{65}$ & 80 & 32 \\
\hline Description & $\begin{array}{l}\text { Sample e66 } \\
\text { stored at 222-s }\end{array}$ & $\begin{array}{l}\text { Sample e66 } \\
\text { stored at 222-s }\end{array}$ & $\begin{array}{l}\text { Sample 019 } \\
\text { stored a 222-s }\end{array}$ & $\begin{array}{l}\text { Sample 19 19. } \\
\text { stored at 222-s }\end{array}$ & $\begin{array}{l}\text { Sample 19 } 19 \\
\text { stored at 222-s }\end{array}$ & $\begin{array}{l}\text { Salmple 199. } \\
\text { Stored a } 222-\mathrm{s}\end{array}$ & $\begin{array}{c}\text { Sample } 607 \\
\text { Stored at 222-s }\end{array}$ & 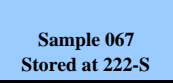 & $\begin{array}{l}\text { Sample } 607 \\
\text { Storerd a 222-s }\end{array}$ & $\begin{array}{l}\text { Sample } 067 \\
\text { stored a 222-s }\end{array}$ & $\begin{array}{l}\text { Sample 817. } \\
\text { Stored a 222-s }\end{array}$ & $\begin{array}{l}\text { Samplele } 177.5 \\
\text { stored at 222-s }\end{array}$ & $\begin{array}{l}\text { Sample 817. } \\
\text { Stored at 222-s }\end{array}$ & $\begin{array}{l}\text { Samplele 817. } \\
\text { Stored a 222-s }\end{array}$ & $\begin{array}{l}\text { Sample 1229. } \\
\text { stored a 222-S }\end{array}$ \\
\hline \multicolumn{16}{|l|}{ 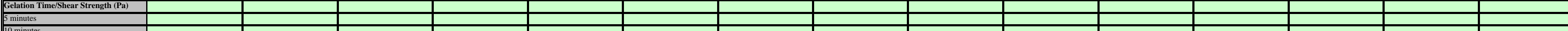 } \\
\hline \multirow{2}{*}{\multicolumn{16}{|c|}{$\begin{array}{l}15 \text { minutes } \\
20 \text { mitues }\end{array}$}} \\
\hline & & & & & & & & & & & & & & & \\
\hline \multicolumn{16}{|l|}{$\begin{array}{ll}3 \\
30 \text { miminues } \\
3\end{array}$} \\
\hline \multirow{2}{*}{\multicolumn{16}{|c|}{ 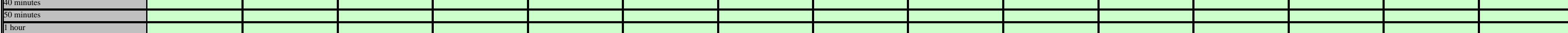 }} \\
\hline \\
\hline & & & & & & & & & & & & & & & \\
\hline \\
\hline$\frac{6 \text { hours }}{24 \text { toris }}$ & & & & & & & & & & & & & & & \\
\hline \multicolumn{16}{|l|}{ 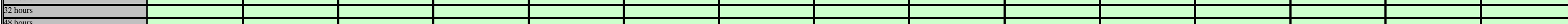 } \\
\hline \multicolumn{16}{|l|}{$\begin{array}{lll}48 \text { nouls } \\
56 \text { hours }\end{array}$} \\
\hline Thionus & 2100 & 530 & 1500 & 640 & 300 & 510 & 111600 & 2640 & 400 & 1400 & 25000 & 4200 & $\overline{10}$ & 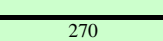 & \\
\hline fifint ime & & & & & & & & & & & & & & & \\
\hline
\end{tabular}




\section{Model Chemical Data Frol}

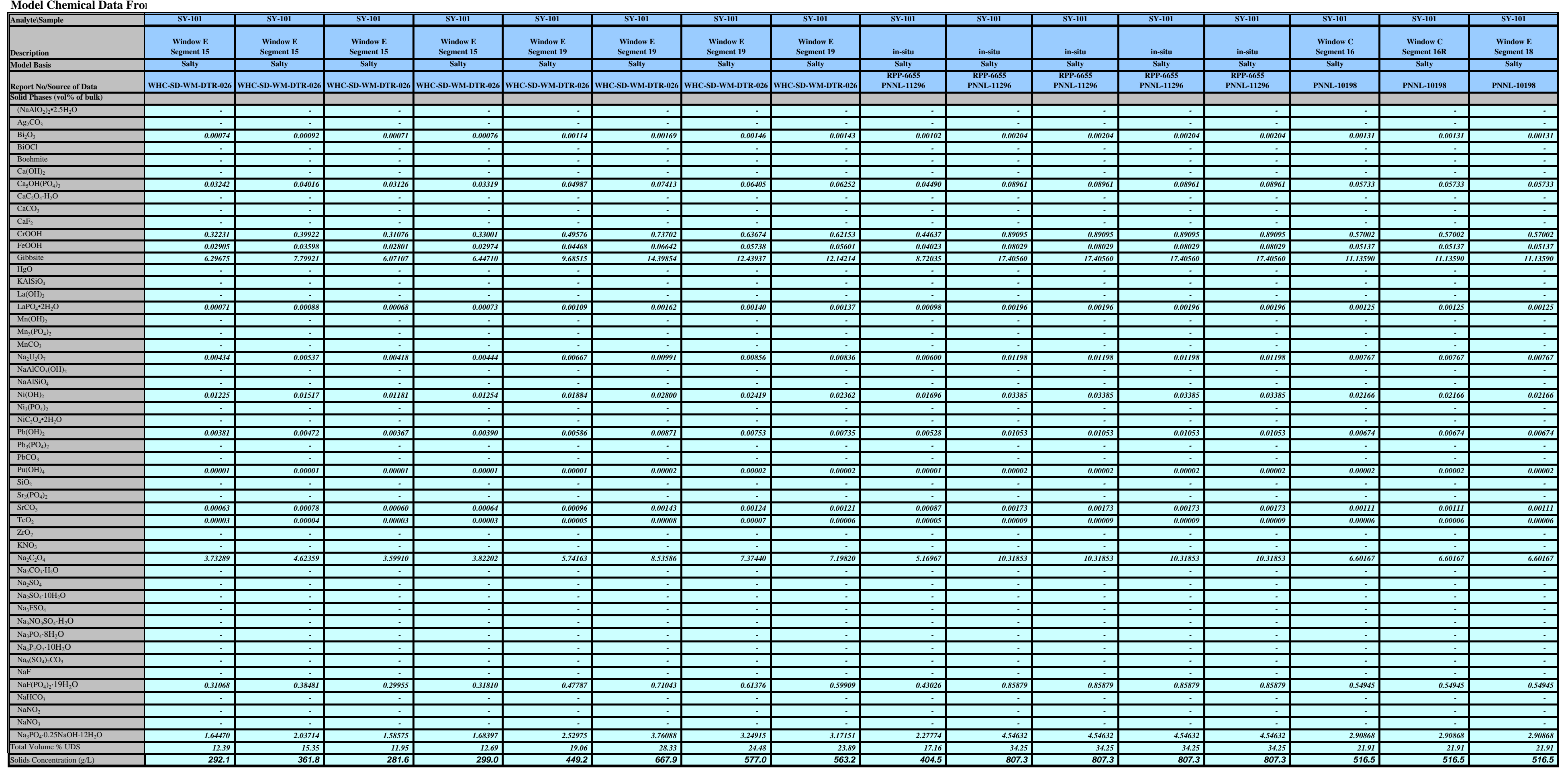




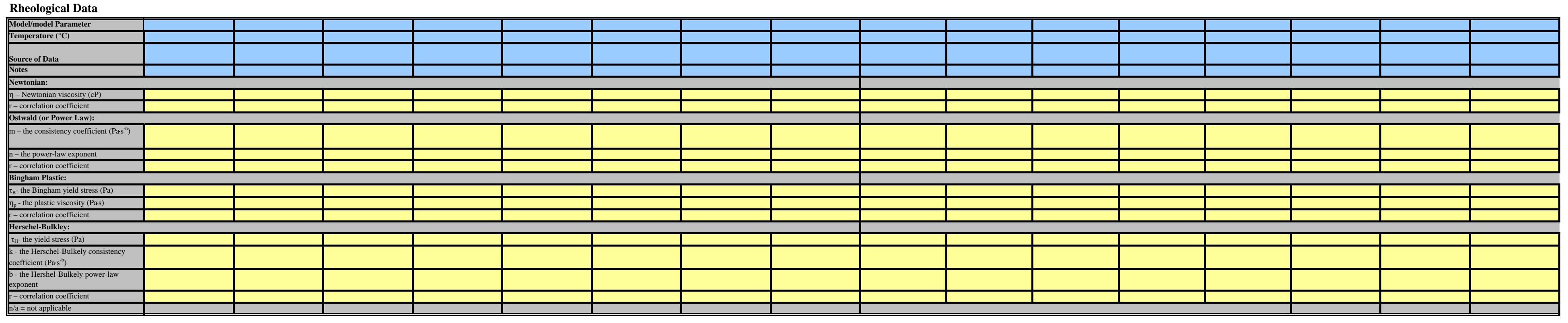

\begin{tabular}{|c|c|c|c|c|c|c|c|c|c|c|c|c|c|c|c|c|}
\hline \multicolumn{17}{|l|}{ Physical Property Data } \\
\hline \begin{tabular}{|l} 
Physical Property \\
\end{tabular} & 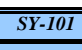 & 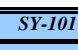 & $\begin{array}{ll}Y Y-101 \\
\end{array}$ & 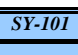 & 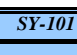 & $\begin{array}{ll}S Y-101 \\
\end{array}$ & 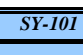 & 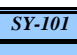 & 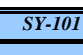 & 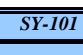 & 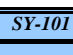 & 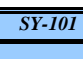 & $\begin{array}{ll}S Y-101 \\
\end{array}$ & 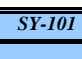 & 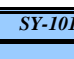 & $\begin{array}{c}S Y-10 \\
\end{array}$ \\
\hline \multicolumn{17}{|l|}{ Notes } \\
\hline Temperature $\left({ }^{\circ} \mathrm{C}\right)$ & ${ }^{32}$ & 50 & 65 & ${ }^{80}$ & 32 & 50 & 65 & ${ }^{80}$ & & & & & & 50 & 50 & 50 \\
\hline \begin{tabular}{|l} 
Zeta Potential (mV) \\
Bull Density (gmL)
\end{tabular} & 1.65 & 1.64 & 1.62 & 1.60 & 1.72 & 1.75 & $\widetilde{\Gamma_{1.74}}$ & 1.72 & 1.63 & 1.78 & 1.78 & 1.78 & 1.78 & 1.68 & 1.68 & 1.68 \\
\hline Fol\% Setrled Solids & 94 & 95 & 90 & 92 & 100 & 95 & 100 & 100 & 100 & 100 & 100 & 100 & 100 & 100 & 100 & 100 \\
\hline Density of Centifituged Solids (g/mL) & $\frac{1.72}{1.72}$ & $\frac{1.78}{1.78}$ & $\frac{1.74}{1.74}$ & $\frac{1.82}{1.82}$ & $\frac{1.76}{1.7}$ & 1.82 & 1.84 & 1.85 & 1.86 & 1.86 & 1.86 & 1.86 & 1.86 & 1.75 & 1.75 & 1.75 \\
\hline \multicolumn{17}{|l|}{ vol\% Centrifuged Solids } \\
\hline \begin{tabular}{|l}
$w \%$ Centrifuged Solids \\
wa
\end{tabular} & 63 & 51 & 46 & 37 & 80 & 77 & 67 & 62 & 77.3 & 77.3 & 77.3 & 77.3 & 77.3 & 80 & 80 & 80 \\
\hline 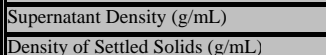 & $\frac{1.55}{1.50}$ & $\frac{1.51}{1010}$ & $\frac{1.52}{1 . \mathrm{m}}$ & 1.49 & 1.57 & 1.51 & $\frac{1.54}{10.9}$ & $\frac{1.52}{1019}$ & 1.48 & 1.48 & 1.48 & 1.48 & 1.48 & 1.49 & 1.49 & 1.49 \\
\hline 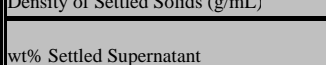 & 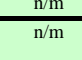 & nim & $\frac{\mathrm{nm}}{\mathrm{nm}}$ & $\frac{\frac{\mathrm{nm}}{\mathrm{nm}}}{\mathrm{nm}}$ & $\frac{n m}{n / m}$ & $\frac{\mathrm{nm}}{\mathrm{nm}}$ & $\frac{\mathrm{nm}}{\mathrm{n}_{\mathrm{m}}}$ & $\frac{\mathrm{nm}}{\mathrm{nm}}$ & & & & & & 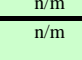 & $\frac{n m}{n / m}$ & $\frac{n \mathrm{~m} m}{\mathrm{n} / \mathrm{m}}$ \\
\hline wo\% dissolved olidid in supenatant & $\mathrm{nm}$ & $\mathrm{n}^{\mathrm{m} m}$ & $\mathrm{n} / \mathrm{m}$ & $\mathrm{n}^{\mathrm{n} / \mathrm{m}}$ & $\mathrm{n}^{\mathrm{m} m}$ & $\mathrm{n} / \mathrm{m}$ & $\mathrm{n} / \mathrm{m}$ & $n$ n/m & & & & & & $\mathrm{nm}$ & $\mathrm{n}^{\mathrm{n} / \mathrm{m}}$ & $\mathrm{n} / \mathrm{m}$ \\
\hline ww\% total solids in Centrifuged Sludge & $\frac{\mathrm{n} / \mathrm{m}}{\mathrm{n}}$ & $\frac{n^{\prime m}}{2 m}$ & $\mathrm{n} / \mathrm{m}$ & $\mathrm{n} / \mathrm{m}$ & $\frac{n}{n / m}$ & $\mathrm{n} / \mathrm{m}$ & $\mathrm{n}^{\mathrm{n} / \mathrm{m}}$ & ${ }^{\mathrm{n} / \mathrm{m}}$ & & & & & & $\mathrm{nm}$ & $n^{n / m}$ & $\frac{\mathrm{n} / \mathrm{m}}{3}$ \\
\hline 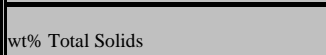 & 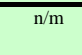 & 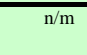 & $\mathrm{n}^{\mathrm{n} / \mathrm{m}}$ & $\mathrm{n} / \mathrm{m}$ & 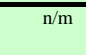 & $\frac{\mathrm{n} / \mathrm{m}}{\mathrm{m}}$ & $\mathrm{n}^{\mathrm{n} / \mathrm{m}}$ & ${ }^{n / m}$ & & & & & & $\frac{\mathrm{nm}}{\mathrm{nm}}$ & $\mathrm{n}^{\mathrm{m} / \mathrm{m}}$ & $\frac{\mathrm{n} / \mathrm{m}}{\mathrm{s}}$ \\
\hline Ww' UDS & $\begin{array}{l}17.70 \\
\end{array}$ & 22.06 & 17,38 & $\begin{array}{l}18.69 \\
\end{array}$ & 26.12 & 38.16 & 33.16 & $\begin{array}{l}332.74 \\
\end{array}$ & $\begin{array}{l}24,81 \\
\end{array}$ & 45.36 & 45.36 & 45.36 & 45.36 & 30.75 & 30.75 & 30.75 \\
\hline $\mid$\begin{tabular}{|l} 
Density of Solids (g/cc) \\
(a)
\end{tabular} & 2.36 & 2.36 & 2.36 & 2.36 & 2.36 & 2.36 & ${ }^{2.36}$ & ${ }^{2.36}$ & 2.36 & 2.36 & ${ }^{2.36}$ & ${ }^{2.36}$ & 2.36 & ${ }^{2.36}$ & 2.36 & $\begin{array}{l}2.36 \\
\end{array}$ \\
\hline
\end{tabular}




\begin{tabular}{|c|c|c|c|c|c|c|c|c|c|c|c|c|c|c|c|c|}
\hline 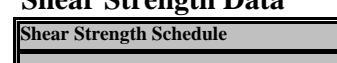 & 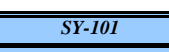 & 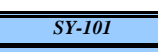 & 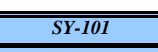 & 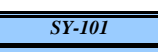 & Str:010 & 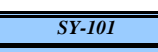 & 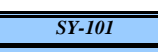 & 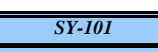 & 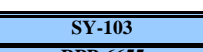 & SYY-133. & 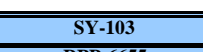 & $\frac{s y=10}{s}$ & 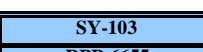 & $\frac{S x-1}{s x-1}$ & 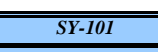 & $\frac{S Y-10}{S-10}$ \\
\hline 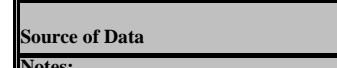 & шнс-вр0628 & 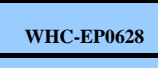 & 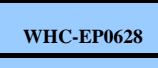 & 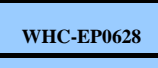 & 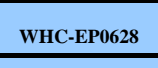 & 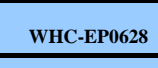 & 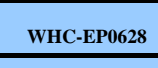 & 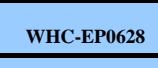 & 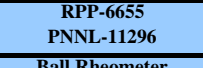 & 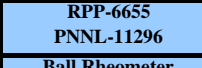 & 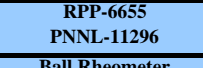 & 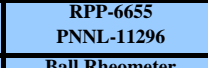 & 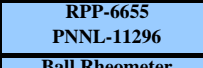 & PNL101998 & $\begin{array}{l}\text { PNLL101998 } \\
\end{array}$ & PNL-10998 \\
\hline 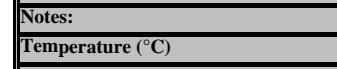 & $\overline{52}$ & 50 & $\frac{65}{6.5}$ & 80 & 32 & $5 \mathrm{~s}_{50}$ & $6{ }_{65}$ & $\overline{50}$ & & & 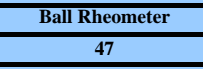 & 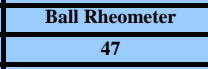 & Ballरn & & & 56 \\
\hline 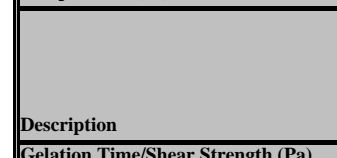 & 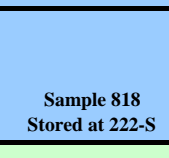 & 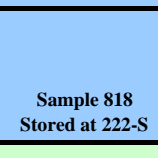 & 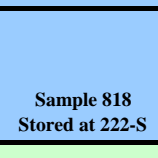 & 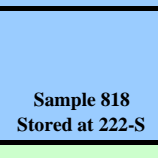 & 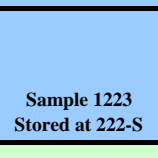 & 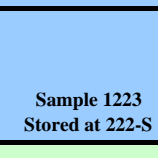 & 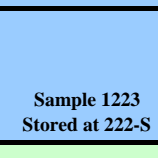 & 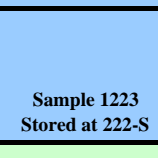 & 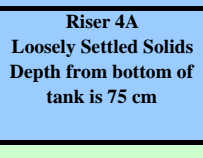 & 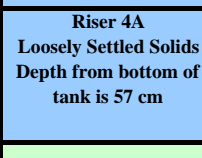 & 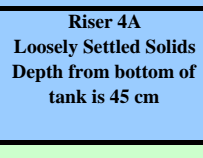 & 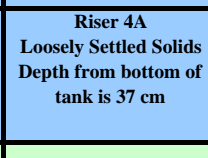 & 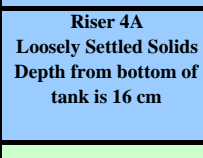 & 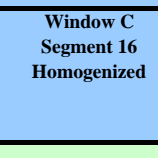 & 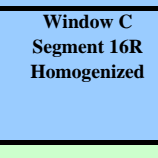 & 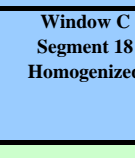 \\
\hline & & & & & & & & & & & & & & & & \\
\hline & & & & & & & & & & & & & & & & \\
\hline & & & & & & & & & & & & & & & & \\
\hline & & & & & & & & & & & & & & & & \\
\hline & & & & & & & & & & & & & & & & \\
\hline & & & & & & & & & & & & & & & & \\
\hline 资 & & & & & & & & & & & & & & & & \\
\hline 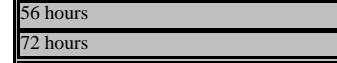 & 17720 & 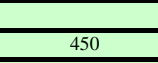 & 790 & 230 & $\frac{71850}{1850}$ & $\frac{370}{300}$ & $\sqrt{730}$ & 670 & 0.77 & 71 & & $\frac{20}{20}$ & 28 & 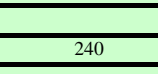 & $\frac{300}{30}$ & 190 \\
\hline
\end{tabular}


Model Chemical Data Fro

\begin{tabular}{|c|c|c|c|c|c|c|c|c|c|c|c|c|c|c|c|}
\hline $\begin{array}{l}\text { Analytelesample } \\
\text { Description } \\
\text { Model Basis }\end{array}$ & 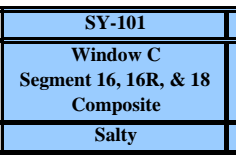 & \begin{tabular}{c|c|} 
SY-101 \\
Widnow C \\
Segment 16, 16R, \& 18 \\
Composite \\
Salty \\
Saly
\end{tabular} & 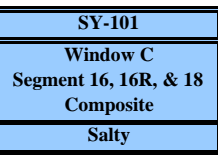 & 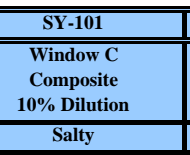 & $\begin{array}{c}\text { SY-101 } \\
\text { WindowC } \\
\text { Cooposite } \\
\text { 10\% Dilution } \\
\text { Salty } \\
\end{array}$ & $\begin{array}{c}\text { SY-101 } \\
\begin{array}{c}\text { Window C } \\
\text { Composite } \\
\text { 10\% Ditution } \\
\text { Salty }\end{array} \\
\end{array}$ & 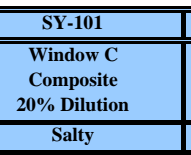 & 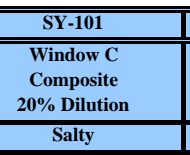 & 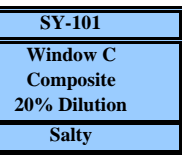 & 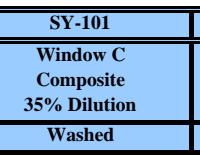 & 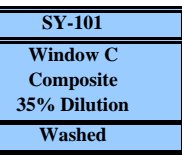 & 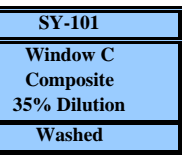 & 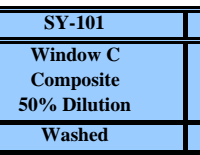 & 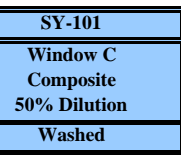 & 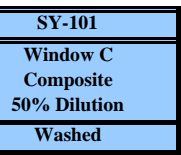 \\
\hline Report No/Source of Data & PNNL-10198 & PNNL-10198 & PNNL-10198 & PNNL-10198 & PNNL-10198 & PNNL-10198 & PNNL-10198 & PNNL-10198 & PNNL-10198 & PNNL-10198 & PNNL-10198 & PNNL-10198 & PNNL-10198 & PNNL-10198 & PNNL-10198 \\
\hline 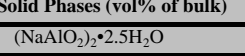 & & & & & & & & & & & & & & & \\
\hline $\begin{array}{l}\mathrm{Ag}_{\mathrm{B}_{2} \mathrm{CO}_{3}} \\
\mathrm{Bi}_{2} \mathrm{O}_{3}\end{array}$ & $\begin{array}{ll}0.00158 \\
\end{array}$ & 0.00083 & 0.00049 & 0.00125 & 0.00047 & 0.00007 & 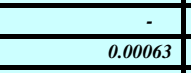 & 0.00014 & 0.00007 & 0.00010 & $\left(\frac{0.0040)}{10}\right.$ & $(0.00050)]$ & 0.00043 & (0.000099) & $\frac{0.000}{(0.000}$ \\
\hline $\begin{array}{l}\text { Biocl } \\
\text { Boolmite }\end{array}$ & 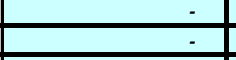 & & $\therefore$ & & & & & & & & 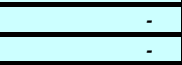 & & & & \\
\hline 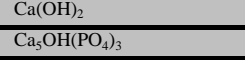 & $\frac{.}{0.0690}$ & 0.03621 & 0.02162 & 0.05495 & 0.02042 & 0.00305 & 0.02780 & 0.00633 & $\begin{array}{l}0.03035 \\
.035\end{array}$ & 0.00438 & $\left(\frac{0.01734)}{4}\right.$ & $(0.02188)$ & 0.01881 & (0.00379) & 0.0310. \\
\hline $\begin{array}{l}\mathrm{CaC}_{2} \mathrm{O}_{4} \mathrm{H}_{2} \mathrm{O} \\
\mathrm{CaCO}_{3}\end{array}$ & & & & & & & & & & & & & & & \\
\hline $\mathrm{Car}_{2}$ & 0.09002 & .0.36001 & 0.21497 & 0.54632 & \begin{tabular}{l|l}
0.20298 \\
\end{tabular} & 0.03035 & 0.27638 & 0.06290 & 0.03035 & 0.04350 & $\frac{0.17243)}{4}$ & (0.21749) & 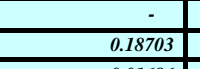 & $\frac{(0.03770)}{2}$ & (0.30886) \\
\hline 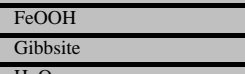 & $\begin{array}{l}0.06213 \\
13.48030 \\
\end{array}$ & $\begin{array}{l}.0 .03244 \\
7.03320 \\
\end{array}$ & $\begin{array}{l}0.01937 \\
4.1957 \\
\end{array}$ & \begin{tabular}{ll|l}
0.04923 \\
10.67290 \\
\end{tabular} & $\begin{array}{l}.01829 \\
3.96549 \\
\end{array}$ & $\begin{array}{l}0.00274 \\
0.592924 \\
\end{array}$ & $\begin{array}{l}0.02491 \\
5.399455 \\
\end{array}$ & $\begin{array}{ll}0.00567 \\
1.22890 \\
\end{array}$ & $\begin{array}{l}0.00274 \\
0.59294 \\
\end{array}$ & $\begin{array}{l}0.00392 \\
0.84977 \\
0.0\end{array}$ & $\begin{array}{l}(0.01554) \\
(3.366599) \\
\end{array}$ & $\begin{array}{l}\left(\frac{0.01960)}{(4.24883)}\right. \\
\end{array}$ & $\begin{array}{l}0.06686 \\
3.65388 \\
\end{array}$ & $\frac{(0.0340)}{(0.73650)}$ & $\frac{(0.02783)}{(6.03396)}$ \\
\hline 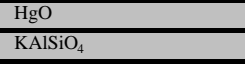 & & & & & & & & & & & & & & & \\
\hline 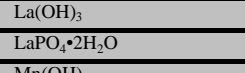 & 0.00152 & 0.00079 & 0.00047 & 0.00120 & \begin{tabular}{l|l}
0.00045 \\
\end{tabular} & 0.00007 & 0.00061 & $\begin{array}{c}- \\
0.00014 \\
\end{array}$ & $\begin{array}{ll}0.00007 \\
\end{array}$ & 0.00010 & $\frac{\cdot}{(0.00038)}$ & $\frac{\dot{ }}{(0.00048)}$ & 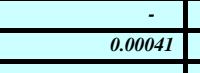 & (0.000008) & $\begin{array}{ll}(0.00668) \\
\end{array}$ \\
\hline 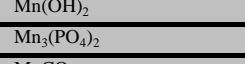 & & & & & & & & & & & C & - & . & $\ldots$ & 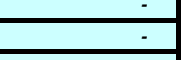 \\
\hline 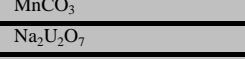 & 0.00928 & 0.00484 & 0.00289 & 0.00735 & 0.00273 & 0.00041 & 0.00372 & $\begin{array}{ll}0.00085 \\
\end{array}$ & $\begin{array}{ll}0.00041 \\
\end{array}$ & 0.00059 & $(0.00232)$ & $\begin{array}{l}(0.00293) \\
\end{array}$ & 0.00252 & $\begin{array}{l}(0.00051) \\
\end{array}$ & (0.00415) \\
\hline$\frac{\mathrm{NaAACl}_{3} \mathrm{OH}_{2}}{\mathrm{NaAHS}_{4}}$ & & & & & & & & & & & & & & & \\
\hline$\frac{\left.\mathrm{N}_{\mathrm{N}(\mathrm{OH}}\right)_{2}}{\mathrm{~N}(\mathrm{n})}$ & 0.02622 & $\begin{array}{ll}0.01368 \\
\end{array}$ & 0.00817 & 0.02076 & 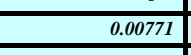 & 0.00115 & 0.01050 & 0.00239 & 0.0015 & 0.00165 & $\begin{array}{l}0.00655) \\
(0.06\end{array}$ & $\begin{array}{ll}(0.00826) \\
\end{array}$ & 0.00711 & $\begin{array}{l}(0.00143) \\
\end{array}$ & $(0.0117$ \\
\hline 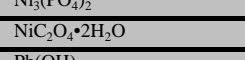 & & & & & & & & & & & & & & & \\
\hline $\mathrm{Pb}_{6}\left(\mathrm{PO}_{0}\right)_{2}$ & o.00816 & $\begin{array}{ll}0.00426 \\
\end{array}$ & 0.00254 & $\stackrel{0.0646}{=}$ & 0.00240 & 0.00036 & 0.00327 & 0.00074 & 0.00036 & 0.00051 & $(0.0220$ & $(0.00257)$ & 0.00221 & $(0.00045)$ & $(0.00$ \\
\hline PbCO & & & & & & & & & & & & & & & \\
\hline$\frac{\mathrm{Pu}(\mathrm{OH})_{4}}{\mathrm{Sig}}$ & 0.00024 & e.00001 & e.00001 & e.00001 & 0.00001 & $\begin{array}{l}0.00000 \\
\end{array}$ & 0.00001 & 0.00000 & 0.00000 & 0.00000 & $(0.000000)$ & $\begin{array}{ll}(0.00001) \\
\end{array}$ & 0.00001 & $(0.000000)$ & $\frac{0.0 .}{0.0}$ \\
\hline 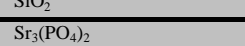 & & & & & & & & & & & & & & & \\
\hline 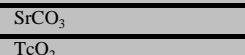 & 0.00134 & $\begin{array}{ll}0.00070 \\
0.00004\end{array}$ & $\begin{array}{l}0.00042 \\
0.0002\end{array}$ & $\begin{array}{ll}0.00106 \\
0.0006\end{array}$ & . 0.00039 & $\begin{array}{l}0.00006 \\
0.0000\end{array}$ & .0.0054 & .0.00012 & $\begin{array}{l}0.00006 \\
0.0000\end{array}$ & 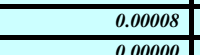 & $\begin{array}{ll}(0.00033) \\
0.000202\end{array}$ & $\begin{array}{ll}(0.00042) \\
(0.00202)\end{array}$ & $\begin{array}{l}0.00036 \\
0.0002\end{array}$ & $\frac{0.00007)}{0.000000}$ & $\frac{(0.00)}{(0.00)}$ \\
\hline 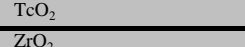 & 0.00007 & 0.00004 & 0.00002 & 0.00006 & 0.00002 & 0.00000 & 0.00003 & 0.00001 & 0.00000 & 0.00000 & & & & & \\
\hline $\mathrm{KNO}_{3}$ & & & & & & & & & & & & & & & \\
\hline $\mathrm{N}_{2} \mathrm{C}_{2} \mathrm{O}_{4}$ & 7.99150 & 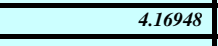 & 2.48963 & $\begin{array}{c}6.32719 \\
\end{array}$ & 2.350055 & 0.35151 & $\begin{array}{ll}3.20095 & -12\end{array}$ & 0.02852 & 0.35151 & & & & & & \\
\hline$\frac{\mathrm{N}_{2} \mathrm{~N}_{2} \mathrm{O}_{3} \mathrm{~S}_{2} \mathrm{O}}{\mathrm{N}_{2} \mathrm{O}_{4}}$ & & & & & & & & & & & & & & & \\
\hline 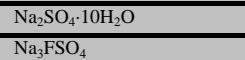 & - & & $\div$ & - & Z & & ב & & & & & & & & \\
\hline $\begin{array}{ll}\mathrm{Na}_{3} \mathrm{NO}_{3} \mathrm{SO}_{4} \mathrm{H}_{2} \mathrm{O} \\
\end{array}$ & & & & & & & & & & & & & & & \\
\hline 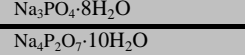 & & & & & & & & & & & & & & & \\
\hline $\mathrm{N}_{a_{6}\left(\mathrm{SO}_{3}\right)_{2} \mathrm{CO}_{3}}$ & & & & & & & & & & & & & & & \\
\hline$\frac{\mathrm{NaF}}{\mathrm{NaF}\left(\mathrm{PO}_{4}\right)} \cdot 19 \mathrm{H}_{2} \mathrm{O}$ & 0.66512 & $\overrightarrow{0.34702}$ & $\overline{0.20721}$ & 0.526600 & o.1956 & D.02926 & 0.26641 & e.06063 & $\stackrel{\square}{a .02926}$ & & & & & & \\
\hline$\frac{\mathrm{NaHCO}_{3}}{\mathrm{NaNON}_{2}}$ & & & & & & & & & & & & & & & \\
\hline & 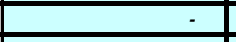 & & & $\bar{n}$ & & & & & & & & & & & \\
\hline 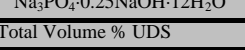 & $\frac{3.52144}{26.53}$ & 1.3.3706 & 1.09692 & 2.78775 & $\begin{array}{l}1.035378 \\
7.80\end{array}$ & 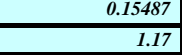 & 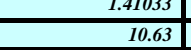 & 告.32099 & 每.54877 & $0.90 \mid$ & (3.59)] & (52)] & 3.89 & $\frac{(0.78)}{(0.78)}$ & $(6.4 .4)$ \\
\hline Solids Concentration (g1) & 625.3. & 326.2] & $194.8 \mathrm{P}$ & 495.0) & 183.9| & 27.5 & $250.4 \mid$ & 5. & 27.5 & 23.3 & (92.4)] & (116.5) & 00.2 & $(20.2)]$ & 165. \\
\hline
\end{tabular}


Rheological Dat

\begin{tabular}{|c|c|c|c|c|c|c|c|c|c|c|c|c|c|c|c|}
\hline $\begin{array}{l}\text { Reoological Data } \\
\text { Model/mode Parameter }\end{array}$ & $\begin{array}{l}\overline{Y Y}-101 \\
\end{array}$ & SY-101 & $\overline{\text { SY-101 }}$ & $\overline{\mathrm{SY}-101}$ & $\overline{\mathrm{SY}-101}$ & $\overline{\text { SY-101 }}$ & strout & SY-101 & $\overline{\overline{\text { SY-101 }}}$ & SY-101 & SY-101 & $\frac{\overline{S Y-101}}{S-1}$ & $\overline{\text { SY-101 }}$ & $\frac{S Y-101}{S Y}$ & $\overline{\overline{\text { SYY-101 }}}$ \\
\hline Temperature $\left({ }^{\circ} \mathrm{C}\right)$ & 50 & 70 & 90 & 50 & 70 & 90 & 50 & 70 & 90 & 50 & 70 & 90 & 50 & 70 & 90 \\
\hline Source of Data & PNNL-10198 & PNNL-10198 & PNNL-10198 & PNNL-10198 & PNNL-10198 & PNNL-10198 & PNNL-10198 & PNNL-10198 & PNNL-10198 & PNNL-10198 & PNNL-10198 & PNNL-10198 & PNNL-10198 & PNNL-10198 & PNNL-10198 \\
\hline \multicolumn{16}{|l|}{ 甚otes } \\
\hline$\frac{\text { Newomanan }}{n-\text { Newtonian viscosity (CP) }}$ & $\overline{96.92}$ & $\overline{74.24}$ & 72.35 & 89.47 & 6190 & 27.16 & $\overline{30.43}$ & 31.68 & 26.33 & $\overline{17.46}$ & 1570 & 970 & 746 & $775,2>>1$ & 386 \\
\hline 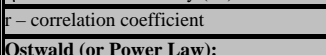 & 1.00 & 1.00 & 0.99 & & 1.00 & 0.99 & & & 0.99 & 1.00 & 1.00 & & 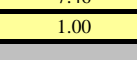 & 1.00 & - \\
\hline \multicolumn{15}{|l|}{ 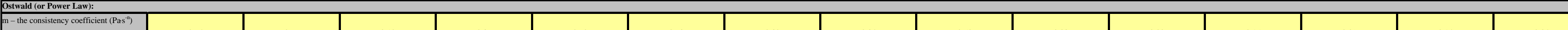 } & \\
\hline 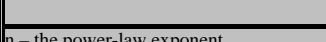 & 0.64 & 0.76 & 2.12 & 0.36 & 0.92 & 0.63 & 0.32 & 0.34 & 0.52 & 0.08 & . 0.22 & 0.15 & 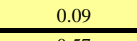 & 0.14 & 0.04 \\
\hline 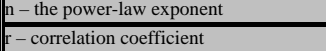 & 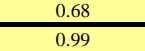 & 0.609 & 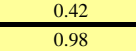 & $\frac{0.76}{1.00}$ & 0.0 .94 & $\frac{0.46}{0.97}$ & 0.609 & 0.009 & $\frac{0.49}{0.98}-3 x-3$ & $\frac{0.75}{0.99}$ & $\frac{0.55}{0.97}$ & 0.0 .93 & 0.0 .97 & 0.51 & 0.59 \\
\hline Bingham Plastic: & {[} & {[} & {[} & {[} & {[} & {[} & {[} & {[} & {[} & {[} & {[} & {[} & - & & \\
\hline $\mid$ & 5.07 & 5.08 & 8.15 & 每399 & 5.20 & 2.68 & 1.31 & 2.17 & 2.63 & 0.71 & 1.29 & 0.81 & 0.57 & 0.70 & 0.27 \\
\hline \multirow{2}{*}{\multicolumn{16}{|c|}{ 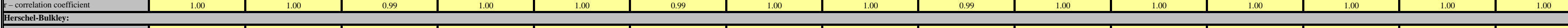 }} \\
\hline & & & & & & & & & & & & & & & \\
\hline 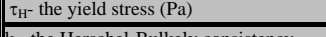 & 4.12 & 4.35 & 6.09 & 2.65 & 4.04 & 2.32 & 1.02 & 1.59 & 2.06 & 0.46 & 1.26 & 0.71 & 0.51 & 0.65 & 0.26 \\
\hline 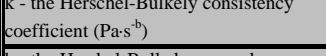 & 0.15 & 0.11 & 0.28 & 0.13 & 0.15 & 0.049 & 0.047 & 0.074 & 0.073 & 0.033 & 0.013 & 0.014 & 0.010 & 0.009 & 0.003 \\
\hline $\begin{array}{l}\text { b- - the Herthel-Bulkely power-law } \\
\text { exponent }\end{array}$ & 0.90 & 0.90 & 0.72 & 0.92 & 0.81 & 0.85 & 0.91 & 0.83 & 0.78 & 0.88 & 0.98 & 0.89 & 0.91 & 0.93 & 0.98 \\
\hline 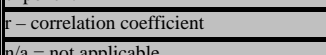 & 100 & & & & & 100 & & & 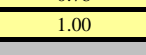 & & & & & 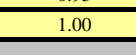 & \\
\hline
\end{tabular}

Notes

Netes

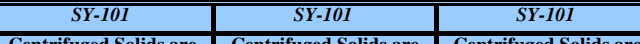

(1) Potential( (Iiv)

wolos Sertede solids

Wro Setled Solids

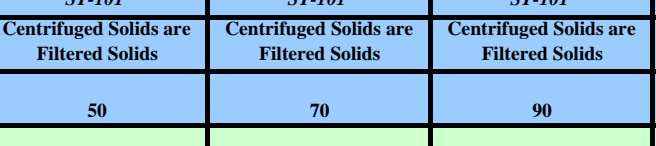

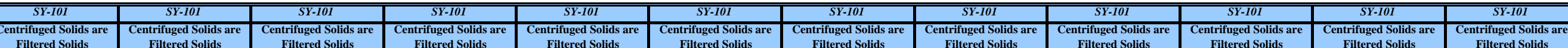
Mog Cenrifituged Solid

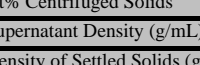

wo\% Serted Supenatant

wo\% dissolved solids in supermatant

1\%\% total solid in Centrifuged Slugge

No\% Toat Solids

W1\% UDS

Density of solid (gyc)

\begin{tabular}{|c|c|c|}
\hline 1.72 & 1.61 & 1.58 \\
\hline & 100 & 100 \\
\hline$\frac{1.74}{1.74}$ & 1.7 & 1.74 \\
\hline & & \\
\hline${ }^{83}$ & 71 & 66 \\
\hline$\frac{1.49}{1.49}$ & 1.49 & 1.51 \\
\hline$\frac{1.72}{\mathrm{n} / \mathrm{m}}$ & $\frac{1.63}{\mathrm{n} m}$ & $\frac{1.58}{n \mathrm{~nm}}$ \\
\hline $\mathrm{n}^{\mathrm{n} m}$ & $\mathrm{n} / \mathrm{m}$ & $n^{n / m}$ \\
\hline $\mathrm{n} / \mathrm{m}$ & $\overline{n / m}$ & $n^{n / m}$ \\
\hline$n / m$ & $\mathrm{n} / \mathrm{m}$ & $\mathrm{n}^{\mathrm{n} / \mathrm{m}}$ \\
\hline 36.35 & 20.26 & 12,33 \\
\hline 2.36 & 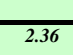 & 2.3 \\
\hline
\end{tabular}

\begin{tabular}{|c|c|c|c|c|}
\hline $\begin{array}{l}\text { Trtugea Sons ar } \\
\text { Filtered Solids }\end{array}$ & $\begin{array}{l}\text { Centiltered Solids } \\
\text { Fild }\end{array}$ & $\begin{array}{l}\text { Centringugesonins are } \\
\text { Filtered Solids }\end{array}$ & 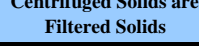 & 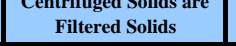 \\
\hline 50 & 70 & 90 & 50 & 70 \\
\hline 1.68 & 1.53 & 1.51 & 1.60 & 1.55 \\
\hline$\frac{100}{100}$ & 98 & 96 & 96 & 99 \\
\hline 1.68 & 1.61 & 1.66 & 1.73 & 1.86 \\
\hline 60 & 61 & 49 & 68 & \\
\hline 1.50 & 1.46 & 1.50 & 1.51 & \\
\hline 1.68 & 1.51 & $\frac{1}{1.55}$ & $\frac{1.59}{1.59}$ & $\frac{1.57}{1.57}$ \\
\hline n/m & & & & \\
\hline $\mathrm{n} / \mathrm{m}$ & $\mathrm{n} / \mathrm{m}$ & $\mathrm{n} / \mathrm{m}$ & $\mathrm{n} / \mathrm{m}$ & $\mathrm{n} / \mathrm{m}$ \\
\hline$\frac{\pi}{n / m}$ & $\frac{n}{n / m}$ & $\overline{n / m}$ & $\frac{n}{n / m}$ & $\frac{n}{n / m}$ \\
\hline $\mathrm{n} / \mathrm{m}$ & $\mathrm{n} / \mathrm{m}$ & $\overline{n l m}$ & $n^{\prime \prime m}$ & $\frac{n}{n / m}$ \\
\hline 29.47 & $\frac{12.02}{1202}$ & $\overline{1.82}$ & 15.65 & 3.68 \\
\hline 2.36 & 2.36 & 2.36 & 2.36 & 2.36 \\
\hline
\end{tabular}

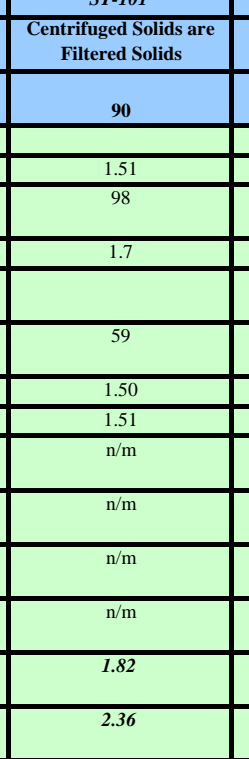

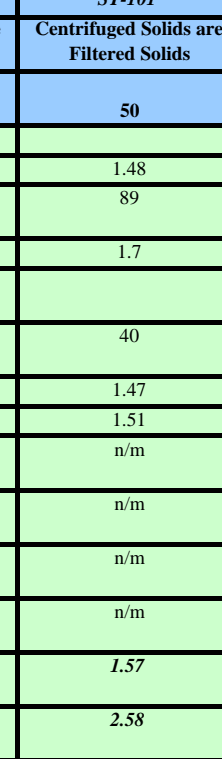

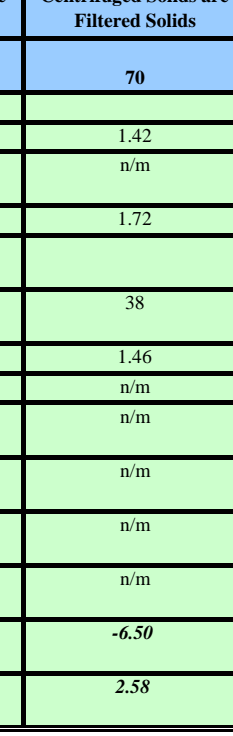

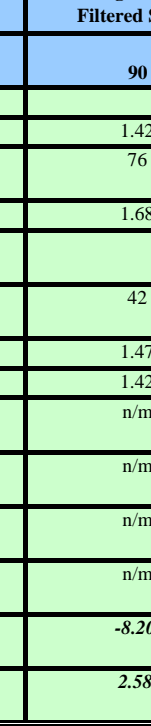

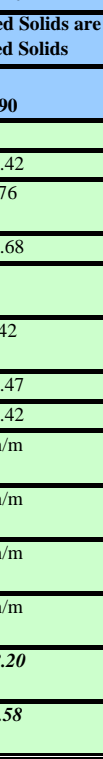

\begin{tabular}{|c|c|c|}
\hline $\begin{array}{l}\text { Fituged ofidids are } \\
\text { Filterd Solds }\end{array}$ & $\begin{array}{l}\text { Centrituged Solidis are } \\
\text { Filtered Solids }\end{array}$ & $\begin{array}{c}\text { Centrifuged Solisisare } \\
\text { Fitlered Solids }\end{array}$ \\
\hline 50 & 70 & 90 \\
\hline 1.34 & 1.29 & 1.25 \\
\hline 34 & ${ }^{34}$ & 33 \\
\hline 1.92 & 1.65 & 1.93 \\
\hline & & \\
\hline 25 & 24 & 24 \\
\hline$\frac{1.29}{1.22}$ & $\frac{1.30}{1.38}$ & $\frac{1.33}{1.25}$ \\
\hline$\frac{n}{n / m}$ & $\frac{n}{n / m}$ & $\mathrm{n} / \mathrm{m}$ \\
\hline$\frac{n}{n / m}$ & $\frac{n}{n / m}$ & $\mathrm{n} / \mathrm{m}$ \\
\hline$\frac{n}{n / m}$ & $\mathrm{~nm}$ & $\mathrm{~nm}$ \\
\hline $\mathrm{n} / \mathrm{m}$ & $\frac{n}{n / m}$ & $\mathrm{n} / \mathrm{m}$ \\
\hline$\overline{7.48}$ & -1.57 & -13.23 \\
\hline 2.58 & 2.58 & 2.58 \\
\hline
\end{tabular}




\begin{tabular}{|c|c|c|c|c|c|c|c|c|c|c|c|c|c|c|c|}
\hline \multirow{2}{*}{\multicolumn{16}{|c|}{$\begin{array}{l}\text { Shear Strength Data } \\
\text { Shear Strength Schedule }\end{array}$}} \\
\hline & $\begin{array}{l}S Y-101 \\
\end{array}$ & $\begin{array}{l}Y Y-101 \\
\end{array}$ & $\begin{array}{l}Y Y-101 \\
\end{array}$ & $\begin{array}{l}S Y-101 \\
\end{array}$ & $\begin{array}{l}S Y Y-101 \\
\end{array}$ & $\begin{array}{l}Y Y-101 \\
\end{array}$ & $\begin{array}{ll}S Y-101 \\
5\end{array}$ & \begin{tabular}{c|c|c|}
$S Y-101$ \\
\end{tabular} & $\begin{array}{l}S Y-101 \\
\end{array}$ & $\begin{array}{ll}S Y-101 \\
\end{array}$ & \begin{tabular}{c|c|c|} 
SY-101 \\
\end{tabular} & 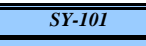 & $\begin{array}{l}S Y-101 \\
S\end{array}$ & \begin{tabular}{c|c|c|}
$S Y-101$ \\
\end{tabular} & $\begin{array}{ll}Y Y-101 \\
\end{array}$ \\
\hline Source of Data & PNL-10198 & PNL-10198 & PNL-10198 & PNL-10198 & PNL-10198 & PNL-10198 & PNL-10198 & PNL-10198 & PNL-10198 & PNL-10198 & PNL-10198 & PNL-10198 & PNL-10198 & PNL-10198 & PNL-10198 \\
\hline $\begin{array}{l}\text { Notes: } \\
\text { Temperature }\left({ }^{\circ} \mathrm{C}\right)\end{array}$ & 50 & & & 50 & & & 50 & 50 & 50 & 50 & 50 & 50 & 50 & 50 & 50 \\
\hline Descripion & \begin{tabular}{|c} 
Window C \\
Segment 16, 16R, \& 18 \\
Composite
\end{tabular} & & & $\begin{array}{c}\text { Window C } \\
\text { Composite } \\
10 \% \text { Diltution }\end{array}$ & & & & & & & & & & & \\
\hline \multirow{2}{*}{\multicolumn{16}{|c|}{ 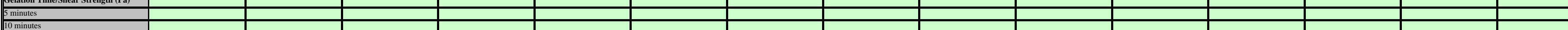 }} \\
\hline & & & & & & & & & & & & & & & \\
\hline \multicolumn{16}{|l|}{ 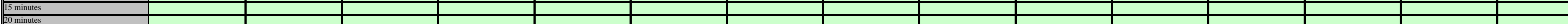 } \\
\hline \multirow{2}{*}{\multicolumn{16}{|c|}{$\begin{array}{l}30 \text { minutes } \\
40 \text { minutes }\end{array}$}} \\
\hline & & & & & & & & & & & & & & & \\
\hline \multicolumn{16}{|l|}{ 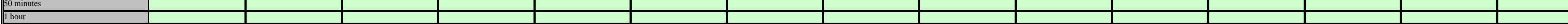 } \\
\hline \multicolumn{16}{|l|}{$\frac{2 \text { hours }}{\text { Bhours }}$} \\
\hline \multirow{2}{*}{\multicolumn{16}{|c|}{$\begin{array}{l}4 \frac{4 \text { hours }}{4 \text { Shours }} \\
\end{array}$}} \\
\hline & & & & & & & & & & & & & & & \\
\hline \multicolumn{16}{|l|}{ 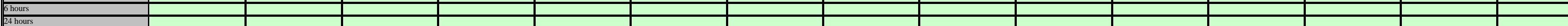 } \\
\hline \multirow{2}{*}{\multicolumn{16}{|c|}{$\frac{3 \text { 2hours }}{48 \text { hours }}$}} \\
\hline & & & & & & & & & & & & & & & \\
\hline 2 h hours & 60 & & & 1110 & & & & & & & & & & & \\
\hline & & & & & & & & & & & & & & & \\
\hline
\end{tabular}


241-SY-102

Model Chemical Data From ESP

\begin{tabular}{|c|c|c|c|}
\hline AnalytelSample & SY-102 & SY-102 & SY-102 \\
\hline Description & Undiluted & $1 \%$ Dilution with $\mathrm{NaNO} 3$ & $\begin{array}{c}48 \% \text { Dilution with 1M } \\
\text { NaNO3 }\end{array}$ \\
\hline Model Basis & Salty & Salty & Salt Free \\
\hline Report No/Source of Data & PNNL-11352 & PNNL-11352 & PNNL-11352 \\
\hline \multicolumn{4}{|l|}{ Solid Phases (vol\% of bulk) } \\
\hline$\left(\mathrm{NaAlO}_{2}\right)_{2} \cdot 2.5 \mathrm{H}_{2} \mathrm{O}$ & - & - & - \\
\hline $\mathrm{Ag}_{2} \mathrm{CO}_{3}$ & - & - & - \\
\hline $\mathrm{Bi}_{2} \mathrm{O}_{3}$ & 0.12803 & 0.12504 & 0.04863 \\
\hline $\mathrm{BiOCl}$ & - & - & - \\
\hline Boehmite & - & - & - \\
\hline $\mathrm{Ca}(\mathrm{OH})_{2}$ & - & - & - \\
\hline $\mathrm{Ca}_{5} \mathrm{OH}\left(\mathrm{PO}_{4}\right)_{3}$ & 0.37952 & 0.37067 & 0.14416 \\
\hline $\mathrm{CaC}_{2} \mathrm{O}_{4} \cdot \mathrm{H}_{2} \mathrm{O}$ & - & - & - \\
\hline $\mathrm{CaCO}_{3}$ & - & - & - \\
\hline $\mathrm{CaF}_{2}$ & - & - & - \\
\hline $\mathrm{CrOOH}$ & 1.49365 & 1.45881 & 0.56734 \\
\hline $\mathrm{FeOOH}$ & 1.03054 & 1.00650 & 0.39144 \\
\hline Gibbsite & 25.37101 & 24.77916 & 9.63684 \\
\hline $\mathrm{HgO}$ & - & - & - \\
\hline $\mathrm{KAlSiO}_{4}$ & - & - & - \\
\hline $\mathrm{La}(\mathrm{OH})_{3}$ & - & - & - \\
\hline $\mathrm{LaPO}_{4} \cdot 2 \mathrm{H}_{2} \mathrm{O}$ & 0.01280 & 0.01250 & 0.00486 \\
\hline $\mathrm{Mn}(\mathrm{OH})_{2}$ & 0.17399 & 0.16994 & 0.06609 \\
\hline $\mathrm{Mn}_{3}\left(\mathrm{PO}_{4}\right)_{2}$ & - & - & - \\
\hline $\mathrm{MnCO}_{3}$ & - & - & - \\
\hline $\mathrm{Na}_{2} \mathrm{U}_{2} \mathrm{O}_{7}$ & 0.05988 & 0.05848 & 0.02274 \\
\hline $\mathrm{NaAlCO}_{3}(\mathrm{OH})_{2}$ & - & - & - \\
\hline $\mathrm{NaAlSiO}_{4}$ & - & - & - \\
\hline $\mathrm{Ni}(\mathrm{OH})_{2}$ & 0.04675 & 0.04566 & 0.01776 \\
\hline $\mathrm{Ni}_{3}\left(\mathrm{PO}_{4}\right)_{2}$ & - & - & - \\
\hline $\mathrm{NiC}_{2} \mathrm{O}_{4} \cdot 2 \mathrm{H}_{2} \mathrm{O}$ & - & - & - \\
\hline$\overline{\mathrm{Pb}(\mathrm{OH})_{2}}$ & 0.03699 & 0.03613 & 0.01405 \\
\hline $\mathrm{Pb}_{3}\left(\mathrm{PO}_{4}\right)_{2}$ & - & - & - \\
\hline $\mathrm{PbCO}_{3}$ & - & - & - \\
\hline $\mathrm{Pu}(\mathrm{OH})_{4}$ & 0.00504 & 0.00492 & 0.00191 \\
\hline $\mathrm{SiO}_{2}$ & - & - & - \\
\hline $\mathrm{Sr}_{3}\left(\mathrm{PO}_{4}\right)_{2}$ & - & - & - \\
\hline $\mathrm{SrCO}_{3}$ & 0.00791 & 0.00773 & 0.00301 \\
\hline $\mathrm{TcO}_{2}$ & 0.00018 & 0.00017 & 0.00007 \\
\hline $\mathrm{ZrO}_{2}$ & 0.00173 & 0.00169 & 0.00066 \\
\hline $\mathrm{KNO}_{3}$ & - & - & - \\
\hline $\mathrm{Na}_{2} \mathrm{C}_{2} \mathrm{O}_{4}$ & 8.07249 & 7.88417 & - \\
\hline $\mathrm{Na}_{2} \mathrm{CO}_{3} \cdot \mathrm{H}_{2} \mathrm{O}$ & - & - & - \\
\hline $\mathrm{Na}_{2} \mathrm{SO}_{4}$ & - & - & - \\
\hline $\mathrm{Na}_{2} \mathrm{SO}_{4} \cdot 10 \mathrm{H}_{2} \mathrm{O}$ & - & - & - \\
\hline $\mathrm{Na}_{3} \mathrm{FSO}_{4}$ & - & - & - \\
\hline $\mathrm{Na}_{3} \mathrm{NO}_{3} \mathrm{SO}_{4} \cdot \mathrm{H}_{2} \mathrm{O}$ & - & - & - \\
\hline $\mathrm{Na}_{3} \mathrm{PO}_{4} \cdot 8 \mathrm{H}_{2} \mathrm{O}$ & - & - & - \\
\hline $\mathrm{Na}_{4} \mathrm{P}_{2} \mathrm{O}_{7} \cdot 10 \mathrm{H}_{2} \mathrm{O}$ & - & - & - \\
\hline $\mathrm{Na}_{6}\left(\mathrm{SO}_{4}\right)_{2} \mathrm{CO}_{3}$ & - & - & - \\
\hline $\mathrm{NaF}$ & - & - & - \\
\hline $\mathrm{NaF}\left(\mathrm{PO}_{4}\right)_{2} \cdot 19 \mathrm{H}_{2} \mathrm{O}$ & 3.09380 & 3.02163 & - \\
\hline $\mathrm{NaHCO}_{3}$ & - & - & - \\
\hline $\mathrm{NaNO}_{2}$ & - & - & - \\
\hline $\mathrm{NaNO}_{3}$ & - & - & - \\
\hline $\mathrm{Na}_{3} \mathrm{PO}_{4} \cdot 0.25 \mathrm{NaOH} \cdot 12 \mathrm{H}_{2} \mathrm{O}$ & 3.94679 & 3.85472 & - \\
\hline Total Volume \% UDS & 43.86 & 42.84 & 10.92 \\
\hline Solids Concentration (g/L) & $1,081.4$ & $1,056.2$ & 294.2 \\
\hline
\end{tabular}

Note: Numbers in italics are calculated values. Numbers in normal font are reported data. 
241-SY-102

Rheological Data

\begin{tabular}{|c|c|c|c|}
\hline Model/model Parameter & SY-102 & SY-102 & SY-102 \\
\hline Temperature $\left({ }^{\circ} \mathrm{C}\right)$ & 25 & 25 & 25 \\
\hline Source of Data & PNNL-11352 & PNNL-11352 & PNNL-11352 \\
\hline Notes & Page 2.8 & Page 2.8 & Page 2.8 \\
\hline \multicolumn{4}{|l|}{ Newtonian: } \\
\hline$\eta$ - Newtonian viscosity (cP) & 16.725 & 16.022 & 0.893 \\
\hline $\mathrm{r}$ - correlation coefficient & 0.959 & 0.991 & 0.980 \\
\hline \multicolumn{4}{|l|}{ Ostwald (or Power Law): } \\
\hline $\mathrm{m}$ - the consistency coefficient $\left(\mathrm{Pa} \cdot \mathrm{s}^{-\mathrm{n}}\right)$ & 1.764 & 1.188 & 0.003 \\
\hline $\mathrm{n}$ - the power-law exponent & 0.135 & 0.201 & 0.782 \\
\hline $\mathrm{r}$ - correlation coefficient & 0.929 & 0.915 & 0.992 \\
\hline \multicolumn{4}{|l|}{ Bingham Plastic: } \\
\hline$\tau_{\mathrm{B}}$ - the Bingham yield stress $(\mathrm{Pa})$ & 2.602 & 2.120 & 0.010 \\
\hline$\eta_{p}$ - the plastic viscosity (Pa-s) & 0.005 & 0.006 & 0.001 \\
\hline $\mathrm{r}-$ correlation coefficient & 0.959 & 0.991 & 0.980 \\
\hline \multicolumn{4}{|l|}{ Herschel-Bulkley: } \\
\hline$\tau_{\mathrm{H}^{-}}$the yield stress $(\mathrm{Pa})$ & 2.377 & 1.882 & 0.000 \\
\hline $\begin{array}{l}\mathrm{k} \text { - the Herschel-Bulkely consistency } \\
\text { coefficient }\left(\mathrm{Pa} \cdot \mathrm{s}^{-\mathrm{b}}\right)\end{array}$ & 0.039 & 0.038 & 0.003 \\
\hline $\begin{array}{l}\text { b - the Hershel-Bulkely power-law } \\
\text { exponent }\end{array}$ & 0.647 & 0.699 & 0.785 \\
\hline $\mathrm{r}$ - correlation coefficient & 0.975 & 0.989 & 0.992 \\
\hline $\mathrm{n} / \mathrm{a}=$ not applicable & & & \\
\hline
\end{tabular}

\section{Physical Property Data}

\begin{tabular}{||l|c|c|c|}
\hline Physical Property & SY-102 & SY-102 & SY-102 \\
\hline Notes & $\begin{array}{c}\text { Letter Report-1990 } \\
\text { RD Scheele } \\
\text { PNNL-11352 }\end{array}$ & & \\
\hline Temperature $\left({ }^{\circ} \mathbf{C}\right)$ & 25 & 25 & 25 \\
\hline Zeta Potential (mV) & -37 & & $\mathbf{1 . 7 9}$ \\
\hline Bulk Density (g/mL) & 1.80 & $\mathrm{n} / \mathrm{m}$ & $\mathrm{n} / \mathrm{m}$ \\
\hline vol\% Settled Solids & 100 & $\mathrm{n} / \mathrm{m}$ & $\mathrm{n} / \mathrm{m}$ \\
\hline Density of Centrifuged Solids (g/mL) & 1.83 & $\mathrm{n} / \mathrm{m}$ & $\mathrm{n} / \mathrm{m}$ \\
\hline vol\% Centrifuged Solids & 92 & $\mathrm{n} / \mathrm{m}$ & $\mathrm{n} / \mathrm{m}$ \\
\hline wt\% Centrifuged Solids & 93.93 & $\mathbf{1 . 2 8}$ & $\mathbf{1 . 1 3}$ \\
\hline Supernatant Density (g/mL) & 1.28 & $\mathrm{n} / \mathrm{m}$ & $\mathrm{n} / \mathrm{m}$ \\
\hline Density of Settled Solids (g/mL) & $\mathrm{n} / \mathrm{m}$ & $\mathrm{n} / \mathrm{m}$ & $\mathrm{n} / \mathrm{m}$ \\
\hline wt\% Settled Supernatant & $\mathrm{n} / \mathrm{m}$ & $\mathrm{n} / \mathrm{m}$ & $\mathrm{n} / \mathrm{m}$ \\
\hline wt\% dissolved solids in supernatant & $\mathrm{n} / \mathrm{m}$ & $\mathrm{n} / \mathrm{m}$ & $\mathrm{n} / \mathrm{m}$ \\
\hline wt\% total solids in Centrifuged Sludge & 67.3 & 59.14 & $\mathbf{2 2 . 6 1}$ \\
\hline wt\% Total Solids & $\mathbf{6 0 . 0 8}$ & & \\
\hline wt\% UDS & & & \\
\hline Density of Solids (g/cc) & & & \\
\hline \hline
\end{tabular}

Note: Numbers in italics are calculated values. Numbers in normal font are reported data. 
241-SY-102

\section{Shear Strength Data}

\begin{tabular}{||l|c|l|l|}
\hline Shear Strength Schedule & SY-102 & & \\
\hline Source of Data & PNNL-11352 & & \\
\hline Notes: & $\begin{array}{c}\text { reference of DiCenso et } \\
\text { al. 1995 }\end{array}$ & & \\
\hline Temperature $\left(^{\circ}\right.$ C) & & & \\
\hline Description & Page 2.11 & & \\
\hline Gelation Time/Shear Strength (Pa) & & & \\
\hline 5 minutes & & & \\
\hline 10 minutes & & & \\
\hline 15 minutes & & & \\
\hline 20 minutes & & & \\
\hline 30 minutes & & & \\
\hline 40 minutes & & & \\
\hline 50 minutes & & & \\
\hline 1 hour & & & \\
\hline 2 hours & & & \\
\hline 3 hours & & & \\
\hline 4 hours & & & \\
\hline 5 hours & & & \\
\hline 6 hours & & & \\
\hline 24 hours & & & \\
\hline 32 hours & & & \\
\hline 48 hours & & & \\
\hline 56 hours & & & \\
\hline 72 hours & & & \\
\hline Infinte time (GRE) & & & \\
\hline
\end{tabular}

Note: Numbers in italics are calculated values. Numbers in normal font are reported data. 
Model Chemical Data From ESP

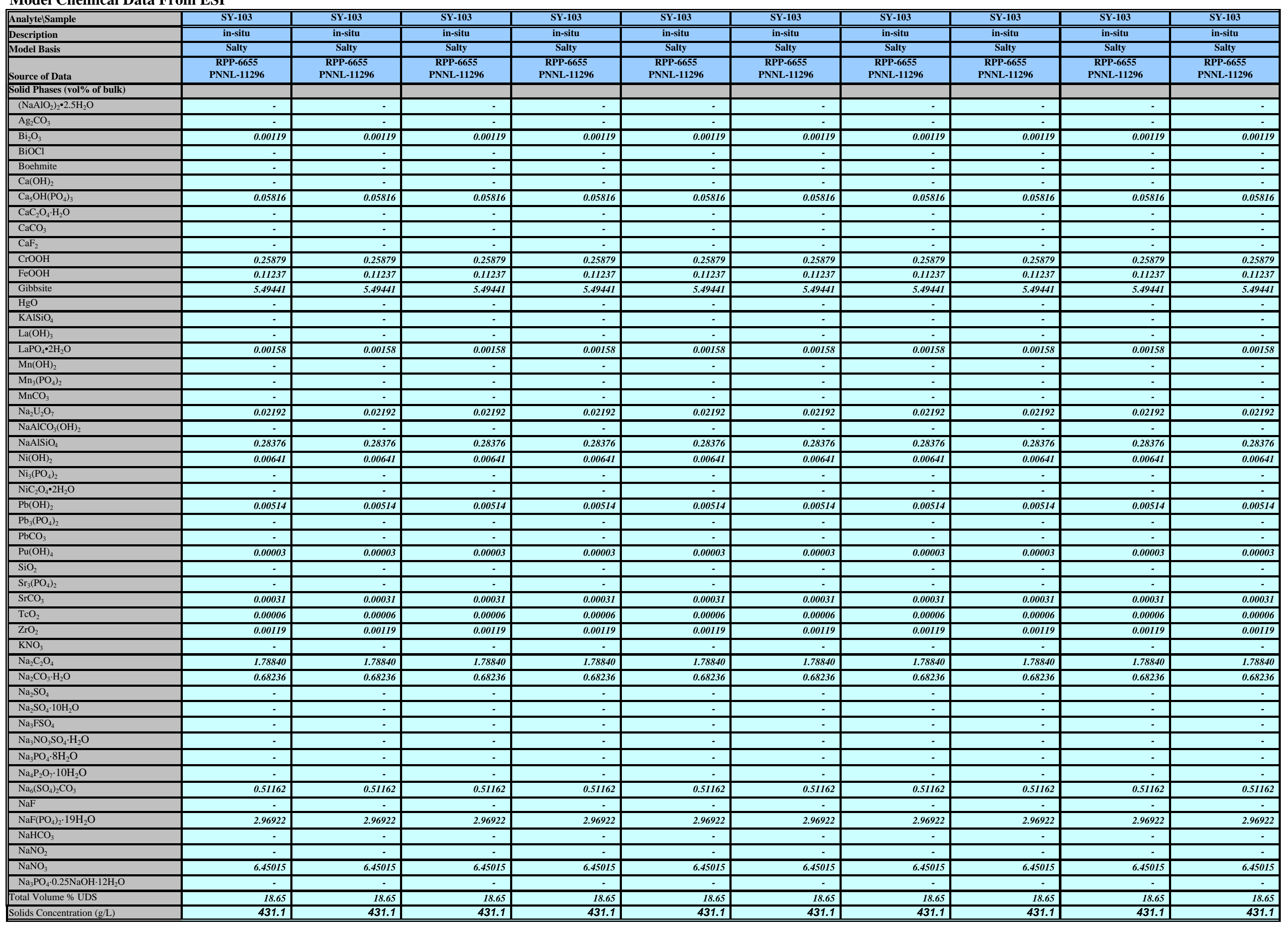




\begin{tabular}{|c|c|c|c|c|c|c|c|c|c|c|}
\hline \multicolumn{11}{|c|}{ Physical Property Data } \\
\hline \begin{tabular}{|l|l|} 
Physical Property \\
\end{tabular} & $\begin{array}{ll}S Y-103 \\
Y\end{array}$ & SYY-103 & 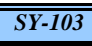 & 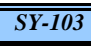 & 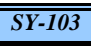 & 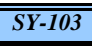 & $\begin{array}{cl}Y-103 \\
\end{array}$ & $\begin{array}{cl}S Y-103 \\
\end{array}$ & 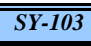 & 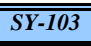 \\
\hline \multicolumn{11}{|l|}{ Source of Data } \\
\hline \multicolumn{11}{|l|}{$\begin{array}{l}\text { Temperature }\left({ }^{\circ} \mathrm{C}\right) \\
\text { Zeta Potential }(\mathrm{mV})\end{array}$} \\
\hline \begin{tabular}{|l} 
Zeta Potential $(\mathrm{mV})$ \\
Bunk Densitg(g/L)
\end{tabular} & 1,57 & 157 & 1,57 & 157 & 157 & 157 & 157 & 157 & 157 & 157 \\
\hline \multirow{2}{*}{\multicolumn{11}{|c|}{$\begin{array}{l}\text { vol\% Settled Solids } \\
\text { Density of Centififuged Solids (g/mL) }\end{array}$}} \\
\hline & & & & & & & & & & \\
\hline \multicolumn{11}{|l|}{ vol\% Centrifuged Solids } \\
\hline \multicolumn{11}{|l|}{ w\%\% Centrifuged Solids } \\
\hline \begin{tabular}{|l} 
Supermatant Density $(\mathrm{g} / \mathrm{mL})$ \\
Density of Settled Solids $(\mathrm{g} / \mathrm{mL})$
\end{tabular} & 1.40 & 1.40 & 1.40 & 1.40 & 1.40 & 1.40 & 1.40 & 1.40 & 1.40 & 1.40 \\
\hline \multicolumn{11}{|l|}{ w\% Settled Supernatant } \\
\hline \multicolumn{11}{|l|}{ wt\% dissolved solids in supernatant } \\
\hline \multicolumn{11}{|l|}{ ww\% total solids in Centrifuged Sludge } \\
\hline \multicolumn{11}{|l|}{ ww\% Total Solids } \\
\hline${ }_{w 1 \%}$ UDS & 27.46 & 27.46 & $\begin{array}{ll}27.46 \\
\end{array}$ & $\begin{array}{ll}27.46 \\
\end{array}$ & $\begin{array}{ll}27.46 \\
\end{array}$ & $\begin{array}{ll}27.46 \\
\end{array}$ & 27.46 & 27.46 & $\begin{array}{ll}27.46 \\
\end{array}$ & $\begin{array}{ll}27.46 \\
\end{array}$ \\
\hline Density of Solids (g/cc) & 2.31 & 2.31 & 2.31 & 2.31 & 2.31 & 2.31 & 2.31 & 2.31 & 2.31 & 2.31 \\
\hline
\end{tabular}

\begin{tabular}{|c|c|c|c|c|c|c|c|c|c|c|}
\hline \multicolumn{11}{|l|}{ Shear Strength Data } \\
\hline Shear Strength Schedule & SY-103 & SY-103 & SY-103 & SY-103 & & SY-103 & SY-103 & SY-103 & $\begin{array}{l}\text { SY-103 } \\
\end{array}$ & $\begin{array}{l}\text { SY-103 } \\
\end{array}$ \\
\hline Source of Data & $\begin{array}{c}\text { RPP-6655 } \\
\text { PNNL-11296 }\end{array}$ & $\begin{array}{c}\text { RPP-6655 } \\
\text { PNNL-11296 }\end{array}$ & $\begin{array}{c}\text { RPP-6655 } \\
\text { PNNL-11296 }\end{array}$ & $\begin{array}{c}\text { RPP-6655 } \\
\text { PNNL-11296 }\end{array}$ & $\begin{array}{c}\text { RPP-6655 } \\
\text { PNNL-1296 }\end{array}$ & $\begin{array}{c}\text { RPP-6655 } \\
\text { PNNL-1296 }\end{array}$ & $\begin{array}{c}\text { RPP-6655 } \\
\text { PNNL-1296 }\end{array}$ & $\begin{array}{c}\text { RPP-6655 } \\
\text { PNNL-11296 }\end{array}$ & $\begin{array}{l}\text { RPP-6655 } \\
\text { PNNL11296 }\end{array}$ & $\begin{array}{l}\text { RPP-6655 } \\
\text { PNNL11296 }\end{array}$ \\
\hline Instrumentation & Ball Rheometer & Ball Rheometer & Ball Rheometer & Ball Rheometer & Ball Rheometer & Ball Rheometer & Ball Rheometer & Ball Rheometer & Ball Rheometer & Ball Rheometer \\
\hline Temperature $\left({ }^{\circ} \mathrm{C}\right)$ & & & & & & & & & & \\
\hline Description & $\begin{array}{c}\text { Riser 17C } \\
\text { Depth from botom of } \\
\text { tank is } 300 \mathrm{~cm}\end{array}$ & \begin{tabular}{|l|} 
Riser 17C \\
$\begin{array}{c}\text { Depht from bottom of } \\
\text { tank is } 250 \mathrm{~cm}\end{array}$
\end{tabular} & $\begin{array}{l}\text { Riser 17C } \\
\text { Depth from bottom of } \\
\text { tank is } 200 \mathrm{~cm}\end{array}$ & $\begin{array}{c}\text { Riser 17C } \\
\begin{array}{c}\text { Depht from bottom of } \\
\text { tank is } 150 \mathrm{~cm}\end{array}\end{array}$ & $\begin{array}{l}\text { Riser 17C } \\
\begin{array}{c}\text { Depth from bottom of } \\
\text { tank is } 100 \mathrm{~cm}\end{array}\end{array}$ & $\begin{array}{l}\text { Riser 22A } \\
\text { Depth from bottom of } \\
\text { tank is } 300 \mathrm{~cm}\end{array}$ & \begin{tabular}{|l|} 
Riser 22A \\
$\begin{array}{c}\text { Depth from bottom of } \\
\text { tank is } 250 \mathrm{~cm}\end{array}$
\end{tabular} & $\begin{array}{l}\text { Riser 22A } \\
\begin{array}{c}\text { Depth from bottom of } \\
\text { tank is } 200 \mathrm{~cm}\end{array}\end{array}$ & \begin{tabular}{|c|} 
Riser 22A \\
$\begin{array}{c}\text { Depth from bottom of } \\
\text { tank is } 150 \mathrm{~cm}\end{array}$
\end{tabular} & $\begin{array}{c}\text { Riser 22A } \\
\begin{array}{c}\text { Depth from botom of } \\
\text { tank is } 100 \mathrm{~cm}\end{array}\end{array}$ \\
\hline Gelation Time/Shear Strenggh (Pa & & & & & & & & & & \\
\hline 5 minutes & & & & & & & & & & \\
\hline 10 minutes & & & & & & & & & & \\
\hline 15 minutes & & & & & & & & & & \\
\hline 20 minutes & & & & & & & & & & \\
\hline $\begin{array}{l}30 \text { minutes } \\
40 \text { mintes }\end{array}$ & & & & & & & & & & \\
\hline 50 minutes & & & & & & & & & & \\
\hline 1 hour & & & & & & & & & & \\
\hline 2 hours & & & & & & & & & & \\
\hline 3 hours & & & & & & & & & & \\
\hline $\begin{array}{l}4 \text { hours } \\
5 \text { hours }\end{array}$ & & & 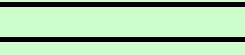 & 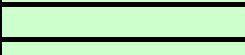 & 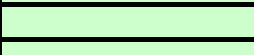 & & & & & \\
\hline$\frac{6 \text { hours }}{6 \text { hours }}$ & & & & & & & & & & \\
\hline 24 hours & & & & & & & & & & \\
\hline 32 hours & & & & & & & & & & \\
\hline $\begin{array}{ll}48 \text { hours } \\
56 \text { bowl }\end{array}$ & & & & & & & & & & \\
\hline $\begin{array}{l}56 \text { hours } \\
7 \text { hours }\end{array}$ & & & & & & & & & & \\
\hline Infinte ime (GRE) & 10 & 45 & 95 & 155 & 228 & 48 & 118 & 180 & 242 & 295 \\
\hline
\end{tabular}


241-T-102

Model Chemical Data From ESP

\begin{tabular}{|c|c|c|c|c|}
\hline AnalytelSample & T-102 & T-102 & 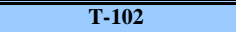 & T-102 \\
\hline Description & $\begin{array}{c}\text { Core } 55 \\
\text { Segment } 2 \text { 1:1 dilution }\end{array}$ & $\begin{array}{c}\text { Core 55 } \\
\text { Segment } 2 \text { 3:1 dilution }\end{array}$ & $\begin{array}{c}\text { Core } 55 \\
\text { Segment } 2 \text { 1:1 dilution }\end{array}$ & $\begin{array}{c}\text { Core 55 } \\
\text { Segment } 2 \text { 3:1 dilution }\end{array}$ \\
\hline Model Basis & Salt Free & Salt Free & Salt Free & Salt Free \\
\hline Report No/Source of Data & $\begin{array}{c}\text { HNF-SD-WM-ER-700 } \\
\text { Rev. } 0 \\
\text { WHC-SD-WM-DP-052 } \\
\text { Rev } 0\end{array}$ & $\begin{array}{c}\text { HNF-SD-WM-ER-700 } \\
\text { Rev. } 0 \\
\text { WHC-SD-WM-DP-052 } \\
\text { Rev } 0\end{array}$ & $\begin{array}{l}\text { HNF-SD-WM-ER-700 } \\
\text { Rev. 0 } \\
\text { WHC-SD-WM-DP-052 } \\
\text { Rev 0 }\end{array}$ & $\begin{array}{l}\text { HNF-SD-WM-ER-700 } \\
\text { Rev. } 0 \\
\text { WHC-SD-WM-DP-052 } \\
\text { Rev } 0\end{array}$ \\
\hline \multicolumn{5}{|l|}{ Solid Phases (vol\% of bulk) } \\
\hline$\left(\mathrm{NaAlO}_{2}\right)_{2} \cdot 2.5 \mathrm{H}_{2} \mathrm{O}$ & - & - & - & - \\
\hline $\mathrm{Ag}_{2} \mathrm{CO}_{3}$ & 0.00083 & 0.00037 & 0.00083 & 0.00037 \\
\hline $\mathrm{Bi}_{2} \mathrm{O}_{3}$ & 0.00292 & 0.00129 & 0.00292 & 0.00129 \\
\hline $\mathrm{BiOCl}$ & - & - & - & - \\
\hline Boehmite & - & - & - & - \\
\hline $\mathrm{Ca}(\mathrm{OH})_{2}$ & - & - & - & - \\
\hline $\mathrm{Ca}_{5} \mathrm{OH}\left(\mathrm{PO}_{4}\right)_{3}$ & 0.00901 & 0.00399 & 0.00901 & 0.00399 \\
\hline $\mathrm{CaC}_{2} \mathrm{O}_{4} \cdot \mathrm{H}_{2} \mathrm{O}$ & - & - & - & - \\
\hline $\mathrm{CaCO}_{3}$ & - & - & - & - \\
\hline $\mathrm{CaF}_{2}$ & - & - & - & - \\
\hline $\mathrm{CrOOH}$ & 0.00011 & 0.00005 & 0.00011 & 0.00005 \\
\hline $\mathrm{FeOOH}$ & 0.07697 & 0.03408 & 0.07697 & 0.03408 \\
\hline Gibbsite & 4.13662 & 1.83152 & 4.13662 & 1.83152 \\
\hline $\mathrm{HgO}$ & - & - & - & - \\
\hline $\mathrm{KAISiO}_{4}$ & - & - & - & - \\
\hline$\overline{\mathrm{La}(\mathrm{OH})_{3}}$ & - & - & - & - \\
\hline $\mathrm{LaPO}_{4} \cdot 2 \mathrm{H}_{2} \mathrm{O}$ & 0.00042 & 0.00019 & 0.00042 & 0.00019 \\
\hline $\mathrm{Mn}(\mathrm{OH})_{2}$ & - & - & - & - \\
\hline $\mathrm{Mn}_{3}\left(\mathrm{PO}_{4}\right)_{2}$ & 0.00897 & 0.00397 & 0.00897 & 0.00397 \\
\hline $\mathrm{MnCO}_{3}$ & - & - & - & - \\
\hline $\mathrm{Na}_{2} \mathrm{U}_{2} \mathrm{O}_{7}$ & 0.02984 & 0.01321 & 0.02984 & 0.01321 \\
\hline $\mathrm{NaAlCO}_{3}(\mathrm{OH})_{2}$ & 0.33528 & 0.14845 & 0.33528 & 0.14845 \\
\hline $\mathrm{NaAlSiO}_{4}$ & 0.07256 & 0.03213 & 0.07256 & 0.03213 \\
\hline$\overline{\mathrm{Ni}(\mathrm{OH})_{2}}$ & 0.00048 & 0.00021 & 0.00048 & 0.00021 \\
\hline $\mathrm{Ni}_{3}\left(\mathrm{PO}_{4}\right)_{2}$ & - & - & - & - \\
\hline $\mathrm{NiC}_{2} \mathrm{O}_{4} \cdot 2 \mathrm{H}_{2} \mathrm{O}$ & - & - & - & - \\
\hline$\overline{\mathrm{Pb}(\mathrm{OH})_{2}}$ & 0.00122 & 0.00054 & 0.00122 & 0.00054 \\
\hline $\mathrm{Pb}_{3}\left(\mathrm{PO}_{4}\right)_{2}$ & - & - & - & - \\
\hline $\mathrm{PbCO}_{3}$ & - & - & - & - \\
\hline$\overline{\mathrm{Pu}(\mathrm{OH})_{4}}$ & 0.00001 & 0.00000 & 0.00001 & 0.00000 \\
\hline $\mathrm{SiO}_{2}$ & - & - & - & - \\
\hline $\mathrm{Sr}_{3}\left(\mathrm{PO}_{4}\right)_{2}$ & - & - & - & - \\
\hline $\mathrm{SrCO}_{3}$ & 0.00018 & 0.00008 & 0.00018 & 0.00008 \\
\hline $\mathrm{TcO}_{2}$ & - & - & - & - \\
\hline $\mathrm{ZrO}_{2}$ & 0.00015 & 0.00007 & 0.00015 & 0.00007 \\
\hline $\mathrm{KNO}_{3}$ & - & - & - & - \\
\hline $\mathrm{Na}_{2} \mathrm{C}_{2} \mathrm{O}_{4}$ & - & - & - & - \\
\hline $\mathrm{Na}_{2} \mathrm{CO}_{3} \cdot \mathrm{H}_{2} \mathrm{O}$ & - & - & - & - \\
\hline $\mathrm{Na}_{2} \mathrm{SO}_{4}$ & - & - & - & - \\
\hline $\mathrm{Na}_{2} \mathrm{SO}_{4} \cdot 10 \mathrm{H}_{2} \mathrm{O}$ & - & - & - & - \\
\hline $\mathrm{Na}_{3} \mathrm{FSO}_{4}$ & - & - & - & - \\
\hline $\mathrm{Na}_{3} \mathrm{NO}_{3} \mathrm{SO}_{4} \cdot \mathrm{H}_{2} \mathrm{O}$ & - & - & - & - \\
\hline $\mathrm{Na}_{3} \mathrm{PO}_{4} \cdot 8 \mathrm{H}_{2} \mathrm{O}$ & - & - & - & - \\
\hline $\mathrm{Na}_{4} \mathrm{P}_{2} \mathrm{O}_{7} \cdot 10 \mathrm{H}_{2} \mathrm{O}$ & - & - & - & - \\
\hline $\mathrm{Na}_{6}\left(\mathrm{SO}_{4}\right)_{2} \mathrm{CO}_{3}$ & - & - & - & - \\
\hline $\mathrm{NaF}$ & - & - & - & - \\
\hline $\mathrm{NaF}\left(\mathrm{PO}_{4}\right)_{2} \cdot 19 \mathrm{H}_{2} \mathrm{O}$ & - & - & - & - \\
\hline $\mathrm{NaHCO}_{3}$ & - & - & - & - \\
\hline $\mathrm{NaNO}_{2}$ & - & - & - & - \\
\hline $\mathrm{NaNO}_{3}$ & - & - & - & - \\
\hline $\mathrm{Na}_{3} \mathrm{PO}_{4} \cdot 0.25 \mathrm{NaOH} \cdot 12 \mathrm{H}_{2} \mathrm{O}$ & - & - & - & - \\
\hline Total Volume \% UDS & 4.68 & 2.07 & 4.68 & 2.07 \\
\hline Solids Concentration (g/L) & 116.1 & 51.4 & 116.1 & 51.4 \\
\hline
\end{tabular}


Rheological Data

\begin{tabular}{|c|c|c|c|c|}
\hline Model/model Parameter & $T-102$ & $T-102$ & $T-102$ & $T-102$ \\
\hline Temperature $\left({ }^{\circ} \mathrm{C}\right)$ & 30 & 30 & 90 & 90 \\
\hline Source of Data & $\begin{array}{l}\text { HNF-SD-WM-ER-700 } \\
\text { Rev. } 0 \\
\text { WHC-SD-WM-DP-052 } \\
\text { Rev } 0\end{array}$ & $\begin{array}{l}\text { HNF-SD-WM-ER-700 } \\
\text { Rev. } 0 \\
\text { WHC-SD-WM-DP-052 } \\
\text { Rev } 0\end{array}$ & $\begin{array}{l}\text { HNF-SD-WM-ER-700 } \\
\text { Rev. } 0 \\
\text { WHC-SD-WM-DP-052 } \\
\text { Rev } 0\end{array}$ & $\begin{array}{l}\text { HNF-SD-WM-ER-700 } \\
\text { Rev. } 0 \\
\text { WHC-SD-WM-DP-052 } \\
\operatorname{Rev} 0\end{array}$ \\
\hline Notes & $\begin{array}{c}\text { Taylor Vorticies } \\
\text { observed fit over } 0-20 \\
1 / \mathrm{sec}\end{array}$ & $\begin{array}{c}\text { Taylor Vorticies } \\
\text { observed fit over } 0-80 \\
1 / \mathrm{sec}\end{array}$ & $\begin{array}{c}\text { Taylor Vorticies } \\
\text { observed fit over } 0-12 \\
1 / \mathrm{sec}\end{array}$ & $\begin{array}{c}\text { Taylor Vorticies } \\
\text { observed fit over } 0-20 \\
1 / \text { sec }\end{array}$ \\
\hline \multicolumn{5}{|l|}{ Newtonian: } \\
\hline$\eta$ - Newtonian viscosity (cP) & 1.840 & 1.167 & 1.130 & 1.154 \\
\hline $\mathrm{r}$ - correlation coefficient & 0.988 & 1.000 & 0.831 & 0.375 \\
\hline \multicolumn{5}{|l|}{ Ostwald (or Power Law): } \\
\hline $\mathrm{m}$ - the consistency coefficient $\left(\mathrm{Pa} \cdot \mathrm{s}^{-\mathrm{n}}\right)$ & 0.003 & 0.002 & 0.008 & 0.010 \\
\hline $\mathrm{n}$ - the power-law exponent & 0.804 & 0.817 & 0.172 & 0.001 \\
\hline $\mathrm{r}$ - correlation coefficient & 0.960 & 0.992 & 0.997 & 1.000 \\
\hline \multicolumn{5}{|l|}{ Bingham Plastic: } \\
\hline$\tau_{\mathrm{B}^{-}}$the Bingham yield stress $(\mathrm{Pa})$ & 0.009 & 0.010 & 0.003 & 0.009 \\
\hline$\eta_{p}$ - the plastic viscosity $(\mathrm{Pa} \cdot \mathrm{s})$ & 0.001 & 0.001 & 0.001 & 0.000 \\
\hline $\mathrm{r}$ - correlation coefficient & 0.988 & 1.000 & 0.831 & 0.375 \\
\hline \multicolumn{5}{|l|}{ Herschel-Bulkley: } \\
\hline$\tau_{\mathrm{H}^{-}}$the yield stress $(\mathrm{Pa})$ & 0.009 & 0.007 & 0.001 & 0.007 \\
\hline $\begin{array}{l}\mathrm{k} \text { - the Herschel-Bulkely consistency } \\
\text { coefficient }\left(\mathrm{Pa} \cdot \mathrm{s}^{-\mathrm{b}}\right)\end{array}$ & 0.001 & 0.002 & 0.005 & 0.003 \\
\hline $\begin{array}{l}\text { b - the Hershel-Bulkely power-law } \\
\text { exponent }\end{array}$ & 0.994 & 0.908 & 0.313 & 0.008 \\
\hline $\mathrm{r}$ - correlation coefficient & 0.987 & 0.998 & 0.993 & 1.000 \\
\hline$n / a=$ not applicable & & & & \\
\hline
\end{tabular}

\section{Physical Property Data}

\begin{tabular}{|c|c|c|c|c|}
\hline Physical Property & $\bar{T} T-102$ & T-102 & T-102 & T-102 \\
\hline \multicolumn{5}{|l|}{ Notes } \\
\hline Temperature $\left({ }^{\circ} \mathrm{C}\right)$ & 30 & 30 & 30 & 30 \\
\hline \multicolumn{5}{|l|}{ Zeta Potential (mV) } \\
\hline Bulk Density (g/mL) & 1.11 & 1.05 & 1.11 & 1.05 \\
\hline vol\% Settled Solids & 15.7 & 8.3 & 15.7 & 8.3 \\
\hline Density of Centrifuged Solids ( $\mathrm{g} / \mathrm{mL}$ ) & $\mathrm{n} / \mathrm{m}$ & $\mathrm{n} / \mathrm{m}$ & $\mathrm{n} / \mathrm{m}$ & $\mathrm{n} / \mathrm{m}$ \\
\hline vol\% Centrifuged Solids & $\mathrm{n} / \mathrm{m}$ & $\mathrm{n} / \mathrm{m}$ & $\mathrm{n} / \mathrm{m}$ & $\mathrm{n} / \mathrm{m}$ \\
\hline wt\% Centrifuged Solids & $\mathrm{n} / \mathrm{m}$ & $\mathrm{n} / \mathrm{m}$ & $\mathrm{n} / \mathrm{m}$ & $\mathrm{n} / \mathrm{m}$ \\
\hline Supernatant Density $(\mathrm{g} / \mathrm{mL})$ & 1.04 & 1.02 & 1.04 & 1.02 \\
\hline Density of Settled Solids ( $\mathrm{g} / \mathrm{mL})$ & $\mathrm{n} / \mathrm{m}$ & $\mathrm{n} / \mathrm{m}$ & $\mathrm{n} / \mathrm{m}$ & $\mathrm{n} / \mathrm{m}$ \\
\hline wt\% Settled Supernatant & $\mathrm{n} / \mathrm{m}$ & $\mathrm{n} / \mathrm{m}$ & $\mathrm{n} / \mathrm{m}$ & $\mathrm{n} / \mathrm{m}$ \\
\hline wt $\%$ dissolved solids in supernatant & 21.72 & 21.25 & 21.72 & 21.25 \\
\hline wt\% total solids in Centrifuged Sludge & $\mathrm{n} / \mathrm{m}$ & $\mathrm{n} / \mathrm{m}$ & $\mathrm{n} / \mathrm{m}$ & $\mathrm{n} / \mathrm{m}$ \\
\hline wt $\%$ Total Solids & $\mathrm{n} / \mathrm{m}$ & $\mathrm{n} / \mathrm{m}$ & $\mathrm{n} / \mathrm{m}$ & $\mathrm{n} / \mathrm{m}$ \\
\hline $\mathrm{wt} \%$ UDS & 10.46 & 4.90 & 10.46 & 4.90 \\
\hline Density of Solids (g/cc) & 2.48 & 2.48 & 2.48 & 2.48 \\
\hline
\end{tabular}


241-T-102

Settling Data

\begin{tabular}{|c|c|c|c|c|}
\hline Settling Schedule & 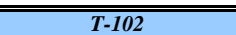 & $\bar{T} T-102$ & & \\
\hline Notes: & $\begin{array}{c}\text { WHC-SD-WM-DP-052 } \\
\text { C08-19 }\end{array}$ & $\begin{array}{c}\text { WHC-SD-WM-DP-052 } \\
\text { C08-24 }\end{array}$ & & \\
\hline Temperature $\left({ }^{\circ} \mathrm{C}\right)$ & 25 & 25 & & \\
\hline Total initial volume & 100 & 100 & & \\
\hline \multicolumn{5}{|l|}{5 minutes } \\
\hline \multicolumn{5}{|l|}{10 minutes } \\
\hline \multicolumn{5}{|l|}{$\mid 15$ minutes } \\
\hline \multicolumn{5}{|l|}{$\mid 20$ minutes } \\
\hline \multicolumn{5}{|l|}{30 minutes } \\
\hline \multicolumn{5}{|l|}{40 minutes } \\
\hline \multicolumn{5}{|l|}{50 minutes } \\
\hline \multicolumn{5}{|l|}{1 hour } \\
\hline 2 hours & 18 & 11 & & \\
\hline \multicolumn{5}{|l|}{3 hours } \\
\hline \multicolumn{5}{|l|}{4 hours } \\
\hline 5 hours & 17 & 10 & & \\
\hline 6 hours & 17 & 10 & & \\
\hline 24 hours & 17 & & & \\
\hline 32 hours & 17 & & & \\
\hline \multicolumn{5}{|l|}{48 hours } \\
\hline \multicolumn{5}{|l|}{56 hours } \\
\hline 72 hours final settled & & & & \\
\hline
\end{tabular}


241-T-107

Model Chemical Data From ESP

\begin{tabular}{|c|c|c|c|c|c|}
\hline AnalytelSample & T-107 & $\overline{T \text { T-107 }}$ & T-107 & $\overline{\bar{T} \text { T-107 }}$ & T-107 \\
\hline Description & "no dilution & 1:1 dilution & 3:1 dilution & 1:1 dilution & "3:1 dilution \\
\hline Model Basis & Salty & Salt Free & Salt Free & Salt Free & Salt Free \\
\hline Report No/Source of Data & $\begin{array}{c}\text { WHC-SD-WM-ER-382, } \\
\text { rev } 0\end{array}$ & $\begin{array}{c}\text { WHC-SD-WM-ER-382, } \\
\text { rev } 0\end{array}$ & $\begin{array}{c}\text { WHC-SD-WM-ER-382, } \\
\text { rev } 0\end{array}$ & $\begin{array}{c}\text { WHC-SD-WM-ER-382, } \\
\text { rev } 0\end{array}$ & $\begin{array}{c}\text { WHC-SD-WM-ER-382, } \\
\text { rev } 0\end{array}$ \\
\hline \multicolumn{6}{|l|}{ Solid Phases (vol\% of bulk) } \\
\hline$\left(\mathrm{NaAlO}_{2}\right)_{2} \cdot 2.5 \mathrm{H}_{2} \mathrm{O}$ & - & - & - & - & - \\
\hline $\mathrm{Ag}_{2} \mathrm{CO}_{3}$ & 0.00008 & 0.00008 & 0.00004 & 0.00008 & 0.00004 \\
\hline $\mathrm{Bi}_{2} \mathrm{O}_{3}$ & 0.11961 & 0.12696 & 0.05809 & 0.12696 & 0.05809 \\
\hline $\mathrm{BiOCl}$ & - & - & - & - & - \\
\hline Boehmite & - & - & - & - & - \\
\hline$\overline{\mathrm{Ca}(\mathrm{OH})_{2}}$ & - & - & - & - & - \\
\hline $\mathrm{Ca}_{5} \mathrm{OH}\left(\mathrm{PO}_{4}\right)_{3}$ & - & - & - & - & - \\
\hline $\mathrm{CaC}_{2} \mathrm{O}_{4} \cdot \mathrm{H}_{2} \mathrm{O}$ & - & - & - & - & - \\
\hline $\mathrm{CaCO}_{3}$ & - & - & - & - & - \\
\hline $\mathrm{CaF}_{2}$ & 0.11698 & 0.12417 & 0.05682 & 0.12417 & 0.05682 \\
\hline $\mathrm{CrOOH}$ & 0.01187 & 0.01260 & 0.00576 & 0.01260 & 0.00576 \\
\hline $\mathrm{FeOOH}$ & 1.04158 & 1.10564 & 0.50589 & 1.10564 & 0.50589 \\
\hline Gibbsite & 3.22914 & 3.42773 & 1.56838 & 3.42773 & 1.56838 \\
\hline $\mathrm{HgO}$ & - & - & - & - & - \\
\hline $\mathrm{KAlSiO}_{4}$ & - & - & - & - & - \\
\hline $\mathrm{La}(\mathrm{OH})_{3}$ & - & - & - & - & - \\
\hline $\mathrm{LaPO}_{4} \cdot 2 \mathrm{H}_{2} \mathrm{O}$ & 0.00010 & 0.00011 & 0.00005 & 0.00011 & 0.00005 \\
\hline $\mathrm{Mn}(\mathrm{OH})_{2}$ & - & - & - & - & - \\
\hline $\mathrm{Mn}_{3}\left(\mathrm{PO}_{4}\right)_{2}$ & 0.02970 & 0.03153 & 0.01443 & 0.03153 & 0.01443 \\
\hline $\mathrm{MnCO}_{3}$ & - & - & - & - & - \\
\hline $\mathrm{Na}_{2} \mathrm{U}_{2} \mathrm{O}_{7}$ & 0.47700 & 0.50633 & 0.23168 & 0.50633 & 0.23168 \\
\hline $\mathrm{NaAlCO}_{3}(\mathrm{OH})_{2}$ & 1.01941 & 1.08210 & 0.49512 & 1.08210 & 0.49512 \\
\hline $\mathrm{NaAlSiO}_{4}$ & 0.92570 & 0.98263 & 0.44961 & 0.98263 & 0.44961 \\
\hline $\mathrm{Ni}(\mathrm{OH})_{2}$ & 0.00496 & 0.00526 & 0.00241 & 0.00526 & 0.00241 \\
\hline $\mathrm{Ni}_{3}\left(\mathrm{PO}_{4}\right)_{2}$ & - & - & - & - & - \\
\hline $\mathrm{NiC}_{2} \mathrm{O}_{4} \cdot 2 \mathrm{H}_{2} \mathrm{O}$ & - & - & - & - & - \\
\hline $\begin{array}{l}\mathrm{Pb}(\mathrm{OH})_{2} \\
\end{array}$ & 0.01135 & 0.01205 & 0.00551 & 0.01205 & 0.00551 \\
\hline $\mathrm{Pb}_{3}\left(\mathrm{PO}_{4}\right)_{2}$ & - & - & - & - & - \\
\hline $\mathrm{PbCO}_{3}$ & - & - & - & - & - \\
\hline $\mathrm{Pu}(\mathrm{OH})_{4}$ & 0.00004 & 0.00005 & 0.00002 & 0.00005 & 0.00002 \\
\hline $\mathrm{SiO}_{2}$ & - & - & - & - & - \\
\hline $\mathrm{Sr}_{3}\left(\mathrm{PO}_{4}\right)_{2}$ & - & - & - & - & - \\
\hline $\mathrm{SrCO}_{3}$ & 0.04453 & 0.04727 & 0.02163 & 0.04727 & 0.02163 \\
\hline $\mathrm{TcO}_{2}$ & - & - & - & - & - \\
\hline $\mathrm{ZrO}_{2}$ & 0.00118 & 0.00125 & 0.00057 & 0.00125 & 0.00057 \\
\hline $\mathrm{KNO}_{3}$ & - & - & - & - & - \\
\hline $\mathrm{Na}_{2} \mathrm{C}_{2} \mathrm{O}_{4}$ & 0.04057 & - & - & - & - \\
\hline $\mathrm{Na}_{2} \mathrm{CO}_{3} \cdot \mathrm{H}_{2} \mathrm{O}$ & - & - & - & - & - \\
\hline $\mathrm{Na}_{2} \mathrm{SO}_{4}$ & - & - & - & - & - \\
\hline $\mathrm{Na}_{2} \mathrm{SO}_{4} \cdot 10 \mathrm{H}_{2} \mathrm{O}$ & - & - & - & - & - \\
\hline $\mathrm{Na}_{3} \mathrm{FSO}_{4}$ & - & - & - & - & - \\
\hline $\mathrm{Na}_{3} \mathrm{NO}_{3} \mathrm{SO}_{4} \cdot \mathrm{H}_{2} \mathrm{O}$ & - & - & - & - & - \\
\hline $\mathrm{Na}_{3} \mathrm{PO}_{4} \cdot 8 \mathrm{H}_{2} \mathrm{O}$ & - & - & - & - & - \\
\hline $\mathrm{Na}_{4} \mathrm{P}_{2} \mathrm{O}_{7} \cdot 10 \mathrm{H}_{2} \mathrm{O}$ & 6.37408 & - & - & - & - \\
\hline $\mathrm{Na}_{6}\left(\mathrm{SO}_{4}\right)_{2} \mathrm{CO}_{3}$ & - & - & - & - & - \\
\hline $\mathrm{NaF}$ & 0.50793 & - & - & - & - \\
\hline $\mathrm{NaF}\left(\mathrm{PO}_{4}\right)_{2} \cdot 19 \mathrm{H}_{2} \mathrm{O}$ & 0.66969 & - & - & - & - \\
\hline $\mathrm{NaHCO}_{3}$ & - & - & - & - & - \\
\hline $\mathrm{NaNO}_{2}$ & - & - & - & - & - \\
\hline $\mathrm{NaNO}_{3}$ & - & - & - & - & - \\
\hline $\mathrm{Na}_{3} \mathrm{PO}_{4} \cdot 0.25 \mathrm{NaOH} \cdot 12 \mathrm{H}_{2} \mathrm{O}$ & - & - & - & - & - \\
\hline Total Volume \% UDS & 14.63 & 7.47 & 3.42 & 7.47 & 3.42 \\
\hline Solids Concentration (g/L) & 360.0 & 229.9 & 105.2 & 229.9 & 105.2 \\
\hline
\end{tabular}


Rheological Data

\begin{tabular}{|c|c|c|c|c|}
\hline Model/model Parameter & 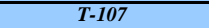 & 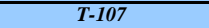 & T-107 & 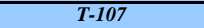 \\
\hline Temperature $\left({ }^{\circ} \mathrm{C}\right)$ & 25 & 25 & 90 & 90 \\
\hline \multicolumn{5}{|l|}{ Source of Data } \\
\hline Notes & $\begin{array}{l}\text { Fit from hardcopies in } \\
\text { Tingey archive file }\end{array}$ & $\begin{array}{l}\text { Fit from hardcopies in } \\
\text { Tingey archive file }\end{array}$ & $\begin{array}{l}\text { Fit from hardcopies in } \\
\text { Tingey archive file }\end{array}$ & $\begin{array}{l}\text { Fit from hardcopies in } \\
\text { Tingey archive file }\end{array}$ \\
\hline \multicolumn{5}{|l|}{ Newtonian: } \\
\hline$\eta-$ Newtonian viscosity (cP) & 9.302 & 4.612 & 8.466 & 2.559 \\
\hline $\mathrm{r}$ - correlation coefficient & 0.949 & 0.980 & 0.940 & 0.987 \\
\hline \multicolumn{5}{|l|}{ Ostwald (or Power Law): } \\
\hline $\mathrm{m}$ - the consistency coefficient $\left(\mathrm{Pa} \cdot \mathrm{s}^{-\mathrm{n}}\right)$ & 0.666 & 0.006 & 0.387 & 0.003 \\
\hline $\mathrm{n}$-the power-law exponent & 0.261 & 0.952 & 0.344 & 1.000 \\
\hline r-correlation coefficient & 0.913 & 0.975 & 0.958 & 0.987 \\
\hline \multicolumn{5}{|l|}{ Bingham Plastic: } \\
\hline$\tau_{\mathrm{B}}$ - the Bingham yield stress $(\mathrm{Pa})$ & 0.852 & 0.049 & 0.630 & 0.000 \\
\hline$\eta_{p}$ - the plastic viscosity (Pa-s) & 0.007 & 0.004 & 0.007 & 0.003 \\
\hline $\mathrm{r}$ - correlation coefficient & 0.949 & 0.980 & 0.940 & 0.987 \\
\hline \multicolumn{5}{|l|}{ Herschel-Bulkley: } \\
\hline$\tau_{\mathrm{H}^{-}}$the yield stress $(\mathrm{Pa})$ & 0.735 & 0.050 & 0.415 & 0.000 \\
\hline $\begin{array}{l}\text { k - the Herschel-Bulkely consistency } \\
\text { coefficient }\left(\mathrm{Pa} \cdot \mathrm{s}^{\text {b }}\right)\end{array}$ & 0.039 & 0.004 & 0.088 & 0.003 \\
\hline $\begin{array}{l}\text { b - the Hershel-Bulkely power-law } \\
\text { exponent }\end{array}$ & 0.710 & 1.000 & 0.574 & 1.000 \\
\hline $\mathrm{r}$ - correlation coefficient & 0.965 & 0.980 & 0.971 & 0.987 \\
\hline $\mathrm{n} / \mathrm{a}=$ not applicable & & & & \\
\hline
\end{tabular}

Physical Property Data

\begin{tabular}{|c|c|c|c|c|c|}
\hline Physical Property & T-107 & $T-107$ & $T-107$ & T-107 & $\overline{T-107}$ \\
\hline \multicolumn{6}{|l|}{ Notes } \\
\hline Temperature $\left({ }^{\circ} \mathrm{C}\right)$ & 30 & 30 & 30 & 30 & 30 \\
\hline \multicolumn{6}{|l|}{ Zeta Potential (mV) } \\
\hline $\mid \overline{B u l k}$ Density $(\mathrm{g} / \mathrm{mL})$ & 1.44 & 1.22 & 1.10 & 1.22 & 1.10 \\
\hline vol\% Settled Solids & 74 & 68 & 32 & 68 & 32 \\
\hline Density of Centrifuged Solids ( $\mathrm{g} / \mathrm{mL}$ ) & 1.53 & 1.44 & 1.32 & 1.44 & 1.32 \\
\hline vol\% Centrifuged Solids & 74 & 36 & 16 & 36 & 16 \\
\hline |wt\% Centrifuged Solids & 79 & 44 & 19 & 44 & 19 \\
\hline | Supernatant Density $(\mathrm{g} / \mathrm{mL})$ & 1.20 & 1.07 & 1.03 & 1.07 & 1.03 \\
\hline Density of Settled Solids $(\mathrm{g} / \mathrm{mL})$ & $\mathrm{n} / \mathrm{m}$ & $\mathrm{n} / \mathrm{m}$ & $\mathrm{n} / \mathrm{m}$ & $\mathrm{n} / \mathrm{m}$ & $\mathrm{n} / \mathrm{m}$ \\
\hline wt\% Settled Supernatant & $\mathrm{n} / \mathrm{m}$ & $\mathrm{n} / \mathrm{m}$ & $\mathrm{n} / \mathrm{m}$ & $\mathrm{n} / \mathrm{m}$ & $\mathrm{n} / \mathrm{m}$ \\
\hline wt $\%$ dissolved solids in supernatant & 23.17 & 20.66 & 19.89 & 20.66 & 19.89 \\
\hline wt\% total solids in Centrifuged Sludge & $\mathrm{n} / \mathrm{m}$ & $\mathrm{n} / \mathrm{m}$ & $\mathrm{n} / \mathrm{m}$ & $\mathrm{n} / \mathrm{m}$ & $\mathrm{n} / \mathrm{m}$ \\
\hline wt\% Total Solids & 47 & $\mathrm{n} / \mathrm{m}$ & $\mathrm{n} / \mathrm{m}$ & $\mathrm{n} / \mathrm{m}$ & $\mathrm{n} / \mathrm{m}$ \\
\hline |wt\% UDS & 25.00 & 18.84 & 9.56 & 18.84 & 9.56 \\
\hline Density of Solids (g/cc) & 2.46 & 3.08 & 3.08 & 3.08 & 3.08 \\
\hline
\end{tabular}


Settling Data

\begin{tabular}{|c|c|c|c|c|}
\hline $\mid$ Settling Schedule & $\overline{T-107}$ & $\overline{T-107}$ & 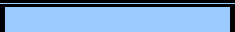 & \\
\hline ||Notes: & $\begin{array}{c}\text { WHC-SD-WM-ER-382 } \\
5-35\end{array}$ & $\begin{array}{c}\text { WHC-SD-WM-ER-382 } \\
5-36\end{array}$ & & \\
\hline $\mid$ Temperature $\left({ }^{\circ} \mathrm{C}\right)$ & 30 & 30 & & \\
\hline Total initial volume & 100 & 100 & & \\
\hline \multicolumn{5}{|l|}{5 minutes } \\
\hline \multicolumn{5}{|l|}{10 minutes } \\
\hline \multicolumn{5}{|l|}{15 minutes } \\
\hline \multicolumn{5}{|l|}{20 minutes } \\
\hline \multicolumn{5}{|l|}{30 minutes } \\
\hline \multicolumn{5}{|l|}{40 minutes } \\
\hline \multicolumn{5}{|l|}{ |50 minutes } \\
\hline \multicolumn{5}{|l|}{1 hour } \\
\hline $\mid 2$ hours & 95 & 65 & & \\
\hline \multicolumn{5}{|l|}{$\mid 3$ hours } \\
\hline \multicolumn{5}{|l|}{4 hours } \\
\hline $\mid 5$ hours & 89 & 56 & & \\
\hline \multicolumn{5}{|l|}{$\mid \overline{6 \text { hours }}$} \\
\hline 24 hours & 72 & 41 & & \\
\hline 32 hours & 70 & 36 & & \\
\hline 48 hours & 68 & 32 & & \\
\hline $\mid 56$ hours & 67 & 32 & & \\
\hline $\mid 72$ hours final settled & & & & \\
\hline
\end{tabular}

Shear Strength Data

\begin{tabular}{|c|c|c|c|c|c|}
\hline |Shear Strength Schedule & T-107 & & & & \\
\hline Source of Data & $\begin{array}{c}\text { WHC-SD-WM-ER-382, } \\
\operatorname{rev} 0\end{array}$ & & & & \\
\hline Notes: & $\begin{array}{l}\text { Page 6-7 } \\
\end{array}$ & & & & \\
\hline Temperature $\left({ }^{\circ} \mathrm{C}\right)$ & Unspecified & & & & \\
\hline Description & $\begin{array}{c}\text { Shear Vane at } \\
\text { unspecified gel time or } \\
\text { temperature }\end{array}$ & & & & \\
\hline Gelation Time/Shear Stre & & & & & \\
\hline $\mid \overline{5 \text { minutes }}$ & & & & & \\
\hline 10 minutes & & & & & \\
\hline 15 minutes & & & & & \\
\hline 20 minutes & & & & & \\
\hline 30 minutes & & & & & \\
\hline 40 minutes & & & & & \\
\hline 50 minutes & & & & & \\
\hline 1 hour & & & & & \\
\hline 2 hours & & & & & \\
\hline $\mid \overline{\text { hours }}$ & & & & & \\
\hline 4 hours & & & & & \\
\hline 5 hours & & & & & \\
\hline $\mid \overline{6 \text { hours }}$ & & & & & \\
\hline 24 hours & & & & & \\
\hline 32 hours & & & & & \\
\hline 48 hours & & & & & \\
\hline 56 hours & & & & & \\
\hline 72 hours & 720 & & & & \\
\hline Infinte time (GRE) & & & & & \\
\hline
\end{tabular}


241-T-110

Model Chemical Data From ESP

\begin{tabular}{|c|c|c|c|c|}
\hline AnalytelSample & T-110 & T-110 & T-110 & T-110 \\
\hline Description & \begin{tabular}{|c|} 
Tank Composite with water \\
added to return to original \\
solids content
\end{tabular} & $\begin{array}{l}30 \mathrm{wt} \% \text { dilution of a core } \\
\text { composite }\end{array}$ & $\begin{array}{l}\text { 1:1 Dilution of a core } \\
\text { composite }\end{array}$ & $\begin{array}{l}\text { 4:1 Dilution of a core } \\
\text { composite }\end{array}$ \\
\hline Model Basis & $\begin{array}{l}\text { Salty } \\
\end{array}$ & Washed & Washed & Washed \\
\hline Source of Data & PNNL-14365 & PNNL-14365 & PNNL-14365 & PNNL-14365 \\
\hline \multicolumn{5}{|l|}{ Solid Phases (vol\% of bulk) } \\
\hline$\left(\mathrm{NaAlO}_{2}\right)_{2} \cdot 2.5 \mathrm{H}_{2} \mathrm{O}$ & - & - & - & - \\
\hline $\mathrm{Ag}_{2} \mathrm{CO}_{3}$ & - & - & - & - \\
\hline $\mathrm{Bi}_{2} \mathrm{O}_{3}$ & 1.15553 & 0.63157 & 0.25860 & 0.05099 \\
\hline $\mathrm{BiOCl}$ & - & - & - & - \\
\hline Boehmite & - & - & - & - \\
\hline$\overline{\mathrm{Ca}(\mathrm{OH})_{2}}$ & - & - & - & - \\
\hline $\mathrm{Ca}_{5} \mathrm{OH}\left(\mathrm{PO}_{4}\right)_{3}$ & 0.00703 & 0.00384 & 0.00157 & 0.00031 \\
\hline $\mathrm{CaC}_{2} \mathrm{O}_{4} \cdot \mathrm{H}_{2} \mathrm{O}$ & - & - & - & - \\
\hline $\mathrm{CaCO}_{3}$ & $\overline{-}$ & $\overline{-}$ & $\overline{-}$ & - \\
\hline $\mathrm{CaF}_{2}$ & 0.32908 & 0.17986 & 0.07365 & 0.01452 \\
\hline $\mathrm{CrOOH}$ & 0.17966 & 0.09820 & 0.04021 & 0.00793 \\
\hline $\mathrm{FeOOH}$ & 2.54317 & 1.39000 & 0.56916 & 0.11221 \\
\hline Gibbsite & - & - & - & - \\
\hline $\mathrm{HgO}$ & - & - & - & - \\
\hline $\mathrm{KAlSiO}_{4}$ & - & - & - & - \\
\hline$\overline{\mathrm{La}(\mathrm{OH})_{3}}$ & $\overline{-}$ & - & $\overline{-}$ & - \\
\hline $\mathrm{LaPO}_{4} \cdot 2 \mathrm{H}_{2} \mathrm{O}$ & 0.06452 & 0.03526 & 0.01444 & 0.00285 \\
\hline $\mathrm{Mn}(\mathrm{OH})_{2}$ & $\overline{-}$ & - & $\overline{-}$ & - \\
\hline $\mathrm{Mn}_{3}\left(\mathrm{PO}_{4}\right)_{2}$ & 0.19524 & 0.10671 & 0.04369 & 0.00861 \\
\hline $\mathrm{MnCO}_{3}$ & - & - & - & - \\
\hline $\mathrm{Na}_{2} \mathrm{U}_{2} \mathrm{O}_{7}$ & - & - & $\overline{-}$ & $\overline{-}$ \\
\hline $\mathrm{NaAlCO}_{3}(\mathrm{OH})_{2}$ & 1.77381 & 0.96950 & 0.39697 & 0.07827 \\
\hline $\mathrm{NaAlSiO}_{4}$ & 0.06315 & 0.03451 & 0.01413 & 0.00279 \\
\hline $\mathrm{Ni}(\mathrm{OH})_{2}$ & 0.01007 & 0.00551 & 0.00225 & 0.00044 \\
\hline$\overline{\mathrm{Ni}_{3}\left(\mathrm{PO}_{4}\right)_{2}}$ & - & - & $\overline{-}$ & - \\
\hline $\mathrm{NiC}_{2} \mathrm{O}_{4} \cdot 2 \mathrm{H}_{2} \mathrm{O}$ & - & - & - & - \\
\hline$\frac{\mathrm{Pb}(\mathrm{OH})_{2}}{4}$ & 0.05860 & 0.03203 & 0.01311 & 0.00259 \\
\hline $\mathrm{Pb}_{3}\left(\mathrm{PO}_{4}\right)_{2}$ & - & - & - & - \\
\hline $\mathrm{PbCO}_{3}$ & $\overline{-}$ & - & $\overline{-}$ & $\overline{-}$ \\
\hline $\mathrm{Pu}(\mathrm{OH})_{4}$ & 0.00012 & 0.00006 & 0.00003 & 0.00001 \\
\hline $\mathrm{SiO}_{2}$ & $\overline{-}$ & - & $\overline{-}$ & - \\
\hline $\mathrm{Sr}_{3}\left(\mathrm{PO}_{4}\right)_{2}$ & - & - & $\overline{-}$ & $\overline{-}$ \\
\hline $\mathrm{SrCO}_{3}$ & 0.05596 & 0.03059 & 0.01252 & 0.00247 \\
\hline $\mathrm{TcO}_{2}$ & 0.00000 & 0.00000 & 0.00000 & 0.00000 \\
\hline$\overline{\mathrm{ZrO}_{2}}$ & 0.00076 & 0.00042 & 0.00017 & 0.00003 \\
\hline$\overline{\mathrm{KNO}_{3}}$ & - & - & $\overline{-}$ & - \\
\hline $\mathrm{Na}_{2} \mathrm{C}_{2} \mathrm{O}_{4}$ & 0.14186 & - & - & - \\
\hline $\mathrm{Na}_{2} \mathrm{CO}_{3} \cdot \mathrm{H}_{2} \mathrm{O}$ & $\overline{-}$ & - & $\overline{-}$ & - \\
\hline $\mathrm{Na}_{2} \mathrm{SO}_{4}$ & - & - & - & - \\
\hline $\mathrm{Na}_{2} \mathrm{SO}_{4} \cdot 10 \mathrm{H}_{2} \mathrm{O}$ & $\overline{-}$ & - & $\overline{-}$ & - \\
\hline $\mathrm{Na}_{3} \mathrm{FSO}_{4}$ & - & - & - & - \\
\hline $\mathrm{Na}_{3} \mathrm{NO}_{3} \mathrm{SO}_{4} \cdot \mathrm{H}_{2} \mathrm{O}$ & - & - & - & - \\
\hline $\mathrm{Na}_{3} \mathrm{PO}_{4} \cdot 8 \mathrm{H}_{2} \mathrm{O}$ & - & - & - & - \\
\hline $\mathrm{Na}_{4} \mathrm{P}_{2} \mathrm{O}_{7} \cdot 10 \mathrm{H}_{2} \mathrm{O}$ & 7.68168 & - & $\overline{-}$ & $\overline{-}$ \\
\hline $\mathrm{Na}_{6}\left(\mathrm{SO}_{4}\right)_{2} \mathrm{CO}_{3}$ & - & - & $\overline{-}$ & $\overline{-}$ \\
\hline $\mathrm{NaF}$ & - & - & - & - \\
\hline $\mathrm{NaF}\left(\mathrm{PO}_{4}\right)_{2} \cdot 19 \mathrm{H}_{2} \mathrm{O}$ & - & - & - & - \\
\hline $\mathrm{NaHCO}_{3}$ & - & - & - & - \\
\hline $\mathrm{NaNO}_{2}$ & $\overline{-}$ & - & $\overline{-}$ & - \\
\hline $\mathrm{NaNO}_{3}$ & - & - & - & - \\
\hline $\mathrm{Na}_{3} \mathrm{PO}_{4} \cdot 0.25 \mathrm{NaOH} \cdot 12 \mathrm{H}_{2} \mathrm{O}$ & $\overline{-}$ & - & - & $\overline{-}$ \\
\hline Total Volume \% UDS & 14.26 & 3.52 & 1.44 & 0.28 \\
\hline Solids Concentration ( $\mathrm{g} / \mathrm{L}$ ) & 436.8 & 160.1 & 65.6 & 12.9 \\
\hline
\end{tabular}

Note: Numbers in italics are calculated values. Numbers in normal font are reported data. 


\section{Rheological Data}

\begin{tabular}{|c|c|c|c|}
\hline$\sqrt{\text { Model/model Parameter }}$ & 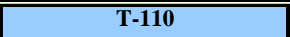 & 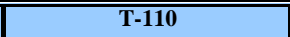 & $\overline{\mathrm{T}-110}$ \\
\hline Temperature $\left({ }^{\circ} \mathrm{C}\right)$ & 25 & 25 & 25 \\
\hline Source of Data & PNNL-14365 & PNNL-14365 & PNNL-14365 \\
\hline |Instrumentation & $\begin{array}{c}\text { Haake RS300 } \\
\text { Concentric Cylinders with a } \\
3 \mathrm{~mm} \text { Gap }\end{array}$ & \begin{tabular}{|c|} 
Haake RS300 \\
Concentric Cylinders with a \\
$3 \mathrm{~mm}$ Gap
\end{tabular} & \begin{tabular}{|c|} 
Haake RS300 \\
Concentric Cylinders with a \\
$3 \mathrm{~mm}$ Gap
\end{tabular} \\
\hline \multicolumn{4}{|l|}{ Newtonian: } \\
\hline$\eta$ - Newtonian viscosity (cP) & $\mathrm{n} / \mathrm{a}$ & $\mathrm{n} / \mathrm{a}$ & 1.41 \\
\hline $\mathrm{r}$ - correlation coefficient & $\mathrm{n} / \mathrm{a}$ & $\mathrm{n} / \mathrm{a}$ & 0.94 \\
\hline \multicolumn{4}{|l|}{ Ostwald (or Power Law): } \\
\hline $\mathrm{m}$ - the consistency coefficient $\left(\mathrm{Pa} \cdot \mathrm{s}^{-\mathrm{n}}\right)$ & $\mathrm{n} / \mathrm{a}$ & $\mathrm{n} / \mathrm{a}$ & $\mathrm{n} / \mathrm{a}$ \\
\hline $\mathrm{n}$ - the power-law exponent & $\mathrm{n} / \mathrm{a}$ & $\mathrm{n} / \mathrm{a}$ & $\mathrm{n} / \mathrm{a}$ \\
\hline $\mathrm{r}$ - correlation coefficient & $\mathrm{n} / \mathrm{a}$ & $\mathrm{n} / \mathrm{a}$ & $\mathrm{n} / \mathrm{a}$ \\
\hline \multicolumn{4}{|l|}{ Bingham Plastic: } \\
\hline$\tau_{\mathrm{B}}$ - the Bingham yield stress $(\mathrm{Pa})$ & 1.54 & 0.14 & 0.02 \\
\hline$\eta_{p}$ - the plastic viscosity (Pa-s) & 0.0059 & 0.0023 & 0.0013 \\
\hline $\mathrm{r}$ - correlation coefficient & 0.99 & 0.99 & 0.95 \\
\hline \multicolumn{4}{|l|}{ |Herschel-Bulkley: } \\
\hline$\tau_{\mathrm{H}^{-}}$the yield stress $(\mathrm{Pa})$ & 1.11 & 0.07 & $\mathrm{n} / \mathrm{a}$ \\
\hline $\begin{array}{l}\text { k - the Herschel-Bulkely consistency } \\
\text { coefficient }\left(\mathrm{Pa} \cdot \mathrm{s}^{-\mathrm{b}}\right)\end{array}$ & 0.02 & 0.01 & $\mathrm{n} / \mathrm{a}$ \\
\hline \begin{tabular}{|l} 
b - the Hershel-Bulkely power-law \\
exponent
\end{tabular} & 0.82 & 0.83 & $\mathrm{n} / \mathrm{a}$ \\
\hline $\mathrm{r}$ - correlation coefficient & 0.99 & 0.99 & $\mathrm{n} / \mathrm{a}$ \\
\hline
\end{tabular}

\section{Physical Property Data}

\begin{tabular}{|c|c|c|c|c|}
\hline$\sqrt{\text { Physical Property }}$ & 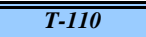 & 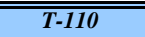 & $\overline{\overline{T-110}}$ & $\overline{\bar{T}-110}$ \\
\hline Source of Data & PNNL-14365 & PNNL-14365 & PNNL-14365 & PNNL-14365 \\
\hline \multicolumn{5}{|l|}{ Temperature $\left({ }^{\circ} \mathrm{C}\right)$} \\
\hline \multicolumn{5}{|l|}{ Zeta Potential (mV) } \\
\hline Bulk Density (g/mL) & 1.50 & 1.26 & 1.13 & 1.04 \\
\hline vol\% Settled Solids & 100 & 87.1 & 46.4 & 22.2 \\
\hline Density of Centrifuged Solids (g/mL) & 1.58 & 1.51 & 1.61 & 1.47 \\
\hline vol\% Centrifuged Solids & 79.9 & 39.9 & 16.4 & 8.1 \\
\hline wt\% Centrifuged Solids & 84 & 48 & 23 & 11 \\
\hline Supernatant Density $(\mathrm{g} / \mathrm{mL})$ & 1.24 & 1.14 & 1.08 & 1.03 \\
\hline Density of Settled Solids (g/mL) & & & & \\
\hline wt\% Settled Supernatant & & & & \\
\hline wt $\%$ dissolved solids in supernatant & & & & \\
\hline wt $\%$ total solids in Centrifuged Sludge & & & & \\
\hline wt $\%$ Total Solids & $\overline{48.4}$ & 29.3 & 15.3 & 6.4 \\
\hline wt \% UDS & 29.12 & $\overline{12.71}$ & 5.80 & 1.24 \\
\hline Density of Solids (g/cc) & 3.06 & 4.55 & 4.55 & 4.55 \\
\hline
\end{tabular}

Note: Numbers in italics are calculated values. Numbers in normal font are reported data. 


\section{Settling Data}

\begin{tabular}{|c|c|c|c|}
\hline Settling Schedule & \multirow[b]{2}{*}{ PNNL-14365 } & \multirow[b]{2}{*}{ PNNL-14365 } & \multirow[b]{2}{*}{ PNNL-14365 } \\
\hline Source of Data & & & \\
\hline \multicolumn{4}{|l|}{ Temperature $\left({ }^{\circ} \mathrm{C}\right)$} \\
\hline Total initial volume & 13.7 & 13.6 & 13.5 \\
\hline 5 minutes & 13.7 & 13.6 & 13.3 \\
\hline 10 minutes & 13.7 & 13.6 & 12.5 \\
\hline 15 minutes & 13.7 & 13.4 & 11.9 \\
\hline 20 minutes & 13.7 & & \\
\hline 30 minutes & 13.7 & 13.3 & 10.9 \\
\hline 40 minutes & 13.7 & & \\
\hline 50 minutes & 13.7 & & \\
\hline 1 hour & 13.7 & 12.2 & 5.9 \\
\hline 2 hours & 13.7 & 10.1 & 4.9 \\
\hline 3 hours & 13.7 & 8.9 & 4.4 \\
\hline 4 hours & 13.7 & 8.9 & 4.1 \\
\hline \multicolumn{4}{|l|}{5 hours } \\
\hline 6 hours & 13.45 & 8.45 & 3.15 \\
\hline \multicolumn{4}{|l|}{24 hours } \\
\hline 32 hours & 12.6 & 6.4 & 3 \\
\hline 48 hours & & & 3 \\
\hline 56 hours & & & 3 \\
\hline 72 hours final settled & 11.9 & 6.2 & 3 \\
\hline
\end{tabular}

\section{Shear Strength Data}

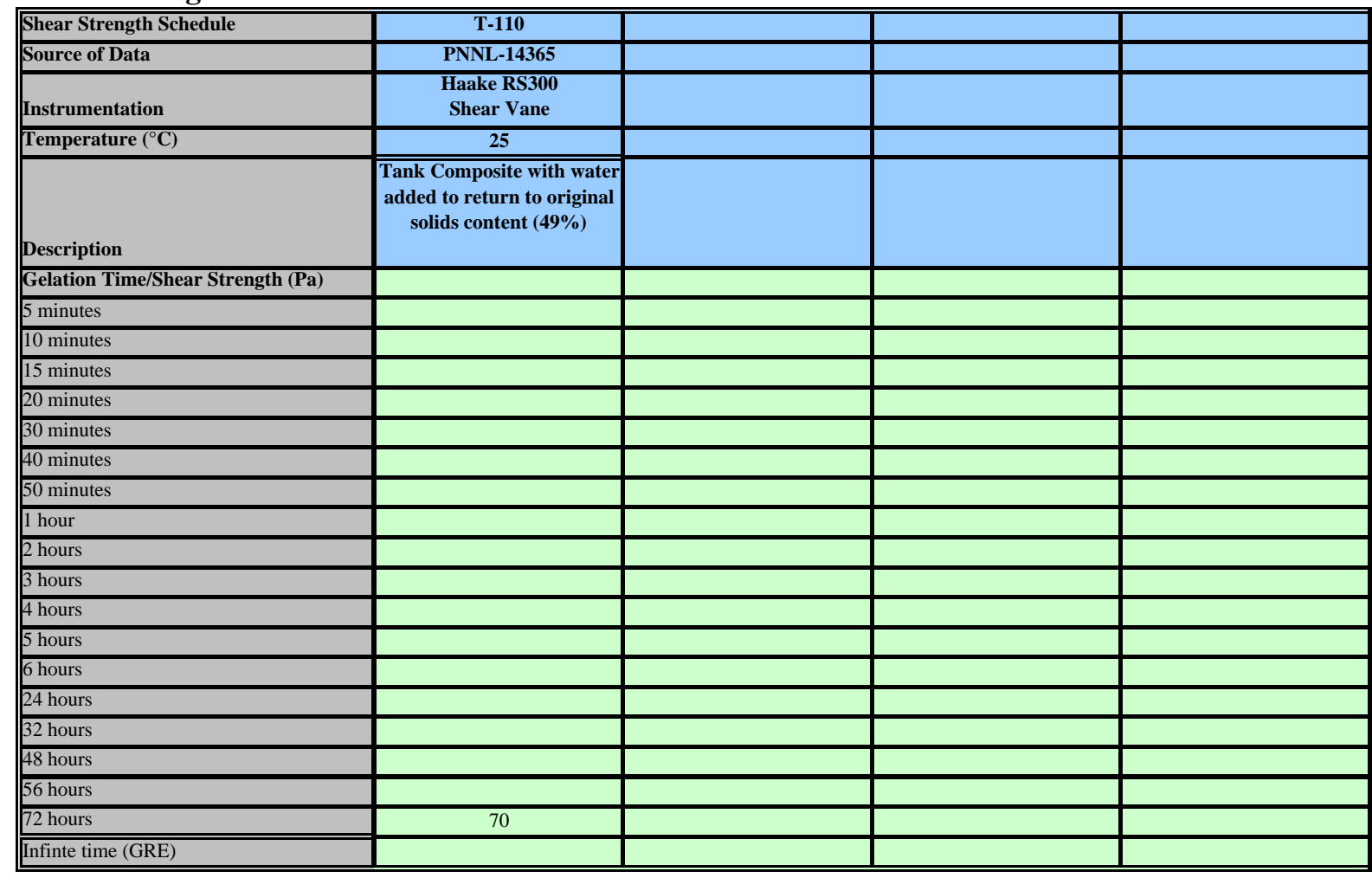

Note: Numbers in italics are calculated values. Numbers in normal font are reported data. 
Model Chemical Data From ESP

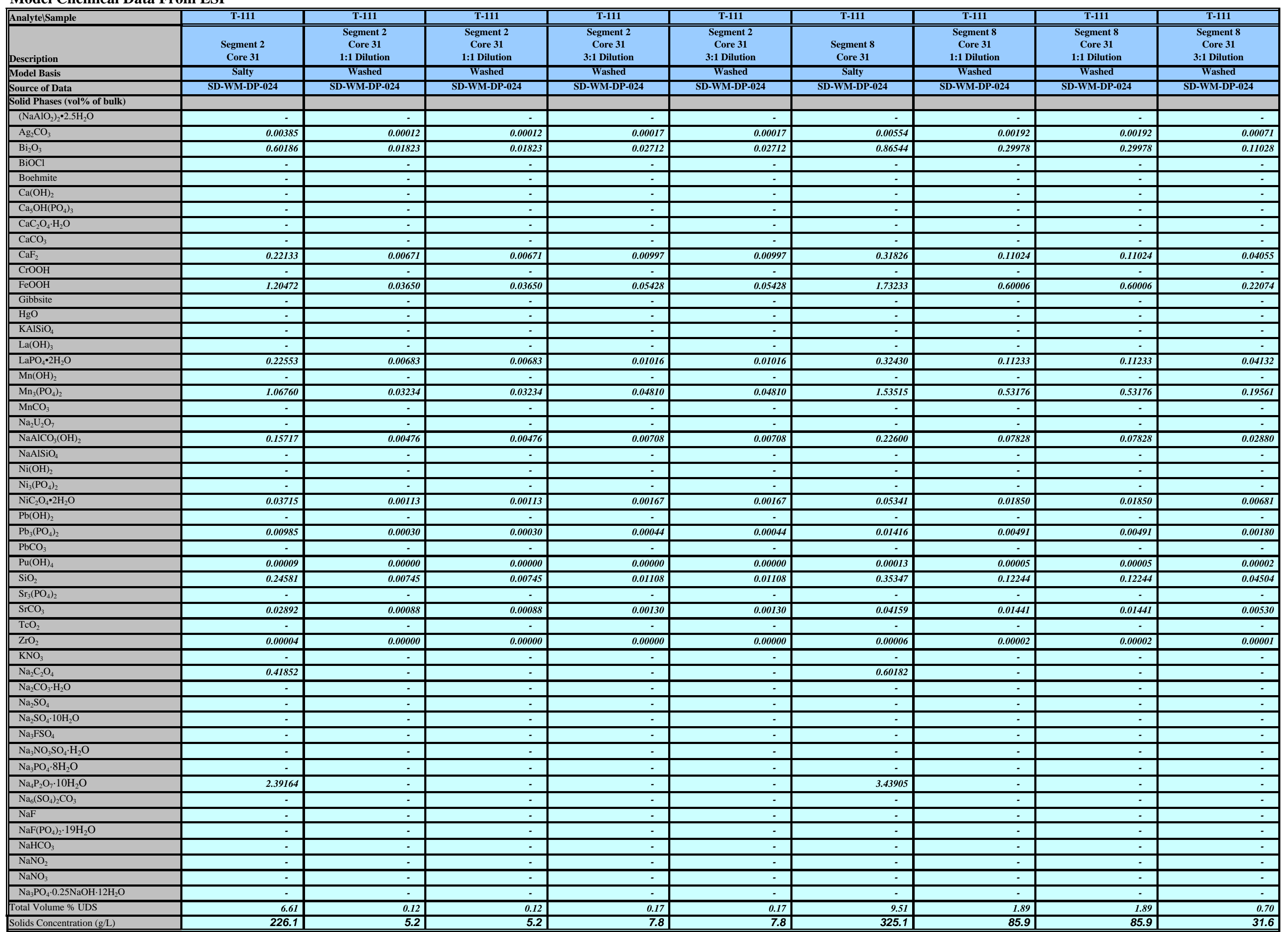


Rheological Data

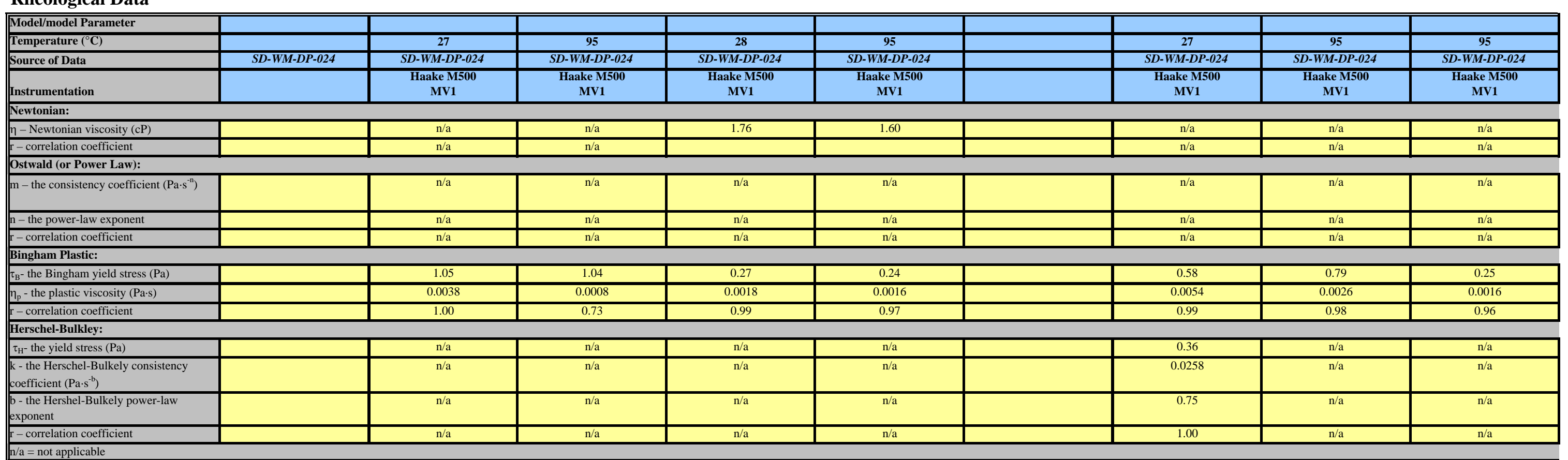

Physical Property Data

\begin{tabular}{|c|c|c|c|c|c|c|c|c|c|}
\hline Physical Property & 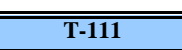 & 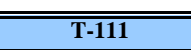 & 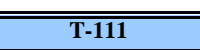 & 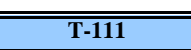 & T-111 & T-111 & T-111 & $\bar{T} \mathrm{~T}-111$ & $\overline{T-111}$ \\
\hline Source of Data & SD-WM-DP-024 & SD-WM-DP-024 & SD-WM-DP-024 & SD-WM-DP-024 & SD-WM-DP-024 & SD-WM-DP-024 & SD-WM-DP-024 & SD-WM-DP-024 & SD-WM-DP-024 \\
\hline Temperature $\left({ }^{\circ} \mathrm{C}\right)$ & & & & & & & & & 28 \\
\hline Zeta Potential $(\mathrm{mV})$ & & & & & & & & & \\
\hline Bulk Density $(\mathrm{g} / \mathrm{mL})$ & $\frac{1.19}{100}$ & $\frac{1.06}{85.7}$ & 1.06 & $\frac{1.03}{55.1}$ & 1.03 & $\frac{1.28}{100}$ & $\frac{1.12}{78.1}$ & 1.12 & $\frac{1.05}{36.4}$ \\
\hline $\begin{array}{l}\text { vol\% Settled Solids } \\
\text { Density of Centrifuged Solids (g/mL) }\end{array}$ & $\frac{1.22}{12}+\mathrm{C}$ & & & & & $\overline{1.22}$ & & & \\
\hline vol\% Centrifuged Solids & 65.8 & & & & & $\frac{1.22}{71.9}$ & & & \\
\hline w\% Centrifuged Solids & 67 & & & & & 76 & & & \\
\hline 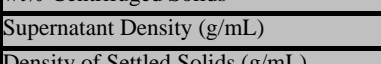 & 1.07 & 1.06 & 1.06 & 1.03 & 1.03 & 1.1 & 1.06 & 1.06 & 1.03 \\
\hline 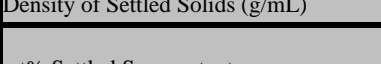 & 0 & & & & & 0 & & & \\
\hline wi\% disolved solids in supermatant & & & & & & & & & \\
\hline wt\% total solids in Centrifuged Sludge & & & & & & & & & \\
\hline w1\% Total Solids & 22.4 & & & & & 29.3 & & & \\
\hline$\left.\right|_{w 1 \%}$ UDS & 19.00 & 0.49 & 0.49 & 0.75 & 0.75 & 25.40 & 7.67 & 7.67 & 3.01 \\
\hline Density of Solidf fa/cl & 3.42 & 4.54 & 4.54 & 4.54 & 4.54 & $\frac{3.42}{3 .}$ & 4.54 & 4.54 & 4.54 \\
\hline
\end{tabular}



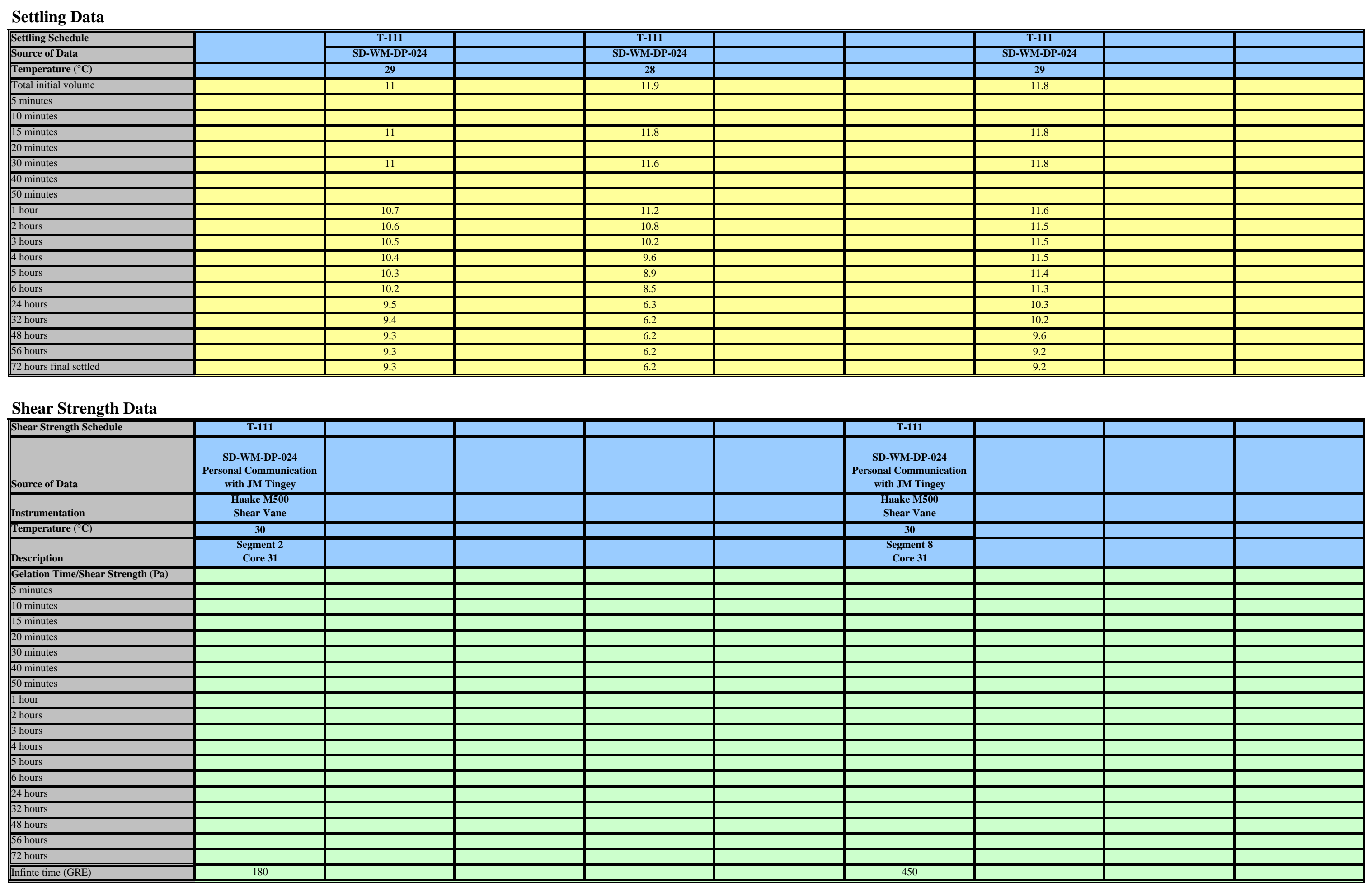
Model Chemical Data From ESP

\begin{tabular}{|c|c|c|c|c|}
\hline AnalytelSample & $\begin{array}{l}\text { T-203 } \\
\end{array}$ & $\begin{array}{l}\text { T-203 } \\
\end{array}$ & $\begin{array}{l}\text { T-203 } \\
\end{array}$ & $\begin{array}{l}\text { T-203 } \\
\end{array}$ \\
\hline Description & $\begin{array}{c}\text { Homogenized Tank } \\
\text { Composite }\end{array}$ & $\begin{array}{c}30 \mathrm{wt} \% \text { dilution of a core } \\
\text { composite }\end{array}$ & $\begin{array}{c}\begin{array}{c}1: 1 \text { Dilution of a core } \\
\text { composite }\end{array} \\
\text { compon }\end{array}$ & $\begin{array}{c}1 \text { Dilution of a core } \\
\text { composite }\end{array}$ \\
\hline Model Basis & $\begin{array}{l}\text { Salty } \\
\end{array}$ & Washed & Washed & Washed \\
\hline Source of Data & PNNL-14365 & PNNL-14365 & PNNL-14365 & PNNL-14365 \\
\hline \multicolumn{5}{|l|}{ Solid Phases (vol\% of bulk) } \\
\hline$\left(\mathrm{NaAlO}_{2}\right)_{2} \cdot 2.5 \mathrm{H}_{2} \mathrm{O}$ & - & - & - & - \\
\hline $\mathrm{Ag}_{2} \mathrm{CO}_{3}$ & 0.00003 & 0.00010 & 0.00004 & 0.00001 \\
\hline $\mathrm{Bi}_{2} \mathrm{O}_{3}$ & 0.33825 & 1.00803 & 0.39795 & 0.13122 \\
\hline$\overline{\mathrm{BiOCl}}$ & - & - & - & - \\
\hline Boehmite & - & - & $\overline{-}$ & - \\
\hline$\overline{\mathrm{Ca}(\mathrm{OH})_{2}}$ & - & - & - & - \\
\hline $\mathrm{Ca}_{5} \mathrm{OH}\left(\mathrm{PO}_{4}\right)_{3}$ & - & - & - & - \\
\hline $\mathrm{CaC}_{2} \mathrm{O}_{4} \cdot \mathrm{H}_{2} \mathrm{O}$ & - & - & - & - \\
\hline$\overline{\mathrm{CaCO}_{3}}$ & - & - & - & - \\
\hline $\mathrm{CaF}_{2}$ & 0.01498 & 0.04465 & 0.01763 & 0.00581 \\
\hline $\mathrm{CrOOH}$ & - & - & - & - \\
\hline $\mathrm{FeOOH}$ & 0.10698 & 0.31881 & 0.12586 & 0.04150 \\
\hline Gibbsite & - & - & - & - \\
\hline $\mathrm{HgO}$ & - & - & - & - \\
\hline $\mathrm{KAISiO}_{4}$ & 0.00888 & 0.02645 & 0.01044 & 0.00344 \\
\hline $\mathrm{La}(\mathrm{OH})_{3}$ & - & - & - & - \\
\hline $\mathrm{LaPO}_{4} \cdot 2 \mathrm{H}_{2} \mathrm{O}$ & 0.20946 & 0.62422 & 0.24643 & 0.08126 \\
\hline $\mathrm{Mn}(\mathrm{OH})_{2}$ & 0.26426 & 0.78753 & 0.31090 & 0.10252 \\
\hline $\mathrm{Mn}_{3}\left(\mathrm{PO}_{4}\right)_{2}$ & - & - & - & - \\
\hline $\mathrm{MnCO}_{3}$ & 0.14380 & 0.42855 & 0.16918 & 0.05579 \\
\hline $\mathrm{Na}_{2} \mathrm{U}_{2} \mathrm{O}_{7}$ & 0.00152 & 0.00452 & 0.00178 & 0.00059 \\
\hline $\mathrm{NaAlCO}_{3}(\mathrm{OH})_{2}$ & - & - & $\overline{-}$ & - \\
\hline $\mathrm{NaAlSiO}_{4}$ & - & - & - & - \\
\hline$\overline{\mathrm{Ni}(\mathrm{OH})_{2}}$ & 0.00402 & 0.01197 & 0.00473 & 0.00156 \\
\hline $\mathrm{Ni}_{3}\left(\mathrm{PO}_{4}\right)_{2}$ & - & - & - & - \\
\hline $\mathrm{NiC}_{2} \mathrm{O}_{4} \cdot 2 \mathrm{H}_{2} \mathrm{O}$ & $\overline{-}$ & - & $\overline{-}$ & $\overline{-}$ \\
\hline $\mathrm{Pb}(\mathrm{OH})_{2}$ & 0.00022 & 0.00067 & 0.00026 & 0.00009 \\
\hline$\overline{\mathrm{Pb}_{3}\left(\mathrm{PO}_{4}\right)_{2}}$ & $\overline{-}$ & - & $\overline{-}$ & $\overline{-}$ \\
\hline $\mathrm{PbCO}_{3}$ & - & - & - & - \\
\hline$\frac{\mathrm{Pu}(\mathrm{OH})_{4}}{}$ & 0.00004 & 0.00013 & 0.00005 & 0.00002 \\
\hline $\mathrm{SiO}_{2}$ & - & - & - & - \\
\hline $\mathrm{Sr}_{3}\left(\mathrm{PO}_{4}\right)_{2}$ & - & $\overline{-}$ & $\overline{-}$ & - \\
\hline $\mathrm{SrCO}_{3}$ & 0.01695 & 0.05051 & 0.01994 & 0.00657 \\
\hline $\mathrm{TcO}_{2}$ & - & - & - & - \\
\hline $\mathrm{ZrO}_{2}$ & 0.00003 & 0.00009 & 0.00004 & 0.00001 \\
\hline $\mathrm{KNO}_{3}$ & - & - & - & - \\
\hline $\mathrm{Na}_{2} \mathrm{C}_{2} \mathrm{O}_{4}$ & $\overline{-}$ & - & $\overline{-}$ & - \\
\hline $\mathrm{Na}_{2} \mathrm{CO}_{3} \cdot \mathrm{H}_{2} \mathrm{O}$ & - & - & - & - \\
\hline $\mathrm{Na}_{2} \mathrm{SO}_{4}$ & $\overline{-}$ & - & $\overline{-}$ & - \\
\hline $\mathrm{Na}_{2} \mathrm{SO}_{4} \cdot 10 \mathrm{H}_{2} \mathrm{O}$ & - & - & $\overline{-}$ & - \\
\hline $\mathrm{Na}_{3} \mathrm{FSO}_{4}$ & $\overline{-}$ & - & $\overline{-}$ & $\overline{-}$ \\
\hline $\mathrm{Na}_{3} \mathrm{NO}_{3} \mathrm{SO}_{4} \cdot \mathrm{H}_{2} \mathrm{O}$ & - & - & - & - \\
\hline $\mathrm{Na}_{3} \mathrm{PO}_{4} \cdot 8 \mathrm{H}_{2} \mathrm{O}$ & - & - & - & - \\
\hline $\mathrm{Na}_{4} \mathrm{P}_{2} \mathrm{O}_{7} \cdot 10 \mathrm{H}_{2} \mathrm{O}$ & - & - & - & - \\
\hline $\mathrm{Na}_{6}\left(\mathrm{SO}_{4}\right)_{2} \mathrm{CO}_{3}$ & - & - & $\overline{-}$ & - \\
\hline $\mathrm{NaF}$ & - & - & - & - \\
\hline $\mathrm{NaF}\left(\mathrm{PO}_{4}\right)_{2} \cdot 19 \mathrm{H}_{2} \mathrm{O}$ & $\overline{-}$ & - & $\overline{-}$ & - \\
\hline $\mathrm{NaHCO}_{3}$ & - & - & $\overline{-}$ & - \\
\hline $\mathrm{NaNO}_{2}$ & - & - & - & - \\
\hline $\mathrm{NaNO}_{3}$ & $\overline{-}$ & - & $\overline{-}$ & $\overline{-}$ \\
\hline $\mathrm{Na}_{3} \mathrm{PO}_{4} \cdot 0.25 \mathrm{NaOH} \cdot 12 \mathrm{H}_{2} \mathrm{O}$ & - & - & - & - \\
\hline Total Volume \% UDS & 1.11 & 3.31 & 1.31 & 0.43 \\
\hline Solids Concentration ( $\mathrm{g} / \mathrm{L}$ ) & 63.0 & 187.7 & 74.1 & 24.4 \\
\hline
\end{tabular}

Note: Numbers in italics are calculated values. Numbers in normal font are reported data. 


\section{Rheological Data}

\begin{tabular}{|c|c|c|c|}
\hline$\sqrt{\text { Model/model Parameter }}$ & 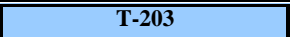 & 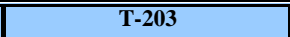 & 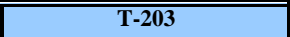 \\
\hline Temperature $\left({ }^{\circ} \mathrm{C}\right)$ & 25 & 25 & 25 \\
\hline Source of Data & PNNL-14365 & PNNL-14365 & PNNL-14365 \\
\hline |Instrumentation & $\begin{array}{c}\text { Haake RS300 } \\
\text { Concentric Cylinders with a } \\
3 \mathrm{~mm} \text { Gap }\end{array}$ & \begin{tabular}{|c|} 
Haake RS300 \\
Concentric Cylinders with a \\
$3 \mathrm{~mm}$ Gap
\end{tabular} & \begin{tabular}{|c|} 
Haake RS300 \\
Concentric Cylinders with a \\
$3 \mathrm{~mm}$ Gap
\end{tabular} \\
\hline \multicolumn{4}{|l|}{ Newtonian: } \\
\hline$\eta$ - Newtonian viscosity (cP) & $\mathrm{n} / \mathrm{a}$ & $\mathrm{n} / \mathrm{a}$ & $\mathrm{n} / \mathrm{a}$ \\
\hline $\mathrm{r}$ - correlation coefficient & $\mathrm{n} / \mathrm{a}$ & $\mathrm{n} / \mathrm{a}$ & $\mathrm{n} / \mathrm{a}$ \\
\hline \multicolumn{4}{|l|}{ Ostwald (or Power Law): } \\
\hline $\mathrm{m}$ - the consistency coefficient $\left(\mathrm{Pa} \cdot \mathrm{s}^{-\mathrm{n}}\right)$ & $\mathrm{n} / \mathrm{a}$ & $\mathrm{n} / \mathrm{a}$ & $\mathrm{n} / \mathrm{a}$ \\
\hline $\mathrm{n}$ - the power-law exponent & $\mathrm{n} / \mathrm{a}$ & $\mathrm{n} / \mathrm{a}$ & $\mathrm{n} / \mathrm{a}$ \\
\hline $\mathrm{r}$ - correlation coefficient & $\mathrm{n} / \mathrm{a}$ & $\mathrm{n} / \mathrm{a}$ & $\mathrm{n} / \mathrm{a}$ \\
\hline \multicolumn{4}{|l|}{ Bingham Plastic: } \\
\hline$\tau_{\mathrm{B}}$ - the Bingham yield stress $(\mathrm{Pa})$ & 35.50 & 3.18 & 0.10 \\
\hline$\eta_{p}$ - the plastic viscosity (Pa-s) & 0.0148 & 0.0031 & 0.0015 \\
\hline $\mathrm{r}$ - correlation coefficient & 0.99 & 1.00 & 1.00 \\
\hline \multicolumn{4}{|l|}{ |Herschel-Bulkley: } \\
\hline$\tau_{\mathrm{H}^{-}}$the yield stress $(\mathrm{Pa})$ & $\mathrm{n} / \mathrm{a}$ & $\mathrm{n} / \mathrm{a}$ & $\mathrm{n} / \mathrm{a}$ \\
\hline $\begin{array}{l}\text { k - the Herschel-Bulkely consistency } \\
\text { coefficient }\left(\mathrm{Pa} \cdot \mathrm{s}^{-\mathrm{b}}\right)\end{array}$ & $\mathrm{n} / \mathrm{a}$ & $\mathrm{n} / \mathrm{a}$ & $\mathrm{n} / \mathrm{a}$ \\
\hline \begin{tabular}{|l} 
b - the Hershel-Bulkely power-law \\
exponent
\end{tabular} & $\mathrm{n} / \mathrm{a}$ & $\mathrm{n} / \mathrm{a}$ & $\mathrm{n} / \mathrm{a}$ \\
\hline $\mathrm{r}$ - correlation coefficient & $\mathrm{n} / \mathrm{a}$ & $\mathrm{n} / \mathrm{a}$ & $\mathrm{n} / \mathrm{a}$ \\
\hline
\end{tabular}

\section{Physical Property Data}

\begin{tabular}{|c|c|c|c|c|}
\hline$\sqrt{\text { Physical Property }}$ & 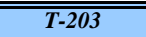 & 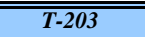 & 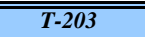 & $\overline{\bar{T}-203}$ \\
\hline Source of Data & PNNL-14365 & PNNL-14365 & PNNL-14365 & PNNL-14365 \\
\hline \multicolumn{5}{|l|}{ Temperature $\left({ }^{\circ} \mathrm{C}\right)$} \\
\hline \multicolumn{5}{|l|}{ Zeta Potential (mV) } \\
\hline Bulk Density (g/mL) & 1.22 & 1.29 & 1.14 & 1.05 \\
\hline vol\% Settled Solids & 100 & 100 & 92.8 & 44.8 \\
\hline Density of Centrifuged Solids (g/mL) & 1.39 & 1.3 & 1.31 & 1.29 \\
\hline vol\% Centrifuged Solids & 87.6 & 71.5 & 38.6 & 15.0 \\
\hline wt \% Centrifuged Solids & 100 & 72 & 44 & 18 \\
\hline Supernatant Density $(\mathrm{g} / \mathrm{mL})$ & 1.17 & 1.14 & 1.08 & 1.03 \\
\hline Density of Settled Solids (g/mL) & & & & \\
\hline wt\% Settled Supernatant & & & & \\
\hline wt $\%$ dissolved solids in supernatant & & & & \\
\hline wt $\%$ total solids in Centrifuged Sludge & & & & \\
\hline wt $\%$ Total Solids & 34.3 & $\overline{30.7}$ & 16.4 & 6.4 \\
\hline wt \% UDS & 5.16 & 14.55 & 6.50 & 2.33 \\
\hline Density of Solids (g/cc) & 5.68 & 5.68 & 5.68 & 5.68 \\
\hline
\end{tabular}

Note: Numbers in italics are calculated values. Numbers in normal font are reported data. 


\section{Settling Data}

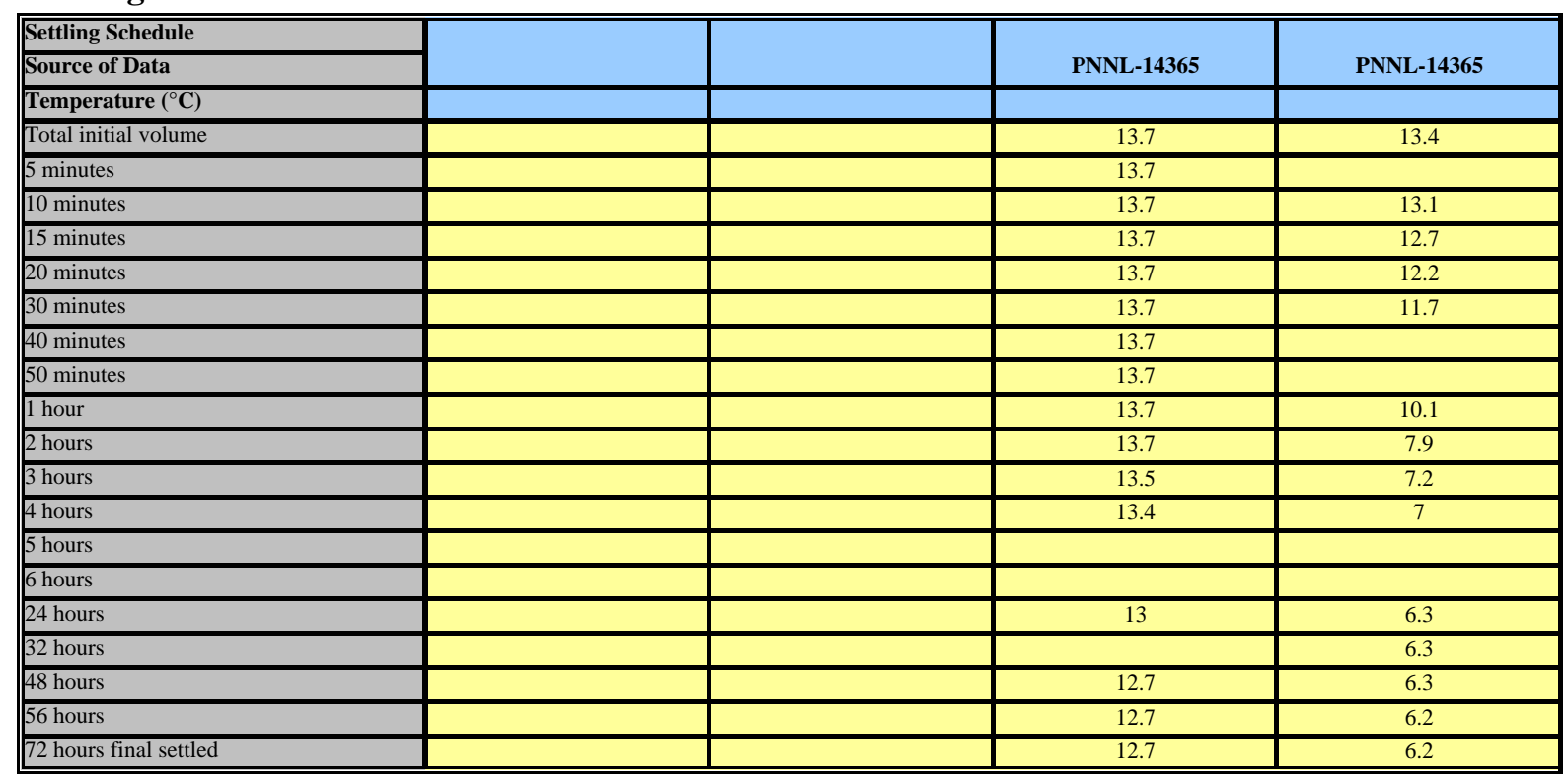

\section{Shear Strength Data}

\begin{tabular}{|c|c|c|c|c|}
\hline Shear Strength Schedule & 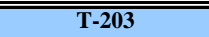 & 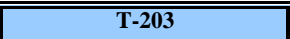 & & \\
\hline Source of Data & PNNL-14365 & PNNL-14365 & & \\
\hline ||Instrumentation & $\begin{array}{c}\text { Haake RS300 } \\
\text { Shear Vane }\end{array}$ & $\begin{array}{c}\text { Haake RS300 } \\
\text { Shear Vane }\end{array}$ & & \\
\hline Temperature $\left({ }^{\circ} \mathrm{C}\right)$ & 25 & 25 & & \\
\hline Description & $\begin{array}{l}\text { Homogenized Tank } \\
\text { Composite }\end{array}$ & $\begin{array}{l}30 \mathrm{wt} \% \text { dilution of the tank } \\
\text { composite }\end{array}$ & & \\
\hline \multicolumn{5}{|c|}{ Gelation Time/Shear Strength (Pa) } \\
\hline \multicolumn{5}{|l|}{5 minutes } \\
\hline \multicolumn{5}{|l|}{10 minutes } \\
\hline \multicolumn{5}{|l|}{15 minutes } \\
\hline \multicolumn{5}{|l|}{20 minutes } \\
\hline \multicolumn{5}{|l|}{30 minutes } \\
\hline \multicolumn{5}{|l|}{40 minutes } \\
\hline \multicolumn{5}{|l|}{50 minutes } \\
\hline \multicolumn{5}{|l|}{1 hour } \\
\hline \multicolumn{5}{|l|}{2 hours } \\
\hline \multicolumn{5}{|l|}{3 hours } \\
\hline \multicolumn{5}{|l|}{4 hours } \\
\hline \multicolumn{5}{|l|}{5 hours } \\
\hline \multicolumn{5}{|l|}{6 hours } \\
\hline \multicolumn{5}{|l|}{24 hours } \\
\hline \multicolumn{5}{|l|}{32 hours } \\
\hline \multicolumn{5}{|l|}{48 hours } \\
\hline \multicolumn{5}{|l|}{56 hours } \\
\hline 72 hours & 3770 & 310 & & \\
\hline Infinte time (GRE) & & & & \\
\hline
\end{tabular}


Model Chemical Data From ESP

\begin{tabular}{|c|c|c|c|c|}
\hline AnalytelSample & $\begin{array}{l}\text { T-204 } \\
\end{array}$ & $\begin{array}{l}\text { T-204 } \\
\end{array}$ & $\begin{array}{l}\text { T-204 } \\
\end{array}$ & $\begin{array}{l}\text { T-204 } \\
\end{array}$ \\
\hline Description & $\begin{array}{c}\text { Homogenized Tank } \\
\text { Composite }\end{array}$ & $\begin{array}{c}30 \mathrm{wt} \% \text { dilution of a core } \\
\text { composite }\end{array}$ & $\begin{array}{c}\begin{array}{c}1: 1 \text { Dilution of a core } \\
\text { composite }\end{array} \\
\text { compon }\end{array}$ & $\begin{array}{c}1 \text { Dilution of a core } \\
\text { composite }\end{array}$ \\
\hline Model Basis & $\begin{array}{l}\text { Salty } \\
\end{array}$ & Washed & Washed & Washed \\
\hline Source of Data & PNNL-14365 & PNNL-14365 & PNNL-14365 & PNNL-14365 \\
\hline \multicolumn{5}{|l|}{ Solid Phases (vol\% of bulk) } \\
\hline$\left(\mathrm{NaAlO}_{2}\right)_{2} \cdot 2.5 \mathrm{H}_{2} \mathrm{O}$ & - & - & - & - \\
\hline $\mathrm{Ag}_{2} \mathrm{CO}_{3}$ & 0.00002 & 0.00011 & 0.00005 & 0.00001 \\
\hline $\mathrm{Bi}_{2} \mathrm{O}_{3}$ & 0.21416 & 1.13735 & 0.49136 & 0.13952 \\
\hline$\overline{\mathrm{BiOCl}}$ & - & - & - & - \\
\hline Boehmite & - & - & $\overline{-}$ & - \\
\hline$\overline{\mathrm{Ca}(\mathrm{OH})_{2}}$ & - & - & - & - \\
\hline $\mathrm{Ca}_{5} \mathrm{OH}\left(\mathrm{PO}_{4}\right)_{3}$ & - & - & - & - \\
\hline $\mathrm{CaC}_{2} \mathrm{O}_{4} \cdot \mathrm{H}_{2} \mathrm{O}$ & - & - & - & - \\
\hline$\overline{\mathrm{CaCO}_{3}}$ & - & - & - & - \\
\hline $\mathrm{CaF}_{2}$ & 0.00419 & 0.02226 & 0.00962 & 0.00273 \\
\hline$\overline{\mathrm{CrOOH}}$ & 0.00512 & 0.02717 & 0.01174 & 0.00333 \\
\hline $\mathrm{FeOOH}$ & 0.05008 & 0.26599 & 0.11491 & 0.03263 \\
\hline Gibbsite & - & - & - & - \\
\hline $\mathrm{HgO}$ & - & - & - & - \\
\hline $\mathrm{KAISiO}_{4}$ & 0.00340 & 0.01806 & 0.00780 & 0.00222 \\
\hline $\mathrm{La}(\mathrm{OH})_{3}$ & - & - & - & - \\
\hline $\mathrm{LaPO}_{4} \cdot 2 \mathrm{H}_{2} \mathrm{O}$ & 0.11411 & 0.60603 & 0.26182 & 0.07434 \\
\hline $\mathrm{Mn}(\mathrm{OH})_{2}$ & 0.23176 & 1.23083 & 0.53175 & 0.15098 \\
\hline $\mathrm{Mn}_{3}\left(\mathrm{PO}_{4}\right)_{2}$ & - & - & - & - \\
\hline $\mathrm{MnCO}_{3}$ & - & - & $\overline{-}$ & - \\
\hline $\mathrm{Na}_{2} \mathrm{U}_{2} \mathrm{O}_{7}$ & 0.00112 & 0.00593 & 0.00256 & 0.00073 \\
\hline $\mathrm{NaAlCO}_{3}(\mathrm{OH})_{2}$ & - & - & $\overline{-}$ & - \\
\hline $\mathrm{NaAlSiO}_{4}$ & - & - & - & - \\
\hline$\overline{\mathrm{Ni}(\mathrm{OH})_{2}}$ & 0.00309 & 0.01641 & 0.00709 & 0.00201 \\
\hline $\mathrm{Ni}_{3}\left(\mathrm{PO}_{4}\right)_{2}$ & - & - & - & - \\
\hline $\mathrm{NiC}_{2} \mathrm{O}_{4} \cdot 2 \mathrm{H}_{2} \mathrm{O}$ & $\overline{-}$ & - & $\overline{-}$ & $\overline{-}$ \\
\hline $\mathrm{Pb}(\mathrm{OH})_{2}$ & 0.00158 & 0.00838 & 0.00362 & 0.00103 \\
\hline$\overline{\mathrm{Pb}_{3}\left(\mathrm{PO}_{4}\right)_{2}}$ & $\overline{-}$ & - & $\overline{-}$ & $\overline{-}$ \\
\hline $\mathrm{PbCO}_{3}$ & - & - & - & - \\
\hline$\frac{\mathrm{Pu}(\mathrm{OH})_{4}}{}$ & 0.00001 & 0.00008 & 0.00003 & 0.00001 \\
\hline $\mathrm{SiO}_{2}$ & - & - & - & - \\
\hline $\mathrm{Sr}_{3}\left(\mathrm{PO}_{4}\right)_{2}$ & - & $\overline{-}$ & $\overline{-}$ & - \\
\hline $\mathrm{SrCO}_{3}$ & 0.00797 & 0.04230 & 0.01828 & 0.00519 \\
\hline $\mathrm{TcO}_{2}$ & - & - & - & - \\
\hline $\mathrm{ZrO}_{2}$ & 0.00001 & 0.00004 & 0.00002 & 0.00000 \\
\hline $\mathrm{KNO}_{3}$ & - & - & - & - \\
\hline $\mathrm{Na}_{2} \mathrm{C}_{2} \mathrm{O}_{4}$ & $\overline{-}$ & - & $\overline{-}$ & - \\
\hline $\mathrm{Na}_{2} \mathrm{CO}_{3} \cdot \mathrm{H}_{2} \mathrm{O}$ & - & - & - & - \\
\hline $\mathrm{Na}_{2} \mathrm{SO}_{4}$ & $\overline{-}$ & - & $\overline{-}$ & - \\
\hline $\mathrm{Na}_{2} \mathrm{SO}_{4} \cdot 10 \mathrm{H}_{2} \mathrm{O}$ & - & - & $\overline{-}$ & - \\
\hline $\mathrm{Na}_{3} \mathrm{FSO}_{4}$ & $\overline{-}$ & - & $\overline{-}$ & $\overline{-}$ \\
\hline $\mathrm{Na}_{3} \mathrm{NO}_{3} \mathrm{SO}_{4} \cdot \mathrm{H}_{2} \mathrm{O}$ & - & - & - & - \\
\hline $\mathrm{Na}_{3} \mathrm{PO}_{4} \cdot 8 \mathrm{H}_{2} \mathrm{O}$ & - & - & - & - \\
\hline $\mathrm{Na}_{4} \mathrm{P}_{2} \mathrm{O}_{7} \cdot 10 \mathrm{H}_{2} \mathrm{O}$ & - & - & - & - \\
\hline $\mathrm{Na}_{6}\left(\mathrm{SO}_{4}\right)_{2} \mathrm{CO}_{3}$ & - & - & $\overline{-}$ & - \\
\hline $\mathrm{NaF}$ & - & - & - & - \\
\hline $\mathrm{NaF}\left(\mathrm{PO}_{4}\right)_{2} \cdot 19 \mathrm{H}_{2} \mathrm{O}$ & $\overline{-}$ & - & $\overline{-}$ & - \\
\hline $\mathrm{NaHCO}_{3}$ & - & - & $\overline{-}$ & - \\
\hline $\mathrm{NaNO}_{2}$ & - & - & - & - \\
\hline $\mathrm{NaNO}_{3}$ & $\overline{-}$ & - & $\overline{-}$ & - \\
\hline $\mathrm{Na}_{3} \mathrm{PO}_{4} \cdot 0.25 \mathrm{NaOH} \cdot 12 \mathrm{H}_{2} \mathrm{O}$ & - & - & - & - \\
\hline Total Volume \% UDS & 0.64 & 3.38 & 1.46 & 0.41 \\
\hline Solids Concentration ( $\mathrm{g} / \mathrm{L}$ ) & 37.3 & 197.9 & 85.5 & 24.3 \\
\hline
\end{tabular}

Note: Numbers in italics are calculated values. Numbers in normal font are reported data. 


\section{Rheological Data}

\begin{tabular}{|c|c|c|}
\hline$\sqrt{\text { Model/model Parameter }}$ & 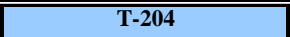 & 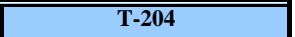 \\
\hline Temperature $\left({ }^{\circ} \mathrm{C}\right)$ & 25 & 25 \\
\hline Source of Data & PNNL-14365 & PNNL-14365 \\
\hline |Instrumentation & \begin{tabular}{|c|} 
Haake RS300 \\
Concentric Cylinders with a \\
$3 \mathrm{~mm}$ Gap
\end{tabular} & \begin{tabular}{|c|} 
Haake RS300 \\
Concentric Cylinders with a \\
$3 \mathrm{~mm}$ Gap
\end{tabular} \\
\hline \multicolumn{3}{|l|}{ Newtonian: } \\
\hline$\eta$ - Newtonian viscosity (cP) & $\mathrm{n} / \mathrm{a}$ & $\mathrm{n} / \mathrm{a}$ \\
\hline $\mathrm{r}$ - correlation coefficient & $\mathrm{n} / \mathrm{a}$ & $\mathrm{n} / \mathrm{a}$ \\
\hline \multicolumn{3}{|l|}{ Ostwald (or Power Law): } \\
\hline $\mathrm{m}$ - the consistency coefficient $\left(\mathrm{Pa} \cdot \mathrm{s}^{-\mathrm{n}}\right)$ & $\mathrm{n} / \mathrm{a}$ & $\mathrm{n} / \mathrm{a}$ \\
\hline $\mathrm{n}$ - the power-law exponent & $\mathrm{n} / \mathrm{a}$ & $\mathrm{n} / \mathrm{a}$ \\
\hline $\mathrm{r}$ - correlation coefficient & $\mathrm{n} / \mathrm{a}$ & $\mathrm{n} / \mathrm{a}$ \\
\hline \multicolumn{3}{|l|}{ Bingham Plastic: } \\
\hline$\tau_{\mathrm{B}}$ - the Bingham yield stress $(\mathrm{Pa})$ & 3.11 & 0.19 \\
\hline$\eta_{p}$ - the plastic viscosity (Pa-s) & 0.0038 & 0.0019 \\
\hline $\mathrm{r}$ - correlation coefficient & 1.00 & 1.00 \\
\hline \multicolumn{3}{|l|}{ Herschel-Bulkley: } \\
\hline$\tau_{\mathrm{H}^{-}}$the yield stress $(\mathrm{Pa})$ & $\mathrm{n} / \mathrm{a}$ & 0.14 \\
\hline $\begin{array}{l}\mathrm{k} \text { - the Herschel-Bulkely consistency } \\
\text { coefficient }\left(\mathrm{Pa} \cdot \mathrm{s}^{-\mathrm{b}}\right)\end{array}$ & $\mathrm{n} / \mathrm{a}$ & 0.0052 \\
\hline \begin{tabular}{|l} 
b - the Hershel-Bulkely power-law \\
exponent
\end{tabular} & $\mathrm{n} / \mathrm{a}$ & 0.83 \\
\hline $\mathrm{r}$ - correlation coefficient & $\mathrm{n} / \mathrm{a}$ & 1.00 \\
\hline
\end{tabular}

\section{Physical Property Data}

\begin{tabular}{|c|c|c|c|c|}
\hline$\longdiv { \text { Physical Property } }$ & $\overline{\bar{T}-204}$ & 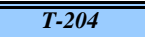 & 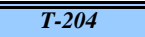 & $\overline{\bar{T}-204}$ \\
\hline Source of Data & PNNL-14365 & PNNL-14365 & PNNL-14365 & PNNL-14365 \\
\hline \multicolumn{5}{|l|}{ Temperature $\left({ }^{\circ} \mathrm{C}\right)$} \\
\hline \multicolumn{5}{|l|}{ Zeta Potential (mV) } \\
\hline Bulk Density $(\mathrm{g} / \mathrm{mL})$ & 1.17 & 1.28 & 1.13 & 1.05 \\
\hline vol\% Settled Solids & 100 & 100 & 88 & 46.2 \\
\hline Density of Centrifuged Solids (g/mL) & 1.4 & 1.36 & 1.3 & 1.35 \\
\hline vol\% Centrifuged Solids & 83.7 & 79.9 & 40 & 16.7 \\
\hline wt \% Centrifuged Solids & 100 & 85 & 46 & 21 \\
\hline Supernatant Density $(\mathrm{g} / \mathrm{mL})$ & 1.14 & 1.12 & 1.06 & 1.03 \\
\hline Density of Settled Solids (g/mL) & & & & \\
\hline wt\% Settled Supernatant & & & & \\
\hline wt $\%$ dissolved solids in supernatant & & & & \\
\hline wt \% total solids in Centrifuged Sludge & & & & \\
\hline wt $\%$ Total Solids & 31.5 & 29.4 & 14.6 & 6.1 \\
\hline wt $\%$ UDS & 3.18 & 15.46 & 7.56 & 2.31 \\
\hline Density of Solids (g/cc) & 5.85 & 5.85 & 5.85 & 5.85 \\
\hline
\end{tabular}

Note: Numbers in italics are calculated values. Numbers in normal font are reported data. 


\section{Settling Data}

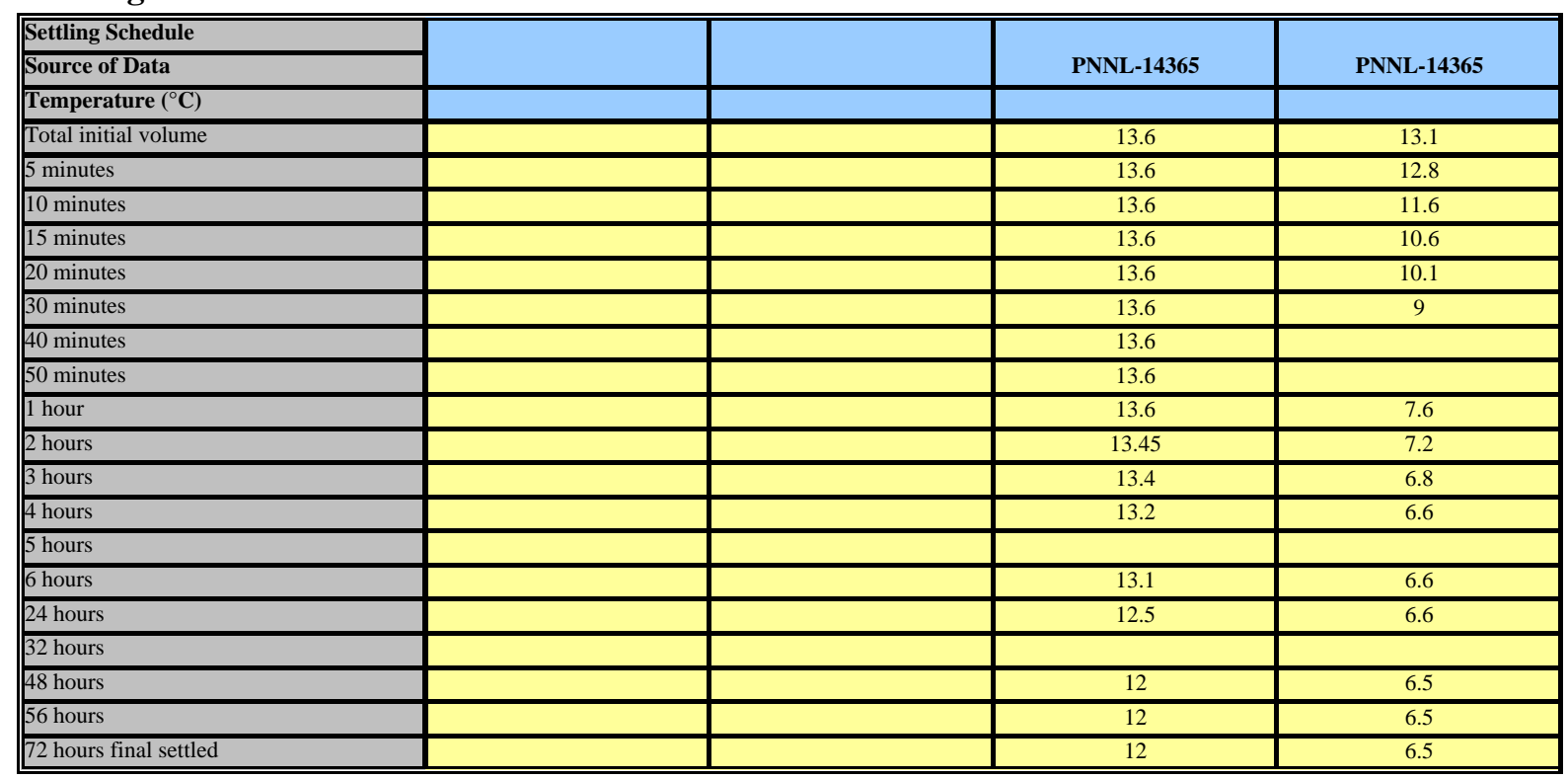

\section{Shear Strength Data}

\begin{tabular}{|c|c|c|c|c|}
\hline Shear Strength Schedule & 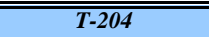 & 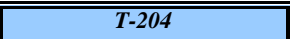 & 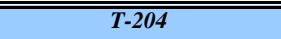 & \\
\hline Source of Data & PNNL-14365 & PNNL-14365 & PNNL-14365 & \\
\hline || Instrumentation & $\begin{array}{c}\text { Haake RS300 } \\
\text { Shear Vane }\end{array}$ & $\begin{array}{c}\text { Haake RS300 } \\
\text { Shear Vane }\end{array}$ & $\begin{array}{c}\text { Haake RS300 } \\
\text { Shear Vane }\end{array}$ & \\
\hline Temperature $\left({ }^{\circ} \mathrm{C}\right)$ & 25 & 25 & 25 & \\
\hline Description & $\begin{array}{l}\text { Homogenized Tank } \\
\text { Composite }\end{array}$ & $\begin{array}{l}30 \text { wt } \% \text { dilution of the tank } \\
\text { composite }\end{array}$ & $\begin{array}{c}\text { Settled Solids from the } 1: 1 \\
\text { Dilution (18.0 wt \% total } \\
\text { solids) }\end{array}$ & \\
\hline \multicolumn{5}{|c|}{ Gelation Time/Shear Strength (Pa) } \\
\hline \multicolumn{5}{|l|}{5 minutes } \\
\hline \multicolumn{5}{|l|}{10 minutes } \\
\hline \multicolumn{5}{|l|}{15 minutes } \\
\hline \multicolumn{5}{|l|}{20 minutes } \\
\hline \multicolumn{5}{|l|}{30 minutes } \\
\hline \multicolumn{5}{|l|}{40 minutes } \\
\hline \multicolumn{5}{|l|}{50 minutes } \\
\hline \multicolumn{5}{|l|}{1 hour } \\
\hline \multicolumn{5}{|l|}{2 hours } \\
\hline \multicolumn{5}{|l|}{3 hours } \\
\hline \multicolumn{5}{|l|}{4 hours } \\
\hline \multicolumn{5}{|l|}{5 hours } \\
\hline \multicolumn{5}{|l|}{6 hours } \\
\hline \multicolumn{5}{|l|}{24 hours } \\
\hline \multicolumn{5}{|l|}{32 hours } \\
\hline \multicolumn{5}{|l|}{48 hours } \\
\hline \multicolumn{5}{|l|}{56 hours } \\
\hline 72 hours & 1520 & 842 & 20 & \\
\hline Infinte time (GRE) & & & & \\
\hline
\end{tabular}




\begin{tabular}{|c|c|c|c|c|c|c|c|c|c|c|c|c|c|c|c|c|c|c|}
\hline 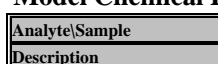 & 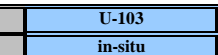 & $\begin{array}{l}\text { U.1-103 } \\
\text { insitit } \\
\end{array}$ & insitim & 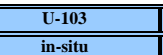 & insititu & 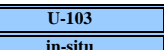 & in-situ & $\begin{array}{ll}\text { U-103 } \\
\text { ins-ititu }\end{array}$ & $\begin{array}{ll}\mathrm{U} \text { U-103 } \\
\text { inistiut }\end{array}$ & $\begin{array}{l}\text { U.1-133 } \\
\text { insitum }\end{array}$ & 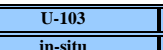 & insititu & $\begin{array}{ll}\text { U-103 } \\
\text { inn-isit }\end{array}$ & in-isi & insiin & insiim & in-situ & ins-sium \\
\hline 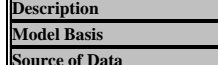 & 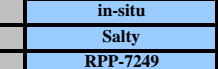 & 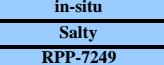 & 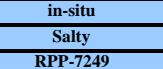 & 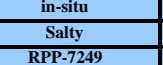 & $\begin{array}{c}\frac{\text { inisiut }}{\text { Saly }} \\
\text { RPP-7249 }\end{array}$ & 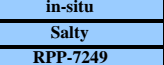 & 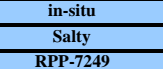 & 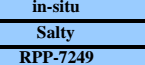 & $\begin{array}{c}\text { in-situ } \\
\text { Salty } \\
\text { RPP.7249 }\end{array}$ & 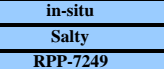 & 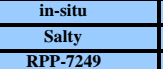 & $\begin{array}{c}\text { ins-situ } \\
\text { Salty } \\
\text { RPP. } 2499 \\
\end{array}$ & $\begin{array}{c}\text { insitu } \\
\text { Salty } \\
\text { RPP-7249 } \\
\end{array}$ & 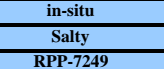 & 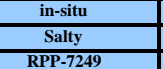 & 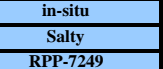 & 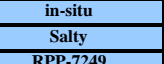 & 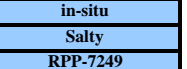 \\
\hline 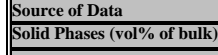 & & & & & & & & & & & & & & & & & & \\
\hline 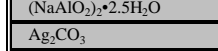 & & & & & & & & & & & & & & & & & & \\
\hline 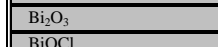 & 0.00074 & 0.00141 & 0.00114 & 0.0012 & 0.00141 & 0.00114 & 0.000105 & 0.000105 & 0.00159 & 0.0015 & 0.0020. & 0.00022 & 0.00159 & 0.00159 & 0.0011 & 0.00132 & 0.0000 & 0.00150 \\
\hline & & & & & & & & & & & & & & & & & & \\
\hline 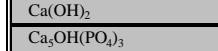 & $\begin{array}{c}0.05 \mathrm{~s} A \mathrm{~B} \\
\end{array}$ & 0.11199 & 0.00957 & $\frac{.}{0.0977}$ & 0.11197 & $\stackrel{.0 .9957}{0.9}$ & $\stackrel{.}{0.08344}$ & $\stackrel{.}{0.08344}$ & $\frac{.}{0.12633}$ & $\frac{-}{0.1262}$ & $\frac{.}{0.1618}$ & $\frac{\dot{1}}{0.001212}$ & $\stackrel{\square}{0.12633}$ & $\frac{\dot{0}}{0.12633}$ & $\stackrel{\square}{0.126}$ & $\frac{\dot{1}}{0.10433}$ & $\stackrel{\square}{0.0014}$ & $\stackrel{\square}{0.1910}$ \\
\hline $\begin{array}{ll}\mathrm{CaC}_{2} \mathrm{O}_{4} \mathrm{H}_{2} \mathrm{O} \\
\mathrm{Cac}_{4}\end{array}$ & & & & & & & & & & & & & & & & & & \\
\hline $\mathrm{at}_{2}$ & & & & & & & & & & & & & & & & & & \\
\hline $\begin{array}{l}\text { CroOH } \\
\text { ProOH }\end{array}$ & 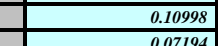 & 0.21059 & 0.17035 & 0.1837 & $\frac{0.21059}{0.12507}$ & 0.17035 & 0.15693 & 0.15693 & 0.03742 & 0.2374 & 0.0 .3049 & 0.0026 & 0.237. & $\frac{0.23742}{0.3620}$ & 0.237 & 0.19717 & $\frac{0.026}{.02612}$ & 0.22400 \\
\hline $\begin{array}{l}\text { Gibbsite } \\
\text { theo }\end{array}$ & $\begin{array}{l}0.00353 \\
1.0353 \\
\end{array}$ & 20.09384 & 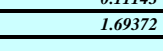 & $\frac{1.1 .2720 .}{1.820 .}$ & 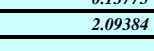 & 1.69372 & 告.56034 & 告.56034 & 告.36059 & 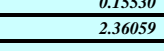 & $\begin{array}{l}0.919276 \\
3.0276 \\
\end{array}$ & $\begin{array}{l}0.0213 \\
0.02654\end{array}$ & $\begin{array}{l}0.15350 \\
2.36059 \\
\end{array}$ & $\begin{array}{l}0.1 .35350 \\
2.36059 \\
\end{array}$ & 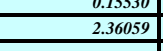 & $\begin{array}{l}0.12968 \\
1.96046 \\
\end{array}$ & 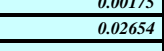 & $\begin{array}{l}0.42635 \\
\text { 2.22721 }\end{array}$ \\
\hline \begin{tabular}{|l}
$\mathrm{HgISO}_{4}$ \\
$\mathrm{KaSO}_{4}$
\end{tabular} & & & & & & & & & & & & & & & & & & \\
\hline 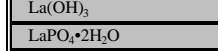 & 0.0002 & 0.00176 & 0.00143 & 0.0015 & 0.00776 & 0 & 0.00131 & 0.001311 & 0.001999 & 0.001999 & $\frac{.}{0.0025}$ & $\underline{0.0002}$ & $\frac{c}{0.0199}$ & $\frac{\pi}{0.0199}$ & $\stackrel{a 019}{0.019}$ & 0.00165 & $\frac{1000}{0.000}$ & 0.00186 \\
\hline 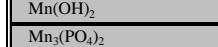 & & & & & & & & & & & & & & & & & & \\
\hline $\mathrm{N}_{\mathrm{N}_{2}, \mathrm{O}_{0},}$ & $\stackrel{\cdot}{0.01533}$ & 0.03548 & $\stackrel{\cdot}{0.02870}$ & 0.03099 & $\stackrel{.03548}{0 .}$ & 0.028770 & $\stackrel{.02644}{0.04}$ & 0.0 .02644 & 0.0 .00000 & $\stackrel{.04000}{0.04}$ & $\frac{\dot{c}}{0.05125}$ & 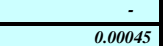 & $\stackrel{a}{0.080}$ & $\stackrel{.04000}{0.04}$ & $\stackrel{a .040}{0}$ & $\overline{0.0332}$ & $\stackrel{\square}{0.0004}$ & $\frac{\cdot 0374}{0.0374}$ \\
\hline 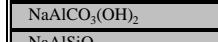 & & & & & & & & & & & & & & & & & & \\
\hline \begin{tabular}{|l}
$\mathrm{NaniOH}_{4}$ \\
$\mathrm{~N}\left(\mathrm{OH} \mathrm{H}_{2}\right.$
\end{tabular} & $\begin{array}{ll}0.2003 \\
0.00565\end{array}$ & $\begin{array}{l}0.02823 \\
0.01033\end{array}$ & $\begin{array}{ll}0.42132 \\
0.00876\end{array}$ & $\frac{0.4611}{0.0094}$ & 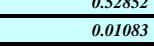 & 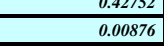 & $\begin{array}{ll}0.35367 \\
0.00807\end{array}$ & 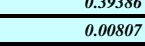 & $\begin{array}{l}0.95353 \\
0.01221 \\
0.121\end{array}$ & 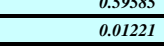 & $\begin{array}{ll}0.06416 \\
0.0156\end{array}$ & $\begin{array}{ll}2.00601 \\
0.00014\end{array}$ & 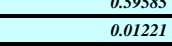 & $\begin{array}{l}0.95353 \\
0.01221\end{array}$ & 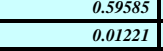 & $\begin{array}{l}0.94966 \\
0.01004\end{array}$ & 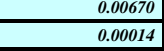 & $\begin{array}{l}\frac{0.5219}{0.0152} \\
0.15\end{array}$ \\
\hline 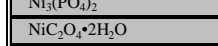 & & & & & & & & & & & $\therefore$ & & & & & & $\therefore$ & \\
\hline 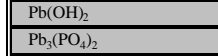 & 0.00222 & 0.00387 & 0.00313 & 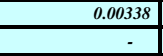 & 0.00387 & 0.00313 & $\begin{array}{ll}0.00288 \\
\end{array}$ & 0.00288 & 0.00346 & 0.00436 & $\begin{array}{ll}0.0056 \\
-\end{array}$ & .0.0005 & 0.00436 & 0.00436 & 0.004 & $\begin{array}{ll}0.0332 \\
-\end{array}$ & $\begin{array}{l}0.00005 \\
-\end{array}$ & - 0.0412 \\
\hline 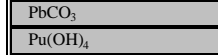 & 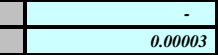 & 0.00005 & 0.00004 & 0.0000. & 0.00005 & 0.00004 & $\frac{1}{0.0004}$ & 0.00004 & . 0.00006 & 0.000066 & 0.000077 & 0.000000 & 0.00006 & $\stackrel{\square}{0.0006}$ & 0.00000 & .0.0005 & 0.00000 & 0.00005 \\
\hline & & - & & & - & & & & & & & & & & & & $=$ & \\
\hline 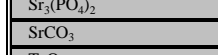 & (0.0001099 & 0.002209 & 0.00169 & .0.0182 & 0.002299 & 0.00169 & 0.00156 & 0.00156 & 0.00236 & 0.00236 & (.0.03032) & D.00003 & 0.00236 & 0.00236 & 0.00237 & 0.001966 & 0.00003 & 0.00222 \\
\hline $2 \mathrm{Ric}$ & 0.00045 & 0.00085 & 0.00069 & 0.00074 & 0.00085 & 0.00069 & 0.00064 & 0.00064 & 0.00096 & 0.00096 & 0.00123 & 0.00001 & 0.00096 & 0.00096 & 0.00098 & 0.00050 & 0.00001 & 0.00091 \\
\hline $\begin{array}{ll}N_{2} C_{2} O_{4} \\
\end{array}$ & 0.039014 & 0.74703 & 0.60427 & 0.05186 & 0.74703 & 0.60627 & 0.55669 & 0.55669 & 0.0821219 & 0.0 .84219 & 1.08012 & 0.00947 & 0.84219 & 0.0 .84219 & 0.0 .82121 & 0.69994 & 0.00947 & $\begin{array}{l}0.79461 \\
\end{array}$ \\
\hline $\begin{array}{ll}\mathrm{N}_{2}, \mathrm{O}_{0} \mathrm{~S}_{4} \mathrm{H}_{3} \\
\end{array}$ & & & & & & & & & & & & & & & & & & \\
\hline 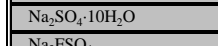 & & & & & & & & & & & & & & & & & & \\
\hline 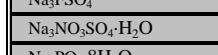 & & & & & & & & & & & & & & & & & & \\
\hline 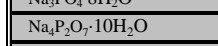 & & & & & & & & & 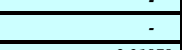 & & 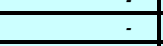 & 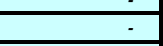 & 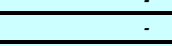 & 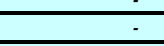 & 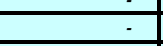 & 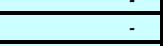 & 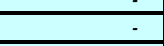 & $\bar{L}$ \\
\hline 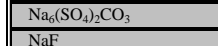 & 1.0743 & 2.05766 & 1.66415 & 1.79552 & 2.05766 & 1.6645 & 1.53338 & 1. 1.5338 & 2.31979 & 2.31979 & . & (.0.02608 & 2.331979 & 2.33999 & 2.3192. & 1.92659 & . & 2.1873 \\
\hline 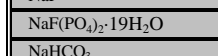 & 1.80837 & 3.46258 & 2.80090 & $\begin{array}{ll}3.02146 \\
3\end{array}$ & 3.46258 & 2.80090 & 2.50034 & 2.50834 & 3.90370 & $\begin{array}{ll}3.90370 \\
3.03\end{array}$ & 5.00055 & $\begin{array}{ll}0.03838 \\
0\end{array}$ & 3.90370 & 3.90370 & 3.0937 & 3224202 & 0.0 .0438 & 3.6334 \\
\hline 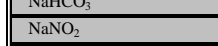 & & & & & & & & & & & & & & & & & & \\
\hline 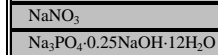 & $\begin{array}{ll}2.18776 \\
1.99871 \\
\end{array}$ & 23.333536 3.8773 & $\begin{array}{lll}18.87608 \\
3.0550\end{array}$ & 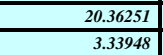 & 23.335363 & 18.8785085 & $\frac{17.38}{2.8 .8}$ & 17.79866 & 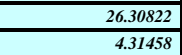 & 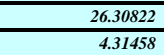 & $\begin{array}{l}3.7 .4333 \\
5.5334\end{array}$ & $\begin{array}{l}0.29575 \\
0.04585\end{array}$ & 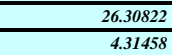 & 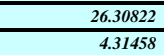 & 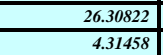 & 21.848944 & $\begin{array}{l}0.29575 \\
.04850\end{array}$ & 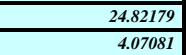 \\
\hline 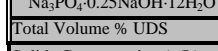 & $\frac{199882.25}{27}$ & 40.029 & $\begin{array}{l}3.252 \\
3\end{array}$ & $\frac{3.535}{738.51}$ & $\frac{30.69}{40.69}$ & $\begin{array}{l}\frac{3232.22}{73} \\
\end{array}$ & 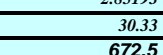 & $\begin{array}{l}3.0303 \\
363\end{array}$ & $\frac{14.58}{10198}$ & $\frac{4588}{1057}$ & $\frac{5384}{15084}$ & $\frac{0.052}{0.52}$ & $\frac{4588}{1458}$ & $\frac{4588}{1058}$ & 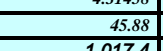 & $\begin{array}{l}38.10 \\
849\end{array}$ & 整.50 & 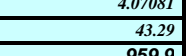 \\
\hline 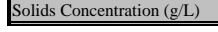 & & & & & & & & & & 1,017.4 & 1,304.8| & & & 1,017.4 & & 844.9 & 12.4 & 959.9 \\
\hline
\end{tabular}




\begin{tabular}{|c|c|c|c|c|c|c|c|c|c|c|c|c|c|c|c|c|c|c|}
\hline \multirow{2}{*}{\multicolumn{19}{|c|}{ 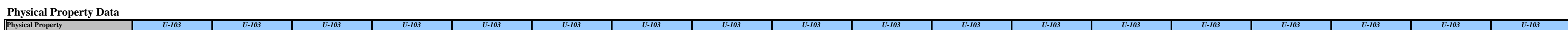 }} \\
\hline & $\begin{array}{ll}-103 \\
-103\end{array}$ & & $\begin{array}{l}-103 \\
\end{array}$ & & $U-103$ & $U:-103$ & $\begin{array}{lll}-103 \\
\end{array}$ & 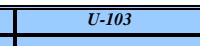 & $U-103$ & $\begin{array}{ll}U-103 \\
\end{array}$ & 0.103 & $\begin{array}{lll}U-103 \\
\end{array}$ & $\begin{array}{ll}U-103 \\
\end{array}$ & $U-103$ & & & & \\
\hline \multicolumn{19}{|l|}{ Source of Data } \\
\hline Temperature ( $(\mathrm{C})$ & & & & & & & & & & & & & & & & & & \\
\hline 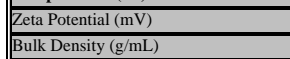 & 1.61 & ${ }_{1,76}$ & $\frac{1,70}{1.0}$ & $\frac{1.7}{1.7}$ & ${ }_{1.76}$ & 1,70 & ${ }_{1.68}$ & 1.68 & ${ }_{1.80}$ & 1.80 & 1.90 & 1.45 & 1.80 & 1.80 & $1.800>190$ & $\frac{1.74}{1.9}$ & 1.45 & 1.78 \\
\hline \multicolumn{19}{|l|}{ 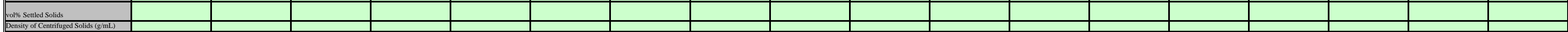 } \\
\hline \multicolumn{19}{|l|}{ vol\%c Cenrifituged Solits } \\
\hline \begin{tabular}{|l|l} 
w1\% Centrifuged Solids \\
\end{tabular} & & & & & & & & & & & & & & & & & & \\
\hline 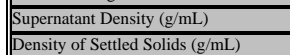 & 1.45 & $\frac{1.45}{4.4}$ & $\frac{1.45}{4.4}$ & $\frac{1.45}{4.9}$ & 1.45 & 1.45 & 1.45 & 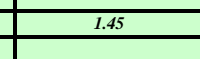 & 1.45 & 1.45 & 1.45 & 1.45 & 1.45 & 1.45 & 1.45 & 1.45 & 1.45 & 1.45 \\
\hline \multicolumn{19}{|l|}{ wwo Serted Supernatart } \\
\hline \multirow{2}{*}{\multicolumn{19}{|c|}{ we\% dissoved solids in sperematant }} \\
\hline \multirow{2}{*}{\multicolumn{19}{|c|}{ 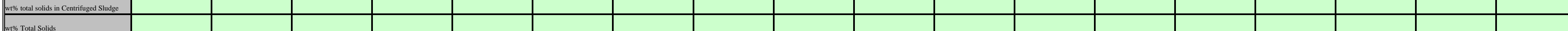 }} \\
\hline & & & & & & & & & & & & & & & & & & \\
\hline we\% UDS & 29.27 & 51.27 & 42.94 & 45.78 & 51.27 & 42,94 & 40.03 & 40.03 & 56.52 & 56.52 & 68.67 & 0.79 & 56.52 & 56.52 & 56.52 & 48.56 & 0.79 & 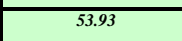 \\
\hline 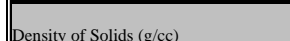 & 2.22 & 222 & 2.222 & 2.22 & 2.22 & 2.22 & 2.22 & 2.22 & 2.22 & & 2.22 & & 2.22 & & 2.22 & 2.2 .2 & $2.2 .2-3$ & \\
\hline
\end{tabular}

\begin{tabular}{|c|c|c|c|c|c|c|c|c|c|c|c|c|c|c|c|c|c|c|}
\hline 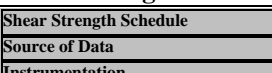 & $\begin{array}{c}\mathrm{U}-103 \\
\mathrm{RPP}-7249 \\
\text { RPR }\end{array}$ & 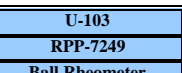 & 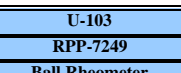 & $\begin{array}{l}\mathrm{U}-103 \\
\mathrm{RPP}-249 \\
\end{array}$ & 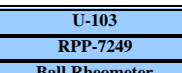 & $\frac{\mathrm{U}-103}{\mathrm{R} P \mathrm{P}-2 \mathrm{29}}$ & 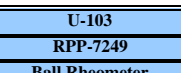 & $\begin{array}{l}\mathrm{U}-103 \\
\mathrm{RPP}-7249 \\
\end{array}$ & 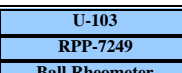 & 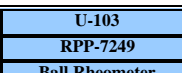 & 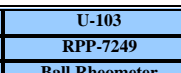 & $\begin{array}{c}\mathrm{U}-103 \\
\mathrm{RPP}-749 \\
\end{array}$ & $\begin{array}{c}\mathrm{U} \cdot 1-13 \\
\text { RPP.7249 } \\
\end{array}$ & $\begin{array}{c}\mathrm{U}-103 \\
\mathrm{RPP}-2.29 \\
\end{array}$ & 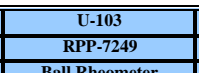 & 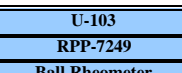 & $\frac{\mathrm{U}-103}{\mathrm{RPP}-2.29}$ & 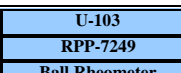 \\
\hline 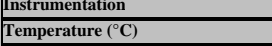 & \begin{tabular}{|l} 
Ball Rheometer \\
26
\end{tabular} & Ball Rreometer & Ball Renemeter & $\begin{array}{l}\text { Ball Rheometer } \\
29\end{array}$ & 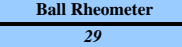 & \begin{tabular}{|l|} 
Ball Rheometer \\
29
\end{tabular} & Ball Rheometer & Ball Rheometer & 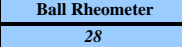 & 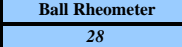 & \begin{tabular}{|l|} 
Ball Rheometer \\
28
\end{tabular} & $\begin{array}{l}\text { Ball Rheometer } \\
26\end{array}$ & 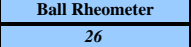 & \begin{tabular}{|l} 
Ball Rheometer \\
\end{tabular} & \begin{tabular}{|l|} 
Ball Rheometer \\
29
\end{tabular} & $\begin{array}{l}\text { Ball Rheometer } \\
29\end{array}$ & 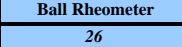 & \begin{tabular}{|l|} 
Ball Rheometal \\
26
\end{tabular} \\
\hline & \begin{tabular}{|c|} 
Riser 13 \\
Depth from botto
\end{tabular} & 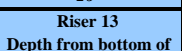 & $\begin{array}{c}\text { Riser } 13 \\
\text { Depht from botto }\end{array}$ & $\begin{array}{c}\text { Riser } 13 \\
\text { Depth from tott }\end{array}$ & \begin{tabular}{|c|} 
Riser 13 \\
Dept from totto
\end{tabular} & \begin{tabular}{|c|} 
Riser 13 13 \\
Depth from obto
\end{tabular} & $\begin{array}{l}\text { Riser } 13 \\
\text { Depht from botte }\end{array}$ & $\begin{array}{l}\text { Riser } 13 \\
\end{array}$ & $\begin{array}{l}\text { Riser } 13 \\
\end{array}$ & \begin{tabular}{|l|l} 
Riser 13 \\
\end{tabular} & \begin{tabular}{|l} 
Riser 13 \\
\end{tabular} & $\begin{array}{c}\text { Rier } 7 \\
\text { Rent }\end{array}$ & $\begin{array}{c}\text { Rier } 7 \\
\text { R. }\end{array}$ & $\begin{array}{l}\text { Riser } 7 \\
\text { Ren }\end{array}$ & 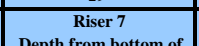 & $\begin{array}{c}\text { Rier } 7 \\
\text { Ren }\end{array}$ & 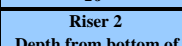 & $\begin{array}{l}\text { Riser } 2 \\
\text { Den }\end{array}$ \\
\hline tion & $\begin{array}{l}\text { Pank is } 160 \text { inchese } \\
\end{array}$ & 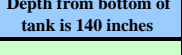 & 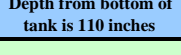 & 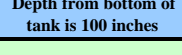 & 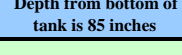 & 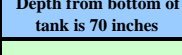 & 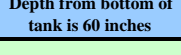 & $\begin{array}{l}\text { Depurinion oitum of } \\
\text { tank is } 0 \text { inches }\end{array}$ & 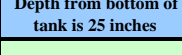 & 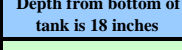 & $\begin{array}{c}\text { Deptintrim ofomom } \\
\text { tank is } 10 \text { inches } \\
\end{array}$ & $\begin{array}{l}\text { Dephnt rom boton of to } \\
\text { tank is } 170 \text { inches }\end{array}$ & 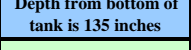 & 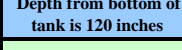 & 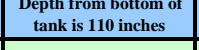 & 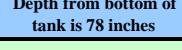 & 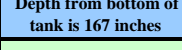 & 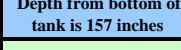 \\
\hline 年 & & & & & & & & & & & & & & & & & & \\
\hline & & & & & & & & & & & & & & & & & & \\
\hline & & & & & & & & & & & & & & & & & & \\
\hline & & & & & & & & & & & & & & & & & & \\
\hline & & & & & & & & & & & & & & & & & & \\
\hline & & & & & & & & & & & & & & & & & & \\
\hline & & & & & & & & & & & & & & & & & & \\
\hline & & & & & & & & & & & & & & & & & & \\
\hline & & & & & & & & & & & & & & & & & & \\
\hline (GRE) & 100 & 200 & $\frac{1750}{1750}$ & 1300 & 500 & 1200 & 800 & 20 & 700 & $\frac{150}{150}$ & 1000 & 50 & 70 & 150 & 200 & $\frac{1000}{1000}$ & 60 & $\frac{250}{250}$ \\
\hline
\end{tabular}


Model Chemical Data From ESP

\begin{tabular}{|c|c|c|c|c|c|c|c|c|c|c|c|c|}
\hline Analytel Sample & U-107 & $\mathrm{U}-107$ & $\begin{array}{l}\mathrm{U}-107 \\
\end{array}$ & $\mathrm{U}-107$ & $\begin{array}{l}\mathrm{U}-107 \\
\end{array}$ & $\mathrm{U}-107$ & $\mathrm{U}-107$ & $\begin{array}{ll}\mathrm{U}-107 \\
\mathrm{n}\end{array}$ & $\mathrm{U}-107$ & $\mathrm{U}-107$ & $\mathrm{U}-107$ & $\begin{array}{ll}\mathrm{U}-107 \\
\end{array}$ \\
\hline Description & $\begin{array}{l}\text { in-situ } \\
\text { Siltw }\end{array}$ & $\begin{array}{l}\text { in-situ } \\
\text { salts } \\
\end{array}$ & $\begin{array}{l}\text { in-situ } \\
\text { Salty } \\
\end{array}$ & $\begin{array}{l}\text { in-situ } \\
\text { Salvy }\end{array}$ & $\begin{array}{l}\text { in-situ } \\
\text { Silver } \\
\end{array}$ & $\begin{array}{l}\text { in-situ } \\
\text { Salty }\end{array}$ & $\begin{array}{l}\text { in-situ } \\
\text { salty }\end{array}$ & $\begin{array}{l}\text { in-situ } \\
\text { Slly } \\
\end{array}$ & $\begin{array}{l}\text { in-situ } \\
\text { Salvy } \\
\end{array}$ & $\begin{array}{l}\text { in-situ } \\
\text { Salvy }\end{array}$ & $\begin{array}{l}\text { in-situ } \\
\text { Salve } \\
\end{array}$ & $\begin{array}{l}\text { in-situ } \\
\text { salte }\end{array}$ \\
\hline 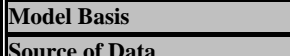 & $\frac{\text { Salty }}{\text { RPP.7249 }}$ & $\frac{\text { SPlty }}{\text { RPP.7249 }}$ & $\frac{\text { Salty }}{\text { RPP-7249 }}$ & 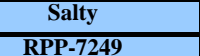 & $\begin{array}{c}\text { Salty } \\
\text { RPP-7249 }\end{array}$ & $\begin{array}{c}\text { Salty } \\
\text { RPP-7249 }\end{array}$ & $\frac{\text { Salty }}{\text { RPP } 7249}$ & $\frac{\text { Salty }}{\text { RPP-7249 }}$ & $\frac{\text { Sally }}{\text { RPP-7249 }}$ & $\frac{\text { Salty }}{\text { RPP-7249 }}$ & $\frac{5 \text { Salty }}{\text { RPP-7249 }}$ & $\frac{\text { Salty }}{\text { RPP-7249 }}$ \\
\hline \multirow{2}{*}{\multicolumn{11}{|c|}{ 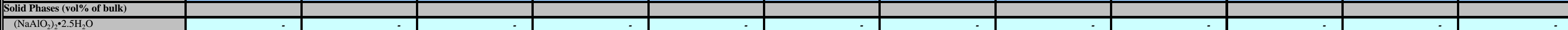 }} & RPP-7249 & RPP-7249 \\
\hline & & & & & & & & & & & & \\
\hline \multicolumn{13}{|l|}{$\mathrm{Ag}_{2} \mathrm{CO}_{3}$} \\
\hline $\mathrm{Bi}_{2} \mathrm{O}_{3}$ & 0.00180 & 0.00180 & 0.00180 & 0.00180 & 0.00180 & 0.00180 & 0.00180 & 0.00193 & 0.00193 & 0.00193 & 0.00168 & 0.00193 \\
\hline \multicolumn{13}{|l|}{\begin{tabular}{|l|} 
BioCl \\
Boehmite \\
\end{tabular}} \\
\hline \multicolumn{13}{|l|}{$\mathrm{Ca}(\mathrm{OH})_{2}$} \\
\hline $\mid \mathrm{Ca}_{5} \mathrm{OH}\left(\mathrm{PO}_{4}\right)_{3}$ & 0.06931 & 0.06931 & 0.06931 & $\begin{array}{ll}0.06931 \\
\end{array}$ & 0.06931 & 0.06931 & $\begin{array}{ll}0.06931 \\
\end{array}$ & 0.07406 & $\begin{array}{ll}0.07406 \\
\end{array}$ & 0.07406 & 0.06455 & $\begin{array}{ll}0.07406 \\
\end{array}$ \\
\hline \multirow{2}{*}{\multicolumn{13}{|c|}{\begin{tabular}{|l|}
$\mathrm{CaC}_{2} \mathrm{O}_{4} \mathrm{H}_{2} \mathrm{O}$ \\
$\mathrm{CaCO}_{3}$ \\
\end{tabular}}} \\
\hline & & & & & & & & & & & & \\
\hline \multicolumn{13}{|l|}{$\mathrm{CaF}_{2}$} \\
\hline \begin{tabular}{|cr}
$\mathrm{CrOOH}$ \\
\end{tabular} & 0.11583 & 0.11583 & 0.11583 & 0.11583 & 0.11583 & 0.11583 & $\begin{array}{ll}0.11583 \\
\end{array}$ & 0.12378 & 0.12378 & 0.12378 & 0.10788 & 0.12378 \\
\hline \begin{tabular}{|l|l|l} 
FeOOH \\
\end{tabular} & 0.14364 & 0.14364 & 0.14364 & 0.14364 & 0.14364 & 0.14364 & 0.14364 & 0.15350 & 0.15350 & 0.15350 & 0.13378 & 0.15350 \\
\hline \begin{tabular}{|l|l|} 
Gibbsite \\
Hon
\end{tabular} & 3.97764 & 3.97764 & 3.97764 & 3.97764 & 3.97764 & 3.97764 & 3.97764 & 4.25066 & 4.25066 & 4.25066 & 3.70462 & 4.25066 \\
\hline \multirow{2}{*}{\multicolumn{13}{|c|}{\begin{tabular}{|l|l|}
$\mathrm{HgO}^{2}$ \\
$\mathrm{KASIO}_{4}$
\end{tabular}}} \\
\hline \multirow{2}{*}{\multicolumn{13}{|c|}{\begin{tabular}{|l|}
$\mathrm{KAlOSOH}_{4}$ \\
$\mathrm{La}(\mathrm{OH})_{3}$
\end{tabular}}} \\
\hline & & & & & & & & & & & & \\
\hline $\mathrm{LaPO}_{4} \cdot 2 \mathrm{H}_{2} \mathrm{O}$ & 0.00153 & 0.00153 & 0.00153 & 0.00153 & 0.00153 & 0.00153 & 0.00153 & 0.00163 & 0.00163 & 0.00163 & 0.00142 & 0.00163 \\
\hline $\mathrm{Mn}(\mathrm{OH})_{2}$ & 0.06228 & 0.06228 & 0.06228 & 0.06228 & 0.06228 & 0.06228 & 0.06228 & 0.06655 & 0.06655 & 0.06655 & 0.05800 & 0.06655 \\
\hline \multicolumn{13}{|l|}{\begin{tabular}{|l|}
$\mathrm{Mn}_{3}\left(\mathrm{PO}_{4}\right)_{2}$ \\
\end{tabular}} \\
\hline \multicolumn{13}{|l|}{\begin{tabular}{|l|l|l}
$\mathrm{MnCO}_{3}$ \\
\end{tabular}} \\
\hline $\mathrm{Na}_{2} \mathrm{U}_{2} \mathrm{O}_{7}$ & 0.01841 & 0.01841 & 0.01841 & 0.01841 & 0.01841 & 0.01841 & 0.01841 & 0.01968 & 0.01968 & 0.01968 & 0.01715 & 0.01968 \\
\hline \multicolumn{13}{|l|}{\begin{tabular}{|l}
$\mathrm{NaAlCO}_{3}(\mathrm{OH})_{2}$ \\
\end{tabular}} \\
\hline \begin{tabular}{|lllll}
$\mathrm{NaAlISO}_{4}$ & \\
\end{tabular} & 0.11722 & 0.11722 & 0.11722 & 0.11722 & 0.11722 & 0.11722 & 0.11722 & 0.12527 & 0.12527 & 0.12527 & 0.10918 & 0.12527 \\
\hline$\sqrt{\mathrm{Ni}(\mathrm{OH})_{2}}$ & 0.00351 & 0.00351 & 0.00351 & 0.00351 & 0.00351 & 0.00351 & 0.00351 & 0.00375 & 0.00375 & 0.00375 & 0.00327 & 0.00375 \\
\hline \multirow{2}{*}{\multicolumn{13}{|c|}{ 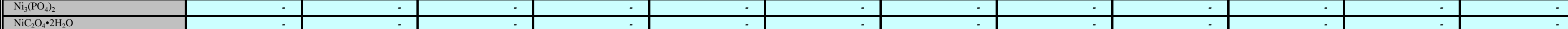 }} \\
\hline & & & & & & & & & & & & \\
\hline $\mathrm{Pb}(\mathrm{OH})_{2}$ & 0.00516 & 0.00516 & 0.00516 & 0.00516 & 0.00516 & 0.00516 & 0.00516 & 0.00552 & 0.00552 & 0.00552 & 0.00481 & 0.00552 \\
\hline \multirow{2}{*}{\multicolumn{13}{|c|}{\begin{tabular}{|l|}
$\mathrm{Pb}_{3}\left(\mathrm{PO}_{4}\right)_{2}$ \\
$\mathrm{PbCO}_{3}$ \\
\end{tabular}}} \\
\hline & & & & & & & & & & & & \\
\hline $\mathrm{Pu}(\mathrm{OH})_{4}$ & 0.00018 & 0.00018 & 0.00018 & 0.00018 & 0.00018 & 0.00018 & 0.00018 & 0.00019 & 0.00019 & 0.00019 & $\begin{array}{ll}0.00017 \\
\end{array}$ & 0.00019 \\
\hline \multirow{2}{*}{\multicolumn{13}{|c|}{\begin{tabular}{|l}
$\mathrm{SiO}_{2}$ \\
$\mathrm{Sr}_{2}\left(\mathrm{PO}_{4}\right)_{2}$
\end{tabular}}} \\
\hline & & & & & & & & & & & & \\
\hline $\mathrm{SrCO}_{3}$ & 0.00075 & 0.00075 & 0.00075 & 0.00075 & 0.00075 & 0.00075 & 0.00075 & 0.00080 & 0.00080 & 0.00080 & 0.00070 & 0.00080 \\
\hline \multicolumn{12}{|l|}{$\mid \overline{\mathrm{TCO}_{2}}$} & \\
\hline $\mathrm{ZrO}_{2}$ & 0.00036 & 0.00036 & 0.00036 & 0.00036 & 0.00036 & 0.00036 & 0.00036 & 0.00038 & 0.00038 & 0.00038 & 0.00033 & 0.00038 \\
\hline \begin{tabular}{|l|l|}
$\mathrm{KNaO}_{3}$ \\
$\mathrm{Na}_{2} \mathrm{C}_{2} \mathrm{O}_{4}$
\end{tabular} & 0.31053 & 0.31053 & 0.31053 & 0.31053 & 0.31053 & 0.31053 & 0.31053 & 0.33184 & 0.33184 & 0.33184 & 0.28921 & 0.33184 \\
\hline $\mathrm{Na}_{2} \mathrm{CO}_{3} \cdot \mathrm{H}_{2} \mathrm{O}$ & & & & & & & & & & & & \\
\hline $\mathrm{Na}_{2} \mathrm{SO}_{4}$ & & & & & & & & & & & & \\
\hline $\mathrm{Na}_{2} \mathrm{SO}_{4} \cdot 1 \mathrm{H}_{2} \mathrm{O}$ & - & - & - & - & - & - & 5 & & - & & - & \\
\hline $\mathrm{Na}_{3} \mathrm{FSO}_{4}$ & - & & & & - & & & & & & & \\
\hline $\mid \mathrm{Na}_{a_{2} \mathrm{NO}_{3} \mathrm{SO}_{4} \mathrm{H}_{2} \mathrm{O}}$ & & & & & & & & & & & & \\
\hline$\| \mathrm{Na}_{3} \mathrm{PO}_{4} 8 \mathrm{HH}_{2} \mathrm{O}$ & & & & & & & & & & & & \\
\hline $\mathrm{Na}_{4} \mathrm{P}_{2} \mathrm{O}_{7} \cdot 10 \mathrm{H}_{2} \mathrm{O}$ & - & - & & & - & & & & & & & \\
\hline $\mathrm{Na}_{6}\left(\mathrm{SO}_{4}\right)_{2} \mathrm{CO}_{3}$ & 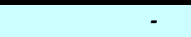 & & & & & & & & & & & \\
\hline & & & & & & & & & & & & \\
\hline $\mid \mathrm{NaF}\left(\mathrm{PO}_{4}\right)_{2} \cdot 19 \mathrm{H}_{2} \mathrm{O}$ & 1.54267 & 1.54267 & 1.54267 & $\begin{array}{ll}1.54267 \\
\end{array}$ & 1.54267 & 1.54267 & $\begin{array}{lll}1.54267 \\
\end{array}$ & 1.64856 & $\begin{array}{ll}.64856 \\
\end{array}$ & 1.64856 & 1.43678 & 1.64856 \\
\hline $\mathrm{NaHCO}_{3}$ & & & & & & & & & & & & \\
\hline $\mathrm{NaNO}_{2}$ & & & & & & & & & & & & \\
\hline $\mid \mathrm{NaNO}_{3}$ & 26.81520 & 26.81520 & 26.81520 & 26.81520 & 26.81520 & 26.81520 & 26.81520 & 28.65579 & 28.65579 & 28.65579 & 24.97461 & 28.65579 \\
\hline$\| \mathrm{Na}_{3} \mathrm{PO}_{4}-0.25 \mathrm{NaOH} \cdot 12 \mathrm{H}_{2} \mathrm{O}$ & 13.81566 & 13.81566 & $\begin{array}{l}13.81566 \\
\end{array}$ & $\begin{array}{lll}13.81566 \\
\end{array}$ & 13.81566 & 13.81566 & 133.81566 & 14.76396 & 14.76396 & 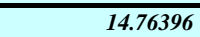 & $\overline{122.86736}$ & 14.76396 \\
\hline Total Volume \% UDS & 47.00 & 47.00 & 47.00 & $\begin{array}{ll}47.00 \\
0.17\end{array}$ & 47.00 & $\begin{array}{ll}47.00 \\
097\end{array}$ & $\begin{array}{ll}47.00 \\
0.07\end{array}$ & 50.23 & 50.23 & 50.23 & 43.78 & 50.23 \\
\hline Solids Concentration (g/1 & 981.7 & 981.7 & 981.7 & 981.7 & 981.7 & 981.7 & 981.7 & $1,049.0$ & 1,049.0 & $1,049.0$ & 914.3 & $\begin{array}{l}1,049.0 \\
\end{array}$ \\
\hline
\end{tabular}




\begin{tabular}{|c|c|c|c|c|c|c|c|c|c|c|c|c|}
\hline $\begin{array}{l}\text { Physical Property Data } \\
\text { Physical Property }\end{array}$ & $\overline{\overline{U-107}}$ & $\overline{\overline{U-107}}$ & $\overline{U U-107}$ & $\overline{U-107}$ & $\overline{\overline{U-107}}$ & $\overline{\mid c U-107}$ & $\begin{array}{ll}U-107 \\
\end{array}$ & 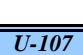 & $\overline{U U-107}$ & 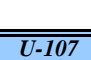 & $\overline{U U-107}$ & $\overline{U U-107}$ \\
\hline \multicolumn{13}{|l|}{ Source of Data } \\
\hline \multicolumn{13}{|l|}{$\mid$\begin{tabular}{|l} 
Temperature $\left({ }^{\circ} \mathrm{C}\right)$ \\
Zeta Potential(mV)
\end{tabular}} \\
\hline \begin{tabular}{|l} 
Zeta Potential $(\mathrm{mV})$ \\
Bulk Density $(\mathrm{g} / \mathrm{mL})$
\end{tabular} & 1.76 & 1.76 & 1.76 & 1.76 & 1.76 & 176 & 176 & 178 & 178 & 178 & 174 & 178 \\
\hline \multirow{2}{*}{\multicolumn{13}{|c|}{\begin{tabular}{|l} 
vol\% Settle Solids \\
Density of Centrifuged Solids (g/mL)
\end{tabular}}} \\
\hline & & & & & & & & & & & & \\
\hline \multicolumn{13}{|l|}{ vol\% Centrifuged Solids } \\
\hline w\% Centrifuged Solids & & & & & & & & & & & & \\
\hline \begin{tabular}{|l} 
Supernatant Density $(g / m L)$ \\
Density of Settled Solids $(g / m L)$
\end{tabular} & 1.47 & 1.47 & 1.47 & 1.47 & 1.47 & 1.47 & 1.47 & 1.47 & 1.47 & 1.47 & 1.47 & 1.47 \\
\hline \multicolumn{13}{|l|}{ wi\% Settled Supernatant } \\
\hline \multicolumn{13}{|l|}{ w1\% dissolved solids in supermatant } \\
\hline \multicolumn{13}{|l|}{ |w1\% total solids in Centrifuged Sludge } \\
\hline \multicolumn{13}{|l|}{ \#w\% Total Solids } \\
\hline |w1\% UDS & 55.78 & 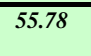 & $\begin{array}{c}55.78 \\
\end{array}$ & 55.78 & 55.78 & 55.78 & 55.78 & 58.93 & 58.93 & $\begin{array}{c}58.93 \\
\end{array}$ & 52.54 & 58.93 \\
\hline Density of Solids (g/cc) & 2.09 & 2.09 & 2.09 & 2.09 & 2.09 & 2.09 & 2.09 & 2.09 & 2.09 & 2.09 & 2.09 & 2.09 \\
\hline
\end{tabular}




\begin{tabular}{|c|c|c|c|c|c|c|c|c|c|c|c|c|}
\hline $\begin{array}{l}\text { Shear Strength Data } \\
\text { Shear Strength Schedule }\end{array}$ & & & & & & & & & & & & \\
\hline 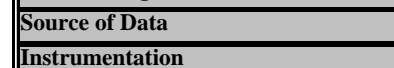 & 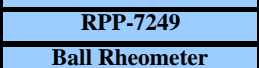 & \begin{tabular}{|c|} 
RPP-7249 \\
Bal Rheometer
\end{tabular} & \begin{tabular}{|c|} 
RPP-7249 \\
Bal Rheometer
\end{tabular} & $\begin{array}{c}\text { RPP.-249 } \\
\text { Ball Rheometer }\end{array}$ & \begin{tabular}{|c|} 
RPP. 2449 \\
Bal Rheometer
\end{tabular} & \begin{tabular}{|c|} 
RPPP-7249 \\
Bal Rheometer
\end{tabular} & \begin{tabular}{|c|} 
RaPP-7249 \\
Bal Rheometer
\end{tabular} & \begin{tabular}{|c} 
RPPP-7249 \\
Ball Rheometer \\
\end{tabular} & \begin{tabular}{|l} 
RPP-7249 \\
Bal Rheometer
\end{tabular} & 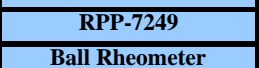 & \begin{tabular}{|l} 
RPP.-7249 \\
Ball Rheometer
\end{tabular} & $\begin{array}{c}\text { RalP-7249 } \\
\text { Ball Rheometer } \\
\end{array}$ \\
\hline $\begin{array}{l}\text { Temperature }\left({ }^{\circ} \mathrm{C}\right) \\
\end{array}$ & & & & & 25 & 25 & 25 & 25 & 25 & 25 & 25 & \\
\hline Descripition & \begin{tabular}{|c|} 
Risior 9 \\
Depth rom bottom of \\
tank is 137 inches
\end{tabular} & \begin{tabular}{|c|} 
Riser 9 \\
Depth from bottom of \\
tankin is 128 inches
\end{tabular} & 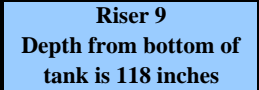 & 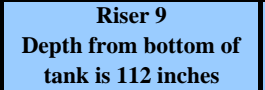 & \begin{tabular}{|c|}
$\begin{array}{c}\text { Riser } 9 \\
\text { Dept from bottom of } \\
\text { tank is } 108 \text { inches }\end{array}$ \\
\end{tabular} & \begin{tabular}{|l|}
$\begin{array}{c}\text { Riser } 7 \\
\text { Dept from botto of } \\
\text { tank is } 31 \text { in inhes }\end{array}$ \\
\end{tabular} & 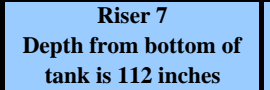 & \begin{tabular}{|c|}
$\begin{array}{c}\text { Riser } 7 \\
\text { Depth rom bottom of } \\
\text { takn is } 102 \text { inches }\end{array}$ \\
\end{tabular} & 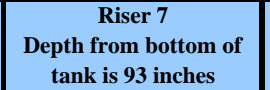 & \begin{tabular}{|c|} 
Riser 7 \\
Depth from bottom of \\
tankin is 8 2 inches
\end{tabular} & \begin{tabular}{|c|}
$\begin{array}{c}\text { Risere } 7 \\
\text { Depth from bottom of } \\
\text { tankin is } 7 \text { gnches }\end{array}$ \\
\end{tabular} & $\begin{array}{c}\text { Riser } 7 \\
\text { Depth from bottom of } \\
\text { tankin is } 7 \text { inches }\end{array}$ \\
\hline 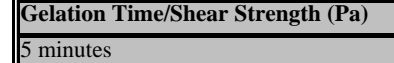 & & & & & & & & & & & & \\
\hline 10 minutes & & & & & & & & & & & & \\
\hline 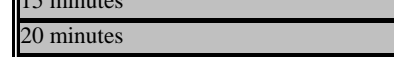 & & & & & & & & & & & & \\
\hline 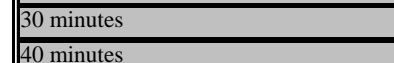 & & & & & & & & & & & & \\
\hline & & & & & & & & & & & & \\
\hline II lour & & & & & & & & & & & & \\
\hline 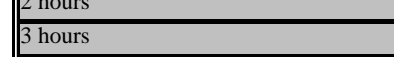 & & & & & & & & & & & & \\
\hline 5 hours & & & & & & & & & & & & \\
\hline & & & & & & & & & & & & \\
\hline 32 hours & & & & & & & & & & & & \\
\hline hiours & & & & & & & & & & & & \\
\hline & & & & & & & & & & & & \\
\hline & & & & & & & & & & & & \\
\hline
\end{tabular}


Model Chemical Data Fro

\begin{tabular}{|c|c|c|c|c|c|c|c|c|c|c|c|c|}
\hline \begin{tabular}{|l|l|} 
AnalytelSample \\
\end{tabular} & $\begin{array}{ll}\mathrm{U}-107 \\
\end{array}$ & $\begin{array}{ll}\mathrm{U}-107 \\
\end{array}$ & $\begin{array}{ll}\mathrm{U}-107 \\
\end{array}$ & $\begin{array}{ll}\mathrm{U}-107 \\
\end{array}$ & $\begin{array}{l}\mathrm{U}-107 \\
\end{array}$ & $\begin{array}{ll}\mathrm{U}-107 \\
\end{array}$ & $\begin{array}{ll}\mathrm{U}-107 \\
\end{array}$ & $\begin{array}{ll}\mathrm{U}-107 \\
\end{array}$ & $\begin{array}{ll}\mathrm{U}-107 \\
\end{array}$ & $\mathrm{U}-107$ & $\begin{array}{ll}\mathrm{U}-107 \\
\end{array}$ & $\begin{array}{ll}\mathrm{U}-107 \\
\end{array}$ \\
\hline Description & $\begin{array}{l}\text { in-situ } \\
\text { Salte } \\
\end{array}$ & $\begin{array}{l}\text { in-situ } \\
\text { salts } \\
\end{array}$ & $\begin{array}{l}\text { in-situ } \\
\text { Salty } \\
\end{array}$ & $\begin{array}{l}\text { in-situ } \\
\text { Salty } \\
\end{array}$ & $\begin{array}{l}\text { in-situ } \\
\end{array}$ & $\begin{array}{l}\text { in-situ } \\
\text { Salty } \\
\end{array}$ & $\begin{array}{l}\text { in-situ } \\
\text { Salty } \\
\end{array}$ & $\begin{array}{ll}\text { in-situ } \\
\text { Slly } \\
\end{array}$ & $\begin{array}{l}\text { in-situ } \\
\text { Salty } \\
\end{array}$ & $\begin{array}{l}\text { in-situ } \\
\text { Salvy } \\
\end{array}$ & $\begin{array}{l}\text { in-situ } \\
\text { Salty } \\
\end{array}$ & 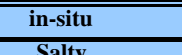 \\
\hline \begin{tabular}{|l} 
Model Basis \\
Surcro of fatat
\end{tabular} & $\begin{array}{ll}\text { Salty } \\
\text { PpP.7249 }\end{array}$ & 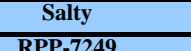 & 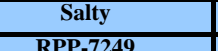 & $\frac{\text { Salty }}{- \text { RPP-7249 }}$ & $\frac{\text { Salty }}{\text { Sap-7390 }}$ & $\frac{\text { Salty }}{\text { RPP.7249 }}$ & $\begin{array}{c}\text { Salty } \\
\text { PP.-7249 }\end{array}$ & $\begin{array}{l}\text { Salty } \\
\text { Rpp.7209 }\end{array}$ & 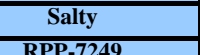 & 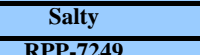 & $\frac{\text { Saly }}{\text { Sply-729 }}$ & $\begin{array}{l}\text { Salty } \\
\text { Spp-7209 }\end{array}$ \\
\hline $\mid \begin{array}{l}\text { Sourre of Data } \\
\text { Sold Phases (v01\% of bulk) }\end{array}$ & & & & & & & & & & & & RPP-7249 \\
\hline \multicolumn{13}{|l|}{ (NaAlO $\left.2_{2}\right)^{2} \cdot 2.5 \mathrm{H}_{2} \mathrm{O}$} \\
\hline \multicolumn{13}{|l|}{$\mathrm{Ag}_{2} \mathrm{CO}_{3}$} \\
\hline $\mathrm{Bi}_{2} \mathrm{O}_{3}$ & 0.00106 & 0.00156 & 0.00156 & $\begin{array}{ll}0.00174 \\
\end{array}$ & 0.00174 & 0.00125 & 0.00236 & 0.00137 & 0.00174 & 0.00217 & 0.00186 & 0.00186 \\
\hline \multirow{2}{*}{\multicolumn{13}{|c|}{\begin{tabular}{|l|l|l} 
Boehmite \\
\end{tabular}}} \\
\hline & & & & & & & & & & & & \\
\hline \multicolumn{13}{|l|}{$\mathrm{Ca}(\mathrm{OH})_{2}$} \\
\hline $\mathrm{Ca}_{5} \mathrm{OH}_{\left(\mathrm{PO}_{4}\right)_{3}}$ & 0.04076 & 0.05979 & 0.05979 & $\begin{array}{l}0.06693 \\
\end{array}$ & 0.06693 & 0.047900 & 0.09071 & 0.05266 & $\begin{array}{l}0.06693 \\
\end{array}$ & 0.08358 & 0.07168 & 0.07168 \\
\hline \multicolumn{13}{|l|}{$\mathrm{CaC}_{2} \mathrm{O}_{4} \cdot \mathrm{H}_{2} \mathrm{O}$} \\
\hline \multicolumn{13}{|l|}{$\mathrm{CaCO}_{3}$} \\
\hline $\mid \mathrm{CaF}_{2}$ & & & & & & & & & & & & \\
\hline $\mathrm{CrOOH}$ & 0.06813 & 0.09993 & 0.09993 & 0.11186 & 0.11186 & 0.08005 & 0.15161 & 0.08800 & 0.11186 & 0.13968 & 0.11981 & 0.11981 \\
\hline FeOOH & 0.08449 & $\overline{0.12392}$ & 0.12392 & 0.13871 & 0.13871 & 0.09928 & 0.18801 & $\overline{0.10914}$ & 0.13871 & 0.17322 & 0.14857 & 0.14857 \\
\hline 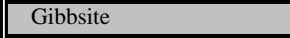 & 2.33950 & 3.43159 & 3.43159 & 3.84113 & 3.84113 & 2.79903 & 5.20625 & 3.02206 & 3.84113 & 4.79671 & 4.11415 & 4.11415 \\
\hline \multicolumn{13}{|l|}{\begin{tabular}{|l|l|} 
HgO \\
KASSOO.
\end{tabular}} \\
\hline \multirow{2}{*}{\multicolumn{13}{|c|}{$\frac{\mathrm{La}(\mathrm{OH})_{3}}{\mathrm{La}}$}} \\
\hline & & & & & & & & & & & & \\
\hline $\mathrm{LaPO}_{4} \cdot 2 \mathrm{H}_{2} \mathrm{O}$ & 0.00090 & 0.00132 & 0.00132 & 0.00147 & 0.00147 & 0.00105 & 0.00200 & 0.00116 & 0.00147 & 0.00184 & 0.00158 & 0.00158 \\
\hline $\mathrm{Mn}(\mathrm{OH})_{2}$ & 0.03663 & 0.05373 & 0.05373 & 0.06014 & 0.06014 & $0.04304 \mathrm{~J}$ & 0.08152 & 0.04732 & 0.06014 & 0.07510 & 0.06442 & 0.06442 \\
\hline \multirow{2}{*}{\multicolumn{13}{|c|}{\begin{tabular}{|l|}
$\mathrm{Mn}_{3}\left(\mathrm{PO}_{4}\right)_{2}$ \\
$\mathrm{M}_{3} \mathrm{C}$
\end{tabular}}} \\
\hline & & & & & & & & & & & & \\
\hline \multicolumn{13}{|l|}{\begin{tabular}{|l|l}
$\mathrm{Na}_{2} \mathrm{U}_{2} \mathrm{O}_{7}$ \\
\end{tabular}} \\
\hline \multicolumn{13}{|l|}{$\mathrm{NaAlCO}_{3}\left(\mathrm{OH}_{2}\right.$} \\
\hline $\mid \mathrm{NaAlSiO}_{4}$ & 0.06895 & 0.10113 & 0.10113 & 0.11320 & 0.11320 & 0.08102 & 0.15343 & 0.08906 & 0.111320 & 0.14136 & 0.12125 & 0.12125 \\
\hline $\mathrm{Ni}(\mathrm{OH})_{2}$ & 0.00206 & 0.00303 & 0.00303 & 0.00339 & 0.00339 & 0.00243 & 0.00459 & 0.00267 & 0.00339 & 0.00423 & 0.00363 & 0.00363 \\
\hline \multicolumn{13}{|l|}{$\begin{array}{ll}\mathrm{Ni}_{3}\left(\mathrm{PO}_{4}\right)_{2} \\
\end{array}$} \\
\hline $\mathrm{NiC}_{2} \mathrm{O}_{4} \cdot 2 \mathrm{H}_{2} \mathrm{O}$ & & & & & & & & & & & & \\
\hline${ }^{\mathrm{Pb}(\mathrm{OH})_{2}}$ & 0.00304 & 0.00445 & 0.00455 & 0.00499 & 0.00499 & 0.00357 & 0.00676 & 0.00392 & 0.00499 & 0.00623 & 0.00534 & 0.00534 \\
\hline \multirow{2}{*}{\multicolumn{13}{|c|}{$\mid$\begin{tabular}{|l|l|}
$\mathrm{Pb}_{3}\left(\mathrm{PO}_{4}\right)_{2}$ \\
$\mathrm{PCCO}$
\end{tabular}}} \\
\hline & & & & & & & & & & & & \\
\hline 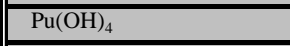 & 0.00011 & 0.00016 & 0.00016 & 0.00018 & 0.00018 & 0.00013 & 0.00024 & 0.00014 & 0.00018 & 0.00022 & 0.00019 & 0.00019 \\
\hline \multirow{2}{*}{\multicolumn{13}{|c|}{\begin{tabular}{|l|}
$\mathrm{SiO}_{2}$ \\
$\mathrm{Sr}_{2}\left(\mathrm{PO}_{4}\right)$
\end{tabular}}} \\
\hline & & & & & & & & & & & & \\
\hline $\mathrm{SrCO}_{3}$ & 0.00044 & 0.00064 & 0.00064 & 0.00072 & 0.00072 & 0.00052 & 0.00098 & 0.00057 & 0.00072 & 0.00090 & 0.00077 & 0.00077 \\
\hline $\mid \mathrm{TCO}_{2}$ & & & & & & & & & & & & \\
\hline $\mathrm{ZrO}_{2}$ & 0.00021 & 0.00031 & 0.00031 & $\begin{array}{ll}0.00034 \\
\end{array}$ & $\begin{array}{ll}0.00034 \\
\end{array}$ & 0.00025 & 0.00047 & 0.00027 & $\begin{array}{ll}0.00034 \\
\end{array}$ & $\begin{array}{ll}0.00043 \\
\end{array}$ & 0.00037 & 0.00037 \\
\hline $\mathrm{KNO}_{3}$ & & & & & & & & & & & & \\
\hline $\mathrm{Na}_{2} \mathrm{C}_{2} \mathrm{O}_{4}$ & 0.18264 & 0.26790 & 0.26790 & 0.29987 & 0.29987 & $0.21461]$ & 0.40644 & 0.23593 & $\begin{array}{ll}0.29987 \\
\end{array}$ & 0.37447 & 0.32118 & 0.32118 \\
\hline $\mathrm{Na}_{2} \mathrm{CO}_{3} \mathrm{H}_{2} \mathrm{O}$ & & & & & & & & & & & & \\
\hline $\mathrm{Na}_{2} \mathrm{SO}_{4}$ & & & & & & & & & & & & \\
\hline $\mid \mathrm{Na}_{2} \mathrm{SO}_{4} \cdot 10 \mathrm{H}_{2} \mathrm{O}$ & & & & & & & & & & & & \\
\hline $\mathrm{Na}_{3} \mathrm{FSO}_{4}$ & & & & & & & & & & & & \\
\hline $\mid \mathrm{Na}_{3} \mathrm{NO}_{3} \mathrm{SO}_{4} \cdot \mathrm{H}_{2} \mathrm{O}$ & & & & & & & & & & & & \\
\hline $\mathrm{Na}_{3} \mathrm{PO}_{4} \cdot 8 \mathrm{H}_{2} \mathrm{O}$ & & & & & & & & & & & & \\
\hline$\| \mathrm{Na}_{\mathrm{N}_{2} \mathrm{P}_{2} \mathrm{O} ;} ; 10 \mathrm{H}_{2} \mathrm{O}$ & & & & & & & & & & & & \\
\hline $\mathrm{Na}_{6}\left(\mathrm{SO}_{4}\right)_{2} \mathrm{CO}_{3}$ & & & & & & & & & & & & \\
\hline NaF & & & & & & & & & & & & \\
\hline $\mid \mathrm{NaF}\left(\mathrm{PO}_{4}\right)_{2} \cdot 19 \mathrm{H}_{2} \mathrm{O}$ & 0.90734 & 1.33089 & 1.33089 & $\begin{array}{l}1.48973 \\
\end{array}$ & $\begin{array}{l}1.48973 \\
\end{array}$ & 1.06617 & 2.01917 & 1.17206 & $\begin{array}{l}1.48973 \\
\end{array}$ & 1.86034 & $\begin{array}{l}.59561 \\
\end{array}$ & 1.59561 \\
\hline$\sqrt{\mathrm{NaHCO}_{3}}$ & & & & & & & & & & & & \\
\hline $\mathrm{NaNO}_{2}$ & & & & & & & & & & & & \\
\hline $\mid \mathrm{NaNO}_{3}$ & 15.77169 & 23.13403 & 23.1.1403 & 25.89491 & 25.89491 & $\begin{array}{ll}18.53256 \\
\end{array}$ & 35.09784 & 20.37315 & 25.89491 & 3233696 & 2773549 & 27.73549 \\
\hline $\mid \mathrm{Na}_{3} \mathrm{PO}_{4} \cdot 0.25 \mathrm{NaOH} \cdot 12 \mathrm{H}_{2} \mathrm{O}$ & 8.12585 & 111.91905 & 11.91905 & 13.34151 & $\overline{13.34151}$ & 9.54830 & 18.08301 & 10.49660 & 13.34151 & 16.66056 & $\overline{14.28981}$ & 14.28981 \\
\hline Total Volume \% UDs & 27.64 & 40.55 & 40.55 & 45.39 & 45.39 & 32.48 & 61.52 & 35.71 & 45.39 & 56.68 & 48.61 & 8.61 \\
\hline Solids Concentration (g) & 577.4 & 846.9 & \begin{tabular}{|c|c|}
846.9 \\
\end{tabular} & 948.0 & 948.0 & 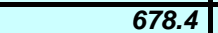 & $\overline{1,284.9}$ & 745.8 & $\overline{948.0}$ & $\overline{1,183.8}$ & $\overline{1,015.3}$ & $\overline{1,015.3}$ \\
\hline
\end{tabular}




\begin{tabular}{|c|c|c|c|c|c|c|c|c|c|c|c|c|}
\hline \multicolumn{13}{|l|}{ Physical Property Data } \\
\hline \begin{tabular}{|l|l|l|} 
Physical Property \\
\end{tabular} & $\overline{U U-107}$ & $\begin{array}{cl}U-107 \\
\end{array}$ & $\begin{array}{c}U-107 \\
\end{array}$ & 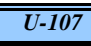 & $\overline{U U-107}$ & 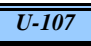 & 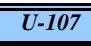 & 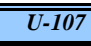 & $\begin{array}{cl}U-107 \\
\end{array}$ & $\begin{array}{cl}-107 \\
\end{array}$ & 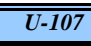 & $\overline{U U-107}$ \\
\hline \multicolumn{13}{|l|}{ Source of Data } \\
\hline \multicolumn{13}{|l|}{$\frac{\text { Temperature }\left({ }^{\circ} \mathrm{C}\right)}{Z \text { Zeta Potential }(\mathrm{mV})}$} \\
\hline $\begin{array}{l}\text { Zeta Potential }(\mathrm{mV}) \\
\text { Bulk Density }(\mathrm{g} / \mathrm{mL})\end{array}$ & 1.64 & 1.77 & 1.72 & 1.75 & 1.75 & 1.67 & 1.85 & 1.69 & 1.75 & 1.82 & 1.77 & 177 \\
\hline \multirow{2}{*}{\multicolumn{13}{|c|}{$\mid$\begin{tabular}{|l}
$\mid$ vol\% Settled Solids \\
Density of Centrifuged Solids (g/mL)
\end{tabular}}} \\
\hline & & & & & & & & & & & & \\
\hline \multicolumn{13}{|l|}{ vol\% Centrifuged Solids } \\
\hline \multicolumn{13}{|l|}{ ww Centrifuged Solids } \\
\hline $\begin{array}{l}\text { Supernatant Density }(\mathrm{g} / \mathrm{mL}) \\
\text { Density of Settled Solids }(\mathrm{g} / \mathrm{mL})\end{array}$ & 1.47 & 1.47 & 1.47 & 1.47 & 1.47 & 1.47 & 1.47 & 1.47 & 1.47 & 1.47 & 1.47 & 1.47 \\
\hline \multicolumn{13}{|l|}{ w1\% Settled Supermatant } \\
\hline \multicolumn{13}{|l|}{ w1\% dissolved solids in supematant } \\
\hline \multicolumn{13}{|l|}{ |w1\% total solids in Centrifuged Sludge } \\
\hline \multicolumn{13}{|l|}{ w1\% Total Solids } \\
\hline ww\% UDS & 35.21 & $\begin{array}{ll}49.24 \\
\end{array}$ & 49.24 & 54.17 & 54.17 & 40.63 & 69.45 & 44.13 & 54.17 & $\begin{array}{c}65.04 \\
\end{array}$ & 57.36 & 57.36 \\
\hline Density of Solids (g/c) & 2.09 & 2.09 & 2.09 & 2.09 & 2.09 & 2.09 & 2.09 & 2.09 & 2.09 & 2.09 & 2.09 & 2.09 \\
\hline
\end{tabular}




\begin{tabular}{|c|c|c|c|c|c|c|c|c|c|c|c|c|}
\hline $\begin{array}{l}\text { Shear Strength Data } \\
\text { Shear Strength Schedule }\end{array}$ & & & & & & & & & & & & \\
\hline 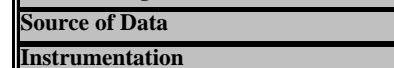 & 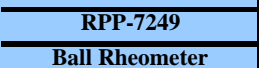 & 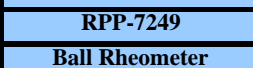 & \begin{tabular}{|c|} 
RPP-7249 \\
Bal Rheometer
\end{tabular} & 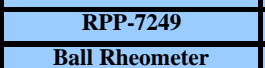 & 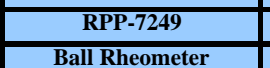 & 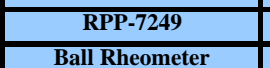 & \begin{tabular}{|c|} 
RaPP-7249 \\
Bal Rheometer
\end{tabular} & 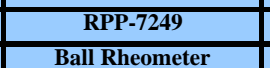 & 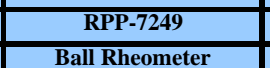 & 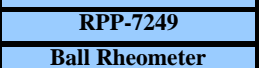 & \begin{tabular}{|l} 
RPP-7249 \\
Bal Rheometer
\end{tabular} & $\begin{array}{c}\text { RalP-7249 } \\
\text { Ball Rheometer } \\
\end{array}$ \\
\hline $\begin{array}{l}\text { Temperature }\left({ }^{\circ} \mathrm{C}\right) \\
\end{array}$ & & & 25 & & 25 & 25 & 25 & 25 & 25 & & & \\
\hline & 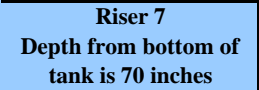 & \begin{tabular}{|c|} 
Riser 7 \\
Depth from bottom of \\
tank kis 58 inches
\end{tabular} & 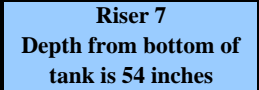 & 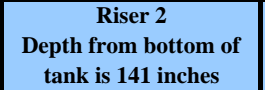 & \begin{tabular}{|c|}
$\begin{array}{c}\text { Riser } 2 \\
\text { Depth routtom of } \\
\text { tank is } 137 \text { inches }\end{array}$ \\
\end{tabular} & \begin{tabular}{|c|}
$\begin{array}{c}\text { Riser } 2 \\
\text { Depth } \\
\text { tron bottom of } \\
\text { tank in } 7 \text { inches }\end{array}$ \\
\end{tabular} & \begin{tabular}{|c|}
$\begin{array}{c}\text { Riser 2 } \\
\text { Depth } \\
\text { trom bottom of } \\
\text { tank is } 6 \text { 2 inches }\end{array}$ \\
\end{tabular} & \begin{tabular}{|c|}
$\begin{array}{c}\text { Risere } 2 \\
\text { Depth rom bottom of } \\
\text { tankin is } 5 \text { sinches }\end{array}$ \\
\end{tabular} & \begin{tabular}{|c|}
$\begin{array}{c}\text { Risere } 2 \\
\text { Depth roum bottom of } \\
\text { tankin is } 5 \text { 5inhes }\end{array}$ \\
\end{tabular} & 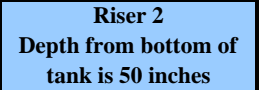 & \begin{tabular}{|c|}
$\begin{array}{c}\text { Risere 2 } \\
\text { Depth from bottom of } \\
\text { tankin is } 4 \text { inches }\end{array}$ \\
\end{tabular} & $\begin{array}{c}\text { Riser } 2 \\
\text { Depth from bottom of } \\
\text { tankin is } 1 \text { 2 inches }\end{array}$ \\
\hline 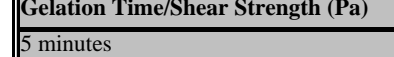 & & & & & & & & & & & & \\
\hline \multicolumn{13}{|l|}{ tomimutes } \\
\hline \multirow{2}{*}{\multicolumn{13}{|c|}{ 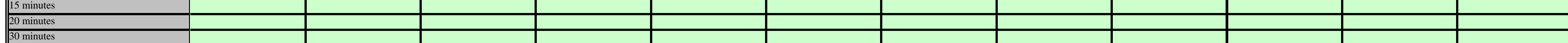 }} \\
\hline \multirow{2}{*}{\multicolumn{13}{|c|}{ 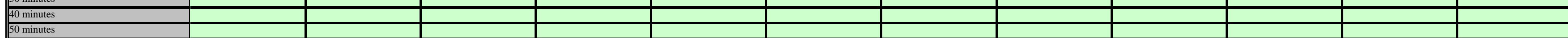 }} \\
\hline & & & & & & & & & & & & \\
\hline \multirow{2}{*}{\multicolumn{13}{|c|}{ 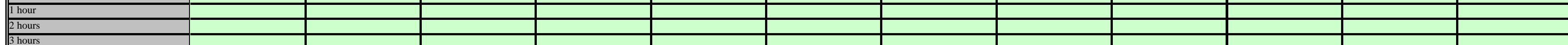 }} \\
\hline & & & & & & & & & & & & \\
\hline \multicolumn{13}{|l|}{$\sqrt{150}$} \\
\hline \multicolumn{13}{|l|}{ hinums } \\
\hline \multicolumn{13}{|l|}{ 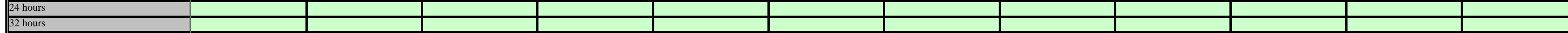 } \\
\hline $\begin{array}{ll}48 \text { hours } \\
5 \text {-hburs }\end{array}$ & & & & & & & & & & & & \\
\hline & & & & & & & & & & & & \\
\hline & & & & & & & & & & & & \\
\hline
\end{tabular}


PNNL-16857

WTP-RPT-154, Rev. 0

\section{Distribution}

No. of

Copies

No. of

No. of

Copies

Copies

OFFSITE

ONSITE

$1 \quad$ Savannah River National Laboratory

Richard Edwards

Savannah River National Laboratory

Westinghouse SA

Aiken, SC 29808-0001

1 Yasuo Onishi Consulting, LLC

Y. Onishi

144 Spengler St.

Richland, WA 99354

$7 \quad$ Pacific Northwest National Laboratory

A. P. Poloski P7-25

J. M. Tingey P7-25

B. E. Wells K7-15

L. A. Mahoney K7-15

G. L. Smith K6-25

D. E. Kurath P7-28

Project Office P7-28

4 Bechtel National Incorporated

D. Alford (2)

H4-02

M. N. Hall H4-02

S. L. Thomson H4-02

4 CH2M HILL Hanford Group, Inc.

M. E. Johnson

H6-19

M. A. Knight

R2-58

J. E. Meacham

R2-58

M. G. Thien

H6-03

1 Office of River Protection

J. J. Davis

H6-60

Distr. 1 